MANFRED ALBRING

EBERHARD WILLE (Hrsg.)

\title{
SZENARIEN IM \\ GESUNDHEITSWESEN
}




\author{
MANFRED ALBRING \\ EBERHARD WILLE (Hrsg.)
}

\title{
SZENARIEN IM GESUNDHEITSWESEN
}

Der Sammelband enthält die erweiterten Referate eines interdisziplinären Workshops über Szenarien im Gesundheitswesen. Im Mittelpunkt stehen die Effekte, die von der europäischen Integration auf das deutsche Gesundheitswesen und hier insbesondere auf die gesetzliche Krankenversicherung (GKV) sowie auf die einzelnen Gesundheitsmärkte ausgehen. Weitere thematische Schwerpunkte bilden integrierte Versorgungsstrukturen sowie ausgewählte Finanzierungsalternativen im Rahmen der GKV. Der Teilnehmerkreis setzte sich aus Vertretern der Ärzteschaft, der Krankenkassen und -versicherungen, der pharmazeutischen Industrie, der Wissenschaft, der Ministerialbürokratie und der Politik zusammen.

Manfred Albring wurde 1943 in Bochum geboren. Studium der Humanmedizin an der Universität Marburg. Bei der Schering AG ist er Leiter Medizin und Gesundheitswesen des Geschäftsbereichs Deutschland. Er ist unter anderem Mitglied der Arbeitsgemeinschaft für dermatologische Forschung, der Deutschen Pharmakologischen Gesellschaft und des Kuratoriums der Deutschen Herzstiftung.

Eberhard Wille wurde 1942 in Berlin geboren. Nach dem Diplom 1966 an der Universität Bonn, der Promotion 1969 und der Habilitation 1973 an der Universität Mainz ist er seit 1975 Professor für Volkswirtschaftslehre und Finanzwissenschaft an der Universität Mannheim. Er ist unter anderem Mitglied des Wissenschaftlichen Beirats beim Bundesministerium für Wirtschaft und des Sachverständigenrates für die Konzertierte Aktion im Gesundheitswesen. 
Szenarien im Gesundheitswesen

Manfred Albring and Eberhard Wille - 978-3-631-75604-1

Downloaded from PubFactory at 01/11/2019 03:10:54AM

via free access 


\section{ALLOKATION IM MARKTWIRTSCHAFTLICHEN SYSTEM}

Herausgegeben von

Heinz König, Hans-Heinrich Nachtkamp,

Ulrich Schlieper, Eberhard Wille

\section{Band 42}

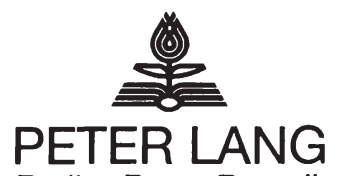

Frankfurt am Main - Berlin - Bern - Bruxelles - New York - Wien 


\section{MANFRED ALBRING \\ EBERHARD WILLE \\ (Hrsg.)}

\section{SZENARIEN \\ IM GESUNDHEITSWESEN}

Bad Orber Gespräche über kontroverse Themen im Gesundheitswesen 5.-7.11.1998

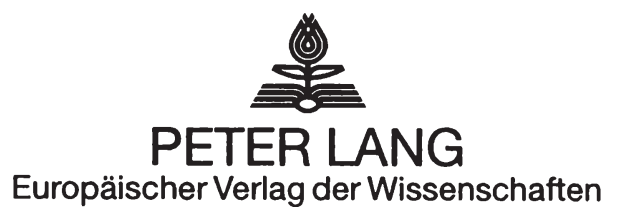


Die Deutsche Bibliothek - CIP-Einheitsaufnahme

Szenarien im Gesundheitswesen/Bad Orber Gespräche über kontroverse Themen im Gesundheitswesen, 5.-7.11.1998. Manfred Albring/Eberhard Wille (Hrsg.). - Frankfurt am Main ; Berlin ; Bern ; Bruxelles ; New York ; Wien : Lang, 1999

(Allokation im marktwirtschaftlichen System ; Bd. 42) Open Access: The online version of this publication is published on www.peterlang.com and www.econstor.eu under the international Creative Commons License CC-BY 4.0. Learn more on how you can use and share this work: http://creativecommons.org/ licenses/by/4.0.

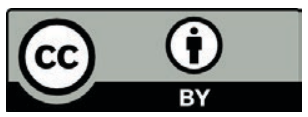

This book is available Open Access thanks to the kind support of ZBW - Leibniz-Informationszentrum Wirtschaft.

ISBN 3-631-34945-9

Gedruckt auf alterungsbeständigem, säurefreiem Papier.

ISSN 0939-7728

ISBN 3-631-34945-9

ISBN 978-3-631-75604-1 (eBook)

(C) Peter Lang GmbH

Europäischer Verlag der Wissenschaften

Frankfurt am Main 1999

Alle Rechte vorbehalten.

Das Werk einschließlich aller seiner Teile ist urheberrechtlich geschützt. Jede Verwertung außerhalb der engen Grenzen des

Urheberrechtsgesetzes ist ohne Zustimmung des Verlages unzulässig und strafbar. Das gilt insbesondere für Vervielfältigungen, Übersetzungen, Mikroverfilmungen und die Einspeicherung und Verarbeitung in elektronischen Systemen.

Printed in Germany 134567 


\section{Danksagung}

Die Planung, Vorbereitung und Durchführung der Bad Orber Gespräche ist mit einem erheblichen Arbeitsaufwand verbunden. Bis zur Fertigstellung des Buches haben alle Beteiligten außerordentlich viel Engagement und Zähigkeit bewiesen. Stellvertretend für die vielen Beteiligten sei hier noch einmal ausdrücklich Martina Kuchenbecker, Irmtraud Block, Susan Kalisch, Ingrid Czemper und Markus Schröder gedankt. 
Manfred Albring and Eberhard Wille - 978-3-631-75604-1

Downloaded from PubFactory at 01/11/2019 03:10:54AM

via free access 


\author{
Frank Münnich \\ Manfred Albring \\ Eberhard Wille \\ Günter Danner
}

Wolfgang Gitter

Helmut Klemm
Doris Pfeiffer

Manfred Zipperer

Hans-Dieter Koring

Hans Günter Verhees

Günter Neubauer

Wolfgang Schmeinck

Frank Diener

Axel Granitza

Alexander P. F. Ehlers

Helmut Laschet

Susanne Renzewitz
Vorwort 3

Begrüßung $\quad 8$

Vor alten und neuen Herausforderungen 13

im Gesundheitswesen

Die europäische Herausforderung für das deutsche Gesundheitswesen - ein Spannungsfeld zwischen nationalrechtlicher Struktur, Vergemeinschaftsdruck, Marktgeschehen und politischer Handlungsnotwendigkeit

\section{Themenkreis I}

Konsequenzen einer Liberalisierung der europäischen Märkte für die deutsche Gesundheitsversorgung

Ärztliche Bedarfsplanung

Ärztliche Bedarfsplanung

Konsequenzen einer Liberalisierung der europäischen Märkte für die deutsche Gesundheitsversorgung

Ärztliche Bedarfsplanung

Konsequenzen einer Liberalisierung der europäischen Märkte für die deutsche Gesundheitsversorgung

Stationäre Leistungen, Kuren und Rehabilitationen

Stationäre Leistungen, Kuren und Rehabilitationen

Stationäre Leistungen, Kuren und Rehabilitationen

Sonstige veranlaßte Leistungen

Sonstige veranlaßte Leistungen

Chancen und Risiken des europäischen

Arzneimittelmarktes - Die Sichtweise der Apotheker

Sonstige veranlaßte Leistungen

Sonstige veranlaßte Leistungen

\section{Themenkreis II}

Veränderte Versorgungsstrukturen

135

Öffnung von Krankenhäusern für die fachärztliche Versorgung

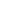

3 
Peter Schwoerer, Johann Öffnung von Krankenhäusern für die

Freund, Elisabeth Simoes, fachärztliche Versorgung

Friedrich Gerhard

Realistische Anforderungen an eine

Weiterentwicklung der Versorgungsstrukturen

Peter Sauermann

Pro und Contra von Hausarztmodellen

162

Wolfgang Brech

Pro und Contra von Hausarztmodellen

Christian Dierks

Pro und Contra von Hausarztmodellen

Axel Munte

Integrierte Versorgungsformen

Gerd W. Zimmermann

Integrierte Versorgungsformen 188

Karl-Heinz Schönbach

Qualitätsgemeinschaften und Praxisnetze

Ulrich Vorderwülbecke

Integrierte Versorgungsformen

Integration ,Vernetzter Praxen' in die GKV

Peter Dewein

Die Pharmaindustrie als Vertragspartner

224

Christoph Straub

Die Pharmaindustrie als Vertragspartner

Dieter Cassel

Die Pharmaindustrie als Vertragspartner

\section{Themenkreis III}

Ausgewählte Finanzierungsalternativen $\quad 234$

Formen der Budgetierung 237

Formen der Budgetierung 243

Formen der Budgetierung 254

Beitragsbemessungs- und Versiche- $\quad 259$

rungspflichtgrenzen

$\begin{array}{ll}\text { Beitragsbemessungs- und Versiche- } & 277\end{array}$

rungspflichtgrenzen

Resümee: Die Suche nach einem effizienten und adäquat finanzierten Gesundheitswesen in einem zusammenwachsenden Europa

Verzeichnis der

Teilnehmer 


\section{Vorwort}

Frank Münnich

Einerseits war es ein geschickter und andererseits ein glücklicher Griff, daß der Veranstalter den Termin für die 3. Bad Orber Gespräche über kontroverse Themen im Gesundheitswesen schon vor Jahresfrist auf den Anfang des November des Wahljahres 1998 gelegt hatte. Zum einen konnte man zwar nicht wissen, aber doch stark vermuten, daß zu diesem Zeitpunkt schon absehbar wäre, wie es in der neuen, 14. Legislaturperiode mit der Gesundheitspolitik weitergehen würde. Zum anderen, und das war der glückliche Zufall, hatte sich durch die Urteile in den Fällen Kohll und Decker gerade in Deutschland im Laufe der Monate ein erheblicher Diskussionsbedarf über das Verhältnis von EU-Recht zu nationalem Sozialrecht aufgestaut. Beide Themen dominieren denn auch diesen Ergebnisbericht.

Lange Zeit wiegten sich die gestandenen Gesundheitspolitiker in der Gewißheit, daß sie von der europäischen Szene nichts zu befürchten hätten. In allen Mitgliedsländern der EU galt mehr oder weniger ausgeprägt das Territorialitätsprinzip, das die Wirksamkeit hoheitlicher Maßnahmen auf das jeweilige Staatsgebiet beschränkt. So hat es denn auch gute und lang bewährte Tradition, daß die Leistungen der Träger der sozialen Sicherung auf das jeweilige Staatsgebiet beschränkt waren. Selbst für Geldleistungen in der Rentenversicherung wurde dieser Grundsatz erst nach 1945 langsam gelockert. Hinzu kam die Überzeugung, daß die europäischen Verträge eine explizite Vorschrift enthielten, daß die Sozialpolitik den einzelnen Mitgliedsstaaten vorbehalten sei. Diese Überzeugung wurde durch zwei Argumentationsketten noch bestärkt: Zum einen sah man in der expliziten Aufnahme des Subsidiaritätsprinzips in die europäischen Verträge eine Garantieerklärung des Rates der Ministerpräsidenten, von der ihnen prinzipiell offenstehenden Möglichkeit, auch die Sozialversicherungssysteme in die Bemühungen um Konvergenz einzubeziehen, keinen Gebrauch zu machen. Und zum anderen hat man wohl die permanent wiederholten gegenseitigen $\mathrm{Be}$ teuerungen, die Systeme seien historisch gewachsen, Ausdruck des nationalen kulturellen Erbes, strukturell und in ihrem Selbstverständnis viel zu unterschiedlich und was es an dergleichen metaphysischen Topoi noch geben mag, schließlich im Sinne der self-fulfilling prophecy selbst geglaubt. Fast alle Beiträge zum Thema Europa spielen auf diesen beiden Klavieren. 
Aus solcherlei Träumen wurde wohl mancher gerissen. Tatsache ist aber, daß der EuGH an der nationalen Prärogative in der Sozialpolitik nicht gerüttelt hat. Im Gegenteil, er hat sie explizite bestätigt. Er hat aber auch bestätigt, daß die im Europavertrag festgeschriebenen Freizügigkeiten, die ja erst den gemeinsamen Markt konstituieren, grundsätzlich immer gelten, wenn nicht die im Vertrag selbst vorgesehenen Ausnahmen zum Tragen kommen. Wie es Herr Schmeinck in seinem Beitrag formuliert: alle Vorschriften der Sozialgesetze fallen weiterhin in die (fast alleinige) Kompetenz der Nationalstaaten, der grenzüberschreitende Waren-, Dienstleistungs- und Erwerbspersonenverkehr fällt in die Kompetenz der EU. Es hätte ja schon seit 1965 auffallen müssen, daß Arzneimittel, und zwar auch solche, die an sozialversicherte Patienten abgegeben werden, von europäischem Recht betroffen sind. Und schon 1992 hatte der EuGH das Recht der Patienten betont, sich unter bestimmten Bedingungen Arzneimittel im EU-Raum auch außerhalb des eigenen Landes zu beschaffen.

Es geht also in beiden Urteilen nicht um die nationale Souveränität in der Sozialpolitik. Es geht auch nicht um Sachleistung oder Kostenerstattung. Ob eine soziale Krankenversicherung so oder so organisiert ist, stand nicht zur Diskussion und fiele auch nicht in die Kompetenz des EuGH. Es geht einzig und allein darum, ob in den verhandelten Fällen einer der Ausnahmetatbestände gegeben war, der eine Abweichung vom Prinzip der Freizügigkeit rechtfertigen würde. Und nur in dem Zusammenhang wird Kostenerstattung erwähnt. Die Regierung Luxemburgs und anderer ihr beigetretener Staaten hatten argumentiert, die finanzielle Stabilität der beiden Luxemburger Krankenkassen sei in Gefahr, wenn ihre Mitglieder ohne vorherige Genehmigung Leistungen im Ausland bezögen. Das, so der EuGH, könne ja wohl nicht sein, wenn die Kasse im Ausland nicht mehr bezahle als im Inland. Gerade die unbedingten Verfechter des Sachleistungsprinzips täten gut daran, sich dieser klaren Logik der Urteile nicht zu verschließen.

Die Diskussionen über die Folgerungen aus den Urteilen konzentrieren sich auf die Frage, ob die Urteile die bewährten „Steuerungsinstrumente“ unterliefen. Man muß dies wohl a priori vermuten, wenn sie hoheitlichen Charakter tragen und ihre Anwendung daher auf das Staatsgebiet beschränkt ist. In vielen Beiträgen wird dies im einzelnen mit mehr oder weniger Trauer im Tonfall belegt. Man sollte sich freilich keinen Illusionen über die Bedeutung dieser Tatsache hingeben: die Beeinträchtigung von Instrumenten zur Kostendämpfung ist kein Ausnahmetatbestand per se, so daß selbst dann, wenn eine solche Beeinträchtigung erwiesen ist, noch nachzuweisen wäre, daß die daraus resultierende Gefährdung der finanziellen Stabilität der sozialen Krankenversicherung "erheblich" ist 
und nicht auf anderem, mit dem EG-Recht konformen Weg beseitigt werden kann.

Die Bedeutung des europäischen Rechts für die deutsche Sozialpolitik beschränkt sich aber nicht auf die Vorschriften über die Freizügigkeiten des gemeinsamen Marktes, die freilich nicht Gegenstand der Beratungen in Bad Orb waren. Wie zwei kürzlich ergangene Urteile in Düsseldorf und Hamburg zeigen, könnte auch das europäische Kartellrecht den Gestaltungsspielraum des deutschen Sozialgesetzgebers empfindlich beschränken. Auch dies hätte man längst, spätestens seit dem Vorlagebeschluß des BSG zum BVG in Sachen Festbeträge, ernst nehmen müssen. Es steht zu vermuten, daß diese juristische Argumentation für die deutsche soziale Krankenversicherung eine ernste Gefahr heraufbeschwören könnte, weil deren Charakteristikum, das kollektivvertragliche Handeln, geradezu einen Musterfall für ein Kartell abgibt und dies ohne tiefgreifende Änderung inrer Struktur im nationalen Alleingang vielleicht gar nicht gelöst werden kann. Es ist nicht von ungefähr, daß sich die Koalition bislang noch nicht auf eine Vorgehensweise verständigen konnte.

Von größerem zeitgeschichtlichen Interesse könnte sich erweisen, daß während der Bad Orber Gespräche die politischen Verhandlungen weiter liefen und einige der Handelnden in Bad Orb anwesend waren. Was lag näher, als mit ihnen Dollpunkte der kommenden Reformen zu diskutieren. Zwei Bereiche stehen dabei im Vordergrund der Reformbemühungen der neuen Koalition: zum einen die neuen Versorgungsformen, vor allem die integrierte Versorgung auf der Basis des Hausarztmodells und einer verbesserten Verzahnung zwischen ambulanter und stationärer Versorgung, und zum anderen das Globalbudget als neues Instrument der finanziellen Steuerung. Demgegenüber tritt das Interesse an den bekannten und bereits auf den 2. Bad Orber Gesprächen artikulierten allgemeinen Finanzierungsproblemen zurück: die Koalition hält zunächst nichts davon, weitere Mittel in das System zu kanalisieren, den Leistungskatalog einzuschränken oder die Teilprivatisierung weiter voranzutreiben. Man wird sehen, wie sich diese Haltung im weiteren Verlauf des Gesetzgebungsverfahrens durchhalten läßt.

Sinn und Zweck einer integrierten Versorgung sind bekannt und im Prinzip wenig strittig. Auch das Für und Wider des Hausarztmodells ist bekannt. Sollte sich die Koalition - und dafür spricht vieles - zu einem sanften Phase-in durchringen und nicht mit der Keule ein rigides Primärarztmodell realisieren, wird es wohl kaum am Widerstand der Fachärzte scheitern. Weitaus problematischer ist die beabsichtigte Integration des stationären Sektors, die bislang hauptsächlich an der ständisch, und das 
heißt und hieß zu allen Zeiten: monetär motivierten Abschottung des ambulanten Sektors scheitert. Liest man als nicht unmittelbar Betroffener die verschiedenen Beiträge zu diesem Thema, so kommen einem die vertretenen Konzepte durchaus ähnlich vor: Was ist wohl der Unterschied zwischen einem „integrierten Dienstleistungszentrum“, einer voll ausgebauten „Praxisklinik“ und einem erweiterten Belegarztsystem, wenn man einmal davon absieht, daß das erstere von einem Krankenhausträger und das letztere von einer Gruppe niedergelassener Ärzte getragen wird? Es wird Zeit, daß bei allen diesen Diskussionen mehr der Wunsch und der Vorteil des Patienten im Vordergrund steht.

Alle bisher vorgelegten Konzeptionen zur Schaffung einer integrierten Versorgung leiden freilich an einem inneren Widerspruch. Einerseits sollen die integrierten Versorgungsformen Wirtschaftlichkeitsreserven erschließen. Das geht, wie alle wissen, nicht ohne einen massiven Kapazitätsabbau im gesamten System. Der wiederum bedeutet den Abbau von Arbeitsplätzen. Das soll aber nicht sein. Wer jedoch glaubt, sich daran vorbeimogeln zu können, leidet an der Illusion, er könne unter Mißachtung aller physikalischen Gesetze fliegen. Der Volksmund hat dafür ein treffendes Sprichwort: Wasch mir den Pelz, aber mach mich nicht naß.

Andererseits werden die Leistungserbringer und Patienten aber nur dann in integrierten Versorgungsformen mitmachen, wenn sie auch etwas davon haben. Der Vorteil für den Patienten könnte in einer verbesserten Versorgung bestehen, worin immer diese sich auch manifestiert. Doch ob die Vorteile der integrierten Versorgung ausschließlich in immateriellen Gütern bestehen können, darf man in Zeiten immer enger werdender Gürtel für füglich bezweifeln. Wie schon Bert Brecht zu berichten wußte: „Nur wer im Reichtum lebt, lebt angenehm“ und kann sich an den Segnungen des Kapitalismus erfreuen. Konkret gesprochen: es geht schon nichts ohne eine zusätzliche „Anschubfinanzierung" durch die Kassen.

Mit anderen Worten: Integrierte Versorgungsformen kommen auf freiwilliger Basis nur dann zustande, wenn sie dank hinreichender Deregulierung so große Produktivitätsfortschritte erbringen, daß aus innen sowohl monetäre Anreize für Versicherte und Leistungserbringer ausgeschüttet werden können als auch darüber hinaus für die Kassen etwas verbleibt. Das geht aber nur, wenn (bei gleichbleibendem Output an Versorgung und Gesundheit) die tradierten Versorgungsformen stärker schrumpfen als die integrierten expandieren. Man darf davon ausgehen, daß alle Insider und alle Repräsentanten der Stände dies wissen. Der bisherige zähe verborgene Widerstand kommt nicht von ungefähr. 
Große Hoffnungen setzen Koalition und viele Kassen auf das geplante Globalbudget als wichtigstem Instrument der Beitragssatzstabilität. Manche Befürworter verweisen in diesem Zusammenhang gern auf die Wirtschaft: viele Unternehmen steuern ihren Wirtschaftsablauf mit Hilfe von Budgets. Allein, die Analogie hinkt in einem entscheidenden Punkt. In Unternehmen gibt es eine Geschäftsführung oder einen Vorstand, dem letztendlich die Entscheidung obliegt. Unsere gemeinsame Selbstverwaltung ist durch eine Vielzahl im Prinzip autonomer Entscheidungsträger gekennzeichnet. Ob mit oder ohne Globalbudget, die Teilbudgets müssen aufeinander abgestimmt werden, und es muß Sanktionsmechanismen geben, um die Überschreitung von globalen Budgets zu ahnden - es sei denn, jede Regierung startet ihre neue Legislaturperiode mit einer Generalamnestie. In einem nationalen Gesundheitsdienst wie dem englischen sind solche Probleme natürlich wesentlich einfacher zu lösen. Es ist a priori auch wenig überzeugend, daß Globalbudgets sektoralen Budgets überlegen sind, weil das Globalbudget in irgendeiner Weise auf die einzelnen Ausgabenbereiche aufgeteilt werden muß und das Ergebnis einer solchen Aufteilung den sektoralen Budgets doch erstaunlich ähnlich sieht. Beim Schreiben dieser Zeilen war die Diskussion noch nicht weiter, als sie es schon in Bad Orb war.

Gerade für den gesundheitspolitischen Enthusiasten, zu denen trotz mancher gegenteiligen Beteuerung wohl nicht nur die meisten Teilnehmer von Bad Orb zu rechnen sind, sondern auch die meisten Leser, bieten die folgenden Beiträge eine breite und kompetente Grundlage für die weiteren Auseinandersetzungen. Dem Chronisten bieten sie ein breites Material, mit dem er später einmal wird darlegen können, daß wir alles schon viel früher gewußt haben. 
Manfred Albring

$\mathrm{Zu}$ unserem 3. Bad Orber Gespräch über kontroverse Themen im Gesundheitswesen darf ich Sie im Namen der Schering Deutschland GmbH sehr herzlich begrüßen. Ich hoffe, daß Sie den Umständen entsprechend eine relativ angenehme Anreise hatten, mit Ihrer Unterkunft zufrieden sind und hier in Bad Orb wie bisher eine Arbeitsatmosphäre vorfinden, die eine aufgeschlossene, sachbezogene und zielorientierte Diskussion erleichtern wird. Mit diesem 3. Workshop setzen wir den Versuch fort, gesundheitspolitische Entwicklungen auf hohem Niveau zu analysieren und zu kommentieren.

Vor zwei Jahren haben wir uns mit dem schillernden und interpretationsbedürttigen Begriff der Innovation im Bereich der Arzneimitteltherapie auseinandergesetzt und mit der Publikation über diese Veranstaltung eine erstaunlich hohe Resonanz bei unseren Partnern im Gesundheitswesen gefunden. Im vergangenen Jahr beschäftigten wir uns mit dem Thema der Reformoptionen im Gesundheitswesen, insbesondere im Bereich der gesetzlichen Krankenversicherung. Dieses Themenspektrum umfaßte alternative Finanzierungsmöglichkeiten, Strukturverträge und Modellvorhaben sowie eine Bewertung des Leistungskataloges der GKV im Licht des Solidaritätsprinzips. Die kritische Beschäftigung mit den Grundlagen unseres Gesundheitssystems hat deutlich gemacht, wie sehr sich auch ein Gesundheitssystem veränderten gesellschaftlichen Bedingungen anpassen kann und muß. Wie sehr aber auch veränderte Paradigmen wie beispielsweise Sozialisierung oder Privatisierung von Risiken oder die veränderte Bedeutung von Solidarität die zukünftige Entwicklung beeinflussen werden. Der Machtwechsel in Bonn ist noch so frisch, die meisten Minister sind ja erst wenige Tage im Amt, daß sich über die operationale Ausgestaltung der mittel- bis längerfristigen Gesundheitspolitik, also der zukünftigen Strukturen im Gesundheitswesen, nur spekulieren läßt. Insofern werden die diesjährigen Bad Orber Gespräche auch deutlich spekulative Elemente aufweisen. Ich sehe das aber als eine attraktive Möglichkeit, ohne Vorurteil Optionen zu diskutieren, die sonst vielleicht nur wenig Chancen hätten, in Betracht gezogen zu werden. Gleichwohl gibt es schon einige Entscheidungen, die längerfristig bedeutend sein könnten:

- Entgegen den Organisationsvorstellungen führender Sozialpolitiker der SPD, die die wichtige Abteilung Krankenversicherung wieder in das Bundesarbeitsministerium eingliedern wollten, wird das Ministe- 
rium für Gesundheit gestärkt. Es bildet nicht nur die Zuständigkeit für die Krankenversicherung, sondern erhält auch noch zusätzlich die Kompetenz für den Bereich der Pflegeversicherung. Es mag sein, daß dieses auch mit den handelnden Personen und insbesondere mit der Person des Bundesarbeitsministers Walter Riester zu tun hat, der seine Aufgabe eher auf die Arbeitsmarktpolitik konzentrieren will. Dieser Umstand ist für die Gesundheitspolitik gleichwohl eine Chance, weil die Zuständigkeit und Verantwortung für die Finanzierung des Gesundheitswesens und für seine Strukturen und seine Qualitätselemente nun doch in einer Hand bleiben. Keineswegs bedeutet dies, daß Gesundheitspolitik losgelöst von der Sozialpolitik betrieben wird. Im Gegenteil, mit den vom Bundeskabinett inzwischen beschlossenen Korrekturen an den Neuordnungsgesetzen werden, wenn auch vorsichtig, neue erste Akzente gesetzt. Aber anerkannt wird nun, daß die Gesundheitspolitik ein ganz eigenständiger Bereich der Sozialpolitik ist, der sich fundamental von dem Transfersystem in der Renten- und Arbeitslosenversicherung sowie der Sozialhilfe darin unterscheidet, daß hinter dem Gesundheitswesen ein hochkomplexes Dienstleistungssystem in allen nur denkbaren Organisationsformen steht.

- Das zweite Faktum ist wohl die unausweichliche Europäisierung der nationalen Gesundheitssysteme durch die Rechtsprechung des Europäischen Gerichtshofes. Diesem Problemkreis ist der erste Block der diesjährigen Bad Orber Gespräche gewidmet.

Nachdem nun Bayern seine Landtagswahlen überstanden und die CSU glanzvoll die von ihr gesteckten Hürden genommen hat, wäre es durchaus sinnvoll, das Thema „Europa“ ohne speziell bayerische Sichtweisen diskutieren zu können. Dies können durchaus spannende Fragen sein, beispielsweise:

- Wie steht es denn mit der Freizügigkeit in Europa, wenn seinen Bürgern faktisch die freie Wahl des Wohnortes dadurch genommen wird, daß der Geltungsbereich des Krankenversicherungsschutzes auf das Inland beschränkt wird?

- Wie muß das Sachleistungssystem der deutschen GKV unter europäischen Bedingungen modifiziert werden, und ist ein Nebeneinander von Sachleistung und Kostenerstattung überhaupt denkbar?

- Wie tragfähig sind die in der EU gefundenen Harmonisierungen der Berufsanforderungen beispielsweise für Ärzte, und müssen sich Leistungsanbieter nicht auf eine völlig neue Dimension des Wettbe- 
werbs einstellen, etwa durch internationale Einkaufsmodelle der Krankenkassen?

In einem zweiten Themenblock werden wir morgen fast den gesamten Tag über neue und noch zu verändernde Versorgungsstrukturen diskutieren. Unmißverständlich haben die beiden Koalitionsparteien vor der Wahl die Richtung künftiger Strukturreformen angegeben. Das Ziel ist eine deutlich stärkere Verzahnung der immer noch stark voneinander abgeschotteten Leistungssektoren im Gesundheitswesen. Das Mittel der Wahl, dies zu realisieren, könnten Einkaufsmodelle zu Gunsten der Krankenkassen und zu Lasten der Organisationen der Leistungserbringer sein.

Hier einige Beispiele, die morgen sicherlich kontrovers debattiert werden:

- Sollen die Krankenhäuser für die ambulante fachärztliche Versorgung geöffnet werden? Werden Krankenhäuser die ambulante Leistung dann als Institution anbieten, oder ist eine Verzahnung so möglich, daß das Krankenhaus bestimmte fachärztliche Leistungen outsourct und einkauft? Vor allem, mit welchem Vergütungssystem wird dies möglich sein?

- Ist nicht schließlich die gesamte kassenärztliche Bedarfsplanung obsolet, wenn sich ambulante und stationäre Leistung immer mehr vermischen und wenn Ärzte in beiden Bereichen tätig sind?

- Sind integrierte ambulante Versorgungsnetze unter Beteiligung von Haus- und Fachärzten ein probates Mittel gegen das unkoordinierte Neben- und Hintereinander medizinischer Leistungen?

- Wie ist es dann mit der freien Arztwahl bestellt, wenn Versorgungsnetze zum Versorgungskartell werden? Ein Kartell beispielsweise gegen Apotheken und Pflegedienste entsteht, vielleicht aber auch gegen den Patienten?

- Sind Hausarztmodelle oder Gate-keeper-Institutionen eine Chance, die immer komplizierter werdende Medizin überschaubar und finanzierbar zu halten?

- Schließlich, wie macht man ein solches Modell, das ja bislang nach meiner Kenntnis von den Versicherten als wenig attraktive Alternative angesehen wird, interessant? Vielleicht mit einem Bonus oder Wahltarifen, die freilich nicht zu den sozialpolitischen Vorstellungen der neuen Bundesregierung passen? 
Bislang sind nur sehr vage Vorstellungen über die zukünftigen finanziellen Rahmenbedingungen bekannt, wie sie die neue Bundesregierung gestalten will. Sicher ist dabei nur eines: der Wille, den Patienten nicht weiter mit Zuzahlungen zu belasten, und der ausgeprägte Wunsch, die paritätische Finanzierung der Beiträge nicht auszuhöhlen.

So erhoffen wir uns dann auch am Samstag vormittag einigen Aufschluß darüber, wie sich beispielsweise ein Globalbudget mit dem Grundsatz der Beitragssatzstabilität vereinbaren läßt. Nach welchen Kriterien das Globalbudget fortentwickelt werden kann und inwieweit medizinische und demographische Parameter dabei eine Rolle spielen werden.

Ich erwarte mit Spannung Antworten auf die Frage, ob sich überhaupt die Ausgabensteuerung mit sektoralen Budgets verträgt mit dem Ziel, die Grenzen der Sektoren zu überwinden.

Letztendlich wollen wir erneut der Frage nachgehen, ob sich bei Besserverdienenden zusätzliche Geldquellen erschließen lassen - die eifrigen Grabungsversuche zu diesem Thema während unserer letzten Tagung hier in Bad Orb im November 1997 hatten in diesem Bereich ja nur einige wenige Krümel erbracht.

Mit Sicherheit werden wir es in den nächsten beiden Tagen nicht schaffen, die Quadratur des Kreises zu erreichen, um die Zukunft auch nur annähernd genau beschreiben zu können. Dennoch - die versammelte Kompetenz der anwesenden Experten aus dem Gesundheitswesen spricht dafür, daß auch dieser Schering-Workshop eine qualitativ hochwertige Auseinandersetzung mit dem anstehenden Thema leisten wird. Ich bin sicher, daß unsere Arbeit in dieser Veranstaltung wiederum Grundlage für eine lesenswerte gesundheitspolitische Publikation sein wird, die bei politischen Entscheidungsträgern und auch bei gesundheitspolitischen Querdenkern Denkanstöße geben kann.

Ich freue mich ganz besonders, daß es uns durch Vermittlung von Herrn Dr. Klusen von der TK gelungen ist, Herrn Dr. Günter Danner, den stellvertretenden Direktor der Europa-Vertretung der deutschen Sozialversicherung in Brüssel, für den Einführungsvortrag der diesjährigen Veranstaltung zu gewinnen. Ich bin sicher, daß Herr Dr. Danner mit bewährter Eloquenz und charismatischen Statements ein flammendes Plädoyer für die Schaffung von EURO-Kompatibilität der nationalen Sozialsysteme halten wird. Wir dürfen alle gespannt sein, wie er sich den Weg zu diesem hohen Ziel vorstellt. 
Wie in den Vorjahren wünsche ich unserer Veranstaltung trotz unterschiedlichster gesundheits-, sozial- oder allgemeinpolitischer Interessenlagen nicht nur einen kontroversen, sondern vor allen Dingen einen fairen und zielorientierten Verlauf. Damit gebe ich das Wort an Herrn Professor Wille. 


\section{Vor alten und neuen Herausforderungen im Gesundheits- wesen}

Eberhard Wille

\section{Zum Themenspektrum der diesjährigen Bad Orber Gespräche}

Zunächst möchte auch ich Sie hier sehr herzlich begrüßen und Ihnen dafür danken, daß Sie uns mit Ihrer Teilnahme immerhin zwei Tage Ihrer sicherlich knapp bemessenen Zeit opfern. Einige von Ihnen nehmen nun schon zum dritten Mal - quasi als Stammgäste - an den Bad Orber Gesprächen über kontroverse Themen im Gesundheitswesen teil, und Herr Dr. Albring und ich erblicken hierin zumindest teilweise eine gewisse Bestätigung unserer bisherigen inhaltlichen Konzeption. Andererseits bildet dieses Vertrauen eine Verpflichtung und eine Herausforderung, mit der wir uns auch in den nächsten zwei Tagen konfrontiert sehen.

Bei der Konzeption des vorliegenden Programms befand sich der Wahlkampf noch in vollem Gange, und es ließ sich schwer prognostizieren, welches Thema nach der Bundestagswahl gesundheitspolitisch im Mittelpunkt stehen würde. In der Furcht, mit einer sehr spezifischen Thematik die gesundheitspolitische Aktualität zu verfehlen, wählten wir für die diesjährige Veranstaltung ein sehr weites inhaltliches Spektrum aus, das sich von den Effekten der europäischen Integration auf die deutsche Gesundheitsversorgung über veränderte Versorgungsstrukturen bis zu ausgewählten Finanzierungsalternativen in der gesetzlichen Krankenversicherung (GKV) spannt. Diese etwas risikoscheue Entscheidung für ein gesundheitsökonomisches Konvolut bedeutete zwangsläufig den Verzicht auf eine stromlinienförmige Thematik, wie sie z. B. bei unseren ersten Bad Orber Gesprächen über „Innovationen in der Arzneimitteltherapie" vorlag. Die Suche nach einem belletristischen Motto für unser weites Themenspektrum führte zu Goethes Faust (Vorspiel auf dem Theater): „Wer vieles bringt, wird manchem etwas bringen, und jeder geht zufrieden aus dem Haus“.

Die Urteile des Europäischen Gerichtshofes (EuGH) in den Rechtsstreitigkeiten Kohll und Decker schienen in Deutschland vielerorts zu überraschen und lösten kontroverse Diskussionen über die Effekte und Herausforderungen aus, die künftig von der europäischen Integration auf die nationalen Sozialversicherungs- und Gesundheitssysteme ausgehen. In Deutschland herrschte offensichtlich die Vorstellung vor, der EG-Vertrag (EGV) klammere die sozialen Sicherungssysteme im Sinne des Subsidiaritätsprinzips von den sonstigen Vorschriften zur Harmonisierung aus 
und erspare den nationalen Systemen somit die Notwendigkeit, die europäische Integration in diesen Bereichen mit Anpassungsmaßnahmen zu begleiten. Im Unterschied zu einigen einschlägigen juristischen Untersuchungen behandelte auch die gesundheitsökonomische Literatur die möglichen Wirkungen, die von der europäischen Integration auf die nationalen Sicherungssysteme ausgehen, arg stiefmütterlich. Dabei kommen bereits bei grober Betrachtung in ökonomischer Hinsicht Zweifel auf, ob in einem zusammenwachsenden Europa mit liberalisiertem Wettbewerb das Gesundheitswesen als autonome nationale Oase eine isolierte Sonderstellung einnehmen kann. Da dieses bisher bestenfalls in Ansätzen diskutierte Thema in den nächsten Jahren an Aktualität zunehmen dürfte, freut es uns ganz besonders, daß wir mit Herrn Danner, Ph. D., und Herrn Dr. Zipperer zwei sachkundige Referenten gewinnen konnten, die hier zum einen aus europäischer Perspektive und zum anderen aus der Sicht der (alten) Bundesregierung in der Tendenz unterschiedliche Positionen vertreten.

Im Unterschied zu den Konsequenzen einer Liberalisierung der europäischen Märkte für die nationalen Gesundheitssysteme standen die Themenkreise „Veränderte Versorgungsstrukturen“ und „Ausgewählte Finanzierungsalternativen" bereits auf dem Programm der letztjährigen Veranstaltung über „Reformoptionen im Gesundheitswesen“. In einer einzigen Veranstaltung kann aber eine erschöpfende Behandlung derart komplexer Problembereiche, die sich zudem empirisch in Fluß befinden, nicht gelingen, und die Suche nach einer effizienten und adäquat finanzierten Gesundheitsversorgung hat zwischenzeitlich eher noch an Aktualität gewonnen. Zudem liegen über Strukturverträge sowie Modellvorhaben inzwischen erste - wenn auch noch rudimentäre - Erfahrungen vor, und die Frage nach der künftigen Finanzierung der GKV erhielt durch den Regierungswechsel zumindest in Teilbereichen, wie z. B. dem Globalbudget und der Beitragsbemessungs- und Versicherungspflichtgrenze, eine andere Akzentuierung. Insofern steht die jetzige Bundesregierung im Rahmen der Gesundheitsversorgung und der Finanzierung der GKV nach innen überwiegend vor alten und nach außen eher vor neuen Herausforderungen.

\section{Das deutsche Gesundheitssystem vor dem Hintergrund von euro- päischer Integration und Globalisierung}

Die Einführung des EURO, der ab dem 01.01.1999 als zugelassenes Buchgeld und ab dem 01.01.2002 als gesetzliches Zahlungsmittel fungiert, markiert unbeschadet von wirtschaftspolitischen Bedenken einen bedeutsamen Schritt in Richtung einer europäischen Integration. Während die nationalen Währungen dann noch ein halbes Jahr als Parallel- 
währungen gelten, bildet der EURO ab dem 01.07.2002 in den Teilnehmerländern der Europäischen Wirtschafts- und Währungsunion (EWU) das alleinige gesetzliche Zahlungsmittel. Die Wirkungen, die vom EURO bzw. der EWU auf das deutsche Gesundheitssystem ausgehen, überlagern sich weitgehend mit Effekten der allgemeinen Globalisierung. Zudem bestehen starke Interdependenzen zwischen den möglichen Folgen, die aus der EWU, der Globalisierung und den jüngsten Urteilen des EuGH in den Rechtsstreitigkeiten Kohll und Decker erwachsen können.

Im obigen Wirkungszusammenhang zeichnet der EURO in den wenigsten Fällen für die entsprechenden Entwicklungen ursächlich verantwortlich, vermag aber vorhandene Tendenzen, wie z. B. die Intensivierung des Standortwettbewerbs im Rahmen der Globalisierung, zu verschärfen. Insofern bietet es sich an, die grundlegenden Effekte des EURO im Zusammenhang mit der Globalisierung zu beleuchten. Sodann erscheint es auf der Grundlage der Normen des EG-Vertrages sinnvoll, bei möglichen Effekten der EWU zwischen den Gesundheitsmärkten, für die im Grundsatz die Vorschriften des freien Waren- und Dienstleistungsverkehrs gelten, und den Sozialsystemen, die im Sinne des Subsidiaritätsprinzips nicht dem Harmonisierungsgebot unterliegen, zu unterscheiden. Die Gesundheitsmärkte stellen nicht nur im nationalen Kontext, sondern auch auf internationaler Ebene eine ökonomisch umkämpfte Wachstumsbranche dar.

Die EWU verlagert die Kompetenzen in der Währungspolitik von der nationalen auf die europäische Ebene. Damit entfallen innerhalb der Teilnehmerländer u. a. Wechselkurse, d. h. Auf- und Abwertungen der Währungen, Devisenverkehrskontrollen und Währungsspekulationen. Die EWU vermag die Effizienz in ihrem Geltungsbereich vor allem durch

- eine Erhöhung der Markttransparenz,

- eine damit einhergehende Verschärfung des Preiswettbewerbs,

- eine Senkung der Transaktionskosten sowie

- eine Verminderung der Planungsrisiken durch den Fortfall der Wechselkurse

zu verbessern.

Wie bereits angedeutet, erzeugt die EWU kaum genuine bzw. unmittelbare Wirkungen auf die nationalen Sozialsysteme, kann aber die schon vorhandenen und sich abzeichnenden Effekte der Globalisierung intensivieren. Schon heute stehen Länder und Regionen um mobile Unternehmen und deren Beschäftigungspotential in einem Standortwettbe- 
werb, der sich ähnlich wie der Preiswettbewerb von international tätigen Anbietern in einem gemeinsamen Währungsraum beinahe zwangsläufig verschärft. Diese Entwicklung förderte bereits in der Vergangenheit weltweite Spezialisierungs- und Konzentrationsprozesse und rückte auch die Sozialabgaben als Belastungen des Faktors Arbeit mit ins wirtschaftspolitische Interesse. Der im Rahmen dieser Globalisierung zunehmende Wettbewerbsdruck kann die nationalen Sozialsysteme unter Umständen zu Anpassungsmaßnahmen zwingen. So liegen z. B. die Lohnzusatzkosten in stärker steuerfinanzierten Gesundheitssystemen niedriger als in überwiegend beitragsfinanzierten. In grenznahen Gebieten erwächst hieraus ein Wettbewerbsnachteil für die heimischen Arbeitskräfte und/oder für die inländische Produktion von arbeitsintensiven Gütern.

Neben öffentlichen Entscheidungsträgern und Unternehmen können im Gesundheitswesen auch Konsumenten, d. h. Versicherte und Patienten, auf die Globalisierungstendenzen reagieren, indem sie das international unterschiedliche Preis- und Qualitätsgefüge von Gesundheitsleistungen für sich nutzen. Schon seit geraumer Zeit suchen wohlhabende Bürger aus Ländern mit einem leistungsschwachen Gesundheitswesen auf eigene Rechnung hochqualifizierte Leistungsanbieter in anderen Ländern auf. Das zunehmende Interesse, auf das die medizinische Behandlung in den Medien stößt, sowie die Möglichkeiten der Telekommunikation lassen in absehbarer Zeit weltweit abrufbare indikationsspezifische Ranglisten der besten Kliniken und Ärzte erwarten. Zudem besitzen die Versicherten im Rahmen der Selbstmedikation und bei Gesundheitsleistungen, die unter die Selbstbeteiligung fallen, einen Anreiz, diese Güter und Dienste in Ländern mit einem niedrigeren als dem heimischen Preisniveau zu erwerben. Nationale Teilmärkte geraten als „HochpreisInseln" auf diese Weise unter internationalen Wettbewerbsdruck. Bestehende Preisdifferenzen treten bei einer einheitlichen Währung viel stärker ins Bewußtsein der Konsumenten, so daß der EURO z. B. die bisher übliche Preisdifferenzierung im pharmazeutischen Bereich erheblich erschwert. Da die möglichen Effekte von EURO und Globalisierung innerhalb der einzelnen medizinischen Behandlungsarten nach Art und Intensität spürbar differieren, behandeln die Beiträge im Rahmen des Themenkreises 1 gesondert die ärztliche Bedarfsplanung, stationäre Leistungen, Kuren und Rehabilitationen und sonstige veranlaßte Leistungen, wie z. B. Arzneimittel.

Die Globalisierungstendenzen, die von den modernen Medien ausgehen, sorgen für eine zügige weltweite Verbreitung neuester medizinischer Forschungsergebnisse und können auf diese Weise auch die nationalen Leistungskataloge beeinflussen. Mit der Kenntnis bestimmter 
Innovationen steigt die Erwartungshaltung der Bevölkerung an die medizinische Behandlung, und es fällt den nationalen Sozialsystemen bzw. deren Entscheidungsträgern dann schwerer, die betreffenden Leistungen aus der Erstattung auszuklammern. Dies empfinden die Versicherten und Patienten vor allem dann als eine unethische Rationierung, wenn Länder mit einer vergleichbaren volkswirtschaftlichen Leistungsfähigkeit ihren Bürgern diese Leistungen im Rahmen ihrer Sozialsysteme gewähren. Die Globalisierung und der EURO konfrontieren die beitragsfinanzierten nationalen Sozial- bzw. Gesundheitssysteme insofern mit einer doppelten Herausforderung, als sie diese von der Einnahmen- und der Ausgabenseite her unter Druck setzen: Der globale Standortwettbewerb verlangt im Sinne internationaler Konkurrenzfähigkeit nach stabilen oder gar sinkenden Beitragssätzen, während das globalisierte Informationsniveau der Bürger gleichzeitig einen Druck in Richtung eines steigenden Niveaus von Gesundheitsleistungen erzeugt.

\section{Veränderte Versorgungsstrukturen über innovative Suchprozesse}

Die Modellvorhaben und Strukturverträge nach $\S \S 63 \mathrm{ff}$. und 73 a SGB V bilden im wesentlichen die gesetzliche Grundlage, um über eine Integration von Managed-Care-Elementen veränderte Versorgungsstrukturen zu erproben. Managed-Care-Ansätze dienen in instrumentaler Hinsicht dazu, die Effizienz und Effektivität der Gesundheitsversorgung zu verbessern, d. h. das Effizienzpostulat umzusetzen. Im Hinblick auf Managed-Care- und Disease-Management-Konzepte verfügen vor allem die USA und die Schweiz über einen Erfahrungsvorsprung, den deutsche Ansätze zumindest partiell nutzen können. Die Integration von ManagedCare-Elementen in eine solidarische GKV setzt allerdings voraus, daß die Finanzierung weiterhin nach dem Solidarprinzip erfolgt, für alle Krankenkassen gegenüber jedem versicherungswilligen und -berechtigten Bürger Kontrahierungszwang und Diskriminierungsverbot besteht und der Risikostrukturausgleich erhalten bleibt. Dies bedeutet aber auch, daß sich die in den USA und der Schweiz gewonnenen Erfahrungen mit Managed-Care-Konzepten nicht eins zu eins bzw. unreflektiert auf das bundesdeutsche Gesundheitswesen übertragen lassen.

Im Rahmen von Modellvorhaben können die Krankenkassen und ihre Verbände Erprobungen

- zur Weiterentwicklung der Verfahrens-, Organisations-, Finanzierungs- und Vergütungsformen der Leistungserbringung (sog. Strukturmodelle) und 
- zu Leistungen zur Verhütung und Früherkennung von Krankheiten sowie zur Krankenhausbehandlung (sog. Leistungsmodelle)

zeitlich befristet, d. $h$. im Regelfall auf längstens acht Jahre, durchführen oder vereinbaren. Bei den Strukturmodellen müssen sich die Krankenkassen im Rahmen ihrer gesetzlichen Aufgabenstellung bewegen, während sich die Leistungsmodelle auf Maßnahmen und Behandlungen beziehen, die (noch) nicht zum Leistungskatalog der GKV gehören. Letztere dürfen allerdings keine Leistungen enthalten, die bereits von den Bundesausschüssen abgelehnt wurden. Im Rahmen der Strukturmodelle eröffnet $\S 63$ Abs. 3 den Modellpartnern die Möglichkeit, das gesamte Leistungserbringerrecht einschließlich der Regelungen des Krankenhausfinanzierungsgesetzes und des Pflegesatzrechtes zu suspendieren.

Krankenkassen unterschiedlicher Kassenarten können ebenfalls gemeinsam Modellvorhaben durchführen und die Kassenärztlichen Vereinigungen ihrerseits die Initiative ergreifen und mit den Krankenkassen und ihren Verbänden entsprechende Vereinbarungen treffen. Sofern die Modellvorhaben die ärztliche Behandlung im Rahmen der vertragsärztlichen Versorgung betreffen, bleiben die Kassenärztlichen Vereinigungen Vertragspartner der Krankenkassen. Dies bedeutet, daß die Krankenkassen auch bei den Modellvorhaben nicht die Möglichkeit besitzen, mit einzelnen Ärzten oder Ärztegruppen selektive Verträge zu schließen. $\S 64$ Abs. 2 SGB V beauftragt die Spitzenverbände der Krankenkassen und die Kassenärztliche Bundesvereinigung, in den Bundesmantelverträgen Grundsätze zur Durchführung von Modellvorhaben mit Vertragsärzten zu vereinbaren. Die Vereinbarungen sollen $u$. a. sicherstellen, $\mathrm{da} ß$ ein Modellvorhaben zustande kommt, wenn mindestens $50 \%$ der Vertragsärzte, die die Voraussetzungen für die Teilnahme erfüllen, die Durchführung des Modellvorhabens befürworten.

Die Krankenkassen können die durch ein Modellvorhaben nachweislich erzielten Einsparungen auch an die daran teilnehmenden Versicherten weiterleiten. Die Modellvorhaben bedürfen einer wissenschaftlichen $\mathrm{Be}-$ gleitung, einer Auswertung hinsichtlich ihrer Zielsetzung durch unabhängige Sachverständige und einer Publikation über die Ergebnisse dieser Evaluation.

Die Strukturverträge, die erst zum Schluß der parlamentarischen Beratungen im Bundestagsausschuß für Gesundheit in das 2. GKV-NOG eingingen, verfolgen ebenfalls das Ziel, Managed-Care-Elemente in das GKV-System zu integrieren. Die Strukturverträge verleihen den Kassenärztlichen Vereinigungen und den Landesverbänden der Krankenkassen sowie den Verbänden der Ersatzkassen die Kompetenz, neue Versor- 
gungs- und Vergütungsstrukturen zu vereinbaren. Letztere können die Verantwortung für die Gewährleistung von Qualität und Wirtschaftlichkeit der vertragsärztlichen Versorgung sowie der ärztlich verordneten und veranlaßten Leistungen insgesamt oder für definierte Teilbereiche auf einen vom Versicherten gewählten Hausarzt oder einen von inm gewählten Verbund haus- und fachärztlich tätiger Vertragsärzte (sog. „Vernetzte Praxen") übertragen. Dabei können die Vertragspartner bei Wahrung der Beitragssatzstabilität ein Budget vereinbaren, das neben den Aufwendungen für die ambulante Behandlung auch die veranlaßten Ausgaben für Arznei-, Verband- und Heilmittel sowie für weitere Leistungsbereiche umfaßt. Das Gesetz eröffnet damit für die beiden Konzeptionen „Hausarztmodell“ und „Vernetzte Praxen“ die Möglichkeit, Behandlungsabläufe sektorübergreifend zu steuern. Das schließt z. B. auch Komplexpauschalen für Behandlungsabläufe ein, die von der ambulanten Behandlung mit der Verordnung von Arzneimitteln über die stationäre Behandlung bis zur Rehabilitation reichen können.

Die Kassenärztliche Bundesvereinigung und die Spitzenverbände der Krankenkassen können Rahmenvereinbarungen zum Inhalt und zur Durchführung der Strukturverträge treffen, deren konkrete Ausgestaltung unter Berücksichtigung regionaler Bedürfnisse dann im wesentlichen der Landesebene überlassen bleibt. Der Gesetzgeber intendiert insofern eine gewisse Einheitlichkeit der Strukturverträge in inhaltlicher und prozeduraler Hinsicht. Dies kommt auch darin zum Ausdruck, daß die Kassenärztliche Bundesvereinigung und die Spitzenverbände der Krankenkassen in den Bestimmungen der Bundesmantelverträge die Voraussetzungen zur Durchführung der Strukturverträge schaffen. Die Mitwirkung von Versicherten und Vertragsärzten erfolgt im Rahmen der Strukturverträge auf freiwilliger Basis.

Wie bereits teilweise angedeutet, stehen im Rahmen veränderter Versorgungsstrukturen derzeit vor allem

- die Öffnung von Krankenhäusern für die fachärztliche Versorgung,

- das Pro und Contra von Hausarztmodellen,

- die Konzipierung integrierter Versorgungsformen und

- die Rolle der Pharmaindustrie als Partner von Erprobungsmodellen

im Mittelpunkt sowohl des Interesses als auch kontroverser Diskussionen. 
Die Strukturverträge betreffen zwar über die von ambulanten Ärzten veranlaßten Leistungen die Arzneimittelversorgung und die stationäre Behandlung, die Arzneimittelhersteller und die Krankenhäuser besitzen aber keine Möglichkeit, solchen Verträgen beizutreten. Die Beteiligung der Krankenhäuser an dreiseitigen Modellvorhaben beschränkt sich bisher auf einige wenige (Sonder-)Fälle. Die Krankenhäuser reklamieren vielmehr eine weitergehende Öffnung für die fachärztliche Versorgung, um auf diesem Gebiet in Konkurrenz zu den niedergelassenen Ärzten zu treten. Da die Arzneimittelhersteller auch bei Modellvorhaben nicht als Vertragspartner mitwirken können, schwindet unter diesen Bedingungen inr Interesse, sich bei Managed-Care-Konzepten zu engagieren. Dabei verfügen pharmazeutische Firmen gerade auf dem Feld des DiseaseManagements über einen hohen Kenntnisstand und teilweise auch über ein beachtliches Innovationspotential.

Im Hinblick auf die Vor- und Nachteile einer Öffnung von Krankenhäusern für die fachärztliche Versorgung und die potentielle Leistungsfähigkeit von Hausarztmodellen folgt der Veranstaltungsablauf auch bei der Auswahl der Referenten dem übergreifenden Motto „Kontroverse Themen im Gesundheitswesen“. Es ist aus unserer Sicht zu erwarten, daß Frau Renzewitz und Herr Dr. Sauermann stärker die jeweiligen Vorzüge herausstellen und Herr Dr. Krimmel und Herr Professor Brech tendenziell eine Gegenposition beziehen. Herrn Dr. Schwoerer und Herrn Dr. Dierks fällt dann die nicht ganz einfache Rolle des „ehrlichen Maklers" zu. Insgesamt gesehen geht es hier wie bei den anderen Themen aber nicht darum, dezisionistisch ein abschließendes Urteil zu fällen, sondern die Meinungsvielfalt zu verdeutlichen und das Argumentationsspektrum herauszuarbeiten.

\section{Finanzierungsfragen als Dauerproblem?}

Die Finanzierung der GKV stand im Zentrum unserer letztjährigen Veranstaltung über „Reformoptionen im Gesundheitswesen“. Die damals aufgezeigten Probleme harren aber noch immer einer konzeptionellen Lösung. Die GKV leidet derzeit global betrachtet weniger an einem exzessiven Ausgabengebaren als an einer Wachstumsschwäche der Einnahmenbasis. Für die unbefriedigende Zunahme der beitragspflichtigen Einnahmen zeichnen neben den „Verschiebebahnhöfen“ innerhalb der Sozialversicherung vor allem das schwache Wirtschaftswachstum, die hohe Arbeitslosigkeit und die gewandelten Arbeitsverhältnisse verantwortlich. Die Steigerungsrate der Grundlohnsumme liegt im Jahr 1998 bei ca. 1,6 \% und im Jahr 1999 voraussichtlich bei 1,7\%, was auf Dauer kaum ausreichen dürfte, um eine Gesundheitsversorgung zu finanzieren, die ausgehend vom heutigen Niveau dem absehbaren demographischen 
Wandel und dem künftigen medizinisch-technischen Fortschritt Rechnung trägt.

Das seit Mitte der siebziger Jahre moderate Ausgabenwachstum der GKV entspringt allerdings keinem sich selbst steuernden Prozeß, sondern geht maßgeblich auf zahlreiche diskretionäre Kostendämpfungsmaßnahmen bzw. Gesundheitsreformen zurück. Da auf der Ausgabenseite der GKV nach wie vor erhebliche Wirtschaftlichkeitsreserven bestehen, gilt zunächst die Devise „Ausschöpfung der Wirtschaftlichkeitsreserven vor Inanspruchnahme neuer Finanzierungsquellen“. Bei einem weiterhin schwachen Wachstum der Einnahmenbasis dürfte die Ausschöpfung des Rationalisierungspotentials allerdings alleine kaum zur Stabilisierung der Beitragssätze ausreichen. Die Ausschöpfung des Rationalisierungspotentials setzt zunächst strukturelle Reformen voraus, die zumeist erst mittelfristig zu Ressourcen-Einsparungen führen. Zudem schlagen sich Maßnahmen zur Verbesserung von Effizienz und Effektivität der Gesundheitsversorgung nicht nur in Ausgabeneinsparungen, sondern auch in Outcome-Erhöhungen nieder.

Sofern in der GKV Budgetdefizite drohen, existieren neben einer Ausschöpfung von Wirtschaftlichkeitsreserven folgende globale Finanzierungsmöglichkeiten:

- Beitragssatzerhöhungen,

- Ausweitung der Selbstbeteiligung,

- Eingrenzung des Leistungskatalogs,

- zusätzliche Mittelaufbringung durch Änderung der Beitragsgestaltung und/oder

- Verlagerung der Finanzierung auf andere öffentliche Ausgabenträger.

Eine Verlagerung von krankenversicherungsfremden Leistungen auf andere öffentliche Ausgabenträger scheitert wohl auf absehbare Zeit an den finanziellen Engpässen der entsprechenden Haushalte. Insofern verbleiben als globale Finanzierungsmöglichkeiten nur eine Einengung des Leistungskatalogs, eine Erhöhung der Selbstbeteiligung oder eine Änderung der Beitragsgestaltung. Die letzte Reformoption besitzt dabei den komparativen Vorzug, nicht die Patienten, sondern die Versicherten stärker zu belasten. 
Vor dem Hintergrund der geltenden Beitragsgestaltung können konkrete Reformoptionen grundsätzlich an der

- GKV-Versicherungspflicht bzw. dem Pflichtversichertenkreis,

- Beitragsbemessungsgrenze,

- Beitragsbemessungsgrundlage,

- beitragsfreien Mitversicherung und/oder

- aufkommensneutralen Änderung der Beitragssatzanteile

ansetzen. Mit Ausnahme der letzten aufkommensneutralen Finanzierungsoption zielen alle anderen derzeit diskutierten Alternativen auf Mehreinnahmen für die GKV, d. h. zum Zeitpunkt der Reform ceteris paribus auf Beitragssatzsenkungen, ab.

Unser Tagungsprogramm greift aus diesem vielschichtigen Komplex von Finanzierungsfragen mit der Beitragsbemessungs- und Versicherungspflichtgrenze sowie den Formen der Budgetierung zwei Themenkreise heraus, die in der kommenden Legislaturperiode auf der politischen Agenda stehen könnten. Dabei kommen wieder jeweils Befürworter und Gegner bzw. Skeptiker der entsprechenden Finanzierungsalternativen zu Wort. So plädierte Herr Professor Pfaff als Politiker und Wissenschaftler zuletzt für eine Anhebung von Beitragsbemessungs- und Versicherungspflichtgrenze auf das Niveau der gesetzlichen Rentenversicherung, während Herr Dr. Klusen als Vertreter einer Krankenkasse, die weit überproportional freiwillig Versicherte und damit Betroffene aufweist, diesen Vorschlag per Saldo eher ablehnt. Innerhalb der Budgetierungsformen interessiert vor allem das Globalbudget, das die neue Bundesregierung für die GKV offensichtlich anvisiert. Das Globalbudget gehörte auch schon vor der Wahl zum Forderungskatalog der Krankenkassen, die in diesem Kontext allerdings seine fiskalischen Implikationen, seine technische Ausgestaltung und seine innere bzw. sektorale Koordinationsfunktion offen ließen. Insofern bietet auch dieses Instrument hinreichenden Zündstoff für interessante Diskussionen. Von letzteren wünsche ich Ihnen und mir trotz kontroverser Standpunkte und Aspekte einen gewinnbringenden Gedankenaustausch in einer entspannten und freundschaftlichen Atmosphäre. 


\section{Die europäische Herausforderung für das deutsche Ge- sundheitswesen - ein Spannungsfeld zwischen national- rechtlicher Struktur, Vergemeinschaftsdruck, Marktge- schehen und politischer Handlungsnotwendigkeit}

\section{Subsidiarität, Zukunftsfähigkeit und Systembehauptung in einem veränderten makroökonomischen Umfeld}

Günter Danner

Mit der Vollendung des europäischen Binnenmarktes und der Währungsunion wird das makroökonomische Umfeld der EU-Mitgliedsstaaten nachhaltig und in einer gegenwärtig kaum in Gänze abschätzbaren Weise beeinflußt. Diese Veränderungen bringen den vernetzten Volkswirtschaften eine Fülle neuer Impulse, verfestigte Währungsstabilität und bieten die Chance, das durch ein erhöhtes Maß sozialer Gerechtigkeit gekennzeichnete "Modell EU“ als Alternative zu den großen Wirtschaftsblöcken der Welt, insbesondere den USA, Japan und Südostasien zu positionieren.

Das historische Schlachtfeld Europa könnte somit nicht trotz, sondern infolge seines höheren sozialen Konsenses zu einer gesellschaftspolitischen Zukunftswirklichkeit führen, die mehr bietet als die Fixierung auf ungezügeltes Wachstum und kurzlebigen Shareholder-value. Diese Entwicklung fällt zeitlich mit einer keineswegs überwundenen Krise in Asien und latent als deren Folge gegenwärtigen und künftigen Problemen in den USA ebenso zusammen wie mit der Permanenzkrise des kriminalitätsbeladenen russischen Wildwuchskapitalismus. Gerade diese Herausforderungen sollten die Verantwortlichen in den Regierungen der EU-Staaten dazu bewegen, statt gelegentlich kleinlichen Prestigegerangels im Wege von mehr Vernetzung und Kooperation stabile Zukunftsvisionen und -strategien zu entwickeln. Wer eine Bürgergesellschaft wünscht, um soziale Reibungsverluste zu minimieren, der wird den Wert eines effizienten sozialen Schutzes nicht verkennen. Konsensgesteuerte gesellschaftliche Modelle sollen dabei nicht über bestehende Interessengegensätze hinwegtäuschen, sondern bemüht sein, im Wege der Definition vorhandener oder konfigurierbarer Schnittmengen an gemeinsamen Interessen neue Dialogformen und Kraftquellen zu erschließen. Unter dem Primat der Sachlichkeit könnte es gelingen, gerade in einem veränderten Umfeld aus alten, fest etablierten Gegensätzlichkeiten von Gruppeninteressen auszubrechen. 
Wie kaum eine andere Generation vor der gegenwärtig aktiven stehen wir in der Pflicht, Zukunftsgestaltung nicht ausschließlich oder auch nur vorwiegend aus den augenblicksgebundenen Interessenlagen heraus zu bewerkstelligen. Der Gesundheitsmarkt und die inn mitgestaltenden Akteure und Institutionen sind von dieser Gemengelage in herausragender Weise betroffen. Einmal spielt sich hier allein in Deutschland ein hochvolumiges Marktgeschehen ab, dessen Auswirkungen nahezu jeden einmal in sehr empfindlicher Weise betreffen. Zum anderen sind es hier wie sonst kaum Einrichtungen des Sozialschutzes, die vollständig oder in wesentlichen Teilen die aufgewendeten Finanzmittel beschaffen und mittelbar verteilen. Im weiten Spektrum von Industrie, Leistungserbringern, Patienteninteressen, öffentlich-rechtlich verfaßten, selbstverwalteten Krankenkassen, privaten oder öffentlichen Krankenanstalten - mit oder ohne Unterstützung durch Bundesländer - erlebt ein sozialrechtlich extrem hochgeregeltes System die Umfeldveränderungen durch Entnationalisierung wirtschaftlicher Gestaltungsmacht.

$\mathrm{Zu}$ allem Überfluß erfolgt dies in einem sozialhistorischen Abschnitt nahezu permanenter Reformgesetzgebung. Dieser Aufsatz kann und wird dem geneigten Leser keine Patentlösungen an die Hand geben. Jeder Versuch, den sich vollziehenden politischen und ökonomischen Gesellschaftswandel im Wege einfacher Schablonen zu erklären, verdiente berechtigterweise Mißtrauen. Es ist vielmehr beabsichtigt, Denkansätze und Überlegungen in einen Prozeß einzuspeisen, der uns auf Sicht begleiten wird und den wir gestalten müssen, sollen nicht künftige Generationen schon bald mit Recht den Vorwurf rückwärtsgewandter Kurzsichtigkeit erheben dürfen. Das Europa von morgen, verstanden als politische Einheit der Mitgliedsstaaten, braucht mehr Demokratie, mehr Bürgerbeteiligung und eine bürgernahe, leistungsfähige und ökonomisch problemadäquate soziale Sicherung im Krankheitsfall, vorzugsweise in subsidiärer Regie der Einzelstaaten unter Ausbildung bislang vernachlässigter Formen grenzüberschreitender Kommunikation. Jede nationalstaatliche Reform mit dem Anspruch auf Seriosität sollte daher diese Entwicklung künftig in sachgerechter Weise berücksichtigen.

\section{Gesundheitssicherung in der EU - eine typologische Übersicht}

\section{Nationale Politik, gesellschaftliche Präferenz und politische Gestal- tung}

Die Systeme der EU-Mitgliedsstaaten sind so unterschiedlich wie die sozialhistorischen Verhältnisse ihrer Entstehungs- und Entwicklungsbedingungen. Kein heutiges System, das nicht über die vielen Jahrzehnte entscheidend von grundsätzlichen gesellschaftlichen Wandlungen be- 
rührt worden ist. Der Zusammenhang zwischen Gesellschaftspolitik, Politikformulierung und Entwicklung eines Gesundheitssicherungssystems liegt somit auf der Hand. Erst seit den Herrschenden auch das Wohl und Wehe des kleinen Mannes und der kleinen Frau zumindest vor Wahlen etwas gelten, steht dabei der Versicherte oder auch Patient nicht mehr als Objekt obrigkeitlicher Huld an letzter Stelle der Relevanzliste. Untrennbar verbunden ist die nur im Wege eines geregelten Sozialstaates mögliche Breitenteilnahme der Bevölkerung am Gesundheitswesen mit dem Erstarken der bürgerlichen Rechte besitzloser und auf ihre Arbeit angewiesener Kreise verknüpft.

Standen die Sozialreformen des kaiserlichen Reichskanzlers Bismarck noch ganz im Zeichen einer gesellschaftlichen Stabilisierung von oben, so zeigen etwa bis heute bestehende Sondergroßzügigkeiten der Basiskrankenversicherung für Bewohner Elsaß-Lothringens, die so anderen Franzosen nicht offenstehen, die politische Absicht nach Wiedereingliederung der bis 1918 zum „Bismarck-Modell“ Deutschlands Zählenden angesichts damals weitgehend ungeregelter sozialer Sicherheit in Frankreich. Auch die staatliche Neuregelung der allgemeinen Pflichtversicherung nach 1945 hat dort an diesem historischen Privileg nichts geändert. Der einst als Modell bekannte britische Wohlfahrtsstaat und sein Gesundheitsdienst (NHS) schließlich wurden auf dem Höhepunkt der britischen Kraftanstrengungen in der Abwehr Hitler-Deutschlands ersonnen und gegen einige Widerstände nach dem Zweiten Weltkrieg als Beispiel politischer Gerechtigkeitssuche zur Stärkung der Gemeinschaft verwirklicht. Sein Gerechtigkeitsnimbus, insbesondere in weniger informierten Teilen der britischen Bevölkerung, wirkt, oft sehr im Gegensatz zur materiellen Realität, bis heute beruhigend auf alle, die auf offiziellen oder semi-offiziellen Wartelisten eingeschrieben sind.

So fest war die Verwurzelung in der Bevölkerung, daß der NHS die neoliberale Umstrukturierung der Thatcher-Jahre und halbherzige Reformen der konservativen Epigonen als Institution überstand. Interessant ist in diesem Zusammenhang die neue Präferenz „aufgeklärter" Neoliberaler für eine rigide beschränkte Armenversorgung, die sich - betriebswirtschaftlich betrachtet - offenbar bestens als Auffangbecken für alle eignet, an denen die zeitgleich munter anbietenden Privatversicherungen kein Interesse haben können. Ergänzt man dies sogar um ein liberales Versicherungsvertragsrecht, wie in Großbritannien und den USA üblich, so können auch ausgemusterte PKV-Kunden der Allgemeinheit und den Wartelisten zugewiesen werden.

Volkswirtschaftlich kann dieses Modell unter Zugrundelegen auch nur minimaler ethischer Wertkategorien eigentlich keinen großen Sinn ma- 
chen, es sei denn, man bemißt den makroökonomischen Wert ausschließlich nach dem kleinstmöglichen Kostenanteil des öffentlichen Gesundheitswesens, bezogen auf das Bruttoinlandsprodukt. Bereits wenige solcher Erwägungen sollten reichen, um ethisch orientierten Zeitgenossen die denkbare Vielfalt der Herangehensweisen bei der Bewertung von breitenzugänglichen Gesundheitswesen zu verdeutlichen. Fallen sozialkulturelle, moralische und ethische Aspekte ebenso weg wie die Hypothese der moralischen Gleichwertigkeit aller Glieder unserer Gesellschaft, so gerät ein umfassendes und sozial ausgewogenes Krankenversicherungssystem sehr schnell auf die Verliererstraße. Die billigende Inkaufnahme einer Zwei-Klassen-Medizin dürfte dann rasch als vermeintlich makroökonomisch sinnvoller Ausweg zur Entlastung des "öffentlichen Sektors" und damit der sogenannten "Lohnnebenkosten“ folgen. Damit würde zusätzlich das Ziel erreicht, die nationalen Gesamtaufwendungen im Medizinbereich möglichst nicht sinken zu lassen und diesen Wachstumsmarkt somit zu erhalten. Schon diese Überlegungen verdeutlichen die Bedeutung einer ethikgestützten Politikformulierung für die Zukunft des sozialen Gesundheitswesens.

$\mathrm{Daß}$ dieser Ansatz nicht unbedingt den Prinzipien der ökonomischen Vernunft zuwiderlaufen muß, ja überdies durchaus zu Leistungen befähigt ist, die ein selektives Marktsystem niemals erreichen kann, unterstreicht den vorhandenen, jedoch relativen Marktcharakter der Gesundheitssicherung und das enge Verhältnis zur Politik. Entsprechend hoch ist die nationalpolitische Verbundenheit der in den EU-Staaten vorhandenen Sicherungsinstrumente. Entsprechend hoch ist die Einbindung nationaler Zielformulierung in nationale politische Tradition und Praxis. Deren Vielfalt und der politische Wunsch, diese heiklen, historisch geprägten Politikbereiche bitte nicht nach Art des fragwürdigen Agrarmarktes durch eine noch zu schaffende Brüsseler Superbürokratie umsetzen zu lassen, begründet die Relevanz einer subsidiären Gestaltung. Dies insbesondere dann, wenn dieser Anspruch mit einer schöpferischen Integration der neuartigen Auswirkungen der europäischen Entwicklungen verbunden wird und sich Herausforderungen stellt, statt diese zu leugnen.

\section{Staatsnahe Systeme in vorwiegender Steuerfinanzierung}

Diese im direkten Vergleich untereinander ebenfalls höchst verschiedenen Modelle finden sich in Großbritannien, Irland, Dänemark, Schweden, Spanien, Portugal, Italien, Griechenland und Finnland. Unabhängig von teilweise vorhandenen Versicherungseinflüssen steht hier der Staat üblicherweise als Aufsichtsinstanz, Politikschöpfer, Organisationsinstanz und vielfach als direkter Realisierer in einer zentralen Rolle. Die Versi- 
cherungsinstitutionen, so es sie überhaupt gibt, sind in ein entsprechendes rechtliches Gerüst eingebunden, das innen kaum individuellen oder gar wettbewerblichen Spielraum läßt. Arbeitet das jeweilige System mit direkter staatlicher Mittelzuweisung, dies findet sich beispielsweise in den am NHS Britanniens ausgerichteten Modellen etwa Schwedens, Dänemarks oder Irlands und Italiens, so gibt der Staat mit dem Geld auch den tatsächlich verfügbaren Leistungsumfang vor. Strenge Definitionen der Behandlungsmöglichkeiten, der Therapievarianten sind ebenso üblich wie Positivlisten für Arzneimittel und weitgehender Ausschluß der freien Arztwahl. Vielerorts ist die ambulante fachärztliche Versorgung nur im Krankenhaus möglich. Solche Systeme sind keineswegs durchgängig billig, wie das Beispiel Schwedens zumindest aus Zeiten vergangener Großzügigkeit deutlich macht. Generell bieten sie aber einfache Möglichkeiten für eine Mangelsteuerung, die bewußt die vorhandenen Ressourcen geringer hält als die bekannte Nachfrage, um im Wege der Verzögerung der Inanspruchnahme eine Kostensenkung verwirklichen zu können. Als Resultat sind in allen benannten Modellen Wartelisten, oft beeindruckender zeitlicher Länge, üblich. Zu kritischen Reaktionen in der Öffentlichkeit kommt es erfahrungsgemäß erst dann, wenn diese Wartelisten mehr verursachen als Schmerzen über lange Zeit. Gerade das Beispiel Schwedens zeigt, daß es dauern kann, bis die Öffentlichkeit einen Premierminister vor der Wahl zu Versprechen „verpflichtet“, „mehr Geld“ für Krankenbehandlung vorsehen zu wollen. Daß es damit, auch im Falle Schwedens, keineswegs getan ist, steht unter solchen Vorzeichen kaum mehr zur Diskussion.

Ein steuerabhängiges Gesundheitswesen in nationaler Regie dürfte zu EURO-Bedingungen, die bemerkenswerterweise für Großbritannien, Schweden und Dänemark nicht gelten, zudem das Problem haben, wahltaktisch opportune ergänzende Mittel jedenfalls nicht mehr durch Inkaufnahme höherer Staatsverschuldung bereitstellen zu können. Nicht von ungefähr lähmte der britische Premier seine Reformfähigkeit für den NHS auch dadurch, daß er bei einem Investitionsstau von rund 5 Mrd. Pfund (rund $15 \mathrm{Mrd}$. DM) und 100.000 fehlenden Klinikbetten schon vor seiner Wahl Steuererhöhungen kategorisch ausschloß. Dieser Grad an Abhängigkeit eines Systems von Wahlkampfaussagen ist eine weitere Facette der Staatsnähe. Oft genug werden solche Entscheidungen vor umfassenden Analysen des finanziellen Zustandes der Gesundheitsversorgung getroffen. Kranke haben in staatsnahen Systemen ohne aktive Selbstverwaltung zudem keine Lobby. Die oft nachrangige politische Priorität der Gesundheitssparte tut ein übriges. 


\section{Nicht-wettbewerbliche Sozialversicherungsmodelle}

Sozialversicherungsmodelle ohne oder mit nur sehr eingeschränktem Wettbewerb finden sich im „zweiten Bismarck-Staat" Österreich und, in völlig anderer Ausgestaltung in „Sécu-Modellen“ Frankreichs, wo der Wettbewerb auf die Ergänzungssparte beschränkt ist, ohne die kaum ein Franzose einen befriedigenden Grad an Deckung der materiellen Folgen der Krankheit verwirklichen kann. Leistet das österreichische Modell infolge der Großzügigkeit seines Leistungskataloges und der strukturellen Nähe zu Deutschland vergleichsweise viel, so kann dies vom „Régime de base" in Frankreich kaum mehr behauptet werden. Zwar konnte die französische Politik Mangelsteuerung weitgehend vermeiden, jedoch ist die Erstattungshöhe der Grundversicherung ohne Komplementärabsicherung unzureichend. Mit einer Vielzahl von Strukturreformen versuchte man in Frankreich, das hohe aufgelaufene Defizit der Krankenversicherung und anderer Sparten abzubauen, da es Maastricht-relevante Formen angenommen hatte. Die seinerzeitig konservative Regierung des Ministerpräsidenten Juppé scheiterte nicht zuletzt an ihrer die Bürgerinnen und Bürger einseitig belastenden Sozialreform, übrigens ganz im Zeichen einer möglichst hohen Staatsregie. Wettbewerb ist sowohl im französischen Basissystem als auch unter Leistungsanbietern weitgehend unbekannt. Die völlige organisatorische Trennung zwischen Einnahmen und Ausgaben hat Teilverantwortlichkeiten und „postamtliche Kleinteiligkeit“ bei der Bewältigung komplexer ökonomischer Prozesse geschaffen, die keine Reformergebnisse aus einem Guß verheiBen. Eine traditionsbedingt „unabänderliche“ korporatistische Kassenvielfalt bei völligem Fehlen wettbewerblicher Charakteristik wirft weitere Fragen auf.

Zur Bewältigung des nach wie vor drückenden Defizits - die Zentralkrankenkasse in Paris kann beim Staat im Fall von Unterdeckung „anschreiben" lassen - wurde dem als Radikalreformer bekannten neuen Kassenchef seitens der zuständigen Ministerin für Solidarität mehr Autonomie in Aussicht gestellt. Eine wie auch immer geartete Lobby für die Versicherten kann eine Kasse, die weder ihren Beitragssatz bestimmen kann, noch Verträge schließt oder Beiträge einzieht, schon mangels Nähe zum Objekt kaum leisten. Der allgegenwärtige Staat, Träger von Krankenhäusern - dem „schwarzen Loch“ in der Finanzwelt der französischen Krankenversicherung -, regelt fast alle Probleme nach Art eines Insichgeschäfts zwischen eigenen Stellen. Nach Auswegen aus dem sich zuspitzenden Dilemma wird dennoch fieberhaft gesucht. Dies um so mehr, da bewährte Methoden der Vergangenheit, etwa inflationsfinanzierte sozialpolitische Großzügigkeit, im Euroland ebensowenig gedeihen wollen wie die verschiedenen kostspieligen Beschäftigungspro- 
gramme, die kaum Aussicht darauf bieten, die Leistungsempfänger tatsächlich und über die Frist staatlichen Tuns hinaus in den Produktionsprozeß eingliedern zu können.

Die bedingt wettbewerblichen Krankenkassen Belgiens bieten zwar Grund- und Ergänzungsversicherung „aus einer Hand“, jedoch mit vergleichsweise höheren nicht gedeckten Eigenanteilen als dies in Frankreich üblich ist. Neue Krankenhaus-Ergänzungsversicherungen ergänzen die bisherigen Angebote und entsprechen sicherlich der örtlichen Nachfrage. Die Aufteilung zwischen beiden Segmenten ist zudem naturgemäß in permanenter Debatte. Ein nachhaltiger Kostendämpfungseffekt ist in keinem Fall direkt daraus ableitbar. Auch ist die Autonomie der wenigen Kassen in deren Verhältnis zum Staat ziemlich schwach entwickelt. Das auch bei uns immer wieder diskutierte Instrument der Kostenerstattung zeigt per se keine kostensteuernde Wirkung. Es ist andererseits allerdings auch nicht automatisch ein Indiz für Absenz sozialstaatsbereiten Handelns. Unstreitig eröffnen alle Kostenerstattungssysteme einen breitenwirksamen Zugang zu medizinischer Behandlung. Dies erfolgt nicht zuletzt, weil die liquidationsfähigen Tarife in aller Regel gesetzlich oder im Verordnungsweg festgelegt sind und die Kostenerstattung somit nicht anbieterseitig als "geschuldetes Minimum“ mit Option auf Ergänzungszahlung durch den Patienten im Abdingungsweg interpretiert werden kann. Ein makroökonomischer oder auch sozialpolitischer Vorteil ist jedoch in der generellen Praxis der Kostenerstattung nicht erkennbar. Die dadurch möglicherweise gewachsene Distanz zwischen Zahlerkollektiven und Leistungserbringern kann, wenn das System Mängel aufweist, die Vereinzelung der medizinischen Nachfrage verstärken und somit die Mitwirkung der Zahler und ihrer Solidargemeinschaften an der Preisbildung weiter erschweren. Ausschließlich praktiziert eröffnete die Kostenerstattung dem Basissystem die Möglichkeit einer nur noch teilweisen Bewirkung von Leistungen und hat somit vordringlich zur Kostenkosmetik beigetragen, da sich der Gesamtpreis nur auf andere Zahlerkonfigurationen verteilt.

Ein Sachleistungssystem ohne Eigenanteile wäre demnach im Interesse einer totalen Erfassung der tatsächlichen Kosten möglicherweise klarer, läßt sich allerdings angesichts der in der frankophonen Welt üblichen Praxis nicht ohne weiteres verwirklichen. Auch sozialverträglich konstruierte Zusatzversicherungen sind, sofern sie nicht den Behandlungszugang der Mehrheit durch Inanspruchnahme knapper öffentlicher Ressourcen weiter verschlechtern, nicht a priori sozial diskriminierend. In verschiedenen EU-Staaten wirken etwa vorwiegend nicht-gewinnstrebende Ergänzungsversicherungen, oft mit der Basiskasse eng verbunden, durchaus nachfragebefähigend. Kennzeichen solcher Angebote 
sind durchweg das Fehlen individueller Risikoprüfungen, eine höchstens nach dem Alter bei Eintritt gestaffelte Pauschalprämie und weitgehender Verzicht auf das Äquivalenzprinzip.

Das gelegentlich hochgelobte Instrument der Eigenbeteiligung schließlich, in Frankreich seit 1928 als "ticket modérateur" geläufig, beschrieb seinerzeit den versichertenseitig zu entrichtenden „Eintritt“ bei einem Arztbesuch. Kostendämpfend hat es sich trotz zeitweiliger Verbote einer 100prozentigen Absicherung durch die Zuwahlkassen nicht bemerkbar gemacht. Es hat nach Meinung seiner Kritiker lediglich dazu beigetragen, den Leistungserbringern den Ernst der Finanzlage der französischen Krankenversicherung nicht so präsent werden zu lassen. Sie wurden jedenfalls bislang von neuartigen Versuchen einer zielführenden, an Qualität und Kostentransparenz orientierten Vergütungspraxis weitgehend verschont.

\section{Gesetzliche Zwangskonvergenz im niederländischen Drei-Säulen- Modell}

Die Niederlande gelten vielen Deutschen, möglicherweise als Folge ihrer niedrigen Arbeitslosigkeit bei allerdings sehr hoher Teilzeitbeschäftigung, geradezu als sozialpolitisches Vorbild. Schnell sind manche mit unverblümten Forderungen nach Kopien des „Poldermodells“ bei der Hand. Im Gesundheitswesen, zweifellos einem sehr wohlgeordneten System, könnten hier dennoch einige Zweifel angebracht sein. Das sogenannte "Dekker-Modell“ in seiner letzten Variante steht einmal mehr vor tiefgreifenden organisatorischen Reformen, diesmal eindeutig zu Lasten des Selbstverwaltungsgedankens.

Mit der Zuweisung Höherverdienender an die traditionell stark etablierte Privatversicherung und die Einbindung dieser Sparte in eine soziale Verpflichtung wurden in der Tat neue Wege beschritten. Kontrahierungszwang für Standardpolicen, Risikostrukturausgleiche und die Absicherung von Großrisiken über den steuerfinanzierten Topf des Gesetzes über besondere Krankheitskosten (AWBZ) haben nach wie vor ihre Verteidiger, auch wenn mittlerweile mancher niederländische Leistungsanbieter von deutschen Verhältnissen und Einkünften träumt. Ein rigides Primärarztmodell, Festpreise für bestimmte Arzneimittel und eine Feinbudgetierung, die alljährlich ein hohes Politikum ist, umreißen die engen Grenzen, in denen der regelnde Staat die Akteure sich bewegen läßt. Auch die Niederlande kennen die Mangelsteuerung in Gestalt von Wartelisten, die so bei uns kaum vertretbar wären. Als neueste Variante wünschen die Krankenkassen auf der Basis der jüngsten EuGH-Urteile in den Rechtssachen Kohll und Decker grenzüberschreitende Verträge, 
um den tatsächlichen Bedarf ohne Inkaufnahme von strukturellen Überkapazitäten abdecken zu können. Mit dieser pragmatischen Reaktion haben sich die Niederländer in bemerkenswerter Weise von der Haltung, etwa der ehemaligen deutschen Bundesregierung abgegrenzt, wie an nachfolgender Stelle noch dargestellt wird. Sie entspricht einer zwar staatsnahen, jedoch bedingt wettbewerblichen Struktur, die jedoch in vielen Einzelheiten nicht übertragbar ist. Das niederländische Gesundheitswesen kämpft nach wie vor an der Kostenfront und zählt nicht eben zu den preiswerten Vertretern in der EU, wenngleich der hohe politische Charakter ziemlich klar hervortritt.

\section{Exkurs: Versicherungspflichtmodell „Schweiz“ und „Freier Markt mit Armenfürsorge in den USA“}

Die EU-Zentriertheit soll nicht verbergen, daß auch außerhalb dieses Blocks Modelle von Krankenversicherung und Gesundheitsmärkten existieren, die bei uns ihre Verfechter haben. Das für viele Reformvorschläge in der deutschen Diskussion der vergangenen Jahre dabei durchaus interessante Modell der Schweiz fußt seit Inkrafttreten des reformierten Krankenversicherungsgesetzes Anfang 1996 auf einer Versicherungspflicht für alle, egal ob bei öffentlich-rechtlichen oder privaten Anbietern. Es fällt dabei auf, daß das Obligatorium in der Schweiz nur von nicht gewinnstrebenden Anbietern durchgeführt wird. Offenbar ist mit der Durchführung sozialer und breitenzugänglicher Krankenversicherung eine Befriedigung von Shareholderinteressen nicht $\mathrm{zu}$ vereinbaren. Die Schweiz kennt weder die beitragsfreie Mitversicherung von Angehörigen noch einen Arbeitgeberbeitrag oder einen entgeltabhängigen Beitragssatz. Sie praktiziert Eigenanteile in Gestalt von sogenannten „Franchisen" und staffelt Leistungen und Prämien traditionswahrend regional. Damit hat sie u. a. eine Vielzahl der Forderungen des ehemaligen bundesdeutschen Sachverständigenrates verwirklicht.

Das in Aussicht gestellte Ziel einer Kostendämpfung konnte jedoch mit keiner der Maßnahmen auch nur ansatzweise befriedigend gelöst werden. Dieses trifft weder den deutschen Sachverständigenrat noch die Akteure der eidgenössischen Krankenversicherung, die es an gutem Rat und Warnungen an die Politik zu keiner Zeit haben fehlen lassen. Eine enorm starke Lobby der Leistungserbringer, vielleicht auch die Existenz einer optionalen „Zweit- und Erstklasseversorgung“ für Solvente sowie eine unglückliche Interessenkonfiguration der regionalisierten Kompetenzen haben Kostenreduktionen problematisch gemacht. Die Option einer Versicherung für die „Gesamtschweiz“, im Unterschied für eine nur im eigenen Kanton, stimmt angesichts der Größe des Landes skeptisch. Im Zeichen eines wünschenswerterweise optimalen Einsatzes von High- 
Tech-Ressourcen muß dies bei einem erheblichen Strukturgefälle zwischen ländlichen Bergkantonen und Metropolen zu einer entbehrlichen Vermehrung bürokratischer Kompetenz- und Kompensationsstreitigkeiten führen. Immerhin verfügen die Schweizer Krankenkassen im Unterschied zu den Niederländern über relativ viel Gestaltungsmacht, die sogar die Besonderheit der ersten kassenbetriebenen Versandapotheke in Soloturn ermöglichte. Die Bekenntnisse des Widerstands der verfaßten Apothekerschaft füllen mittlerweile Bände. Die Industrie, gelegentlich aus Sicht der Zahlerkollektive vorschnell alleiniger Sündenbock für die endgültigen, oft kräftig überhöhten Abgabepreise, kann damit offenbar ganz gut leben. Ähnliche Experimente werden auch im Bereich der „Managed Care" in Gestalt von Erprobungen mit HMO oder Disease Management veranstaltet.

Die vergleichsweise schwache Position des Schweizer Staates hat im Unterschied zum niederländischen Modell durchaus für Interesse auch in Kreisen deutscher Szenekenner gesorgt. Daß es dabei ernstlich nicht, wie manchmal zu lesen steht, um die baldige Abschaffung des Arbeitgeberbeitrages bei uns gehen kann, diesen gab es in der Eidgenossenschaft so wie bei uns nie, sollte allerdings nicht überraschen.

Anders im Land der unbegrenzten Möglichkeiten. Ohne eine geregelte Pflichtversicherung für die materiellen Folgen der Krankheit sind, je nach Grenzziehung, rund 50 Millionen Amerikaner nicht, nicht durchgängig oder unvollständig geschützt. Erst völlige Mittellosigkeit oder der Rentenempfängerstatus eröffnen die Möglichkeiten von Medicaid und Medicare. Ein weltweit nahezu beispielloses Preisniveau für medizinische Dienstleistungen und Produkte hat dazu geführt, daß selbst die klassische private Vollversicherung kaum mehr bezahlbar ist. Als Folge entstanden HMOs unter der Anleitung von Versicherungsgesellschaften und gestatteten vertretbare Prämien bei rigider Beschränkung des Zugangs und des Umfangs der Versorgung. Ein ausgeklügeltes Standardisierungswesen im Therapiebereich hat neben atemberaubenden Haftpflichtpraktiken jedes Abweichen von definierten Standards für den Leistungserbringer zum Hasardeurspiel gemacht.

Die vorwiegend arbeitgeberfinanzierten HMOs sind durch Jobgebundenheit und Einschränkungen der Therapiefreiheit ebenso gekennzeichnet wie durch Fixierung auf sogenannte "In-network-doctors“. Jedes Abweichen, etwa in einem Notfall ohne Netzpräsenz in der Nähe, erfordert stringente Begründungen oder setzt die Leistungsverpflichtung aufs Spiel. Dies hat $u$. a. dazu geführt, daß ein wichtiges Element der amerikanischen gesellschaftlichen Leistungskraft, die ungewöhnlich hohe örtliche Mobilität der Beschäftigten und Arbeitsuchenden, in nicht eben ge- 
ringem Umfang gefährdet wurde. Wer immer mit seinem Arbeitgeber auch seine Krankenversicherung bzw. deren Tarif wechselt, tut dies dort zu den üblichen PKV-Bedingungen, d. h. mit neuerlicher Gesundheitsprüfung und alters- und vorerkrankungsbedingten Risiken. Ein überaus anbieterorientiertes Vertragsrecht gestattet zudem eine Reihe von bei uns unvorstellbaren Risikoausschlüssen, Therapieeinschränkungen, Begrenzungen auf ein erkrankungsgebundenes Leistungsmaximum oder eine Gesamtbeschränkung auf ein „Lebenszeitmaximum“ an finanziellen Lasten für den Versicherer. Mit diesem „life-time maximum" sind nahezu alle aktuarischen, bezifferbaren Risiken auf den Versicherungsnehmer übergegangen.

Trotz aller liberaler Freiheiten ist das US-amerikanische Gesundheitswesen - bei enormer Potenz im Bereich Forschung, Therapie und HighTech-Medizin für diejenigen, die dorthin Zugang haben - vergleichsweise außergewöhnlich teuer und leistet sozialpolitisch wenig. Krankheit ist Verarmungsursache Nr. 1 für bürgerliche Haushalte, insbesondere für Familien, dem Kultobjekt gerade konservativer politischer Ideologie in den USA. Allen politischen Bekenntnissen zur Familie zum Trotz konnte ein großer medizinisch-industrieller Komplex mit feinverschachtelten Interessenverschränkungen zwischen der Medizinindustrie, den Anteile haltenden Ärzten, der Pharmaindustrie und einer von diesen Interessengruppen gesteuerten Politik das Clinton-Projekt kippen. Dessen mangelhafte Konstruktion und möglicherweise auch fehlende Aufrichtigkeit verringerte die politischen Chancen. Mit einer wachsenden Kluft zwischen Wohlstand und Armut, einem durch Zweit-, Dritt- und Zusatzjobs geprägten Arbeitsmarkt gelingt die Integration großer Teile der Gesellschaft immer weniger. Perspektivlosigkeit, oft bereits von der Geburt an, bestimmen die Wirklichkeit in den "tough-areas" amerikanischer Großstädte keineswegs nur für "lllegale“.

Mit der Rückführung der letzten Sozialgesetze des „New Deal“ aus den 30er Jahren steht Amerika am politischen Scheideweg. Eine umfassende Wachstumseuphorie vermag noch die ärgsten Widersprüche zu bemänteln; fällt diese Option eines Tages, etwa durch Abfluß gigantischer asiatischer Kapitalinvestitionen, so wird sich zeigen, wie krisenfest die amerikanische Gesellschaft, deren Verfassung als einzige das „Streben nach Glück" garantiert, wirklich ist. Kritiker erklären die politische Abstinenz der US-amerikanischen Bevölkerung gern mit dem hohen Engagement im persönlichen Alltag und einem durch private Fernsehanstalten und deren Informationsstil geprägten sozialkulturellen Bewußtsein.

Geringe Wahlbeteiligung paart sich mit hohem Interesse an tatsächlichen oder vermeintlichen Skandalen und Sensationen. Sozialpolitik, ein 
an sich recht trockenes Geschäft, vermag in diesem Wettstreit um die Aktualität des Augenblicks kaum mitzuhalten. Eine politische Opposition zum ungezügelten Wirtschaftsliberalismus fehlt ohnehin ebenso wie eine politisch präsente Arbeitnehmerbewegung als Gegenmacht der auf ihre Arbeitskraft Angewiesenen.

\section{Leistungsparameter für soziale Krankenversicherung}

Für einen auch nur halbwegs ansprechenden Definitionsversuch der EUEinflußgrößen auf die existierenden Sozialversicherungssysteme ist es von Belang, Qualitätskriterien für derartige Einrichtungen in gebotener Kürze allgemein zu streifen.

Ein zu EU-Bedingungen gut funktionierendes System sollte insbesondere folgende Faktoren aufweisen, die sich zwar nach politischen Akzenten verschieben, jedoch nicht gegenseitig ersetzen können:

- Qualität als Determinante für Preiswürdigkeit

- Nachfrageorientierte Verfügbarkeit

- Innovationsvermögen

- Soziale Gerechtigkeit und Zugänglichkeit

- Makro- und mikroökonomische Sozialverträglichkeit

- Binnenmarktkompatibilität aus Sicht der Patienten, Versicherten, Produkte und Dienstleistungen

Fehlen diese Eigenschaften, so besteht die Gefahr, daß beispielsweise zwar zu geringem Aufwand ein Basisschutz vorgehalten wird, dessen eingeschränkte Verfügbarkeit jedoch über Wartelisten sowohl die Qualität reduziert, als auch das Gebot sozialer Gerechtigkeit verletzt. Kommt es zu regelmäßigen Überkapazitäten und einem anbieterorientierten Absatz von Produkten und Dienstleistungen, so ist die finanzielle Überforderung des Systems mit allen Folgen absehbar. Das sozialökonomische Dilemma wird nicht geringer durch den Umstand, daß die vielkritisierten Beiträge oder Abgaben vermindert um die allgemeinen Verwaltungskosten der Kostenträger als Nettoliquidität für Entlohnung von Nachfrage zur Verfügung steht. Ein makroökonomisch „bedenklicher" Betrag wird also durch Verminderung um einen Prozentwert für Verwaltungskosten zu einer Summe, die Einkommen, Arbeit, Steuereinnahmen, Innovationen und Wohlstand neben der reinen Gesundheitssicherung abwirft.

Hier zu erkennen, wo zu welchen Bedingungen eine wünschenswerte Interessenverteilung vorliegt, ist die hohe Schule der Gesundheitsöko- 
nomie. Ein Konflikt zwischen makroökonomischen und betriebswirtschaftlichen Interessen ergänzt diese Aufgabe, wie jeder verfolgen kann, der eine neue Runde an Reformgesetzgebung beobachtet. Nicht von ungefähr hat der Staat in allen EU-Systemen, vielleicht auch aus Alternativlosigkeit, in wachsendem Umfang Definitions- und Entscheidungskompetenz übernommen. Im Wege gesetzgeberischer Verfügung gelang es jedoch kaum, essentielle Fragen der gesellschaftlichen Bedürfnisbefriedigung und Mittelverwendung dauerhaft zur mehrheitlichen Freude zu regeln. Das hier voreilig vielgeschmähte Deutschland macht noch eine bemerkenswerte Ausnahme, wiewohl der Trend unverkennbar ist, übrigens unabhängig von der politischen Couleur der Regierenden.

\section{Die europäische Dimension unter Bedingungen der Währungsunion}

Die Vollendung des Binnenmarktes ist irreversibler Souveränitätstransfer, genauso wie die Vernetzung ehemals unabhängiger nationaler Volkswirtschaften im verbindlichen und damit DM-ähnlichen Stabilitätsrahmen „Euroland“ die „Nationalökonomie“ ablöst. Wesentliche makroökonomische Schlüsselfragen können daher unter ungewollter oder gewollter Außerachtlassung europäischer Komponenten nicht mehr seriös beantwortet werden. Mithin erzwingt die Währungsunion politische Kompetenzverlagerungen struktureller und inhaltlicher Art. Dies ist angesichts der unverändert bestehenden eklatanten politischen Schwäche der EU keineswegs unproblematisch. Man denke nur an die Kalamitäten beim Formulieren einer kohärenten Außenpolitik oder an schädliche Personalstreitigkeiten, gesteuert durch den Wunsch nach nationaler Geltung. Politische Schwäche kann durchaus Raum für wirtschaftliche Stärke geben. Problematisch wird dies jedoch dann, wenn etwa im Bereich zukunftsfähiger Sozialpolitik ein Gegengewicht zu wirtschaftlichen Machtverhältnissen politisch gesichert werden muß.

Ohne soziales Steuerungsprogramm wird der Gesundheitsmarkt zwangsläufig den Patienten als unvollständigen Marktteilnehmer - weitgehend ohne die Möglichkeit eines Verzichts auf Nachfrage oder auch nur deren abschließende Definition - in ethisch unzumutbarer Weise benachteiligen. Diese auf nationaler Ebene durchaus geregelte Frage gewinnt im supranationalen Bereich neue Aktualität. Eine Harmonisierung der bestehenden Gesundheitssicherungssysteme wäre nur auf dem kleinsten gemeinsamen Nenner - etwa zwischen Griechenland und uns - denkbar. Sie würde zudem einen nicht mehr vermittelbaren Qualitätsverlust, gepaart mit undurchschaubaren Transferbürokratien in Brüssel, unvermeidlich machen. Scheidet die Harmonisierung also aus - ernstlich wird sie derzeit nirgends gefordert -, so müssen in nationaler subsidiärer Regie geeignete Synchronisierungsstrategien für die beste- 
henden Systeme geschaffen werden, die den veränderten makroökonomischen Bedingungen entsprechen. Eine Erhöhung der Europakompatibilität des eigenen Gesundheitssystems ist im Wege primär nationaler Reformanstrengungen gerade für unser staatsfernes System vergleichsweise einfach. Staatsnah betriebene Varianten werden hier ungleich mehr Probleme zu lösen haben, können sich jedoch vermutlich mit Aussicht auf Erfolg länger taub stellen.

\section{Aspekte eines europäischen Gesundheitsmarktes}

Mit den mittlerweile bei uns berühmten EuGH-Urteilen und künftigen Entscheidungen ähnlicher Richtung hat der EuGH die national Verantwortlichen, oft zu deren Schmerz, daran gemahnt, daß es nur wenig Hoffnung darauf gibt, im Wege von rechtlichen Bereichsausnahmen den 300 Mrd. DM/Jahr umfassenden nationalen GKV-Gesundheitsmarkt nationalen Anbietern vorzubehalten. Diese unter EU-Kriterien wenig überraschende Entscheidung legte den Finger auf die Schnittstelle aus nationalstaatlicher, subsidiärer Regelungskompetenz zu Strukturfragen der sozialen Krankenversicherung einerseits und dem EU-Freiheiten unterworfenen Waren- und Dienstleistungscharakter medizinischer Angebote. Die bekannten, heftigen Reaktionen der ehemaligen Bundesregierung, aber auch anderer Systemteilnehmer und Politiker sind in den zurückliegenden Monaten durchaus verhaltener und damit adäquater geworden.

Die stammtischfähige Forderung nach „Infragestellung des EuGH“ wurde im Verlauf dieses Prozesses über den Umweg einer Bemühung der Regelvergütungspraxis für die Geltung europarechtlicher Entscheidungen zu einem abwartenden Beobachten mit hoffentlich konstruktivem künftigen Herangehen verändert. Es bleibt zu hoffen, daß die neue Regierung hier Handlungskompetenz durch Wahrung eines höchstmöglichen Spielraumes auch für anstehende nationale Strukturreformen wahrt. Erste quantitative Ernüchterungen und öffentlich diskutierte Qualitätsprobleme von Versicherten beim "Hau-Ruck-Zahnersatz" unter Palmen haben erkennen lassen, daß es hier nicht um ein zur raschen Aktion ermutigendes Sujet geht. Vielmehr werden Ausweitungen der vertraglichen Verbindungen im EU-Bereich sinnvollerweise auch den europäisch schon recht weit fortgeschrittenen Zertifizierungs- und Standardisierungsprozeß für Qualitätsmessung im medizinischen Bereich einbeziehen müssen.

Die EuGH-Entscheidungen gewinnen ihre Brisanz denn auch bestimmt nicht aus einer massenweise Inanspruchnahme weitgehend unbekannter ausländischer Anbieter durch deutsche Versicherte zu privatrechtlichen Schuldbedingungen und unter Fehlen jeder Kostentransparenz. 
Der eigentliche Sprengstoff liegt im Wetterleuchten einer denkbaren neuen Wettbewerblichkeit zwischen Leistungsanbietern, wie er über den europäischen Umweg auch die künftige Gesundheitsreform beeinflussen könnte. Hier fühlen sich, ob berechtigt oder nicht soll hier nicht erörtert werden, Institutionen und Personen in Frage gestellt und mit Unbekanntem konfrontiert. Es liegt auf der Hand, daß ein Wachstumsmarkt der Dimension des Gesundheitswesens nicht mit den Instrumenten des nachrangigen nationalen Sozialrechts gegen EU-Freiheiten abgeschottet werden kann, ohne daß tatsächliche und schwerwiegende Gefährdungstatbestände nachgewiesen werden.

Die deutschen Überkapazitäten in fast allen Bereichen lassen hier derzeit nur Spekulationen zu, die kaum hinreichende Überzeugungskraft entfalten dürften. Eine Angst der Leistungsanbieter Deutschlands wäre, gemessen an dem Postulat ihrer für jeden Vergleich gerüsteten Qualität und bestehender Unterversorgung in Nachbarstaaten, kaum begründet. Schon liegen aus den Niederlanden erste Wünsche der Krankenkassen nach grenzüberschreitenden Verträgen vor, die vom Bettenabbau betroffene deutsche Krankenhäuser in zumutbarer Grenzentfernung bestimmt gern zur Kenntnis nehmen. Daß angesichts unterschiedlicher Krankenhausfinanzierungspraxis in den Niederlanden und bei uns hier europarechtliche Folgewirkungen entstehen können, sei am Rande bemerkt. Fehlen den niederländischen Anstalten infolge der nominell „teureren" monistischen Finanzierung dereinst die Patienten, so könnte sich die Frage stellen, ob „dual-finanzierte“ - d. h. mit öffentlichen Zuwendungen, „verbilligte“ - deutsche Mitbewerber nicht im Wege unzulässiger Subventionen einen Vorteil erlangen.

Der Wunsch der Krankenhauslobby nach geteilter Abrechnungspraxis und unterschiedlichen Preisen zwischen Inländern und EU-Ausländern dürfte ebenfalls Probleme bereiten. Bliebe das Überdenken der wenig europakompatiblen dualen Finanzierung, wie es ja bereits von nicht eben wenigen in Deutschland aus anderen Gründen gefordert wird.

\section{Ausländische Reaktionen auf die EuGH-Urteile}

Diese entsprechen üblicherweise dem jeweils vorherrschenden Meinungsbild der zuständigen Ministerialbürokratie und den hierdurch beeinflußten Institutionen. Die Niederlande reagieren mit der aktiven Variante in Gestalt des Wunsches nach grenzüberschreitenden Verträgen. Allerdings wird zugleich der Versuch unternommen, die Verpflichtung zur Erstattung von im Ausland entstandenen Kosten mit dem Hinweis auf das national ausschließlich angewendete Sachleistungsprinzip zu verneinen. Dieser Widerspruch ist nur scheinbar schwerwiegend, da die 
Masse der an ausländischen Behandlungen interessierten Versicherten auf niederländischen Wartelisten sicherlich für kanalisierende Zuweisungen an qualitätsgeprüfte und verläßliche Vertragspartner eher dankbar sein dürfte. Daß ein Verweis auf ausschließlich praktizierte Sachleistung allein genügte, um Europa auszublenden, darf getrost bezweifelt werden. Vorausschauend stellt die niederländische Reaktion jedoch in wachsendem Umfang auf die Wahlfreiheit der Patienten ab, die nicht blockiert werden dürfte. Der allgemeine Wunsch nach aktiver, subsidiärer Bewältigung des Problems, statt entrüsteten Schulterzuckens und verstimmten Abwartens neuer Urteile, charakterisiert eine pragmatische Linie.

Frankreich ist zwar der Auffassung, daß die Urteile befolgt und umgesetzt werden müssen, hat jedoch bislang kaum aktiv eine offizielle Position vertreten. Man sieht im Unterschied zu heimischen Deutungen gerade die Sachleistungsstaaten als eigentliche „Zielscheibe“, die dann naturgemäß auch als betroffen gelten. Frankreich möchte die Subsidiarität wahren, erkennt jedoch zugleich die Notwendigkeit, auf der Basis bestimmter Übereinkommen die Krankenversicherungssysteme zu „öffnen", ohne sie ihrer nationalen Inhalte zu berauben. Keinesfalls sollte durch rückwärtsgewandtes Leugnen eigener nationaler Betroffenheit die Gestaltungskompetenz nolens volens auf den EuGH im Wege von Einzelentscheidungen übergehen. Auch nationale Gesundheitsdienste, etwa diejenigen Spaniens oder Großbritanniens, müßten sich nach französischer Auffassung diesen Urteilen unterwerfen. Im Wege aktiver Politikformulierung solle man bestrebt sein, „das Heft des Handelns“ in der Hand zu behalten.

Österreich hat mit seiner lange bewährten Erstattungsregelung für im Ausland als Privatzahler auftretende sozialversicherte Patienten naturgemäß keine Probleme mit den EuGH-Urteilen. Generell habe diese schon seit geraumer Zeit übliche Vorgehensweise zu keinen Krisen oder Anspannungen geführt. Expressis verbis wird bezweifelt, daß eine Ratsentschließung mit der Zielsetzung, die Gesundheitsmärkte von den EU-Freiheiten auszunehmen, mehrheitsfähig oder überhaupt nur sinnvoll wäre. An klaren politischen Willensbekundungen zur zeitgerechten Weiterentwicklung der sozialen Krankenversicherung sei auch deren Zukunftsfähigkeit zu messen.

Finnland mit seinem dualen Gesundheitssystem, dessen staatlicher Teil dem NHS Großbritanniens ähnelt, sieht keine Gefahren aus der neuen Rechtsprechung. Sowohl die nach Art eines Gesundheitsdienstes organisierte Versorgung als auch die im Wege teilweiser Kostenerstattung vergüteten privaten Anbieter könnten durch eine grenzüberschreitende 
Ergänzungsversorgung nicht gefährdet werden. Bislang galt ein strenger Genehmigungsvorbehalt bei Inanspruchnahme ausländischer Behandlungskapazitäten. Eine Anpassung an die Luxemburger Urteile sei geplant. Man denkt derzeit daran, die Kostenerstattung für eine Behandlung im EU-Ausland mit derjenigen für inländische private Anbieter gleichzusetzen.

Italien mit seinem nationalen Gesundheitsdienst hat sich bislang nicht zu einer vertiefenden Analyse einer möglichen eigenen Betroffenheit entschlossen. Man sieht sich infolge der nationalen Eigenarten nicht betroffen. Inländische Kritiker des weitgehend maroden italienischen Gesundheitswesens erklären diese Sorglosigkeit als Ergebnis einer, auch wenn rechtlich erzwungen, denkbar geringen Erstattungslast. Wahrscheinlicher sei, daß die Patienten in Ermangelung ausreichender und qualitativ hochwertiger inländischer Kapazitäten in manchen Landesteilen möglicherweise eher auf fingierte Notfälle bei Aufenthalten im EUAusland auswichen, wie dies in Frankreich bereits seit einiger Zeit beobachtet wird. Eine solche Verhaltensweise ermöglicht auf der Grundlage der EWG-Verordnung 1408/71 über die Koordinierung des zwischenstaatlichen Sozialrechts die Auslandsbehandlung als quasi inländischer Sozialversicherter mit der Gewißheit, daß die heimische Institution den Preis in voller Höhe erstatten muß und zudem Vorauslagen bei Inanspruchnahme des Auslandsberechtigungsscheines vermieden werden können.

Großbritannien glaubt offiziell, mit seinem wohnortgebundenen nationalen Gesundheitsdienst durch diese Urteile nicht berührt zu sein. Es gäbe dort keine medizinischen Dienstleistungen im Sinne des Artikels 60, Absatz 2 des EG-Vertrages. Zum Charakter der auch dort handelsüblich verkauften Arzneimittel wird nicht Stellung genommen. Kritiker meinen, daß britische Politiker, ohnehin von wenig EU-Begeisterung beflügelt, die Bedeutung der Urteile schlichtweg verkennen. Man möchte jede Veränderung des Bestehenden vermeiden und erhofft sich von einem Verschieben des Problems auf die „Arbeitsebene“ zumindest einen Zeitgewinn. Tatsächlich wäre britischen NHS-Begünstigten mit einer Erstattung von im EU-Ausland bezogenen medizinischen Dienstleistungen angesichts künstlich niedriger heimischer Tarife nur wenig gedient. Die Frage, ob ein sofortiger Zugang zu bestimmten Therapien im EU-Ausland ohne spätere Abbruchmöglichkeit daheim (z. B. Zugang zur Erstdialyse) eine Möglichkeit darstellte, die teilweise skandalösen Wartelisten zu umgehen, wird erst gar nicht erörtert. Interne Gespräche vermitteln das Bild einer sich in der Sicherheit vermeintlicher Nichtbetroffenheit wiegenden Politik, die - vordringlich bemüht, sich mehr von der EU zu distanzieren als den Brückenschlag zu suchen - isolationisti- 
schen Wunschvorstellungen anhängt. Das bröckelnde Bild des „neuen Wirtschaftswunderstandortes" außerhalb der EURO-Zone trägt ebenfalls nicht dazu bei, europäische Dimensionen kreativ in nationales Handeln einzubeziehen.

Dänemark, ein dezentralisierter staatlicher Gesundheitsdienst mit öffentlichem Versorgungszugang, hat bislang keine vertiefenden Analysen veröffentlicht. Man wünscht keine weiteren Urteile des EuGH, vermag aber nicht zu erklären, wie dies denn zu erreichen wäre.

Ähnlich verhält es sich mit Schweden, das durch verschiedene Wartelisten für eine Reihe von medizinischen Behandlungen ähnlich geprägt ist wie sein skandinavischer Nachbarstaat. Vorschläge, die eine Antwort auf die Herausforderungen des europäischen Gesundheitsmarktes in einer Veränderung der bestehenden zwischenstaatlichen Rechtsvorschriften der EWG-VO 1408/71 (Stichwort: Auslandskrankenschein) sehen, offenbaren einmal mehr, wie weit man davon entfernt ist, den Marktcharakter der medizinischen Güter und Dienstleistungen unabhängig von ihrer nationalrechtlich gestaltbaren Vergütung- und Begrenzungspraxis in seinen grundsätzlichen Auswirkungen zu erfassen.

Die Europäische Kommission möchte sichergestellt wissen, daß Gesundheitsgrundsätze weiterhin gegen ökonomische Ansätze die Oberhand behalten. Das Subsidiaritätsprinzip sei nicht gefährdet. Es gelte jedoch, eine bessere Verknüpfung der verschiedenen Gesundheitssysteme sicherzustellen. Dazu sei eine Messung von Quantität und Qualität nebst einer Bewertung von Leistungen erforderlich, um geeignete Maßeinheiten zu schaffen. Die Kommission möchte die Mitgliedsstaaten dabei durch Material und Informationen unterstützen. Mehr Transparenz und mehr wechselseitiges Verständnis seien ein wichtiger Schritt. Statt defensiver Einstellungen favorisiert Brüssel ganz offenbar eine intelligente Verbindung der freiheitstiftenden Elemente des Binnenmarktes mit kreativer Gestaltungskompetenz in nationaler Regie und neuen eigenen Kompetenzen in der Zusammenführung von Gesundheitspolitiken außerhalb des Sektors der Sozialversicherung. Mehr Dialog, Personalaustausch und partnerschaftliches Zusammenwirken zwischen nationalen Akteuren und Kommissionsdienststellen könnte dann auch das künftige Bild prägen.

\section{Zur Entwicklung von Arbeit und Beschäftigung}

Aus verschiedenen Gründen steht die Sorge um mehr Beschäftigung ganz oben auf der politischen Agenda. Diese Präferenz wird kaum verwundern und entspricht auch einer neuen Option auf synchronisierte Po- 
litikformulierung statt isolierter nationaler Beschäftigungspolitik. Beschäftigung, namentlich solche, die versicherungspflichtig und gegen Arbeitsentgelt erfolgt, ist die Finanzgrundlage der deutschen GKV. Verändern sich also infolge des Transparenz- und Mobilitätsgewinns der Währungsunion die traditionellen Beschäftigungsformen, so muß dies vorausschauend erkannt und politisch bewältigt werden. Es steht zu vermuten, daß zu Bedingungen eines rasanten Strukturwandels am Arbeitsmarkt durch den EURO keine Arbeitsplatzlawine ausgelöst wird. Insbesondere das Nachfragegefälle zwischen höchstqualifizierten und durchschnittlich oder geringer Befähigten wird weiterhin für Probleme sorgen. Mit dem eher schlichten Argument einer möglichst breiten Erhöhung der Renditeaussichten für Investivkapital ist in der Vergangenheit keine auffällige Besserung der heimischen Beschäftigungslage verwirklicht worden.

Trotz „Lohnmäßigung" - was immer außer Lohnkürzungen darunter auch verstanden wird - und Reallohnverzichts fanden sich durchaus gewinnträchtigere Investitionsoptionen als der Ausbau des heimischen Personalbestandes. Die zeitgleich bemerkenswerte Divergenz zwischen Produktivität und Beschäftigung sollte allen zu denken geben, die tatsächlich auf einen echten Trendwechsel setzen. Beschäftigung könnte durchaus in nicht allzu ferner Zeit höchst unterschiedlich zusammengesetzte Erwerbsbiographien eher meinen als versicherungspflichtige Daueranstellungen traditionellen Zuschnitts. Dafür ist unser Sozialversicherungssystem derzeit kaum gerüstet. Im Unterschied zu Pflichtversicherungsmodellen - hier wird zwischen Selbständigkeit und Arbeitnehmereigenschaft nicht unterschieden - und naturgemäß steuerfinanzierten Diensten, die eine andere Finanzierungsgrundlage aufweisen, besteht bei uns daher vorausschauender Denkbedarf. Die auch bei nur flüchtiger Betrachtung auffälligen Schnittstellen zwischen unterschiedlichen Politiksparten - man denke nur an Bildungs- und Sozialpolitik erfordert künftig möglicherweise mehr als heute schon ganzheitliche gesellschaftspolitische Konzepte. Der makroökonomische Rahmen des neuen Eurolandes wird durch mehr Transparenz und die Macht des Faktischen hier schon kurzfristig neue politische Visionen erforderlich machen, die sich von bekannten Verteilungskämpfen der Vergangenheit qualitativ unterscheiden sollten.

\section{Gedanken zur Marktcharakteristik der EU-Gesundheitsmärkte}

Bei allen strukturellen Unterschieden zwischen den Gesundheitssystemen der EU zeigen sich doch viele deckungsgleiche Probleme. Beklagt wird insbesondere das Fehlen einer wirkungsvollen Einflußnahme auf die Preisbildung für medizinische Dienstleistungen und Produkte durch 
die einkaufsbefähigenden Nachfragerkollektive. Dies gilt in Verbindung mit einer Einzelleistungsvergütung in vielen EU-Systemen als Ursache der mangelnden Kostensteuerung. Derzeit leiden die Systeme in nahezu völliger Abgeschlossenheit voneinander still vor sich hin. Im Unterschied zur Industrie sind die begrifflichen und sprachlichen Kommunikationsprobleme isolationsbedingt keineswegs überwunden. Staatsferne wie in Deutschland ist nur wenigen gegeben. Zwangsläufig verwahren sich staatliche Bürokratien in solchen Ländern gegen grenzüberschreitende Kontakte durch die Akteure selbst bzw. versuchen, diese auf ein leicht kontrollierbares Spektrum an detaillierten Fragestellungen zu begrenzen.

Deutschland hat bis heute die einzige Systemvertretung auf europäischer Ebene, die allerdings in verstärktem Maß durch Einrichtungen anderer Mitgliedsstaaten kontaktiert wird. Ein Blick auf die Traditionen der nationalen Reformansätze zeigt ein durchaus technisch begrenztes Spektrum an Reformoptionen. Der etatistische Weg einer gesetzlichen Ausgabenunterdrückung bis hin zur Mangelsteuerung überwiegt dabei ebenso wie eine scheinbar nur schwer zu ändernde Anbieterorientierung und nacheilende Versuche zur einnahmeseitigen Korrektur ausgabenindizierter Probleme, unter Bedingungen hoher Arbeitslosigkeit und sinkender Reallöhne ein weitgehend hoffnungsloses Unternehmen. Insellösungen drohen als Ergebnis politisch nicht hinreichend vernetzter Prozesse. Daß es derlei schon gibt, zeigt sich unter anderem an der Vielzahl unterschiedlicher Auslandsbegriffe in der deutschen Rechtsordnung, gültig im Euroland kurz vor der Jahrtausendwende.

Politische Willensbildung tendiert dazu, aktuellen Entwicklungen hinterherzulaufen. Je schneller das Tempo der Erneuerungen, desto fühlbarer kann dies werden. Wird hier nicht gegengesteuert, so gerät die Politik in Europa kann dies exemplarisch besichtigt werden - ins Hintertreffen. Wirtschaftliche Entscheidungsprozesse gestalten sich insbesondere in der Betriebswirtschaft eben ungleich einfacher. Der rechtlich in vielfacher Weise geregelte, manche meinen auch übermäßig geregelte Gesundheitsmarkt bestimmt, dort wo zugelassen, gern länger Vorhandenes zum Bewährten, gelegentlich verbunden mit dem Anspruch auf Unveränderbarkeit. Ewigkeitsklauseln vertragen sich mit der Dynamik der heutigen Zeit und der gesellschaftlichen Tendenz zur emotionslosen Bewertung von Zweckmäßigkeit nur schwer. Zu solchen sozialökonomischen Atavismen zählen Beobachter u. a. ein steinaltes „Fremd- und Mehrbesitzverbot" für Apotheken, das Verbot von Versandapotheken oder das auffällige Festhalten niedergelassener Ärzte an einer "freien“ Einzelpraxis mit möglichst geringer Vernetzung. In den USA gehört diese Institution, salopp formuliert die "Dr.-Emma-Praxis“, schon aus Investitions- und Renditeerwägungen überwiegend der Vergangenheit an. 
Es bleibt abzuwarten, wie sich diese und andere Phänomene in der durchökonomisierten Welt des Eurolandes positionieren.

Die soziale Zukunftsbewältigung wird auf politisch gesteuerte, jedoch ökonomisch fundierte Reformanstrengungen nicht verzichten können. Verbunden mit einem nationalen politischen Kurswechsel kann sich dies aber sinnvollerweise nicht in Rückführungen unwillkommener ehemaliger Reformschritte erschöpfen, wiewohl dies in Einzelfällen sozial und politisch angezeigt sein mag. Märkte fordern für ihre Fortschreibung eine möglichst mehrseitige Interessenbefriedigung der unterschiedlichen Teilnehmer. Daraus lassen sich durchaus Konsenspotentiale ableiten, die dereinst an die Stelle ritualisierter Verkündungen weitgehend bekannter Positionen treten können. Der zu vielschichtigem Konsens zwingende EU-Einfluß dürfte hierzu gerade unter den Bedingungen eines verbreiteten und berechtigten Wunsches nach subsidiärer Gestaltungskompetenz anregen.

\section{Szenarien, Perspektiven und Provokationen}

Die EU fungiert derzeit als „ungeliebter Spiegel“ für nationale Systemwidersprüche. Will man ein hochgeregeltes Verteilungssystem durch einen sozialpolitisch gesteuerten Markt auch nur in Teilen ersetzen, so sollten die aktuellen und künftigen Einwirkungen aus Europa berücksichtigt werden. Gefahr droht dem Vorhandenen weniger von der EU als durch die vorwiegend selbstkonstruierte Fortschrittsfalle. Ein mehr oder weniger rigides Budgetsystem nebst dazu eigentlich kontraproduktiver Vergütung von Einzelleistungen steht neben einem politisch problematisch zu beherrschenden Krankenhaussektor. Letzterer weist, dies sei am Rande bemerkt, die Eigenart auf, daß ein Teil der mittelbaren oder unmittelbaren Krankenhauseigentümer als Bundesländer an der sie direkt betreffenden Gesundheitsgesetzgebung mitwirken, wohingegen anderen Hospitaleignern dies verwehrt ist. Viele dieser Instrumente sind in anderen EU-Staaten ungleich deutlicher ausgeprägt als bei uns. Jede Systemveränderung wäre dort mit noch höheren Dominowirkungen verbunden. Aus Sicht des vielfach unterrepräsentierten Patienten sind aber Anstrengungen zur Zukunftssicherung des Systems unverzichtbar. Der einfach erklärbare Wunsch nach „mehr Geld“ in einem schon für das Vorhandene unzureichenden Regelkreis ist dabei ebenso unökonomisch wie hilflos.

"Mehr Geld für Qualität an die richtige Adresse“ wäre schon ein Schritt vorwärts. Ein schwieriger allerdings, weil sich jeder automatisch selbst als Adressat versteht. Die Furcht vor dem Administrationsmoloch „Brüssel" ist weitgehend unbegründet. National gestaltbar bleibt, was sich EU- 
kompatibel verkauft und auf institutionelle Bunkermentalität und aussichtsloses Verzögernwollen zugunsten aktiver Herangehensweisen verzichtet. Personen, Politiker und Institutionen benötigen eine vorwärtsgerichtete EU-Strategie, um begreiflichen Ängsten um die eigene Fortexistenz erfolgreich begegnen zu können. Die Gesundheitsmärkte werden zögerlich, aber wohl unaufhaltsam einen sozialverträglichen Marktcharakter ausprägen, dessen Umfang sehr von der nationalen Politikgestaltung und deren Vernetzung mit Partnern abhängen wird. Mehr Zusammenwirken der Akteure ist auch und gerade für Institutionen erforderlich, die es so nur daheim gibt, weil sie in der EU keine Entsprechung haben. Daraus folgt unmittelbar der Wert ungewöhnlichen Meinungsaustausches, auch und gerade über materiell Trennendes hinweg.

Jede intellektuelle Erstarrung in Ritualen gefährdet die Qualität der Auseinandersetzung und erschwert das Finden von Schnittmengen gemeinsamer Interessen. Dies motiviert unter den Besonderheiten der Sozialbindung des Gesundheitswesens den Gesetzgeber zu Zwangslösungen, von denen gerade andere EU-Systeme schon ungleich stärker als wir geprägt sind. Der rechtzeitige Verzicht auf simplifizierende DuBotschaften, institutioneller Kompetenzgewinn durch Vorwärtsstrategien und aktive Mitwirkung am Aufbrechen nationaler und supranationaler Verkrustungen im Interesse eines Mitgestaltens der fremden und der eigenen Zukunft könnten viel bewirken. Jeder Kompetenztransfer nach Brüssel muß daheim institutionell vorbereitet werden, jede künftige Kompetenzbewahrung in Berlin ist EU-kompatibel zu organisieren. Die Herausforderungen der EU werden sich verstetigen. Große Kapitel, beispielsweise die Osterweiterung, sind dabei, den Bereich der sozialen Sicherung zu erfassen. Ein Umstand, der noch nicht eben seit langer Zeit politisch diskutiert wird. Staatsferne Systeme bewahren ihren Charakter besonders überzeugend, wenn sie in Gestalt aktiver Politikberatung die Rolle des Gesetzgebers flankieren, basisnahes Know-how anbieten und durch Beziehungsfähigkeit der unterschiedlichen Akteure geprägt sind. $\mathrm{Zu}$ innen gibt es in der EU-Vielfalt eigentlich keine Alternative, bestimmt nicht aus Sicht der Patienten oder der Leistungserbringer.

Der Aufbau der Europäischen Union fällt mit national dominierten politischen Reformen zusammen und erzwingt einen geistigen Brückenschlag. Verständlicherweise dominieren Gegenwartsängste und Unsicherheiten. Es sollte jedoch gerade kurz vor Beginn des neuen Millenniums nicht vergessen werden, daß noch unsere Groß- und Urgroßväter sich weder ein mit dem Erreichten auch nur in Bruchstücken vergleichbares Gesundheitswesen noch einen friedlichen europäischen Vereinigungsprozeß auch nur vorstellen konnten. Soziale Ausgrenzung, rigide Klassengesellschaften, Massenarmut und die Allgegenwart von Krieg, 
Not und Nationalismus kennzeichneten den Alltag vor wenig mehr als 80 Jahren. Um unsere deutsche gesellschaftliche Realität von heute beneiden uns berechtigterweise viele Völker der Welt. Ein soziales Glück in Isolation ist allerdings kaum mehr praktikabel. Das Konsensmodell EU hat Staaten eine soziale Perspektive gegeben, die noch vor einem Vierteljahrhundert zu quasi mittelalterlichen Despotien zählten. Wenn wir die Zukunft vorurteilsfrei, sachlich und entschlossen zu bewältigen trachten, so erhöhen wir die Wahrscheinlichkeit eines lebenswerten gesellschaftlichen Umfeldes für nachfolgende Generationen. 


\section{Themenkreis I}

\section{Konsequenzen einer Liberalisierung der europäischen Märkte für die deutsche Gesundheitsversorgung}

Wolfgang Gitter

Der Themenkreis 1 bezieht sich auf Konsequenzen einer Liberalisierung der europäischen Märkte für die deutsche Gesundheitsversorgung. Nun fällt es einem Juristen an sich schwer, auf eine Interpretation und Auseinandersetzung mit den drei Urteilen, die sich auf unsere Themenstellung beziehen, zu verzichten. Aber ich werde es dennoch tun, zum einen, weil hier ein sachverständiger Kreis versammelt ist, und zum anderen, weil vorhin schon darüber gesprochen worden ist. Wenn wir in der Diskussion darauf eingehen, würde ich das natürlich sehr begrüßen.

Diese drei Entscheidungen haben in der Presse, wie Sie wissen, eine große Beachtung gefunden. Es hat Schlagzeilen gegeben wie "Freier EG-Binnenmarkt rüttelt an den nationalen Sozialversicherungssystemen" oder „Müssen sich die Krankenversicherungen dem europäischen Markt öffnen?“ oder „Wann bricht der Damm des Exportverbots für andere Leistungen?". Auch in der versicherungsrechtlichen Literatur hat es eine rege Auseinandersetzung mit diesen Entscheidungen gegeben. Ich zitiere einmal aus der Zeitschrift Die Krankenversicherung. Da heißt es: „Die Verunsicherung in der Krankenkassenlandschaft ist groß. Zahlreiche Versicherte stellen bereits Kostenübernahmeanträge für Auslandsbehandlungen. Ausländische Leistungserbringer beantragen Versorgungsverträge mit inländischen Krankenkassen. Einige von innen entdecken die Kostenerstattung für Auslandsbehandlungen als Wettbewerbsfeld."

Außer acht gelassen wird hierbei, welche Folgewirkungen eine solche Kostenübernahme hat. Völlig ungeklärt ist beispielsweise, welche Anforderungen an die ausländischen Leistungserbringer gestellt werden können und müssen. Sind die inländischen Qualitätsstandards uneingeschränkt auf Auslandsbehandlungen übertragbar? Welche Instrumentarien müssen geschaffen werden, um die Qualität der Leistungserbringung im Ausland zu prüfen? Wer trägt die hiermit verbundenen Kosten? Wie wirkt sich eine Kostenerstattung auf inländische Zulassungs- und Bedarfsplanregelungen aus?

All diese Problemstellungen sind von dem Veranstalter unserer Tagung thematisiert worden. Das erste Unterthema, die ärztliche Bedarfspla- 
nung, geregelt in den $\S \S 99 \mathrm{ff}$. SGB V, ist ein viel diskutiertes und umstrittenes Feld. Das gilt insbesondere für § 102 SGB V, der ab 01.01.1999 die Zulassung aufgrund von gesetzlich festgelegten Verhältniszahlen vorsieht. Umstritten sind aber auch die gesetzlichen Altersgrenzen in $\S 98$ Abs. 2 Ziff. 12 SGB V, die einen Ausschluß der Zulassung von Ärzten, die das 55. Lebensjahr vollendet haben, vorsieht und $\S 95$ Abs. 7 Satz 2 SGB V, nach dem ab 01.01.1999 die Zulassung am Ende des Kalendervierteljahres endet, an dem der Vertragsarzt sein 68. Lebensjahr vollendet. Ich gehe davon aus, daß die Referenten aus verfassungs- und europarechtlicher Sicht die im deutschen Recht geregelte Bedarfsplanung und -zulassung prüfen werden. Selbst wenn man die Zulässigkeit bejaht, was ja höchst umstritten ist, stellt sich doch die Frage, ob man den deutschen Arzt von der kassenärztlichen Versorgung ausschließen kann, wenn man dem ausländischen Arzt ohne weitere Prüfungen diese Leistungen eröffnet. Grundsätzlich fehlen ja für ausländische Leistungen der ärztlichen Behandlung die im deutschen Recht vorgesehenen Sicherungs- und Kontrollinstrumente. Ferner entstehen Finanzierungsprobleme, da sich die Kostenerstattung ausländischer Arztleistungen doch auch auf die Finanzierung der inländischen Leistungen auswirken würde. Hier könnte die Rechtsprechung des EuGH Bedeutung gewinnen, die als Rechtfertigungsgrund für mögliche Beschränkungen ausländischer Leistungserbringung die erhebliche Gefährdung des finanziellen Gleichgewichts von sozialen Sicherungssystemen nennt. Es ergeben sich also nach meiner Auffassung bei den deutschen Bedarfsplanregelungen auch erhebliche Probleme im Hinblick auf die ausländische Leistungserbringung.

Bei den weiteren Unterthemen geht es um einzelne Leistungsbereiche wie die stationären Leistungen, Kuren und Rehabilitation. Man wird auch hier fragen müssen, ob selbst dann, wenn eine Beschränkung der Kostenerstattung auf inländische Pflegesätze erfolgt, sich Auswirkungen auf das nationale Planungs- und Finanzierungssystem ergeben. Es könnte bei der Erbringung der genannten Leistungen im Ausland das System der Bedarfsplanung mit der Garantie flächendeckender Versorgung gefährdet werden. Dadurch wäre möglicherweise wiederum ein Rechtfertigungsgrund gegeben, den der EuGH ebenfalls für die Beschränkung der Öffnung für ausländische Leistungen nennt, nämlich die Gefährdung einer ausgewogenen, allen zugänglichen ärztlichen und klinischen Versorgung. Bei Kuren und Rehabilitationen wäre aufgrund der Attraktivität von Auslandsaufenthalten eine verstärkte Inanspruchnahme ausländischer Einrichtungen zu erwarten. Die Folge davon wäre, daß inländische Einrichtungen z. T. nicht mehr ausgelastet wären. Eine Schließung der Einrichtungen wäre aber wohl nur eingeschränkt möglich, da flächendeckende Versorgungsstrukturen für die Versicherten un- 
verzichtbar sind. Die Folge wäre auch in diesem Bereich eine erhebliche Kostenzunahme.

Ein weiteres Unterthema sind die sonstigen veranlaßten Leistungen. Ich gehe davon aus, daß von den Referenten das Thema „Arznei-, Heil- und Hilfsmittel" angesprochen wird. Bei im Ausland eingekauften Arzneimitteln könnte wohl, soweit im Inland Festbeträge festgesetzt sind, eine Kostenerstattung nur in dieser Höhe erfolgen. Außerdem wäre zu erörtern, ob und wie man die Zuzahlung sicherstellen will. Im Rahmen der derzeitigen Arzneimittelbudgets könnte es ferner zu Mehrbelastungen kommen, wenn es nicht gelingt, eine Herausrichtung der Kosten für ausländische Arzneimittel aus den Budgets zu vereinbaren. Bei Heilmitteln entstehen ähnliche Probleme, da auch hier Budgetierungen bzw. arztgruppenspezifische Richtgrößen bestehen. Es müßte also auch hier auf vertragspolitischem Wege versucht werden, die ausländischen Leistungen aus dem Verordnungsvolumen herauszunehmen, da sonst zusätzliche Kosten entstehen. Bei Hilfsmitteln schließlich müßte die Kostenerstattung auf den nationalen Festbetrag bzw. nationale Preisvereinbarungen beschränkt werden. Probleme sehe ich hier auch im Hinblick auf die Durchsetzung von Gewährleistungsansprüchen. Bei fehlender Gewährleistung würden den Kassen zusätzliche Kosten entstehen.

Lassen Sie mich damit meinen kurzen Überblick über den Themenkreis 1 und einige Problemfelder abschließen. Wir Juristen sind ja immer ein wenig Bedenkenträger und sehen überall Problemfelder entstehen, aber ich hoffe, daß unsere Diskussion zur Klärung der Problemfelder beiträgt. Eine rechtspolitische Diskussion, bei der ein europäisches Gesundheitsversorgungssystem zugrunde gelegt wird, hielte ich allerdings aufgrund der erheblichen nationalen Unterschiede gegenwärtig nicht für sinnvoll, und ich habe es begrüßt, daß dies bereits in den einleitenden Statements so klar zum Ausdruck gebracht worden ist. Es dürfte auch noch einige Zeit bei der gegenwärtigen Rechtslage bleiben, nach der aufgrund von Art. 51 EG-Vertrag nur eine Koordinierung der nationalen Sozialversicherungssysteme, aber kein Gemeinschaftssystem geschaffen werden kann. Wie diese Koordinierung und Vernetzung erfolgen kann, wird Gegenstand unserer Erörterungen sein, und ich darf den ersten Referenten, Herrn Dr. Klemm, bitten, das Wort zu seinem Vortrag „Ärztliche Bedarfsplanung“ zu ergreifen. 


\section{Ärztliche Bedarfsplanung}

Helmut Klemm

„Die Kassenärtlichen Vereinigungen haben im Einvernehmen mit den Landesverbänden der Krankenkassen und den Verbänden der Ersatzkassen sowie im Benehmen mit den zuständigen Landesbehörden nach Maßgabe der von den Bundesausschüssen erlassenen Richtlinien auf Landesebene einen Bedarfsplan zur Sicherstellung der vertragsärztlichen Versorgung aufzustellen und jeweils der Entwicklung anzupassen.

Die Ziele und Erfordernisse der Raumordnung und Landesplanung sowie Krankenhausplanung sind zu beachten. Der Bedarfsplan ist in geeigneter Weise zu veröffentlichen."

So, meine sehr verehrten Damen und Herren, steht es im § 99 SGB V niedergeschrieben. Ärztliche Bedarfsplanung? Welche semantischen Wortspielereien verbergen sich dahinter?

Über die Interpretation dieses Begriffes läßt sich trefflich diskutieren.

Zunächst einmal ist es unstreitig, daß der Gesetzgeber damit versucht, die Zahl an Ärzten zu definieren, die nach seiner Meinung notwendig, ausreichend und zweckmäßig für die ambulante ärztliche Versorgung der Bevölkerung vorzuhalten ist.

Vielleicht könnte aber auch damit gemeint sein - und erlauben Sie mir diese gedanklichen Ausflüge -, daß Ärzte den Bedarf planen, wobei Kritiker sofort die Frage stellen würden: Meinen Sie den Bedarf an notwendig zu erbringenden Leistungen aufgrund klar definierter Indikationsstellungen oder verstehen Sie eher darunter, den eigenen Bedarf an finanziellen Ressourcen zu sichern?

Es läßt sich aber gar eine dritte Interpretationsvariante diskutieren, nämlich aus der Sicht des Patienten, der da fragen möge: Wieviel Ärzte und welche Art von Ärzten benötige ich als Patient, um allzeit eine gute Gesundheit zu genießen?

Die hier aufgeworfenen Fragen lassen erkennen, welch großer Spannungsbogen sich einerseits von staatlich reglementierender, gesetzlich verankerter Bedarfsplanung bis hin zur optimalen Inanspruchnahme aller Dienstleistungssparten im Gesundheitswesen durch den Patienten spannen läßt. 
Der „allgemein bedarfsgerechte Versorgungsgrad“ ist das grundlegende Strukturelement des neuen Planungssystems. Versorgungsgrad ist eine Relation, welche $\S 101$ SGB V als „Verhältnis“ beschreibt. Was in ein Verhältnis zu setzen ist, regelt $\S 101$ allerdings nicht. Gemeint ist wohl das Verhältnis von Arztzahlen zu Bevölkerungszahlen. Damit steht eindeutig fest, daß es sich hier um eine rein statistische Ausgangsgröße handelt, die den allgemein bedarfsgerechten Versorgungsgrad definiert. Um die vom Gesetzgeber so definierte ärztliche Bedarfsplanung nicht gänzlich zur statistischen Zahlenspielerei verkommen zu lassen, war der Gesetzgeber so klug, auch qualitative Ausnahmeregelungen zuzulassen, nach denen ausnahmsweise eine Zulassung trotz Zulassungsbeschränkung gestattet werden kann, wenn zur Wahrung der Qualität der ärztlichen Versorgung in einem Versorgungsbereich dieses unerläßlich erscheint. Bereits an dieser Stelle sei die Frage erlaubt, ob die §§ 99, 101 und 102 SGB V inhaltlich ihre Aufgaben erfüllt haben. Bei kritischer Würdigung muß diese Frage eigentlich mit einem klaren „Nein“ beantwortet werden, denn die Entwicklung der Zahl der Vertragsärzte ist disproportional zur Entwicklung der Zahl der Mitglieder der gesetzlichen Krankenversicherung verlaufen. Auch wenn aus Sicht des Bundesverfassungsgerichts die Zulassungssteuerung im Hinblick auf die Finanzsituation der gesetzlichen Krankenversicherungen unausweichlich war, so hat die Regelung faktisch keinen Beitrag zur finanziellen Stabilisierung der gesetzlichen Krankenversicherung geleistet, da es - wie bekannt ist - durch die Übergangsregelung zu einem weit überproportionalen Zustrom von Vertragsärzten in die vertragsärztliche Versorgung 1993 gekommen ist.

\section{Statistische Betrachtung}

Kamen 1960 auf einen berufstätigen Arzt 779 Einwohner, so wurden 1997290 Einwohner von einem berufstätigen Arzt versorgt. In absoluten Zahlen ausgedrückt bedeutet dies, daß sich die Zahl der berufstätigen Ärzte 1960 von 93.905 auf 282.737 im Jahr 1997 verändert hat.

Bei den Zahnärzten sind derartige dramatische Entwicklungen nicht festzustellen: Kam 1960 auf 1.882 Einwohner ein Zahnarzt, so stand 1997 ein Zahnarzt 1.323 Einwohnern zur Verfügung. Hier wurden längst nicht in dem Ausmaß die Überkapazitäten aufgebaut, die heute in der vertragsärztlichen Landschaft der Humanmedizin bestehen. Spätestens die statistischen Zahlen beweisen eindeutig, daß zu viele Ärzte im System sind. Diese Aussage gilt allerdings nur unter rein statistischen Gesichtspunkten. Da das Arzt-Patienten-Verhältnis zunächst einmal grundsätzlich ein subjektiv gefärbtes ist, wird es auch bei subtilster, staatlich verordneter Bedarfsplanung nach wie vor sehr stark frequentierte Arzt- 
praxen geben und weniger gut besuchte. Damit kann auch belegt werden, daß die vom Gesetzgeber verordnete ärztliche Bedarfsplanung den individuellen Bedürfnissen der zu versorgenden Patienten nicht gerecht werden kann, vielleicht auch gar nicht will, da der Gesetzgeber ausschließlich übergeordnete vom Gemeinwohl getragene Interessen zu verfolgen hat.

Es gilt festzuhalten, daß die gesetzlich bestimmte ärztliche Bedarfsplanung nicht zur finanziellen Sicherung des GKV-Systems in dem Sinne beiträgt, wie das inre Initiatoren sich gewünscht hatten. Allerdings wird das Mindestziel erreicht, daß eine bevölkerungs- und gemeindeorientierte Versorgung im Gesundheitswesen weitestgehend garantiert wird. Das drückt sich allein durch einen in Deutschland erzielten Versicherungsgrad von knapp $90 \%$ aus.

Bevölkerungsbezogene Versorgungsplanungen haben im europäischen System eine Tradition, die allerdings weitestgehend systemabhängig ist. Auf der Angebotsseite gibt es in Deutschland im ambulanten Bereich eine landesbezogene Planungsebene. Die Kassenärztlichen Vereinigungen der Länder und die Krankenkassen üben gemeinsam die Bedarfsplanungskompetenz für den ambulanten Sektor aus. Dabei gilt es allerdings, eine Besonderheit zu beachten. Diese Systembesonderheit besteht in einer Mixtur aus öffentlichen und privaten Strukturelementen.

Einerseits haben wir öffentlich regulierte Verhältniszahlen von Vertragsärzten zu Einwohnern in verwaltungsbezogenen Planungseinheiten. Andererseits benutzen die Ärzte auf der Anbieterseite privatwirtschaftliche Komponenten mit Entscheidungsfreiheit hinsichtlich der Niederlassung und der zu tätigenden unternehmerischen Praxisinvestitionen.

Ärztliche Bedarfsplanung zu verstehen als eine von Ärzten definierte Bedarfsplanung könnte auch synonym als anbieterorientiertes Gesundheitsmanagement definiert werden. Dafür gibt es allerdings keine wissenschaftlich entwickelten Grundlagen im Sinne einer realen Bedarfsforschung. Einen echten Bedarfsbegriff qualitativer Art gibt es nicht. Denn die bei anbieterorientierten Planungsprozessen gegenwärtig überwiegend einbezogenen Expertenmeinungen weisen erhebliche Varianzen und prognostische Unsicherheiten sowie Interessenbindungen auf. Wenn die Bedarfsplanung z. B. durch Experten erfolgt, die selbst Anbieter von Gesundheitsleistungen sind, so hat die Erfahrung gezeigt, daß z. B. bei einer bestehenden Einzelleistungsvergütung der auf diese Weise professionell definierte Bedarf tendenziell stets über dem realen Bedarf liegt. 
Ärztliche Bedarfsplanung aus der Sicht des Patienten stellt sich wiederum anders dar. Da die Bedürfnisse der Patienten individuell stark verschieden sind, ist eine einheitliche Definition der subjektiven Patientenbedürfnisse zur ärztlichen Bedarfsplanung sehr schwierig. Zwei Grundtypen von Patienten lassen sich dabei unterscheiden: Die einen suchen und verlangen mehr Information über ihr Problem, die anderen vermeiden eine weitgehende Aufklärung über ihre Krankheit, insbesondere dann, wenn es sich um negative Prognosen handeln könnte.

Patienten sehen als eines ihrer zentralen Rechte die freie Arztwahl an. Die freie Arztwahl ermöglicht allen Versicherten, einen Arzt zu wählen, der ihren Präferenzen entspricht und den Arzt bei einer Vertrauenskrise auch zu wechseln. Dieses Recht, das durch die Einführung der Krankenversichertenkarte noch gestärkt worden ist, nimmt entsprechend bei der Bevölkerung einen hohen Stellenwert ein. Rund 90 \% der Bevölkerung hält es für nicht vertretbar, dieses Recht aus Kostengründen einzuschränken, wobei es zwischen den einzelnen Bevölkerungsgruppen keine nennenswerten Unterschiede gibt. Auch ohne tatsächliche Ausübung eines Arztwechsels, der faktisch nur eine geringe Rolle spielt, sichert allein die Möglichkeit dieses Arztwechsels die Patientenautonomie. Dieses von den Patienten so hoch eingeschätzte Gut der freien Arztwahl leitet über zur Frage des systemorientierten Managements bei der ärztlichen Bedarfsplanung. Es führt zu der Frage, ob die Einführung eines Primärarztsystems unter Wahrung qualitativer Aspekte langfristig ein Beitrag zur Finanzierbarkeit der gesetzlichen Krankenversicherung sein kann. Dem Primärarztkonzept liegt die Vorstellung zugrunde, daß ein Hausarzt in der Lage ist, ernste Erkrankungen von Befindlichkeitsstörungen zu trennen, aber auch ernste unter scheinbar trivialen Erkrankungen herauszufinden, und daß er weitergehende medizinische Untersuchungen nur dann veranlaßt, wenn sie streng indiziert sind.

Bis heute konnte, zumindest gilt das für Deutschland, an keiner Stelle der Beweis erbracht werden, daß ein Primärarztsystem tatsächlich kostensparend sein kann. Vielleicht hilft hier der Blick über die Grenzen weiter.

In England sind $99 \%$ der Bevölkerung bei einem der mehr als 30.000 Allgemeinärzte eingeschrieben. Im Durchschnitt versorgt ein Allgemeinarzt jeweils ca. 1.900 Versicherte. Die Versicherten können einen Arzt wählen, sofern dieser noch Platz auf seiner Liste hat. Jeweils nach einem Jahr ist ein Wechsel zu einem anderen Arzt ohne Begründung möglich. Die Tätigkeiten des Allgemeinarztes umfassen: 
- Kurative Medizin, einschl. Pädiatrie und Gynäkologie,

- Präventions- und Rehabilitationsleistungen,

- Screening-Untersuchungen, wie z. B. gynäkologische Vorsorgeuntersuchungen,

- kleine Chirurgie sowie Basislabor.

Die dafür notwendige technische Ausstattung der Praxen liegt deutlich unter den Gegebenheiten in Deutschland. Ein EKG ist in der Regel nicht obligat, schon gar nicht ein Sonographiegerät. Die Vergütung erfolgt weitestgehend nach Pauschalen. Da eine Pauschalvergütung die Gefahr der Unterversorgung von Patienten in sich trägt, werden neben Pauschalen Impfungen, Schwangerenvorsorgen, Nachtbesuche und chirurgische Leistungen als Einzelleistungen vergütet.

Das System hat allerdings ein großes Problem: Das sind die eingeschränkten Kapazitäten im fachärztlichen Bereich. Selbst für Patienten mit schwersten Erkrankungen werden Wartelisten geführt, und nicht selten kommt es vor, daß die Patienten versterben, bevor sie den Spezialisten aufsuchen konnten.

Von diesen Mängeln des staatlichen Gesundheitsdienstes hat in jedem Fall der private Sektor profitiert, da eine Behandlung in Privatkliniken und bei Privatärzten eine Möglichkeit darstellt, die Wartelisten zu umgehen. Annähernd $15 \%$ der britischen Bevölkerung sind zwischenzeitlich privat versichert.

In den Niederlanden erfolgt die ambulante ärztliche Versorgung fast ausschließlich durch Allgemeinärzte, die im Durchschnitt knapp über 2.000 Patienten betreuen. Der Allgemeinarzt in den Niederlanden wird als echter Gatekeeper im System gesehen. Jede Überweisung zum Spezialisten oder ins Krankenhaus muß durch den Allgemeinarzt erfolgen. Dies gilt sowohl für die Sozialversicherten als auch für die ca. $30 \%$ Privatversicherten. Die Aufgaben des Hausarztes sind in erster Linie die Akutversorgung, die Dauerbehandlung chronisch Kranker und die häusliche Betreuung terminal kranker Patienten. Im Gegensatz zu Deutschland genießt die Allgemeinmedizin in den Niederlanden bereits während der Ausbildung an den Hochschulen einen hohen Stellenwert. In allen medizinischen Ausbildungsstätten gibt es einen Lehrstuhl für Allgemeinmedizin. Bereits bei der Vergabe der Weiterbildungsstellen nach Abschluß des Studiums wird darauf geachtet, daß nur motivierte Bewerber für die Allgemeinmedizin zugelassen werden. 
Für zahlreiche Krankheitsbilder, die in der ambulanten Versorgung von Bedeutung sind, gibt es klar definierte Leitlinien. Auch in den Niederlanden ist die personelle und apparative Ausstattung des Allgemeinarztes im Gegensatz zu Deutschland sehr bescheiden. Im Durchschnitt kommen 0,8 Hilfspersonen auf einen Allgemeinarzt. In Deutschland beträgt die Durchschnittszahl 2,3 Hilfskräfte pro Arzt.

Die Fachärzte sind in den Niederlanden fast ausschließlich an den Kliniken tätig. Es gibt allerdings im Gegensatz zu England keine Wartezeiten. Bei den sozialversicherten Patienten erhält der Allgemeinarzt eine Pauschale, bei Privatpatienten eine Einzelleistungsvergütung. Die Pauschale ist entsprechend der Patientenzahl gestaffelt und nimmt bei höheren Patientenzahlen prozentual ab.

Ohne auf weitere Beispiele in anderen Ländern eingehen zu wollen, können folgende Vor- und Nachteile eines Primärarztsystems im europäischen Raum beschrieben werden: Unabhängig davon, ob ein Patient aktuell erkrankt ist oder nicht, führt die Einschreibung bei einem Primärarzt in der Regel zu einer positiven Bindung zwischen Arzt und Patient. Der Versicherte verfügt über einen kompetenten Ansprechpartner in allen Dingen, die Gesundheit und Krankheit betreffen. Im Fall des erneuten Arztkontaktes kann im Primärarztsystem auf die bereits vorliegende Krankengeschichte zurückgegriffen werden. Doppeluntersuchungen können vermieden werden. Der Primärarzt fungiert somit als eine ärztliche Koordinierungsinstanz, bei der sämtliche Verordnungen für einen Patienten, Vor- und Begleiterkrankungen dokumentiert und zusammengeführt werden. Diesen Vorteilen eines Primärarztsystems steht der Verzicht auf freie Arztwahl gegenüber, das als Prinzip überzeugt und für den Aufbau eines tragfähigen Vertrauensverhältnisses auch sachlich begründet werden kann.

Es erhebt sich an dieser Stelle die Frage, ob ein Primärarztsystem als Instrument ärztlicher Bedarfsplanung für die Weiterentwicklung des Gesundheitswesens in Deutschland gelten kann.

Berücksichtigt man, welche große Bedeutung die Gewöhnung an ein System für die Patientenzufriedenheit hat, würde eine plötzliche Umstellung in Deutschland auf ein Primärarztsystem wahrscheinlich auf einen großen Widerstand in der Bevölkerung stoßen.

Dagegen spricht auch, daß in Deutschland die Qualifikation im Gegensatz zu Holland von praktischen Ärzten und Allgemeinmedizinern zu uneinheitlich ist, um das Gesamtsystem entsprechend umzuorientieren. 
Denn die Einführung eines Primärarztsystems setzt unabdingbar ein hohes Weiterbildungsniveau in der Allgemeinmedizin voraus.

Deshalb gewinnt der Vorschlag der Kassenärztlichen Bundesvereinigung im Eckpunktepapier zunehmend an Bedeutung. Danach sollte jeder Versicherte pro Quartal einen Arzt seines Vertrauens im Sinne der freien Arztwahl sowohl für den hausärztlichen Bereich als auch für den fachärztlichen Bereich auswählen können. Die Mehrfachinanspruchnahme von Ärzten derselben Fachgruppe im selben Quartal durch eigene Entscheidung des Versicherten müßte danach jedoch im finanziellen Verantwortungsbereich des einzelnen zwangsmäßig verankert werden, um das Gesamtsystem zu entlasten.

Die ärztliche Bedarfsplanung in Deutschland muß auch unter dem jüngst ergangenen Urteil des Europäischen Gerichtshofes (EuGH) in den Rechtssachen Kohll und Decker betrachtet werden. Der frühere Bundesgesundheitsminister Horst Seehofer hat im Rahmen des ergangenen Urteils immer von einer erheblichen Gefährdung des finanziellen Gleichgewichts des Systems der gesetzlichen Krankenversicherung in Deutschland gesprochen. Ob die praktischen Auswirkungen der Urteile tatsächlich so weitreichend sind, um damit eine Einschränkung der Vertragsfreiheiten zu rechtfertigen, läßt sich z. Z. nicht absehen. Sprachbarrieren und Reisekosten lassen jedoch erwarten, daß sich die Leistungen auf deutsche Tourismuszentren und grenznahe Regionen beschränken werden. Nach Angaben des Bundesgesundheitsministeriums hat die gesetzliche Krankenversicherung 1997 für sämtliche im Ausland gegenüber gesetzlich Versicherten erbrachten Leistungen knapp 700 Mio. DM aufgewendet. Diese Aufwendungen stellen gemessen an den gesamten Ausgaben für Leistungen der gesetzlichen Krankenversicherung nur ca. $0,3 \%$ dar. Somit werden bereits jetzt Auslandsleistungen, die außerhalb der inländischen Steuerungsmechanismen erbracht werden, von den Kassen erstattet, ohne daß gleichzeitig behauptet werden könnte, es trete eine Systemgefährdung insgesamt ein.

Die derzeitige Rechtslage beschränkt die Krankenkassen darauf, nur mit inländischen Leistungserbringern in Vertragsbeziehungen treten zu können. Die Möglichkeit vertraglicher Vereinbarungen mit Ärzten aus anderen EG-Mitgliedsstaaten zur Leistungserbringung gegenüber Versicherten scheidet somit aus. Es kommt damit nur die durch die EuGH-Urteile vorgesehene Kostenerstattung nach Inlandssätzen in Betracht. Allerdings könnte der Gesetzgeber im Rahmen einer gesamteuropäischen Harmonisierung die Kassen gesetzlich dazu ermächtigen, Verträge auch mit Leistungserbringern aus anderen EG-Mitgliedsstaaten abschließen zu dürfen. Nähme ein deutscher Kassenpatient dann in einem anderen 
EG-Mitgliedsstaat Leistungen eines solchen EG-Vertragsarztes in Anspruch, so empfänge er Sachleistungen, die im Verhältnis zwischen Arzt und Krankenkasse abgerechnet würden.

Auch für die inländischen Ärzte in Deutschland eröffnen die EuGHUrteile Chancen, Patienten aus anderen EG-Mitgliedsstaaten zur Behandlung anzunehmen. Damit könnte sich den deutschen Ärzten ein neues Klientel erschließen.

Mittelfristig ist allerdings nicht davon auszugehen, daß die Z.Z. gültige EuGH-Rechtsprechung die national geregelte ärztliche Bedarfsplanung in Deutschland beeinflussen wird.

\section{Fazit}

1. Gesetzlich bestimmte ärztliche Bedarfsplanung hat nicht verhindern können, daß zu viele Ärzte im System sind.

2. Auch das Psychotherapeutengesetz bringt zusätzliche Leistungserbringer in großer Zahl ins System bei gleichzeitig begrenzten finanziellen Mitteln, die - so ist zu befürchten - die Finanzierbarkeit sprengen werden, so daß auch dadurch bewiesen ist, daß staatliche Reglementierung nicht den tatsächlichen Bedarf regeln kann, wenn nicht gleichzeitig gesellschaftspolitisch entschieden wird, wieviel Geld für welche Leistungen zur Verfügung stehen soll.

3. Ein Primärarztsystem als Instrumentarium einer systemorientierten Bedarfsplanung ist derzeit in Deutschland nicht durchführbar, da die qualitativen Voraussetzungen auf der Anbieterseite einerseits und die gewohnte Erwartungshaltung der Bevölkerung andererseits in einem solchen System nicht in Einklang zu bringen sind.

4. Eine indirekte Steuerung der ärztlichen Bedarfsplanung durch eine politisch verantwortete sektorale Budgetierung der einzelnen Leistungsbereiche ist schon deshalb abzulehnen, weil sie eine ausschließliche Kostendämpfungsmaßnahme darstellt, die dem tatsächlichen Bedarf der medizinischen Inanspruchnahme auf hohem Niveau nicht gerecht werden kann.

5. Die Ärzteschaft selbst muß inren eigenen Beitrag zur Implementierung neuer Versorgungsstrukturen liefern, indem Netzstrukturen oder Verbundstrukturen durch sie selbst konzipiert werden, um unter Rückbesinnung auf ihr Anbietermonopol einerseits eine wirtschaftliche Verwendung der finanziellen Mittel zu gewährleisten, 
andererseits aber auch ihre zentrale Bedeutung im Gesundheitssystem unter Beweis zu stellen.

6. Im Rahmen derartiger Wandlungsprozesse wird es zwangsläufig zu einem Selektionsprozeß innerhalb der Ärzteschaft kommen, da nicht für jeden Arzt in jedem Netz ein Platz frei sein wird.

7. Nur durch die Schaffung neuer Strukturen wird sich die vertikale Konkurrenzsituation zwischen einzelnen Arztgruppen und insbesondere zwischen niedergelassenen Fachärzten und dem Krankenhaus auf Dauer vermeiden lassen. Darüber hinaus würden diese Strukturen dem Patienten weiterhin unter dem Aspekt der freien Arztwahl den Zugang zu einem wirklich breiten Angebot qualifizierter ärztlicher Leistung ermöglichen. Ärzte müssen sich deshalb verstärkt für die Erprobung neuer Modelle einsetzen. Nur so können sie Handlungsvollmacht zurückgewinnen und Selbstbestimmung statt Fremdbestimmung erreichen. Es ist sowohl ökonomisch als auch medizinisch der falsche Weg, wenn permanent versucht wird, mit einer Verschärfung der bürokratischen und staatlichen Regulierung, mit Budgets und Richtgrößen sowie einer ärztlichen Bedarfsplanung im Sinne einer Zulassungssperre für Ärzte die Ausgabenentwicklung im Gesundheitswesen in Deutschland in den Griff bekommen zu wollen. Alle Beteiligten im Gesundheitswesen haben nicht mehr viel Zeit, nach Lösungen zu suchen. Da die meiste Zeit allerdings damit verlorengeht, daß man nicht zu Ende denkt, sollte sich keine Gruppierung mehr einer aussichtsreichen Lösung verweigern. 


\section{Ärztliche Bedarfsplanung}

\section{Konsequenzen einer Liberalisierung der europäischen Märkte für die deutsche Gesundheitsversorgung}

Doris Pfeiffer

\section{Die Urteile des Europäischen Gerichtshofes (EuGH) in den Fällen Kohll/Decker}

Der Europäische Gerichtshof hat mit zwei Urteilen vom 28.04.1998 (Fälle Kohll/Decker) entschieden, daß eine nationale Regelung, die die Erstattung der Kosten für Zahnbehandlung durch einen Zahnarzt in einem anderen Mitgliedsstaat nach den Tarifen des Versicherungsstaates von der Genehmigung der Krankenkasse abhängig macht, gegen Artikel 59 und 60 EG-Vertrag (Freiheit des Dienstleistungsverkehrs) verstößt. Gegen Artikel 30 und 35 EG-Vertrag (Freiheit des Warenverkehrs) verstößt eine Regelung, die eine Kostenerstattung für eine im EU-Ausland gekaufte Brille von einer vorherigen Genehmigung der Krankenkasse abhängig macht.

In beiden Entscheidungen wiederholt der EuGH seine ständig vertretene Auffassung, daß das Gemeinschaftsrecht die Zuständigkeiten der Mitgliedsstaaten zur Ausgestaltung inrer sozialen Sicherungssysteme unberührt läßt. Bei der Ausübung dieser Rechte müssen die Mitgliedsstaaten jedoch das Gemeinschaftsrecht beachten. Die Tatsache, daß die streitigen Regelungen zum Bereich der sozialen Sicherung gehören, schließt die Anwendung der Grundfreiheiten des freien Warenverkehrs und der Dienstleistungsfreiheit nach Artikel $30 \mathrm{ff}$. und $59 \mathrm{ff}$. EG-Vertrag nicht aus. Nach Auffassung des EuGH sind deshalb Genehmigungsvorbehalte, die eine Krankenversicherung bei der Inanspruchnahme von Leistungen im EU-Ausland macht, Eingriffe in den Anwendungsbereich der Grundfreiheiten, weil damit die Ausübung dieser Freiheiten unmittelbar oder mittelbar, tatsächlich oder potentiell behindert oder erschwert wird.

Der EuGH sieht allerdings den Geltungsbereich der Grundfreiheiten nicht unbegrenzt. Aufgrund von Rechtfertigungsgründen, deren Vorliegen zu prüfen ist, kann der Geltungsbereich beschränkt werden. Einer der Rechtfertigungsgründe ist eine erhebliche Gefährdung des finanziellen Gleichgewichts des Systems der sozialen Sicherung. Dabei müßten wesentliche Auswirkungen auf die Finanzierung der Systeme der sozialen Sicherung, die zudem nachzuweisen sind, zu erwarten sein. In den beiden vorliegenden Verfahren sah der EuGH eine solche Ge- 
fährdung nicht als gegeben. Darüber hinaus könnten Gründe der öffentlichen Gesundheit (Art. 36 und 66 i. V. m. 56 EG-Vertrag) für eine Einschränkung der Grundfreiheiten maßgebend sein. Darunter faßt der EuGH sowohl die Qualitätssicherung als auch die Aufrechterhaltung einer ausgewogenen, allen zugänglichen ärztlichen und klinischen Versorgung sowie die Gefährdung der öffentlichen Sicherheit und Ordnung. All diese Gründe sieht der EuGH jedoch in den vorliegenden Fällen nicht als gegeben an.

Die Entscheidungen des EuGH haben für erheblichen Wirbel in der deutschen Krankenversicherungsszene - ebenso wie in den Medien gesorgt. Dabei geht es zentral um die Frage, inwieweit die Urteile Auswirkungen auf die Praxis im deutschen Gesundheitswesen haben. Zunächst handelt es sich um Entscheidungen über luxemburgische Fälle, die rein formal nur zwischen den Prozeßparteien wirken. Allerdings entscheidet der EuGH gemäß Art. 177 EG-Vertrag über die Auslegung dieses Vertrages. Gesetzgebung, Rechtsprechung und Verwaltung der Mitgliedsstaaten haben die Entscheidungen bei der Auslegung und Anwendung europäischen Rechts zu würdigen und zu berücksichtigen.

Das Bundesgesundheitsministerium - noch unter der Leitung von Horst Seehofer (CSU) - forderte kurz nach der Entscheidung eine sehr restriktive Handhabung von eventuell seitens der Versicherten an die deutschen Krankenkassen herangetragenen Erstattungsansprüchen, was sich in einem entsprechend formulierten Schreiben des Bundesversicherungsamtes als Aufsichtsbehörde der bundesweit tätigen Krankenkassen niederschlug. Gleichzeitig wurde von seiten der - überwiegend SPD-geführten - Bundesländer, die die Aufsicht über die regional tätigen Krankenkassen führen, eine vermeintlich versichertenfreundliche Auslegung der Urteile im Sinne einer großzügigen Erstattungspraxis favorisiert. Sowohl das Bundesgesundheitsministerium und die Spitzenverbände der Krankenkassen als auch die Gesundheitsministerkonferenz der Länder sahen es als notwendig an zu klären, wie die konkreten Auswirkungen der Urteile eingeschätzt werden. Ziel einer gemeinsamen Arbeitsgruppe sollte es vor allem sein, eine gemeinsame Position zu erzielen, um eine unterschiedliche Behandlung von Versicherten regionaler und bundesweiter Krankenkassen im Wettbewerb durch die jeweiligen Aufsichtsbehörden zu vermeiden.

Zwischenzeitlich wurde deutlich, daß weder die eine noch die andere Extremposition aufrechtzuerhalten war. Zum einen wurde deutlich, daß der Umfang der im EU-Ausland in Anspruch genommenen Leistungen (noch) relativ gering ist. Zur Zeit entstehen nach Angaben des Bundesgesundheitsministeriums für die Inanspruchnahme von Gesundheits- 
leistungen im Ausland und die Erstattung durch inländische Krankenkassen Ausgaben in Höhe von ca. 760 Mio. DM pro Jahr, d. h. ca. 0,3\% der Gesamtausgaben der gesetzlichen Krankenversicherung. Vermutlich führt die durch die Einführung des EURO zu erwartende Transparenz zu einem verstärkten Interesse der Versicherten an Leistungsinanspruchnahme im EU-Ausland. Dennoch ist aufgrund der fehlenden finanziellen Anreize, der organisatorischen Hemmnisse (notwendige Reisen) und der Sprachbarrieren auch zukünftig nicht mit einer erheblichen Ausweitung zu rechnen. Dennoch sind die Konsequenzen der Übertragung der EuGH-Urteile auf die deutschen Verhältnisse nicht zu vernachlässigen, dann nämlich, wenn die Wirkungen auf die inländischen Steuerungsmechanismen vor allem im Rahmen des Sachleistungsprinzips untersucht werden. Eine wesentliche Problematik wird dabei in der von Leistungserbringerseite eingebrachten Argumentation der Inländerdiskriminierung durch restriktive Steuerungsinstrumente, wie z. B. die Bedarfsplanung, gesehen. Auch wenn die rechtliche Relevanz nicht gegeben ist, so muß doch mit erheblichem politischen Druck gerechnet werden.

Die Arbeitsgruppe verständigte sich in ihren Beratungen auf eine Anwendung derart, daß die EuGH-Urteile unmittelbar für alle Leistungen gelten, die im Rahmen der Kostenerstattung gewährt werden. Dabei handelt es sich um Leistungen, bei denen der Gesetzgeber den Versicherten explizit auf die Beschaffung der Gesundheitsleistungen am Markt verweist. In diesen Fällen erscheint die Beschränkung der wirtschaftlichen Grundfreiheiten nicht gerechtfertigt. Stellt jedoch das nationale Krankenversicherungssystem dem Versicherten die notwendigen Gesundheitsleistungen im Rahmen eines Sachleistungssystems, d. h. aufgrund öffentlich-rechtlicher Regelungen, zur Verfügung, so werden diese Leistungen nicht vom Geltungsbereich der Grundfreiheiten erfaßt.

Da die Urteile des EuGH vor dem Hintergrund eines durch die Kostenerstattung geprägten Krankenversicherungssystems ergangen sind, kam die gemeinsame Arbeitsgruppe zu dem Ergebnis, daß es keinen Anlaß gibt, von der bisherigen Rechtspraxis der territorialen Begrenzung von Sachleistungsansprüchen abzuweichen. Entscheidend für zukünftige Entscheidungen des EuGH wird es sein, ob es gelingt, auf europäischer Ebene eine Diskussion und Klarstellung darüber zu erreichen, wie das Verhältnis von Grundfreiheiten des Binnenmarktes und nationalem Gestaltungsanspruch in den Systemen der sozialen Sicherung von den Mitgliedsstaaten gesehen wird. Dieser politische Ansatz soll im Rahmen der seit 1. Januar 1999 bestehenden deutschen EU-Ratspräsidentschaft in die Diskussion gebracht werden. Zugleich herrschte Einvernehmen, daß die Möglichkeiten für die Versicherten, im grenznahen Bereich grenzüberschreitend Leistungen in Anspruch nehmen zu können, ver- 
bessert werden sollen. Bereits heute bestehen umfangreiche Regelungen für sogenannte Wanderarbeitnehmer im Rahmen der EWG-Verordnung 1408/71 sowie grenzüberschreitenden Projekten wie EUREGIOs. Darüber hinaus sollten die Möglichkeiten untersucht werden, wie z. B. durch vertragliche Lösungen inländischer Krankenkassen mit ausländischen Leistungsanbietern bei der Inanspruchnahme im Ausland Steuerungsaspekte hinsichtlich Qualität, Preis und Umfang der Leistungen zum Tragen kommen könnten.

\section{Auswirkungen auf die Steuerungsinstrumente im ambulanten Sek- tor}

Wenn auch die Inanspruchnahme von Leistungen im EU-Ausland nicht als sehr umfänglich eingeschätzt wird, so muß dennoch mit Wirkungen auf die inländische Versorgung gerechnet werden. Gerade im ambulanten ärztlichen Sektor wirkt eine Reihe von Steuerungsinstrumenten, deren Funktionsfähigkeit durch die Übertragung der EuGH-Urteile auf Sachleistungen zumindest in Frage gestellt ist. Zu den Steuerungsinstrumenten gehören die Zulassung zur vertragsärztlichen Versorgung bzw. Zwangsmitgliedschaft in der Kassenärztlichen Vereinigung, Bedarfsplanung, Gesamtverträge bzw. Gesamtvergütung (mit Obergrenze), Richtlinien des Bundesausschusses und Wirtschaftlichkeitsprüfungen. Zudem sind Auswirkungen auf die veranlaßten Leistungen zu beachten.

Die Zulassung von Vertragsärzten - wie die Bedarfsplanung - werden zunächst nicht von den Auswirkungen der EuGH-Urteile tangiert. Allerdings stellt sich die Frage, ob die Erstattung von Kosten, die von einem inländischen Nichtvertragsarzt verursacht wurden, verweigert werden kann. Zwar sieht § 13 SGB V eine Kostenerstattung nur für Vertragsleistungserbringer vor. Wenn jedoch nicht sichergestellt werden kann, daß auch im Ausland nur Leistungen von "Vertragsärzten" erstattet werden können, wird der inländische Nichtvertragsarzt eine Diskriminierung gegenüber dem ausländischen geltend machen.

In den Gesamtverträgen zwischen Kassenverbänden und Kassenärztlichen Vereinigungen (KVen) werden die Gesamtvergütungen vereinbart, die an die KVen mit befreiender Wirkung gezahlt werden. Um die in den EuGH-Urteilen diskutierte Gefährdung der finanziellen Stabilität des Systems zu vermeiden, müßte zwingend eine Anrechnung der Kosten für ambulante ärztliche Behandlung auf die an die KVen zu zahlende Gesamtvergütung erfolgen. Erfolgt eine solche Anrechnung in größerem Umfang, werden die Kassenärztlichen Vereinigungen andererseits geltend machen, daß sie nicht mehr imstande sind, den Sicherstellungs- 
auftrag zu erfüllen, da ihnen die dazu notwendigen Mittel entzogen werden.

Zusätzliche finanzielle Belastungen sind darüber hinaus aufgrund der Mengenausweitungstendenzen im Rahmen der angebotsinduzierten Nachfrage von medizinischen Leistungen zu erwarten. Gerade bei ärztlichen Dienstleistungen ist nicht damit zu rechnen, daß im Ausland erbrachte Leistungen zu einer entsprechenden Reduktion der Leistungsmenge im Inland führen.

Die Richtlinien des Bundesausschusses Ärzte/Krankenkassen sollen eine ausreichende, zweckmäßige und wirtschaftliche Versorgung der Versicherten gewährleisten. Sie regeln damit u. a., welche Leistungen im Rahmen der vertragsärztlichen Versorgung erbracht und abgerechnet werden dürfen. Nach den Urteilen des EuGH gilt die Kostenerstattung für im EU-Ausland erbrachte Leistungen nur für die im Rahmen der inländischen „Tarife“ vorgesehenen Leistungen. Daher ist davon auszugehen, daß auch nur die Leistungen erstattungsfähig sind, die im deutschen vertragsärztlichen System abrechnungsfähig sind. Es stellt sich allerdings die Frage, ob und wie eine Überprüfung der Einhaltung der Richtlinien des Bundesausschusses bei im Ausland erbrachten Leistungen möglich ist.

Bedeutsamer sind jedoch die Reaktionen der inländischen Leistungserbringer. Insbesondere eine restriktive Bedarfsplanung mit Zulassungsbeschränkungen nach Verhältniszahlen würde von deutschen Ärzten als Diskriminierung gesehen werden, sofern die Versicherten ohne Einschränkung und in erheblichem Umfang Ärzte im europäischen Ausland in Anspruch nehmen können. Es muß damit gerechnet werden, daß diejenigen, die sich im Inland nicht (mehr) niederlassen können, sich entweder im grenznahen Ausland niederlassen oder aber entsprechende Klageverfahren zur Erlangung der Kostenerstattung auch im Inland anstreben werden. Dabei ist allerdings fraglich, ob derartige Klageverfahren Aussicht auf Erfolg haben könnten. Solange es um eine Beziehung zwischen einem inländischen Arzt und einem inländischen Patienten geht, könnte der EuGH nicht angerufen werden, da dieser nur bei grenzübergreifenden Sachverhalten zuständig ist.

Denkbar wäre jedoch der Weg zum Bundesverfassungsgericht mit dem Hinweis auf die Inländerdiskriminierung durch die Ungleichbehandlung der inländischen Ärzte gegenüber denjenigen aus den EU-Mitgliedsstaaten. Das Bundesverfassungsgericht könnte einer derartigen Klage nur dann stattgeben, wenn es sich um eine Ungleichbehandlung von gleichen Sachverhalten handelt. Bei der sogenannten umgekehrten Dis- 
kriminierung, d. h. Inländer werden schlechter behandelt als Ausländer, liegt jedoch in aller Regel kein Verstoß gegen den Gleichbehandlungsgrundsatz des Grundgesetzes (Art. 3) vor.

Erste Hinweise für die rechtliche Würdigung gibt ein Verfahren, in dem das Landessozialgericht (LSG) München (Az. L 4 KR 77/96 vom 25. Juni 1998) bereits in einem ähnlich gelagerten Fall entschieden hat. Dort begehrte eine deutsche Versicherte für die Behandlung bei einem deutschen nicht zugelassenen Zahnarzt die Erstattung der entstandenen Kosten. Unter ausdrücklicher Berücksichtigung der neuesten EuGHRechtsprechung entschied das LSG München, daß kein Anspruch auf eine Kostenerstattung besteht.

\section{Bedarfsplanung im ambulanten Sektor}

Eines der wesentlichen Steuerungsinstrumente, das durch die Auswirkungen der EuGH-Rechtsprechung gefährdet sein könnte, ist die Bedarfsplanung im ambulanten ärztlichen Sektor. Die durch das Kassenarzturteil von 1960 in ihrer Wirkung massiv reduzierte ärztliche Bedarfsplanung wurde erst durch das Gesundheitsstrukturgesetz (GSG) von 1993 wieder verschärft. Zwar steht die verfassungsrechtliche Klärung, ob die heutige Regelung gegen das Recht auf freie Berufsausübung (Art. 12 Abs. $1 \mathrm{GG}$ ) verstößt, noch aus, dennoch steht zu vermuten, daß die 1960 vom Bundesverfassungsgericht angeführten Gründe, daß zunächst andere Maßnahmen zur Steuerung der Gesundheitsausgaben ergriffen werden müßten, heute nicht mehr Gültigkeit haben. Zudem hat die nicht vollzogene - weil von Ex-Bundesgesundheitsminister Seehofer aufgrund verfassungsrechtlicher Bedenken nicht realisierte - Zulassung nach strikten Verhältniszahlen ( $\$ 102$ SGB V) und einige Lockerungen im 2. GKV-Neuordnungsgesetz zu einer Aufweichung der Bedarfsplanung geführt. Es bleibt abzuwarten, wie die neue Bundesregierung sich in dieser Frage verhält.

Bei der Untersuchung der Auswirkungen der EuGH-Rechtsprechung auf die ärztliche Bedarfsplanung stellt sich ganz entscheidend die Frage nach deren grundsätzlicher Notwendigkeit. Die Bedarfsplanung als bürokratisches, regulierendes Instrument ist nur deshalb notwendig, weil unter den Bedingungen des Kollektivvertrages der Zugang zur vertragsärztlichen Versorgung gesteuert werden muß. Entsprechend zeigt die Arztzahlentwicklung eine ständig steigende Tendenz. Zusätzlich zu den ohnehin jährlichen Nettozugängen war durch die Einführung der konkreten Bedarfsplanung 1993 und den damit verbundenen Übergangsregelungen ein weiterer Anstieg zu verzeichnen (Abb. 1). Da unter den Budgetierungsbedingungen des GSG die ärztliche Gesamtvergütung 
maximal entsprechend der Entwicklung der beitragspflichtigen Einnahmen wachsen konnte, blieb der Arztzahlanstieg bei den ärztlichen Honoraren für die Krankenkassen ohne Ausgabenwirkung. Gleichzeitig stieg aber der Konkurrenzdruck innerhalb der Ärzteschaft, der zu Mengenausweitungen bei weiter fallenden Punktwerten führte (,HamsterradEffekt").

Abb. 1: Zu- und Abgang von Kassen-Nertragsärzten 1980 - 1997 - alte Bundesländer

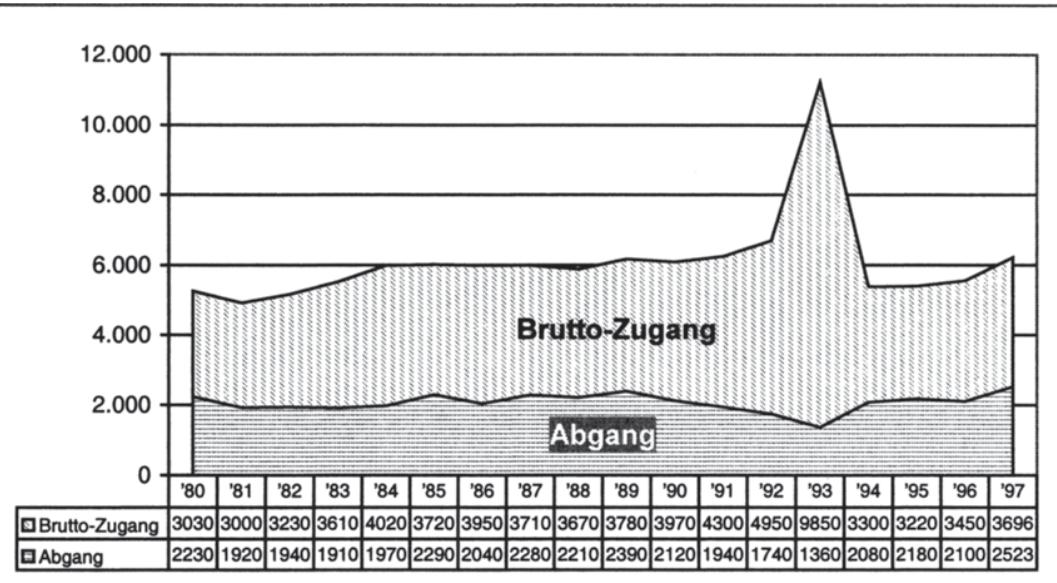

Auch für die Krankenkassen blieben die gestiegenen Arztzahlen nicht ohne Konsequenzen für die Ausgaben. Während die ärztlichen Honorare auch nach Ende der gesetzlichen Budgetierung moderate Zuwächse im Rahmen der zwischen Krankenkassen und KVen vereinbarten Gesamtvergütungen verbuchten (Abb. 2), stiegen die veranlaßten Leistungen um so stärker an. Der Konkurrenzdruck unter den niedergelassenen Ärzten beförderte die Verordnung von Arznei- und Heilmitteln sowie die Krankenhauseinweisungen, was eine entsprechende Ausgabenentwicklung zur Folge hatte (Abb. 3). 
Abb. 2: GKV-Leistungsausgaben und Vertragsärzteindex $(1985=100) 1985-1997$ - alte Bundesländer

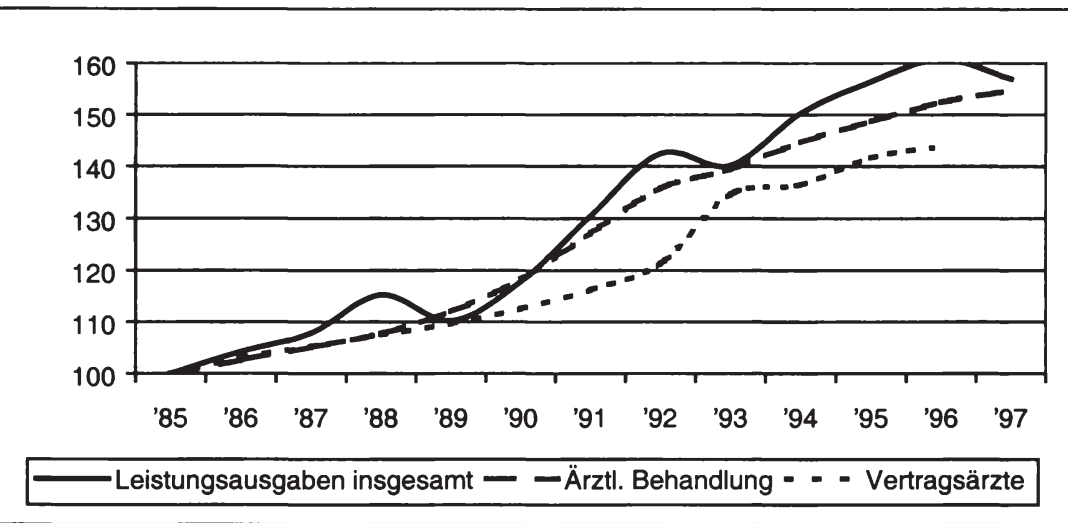

Abb. 3: Veranlaßte Leistungen in der GKV und Vertragsärzte Index $(1985=100)$ 1985 - 1997 - alte Bundesländer

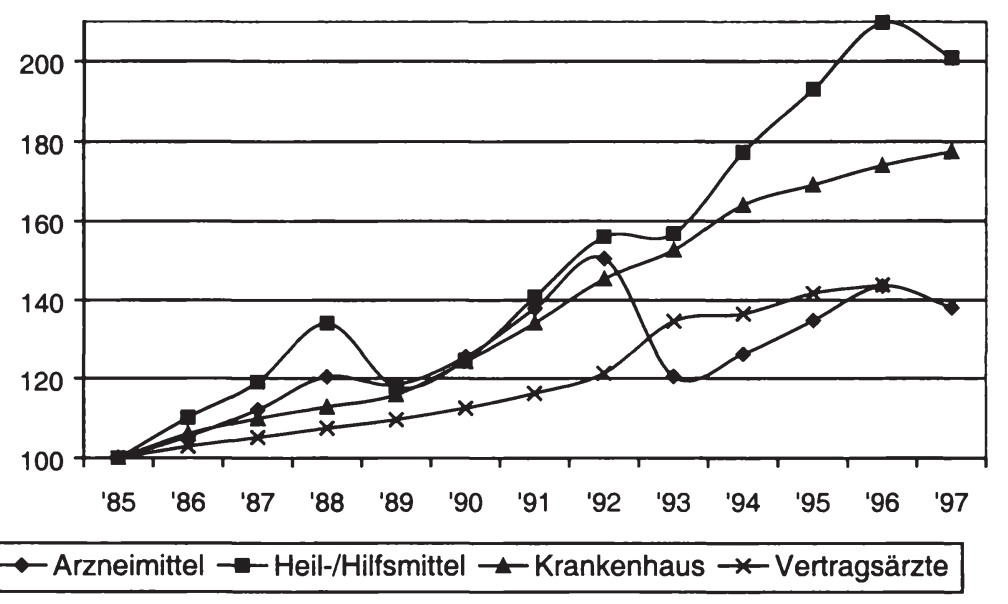

Quelle: Eigene Berechnungen und Darstellung nach: BMG, KJ 1 und KBV

Das Instrument der Bedarfsplanung soll diesen Effekten entgegenwirken, birgt jedoch neben der rechtlichen Problematik die Schwierigkeit, den tatsächlichen „Bedarf“ auch zu definieren. Die Erfahrung zeigt, daß derartige planerische Ansätze (auch) im Gesundheitswesen in der Regel 
scheitern. Eine Sperrung der Zulassung führt letztlich zu einer Diskriminierung junger Ärztinnen und Ärzte, denen die Option zur Niederlassung verbaut wird. Allerdings ist unter den Bedingungen eines Kollektivvertrages mit Kontrahierungszwang kein alternatives Steuerungsinstrument denkbar. Andere Ansätze setzen generell veränderte Strukturen im Gesundheitswesen voraus.

\section{Wettbewerbsmodell ohne Bedarfsplanung}

Derartig geänderte Strukturen sind im Modell einer solidarischen Wettbewerbsordnung vorgesehen. Der Wettbewerb auf der Krankenkassenseite wurde bereits mit Wahlfreiheit und Risikostrukturausgleich im GSG 1993 intensiviert. Ausgeblieben ist jedoch bis heute die entsprechende Flexibilisierung auf der Angebotsseite, da nur so ein sinnvoller Wettbewerb um effizientere und qualitativ hochwertige Versorgungsstrukturen ermöglicht wird. Dazu bedarf es in erster Linie der Aufhebung des Kontrahierungszwanges im ambulanten wie im stationären Sektor.

Damit würde das Vertragsmonopol der Kassenärztlichen Vereinigungen aufgebrochen. Der Sicherstellungsauftrag ginge zurück an die Krankenversicherung bzw. würde von Ärzten und Krankenversicherung im Rahmen vertraglicher Vereinbarungen ausgefüllt. In einem derartigen System könnten die Krankenkassen mit Gruppen von Ärzten, und zwar in dem Umfang und der Qualität, wie sie für die Versorgung der Versicherten notwendig sind, Verträge über die ambulante medizinische Behandlung schließen.

Um den Solidarcharakter der gesetzlichen Krankenversicherung sicherzustellen, ist in einem derartigen wettbewerblich organisierten System jedoch eine Rahmenordnung notwendig, die u. a. einen einheitlichen Leistungskatalog für alle Krankenkassen vorsieht. Ziel einer solidarischen Wettbewerbsordnung ist nämlich die effizientere Erstellung medizinischer Leistungen in unterschiedlichen Organisationsstrukturen, nicht aber der Wettbewerb um Versicherte durch mehr Leistungen. Zwingend ist in einem solchen Modell ebenso die solidarische Finanzierung durch einkommensbezogene Beiträge und die Solidarfunktionen gegenüber Alten, Kranken, Familien und Geringverdienenden. Ebenso notwendig sind Mindestvoraussetzungen bezüglich Qualität bzw. Qualifikation der Leistungserbringer. Der Wettbewerb sorgt dann dafür, daß die Leistungserstellung den Kriterien der Effizienz genügt, was angesichts generell knapper Ressourcen in einer begrenzten Welt auch eine Verpflichtung gegenüber den Beitragszahlern ist, um so mehr, wenn sie als Pflichtversicherte erhebliche Teile ihres Einkommens zwangsweise an die gesetzliche Krankenversicherung abführen müssen. 
In einem derartigen wettbewerblich organisierten Gesundheitswesen ist eine Bedarfsplanung überflüssig. Durch den Wegfall des Kontrahierungszwanges können bedarfsgerechte Versorgungsstrukturen geschaffen werden, indem gerade die Kapazitäten unter Vertrag genommen werden, die für die Versorgung der Versicherten erforderlich sind.

\section{Verträge mit ausländischen Anbietern}

Auf vertragliche Bindung im Sinne eines Einkaufsmodells mit ausländischen Anbietern setzt auch der Ansatz, den die niederländische Krankenversicherung in bezug auf die Wirkungen der europäischen Rechtsprechung bereits praktiziert. Hier werden die niederländischen Versicherten im Ausland auf Anbieter verwiesen, mit denen die niederländische Krankenversicherung eine vertragliche Vereinbarung geschlossen hat. Ihre Legitimation hierzu ziehen die niederländischen Krankenkassen aus der Tatsache, daß die Niederländer auch im Inland auf die Leistungserbringer, die im Rahmen eines Einkaufsmodells unter Vertrag stehen, verwiesen werden.

Nach heutigem deutschen Recht ist eine derartige Verweisung nicht möglich. Ob eine derartige Verweisung unter den Bedingungen eines Einkaufsmodells möglich wäre, muß dahingestellt bleiben. Denn für den Fall, daß ein Versicherter sich auch unter diesen Bedingungen bei einem anderen, $d$. $h$. nicht vertraglich gebundenen, Leistungserbringer behandeln läßt, könnte der EuGH auch hier auf die Beschränkung der Grundfreiheiten verweisen.

Selbst wenn diese generelle Verweisung nicht möglich ist, so bleibt dennoch in einem derartigen Vertragsmodell die Option, den Versicherten eine Inanspruchnahme der Vertragspartner zu empfehlen. Dies böte einen Ansatz zur Steuerung von Preisen, Qualität und Mengen der Leistungen. Der Hinweis auf gesicherte Qualität und die entfallende oder aber zumindest kalkulierbare Eigenbelastung des Versicherten, für den Fall, daß die Kosten die in Deutschland üblichen Sätze übersteigen, könnte das Problem zumindest quantitativ abmildern. Zu denken wäre an Verträge mit Leistungserbringern im grenznahen Bereich oder in besonders frequentierten Urlaubsregionen. Offen ist allerdings, ob die jeweiligen Leistungserbringer zu solchen Verträgen bereit wären, da sie damit möglicherweise ihre eigenen Behandlungs- und Abrechnungsmöglichkeiten beschränken würden. 


\section{Ausblick}

Grundsätzlich ist - vor allem von seiten der Politik - die Frage zu beantworten, ob und wie der europäische Einigungsprozeß vorangetrieben werden soll. Zwar beinhaltet der Amsterdamer Vertrag das Subsidiaritätsprinzip, jedoch werden auch für den Bereich der Gesundheitspolitik teilweise Felder einer Gemeinschaftspolitik gesehen (im Grundsatz nur bei Einstimmigkeit). Auch wenn Konsens besteht, daß in diesem Feld keine Harmonisierung erfolgen soll, so ist dennoch davon auszugehen, daß eine allmähliche Annäherung der Systeme durch Konvergenz kaum aufzuhalten ist.

Wichtig ist allerdings, daß der europäische Einigungsprozeß politisch gestaltet werden muß. Es darf nicht der Rechtsprechung überlassen bleiben, ob im Grundsatz richtige und der europäischen Einigung förderliche Prinzipien des Binnenmarktes - Freiheit des Warenverkehrs und Dienstleistungsfreiheit - die Entwicklungen in den sozialen Sicherungssystemen überrollen und zu Ergebnissen führen, die keine der nationalen Regierungen anstrebt. Hier muß eine Diskussion über Ziele und Maßnahmen und vor allem über die Bewertung des Spannungsverhältnisses von sozialen und ökonomischen Fragen geführt werden. Daher ist es von zentraler Bedeutung, daß auch die deutsche Krankenversicherung sich an diesem Diskussionsprozeß beteiligt und Gestaltungsrechte für die Zukunft einer Sozialunion einfordert und einbringt. 


\section{Ärztliche Bedarfsplanung}

Konsequenzen einer Liberalisierung der europäischen Märkte für die deutsche Gesundheitsversorgung

Manfred Zipperer

Wir befassen uns heute abend mit den Konsequenzen einer Liberalisierung der europäischen Märkte für die deutsche Gesundheitsversorgung, speziell im Hinblick auf die Auswirkungen der ärztlichen Bedarfsplanung. Was sagt das europäische Recht dazu? Das geltende primäre Gemeinschaftsrecht enthält unmittelbar keine Aussagen zur Gesundheitsversorgung. Zwar befaßt sich Titel X in Artikel 129 des Maastrichter Vertrages mit dem Gesundheitswesen, behandelt aber nur die Förderung der Zusammenarbeit und die Verhütung von Krankheiten. Fördermaßnahmen dürfen nur „unter Ausschluß jeglicher Harmonisierung der Rechts- und Verwaltungsvorschriften der Mitgliedsstaaten“ beschlossen werden.

Titel XIII wiederholt dies in Artikel 152 des Amsterdamer Vertrages und betont in dessen Absatz 5 ausdrücklich:

„Bei der Tätigkeit der Gemeinschaft im Bereich der Gesundheit der Bevölkerung wird die Verantwortung der Mitgliedsstaaten für die Organisation des Gesundheitswesens und die medizinische Versorgung im vollen Umfang gewahrt."

Das bedeutet, daß das Gesundheitswesen nach wie vor ausschließliche Domäne der Mitgliedsstaaten ist und die Europäische Union und ihre Institutionen auf diesem Gebiet keine Zuständigkeit haben.

Diese Rechtslage mutet um so merkwürdiger an, als die Rechtsgebiete um die gesetzliche Krankenversicherung herum bereits weitgehend durch das Recht der Europäischen Union geprägt sind, z. B. bei Arzneimitteln und Medizinprodukten, bei der Ausbildung von Ärzten/Zahnärzten und medizinischem Hilfspersonal.

Das gilt auch für die Koordinierung der verschiedenen Systeme der sozialen Sicherheit der Mitgliedsstaaten. Artikel 42 des Amsterdamer Vertrages (Art. 51 des Maastrichter Vertrages) gibt nämlich dem Rat die Befugnis, die auf dem Gebiet der sozialen Sicherheit für die Herstellung der Freizügigkeit der Arbeitnehmer notwendigen Maßnahmen zu beschließen. Diese Regelung, die im Titel III „Freizügigkeit, freier Dienstleistungs- und Kapitalverkehr" steht, ist die Rechtsgrundlage für sekundä- 
res Gemeinschaftsrecht, insbesondere für die beiden EG-Rechtsverordnungen 1408/71 und 574/72.

Die darin enthaltenen Vorschriften betreffen aber nur Koordinierungsregelungen, die dem Erhalt von Rechten dienen, wenn Staatsangehörige von Mitgliedsstaaten der Europäischen Union als Arbeitnehmer den Mitgliedsstaat wechseln, d. h. Sachverhalten, bei denen Leistungen im Krankheitsfall in einem anderen Mitgliedsstaat in Anspruch genommen werden als im Mitgliedsstaat des Versicherungs- und Beschäftigungsverhältnisses.

Diese Koordinierungsregelungen machen die Inanspruchnahme von Leistungen - von Akutfällen und Grenzgängern abgesehen - von der Genehmigung der Krankenkasse abhängig. Das ist verständlich, denn das System eines Mitgliedsstaates zur Sicherung im Krankheitsfall ist ausgerichtet auf die Inanspruchnahme von Leistungen im Inland, auf die dort bestehende Risiko-, Angebots- und Nachfragestruktur und die damit im Zusammenhang stehenden Steuerungsmechanismen.

Auch das System der deutschen gesetzlichen Krankenversicherung verlangt nach diesem Genehmigungsvorbehalt. Wir haben in Deutschland ein Sachleistungssystem, bei dem die Krankenkasse die Leistungen durch Verträge mit Leistungsanbietern zur Verfügung stellt, und zwar wegen des Sachleistungsprinzips ausschließlich mit inländischen Leistungsanbietern. Deshalb können Leistungen auch nur im Inland angeboten und in Anspruch genommen werden. Dies ist Sinn und Zweck des Territorialitätsprinzips. Bei Aufenthalt im Ausland ruhen die Leistungen grundsätzlich. Da das Vertragsrecht mit öffentlich-rechtlichen Rechten und Pflichten verbunden ist, die nur im Inland wirken und durchsetzbar sind, erstrecken sich die Leistungszusagen der gesetzlichen Krankenversicherung nur auf das Inland.

Die Ausnahmen des sekundären Gemeinschaftsrechts bauen auf diesem Grundmuster auf. Sie sehen Sachleistungsaushilfe vor: Der ausländische Versicherungsträger gewährt die Leistung im Aushilfswege wie einem eigenen Versicherten, also unter denselben Konditionen. Das bedeutet Inanspruchnahme nur von Vertragsärzten oder Vertragskrankenhäusern, Zuzahlungen, Leistungsausschlüsse. Eine Ausnahme besteht nur für die Geldleistungen, die die Krankenkasse des Versicherten ohne Begrenzung direkt an diesen ins Ausland zahlt.

Diese Rechtslage ist für die finanziellen Leistungen der Pflegeversicherung von grundlegender Bedeutung. 
Begibt sich jemand zur Behandlung ins Ausland und nimmt er dort Leistungen in Anspruch, ohne daß es sich um einen Akut- oder Grenzgängerfall handelt, muß die nationale Krankenkasse diese Inanspruchnahme von Leistungen genehmigen. Diese Genehmigung hebt das Ruhen der Leistung auf. Auch die EG-Verordnung 1408/71 sieht diese Regelung vor; sie geht insoweit nicht über unser nationales Recht hinaus. Mit dieser Genehmigungspflicht schützen sich die nationalen Versicherungsund Versorgungssysteme vor einer nicht kontrollierbaren und deshalb finanziell problematischen Inanspruchnahme.

In diese heile Welt der Leistungskoordinierung bricht nun der Europäische Gerichtshof mit seinen Entscheidungen in den beiden Rechtssachen Kohll und Decker ein. Er sieht in dem Genehmigungsvorbehalt einen Verstoß gegen die Grundfreiheiten des freien Waren- und Dienstleistungsverkehrs; der Leistungsanspruch sei von der Genehmigung der Krankenkasse nicht abhängig.

Die beiden Entscheidungen betreffen zunächst nur den Leistungsanspruch des Versicherten: Er kann die Leistungen, die ihm seine Krankenkasse zur Verfügung stellt, im gesamten Hoheitsgebiet der Europäischen Union in Anspruch nehmen, muß sich allerdings mit dem Leistungsniveau seiner nationalen Krankenkasse zufrieden geben. Diese Rechtsprechung wirkt sich aber auf das gesamte Versorgungssystem aus, weil der Schutzzaun, den das Territorialitätsprinzip aus wohlerwogenen Gründen um das nationale System gezogen hat, damit durchlöchert und in seinem Instrument unterlaufen wird.

Ich will dies am Beispiel der ärztlichen Bedarfsplanung erläutern:

Ein Anspruch auf Leistungen der ärztlichen bzw. zahnärztlichen Behandlung besteht nach geltendem Recht nur, wenn die Behandlung von einem Vertragsarzt/Vertragszahnarzt durchgeführt wird. Zwar hat der Versicherte die freie Arztwahl, aber eben nur unter den für die Behandlung von Versicherten zugelassenen Ärzten. Diese Beschränkung hängt mit dem öffentlich-rechtlichen Sicherstellungsauftrag der Kassenärztlichen Vereinigungen zusammen, deren Mitglieder die Vertragsärzte sind. Die Begrenzung gilt nicht nur, soweit die Leistungen als Sachleistung erbracht werden, sondern auch bei Kostenerstattung.

Mit dem Sicherstellungsauftrag übernimmt die Kassenärztliche Vereinigung die ärztliche Versorgung der Versicherten in inrem regional abgegrenzten Wirkungsbereich. Die Bedarfsplanung legt fest, wieviel Ärzte und Fachärzte in einem Planungsbezirk erforderlich sind, um diesen Auftrag zu erfüllen. Die Bedarfsplanung deckt den in der Region benö- 
tigten ambulanten ärztlichen Bedarf ab, sowohl in der Gesamtzahl als auch hinsichtlich der benötigten medizinischen Disziplinen.

Diese Bedarfsplanung müßte geändert werden, wenn in nicht unbeträchtlichem Umfang Ärzte in anderen Mitgliedsstaaten in Anspruch genommen würden. Das heißt, daß die Zahl der inländischen Vertragsärzte ggf. verringert werden müßte. Es ist aber auch denkbar, daß die Ärzte in Deutschland in vermehrtem Umfang von Ausländern aus der übrigen Europäischen Union in Anspruch genommen werden. Müßte dann die Planung angepaßt werden, obwohl der Sicherstellungsauftrag nur Versicherte der deutschen gesetzlichen Krankenversicherung betrifft?

Viel wichtiger erscheint mir noch die Antwort auf eine andere Frage:

Müssen die Versicherten der deutschen gesetzlichen Krankenversicherung bei der Inanspruchnahme von ärztlicher Behandlung im EUAusland Ärzte in Anspruch nehmen, die für das dort geltende System arbeiten? Solange die Leistung im Wege der Sachleistungsaushilfe über den sog. europäischen Krankenschein erbracht wird, wird sie im Vertragssystem abgegeben. Was geschieht aber, wenn ein Versicherter einen nicht dem System angeschlossenen Arzt in Anspruch nimmt? Wie prüft die Krankenkasse die nach nationalem Recht geforderte Voraussetzung nach?

$\S 13$ SGB V sieht eine Kostenerstattung nur für Vertragsleistungserbringer vor.

Noch weiter gefragt: Läßt sich eine restriktive Bedarfsplanung mit Zulassungsbeschränkungen nach Verhältniszahlen auf Vertragsärzte in Deutschland noch rechtfertigen, wenn Versicherte ohne diese Einschränkung und in erheblichem Umfang Ärzte/Zahnärzte im EU-Ausland in Anspruch nehmen können? Muß nicht damit gerechnet werden, daß sich Ärzte, die im Inland keine Zulassung mehr erhalten haben, im grenznahen Ausland niederlassen? Diese Fragestellungen sind für die Aufrechterhaltung unserer nationalen Bedarfsplanung von entscheidender Bedeutung, weil die Rechtfertigung dieser Beschränkung gegenüber dem Bundesverfassungsgericht in den dort anhängigen Verfahren eine zentrale Rolle spielt.

Falls man die Leistungserbringung durch Ärzte im EU-Ausland ohne Beschränkung zuließe, könnten Vertragsärzte in Deutschland aus dieser Diskriminierung keine Rechte herleiten, weil die sog. umgekehrte Diskriminierung, die Inländer schlechter behandelt als Ausländer, nicht gegen Artikel 3 Grundgesetz verstößt. Ich verkenne allerdings nicht, daß 
auch dann, wenn die rechtliche Argumentation hier nicht tragfähig ist, mit einer politischen Diskussion in dieser Richtung gerechnet werden muß.

Welches Interesse haben die Krankenkassen an einer Liberalisierung? Im Vordergrund steht natürlich der Gesichtspunkt, daß Leistungen, die im EU-Ausland in Anspruch genommen werden, preiswerter sind als in Deutschland, so daß Kosten eingespart werden können. Diese Argumentation und Erwartung ist in der Diskussion um den Zahnersatz eingesetzt worden („Zahnersatz auf Mallorca"). Auch ließe sich das umständliche Verfahren der Sachleistungsaushilfe durch die direkte Kostenerstattung wesentlich vereinfachen. Ich könnte mir aber auch vorstellen, daß die Krankenkassen die Inanspruchnahme ausländischer Leistungserbringer als Argument bei der Absenkung der Gesamtvergütung oder der Ânderung der Bedarfsplanung einsetzen. Und dann besteht auch noch die Überlegung, mit ausländischen Ärzten Verträge auBerhalb der Kassenärztlichen Vereinigung und ohne sie zu schließen. Hierbei ließe sich das Einkaufsmodell, dessen Realisierung im Inland wesentliche Hindernisse entgegenstehen, in gewissem Umfang doch noch verwirklichen. Soweit solche Verträge nicht flächendeckend wären, müßten die Versicherten auf bestimmte Vertragsärzte verwiesen werden, wollen sie nicht mit Belastungen rechnen.

Mit welchen tatsächlichen Auswirkungen der beiden Urteile des Europäischen Gerichtshofs muß gerechnet werden? Vorerst ist eine vermehrte Inanspruchnahme von Vertragsärzten/Zahnärzten in Deutschland wohl nicht zu erwarten. Zum einen darf die nationale Krankenkasse nur höchstens die Aufwendungen erstatten, die nach ihrem Recht vorgesehen sind. Soweit also die Vergütung der Vertragsärzte in Deutschland höher ist als etwa in Frankreich oder in Belgien, müssen die dort Versicherten den Differenzbetrag aus eigener Tasche zahlen. Sicher werden auch die Sprachbarriere, die Barzahlung und die Anreise die Inanspruchnahme bremsen, allerdings weniger in grenznahen Gebieten. Man wird jedoch mit erheblichen Änderungen rechnen müssen, wenn die totale Preistransparenz im Rahmen der EURO-Umstellung hergestellt ist und die Krankenkassen verstärkt auf Versorgungsangebote aus dem EUAusland reagieren.

Vergleichbares gilt für die Inanspruchnahme ausländischer Ärzte durch Versicherte der deutschen gesetzlichen Krankenversicherung. Ich halte es allerdings durchaus für möglich, daß im grenznahen Bereich Versicherte ausländische Ärzte auch deshalb aufsuchen, weil sie sich von ihnen eine großzügigere Verordnung von veranlaßten Leistungen und ggf. Leistungen erhoffen, bei denen sie im Inland wegen Bedarfsplanung (Wartezeit) oder Budgetierung Probleme bekommen hätten. Gegen eine 
solche Mehrfachinanspruchnahme und gegen solche Verstöße gegen das Wirtschaftlichkeitsprinzip können die deutschen Krankenkassen nicht viel ausrichten.

Diese in meinen Ausführungen zum Ausdruck gekommene restriktive Haltung gegenüber den beiden Entscheidungen des Europäischen Gerichtshofes hat in der Öffentlichkeit viel Kritik gefunden. Inzwischen wird man sich offenbar bei den Beteiligten im deutschen Gesundheitswesen wie in zahlreichen Mitgliedsstaaten bewußt, daß die Nachteile und Gefahren aus der Liberalisierung der europäischen Märkte im Gesundheitswesen die Vorteile möglicherweise noch überwiegen. Solange die Organisation und Finanzierung der Gesundheitssysteme in den Mitgliedsstaaten noch so grundlegende Unterschiede aufweisen, wird es sehr schwierig sein, grenzüberschreitende Formen der Versorgung und der Zusammenarbeit zu entwickeln - so wünschenswert dies für das Zusammenwachsen Europas auch wäre. 


\section{Stationäre Leistungen, Kuren und Rehabilitationen}

\section{Hans-Dieter Koring}

Im Leitthema dieses Vortrags- und Diskussionsblocks findet sich ein Paradoxon, das sich allerdings erst erschließt und offenbar wird, wenn man die Verhältnisse in Deutschland richtig einordnet.

Es werden zum einen die Liberalisierung europäischer Märkte, zum anderen die deutsche Gesundheitsversorgung angesprochen.

Grundsätzlich verstehen wir unter Versorgung sowohl die private wie auch die staatliche Existenzsicherung, die über Märkte oder Zuteilung erreichbar ist. Im sozialstaatlich abgeleiteten System der gesetzlichen Krankenversicherung in Deutschland ist die Versorgung weitgehend nicht mit marktwirtschaftlichen Mitteln, sondern im Rahmen korporatistischer Zuteilung sichergestellt.

Unsere gesetzliche Krankenversicherung ist in erster Linie nicht Versicherung, sondern Versorger in mittelbarer Selbstverwaltung: Sie hat die Aufgabe, die Gesundheit der Versicherten zu erhalten, wiederherzustellen oder ihren Gesundheitszustand zu bessern (1 SGB V).

Aus diesem Zusammenhang werden die teilweise heftigen Reaktionen der seinerzeitigen Bundesregierung, der Bundestagsfraktionen und aus den Parteien verständlich. Offensichtlich besteht übergreifend Konsens, daß die gesetzliche Krankenversicherung in Deutschland als umfassende sozialstaatliche Sicherung der Existenz in bezug auf die Gesundheit jedes einzelnen Bürgers - soweit er einer Versicherungspflicht unterliegt - wirkt.

Um so erstaunlicher mutet die politische Diskussion der letzten Jahre zur Förderung des Wettbewerbs bei den Leistungserbringern im Gesundheitswesen an.

In diese Gemengelage hinein gibt der Spruch des Europäischen Gerichtshofes einfache, klare Antworten. Das, was viele Experten, Wissenschaftler und Politiker hier und da zum Teil vehement vertreten haben, nämlich, daß Gesundheitsdienstleistungen und -güter marktlicher Organisation zugänglich seien, wird noch übertroffen.

Auch diese mit Besonderheiten, Tabus und Einschränkungen belegten Dienstleistungen und Güter unterliegen dem Gebot des freien Binnen- 
marktes. Nicht die sozialstaatliche Zuteilung steht im Vordergrund, sondern ausschlaggebend ist die Dienstleistung und Güterversorgung im Anbieter/Leister-Kunden-Verhältnis.

Die finanzielle Absicherung und Kostenübernahme in solidarischer Gemeinschaft oder sozialstaatlicher Fürsorge ist nationalstaatliche Aufgabe und Regelungsbefugnis.

Damit ist der Dualismus der europäischen Gesundheitssysteme - gleich, ob steuerlich oder beitragsfinanziert - klar definiert:

a) Die Finanzierung in Art, Form und Inhalt ist nationalstaatlich zu entscheiden.

b) Die Organisation der Leistungserbringung unterliegt den Regeln des europäischen Wettbewerbsrechtes; die Besonderheiten des Marktes sowohl auf der Anbieter- wie auch auf der Nachfragerseite können und müssen ordnungspolitisch gelöst werden wie in anderen existentiellen Bereichen (z. B. Landwirtschaft, Energie).

Nun heißt Liberalisierung aber auch freie Gestaltung, also nicht nur Freizügigkeit, sondern auch Freiwilligkeit. Die Versicherten sollen künftig europaweit wählen können. Die Krankenkassen haben diese Möglichkeiten bereits weitgehend - jedenfalls in den vom EuGH beurteilten Segmenten - zugelassen.

Gleichwohl werden die Angebote im europäischen Ausland noch überaus behutsam - eben pragmatisch - in Fällen der Zweckmäßigkeit und soweit es im Einzelfall sinnvoll ist genutzt. Ein gleicher Pragmatismus sollte die Politik bei ihren notwendigen Reaktionen leiten; es darf aber kein Zweifel bleiben, daß auf Dauer die im deutschen System verinnerlichten korporatistischen Ausschließlichkeitsfestlegungen, Sicherstellungsaufträge und Kontrahierungszwänge in Frage stehen.

Es bleibt deshalb - gerade im Rahmen der geplanten grundsätzlichen Strukturreform im Gesundheitswesen - die Herausforderung, den Markt der Gesundheitsdienstleistungen und -sachgüter wegen der Besonderheiten der Existenzsicherung mit den Mitteln der Ordnungspolitik und des Verbraucherschutzes zu ordnen und zu sichern.

Auch die Krankenkassen müssen pragmatisch vorgehen im Sinne einer Kundenorientierung. Je nach Bedarfslage und Angebot kann es angezeigt sein, den Präferenzen der Versicherten mit vertraglichen Absiche- 
rungen auch im europäischen Ausland (eventuell in Kooperation mit entsprechenden Versicherern) zu folgen.

Dieses steht in den beurteilten Bereichen nicht akut an; im folgenden will ich einen (Aus-)Blick auf stationäre Leistungen, Kuren und Rehabilitation werfen.

Dazu will ich stationäre Leistungen und Rehabilitation in einem Zusammenhang behandeln, weil sie zusammengehören oder sich zumindest gegenseitig unmittelbar ergänzen. Am deutlichsten wird das bei den medizinischen Anschlußrehabilitationen, die ich als ausgelagerte Genesungs- und Qualitätssicherungsphase des Krankenhauses einordne. Auch für soziale und berufliche Rehabilitation gilt ähnliches.

Dieser integrierende Blickwinkel ist im übrigen nur deshalb ungewöhnlich, weil Trennungslinien - wirkliche Schnittstellen - durch verschiedene Zuständigkeiten in der Kostenträgerschaft (Krankenversicherung/Rentenversicherung/Arbeitslosenversicherung) historisch entstanden sind und wirken.

Wenn wir Krankenkassen in diesem Bereich, wie eben beschrieben, pragmatisch vorgehen, müssen wir konstatieren, daß bei dem Ausbaustand unserer Hospitäler und Kliniken sicher nicht von Mangel gesprochen werden kann, den wir beheben müßten. Das war in vergangenen Zeiten z. B. in der Herzchirurgie anders.

Daraus können wir allerdings auch lernen, daß Märkte - auch die Märkte der Gesundheitsdienstleistungen-sehr flexibel auf Angebotslücken reagieren können. In der Schweiz, in England, in Belgien, den Niederlanden und in Dänemark wurden sehr schnell gute herzchirurgische Leistungen (Beratung und Begleitung natürlich nach den Wünschen und Erfordernissen des Patienten!) bereitgestellt. Insofern müssen wir schon beobachten, welche Angebotsstrukturen in Europa sich in ihrer Tendenz an unsere Versicherten wenden, damit wir uns verbraucherschützend einsetzen können, insbesondere zur Qualität und zur Wirtschaftlichkeit der Leistungen. Wenn ich beispielsweise daran denke, daß die Deutschen über Eurotransplant seit Jahren Organempfänger aus Europa in erheblichem Umfang sind, wäre vorstellbar, daß sich die Transplantationsmedizin zu den gewonnenen Organen hin verlagert; Herausforderung für die GKV in Sachen Abstimmung und Gestaltung!

Der Beitrag der Politik und des Gesetzgebers ist natürlich gefordert. Monistische Krankenhausfinanzierung, in der Rehabilitation fest etabliert, ist unausweichlich. Damit ist aber auch Planung obsolet, sowohl der Län- 
der, aber auch etwa der Krankenkassen (wie häufig diskutiert), und die Krankenhäuser werden in einen Qualitäts-, Leistungs- und Effizienzwettbewerb entlassen, der der Versorgung nur gut bekommen kann - auch als Beitrag für den Abbau von Überversorgung. Zum europäischen Kontext wird den guten Spitälern die Öffnung des Binnenmarktes nach meiner Einschätzung gut bekommen; die Potentiale für den Export von Leistungen durch den Import von Patienten sind nicht zu unterschätzen.

Nun zu den Kuren, dem deutschen Spezifikum.

Je mehr die Ortsgebundenheit von Kurmitteln in Frage steht, desto mehr werden Anbieter von stationären und ambulanten Kuren die Etablierung im europäischen Ausland favorisieren - aus dem Blickwinkel des Anbieters einerseits, aus Gründen von bestimmenden Kostenfaktoren andererseits.

Wir wissen natürlich, daß die deutschen Anbieter erhebliche Konkurrenz haben im europäischen Ausland, natürlich auch deshalb, weil sie in klimatisch begünstigten Gegenden liegen.

Rückgänge im Kurbereich waren in den letzten Jahren regelmäßig (im übrigen ohne europäischen Einfluß!) von Protesten der Einrichtungen wie auch der Kurorte und der Bundesländer begleitet. Es handelt sich nicht selten um strukturschwache Regionen, in denen Kureinrichtungen durchaus einen spürbaren Wirtschaftsfaktor darstellen.

Bei Kuren stehen andere Erwägungen im Vordergrund als bei ärztlicher Behandlung oder dem Krankenhausbereich. Klimatische Erwägungen sind das eine, aber auch die sprachliche Barriere dürfte eine geringere Bedeutung haben als im Krankenhaus, zumal man im Ausland sehr schnell auf den Gedanken kommen wird, sich die deutsche Leistungsart „Kur" zunutze zu machen und auch für deutschsprachiges Personal zu sorgen.

Ein sehr viel größerer Personenkreis als bei stationärer Krankenhausbehandlung wird die Frage nach Pragmatismus und Freiwilligkeit für sich bejahen - mit entsprechender Nachfrage dürtte zu rechnen sein.

Die Patienten werden vermutlich keinen Nachteil haben - im Gegenteil. Nachteilig wird diese Entwicklung für die Kurorte und deren Einrichtungen, weil sie mit einer Kompensation durch den öffentlich-rechtlichen Sektor nicht rechnen können. Die Vorboten erleben wir schon heute. Diesen öffentlichen Druck wird die Politik aushalten oder aber sich inm beugen müssen. In jedem Fall sollte sie inn kommen sehen. 
Die Kureinrichtungen selbst sind gut beraten, inr Angebot der Entwicklung anzupassen und verstärkt auf private Zahler zu setzen - das Potential des Wellness-Marktes ist bei weitem noch nicht ausgeschöpft.

Das Fazit, das ich also aus den Urteilen des Europäischen Gerichtshofes ziehe, ist das folgende: Die Rechtsprechung hat das deutsche Gesundheitswesen nicht in einer Weise verändert, die sich in Mark und Pfennig ausdrücken läßt oder in der Zahl von Patienten, die im EUAusland Leistungen in Anspruch nehmen.

Sie haben aber Blickweisen verändert, Denkverbote aufgehoben und neuen Wind in strukturelle Überlegungen gebracht.

Vertragliche und Angebotsstrukturen werden sich ändern müssen - für diejenigen, die die Fragen nach Pragmatismus und Freiwilligkeit für sich bejahen. 


\section{Stationäre Leistungen, Kuren und Rehabilitationen}

\section{Hans Günter Verhees}

Die Brille aus Paris, der Zahnersatz aus Venedig und die Reha-Kur auf Mallorca: Sind das konkrete und realistische Visionen für „AOK - Die Gesundheitskasse“, zumal - einmal mehr ganz "trendy“ - sie auf Mallorca schon eine Geschäftsstelle hat?

Aus Sicht der Krankenkasse stellt sich unter monetären Aspekten bei der Bewertung europäischer Komponenten zuallererst die Frage: Sind die Urteile des EuGH vom 28.04.1998 eigentlich mehr Chance oder mehr Risiko?

Chance oder Risiko - für wen denn eigentlich? Auch für den Patienten? Auch für den Leistungserbringer? Oder nur für die Krankenkasse?

Es sind viele kluge Worte, gestern und auch heute schon, zum Thema gesagt worden, wie mit den europäischen Weiterungen und seinen Auswirkungen auf das deutsche Gesundheitswesen umzugehen ist. Ich kann wenig tun, um die Brillanz der Gedanken und die Reichweite der Ideen, die schon erörtert worden sind, noch zu übertreffen.

Ich muß es aber tun, denn per Fax vom 02.11.1998 ermahnte mich Herr Dr. Albring - gestützt auf die zusätzliche Autorität von Herrn Professor Wille -, mindestens 15 und höchstens 20 Minuten zum Thema zu sprühen - vor Ideen, versteht sich.

Oft wird eingewandt, die Urteile Kohll und Decker des Europäischen Gerichtshofs vom 28. April 1998 seien ohne jede Bedeutung, da sie sich lediglich auf ein Brillengestell und ein Stückchen Zahnersatz bezogen hätten.

Darüber hinaus seien insbesondere die stationären Leistungen im Urteil ja ausdrücklich von dieser Rechtsprechung ausgenommen. Es gibt allerdings auch mehr resignative Stimmen, die argwöhnen, daß Krankenhäuser - sozusagen grundsätzlich - von jeder Limitierung ausgenommen seien.

Der ehemalige Gesundheitsminister hat sich sogar zu der schriftlichen Festlegung verleiten lassen, diese Urteile hätten deshalb überhaupt keine Auswirkungen, da sie ja „förmlich nur zwischen den Prozeßparteien“ wirken. Die „den Entscheidungen zugrunde liegenden Sachverhalte 
(seien) mit der Sach- und Rechtslage hinsichtlich der vom Sachleistungsprinzip geprägten deutschen gesetzlichen Krankenversicherung nicht vergleichbar".

Einem juristischen Seminaristen (selbst eines früheren Semesters) wären solche Ausführungen schlecht bekommen. Der Europäische Gerichtshof hat nämlich nichts anderes getan, als in zwei ganz spezifischen Fallkonstellationen die Frage des europäischen Rechtes, seine Auswirkung auf die nationalen Rechtssysteme und das Verhältnis zwischen den nationalen Sozialversicherungssystemen und der europaweiten Waren- und Dienstleistungsfreiheit zu bewerten. Er hat dies in aller Kürze und Konsistenz zu seiner bisherigen Judikatur getan, denn aus europäischer Sicht ist diese Konstellation gar nicht rechtlich problematisch. So wenig problematisch wie das Bosman-Urteil.

Die Crux ist doch, daß der eherne Grundsatz des Sozialrechts, nämlich die grenzüberschreitende Leistungsgewährung als „Verbot mit Erlaubnisvorbehalt" zu behandeln, von Europa- und Wirtschaftsrechtlern als schlicht anstößig empfunden wird. Ganz im Kontrapunkt hierzu sorgt die Vorstellung vom europaweiten, freien Waren- und Dienstleistungsverkehr beim Sozialrechtler für blankes Entsetzen.

Aber ich meine, hier irrt der traditionelle Sozialrechtler: Auch vor dem 28.04.1998 war die Vorstellung vom Sozialrecht als der letzten Enklave und dem verbliebenen Refugium des nur scheinbar souveränen Nationalstaates eine Fiktion. Die Zeiten, in denen sich europäisches Sozialrecht auf die spannenden Rechtsfragen zur Stellung des „Wanderarbeitnehmers" und des „E 111“ reduzieren ließen, sind nun mal vorbei.

Die aus EWG und EG hervorgegangene EU war in erster Linie immer ein ökonomisches Prinzip und wollte gerade Selbständigen - eben auch Optikern und Zahnärzten - ein Grundrecht auf Entfaltung grenzüberschreitender Tätigkeiten eröffnen.

Auch wenn der EuGH in ständiger Rechtsprechung - zutreffend zitiert von Seehofer - konstatiert hat, daß das Gemeinschaftsrecht die Zuständigkeit der Mitgliedsstaaten zur Regelung der Systeme sozialer Sicherheit unberührt läßt: Bei der Ausgestaltung der Marktchancen von europäischen Marktteilnehmern macht er Ernst mit der Durchsetzung der europarechtlichen Grundfreiheiten.

Das „Fatale“ daran ist, daß die rechtlichen Grundsätze, die der Europäische Gerichtshof aufgezeigt hat, eben nicht nur für den Erwerb einer Brille oder die Schließung einer Zahnlücke gelten. Sie gelten auch für 
alle anderen Bereiche des Dienstleistungsrechts. Und trotz aller planungs- und förderrechtlichen Spezifika mittelfristig auch für RehaKliniken und Krankenhäuser.

Ich meine, daß die mit der 5 . Änderungs-VO zur BPfVO begonnene Liberalisierung der pflegesatzrechtlichen Vorschriften nun der Auftakt war. Elektive Eingriffe - und nur hier funktioniert Markt - lassen sich organisatorisch allemal in die übrige Versorgung integrieren.

Im übrigen gilt: Überkapazitäten sind in einem erweiterten Markt auch Produktionsreserven.

Zu hoffen bleibt, daß die europäische Billigung des Brillenerwerbs dazu beiträgt, unsere Kurzsichtigkeit zu diesem Thema zu kurieren.

Ist das jetzt die Apokalypse im deutschen Gesundheitswesen? Ich meine: Nein. Denn: Wer die Einheit Europas will, muß auch bereit sein, die Konsequenzen zu ertragen. Ein einheitlicher Binnenmarkt führt systemimmanent langfristig zur Harmonisierung der Wirtschafts- und Sozialsysteme, aber nicht zwangsläufig auf dem Niveau des kleinsten gemeinsamen Nenners.

Lassen Sie mich meine Position kurz umreißen: Die europäischen Grundfreiheiten gelten universell, sie sind ein wesentliches Grundprinzip des Zusammenlebens in Europa. Die Freiheit der wirtschaftlichen Betätigung ist nicht davon abhängig, ob ein Staat gewisse Bereiche seines Wirtschaftslebens protektionistisch abschirmen will. Die einzige Schranke, und die resultiert aus dem europäischen Vertragsrecht selber, liegt in der Frage der Legalität und gesellschaftlichen Zumutbarkeit einer wirtschaftlichen Betätigung.

Die in der Diskussion zum Schutz der nationalen Sozialsysteme reklamierten Restriktionen lauteten „Kostenexplosion“, „Qualitätssicherung“ und - last but not least - „Existenzgefährdung“.

Das Argument von der Kostenexplosion überzeugt schon in tatsächlicher Hinsicht wenig, als doch die zu ersetzenden Kosten auf das Niveau des Heimatlandes in zuverlässiger Weise limitiert werden.

Den Qualitätssicherungsaspekt überzubewerten, verrät ein übergroßes $\mathrm{Ma} ß$ an Hybris. Es widerspricht zumindest den politischen Bemühungen Deutschlands im europäischen Einigungsprozeß, von der grundsätzlichen Gleichwertigkeit der Leistungserbringung in sämtlichen Mitglieds- 
staaten auszugehen und die beruflichen Qualifikationen im Rahmen der Niederlassungsfreiheit anzuerkennen.

Das „Bestandsgefährdungsargument" schließlich suggeriert den drohenden Untergang einzelner nationaler Systeme sozialer Sicherheit. Dies ist eine Begründung aus der Spezies sogenannter K.-o.-Argumente, die meist aber schon deshalb nicht stichhaltig sind, als sie vorliegend die geradezu massenhafte Abkehr von den Leistungen des an sich zuständigen Staates unterstellen, die im Falle der Reha-Kuren allerdings schon in Ermangelung einer wirklich vergleichbaren qualitativ hochwertigen Reha-Landschaft anderen Orts kaum eintreten kann. Etwas Erdnähe in der Argumentation täte zuweilen gut!

Dies heißt mit anderen Worten, daß selbstverständlich auch Anbieter stationärer Gesundheitsleistungen ein Anrecht haben, ihre Dienstleistung grenzüberschreitend anzubieten. Nach allen Regeln der-juristischen - Kunst kann kein EU-Nationalstaat sich auf Dauer gegen diese Form der wirtschaftlichen Betätigung wehren, insbesondere nicht durch nationale gesetzgeberische Maßnahmen. Eine solche Auffassung hat bislang nicht einmal das Bundesverfassungsgericht vertreten, obwohl es in dieser Dekade eine merkliche Kehrtwendung hin zur stärkeren Behauptung nationaler Souveränität vollzogen hat.

In der mit vielen Emotionen gespickten Diskussion muß auf einen rechtlichen Gesichtspunkt immer wieder mit aller Deutlichkeit hingewiesen werden: Aus der Sicht des europäischen Rechts ist zwischen dem nationalen Gesundheitswesen als System der sozialen Sicherheit und der Teilnahme eines Dienstleisters am Gesundheitsmarkt in aller Schärfe zu unterscheiden. Beide Fragen haben nach der Logik des europäischen Vertragsrechts nichts miteinander zu tun.

Die europäischen Verträge, so die übereinstimmende Auffassung aller Rechtsgelehrten zu diesem Thema, vermeiden Einflußnahme auf die Ausgestaltung eines nationalen Systems der sozialen Sicherheit.

Wie ein Staat seine Bürger absichert, ob ein höheres oder niedriges $\mathrm{Maß}$ der sozialen Sicherheit gewährleistet wird, in welcher Form es finanziert wird, ist demnach zunächst keine Sache des europäischen Rechts.

Aber diese Frage der Ausgestaltung des sozialen Sicherungssystems betrifft nicht die Frage, ob ein Zahnarzt bohren und ein Chirurg schneiden darf und ob er diese Leistungen beim Kunden im Wege der Kostenerstattung oder im Wege der Direktabrechnung mit dem Sozialleistungs- 
träger abrechnen darf. Die Freiheit zur Ausgestaltung des sozialen Sicherungssystems betrifft allein die Beziehung zwischen Sozialleistungsträger und Versichertem. Wiederholt hatte der Gerichtshof bis in die jüngste Zeit Gelegenheit, sich mit der Frage europäischer Wahlfreiheit zu einem beliebigen sozialen Sicherungssystem auseinanderzusetzen. Er hat bei diesen Gelegenheiten klargestellt, daß nur der jeweilige Aufenthalts- bzw. Beschäftigungsstaat entscheidend ist für die Frage, welchem Sicherungssystem ein Bürger angehört. Ein Wahlrecht besteht insofern eben nicht. Konvergenz zwischen den sozialen Sicherungssystemen wird durch die Regelungen zur Zusammenarbeit und den individuellen Transfer in den maßgeblichen Verordnungen, insbesondere der Verordnung 1408/71, gewährleistet.

Gänzlich anders sieht das europäische Recht die Position des Leistungserbringers. Dem liegt eine schlichte - aber wahre - Einsicht zugrunde: Das Solidarsystem ist nicht zur Gesunderhaltung und wirtschaftlichen Absicherung der Leistungserbringer geschaffen. Demzufolge handelt es sich bei der Handreichung eines Arztes, bei der Aufnahme in ein Krankenhaus, bei Operationen, Physiotherapie und dem Verkauf von Hilfsmitteln nicht um Solidaritätsverpflichtungen, die der jeweilige Nationalstaat gegenüber seinen Bürgern unmittelbar einzulösen hat. Es handelt sich schlicht und einfach um eine normale wirtschaftliche und entgeltliche Tätigkeit.

Die Inanspruchnahme von Behandlungen jeder Art durch die Berechtigten, also unserer Versicherten, unterliegt unter allen denkbaren sozialrechtlichen Gestaltungen dem Privatrecht. Lediglich die Finanzierung der Inanspruchnahme ist durch nationales Sozialrecht unterschiedlich geregelt.

Teilhabe am Gesundheitsmarkt ist - wer wüßte das besser als ein von Kostenexplosionen gebeutelter „Kostenträger" - erwerbswirtschaftliche, weil vergütete Tätigkeit. Anders könnte die europäische Diskussion nur geführt werden, wenn das karitative Moment bei der Erbringung von Behandlungsleistungen überwiegen würde. Dieser Nachweis kann zur Zeit aber kaum gelingen.

Nach alledem gilt, daß auch ein im Gesundheitswesen agierender Marktteilnehmer natürlich seine Dienstleistung grenzüberschreitend anbieten kann. Es kann einem Patienten nicht verwehrt werden, sich jenseits seiner Nationalstaatsgrenzen behandeln zu lassen. Auch kann einem Krankenhaus nicht verwehrt werden, Patienten aus anderen EUStaaten aufzunehmen und zu behandeln. Es kann einer Rehabilitationseinrichtung nicht verwehrt werden, um Patienten aus anderen EU- 
Staaten zu werben. Nicht einmal das ärztliche Standesrecht limitiert Marketingaktivitäten stationärer Einrichtungen.

Ein letztes - zutiefst rheinisches - verbleibendes Standardargument gegen die Öffnung des Gesundheitsmarktes lautet: „Wenn dat alle mache däten - da könnt' ja jeder kommen." Genau.

Ich will nicht verhehlen, daß ich der festen Überzeugung bin, daß - ähnlich wie in anderen Bereichen des Gesundheitsmarktes-diese Entwicklung in Richtung völliger Liberalisierung auch für das Angebot stationärer Leistungen nicht aufzuhalten sein wird. Bosman läßt grüßen!

Ich denke, der Konvergenzprozeß wird stufenweise erfolgen. Er wird zunächst den ambulanten Bereich, dann die medizinische Rehabilitation, die Finanzierung, das Krankenhaus und zuletzt mit der Koordinierung und Synchronisierung der Organisation der Gesundheitssysteme sein Ziel erreichen.

Aus deutscher Sicht wird gerne argumentiert, daß die deutsche Qualität so einzigartig in der Welt sei, daß andere Angebote schon aus fürsorgerischen Schutzerwägungen vom deutschen Patienten ferngehalten werden müßten.

Ich bin allerdings von der Robustheit des Deutschen und der Qualität freiwillig aufgesuchter anderer europäischer Anbieter immerhin so überzeugt, daß ich glaube, daß das, was einen Franzosen oder einen Spanier nicht umbringt, per se auch für den Deutschen nicht abträglich sein kann. Eine ähnliche Diskussion haben wir in Sachen Reinheitsgebot des Bieres und - mit Loriot-zur Qualität der deutschen Nudel übrigens schon einmal geführt. Und uns im übrigen auch damals nicht durchgesetzt!

Eingangs habe ich die Frage gestellt, ob die Entscheidungen Kohll/Decker vom 28.04.1998 nun mehr Chance oder mehr Risiko darstellen.

Ich meine, in einer Zeit, in der Sozialleistungsansprüche als Rechtsansprüche verstanden wurden und die europäischen Grundfreiheiten zutreffenderweise als Freiheiten von Konsumenten und Leistungserbringern begriffen wurden, ist kein Raum mehr für protektionistische und archaische Schutzzäune. 
Der Anteil von Gesundheitsleistungen am gesamten europäischen Bruttosozialprodukt beträgt fast $10 \%$, allein der GKV-Markt in Deutschland fast $300 \mathrm{Mrd}$. DM.

Nennen Sie mir einen guten Grund, der den Entzug dieses bedeutenden Wachstumsmarktes aus den allgemein anerkannten - und gewollten! Regeln über den europäischen Binnenmarkt legitimieren könnte. Die Alleinverantwortung des nationalen Mitgliedsstaates für "sein" Sozialrecht leistet diese Argumentation bei weitem nicht, weil das Sozialrecht - wie gezeigt - eben nur die Bezahlung, nicht aber die Erbringung der Leistung regeln darf.

Konkret zu den Chancen.

Jeder von uns dürfte schon einmal in allfälligen Sonntagsreden die unübertreffliche Leistungsfähigkeit und Einmaligkeit des deutschen Gesundheitssystems gepriesen haben. Wie paßt eigentlich zu diesen selbstüberschätzend anmutenden Huldigungen unsere kleinmütige Verzagtheit, wenn Generalanwalt Giuseppo Tesauro und ihm folgend die Richter des EuGH ein winzig kleines Wettbewerbsfensterchen zu den qualitativ so unterlegenen anderen Ländern aufmacht? Warum eigentlich sollten uns scharenweise die Patienten weglaufen, wo sie doch überall von geringwertiger und überteuerter Medizin bedroht sind? Ich erinnere an das Beispiel der Hüftendoprothese, die bei uns als Fallpauschale um die 15.000,00 DM, in den USA hingegen ca. 50.000,00 \$ kostet. Wer einerseits Superlative zur Beschreibung unseres deutschen Gesundheitssystems bemüht und gleichzeitig infolge der EuGH-Rechtsprechung den Untergang der deutschen Krankenversicherung befürchtet, stellt an die innere Konsistenz seiner Argumentation allenfalls Ansprüche, die nicht einmal zur Lufthoheit über den Stammtischen befähigt.

Und nie vergessen: Einem Trierer Zahnarzt wurde durch das Kohll-Urteil die Abrechnung mit der luxemburgischen „Caisse de maladie“ eröffnet. Ausgerechnet einem Zahnarzt, also einem Angehörigen einer Gruppe besonders notleidender und unterprivilegierter Marktteilnehmer.

Mit dem Urteil hat sich jedenfalls der Wind gedreht. Wir werden die Windrichtung schon deshalb nicht ändern können, weil es gegen EuGHUrteile nicht einmal Rechtsmittel gibt. Aber wir können die Segel richtig setzen - und das sollten wir gemeinsam tun! 


\section{Stationäre Leistungen, Kuren und Rehabilitationen}

Günter Neubauer

Der nachfolgende Beitrag behandelt in erster Linie die stationäre Krankenversorgung durch Krankenhäuser. Die Versorgung in Rehabilitationskliniken wird lediglich im letzten Punkt angesprochen. Diese nachrangige Betrachtung der Rehabilitationskliniken entspricht bei weitem nicht deren tatsächlicher Aufgabenstellung im System. Es sind Zeitgründe, weshalb die Rehabilitationskliniken nur in ihrer Funktion als Komplementäreinrichtung zu den Krankenhäusern betrachtet werden. Tatsächlich verdienten die Rehabilitationseinrichtungen einen eigenen Beitrag.

\section{Problemstellung}

Die Krankenhäuser gleichen heute Schwimmern, die versuchen, gegen die Flußströmung anzukämpfen. Sie sind - um im Bild zu bleiben - jeden Abend völlig erschöpft, gleichwohl erhalten sie von neutralen Beobachtern das Urteil, daß sie nicht vom Fleck gekommen sind. Selbst die Krankenkassen, die die Schwimmer nach getaner Arbeit zu verköstigen haben, schließen sich mehr und mehr dieser Meinung an. Auch sie stellen fest, daß trotz ausreichender Ernährung der Schwimmer diese nicht weiterkommen. Doch die offizielle Gesundheitspolitik heißt dieses „Gegen-den-Strom-schwimmen" immer noch für richtig, obwohl auch hier die Einsicht zunimmt, daß keine rechten Fortschritte erzielt werden. Mit der Vereinigung Europas nimmt die Fließgeschwindigkeit des Flusses weiter zu. Es bleibt abzuwarten, wie lange die Schwimmer weiterhin meinen, gegen die Flußrichtung durchhalten zu können. Worauf es ankommt ist, die Richtung zu ändern, da offensichtlich gegen den Strom keine Fortschritte zu erzielen sind.

Das Fatale ist, daß die Betroffenen, nämlich die Krankenkassen wie auch die Krankenhäuser, noch immer mehrheitlich der Meinung sind, die Richtung würde stimmen. Allerdings stellen mehr und mehr Krankenhäuser und Krankenkassen fest, daß es offensichtlich vernünftiger wäre, die Schwimmrichtung zu ändern, nämlich die staatliche Planwirtschaft durch ein wettbewerbliches System zu ersetzen. Doch bevor wir zu dieser Schlußfolgerung kommen, soll das derzeitige System der deutschen Krankenhausversorgung aus ökonomischer Sicht beschrieben werden. Wie sieht nun das derzeitige System der deutschen Krankenhausversorgung aus ökonomischer Sicht aus? 


\section{Die deutsche Krankenhausversorgung: ein Beispiel staatlicher Planwirtschaft}

Die deutsche Krankenhausversorgung beinhaltet im Kern eine staatliche Angebotsplanung. Wie in der nachfolgenden Abbildung (Abb. 1) dargestellt, geht dieser Angebotsplanung eine staatliche Bedarfsermittlung voraus. Die Bedarfsermittlung erfolgt derzeit mit Hilfe der sog. analytischen Formel. Die Angebotsplanung wird über eine staatliche Investitionslenkung umgesetzt. Diese staatliche Investitionslenkung wird von Steuermitteln getragen und bedarf daher der parlamentarischen Legitimation. Aus diesem Grund ist auch ein Investitionsplan notwendig, der aufzeigt, welche Mittel wofür verwendet werden, und der im Grundsatz über die demokratischen Organe bewilligt wird.

Abb. 1: Die staatliche Angebotsplanung der deutschen Krankenhausversorgung

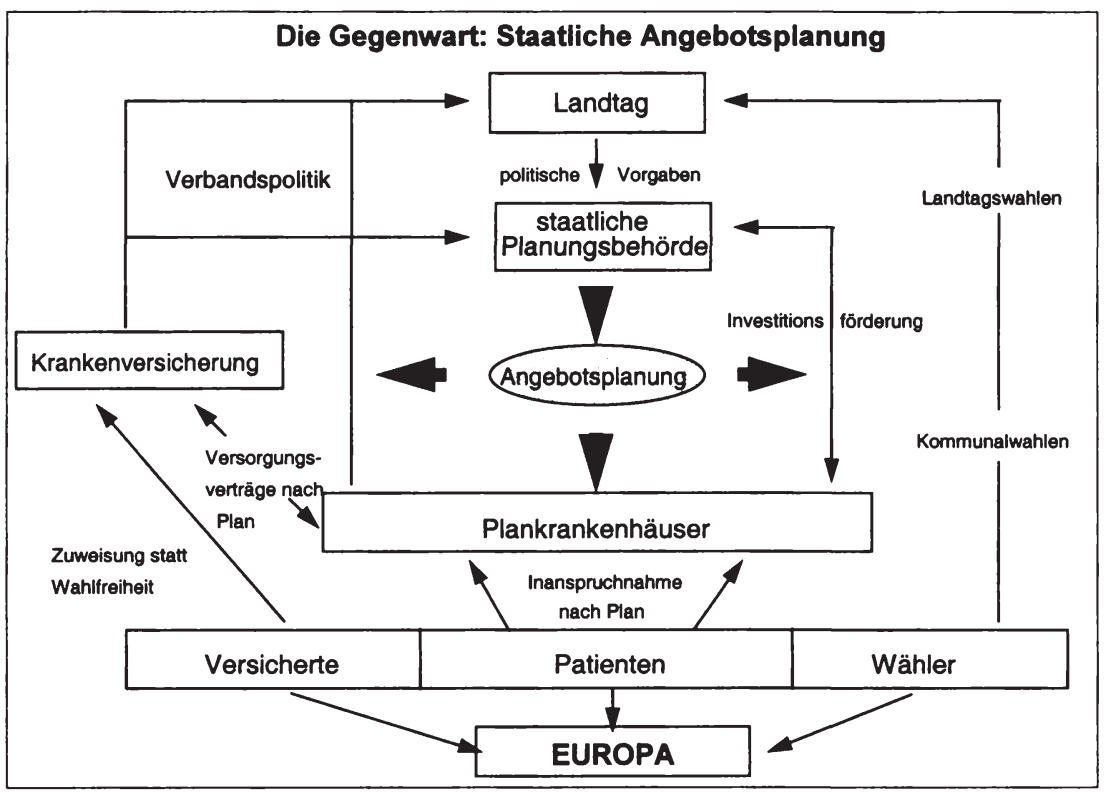

Die staatliche Angebotsplanung bezieht sich nicht nur auf die Krankenhäuser, sondern auch auf die Krankenkassen. Für die Krankenhäuser bedeutet die Aufnahme in den Plan so etwas wie den „Ritterschlag“ und damit die Aufnahme in den „Adelsstand“. Plankrankenhäuser sind nämlich in ihrer Existenz quasi gesichert. Sie haben Anspruch auf Versor- 
gungsverträge gegenüber den Krankenkassen. Offen bleibt zwar, in welcher Höhe die Krankenkassen die Versorgungsleistungen zu honorieren haben. Doch sind auch hier die Krankenkassen nicht frei, da im Streitfall Schiedsämter eingeschaltet werden, die entscheiden können. Zwar bleibt dann immer noch eine gerichtliche Überprüfung der Entscheidung des Schiedsamtes offen, doch ist diese in der Regel mit vielen Unwägbarkeiten verbunden.

Die Krankenversicherungen sind in diesem System quasi Vollzugsgehilfen staatlicher Planung. Mit viel Mühe haben sich die Krankenkassen das Recht erkämpft, auch Plankrankenhäusern kündigen zu dürfen, falls diese nicht bedarfsgerecht und unwirtschaftlich sind. Allerdings muß in diese Kündigung das Land einwilligen, andernfalls tritt die Kündigung nicht in Kraft.

Ein Drittes kommt hinzu: Auch die Patienten haben in einem System staatlicher Planung keine freie Wahl, sondern müssen sich an das von den einweisenden Ärzten angegebene Krankenhaus halten. Die einweisenden Ärzte wiederum müssen nach $\S 73$ Abs. 4 SGB V die Patienten in eines der beiden nächstgelegenen, geeigneten Plankrankenhäuser einweisen. Das heißt also: Patienten und einweisende Ärzte haben keine freie Wahl der Krankenhäuser, sondern müssen sich in die staatlich vorgeplante Versorgungsstruktur einfügen.

Spätestens an dieser Stelle erkennt man, daß dieses System in einer modernen Welt nicht mehr aufrechtzuerhalten ist. Seit nämlich der „Bazillus" der freien Wahl in das System der gesetzlichen Krankenversicherung eingedrungen ist - in Form der freien Wahl der Krankenkasse -, kann auch schwerlich eine freie Wahl der Krankenhäuser verwehrt werden. Heute kommt noch hinzu, daß auch den Patienten Wahlrechte gegenüber allen geeigneten Einrichtungen in Europa eingeräumt werden müssen. Es kann nicht gewollt sein, daß Europa sich eine einheitliche Währung gibt, andererseits aber den Verbrauchern die freie Wahl verwehrt. Von daher wird auch der Krankenhaussektor in nächster Zeit nicht länger als nationale Angelegenheit betrachtet werden können. Es gilt, das Gesundheitswesen insgesamt für Europa zu öffnen und damit auch in einen europaweiten Wettbewerb einzubinden. Daß dieser aufgrund des Dienstleistungscharakters von medizinischen Diensten regional begrenzt bleiben wird, ist kein Gegenargument.

Aber auch für eine Demokratie ist die periodische Wahlmöglichkeit ein Grundelement. Es ist nicht einzusehen, warum nicht auch ähnliche Wahlmöglichkeiten dem Patienten einzuräumen sind. Mit anderen Worten: Wir sind der Meinung, daß eine staatliche Planwirtschaft heute auch 
demokratischen Grundprinzipien widerspricht. Hier kann nicht das Argument eingebracht werden, daß doch viele Menschen für die Wahl gar nicht geeignet sind. Auch wenn in einer Demokratie nur ein kleiner Teil zur Wahlurne geht, werden die Wahlen als gültig angesehen. So ähnlich ist es im Gesundheitssektor. Selbst wenn nur ein kleiner Teil der Patienten seine Wahlrechte ausübt, kann dies ein wichtiges Signal für alle Krankenhäuser sein, da ein Wechsel von 10-15\% der Patienten für jedes Krankenhaus einen spürbaren Verlust bzw. Zugewinn darstellt. Das heißt, das Wahlrecht muß immer und jederzeit direkt ausgeübt werden können. Dem widerspricht nicht, wenn ein Versicherter freiwillig seine Wahlmöglichkeiten für eine bestimmte Periode aufgibt, um damit andere Vorteile zu erlangen.

Es gibt aber noch mindestens zwei weitere Gründe, die gegen ein System der staatlichen Angebotsplanung sprechen. Zunächst wäre hier die rasche medizinische und ökonomische Weiterentwicklung zu nennen. Schon heute werden immer mehr und mehr Eingriffe schonender vorgenommen, so daß die Krankenhausverweildauer deutlich verkürzt werden kann. Eine Konsequenz daraus ist, daß die Planung der Kapazitäten revidiert werden müßte. Nun werden aber Krankenhäuser mit einer Lebensdauer von etwa 30 Jahren geplant und gebaut, während sich auf der anderen Seite die medizinische und organisatorische Technologie quasi täglich ändert. $\mathrm{Daß}$ dies zu einem inneren Widerspruch führen muß, liegt auf der Hand. Entweder die Planung muß ständig nachgebessert werden oder die Krankenhäuser werden auf Strukturen festgehalten, die nicht mehr zeitgemäß und bedarfsorientiert sind. Wir können heute in Deutschland beides feststellen: Sowohl die Krankenhausstrukturen sind überdimensioniert als auch die betrieblichen Strukturen selbst sind nicht mehr zweckmäßig angelegt.

Wie wenig die staatliche Planung die Realität trifft, kann die nachstehende Tabelle (Tab. 1) belegen. Wir haben in der ersten Spalte dieser Tabelle für die Jahre 1993 bis 1997 die jeweils geplante Grundlohnsummenentwicklung eingetragen, nach deren Maßgabe die Ausgaben für Krankenhäuser maximal wachsen dürfen. Die tatsächlichen Zahlen, die ein Jahr später ermittelt wurden, finden sich in der zweiten Spalte. Es zeigt sich, daß selbst bei einer Vorausplanung von nur einem Jahr gravierende Abweichungen auftreten. Diese haben wir in der dritten Spalte daneben gestellt. Die Abweichungen zwischen Planung und Realität schwanken sowohl für die alten wie für die neuen Bundesländer zwischen $19 \%$ und $91 \%$. Wenn aber staatliche Planung selbst bei der Vorhersage für ein Jahr derartig weit von der Realität entfernt ist, kann man sich vorstellen, welche Abweichungen sich bei einer Planung von Kapazitäten für 30 Jahre einstellen. 
Tab. 1: Die Entwicklung der Grundlohnsumme in Planung und Realität

\begin{tabular}{|c|c|c|c|c|c|c|}
\hline & \multicolumn{3}{|c|}{ alte Bundesländer } & \multicolumn{3}{c|}{ neue Bundesländer } \\
\hline Jahr & Planung & Realität & $\begin{array}{c}\text { Abwei- } \\
\text { chung }\end{array}$ & Planung & Realität & $\begin{array}{c}\text { Abwei- } \\
\text { chung }\end{array}$ \\
\hline 1993 & 3,1 & 4,0 & $29 \%$ & 9,5 & 16,5 & $74 \%$ \\
\hline 1994 & 3,2 & 2,4 & $25 \%$ & 7,5 & 8,9 & $19 \%$ \\
\hline 1995 & 1,7 & 0,7 & $59 \%$ & 3,5 & 0,3 & $91 \%$ \\
\hline 1996 & 1,7 & 1,0 & $41 \%$ & 4,5 & 1,7 & $62 \%$ \\
\hline $\begin{array}{c}1997 \\
(1 . \text { HJ) }\end{array}$ & 1,1 & 0,1 & $91 \%$ & 1,5 & 0,7 & $53 \%$ \\
\hline
\end{tabular}

Ein zweites, systemimmanentes Manko stellt die starke Politisierung der Krankenhausplanung dar. Selbst wenn die jeweiligen staatlichen Krankenhausadministrationen die Pläne einigermaßen treffsicher aufstellen, ist damit noch nicht gewährleistet, daß diese Pläne auch sachgerecht umgesetzt werden. Denn staatlich geförderte Krankenhäuser sind auch ein Stück Politik. So hat die Nachkriegsgeschichte gezeigt, daß es den politischen Entscheidungsträgern relativ leicht fält, neue Krankenhäuser zu eröffnen oder alte Krankenhäuser zu sanieren. Schier unmöglich ist es jedoch, überschüssige Krankenhauskapazitäten abzubauen.

Hierfür kann Berlin ein treffliches Beispiel sein. Der Autor war erst wenige Tage vor diesem Beitrag als Sachverständiger nach Berlin eingeladen, um zu der notwendigen Reduktion von Krankenhausbetten in Berlin Stellung zu nehmen. Es stellte sich dabei in der Diskussion heraus, daß sich zwar alle Entscheidungsträger einig waren, daß Kapazitäten abgebaut werden müssen, jedoch niemand bereit war, in seinem Wahlkreis einer solchen Bettenreduktion zuzustimmen. Nicht selten hatten die einzelnen Krankenhäuser in jeder Senatsfraktion einen „Schutzpatron" sitzen, der Unheil von seinem Krankenhaus abzuwenden geschworen hat.

Im Ergebnis haben wir somit eine politische Durchdringung eines Wirtschaftssektors von hoher Bedeutung. Geboten ist, die politische Entscheidungsträgerschaft aus dem Krankenhaussektor so weit wie möglich zurückzuziehen. Bevor wir nun der Frage nach der Alternative zur staatlichen Angebotsplanung nachgehen, wollen wir noch auf ein Paradoxon aufmerksam machen: Die privaten Krankenhäuser scheinen mit der staatlichen Angebotsplanung besser zurechtzukommen als die öffentlichen! 


\section{Die Rolle der privaten Krankenhausunternehmen}

Betrachtet man die Entwicklung der Umsatzzahlen von drei Krankenhausunternehmen, die zu den fünf größten in Deutschland gehören, so stellt man fest, daß diese Krankenhausunternehmen sich deutlich schneller als der Markt insgesamt entwickeln. In der nachfolgenden Abbildung haben wir diese Entwicklung seit 1990 graphisch dargestellt (Abb. 2). Wie man sieht, haben sich die privaten Krankenhausunternehmen weit von der generellen Marktentwicklung abgehoben. Man kann schon von den Zahlen her vermuten, daß die schnelle Entwicklung der Privaten zu Lasten des übrigen Marktes geht. Mit anderen Worten: Es findet derzeit eine Umstrukturierung des Marktes statt.

Abb. 2: Umsatzentwicklung der drei größten Krankenhausunternehmen und Krankenhausausgaben der GKV im Vergleich $(1990=100)$

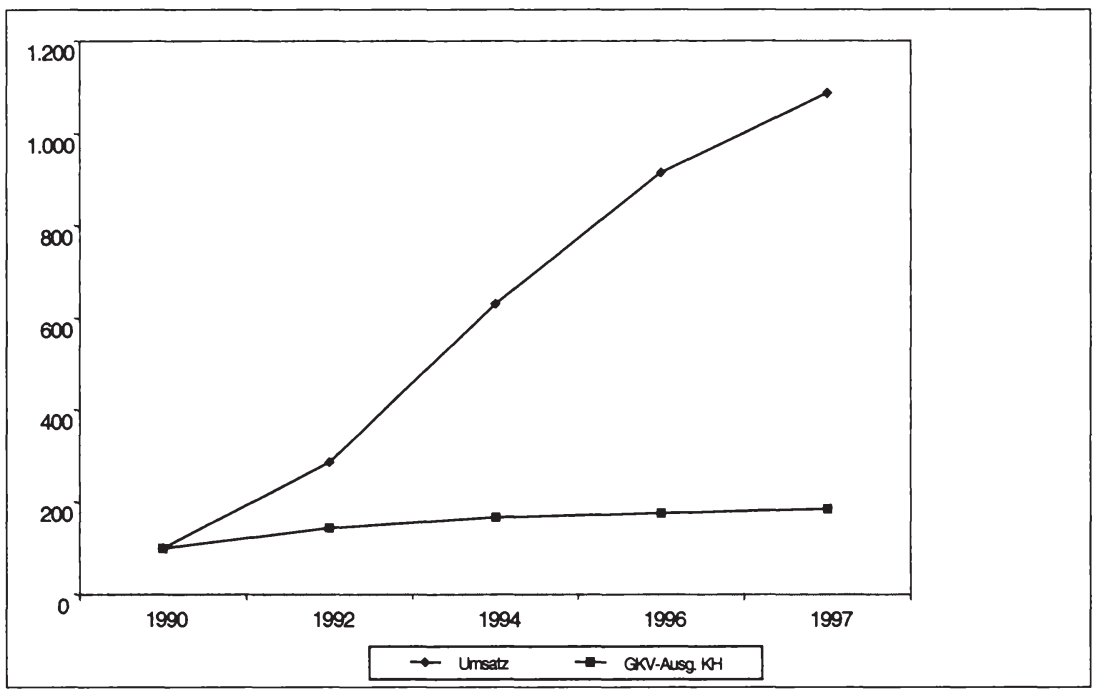

Das Paradoxon an dieser Entwicklung ist, daß die privaten Krankenhausunternehmen weitgehend auf die staatlichen Fördermaßnahmen verzichten, obwohl sie in der Regel selbst Plankrankenhäuser leiten und damit Anspruch auf staatliche Investitionsförderung hätten. Auf der anderen Seite fallen die aus öffentlichen Steuergeldern über den Kapitalmarkt finanzierten Krankenhäuser zurück. Es wird hier deutlich, daß öffentlich geförderte Krankenhäuser offensichtlich im Wettbewerb mit privat finanzierten Einrichtungen nicht Schritt halten können. Hierfür lassen sich zwei Gründe anführen: Zum einen ist die staatliche Mittelvergabe 
mit vielen Auflagen und hohem bürokratischen Aufwand verbunden, so daß eine öffentliche Finanzierung wesentlich mehr Zeit benötigt als eine Privatfinanzierung. Oft ist aber ein Zeitvorsprung entscheidend für die Marktposition. Als zweites kommt hinzu, daß die staatlichen Fördermittel nicht ausreichend fließen. Krankenhäuser, die weitgehend auf die öffentliche Förderung angewiesen sind, sind damit nicht nur langsamer, sondern sie sind auch unterfinanziert. Im Ergebnis sieht man, daß private Unternehmen, die ihre Investitionen aus Erträgen zu finanzieren haben, im Markt besser liegen als steuerfinanzierte Konkurrenten, die ihre Investitionsmittel nicht selbst zu verdienen brauchen.

Ein Nebeneffekt dieser Entwicklung ist, daß öffentliche Häuser und die in innen Beschäftigten automatisch als weniger fähig gelten, obwohl sie im Grunde genommen nur Sklaven ihrer Umweltbedingungen sind, die sie aus eigener Kraft nicht ändern können. Aus diesem Grund wechseln heute immer mehr Krankenhäuser sowohl die Rechtsform als auch die Unternehmensführung in Richtung Privatunternehmen. Die duale Krankenhausfinanzierung, die vorsieht, daß die Investitionen aus Steuergeldern aufgebracht werden, hat damit letztendlich versagt. Doch wird diese Erkenntnis immer noch nicht weit genug wahrgenommen, wenngleich die Ergebnisse immer deutlicher zu Tage treten. Hier wird besonders deutlich, wie Krankenhäuser gegen den Strom schwimmen, ohne daß sie von der Stelle kommen, während ihre Konkurrenten bereits längst die Richtung geändert haben. Provokativ könnte man formulieren: Die staatliche Angebotsplanung läßt ihre Kinder, nämlich die öffentlichen Krankenhäuser, von den Privatunternehmen fressen. Letztere nutzen die Vorteile der Planung und vermeiden deren Nachteile, indem sie über den privaten Kapitalmarkt Investitionsmittel aufnehmen.

\section{Die Alternative: Wettbewerbliche Nachfragesteuerung}

In einem Modell der wettbewerblichen Nachfragesteuerung treten die Krankenkassen als Nachfrager von Krankenhausleistungen auf und schließen mit verschiedenen Krankenhäusern Verträge für die Versorgung ihrer Patienten. Damit stehen die Krankenkassen in den Augen der Versicherten in einem Wettbewerb. Jene Krankenkasse, welche die geeigneten Versorgungsverträge schließt, darf auch damit rechnen, daß sie entsprechend Versicherte anwerben kann (Abb. 3). 
Abb. 3: Wettbewerbliche Nachfragesteuerung in der Krankenhausversorgung

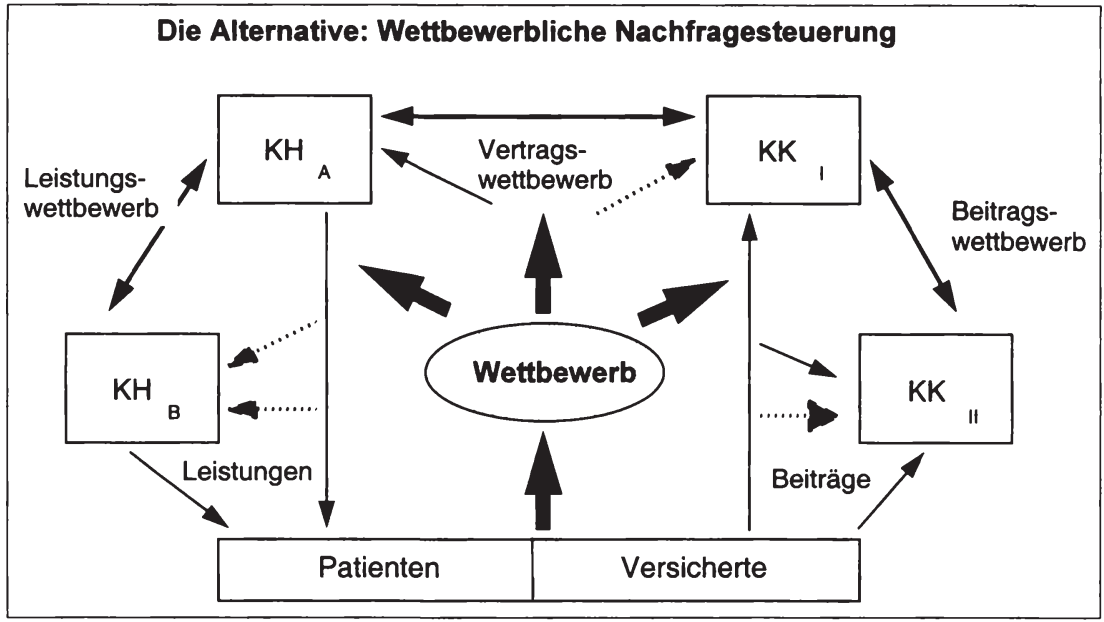

Auf der anderen Seite stehen auch die Krankenhäuser in einem Wettbewerb um Versorgungsverträge mit den Krankenkassen. Jene Krankenhäuser, die in den Augen der Krankenkassen am ehesten geeignet sind, eine adäquate Versorgung im Krankheitsfall sicherzustellen, werden vorrangig unter Vertrag genommen. Auf diese Art und Weise gelingt es, quasi nebenbei Überkapazitäten durch eine entsprechende Vertragspolitik abzubauen. Entweder erhalten überzählige Krankenhäuser überhaupt keine Verträge mehr oder nur noch von wenigen Krankenkassen, so daß sie nicht mehr ihre gesamte Kapazität nutzen können.

Den Krankenhäusern selbst bleibt aber auch der Weg, die Patienten direkt an sich zu binden. Denn je besser es den Krankenhäusern gelingt, die Patienten und ihre einweisenden Ärzte direkt an sich zu binden, um so weniger sehen sie sich dem Vertragswettbewerb gegenüber den Krankenkassen ausgesetzt. In jedem Fall werden sowohl die Krankenkassen versuchen, via Vertragspolitik Versicherte zu gewinnen, als auch die Krankenhäuser versuchen werden, im Leistungswettbewerb Patienten an sich zu ziehen, um so den Vertragswettbewerb zu reduzieren.

In einer derart wettbewerblich gesteuerten Krankenhausversorgung ist der Kranke dem Wettbewerb nicht ausgesetzt, solange er sich auf die Vertragspolitik seiner Krankenkasse verläßt. 
Im heutigen System haben wir bereits den Beitragswettbewerb der Krankenkasse um Versicherte. Was noch fehlt, ist der Vertragswettbewerb der Krankenhäuser untereinander mit den Krankenkassen. Rudimentär entwickelt ist der Leistungswettbewerb der Krankenhäuser um Patienten. Beide Felder werden sich aber in der nächsten Zeit öffnen, so daß sich auch hier auf längere Sicht eine wettbewerbliche Ausrichtung durchsetzen wird. Ein großes Hindernis hierbei sind die Krankenhauspläne der Länder, die einem Wettbewerbssystem diametral entgegenstehen.

\section{Rehabilitationskliniken als Komplementäreinrichtungen}

Die medizinische Rehabilitation richtet sich überwiegend auf solche $\mathrm{Pa}$ tienten, die eine Akutbehandlung erhalten haben und zur Ausheilung dieser Erkrankung sowie zur Wiederherstellung ihrer täglichen Funktionsfähigkeit in eine Rehabilitationsklinik überwiesen werden. Davon zu unterscheiden sind Patienten in sog. Heilverfahren, bei denen keine Akuterkrankung im Mittelpunkt steht, sondern eine drohende Minderung oder ein drohender Verlust der Erwerbsfähigkeit. Heilverfahren sind damit vor allen Dingen auf den Arbeitsmarkt gerichtet, während medizinische Rehabilitation einen Teil der medizinischen Behandlung darstellt.

Heute stellen wir fest, daß die Akutbehandlungen immer kürzer werden, weil die Medizin schonendere Behandlungsverfahren entwickelt und die Vergütungssysteme eine kürzere Verweildauer belohnen. Andererseits werden Akutpatienten immer älter, weil der medizinische Fortschritt zum großen Teil den älteren Patienten zugute kommt. Dies hat zur Folge, daß immer mehr Patienten in kürzerer Zeit in den Krankenhäusern behandelt werden. Diese Patienten werden in der Regel noch nicht „entlassungsfähig“ nach Hause geschickt, obwohl sie einer weiteren Nachbehandlung bedürfen.

Eine solche Nachbehandlung bieten die medizinischen Rehabilitationskliniken an. Auf längere Sicht werden diese Einrichtungen weiterhin an Bedeutung als Komplementäreinrichtung zur Akutbehandlung gewinnen. Immer häufiger schließen sich heute Akutkrankenhäuser und Rehabilitationskliniken zu einem Leistungsverbund zusammen, sei es in einer Trägerschaft oder sei es auch nur als kooperative Partnerschaft. Das ökonomische Ziel solcher Zusammenschlüsse ist es, den Übergang des Patienten von der einen in die andere Einrichtung zu optimieren. Denn je besser die Rehabilitationsklinik ausgestattet ist, um so früher kann sie die Patienten aus der Akutbehandlung übernehmen. Auf der anderen Seite kann die Akutbehandlung sich auf ihre eigentliche Kernkompetenz, nämlich die medizinisch-ärztliche Behandlung, konzentrieren und die 
pflegerische und die rehabilitative Komponente an die Rehabilitationsklinik abgeben.

Eine logische Konsequenz einer solchen Kooperation ist, daß auch das Vergütungssystem seitens der Krankenkassen entsprechend zusammengefaßt wird. Heute werden zu diesem Zweck sog. Komplexpauschalen entwickelt, welche die Akutbehandlung und die Rehabilitation in Form einer Pauschale abgelten. Solche Komplexpauschalen stoßen zwar noch auf eine Reihe von Widerständen, gleichwohl gibt es mittlerweile in Deutschland bereits mehrere Pilotversuche. Auf längere Sicht dürfte sich ein solches Vergütungsmodell gegenüber dem alten, additiven Vergütungssystem als überlegen erweisen.

Die Patienten in Heilverfahren, zuweilen auch als Kurpatienten bezeichnet, werden hingegen bei der anhaltenden Arbeitslosigkeit an Bedeutung verlieren. Schließlich ist es auch schwer einzusehen, 55jährige für einen Arbeitsmarkt zu rehabilitieren, auf dem sie nur geringe Wiederbeschäftigungschancen haben. Es kommt hinzu, daß es bei rund 4 Mio. gesunden Arbeitslosen auch unter Kosten-Nutzen-Aspekten als wenig ertragreich betrachtet werden kann, insbesondere ältere Arbeitnehmer für den Arbeitsmarkt zu rehabilitieren.

\section{Ausblick: Europa als Anstoß}

Die jüngste Rechtsprechung des Europäischen Gerichtshofes hat deutlich gemacht, daß auch der Gesundheitssektor dem Gebot der Europäisierung zu unterziehen ist. Ein in sich geschlossenes nationales System, wie es die deutsche Krankenhausversorgung darstellt, muß damit in Konflikt geraten. Es bleibt abzuwarten, bis auch für den Dienstleistungsbereich, zu dem der Krankenhaussektor zu zählen ist, der Europäische Gerichtshof wiederum die Initiative ergreift und eine entsprechende Öffnung verlangt. Daß der Krankenhaussektor immer weniger als ein geschlossenes System gelten kann, wird auch dadurch deutlich, daß die Länder ihre Krankenhäuser ermuntern, sich um ausländische Patienten zu bemühen. Wenn ausländische Patienten außerhalb des Budgets behandelt werden dürfen, ist dies der - angenehme - Anfang zur Öffnung.

Aus mittlerer Sicht kann es nicht angehen, daß sich die Gesundheitsmärkte der Öffnung nach Europa entziehen. Dies kann nicht sein und braucht auch nicht zu sein. Zumindest sind keine Nachteile für die Patienten und letztlich auch für die Krankenversicherungen zu erwarten. Es ist auch nicht einzusehen, warum die leistungsstarken deutschen Gesundheitsleistungsanbieter den europäischen Wettbewerb scheuen sollten. Hier hat die deutsche Pharmaindustrie schon vor langer Zeit ge- 
zeigt, wie man in einem internationalisierten Markt bestehen kann. Vielleicht sollten die übrigen Leistungserbringer hier ein wenig von der Pharmaindustrie lernen. 
Wolfgang Schmeinck

Ich habe den Eindruck, als wenn das Thema Binnenmarkt sowie europäische Rechtsprechung und deren Auswirkungen auf das deutsche Gesundheitswesen nun durch die vielen Referenten, vor allem durch die, die sich an das Thema gehalten haben, weitestgehend abgearbeitet ist. Ich frage mich, was ich Ihnen noch erzählen soll unter dem Aspekt "Sonstige veranlaßte Leistungen“, ohne langweilige Wiederholungen zu produzieren.

Der bisherigen Diskussion habe ich das Vorhandensein zweier Lager entnommen: Das eine Lager besteht aus Skeptikern mit kleinen Einschlüssen von Verzagtheit, das andere Lager aus notorischen Optimisten. Ich will mich outen als einer von denen, die aus Sicht der Patienten und aus Sicht der Kostenträger in der europäischen Entwicklung mehr Chancen als Risiken sehen. Ich räume aber ein, daß man das, wenn man sich nur mit dem Thema "Sonstige Vertragspartner" beschäftigen muß, auch leichter sagen kann, als wenn man sich über Krankenhausbedarfsplanung und Ärzte äußern muß. Und ich füge gleich hinzu, daß diese optimistische Einschätzung bei meinen Kollegen von den anderen Kassenverbänden durchaus nicht durchgängig vorhanden ist; auch dort gibt es Systemkonservative, die gute Gründe für ihre Haltung auffahren können.

Meine Position erklärt sich aus einer einfachen Überlegung: Ich trenne zwei Problemebenen: Die eine ist die, auf der die Versicherung gegen die Folgen von Krankheit organisiert wird, die zweite Ebene ist die, auf der die Gesundheitsleistungen organisiert werden.

Die Ebene, auf der die Versicherung gegen Krankheit organisiert wird, in der möglichst offene Zugangsbedingungen zu Versicherungen festgelegt, in der faire Beiträge abgestimmt und in der gerechte Subventionszahlungen zwischen den Beteiligten organisiert werden, diese Ebene sehe ich durch die europäische Binnenmarktentwicklung und auch durch die EuGH-Rechtsprechung nicht beeinträchtigt. Das, was wir in Deutschland als unsere solidarische Krankenversicherung schätzen, wird auf absehbare Zeit nicht gefährdet; es schützt uns die vorhandene Vielfalt der Systeme in Europa, die Herr Danner gestern beschrieben hat.

Auf der zweiten Ebene aber, auf der es darum geht, Gesundheitsleistungen effizient zu organisieren, wünsche ich mir geradezu Wettbewerb, 
wünsche ich mir Markt als Instrument zum Organisieren und Verbessern von Qualität und Effizienz. Ich gehe davon aus, daß insbesondere im Bereich „Sonstige veranlaßte Leistungen“, also konkret Arznei- und Hilfsmittel, ein europäischer Markt mehr Wettbewerb, mehr Druck und mehr Alternativen erzeugen hilft, als dies auf einem nur nationalen Binnenmarkt möglich wäre.

Ich kann nachvollziehen, daß von seiten der Leistungserbringer und gelegentlich auch von seiten der Krankenversicherung als Gefahr gesehen wird, daß Leistungen additiv und nicht substitutiv importiert werden. Gegen diese Besorgnis gilt das Argument aus der gestrigen Diskussion mit Herrn Professor Pfaff: Entweder sind wir im deutschen Gesundheitswesen so effizient, wie wir zu behaupten gewohnt sind; dann muß es doch möglich sein, nationale Gesundheitsleistungen auch zu exportieren, dann darf der europäische Binnenmarkt eben keine Einbahnstraße gegen uns sein. Ist das deutsche Gesundheitswesen aber nicht so effizient, möglicherweise auch deshalb nicht, weil die deutschen Krankenkassen Preis-Leistungs-Verhältnisse zugelassen haben, die schlechter sind als die unserer Nachbarn, muß ich als Vertreter der Krankenversicherung geradezu ein Interesse daran haben, daß es zu qualifiziertem Leistungsimport kommt.

Natürlich sehe ich, daß wir gegenwärtig als Krankenversicherung Überkapazitäten in nahezu allen Leistungsbereichen alimentieren. Ich kann die gestern von Herrn Professor Gitter gestellte Frage, wie beispielsweise der Kauf eines Arzneimittels in Belgien in Einklang zu bringen sei mit einem deutschen sektoralen Arzneimittelbudget, konkret nicht beantworten. Auf mittlere Sicht bedrückt mich dies allerdings nicht. Ich bin nämlich davon überzeugt, daß es mit Hilfe von Wettbewerb eher gelingen wird, Überkapazitäten abzubauen, als dies mit nationalen gesetzlichen Maßnahmen zu erreichen wäre. Dafür sprechen die Erfahrungen mit sehr vielen ernsthaften Reformversuchen der letzten Jahre, die Überkapazitätsprobleme nicht gelöst haben. Auch diejenigen, die nicht dieser Auffassung sind, die Wettbewerb und Markt im Gesundheitswesen eher als Fremdkörper ansehen, kommen im übrigen nicht an der Tatsache vorbei, daß es diesen europäischen Markt mindestens für Arznei- und Hilfsmittel längst gibt. Herr Danner hat gestern Gründe dafür genannt:

Arzneimittel, aber auch Hilfsmittel haben viel zu sehr Warencharakter, sind viel zu einfach transferierbar von einem nationalen Markt in den anderen, als daß man glauben könnte, solche Transfers wären auf Dauer zu verhindern. Für Arzneimittelhersteller lohnt es sich offensichtlich, über nationale Märkte hinauszudenken bei der Investition, bei der Preisbil- 
dung, beim Marketing. Diese Anpassungsgeschwindigkeit der Herstellerseite bringen die Sozialversicherungsträger als auf Konsens hin organisierte öffentlich-rechtliche Bereiche bislang nicht auf.

Derzeit ist Deutschland meines Wissens das einzige europäische Land, in dem es im Arzneimittelbereich keinen speziellen Krankenkassenmarkt gibt, keine nennenswerte zweite Zugangsschwelle zum Leistungsspektrum der gesetzlichen Krankenversicherung. Wenn das so bliebe, würde ich von einem zunehmend funktionierenden europäischen Binnenmarkt ganz überwiegend positive Effekte erwarten. Ich denke daran, daß es eine wie auch immer geartete Annäherung der Preisniveaus geben wird; wir haben in Deutschland immer noch ein relativ hohes Preisniveau. Ich kann mir auch vorstellen, daß es in einem europäischen Arzneimittelmarkt eine Straffung der Angebotsstrukturen gibt; vermutlich lohnt es sich für einen Hersteller dann, wenn die Marktspaltung nicht mehr funktioniert, nicht mehr, unterschiedliche Produktformen auf unterschiedlichen nationalen Märkten anzubieten. Das kann meines Erachtens nur transparenzfördernd und qualitätssichernd sein. Vielleicht wird ja auch irgendwann einmal die Frage der länderunterschiedlichen Mehrwertsteuerbelastung von Arzneimitteln diskutiert, wobei ich allerdings einräume, daß man bei einer Diskussion dieses speziellen Themas auch dem gegenwärtigen Ist-Zustand in Deutschland Gerechtigkeit widerfahren lassen müßte. Für ein Land, das sozusagen keinen Schutzwall für sein Sozialversicherungssystem hat, wären solche Entwicklungen über den Binnenmarkt ganz heilsam.

Wenn es auch in Deutschland, sei es durch eine Positivliste oder ähnliche Regelungen, zu einem Zweitmarkt, einem speziellen Markt für Krankenkassen kommen sollte, würde - abhängig von seiner Ausgestaltung-dieses Instrumentarium die Straffungs-, Preissteuerungs- und -senkungsfunktion wahrnehmen, die ich ansonsten vom europäischen Binnenmarkt erwarte. Auch diese Effekte sollten möglichst supranational synchronisiert werden: Die nationalen Krankenversicherungssysteme in Europa sind gut beraten, wenn sie ihre jeweiligen speziellen Kassenmärkte mit ihren Nachbarn zunehmend abstimmen. Im Lager der Krankenversicherungen gibt es dazu erste, offen gestanden zur Zeit noch ein wenig unbeholfene Versuche; diese Koordinierung auf Kassenseite kommt langsam in Gang - Geduld ist erforderlich.

Wird eine Annäherung unterschiedlicher Preisniveaus auf dem europäischen Arzneimittel- und vor allem Hilfsmittelmarkt nicht schnell genug von der Herstellerseite erzeugt, werden sich die Kostenträger um Arbitrageverfahren bemühen, sobald und soweit innen dies rechtlich möglich ist. Eine solche Arbitrage könnte z. B. darin bestehen, daß verstärkt 
Reimport oder Parallelimport stattfindet, entweder durch die klassischen Vertriebswege, im Arzneimittelbereich durch deutsche Apotheken oder vor dem Hintergrund der EuGH-Rechtsprechung auch zunehmend individuell durch deutsche Versicherte, die in ausländischen Apotheken einkaufen. Ich kann mir vorstellen, daß deutsche Krankenkassen mit effizienten Hilfsmittellieferanten jenseits der deutschen Grenzen Verträge abschließen. Vielleicht müssen Kassen ja sogar in nicht zu ferner Zeit Lieferverträge europaweit ausschreiben.

Auf lange Sicht stellt sich im übrigen noch eine ganz andere Frage: Wenn sich die Preise im europäischen Binnenmarkt so annähern, wie ich es erhoffe, ist es dann eigentlich sinnvoll, so unterschiedliche Systeme wie die deutschen Festbeträge und zum Beispiel auf französische Art administrierte Preise veranlaßter Leistungen für Kassen nebeneinander bestehen zu lassen, oder müßte man auch hier an eine Synchronisierung der Instrumente denken?

Auf kürzere Sicht wird es konkreter: Das OLG Düsseldorf hält die Bildung der deutschen Arzneimittelfestbeträge für im Widerspruch zum europäischen Kartellrecht befindlich. Nach gegenwärtigem Recht haben wir für Hilfsmittel nicht nur keine europaweiten, sondern nicht einmal deutschlandweite Festbeträge. Ich habe mich immer schon gefragt, welchen Sinn es macht, daß z. B. Inkontinenzprodukte in Hessen andere Festbeträge haben als in Württemberg; diese gesetzliche Regelung ist wahrscheinlich eine Verbeugung vor dem Föderalismus gewesen. Vor europäischem Hintergrund erinnern bundeslandindividuelle Festbeträge sehr an Kleinstaaterei. Auch im Bereich der Vertriebsstrukturen gibt es einige nationale deutsche Besonderheiten, die wahrscheinlich auf Dauer kaum zu halten sein werden, mögen sie auch irgendwann noch so sinnvoll gewesen sein. Weil nach mir ein Vertreter der Apotheker spricht, nenne ich speziell das Fremd- und Mehrbesitzverbot, das früher durchaus eine qualitätssichernde Maßnahme gewesen sein mag, inzwischen aber zunehmend sinnleer wird.

Seit einiger Zeit beobachte ich europäische Sozial- und Gesundheitspolitik und habe schon häufiger für mich Prognosen gemacht, in welchem Zeitraum eine vernünftigerweise zu erwartende Entwicklung abgeschlossen sein wird. Meine Erfahrung mit den eigenen Prognosen: Die Angleichung von Märkten, organisatorische Änderungen, Synchronisierungen, all dies kommt, aber es dauert drei- oder viermal länger als ungeduldige Menschen dies voraussehen. 


\title{
Sonstige veranlaßte Leistungen
}

\author{
Chancen und Risiken des europäischen Arzneimittelmarktes \\ - Die Sichtweise der Apotheker -
}

Frank Diener

\section{Spezifitäten der Arzneimittelversorgung auch im gemeinsamen Binnenmarkt}

Gerade unter europäischen Aspekten ist es Mode geworden, "marktkonforme" Sichtweisen zu vertreten und die fundamentalen Freiheiten des Waren- und Dienstleistungsverkehrs zu betonen. Oft hat man den Eindruck, daß „Markt“ als Synonym für das „Fehlen von Regulierung“ verstanden wird. Es ist aber selbst bei ordoliberalen Ökonomen, die das Ideal des „fernen Staates" postulieren, Konsens, daß eine Marktordnung ohne Regulierung nicht funktioniert. Conditio sine qua non für einen funktionierenden Markt ist unter anderem die Gewährleistung einer stabilen Rechts- und Eigentumsordnung - erst damit ist das „Transaktionieren" von Gütern und Diensten überhaupt möglich. Ökonomen lernen und lehren aber auch seit jeher, daß - wenn man den regulativen Kanon beschränkt oder ganz auf ihn verzichtet-unter gewissen Umständen Marktversagen auftritt. Die sog. „Neue Institutionenökonomik“, eine noch vergleichsweise junge volkswirtschaftliche Schule, befaßt sich etwa seit Anfang der 80er Jahre intensiv mit der Frage, inwieweit zur „Transaktionierung" von Waren und Diensten besondere regulative Erfordernisse im Sinne einer optimalen Allokation von Ressourcen bestehen oder nicht. Für die Protagonisten dieser Schule sind nicht "der Staat", „der Markt" oder „die Unternehmung“ per se als superiore Lösungsmechanismen der Transaktionierung von Gütern und Diensten zwischen Wirtschaftssubjekten gegeben, vielmehr wird geprüft, ob es im konkreten Fall „Besonderheiten" - sog. Spezifitäten - gibt, die maßgeschneiderte Lösungsmechanismen (sog. "governance structures") im Sinne eines reduzierten oder erhöhten regulativen Eingriffniveaus erforderlich machen. Die "Neue Institutionenökonomik" bezieht in ihre Analysen Unsicherheit, Risiko, asymmetrische Information und anderes mehr ein. Sie eignet sich zur Anwendung auf verschiedenste ökonomische Themengebiete.

Überträgt man diesen Denkansatz auf den Arzneimittelmarkt, ergibt sich, daß er von einer gleich dreifach-simultanen Spezifität gekennzeichnet ist: 
- Arzneimittel sind im menschlichen Körper oft hochwirksame Produkte mit einem schmalen therapeutischen Spektrum. Für sich genommen „harmlose“ Arzneistoffe können im Verbund mit anderen Arzneistoffen erhebliche unerwünschte Wechselwirkungen haben. Zum Teil können diese Wechselwirkungen bereits dadurch vermieden werden, daß die Einnahme zweier verschiedener Substanzen zeitversetzt erfolgt. Zum Teil dürfen entsprechende Substanzen aber keinesfalls im gleichen Zeitraum angewandt werden. Auch Kontraindikationen etc. sind zu berücksichtigen. Arzneimittel sind also aufgrund ihres ambivalenten Charakters in besonderem Maße beratungsintensiv und erklärungsbedürftig. Erst mit dem Apotheker wird das Arzneimittel zur Medizin. Mithin gibt es bei Arzneimitteln eine Produktbesonderheit.

- Die Patienten, die Arzneimittel benötigen, sind krankheitsbedingt in ihren Möglichkeiten eingeschränkt. Sie haben krankheitsbedingt ein „Handicap“ - sonst bräuchten sie keine Arzneimittel. Die Patienten, die Arzneimittel benötigen, sind über das verfügbare Spektrum von Arzneimitteln und deren adäquate Einsatzmöglichkeiten nur unzulänglich informiert. Das überrascht nicht sonderlich, denn Arzneimittel sind - in der Terminologie der "Neuen Institutionenökonomik" - sog. „option demands“, also Produkte, die nicht kontinuierlich, sondern nur unter gewissen Bedingungen nachgefragt werden und dem Nachfrager insofern nicht wie normale Gebrauchsgüter vertraut sind. Sogar chronisch kranke Patienten, die oft über "ihr" Arzneimittel gut informiert sind, haben erhebliche Wissenslücken über das Zusammenspiel „ihres“ Arzneimittels mit solchen, die sie nicht im Zusammenhang mit ihrer chronischen Krankheit benötigen. Im Vergleich zu den Heilberufen sind die Patienten daher in einer Situation der asymmetrischen Information. Beides zusammengenommen, das krankheitsbedingte Handicap und die Problematik der asymmetrischen Information, bedeuten aber das Vorliegen einer Patientenbesonderheit.

- Die Arzneimittelversorgung wie auch die Gesundheitsversorgung insgesamt kann angesichts der damit verbundenen Kostenrisiken aus sozialen Gründen nicht ohne Versicherungsschutz gesamtgesellschaftlich zufriedenstellend bewerkstelligt werden. Insofern haben alle modernen Staaten einen Versicherungsschutz für den Krankheitsfall entwickelt. Jeder Versicherung, wie auch immer sie im nationalen Einzelfall organisiert sein mag, ist aber die Problematik immanent, daß der Veranlasser einer Leistung nicht der Empfänger der Leistung ist, der Empfänger der Leistung nicht ihr Bezahler ist und der Bezahler die Leistung weder empfängt noch veranlaßt oder erbringt. Anders als im bilateralen Tausch von Leistung und Gegenleistung handelt es 
sich hier um eine mindestens trilaterale Beziehungsstruktur mit all ihren potentiellen Folgen. Es liegt also auch eine Versicherungsbesonderheit vor.

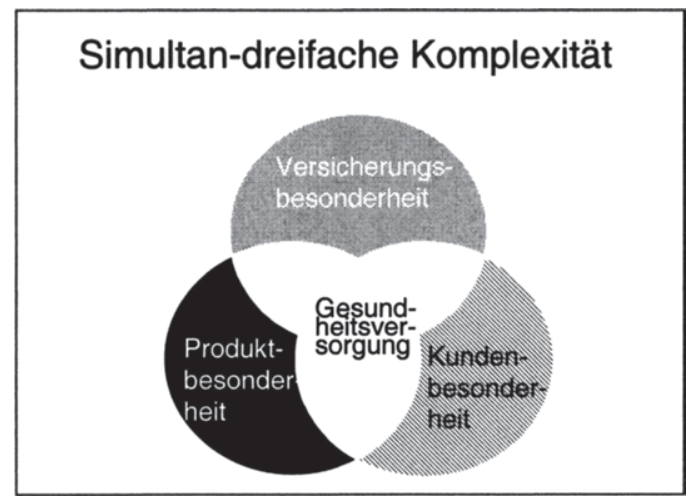

Diese drei Besonderheiten stellen sich bereits in jedem nationalen Arzneimittelversorgungssystem. Es gibt viele Wege, mit dieser mehrfachen Komplexität umzugehen - sicher ist nur, daß der pure „Marktmechanismus“ zu Marktversagen und inferioren Allokationen führen würde. In praktisch allen Industriestaaten wird deshalb die Arzneimittelversorgung mit regulativen Maßnahmenbündeln organisiert, die auf die Anspruchsniveaus sowie die wirtschaftlichen Leistungsfähigkeiten des betreffenden Nationalstaats maßgeschneidert entwickelt worden und nicht zuletzt auch vor dem jeweiligen historisch gewachsenen soziokulturellen Hintergrund zu verstehen sind. Auch wenn es Ähnlichkeiten gibt, so sind doch die Systeme der Arzneimittelversorgung insgesamt eher durch Unterschiedlichkeiten gekennzeichnet.

Bei der Schaffung eines europäischen Arzneimittelmarktes ergibt sich daraus auf absehbare Zeit die Notwendigkeit und Bedeutung des Subsidiaritätsprinzips für die Arzneimittelversorgung - zumindest so lange, bis ein vollständig harmonisiertes Gesundheitswesen in einem auch finanzwirtschaftlich weitgehend koordinierten und auch harmonisierten gemeinsamen Binnenmarkt besteht. Dies schlägt sich auch im EG-Vertrag nieder.

Grundprinzip der Arzneimittelpolitik in einem zusammenwachsenden Europa ist deshalb, daß die EU das regelt, was den supranationalen Handel im gemeinsamen Markt betrifft, und sich aus allem heraushält, was 
"nur" den intranationalen Handel im jeweiligen Mitgliedsstaat betrifft. Ganz konkret heißt das, daß die Arzneimittelhersteller- und Großhandelsstufe in den Fokus der EU-Politik fallen, während die Letztvertriebsund Endverbraucherstufe in nationaler Hoheit organisiert werden.

Deshalb wird im Arzneimittelbereich beispielsweise die

- Zulassung,

- Entwicklung,

- Herstellung,

- Packungskennzeichnung und Packungsbeilage,

- Herstellerwerbung und

- Herstellerhaftung

von der EU geregelt, und die EU hat auch die Kompetenz, in Beschränkungen bei der Herstellerpreisbildung einzugreifen.

Nicht bei der EU, sondern in nationaler Hoheit liegen dagegen alle Regelungen über

- die Bildung der Endverbraucherpreise bei Arzneimitteln,

- die Erstattung von Arzneimittelkosten in den nationalen Versicherungssystemen und

- die Bindung der Arzneimittel an bestimmte Vertriebswege,

denn diese Regelungen betreffen nicht den zwischenstaatlichen Handel, sondern ausschließlich die Art und Weise der innerstaatlichen Arzneimittelversorgung und deren Finanzierung in den nationalen Sozialversicherungssystemen.

Anders gesagt: Jeder Hersteller hat mit seinen zugelassenen Arzneimitteln ungehinderten Zugang zu jedem Teilmarkt - auf diesem Teilmarkt gelten aber für die Letztvertriebs- und Endverbraucherstufe die nationalen Spielregeln. In der Folge gibt es in den Mitgliedsstaaten der EU unterschiedliche Regelungen über

- die Verordnungsfähigkeit von Arzneimitteln zu Lasten der Kostenträger (z. B. Positiv- oder Negativlisten),

- die Regelungen zur Arzneimittelabgabe auf der Letztvertriebsstufe (Apothekenpflicht, Freiverkauf, Apothekengesetz, Apothekenbetriebsordnung etc.), 
- die Arzneikostenerstattung (Sachleistungs- oder Kostenerstattungsprinzip und/oder Festbetragssysteme),

- die Endverbraucherpreisbildung (z. B. Arzneimittelpreisverordnung)

- Mehrwertsteuerbelastung von Arzneimitteln oder

- Zuzahlungen und/oder Selbstbehalte der Patienten bei verordneten Arzneimitteln.

\section{Ausgewählte Aspekte}

\section{Kostenprobleme in den nationalen Gesundheitssystemen}

Die steigenden Kosten in den nationalen Gesundheitssystemen sind nur zum Teil durch Arzneimittel verursacht. In Deutschland sind beispielsweise die Ausgaben der gesetzlichen Krankenversicherung 1997 mit 32 Mrd. DM immer noch rund 1 Mrd. DM niedriger gewesen als 1992. Der Anteil der Arzneimittelausgaben an den Gesamtausgaben der gesetzlichen Krankenkassen ist in Deutschland von über $15 \%$ Anfang der 90er Jahre auf gegenwärtig 12 \% zurückgeführt worden. 1999 sollen die Ausgaben für Arzneimittel gegenüber 1998 aufgrund des neuen Solidaritätsstärkungsgesetzes um 1 Mrd. DM reduziert werden. Die kostensteigernden Effekte gingen - jedenfalls in Deutschland - in den 90er Jahren nicht vom Arzneimittel, sondern von anderen Leistungsbereichen des Gesundheitswesens aus. Es darf deshalb nicht der verfälschende Eindruck erweckt werden, als wären kostendämpfende Regelungen im Bereich des Arzneimittels in besonderer Weise veranlaßt und eine herausragende Lösung zur Bewältigung sonstiger Ausgaben- und Finanzierungsprobleme im Gesundheitswesen. Probleme sollten dort gelöst werden, wo sie entstehen. 


\begin{tabular}{|c|c|c|c|c|}
\hline Daten für 1997 & $\begin{array}{l}\text { Gesundheits- } \\
\text { ausgaben } \\
\text { (\% BIP) }\end{array}$ & $\begin{array}{c}\text { Arzneimittel- } \\
\text { ausgaben } \\
(\% \mathrm{BIP})\end{array}$ & $\begin{array}{l}\text { Arzneimittel- } \\
\text { ausgaben } \\
\text { (\% Gesundheits- } \\
\text { ausgaben) }\end{array}$ & $\begin{array}{c}\text { Arzneimittel- } \\
\text { ausgaben } \\
\text { (\$ pro Kopf) }\end{array}$ \\
\hline Belgien & 7,6 & 1,4 & 13 & 267 \\
\hline Dänemark & 7,7 & 0,7 & 12 & 215 \\
\hline Deutschland & 10,4 & 1,3 & 11 & 269 \\
\hline Griechenland & 7,1 & 1,8 & 25 & 118 \\
\hline Spanien & 7,4 & 1,5 & 16 & 193 \\
\hline Frankreich & 9,9 & 1,7 & 17 & 435 \\
\hline Irland & 7,0 & 0,7 & 10 & 111 \\
\hline Italien & 7,6 & 1,4 & 14 & 209 \\
\hline Luxemburg & 7,1 & 0,8 & 12 & 260 \\
\hline Niederlande & 8,5 & 0,9 & 13 & 272 \\
\hline Österreich & 7,9 & 1,1 & 10 & 260 \\
\hline Portugal & 8,2 & 2,2 & 18 & 127 \\
\hline Finnland & 7,3 & 1,1 & 11 & 192 \\
\hline Schweden & 8,6 & 1,1 & 16 & 315 \\
\hline Vereinigtes & 67 & & 10 & \\
\hline Königreich & 6,7 & 1,2 & 10 & 143 \\
\hline Vereinigte Staaten & 14,0 & 1,1 & 7 & 319 \\
\hline Japan & keine Angaben & keine Angaben & 20 & 425 \\
\hline Schweiz & 10,2 & 0,8 & 11 & 396 \\
\hline
\end{tabular}

Quelle: EU-Kommission, Mitteilung über den Binnenmarkt für Arzneimittel, Anhang 2, KOM (1998) 588 endg.

\section{Arzneimittelverbrauch}

Der Pro-Kopf-Arzneimittelverbrauch in Deutschland liegt mit etwa 20 Packungen p.a. im europäischen Mittelfeld. Die insgesamt in Deutschland in Apotheken abgegebene Packungszahl lag 1997 trotz gestiegener Bevölkerungszahl um etwa $15 \%$ niedriger als 1992. Etwa 1/3 davon entfällt auf rezeptfrei in Apotheken abgegebene Produkte und 2/3 aufgrund ärztlicher Verordnung abgegebene Produkte. 


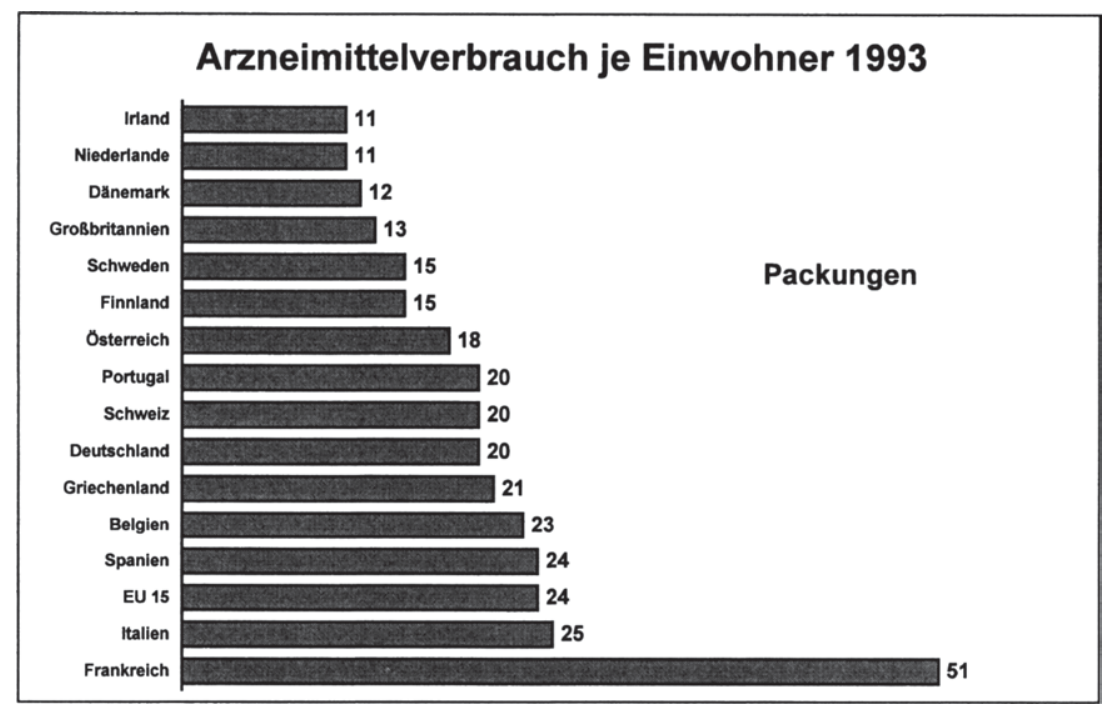

\section{Herstellerpreise}

Anders als in einer Reihe von EU-Staaten kann in Deutschland der Arzneimittelhersteller seinen Abgabepreis frei festsetzen, und zwar sowohl bei verschreibungspflichtigen als auch bei nicht-rezeptpflichtigen Produkten. Der Vorschlag der EU-Kommission in ihrer "Mitteilung zum gemeinsamen Binnenmarkt für Arzneimittel", auf Preiskontrollen bei nichtverschreibungspflichtigen Produkten zu verzichten, begegnet daher seitens der deutschen Apotheker keinen Bedenken, sofern er auf die Herstellerstufe beschränkt bleibt. Strikt abzulehnen ist dagegen eine Preisfreigabe bei allen Arzneimitteln auf der Endverbraucherstufe. Dies hat insbesondere zwei Gründe:

- Sehr viele Arzneimittel, die aus der Verschreibungspflicht entlassen worden sind, weil sie mit entsprechender Beratung in den Apotheken auch zur Selbstmedikation abgegeben werden können, sind weiterhin bedeutsam für die ärztliche Therapie im Rahmen der gesetzlichen Krankenversicherung. Die Abwicklung der Versorgung mit diesen Arzneimitteln kann im Rahmen der gesetzlichen Krankenversicherung bei einem Sachleistungsprinzip nur mit einer Preisregulierung auf der Endverbraucherstufe kostengünstig administriert werden, weil ansonsten bei jeder einzelnen Arzneimittelabgabe zwischen der Versicherung und der Apotheke separate Preisverhandlungen nötig wären. Ei- 
ne staatliche Regulierung der Apothekenabgabepreise, wie sie die deutsche Arzneimittelpreisverordnung vorsieht, ist daher einer fallweisen Preisverhandlung oder einseitigen Preisfestsetzung durch marktbeherrschende Krankenversicherungssysteme weit überlegen.

- Eine Preisfreigabe auf der Endverbraucherstufe würde Kranke vor die unzumutbare Notwendigkeit stellen, Preisvergleiche vorzunehmen. Zugleich würde über Marketingmaßnahmen ein medizinisch nicht indizierter Mehrverbrauch drohen, der gerade wegen der ambivalenten Struktur von Arzneimitteln Kosten an anderer Stelle im Gesundheitswesen zur Folge hätte. Mit einer Preisfreigabe würde zudem provoziert, daß Notsituationen der Patienten merkantil ausgenutzt würden. Das wäre dann zwar marktkonform, aber unter sozialen Gesichtspunkten unverantwortlich.

\section{Preisniveau}

Bei „alten“ Arzneimitteln, bei denen kein Patentschutz mehr besteht, entscheidet die Konkurrenzlage über den Preis. Hier üben in Deutschland die Arzneimittelfestbeträge in Verbindung mit dem im EU-Vergleich hohen Generikawettbewerb einen nachhaltigen Preisdruck aus. Bei den 338 Wirkstoffen, für die es neben den Originalprodukten auch Generika gibt, beträgt der Verordnungsanteil der Generika $67 \%$ (1996). Bei etwa 100 dieser Wirkstoffe liegt der Verordnungsanteil der Generika sogar über $90 \%$, teilweise sogar bei $100 \%$. Das deutsche Arzneimittelpreisniveau liegt derzeit immer noch unter dem des Jahres 1988.

Bei vielen europäischen Arzneimittelpreisstudien werden lediglich die Preise von Originalprodukten und dies auch nur für einen eingeschränkten, nicht repräsentativen Warenkorb verglichen (Beispiel: OECD-Preisstudie). Studien mit einem umfassenderen Warenkorb, die auch Generikaprodukte enthalten, zeigen dagegen, daß Deutschland bei den 15 EU-Mitgliedsstaaten im Mittelfeld liegt. Es ist zu beobachten, daß bei „neuen“, noch patentgeschützten Produkten zunehmend ein enger „EURO-Preiskorridor" eingehalten wird. 


\section{Europäischer Arzneimittelvergleich}

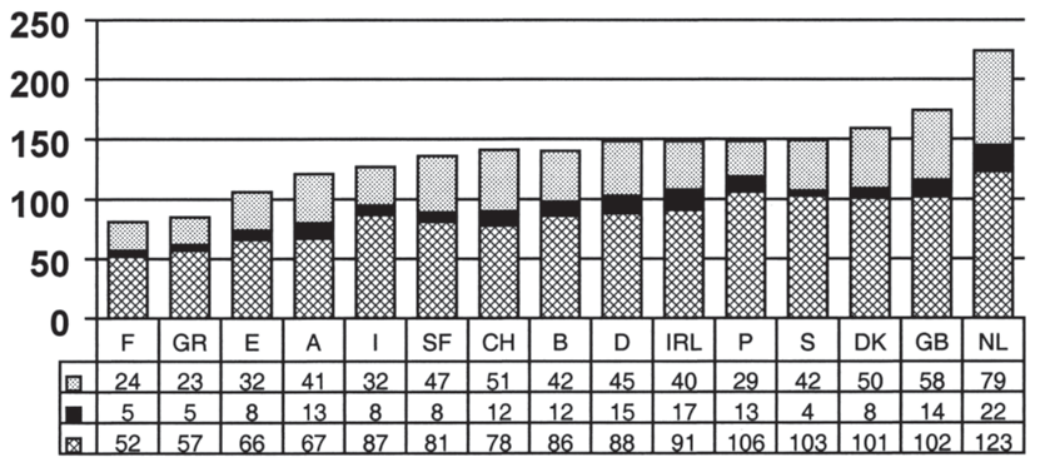

Durchschnittspreisvergleich 1995 in Schilling pro Packung gewichtet nach Kaufkraftparitäten Studie des Industriewissenschaftlichen Instituts der Universität Wien

\section{Parallelimporte}

Parallel- und Reimporte namensgleicher Arzneimittel machen in Deutschland etwa $2 \%$ der GKV-Verordnungen und der GKV-Arzneimittelausgaben aus. Importarzneimittel werden bei etwa 50 Wirkstoffen meist in den Standardpackungsgrößen angeboten. Wegen der Festbetragsregelung sind in Deutschland die Preisdifferentiale zwischen Originalprodukten und namensgleichen Re- und Parallelimporten seit 1989 geschmolzen. Darüber hinaus hat die permanent angewachsene Generikaquote einen weiteren Anstieg der Importquote gebremst, denn Importarzneimittel werden überwiegend bei Wirkstoffen angeboten, die einem regen generischen Wettbewerb unterliegen, so daß für den Arzt anstelle des Importarzneimittels meist preisgünstigere wirkstoffgleiche Generika zur Verfügung stehen.

\section{Distributionskosten und Mehrwertsteuer}

Die Distributionskosten für Arzneimittel, also die Wertschöpfungsanteile des Großhandels und der Industrie am Apothekenverkaufspreis, liegen in Deutschland im europäischen Mittelfeld. Im GKV-Bereich beträgt die Apothekenspanne am Apothekenverkaufspreis unter Berücksichtigung des 5\%igen Zwangsabschlags 21,9\% (1997) - mit weiterhin fallender Tendenz. Zusammen mit der Großhandelsspanne von 8,8 \% ergibt sich 
im deutschen GKV-Bereich somit ein Distributionskostenanteil von insgesamt $30,7 \%$. Im hochpreisigen, mit innovativen HochtechnologiePräparaten besetzten Preisbereich sind mit der Novellierung der Arzneimittelpreisverordnung seit 01.01.1998 die Handelsspannen der Apotheken und Großhändler so deutlich reduziert worden, daß sie auch bei den in der öffentlichen Diskussion häufig zitierten Extrembeispielen nunmehr zusammen weniger als $6 \%$ des Apothekenverkaufspreises betragen.

\section{Komponente des Arzneimittelpreises}

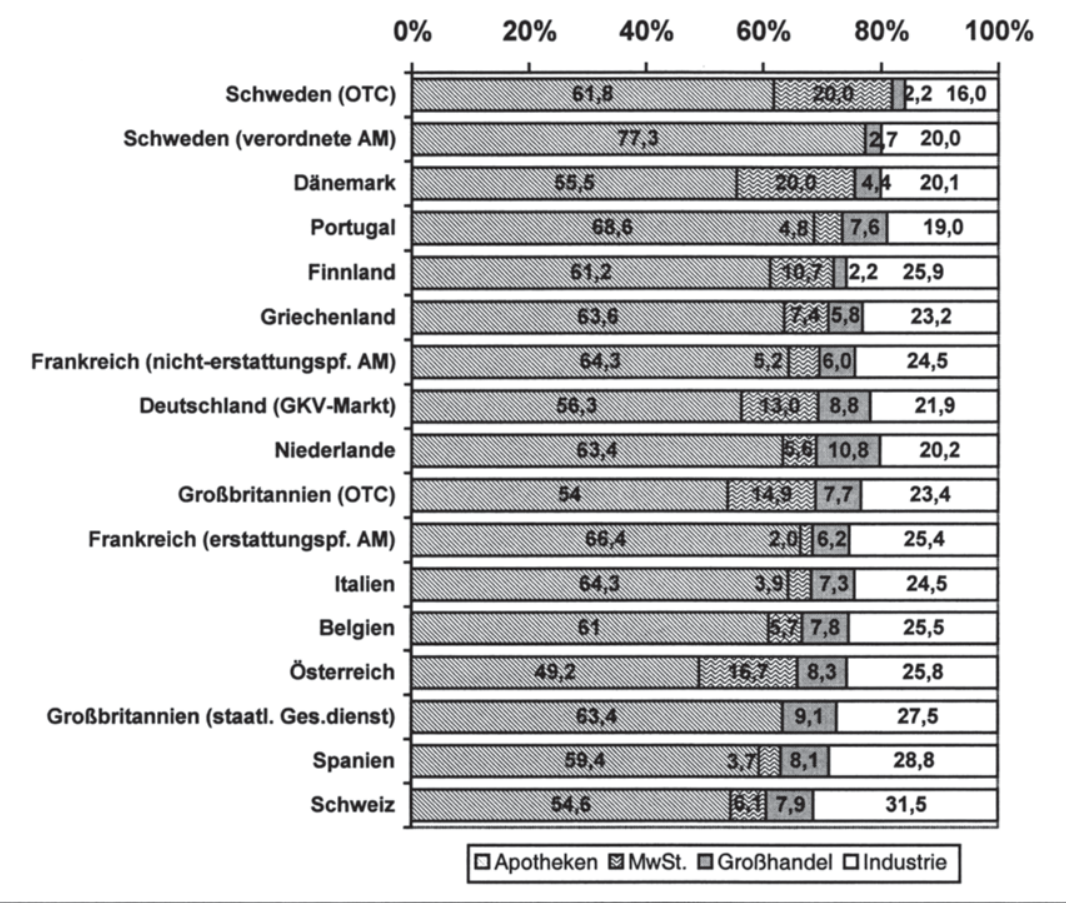

Nicht statthaft, aber regelmäßig von interessierten Kreisen praktiziert, ist es übrigens, die Mehrwertsteuer zu den Distributionskosten zu rechnen. In Deutschland könnte der Endverbraucherpreis erheblich reduziert wer- 
den, wenn hier wie sonst in der EU nur der reduzierte MwSt.-Satz auf Arzneimittel angewandt werden könnte.

\section{Vertriebswege und Elektronischer Handel}

Mit der Fernabsatzrichtlinie (Richtlinie 97/7/EG über den Verbraucherschutz bei Vertragsabschlüssen im Fernabsatz) sieht die EU vor, daß jeder Mitgliedsstaat das Recht hat, den Versandhandel mit Arzneimitteln zu untersagen. Die überwiegende Zahl der Mitgliedsstaaten hat davon Gebrauch gemacht, um die mit Arzneimittelfälschungen, Verwechslungen oder Transportbeschädigungen einhergehenden Risiken von $\mathrm{Pa}$ tienten fernzuhalten. Dieses Recht, den Versandhandel in einem Mitgliedsstaat zu untersagen, bedingt sachlogisch zugleich die Verpflichtung für die anderen Mitgliedsstaaten, diese Entscheidung zu respektieren und sie nicht dadurch unterlaufen zu lassen, indem sie den Versand aus ihrem Land in den betreffenden Mitgliedsstaat tolerieren oder gar explizit gestatten.

Der Deutsche Bundestag hat mit Zustimmung des Bundesrates 1998 in der 8. AMG-Novelle das in Deutschland bereits bestehende Versandhandelsverbot für Arzneimittel bekräftigt und lückenlos ausgeweitet.

Beim gleichwohl praktizierten, illegalen Versandhandel mit Arzneimitteln geht es nicht wirklich um die Ausnutzung von Preisdifferentialen - diese können ja auch dadurch wahrgenommen werden, daß man beispielsweise in der deutschen Apotheke das entsprechende Produkt als Importarzneimittel nachfragt und es dann in der Regel auch sofort oder jedenfalls schneller als über einen Versandhändler erhält sowie auch porto- und versandkostenfrei. Vielmehr dreht es sich bei den im Markt zu beobachtenden Versandangeboten darum, verschreibungspflichtige Arzneimittel ohne ärztliches Rezept gegenüber dem Endverbraucher zu handeln. Eine besondere Gefährdung besteht in der Banalisierung des Arzneimittels über die Vertriebsschiene Versandhandel. Deshalb warnen die Apotheker nachdrücklich vor der Nutzung oder Tolerierung solcher illegalen Vertriebswege: Die Folgekosten von kontraindiziertem Arzneimittelgebrauch würden unmittelbar auch die Haushalte der Krankenversicherungssysteme belasten.

In diesem Zusammenhang ist auch der elektronische Handel - electronic commerce - differenziert zu sehen:

Die Warenwirtschaftssysteme der Apotheken sind mit den EDVSystemen ihrer Vorlieferanten vernetzt. Elektronischer Handel innerhalb der Vertriebskette vom Hersteller über den Großhandel zur Apotheke 
findet in Deutschland schon seit langem und auf hohem technischen Niveau statt, ohne Bedenken zu begegnen.

Abgelehnt wird von uns dagegen jede Art des electronic commerce mit Arzneimitteln gegenüber dem Endverbraucher, da er ohne additiven Versandhandel nicht praktizierbar ist und letzterer den Patienten unverantwortlichen Risiken aussetzt. Die Kombination von electronic commerce und Versandhandel umgeht Arzt und Apotheker, verzichtet also auf eine individuelle pharmazeutische Beratung und überantwortet damit die Entscheidung über die therapeutische Veranlassung des Arzneimitteleinsatzes mit all ihren Risiken dem Patienten.

Nicht nur wir haben diese Sorgen. In einem Entwurf vom 8. Mai 1998 zu einer Öffentlichkeitsinformation der WHO zum Bezug medizinischer Produkte über das Internet heißt es hierzu wörtlich:

Be cautious about medical products

Medical products (medicines, diagnostics, medical devices, etc.) are often offered for sale on the Internet. However, this is not the right way to obtain them. Use of medical products obtained through the Internet may represent a danger on your health. You are strongly adviced to obtain your medicine only through the approbiate channels. This means: See your doctor if you think you need a medicine; and go to the pharmacy with the prescription given to you by your doctor. Also, be careful about medicines that are available without medical prescripton, such as simple painkillers, or cough syrup: buy your self-medication medicines only at the pharmacy or drug store.

Auch die vom EU-Parlament angenommenen Änderungen zu einem Vorschlag für eine Entscheidung des Rates über die Annahme eines mehrjährigen Aktionsplans der Gemeinschaft zur Förderung der sicheren Nutzung des Internets (KOM(97)0582-C40042/98-97/0377(CNS) beziehen den Gesundheitsschutz ebenfalls in die besonders schutzwürdigen Belange ein.

Die international wachsenden Bedenken gegen elektronischen Handel mit Arzneimitteln beruhen nicht nur darauf, daß damit sämtliche gesundheitspolitisch gewünschten Sicherheitsvorkehrungen im Falle ärztlicher und pharmazeutischer Konsultation durch den Patienten unterlaufen würden, sondern auch auf der zunehmenden Sorge, daß electronic commerce und Versandhandel mit Arzneimitteln aufgrund der damit verbundenen Gesundheitsrisiken einen unmittelbaren Reflex auf die Kosten in den nationalen Gesundheitssystemen haben. Auch wenn diese Ko- 
sten nur schwer meßbar sind, so kann doch ihre Existenz nicht negiert werden. Deshalb erscheint es geradezu als unverantwortlich, den Versandhandel unter Hinweis auf die Länder, die inn haben, als unbedenklich und kostensparend anzusehen.

\section{Grenzüberschreitende Leistungsinanspruchnahmen}

Im Frühjahr 1998 hat der Europäische Gerichtshof in zwei Fällen zur grenzüberschreitenden Inanspruchnahme von Gesundheitsleistungen im Rahmen von Sozialversicherungssystemen geurteilt. Die luxemburgische Sozialversicherung wurde verurteilt, Patienten, die Leistungen in Deutschland in Anspruch genommen hatten, die Kosten dafür bis zur Höhe der in Luxemburg vorgesehenen Grenzen zu erstatten. In den Medien wurde gleich gemutmaßt, daß nun auch jegliche verordnete Arzneimittel im Ausland zu Lasten der Krankenkassen bezogen werden könnten.

Das Bundesgesundheitsministerium hat diese „Euphorie“ gedämpft, indem es darauf verwies, daß das Urteil zunächst auf zwei konkrete Fälle aus dem Hilfsmittel- und zahnärztlichen Bereich beschränkt sei und zudem auch aus dem Urteil hervorgehe, daß die nationale Hoheit für die Organisation, Steuerung und Finanzierung des Gesundheitssystems nicht gefährdet werden dürfe. Die Freiheiten des Verkehrs von Waren und Dienstleistungen in einem gemeinsamen Binnenmarkt dürften schließlich nicht das Subsidiaritätsprinzip in inakzeptabler Weise relativieren. Wenn sich ein nationales Gesundheitswesen für das Kostenerstattungsprinzip entscheidet, kann es die Kostenerstattung nicht auf sein nationales Territorium begrenzen, sondern muß es im gesamten Binnenmarkt gegen sich gelten lassen. Ursache ist, daß es im Kostenerstattungssystem nur eine bilaterale Beziehung zwischen Patient und Kostenträger, aber keine Vertragsbeziehung zwischen Kostenträger und Leistungserbringer gibt. In einem Kostenerstattungssystem wird also bewußt auf eine Fülle von Steuerungsmöglichkeiten verzichtet, die in einem Sachleistungssystem bestehen, weil es dort eine unmittelbare Vertragsbeziehung zwischen dem Kostenträger und dem Leistungserbringer gibt. Wenn also ein nationales Gesundheitswesen auf der Grundlage eines Sachleistungsprinzips operiert, wird damit dokumentiert, daß die nationale Steuerung im bilateralen Kontakt zwischen Kostenträger und Leistungserbringer gewollt ist. Und eben diesen Weg hat die neue Bundesregierung mit dem zum 01.01.1999 in Kraft getretenen Solidaritätsstärkungsgesetz betont, weil damit Kostenerstattungselemente bewußt zurückgenommen werden. Eine grenzüberschreitende Leistungsinanspruchnahme hätte jedenfalls negative Auswirkungen auf die nationale Steuerbarkeit des Gesundheitssystems: Dies begänne mit der wegfal- 
lenden Datentransparenz und würde damit enden, daß ausländische Leistungserbringer nicht auf die Einhaltung derjenigen Normen und Regularien verpflichtet werden können, denen die inländischen Anbieter unterliegen. Dies würde also entweder die Diskriminierung inländischer gegenüber ausländischen Anbietern bedeuten oder aber den Verzicht auf Steuerungsinstrumente wie Arzneimittelbudgets etc. implizieren.

Ohnehin ist die grenzüberschreitende Leistungsinanspruchnahme für den Patienten in mehrfacher Hinsicht unbequem. Er muß sich ins Ausland begeben, er muß den kompletten Rechnungsbetrag vorfinanzieren und inn sich hinterher mühselig von seiner Krankenkasse zurückholen. Nachdenkliche Krankenkassenvertreter haben auch zur Vorsicht gemahnt, denn wenn den Patienten der Leistungsbezug im Ausland erst einmal großzügig gestattet wird, wird man ihn auch nicht mehr lange zwingen können, in der deutschen Krankenversicherung Zwangsmitglied zu sein, und man wird inm dann auch erlauben müssen, sich den Versicherungsschutz kostengünstig im Ausland zu besorgen.

\section{EURO}

In der Übergangsphase vom 01.01.1999 bis zum 31.12.2001 haben alle Wirtschaftsteilnehmer die Möglichkeit, entweder den EURO oder die DM als Buchgeld zu benutzen; als Bargeld steht der EURO erst ab dem 01.01.2002 zur Verfügung. Es ist natürlich klar, daß nur mit einer koordinierten Nutzung dieses Wahlrechts im komplexen Arzneimittelmarkt ab Januar 1999 ein völliges Chaos vermieden werden kann.

Was die Einführung des EURO anbelangt, so sind im deutschen Arzneimittelmarkt die "Hausaufgaben“ 1998 erledigt worden. Die von der ABDA initiierte Expertengruppe „EURO“ hat gemeinsam mit allen maßgeblich beteiligten Organisationen im Arzneimittelmarkt - auch den Kostenträgern - eine Konsenslinie zur Einführung des EURO im Arzneimittelbereich erarbeitet, die im „EURO-Leitfaden für Apotheker" detailliert festgehalten worden ist.

Im Kern beinhaltet diese Konsenslinie, daß im Arzneimittelbereich in der Übergangsphase vom 01.01.1999 bis zum 31.12.2001 ausschließlich die Denomination DM benutzt und ab dem 01.01.2002 schlagartig im Sinne eines Big Bang auf den EURO umgestellt wird. Arzneimittelpreisverordnung, Zuzahlungsregelungen, Warenwirtschaftssysteme, Krankenkassenabrechnungen etc. bleiben also bis zum 31.12.2001 vollständig „DMbasiert" und werden dann „über Nacht" auf EURO umgestellt. 
Damit wird das Prinzip „Kein Zwang, aber auch keine Behinderung“ ideal umgesetzt: Ein Hersteller, der unbedingt sein Produkt in EURO auspreisen möchte, denominiert gegenüber der IFA seinen Produktpreis in EURO, diese rechnet gemäß gesetzlichem Umrechnungskurs den EURO-Betrag in DM um. Aufgrund der „DM-basierten“ Arzneimittelpreisverordnung ergeben sich dann der Großhandels- und Apothekenabgabepreis, der in das Warenwirtschaftssystem der Apotheke eingespielt wird. Da in den Warenwirtschaftssystemen der Apotheken bereits mit den Update-Auslieferungen im Januar 1999 entsprechende EUROModule mitgeliefert werden, ist sichergestellt, daß in der Apotheke auf Wunsch „per Tastendruck“ dem Patienten auf dem Kassenbon der Rechnungsbetrag nachrichtlich in EURO ausgedruckt werden kann.

\section{Rolle der Apotheker}

In fast jedem zweiten Medikationsfall ist die Patienten-Compliance suboptimal - das ist kein deutsches Phänomen, sondern auch in allen anderen europäischen Ländern Realität. Zugleich treffen die Ärzte ihre Therapieentscheidung regelmäßig nur in Kenntnis der von innen veranlaßten Medikation; die Selbstmedikation und die Verordnungen anderer Ärzte bleiben ganz oder teilweise unberücksichtigt. Das kann nicht optimal sein. In einer Reihe von Mitgliedsstaaten der EU erkennen die Kostenträger zunehmend, daß es zielführend ist, die pharmazeutische Kompetenz der Apotheker nicht vermindert, sondern verstärkt zu nutzen. Die deutschen Apotheker sind beispielsweise gern bereit, zusätzliche pharmazeutische Dienstleistungen "vor" und "nach" der Arzneimittelabgabe gemeinsam mit Ärzten und Krankenkassen zu erproben. Die Stichworte sind dabei unter anderem „Pharmazeutische Betreuung“ und „Telematik im Gesundheitswesen“. Der Unterschied zwischen Deutschland und anderen europäischen Staaten ist, daß hier versucht wird, am Apotheker zu sparen, während andere mit dem Apotheker sparen. Vielleicht gibt Europa hier ja positive Impulse in die deutsche GKV. 
Axel Granitza

Politische Wellen geschlagen hat das EuGH-Urteil vom 07.02.1998 in Sachen Kohll/Decker.

Das Urteil hat klargestellt, daß

- trotz nationaler Zuständigkeit für Systeme der sozialen Sicherheit Grundsätze des FWV (Freier Warenverkehr) zu beachten sind,

- somit FWV auch für Dienstleistungen und für Waren gilt,

- nationale Inhalte von Leistungsbegrenzungen auch bei FWV durchaus erhalten bleiben (Festbeträge, Negativliste etc.),

- aber nicht jeder Verlust von Steuerungsmöglichkeiten durch die Nationalstaaten (Kassenzulassungen, Budgets, Richtgrößen) eine erhebliche Gefährdung der sozialen Sicherheit bedeutet (nur in einem solchen Fall könnte der FWV und der FDV [Freier Dienstleistungsverkehr] begrenzt werden),

- Sachleistung keine heilige Kuh ist und nicht als Argument verwendet werden kann, um den freien Verkehr gesundheitlicher Leistungen zu beschränken.

Die Pharmaindustrie hat die Schreckensrufe der Politiker nach dem Urteil - dessen Auswirkungen ich übrigens für bedeutender halte, als von vielen angenommen - mit einiger Verwunderung zur Kenntnis genommen.

Denn was unterscheidet den FV von Dienstleistungen oder den FWV mit Brillen von dem FWV mit Arzneimitteln? Ist bei den Reimporten von Arzneimitteln nicht stets der Jubel der Politiker, der Kassen etc. zu hören gewesen? Warum in dem anderen Fall die Aufregung?

Welcher Unterschied wird gesehen zwischen den Warnungen der pharmazeutischen Industrie vor einem Europa des Handels, der allein durch Reimporte begünstigt wird (nicht die Patienten) und den Angstrufen der nationalen Politiker, die ihre Steuerungsmöglichkeiten gefährdet sehen? Ist nicht Sicherstellung der Forschung im Arzneimittelbereich ein genauso wichtiges oder gar wichtigeres Ziel als der Erhalt nationaler Steuerungskompetenz? 
Und warum wird das Qualitätsargument (das Ausland hat nicht unseren Standard) bei anderen Gesundheitsleistungen ernster genommen als bei Arzneimitteln?

Ich will damit nun nicht etwa im Sinne einer Gleichbehandlung einem schrankenlosen FWV bei allen Dienstleistungen das Wort reden, ich will nur auf erstaunliche Unterschiede in der Wahrnehmung aufmerksam machen.

Um es klarer zu sagen: Ich meine, daß wir, solange wie das Entgelt für Dienstleistungen oder Preise für Waren, z. B. Arzneimittel, staatlich administriert wird, einer unbeschränkten FWV oder FDV nicht das Wort reden können.

Solange die Staaten glauben, marktwirtschaftliche Prinzipien bei der Preisbildung für Arzneimittel nicht zuzulassen, solange müssen wir nach Wegen suchen, den FWV sinnvoll zu limitieren.

Um es aber klar zu sagen: Ich rede von einer Zeit, die hoffentlich eine Übergangszeit ist, deren Dauer im einzelnen Segment vielleicht auch unterschiedlich ist (OTCs werden früher dereguliert?). Im Grundsatz - wenn die Übergangszeit nach Abbau von Regulierungen nicht mehr notwendig ist - bin ich ein vehementer Verfechter des FWV und des FDV.

$\mathrm{Daß}$ die Wahrnehmungsfähigkeit $m$. E. bei den Politikern aller Parteien etwas getrübt ist, ergibt sich aus den recht aufgeregten seinerzeitigen Reaktionen von Herrn Seehofer, aber genauso aus der Koalitionsvereinbarung der neuen Regierungsparteien.

Dort wird in dem Abschnitt, der das Gesundheitssystem behandelt, gesagt: „Zu den notwendigen Strukturreformen zählen darüber hinaus ... Neuordnung des Arzneimittelmarktes (Positivliste, Reimporte)."

Da sind sie wieder die unseligen Reimporte, und niemand sage oder versuche mit Zahlen zu beweisen, daß Reimporte ein geringes Ausmaß hätten und von der Pharmazeutischen Industrie hochgespielt würden. Es gibt in Europa auch ein Reimportdenken, das einen grenzüberschreitenden realen Transport von Waren gar nicht mehr im Auge hat. Was sind Preisvergleiche und politische Argumentationen auf Basis solcher zweifelhaften Vergleiche (die oft nicht einmal die unterschiedlichen Distributionskosten und Mehrwertsteuersätze in Europa berücksichtigen) anders als gedanklicher Reimport; ohne Ortswanderung der Waren? 
Ich will versuchen, Ihnen zu begründen, weshalb ich meine, daß man konsequenterweise so lange "territorial“, national staatlich im Hinblick auf alle Leistungen im Gesundheitswesen denken muß, wie die Mitgliedsstaaten der Gemeinschaft ihre nationale Regelung und Administrierung und Steuerungskompetenz auf dem Gebiet der gesundheitlichen Versorgung beanspruchen. Solange die Staaten hier nicht wirklich dazu beitragen, daß sich ein europäischer Markt für Waren und Dienstleistungen entwickelt, ist es $m$. E. unverständlich (unredlich), einerseits dem europäischen FWV (mit Arzneimitteln) das Wort zu reden, andererseits aber mit Klauen und Zähnen nationalen Dirigismus, nationale Steuerungskompetenzen zu verteidigen.

Ich plädiere also für ein striktes „Territorialitätsprinzip“ für eine - hoffentlich kurze - Übergangszeit, in der die Mitgliedsstaaten administrieren statt zu deregulieren.

Lassen sie mich den Versuch machen, dies kurz zu begründen - wobei ich die sehr überzeugende juristische Begründung, die uns der Europarechtler Professor Schwarze aus Freiburg geliefert hat, einmal ausklammere. (Vgl. Professor J. Schwarze: „Die Bedeutung des Territorialprinzips bei mitgliedsstaatlichen Preiskontrollen auf dem europäischen Arzneimittelmarkt" in „Unverfälschter Wettbewerb für Arzneimittel im europäischen Binnenmarkt/Nomos Verlag, Baden-Baden, 1. Auflage 1998).

Bekanntlich nehmen die Einzelstaaten für ihre nationalen Kostendämpfungsmaßnahmen, die europaweit sehr unterschiedlich sind, Souveränitätsrechte in Anspruch, die innen nach ihrer Interpretation der Römischen Verträge auf dem Gebiet der Sozialpolitik zustehen.

Dabei weisen sie zu Recht darauf hin, daß ihre spezifisch nationalen ökonomischen Rahmendaten und Rahmengegebenheiten ihr jeweiliges Kostendämpfungsmodell rechtfertigen. Jedoch: Durch den freien Warenverkehr sind ihre Kostendämpfungsmaßnahmen nicht mehr auf den nationalen Raum beschränkt, sondern weiten sich nach Europa aus. Mit jeder Arzneimittelpackung, jedem Preisvergleich kursiert auch ein nationales Gesundheitssystem europaweit. Portugal mag gute Gründe (Kaufkraft, Produktivität, Lohnstückkosten, Lohnniveau etc.) für die Festsetzung bestimmter Preise und Erstattungslimits haben, nur: für Deutschland oder Dänemark gelten diese Gründe eben nicht.

Die nationalstaatliche Administration im Bereich Preise und Erstattung erzeugt meines Erachtens im Kern ein Europa der Beliebigkeit, des Zufalls, ja des Chaotischen. Nationale Regelungen sind bei einem freien Warenverkehr nicht per se an den Grenzen aufhaltbar. Volkswirtschaftli- 
cher Unsinn ist die Folge: Was in Portugal in bezug auf Arzneimittelpreise von den Behörden entschieden wird, wird über das Vehikel des freien Warenverkehrs auf ganz Europa ausgebreitet. Was portugiesischen Krankenkassen an Belastung zugestanden wird bzw. was an Mitgliedsbeiträgen für portugiesische Arbeitnehmer adäquat ist, hat europaweite Gültigkeit. Und dies unter Umständen in Konkurrenz mit der Diffusion des spanischen oder griechischen Systems - ein Europa der Beliebigkeit, losgelöst von jeder wirtschaftlichen Rechtfertigung. Das wäre so, als wenn man die preisadministrierten Straßenbahnfahrscheine von Lissabon oder Athen beliebig in Kopenhagen, London oder Berlin gelten lassen würde oder die Ministergehälter von Portugal oder Griechenland auch in Dänemark oder Deutschland übernehmen würde.

Ich glaube auch, daß die Portugiesen ihre Arzneimittelpreise nur für ihr Land regeln wollen. Der Sekundäreffekt, d. $h$. die Ausstrahlung des portugiesischen Administrationssystems, ist einfach eine tatsächliche Folge, die nicht von Portugal für ganz Europa gewollt ist. Die portugiesische Bürokratie will überhaupt nicht über den Arzneimittelpreis in anderen Ländern entscheiden, sie hat nur ihr Land im Auge.

Der irische Gesundheitsminister Michael Noonan hat bereits bei der Bangemann-Runde am 9. Dezember 1996 in Frankfurt gesagt: „The reality, and I think we have to face it, is that unrestricted free movement is not compatible with unrestricted Government intervention in the domestic market. One or the other must give ground. Having said that, Member States cannot give ground on their prerogative to set health policy within their own jurisdiction. Nor is it acceptable that one country can or should impose its choice of health policy on its neighbours by the action of parallel trade. This would seem to point to the need for both the Commission and the Member States to show flexibility in the application of free movement where national price controls apply."

Selbst wenn man davon ausgeht, daß in bezug auf die gegenwärtigen Mitgliedsstaaten die volkswirtschaftlichen Rahmendaten in Europa, also z. B. Lohnniveau und Produktivität (z. B. Lohnstückkosten), sich immer mehr angleichen werden, wird aber meines Erachtens das Thema der mittelbaren extraterritorialen Erstreckung rein national gerechtfertigter Regelungen durch den freien Warenverkehr eher noch dramatischer.

Wir alle hoffen, daß die Beitrittsverhandlungen mit den mittel- und osteuropäischen Ländern nicht nur der ersten Welle zu einem guten Abschluß kommen. Wir alle hoffen auch, daß es zu tragbaren Übergangsregeln in den Vereinbarungen kommt, etwa was den Patentschutz für pharmazeutische Produkte angeht, aber ebenso auch Zulassungsverfahren und 
Bestimmungen zur Arzneimittelsicherheit und Arzneimittelqualität. (Dabei ist das seinerzeit mit Spanien und Portugal zum Patentschutz Vereinbarte kein ausreichender Maßstab.)

Aber selbst wenn das gelingt: Das auf Administration beruhende Arzneimittelpreisniveau in den genannten Ländern ist sehr niedrig, und auch das mag seine Rechtfertigung in den singulären ökonomischen Rahmenbedingungen des jeweiligen Landes finden, aber eben nur und allein darin. Für jede Geltung der Preise oder des Erstattungssystems außerhalb des jeweiligen Landes fehlt jede innere Rechtfertigung. Warum sollte die deutsche oder dänische Krankenkasse davon profitieren, daß der polnische oder tschechische Arbeitnehmer bei geringerer Produktivität weit weniger verdient als der deutsche? Und-polemisch überspitzt - wenn denn Polen der Maßstab sein sollte: Warum senken wir nicht unsere Straßenreinigungsgebühren, Abwasserabgaben, Steuern etc. auf das polnische Niveau?

Wenn wir uns einmal das Sozialprodukt in einigen Ländern ansehen, die Beitrittskandidaten sind: Da gibt es gerade bei künftigen EU-Ländern einen weiten Abstand etwa zu Deutschland, Frankreich und Großbritannien. Die Einkommensunterschiede sind groß. Entsprechend ist auch die Leistungskraft von Krankenversicherungen, die meist primär von Mitgliedsbeiträgen gespeist werden, unterschiedlich; ebenso wie die Leistungskraft des Staates, falls er es ist, der die Krankheitskosten finanziert. Dies schlägt auf die Preisfestsetzungen dieser Länder durch.

Die durch den freien Warenverkehr geförderte chaotische Situation in Europa - d. h. die beliebig über die einzelnen Mitgliedsstaaten hinausgehende Erstreckung der administrativen Systeme - muß sich natürlich auch auf das Agieren der pharmazeutischen Unternehmen auswirken. Wenn der Beamte in Lissabon oder später in Budapest mit seiner administrativen Handlung (z. B. Preisfestsetzung) bewußt oder unbewußt einen Sachverhalt regelt, der nicht nur Portugal oder Ungarn betrifft (das wäre legitim), sondern ganz Europa angeht, dann muß auch der europäische Pharmaunternehmer in Portugal oder Ungarn so reagieren, wie es seine europäischen Interessen, d. h. nicht nur portugiesische oder ungarische Interessen, effordern, dies unter Umständen zu Lasten der Patienten in Ungarn oder Portugal. Obwohl er z. B. bereit wäre, sein Produkt in Portugal und Ungarn und nur dort zu den dortigen Bedingungen einzuführen, wird er davon unter Umständen mit Rücksicht auf seine europäischen Interessen Abstand nehmen. Es gibt Situationen, in denen das ethisch nicht zu verantworten ist. So ist es meines Erachtens z. B. nicht vertretbar, ein innovatives Arzneimittel zur Linderung oder Heilung schwerer Krankheiten einem Teil der Europäer vorzuenthalten. Der Un- 
ternehmer hat also in einem solchen Fall nicht die Freiheit, ein Arzneimittel partiell nicht in den Verkehr zu bringen.

Auch hier zeigt es sich, daß nur die Anwendung des Territorialitätsprinzips Abhilfe schaffen kann.

Das Territorialitätsprinzip steht in administrierten „Märkten“ (wenn man von Märkten überhaupt sprechen kann) aus rechtlichen Gründen dem echten und dem gedanklichen Parallel- und Reimport entgegen. Diese rechtlichen Gründe werden durch die unterschiedlichen volkswirtschaftlichen Rahmendaten verstärkt, ja legitimiert.

Nun kann man sich fragen: Was kann die EU insbesondere bei dieser Sachlage und in Anbetracht der von den Nationalstaaten beanspruchten Souveränität in gesundheits- und sozialpolitischen Fragen tun? Welche Kompetenz hätte sie? Welche Pflichten hat sie?

Ich sehe mehrere Ansatzpunkte:

1. Nach den Römischen Verträgen ist der gemeinsame Markt zu realisieren. Herstellung eines Marktes heißt Schaffung von Wettbewerb, Deregulierung in bezug auf administrative Maßnahmen. Ich will also nicht einer europäischen Harmonisierung sozialrechtlicher oder preisadministrativer Maßnahmen das Wort reden. Gerade dazu hätte Europa - die Kommission - keine Kompetenz (Prinzip der Subsidiarität). Die Perspektive eines administrierten europäischen Einheitssystems wäre höchst gefährlich, weil es mit Sicherheit eine Kumulation aller Systeme wäre-PPRS (Pharmaceutical Price Regulation Scheme, Positiv- und Negativlisten, Kostenkontrollen, Festbeträge, Vergleichssysteme). Diese Kombination wäre ein Horrorszenario und hätte mit einem gemeinsamen Markt nichts mehr zu tun.

2. Ich meine, daß neben der Deregulierung und Wettbewerbsförderungskompetenz auch eine gewisse Ordnungskompetenz eine Rolle spielen muß. Es ist eben gerade kein Eingriff in nationalstaatliche Souveränität, wenn mit Autorität innerhalb Europas gesagt werden kann:

Jeder Mitgliedsstaat kann seine sozialrechtlichen Verhältnisse administrieren, nur eben hört seine Regelungskompetenz an den Grenzen so lange auf, wie die Mitgliedsstaaten jenseits seiner Grenzen gleichermaßen administrative Regelungskompetenzen beanspruchen. 
Gemäß dem Europäischen Gerichtshof in seiner Entscheidung Merck .l. Primecrown vom Dezember $1996, \ldots$ sind Verzerrungen, die durch eine unterschiedliche Preisregelung in einem Mitgliedsstaat verursacht werden, durch Maßnahmen der Gemeinschaftsbehörden auszuschalten ..... Durch eine solche Ordnungsrolle der EU würde Europa gestärkt, ebenso wie die Rolle der Mitgliedsstaaten auf sozialpolitischem Gebiet.

3. Und schließlich: Die EU muß - schon um im Wettbewerb mit den USA, Japan etc. zu bestehen - auch Industrie- und Forschungsförderungspolitik für den Standort Europa betreiben, d. h., sie sollte keine Verhältnisse zulassen, bei denen der Forschung Mittel entzogen werden, die nicht dem Patienten und der Forschung zugute kommen, sondern nur dem Handel oder der Distribution.

Aus alledem folgt für mich: Solange die Arzneimittelpreisbildung in Europa durch die Mitgliedsstaaten administriert ist, muß das Territorialitätsprinzip gelten, das juristisch, volkswirtschaftlich und industriepolitisch vernünftig ist.

Reimporte - auch gedankliche - zuzulassen und den Verlust von Steuerungskompetenz im Gesundheitsbereich zu beklagen, das geht nicht.

Territoriale Begrenzung von Dienstleistung und Waren oder Akzeptanz eines echten Marktes im wahrsten Sinne des Wortes: Das ist m. E. die Alternative. 
Alexander P. F. Ehlers

\section{Einleitung}

Der Gesundheitsmarkt der EG ist in seiner Gesamtheit einer der größten Märkte der EG. Sein Anteil stellt ca. $8 \%$ am Bruttosozialprodukt dar1. Um so erstaunlicher mutet es auf den ersten Blick an, daß gerade in der Gesundheitspolitik bisher von einem gemeinsamen europäischen Konzept nicht die Rede sein kann. Selbstverständlich existierten bereits vielfältige Projekte, die EU-weit durchgeführt wurden2 2 . Alle Aktionen in diesem Bereich waren jedoch einzelne, nicht auf einem gemeinsamen, umfassenden Grundkonzept fußende Tätigkeiten.

Die Mitgliedsstaaten in der Europäischen Union wachsen immer mehr zusammen. Durch die bevorstehende Währungsunion setzt ein weiterer Harmonisierungsprozeß ein. Bislang wird der Harmonisierungsgedanke vom Bereich der Gesundheitspolitik strikt ferngehalten. Das Gesundheitswesen liegt nach wie vor im Bereich der Zuständigkeiten der einzelnen EU-Mitgliedsstaaten. Dies steht im Einklang mit der ständigen Rechtsprechung, wonach die Zuständigkeit der Mitgliedsstaaten zur Ausgestaltung ihrer Systeme der sozialen Sicherheit unberührt vom Gemeinschaftsrecht bleibt ${ }^{3}$.

In Ermangelung einer Harmonisierung auf Gemeinschaftsebene bestimmt daher das Recht eines jeden Mitgliedsstaates, unter welchen Voraussetzungen zum einen ein Recht auf Anschluß an ein System der sozialen Sicherheit oder eine Verpflichtung hierzu und zum anderen Anspruch auf Leistung besteht 4 .

Der nationale Gesundheitsmarkt soll dadurch vor jedwedem fremdländischen Einfluß, vor jeder grenzüberschreitenden Erweiterung geschützt werden 5 .

1 Sendler, Arbeit und Sozialpolitik 1995, Heft 5/6, 48 ff., 53

2 Vgl. zur beispielhaften Aufzählung Hanika, MedR 1998, 193 (194)

3 EuGH, Urteil vom 28.04.1998 Rechtssache Decker ./. Union des caisses des maladies, abgedruckt in MedR 1998, 317 (318) mit konkreten Nachweisen zur Rechtsprechung

4 Ebenda

5 So Everling, Veränderung der Krankenkassen-Landschaft. In Das Deutsche Gesundheitswesen im vereinten Europa, Teltower Kreis, Radebeul 1997, 6 (16/17) 
Bis zum sog. Maastrichter Vertrag vom 7. Februar 1992 gab es keine unmittelbare und selbständige Kompetenz der EG zur Gesundheitspolitik6. Erst mit erwähntem Vertrag und durch die Einführung des Art. 129 in den EG-Vertrag wurde für die Gesundheitspolitik der Gemeinschaft eine eigene Kompetenz- und Rechtsgrundlage geschaffen 7 . Seit Maastricht wurde als Ziel der europäischen Gesundheitspolitik die Orientierung gemeinschaftlicher Maßnahmen an einem hohen Gesundheitsschutzniveau ausgerufen. Der am 2. Oktober 1997 unterzeichnete Vertrag von Amsterdam enthält eine Neufassung der Gesundheitskompetenz der Gemeinschaft. Der Vertrag ist noch nicht von den Mitgliedsstaaten ratifiziert. Geplant ist eine Ratifizierung bis zum 01.01.19998.

Zwar wird durch die Neufassung des Art. 152 die nationale Verantwortung der einzelnen Mitgliedsstaaten nicht gekürzt, dennoch wird die Position der EU im Bereich der Gesundheitspolitik gegenüber der bisherigen Fassung deutlich gestärkt. Das bisher auch beibehaltene Prinzip der Subsidiarität schließt den Fortbestand der uneingeschränkten Kompetenz der Mitgliedsstaaten ein, ihre eigenen Gesundheitssysteme selbst zu organisieren und zu steuern' 9 . Auch nach dem neuen Wortlaut des Art. 152 erfolgt daher keine Harmonisierung der nationalen Gesundheitssysteme.

Bisher existiert für die Harmonisierung keine Rechtsgrundlage. Sie wird daher nur über den faktischen Weg und möglicherweise durch die Hintertür der Rechtsprechung des EuGH schrittweise vollzogen.

Durch die hier im Mittelpunkt stehenden Entscheidungen des EuGH im Fall Decker und Kohll bewegt sich die Harmonisierungsbestrebung unaufhaltsam auch auf die europäische Gesundheitspolitik zu. Die Auswirkungen auf die nationalen Gesundheitssysteme und schließlich auf die deutsche Gesundheitspolitik sind es wert, beleuchtet zu werden.

Die EuGH-Urteile im Fall Decker und Kohll sind Folge des Prozesses des Zusammenwachsens der Mitgliedsstaaten. Im Zuge dieses Prozesses treten Probleme auf, deren Lösung die Aufgabe der Zukunft sein wird. Eines davon ist die Inanspruchnahme von Leistungen der Gesundheitsversorgung von EU-Bürgern in anderen Mitgliedsstaaten und deren

6 Hanika, MedR 1998, 193 (194)

7 Hanika, MedR 1998, 193 (194)

8 Hilf/Pache, NJW 1998, 705

9 Hanika, MedR 1998, 193 (195) 
Abwicklung mit dem eigenen Gesundheitssystem, was Gegenstand der hier im folgenden zu besprechenden EuGH-Urteile war. Insofern sind die Urteile des EuGH voraussichtlich von indizieller Bedeutung für den weiteren Verlauf der europäischen Gesundheitspolitik.

\section{Die EuGH-Entscheidungen}

Der EuGH hat mit seinen Entscheidungen in den Rechtssachen Decker (Urteil vom 28.04.1998 - C -120/95) und Kohll (Urteil vom 28.04.1998 $\mathrm{C}-158 / 96$ ) in Deutschland für große Aufregung gesorgt. In beiden Entscheidungen handelte es sich bei den Klägern um luxemburgische Staatsangehörige.

Quintessenz des Falles Decker ist die Feststellung, daß ein Gemeinschaftsbürger das Recht hat, eine ihm im eigenen Mitgliedsstaat verschriebene Brille in einem anderen Mitgliedsstaat zu erwerben und die Kosten dafür von seiner Krankenversicherung ersetzt zu bekommen.

Im Fall Kohll entschied der EuGH, daß ein Gemeinschaftsbürger zahnärztliche Leistungen in einem anderen Mitgliedsstaat in Anspruch nehmen darf und die hierdurch entstandenen Kosten bei dem Sozialversicherungsträger seines Heimatstaates nach den dort geltenden Tarifen in Form der Kostenerstattung geltend machen kann.

\section{Der Fall Decker}

Der Luxemburger Nikolas Decker kaufte eine inm von einem Luxemburger Augenarzt verschriebene Brille mit Korrekturgläsern bei einem Optiker in Belgien. Dabei hatte er die in Luxemburg erforderliche vorherige Genehmigung des zuständigen Sozialversicherungsträgers nicht beantragt und infolgedessen auch nicht erhalten. Nach luxemburgischem Sozialversicherungsrecht hat der Versicherte nur dann einen Anspruch auf Kostenerstattung einer Behandlung im Ausland, wenn der Sozialversicherungsträger vorher seine Genehmigung erteilt hat - ausgenommen es liegt ein Fall der Ersten Hilfe im Rahmen eines im Ausland erlittenen Unfalls oder einer dort aufgetretenen Krankheit vor.

Begründet wird dieser Genehmigungsvorbehalt von der luxemburgischen Regierung mit dem Schutz der öffentlichen Gesundheit. Die Regelung sei erforderlich, um die Qualität der ärztlichen Leistungen zu gewährleisten. Diese könne bei denjenigen, die sich in einen anderen Mitgliedsstaat begäben, nur im Zeitpunkt des Antrages auf Genehmigung überprüft werden. Des weiteren solle das luxemburgische Krankenversicherungssystem eine ausgewogene, allen Versicherten offenstehende 
ärztliche und klinische Versorgung sicherstellen. Darüber hinaus führt die Regierung dringende Gründe des Allgemeininteresses an; die Wirtschaftlichkeit und Finanzierbarkeit des Gesundheits- und Krankenversicherungssystems müsse gesichert werden, um in einer bestimmten Region jedermann den Zugang zum System ermöglichen zu können.

Der Sozialversicherungsträger lehnte die Kostenerstattung ab, weil die besagte Genehmigung zuvor nicht eingeholt worden war.

\section{Der Fall Kohll}

Der Luxemburger Raymond Kohll beantragte für seine Tochter die Genehmigung zur Erstattung der Kosten einer zahnregulierenden Behandlung, die bei einem deutschen Zahnarzt in Trier durchgeführt werden sollte.

Hier lehnte der Sozialversicherungsträger die Zusage der Kostenerstattung ab, weil die Behandlung nicht dringend sei und auch in Luxemburg erbracht werden könne.

\section{Die Begründung des EuGH}

Letztendlich ging es bei beiden Fällen um die Frage der Zulässigkeit des Genehmigungsvorbehalts. Der EuGH kam zu dem Ergebnis, daß die luxemburgische Vorschrift aus dem Sozialrecht über den Genehmigungsvorbehalt gegen den Grundsatz des freien Warenverkehrs, Art. 30 und 36 des EG-Vertrages, sowie gegen den Binnenmarktgrundsatz des freien Dienstleistungsverkehrs, Art. 59 und 60 des EG-Vertrages, verstößt.

Die Vorschrift hindere zwar den Versicherten nicht daran, medizinische Erzeugnisse in einem anderen Mitgliedsland zu erwerben. Die Erstattung der Kosten werde aber von einer Genehmigung abhängig gemacht und versagt sie den Versicherten, die keine Genehmigung haben. Dagegen würden Kosten, die im Versicherungsstaat selbst anfallen, nicht solchen Genehmigungen unterliegen.

Es gebe keine Rechtfertigung für den diese Grundsätze behindernden Genehmigungsvorbehalt. Rein wirtschaftliche Gründe könnten eine Beschränkung des elementaren Grundsatzes des freien Waren- bzw. Dienstleistungsverkehrs nicht rechtfertigen. Jedoch könne eine erhebliche Gefährdung des finanziellen Gleichgewichts des Systems der sozialen Sicherheit einen zwingenden Grund des Allgemeininteresses dar- 
stellen ${ }^{10}$. Von einer solchen Gefährdung sei aber hier nicht auszugehen gewesen.

Eine Gefährdung der öffentlichen Gesundheit durch die Behandlung durch einen Arzt eines anderen EU-Landes wird vom EuGH abgelehnt. Aufgrund der bisherigen Harmonisierung auf dem Gebiet der medizinischen Berufe und Erzeugnisse sei dafür gesorgt, daß Patienten in allen Mitgliedsstaaten gleiche Garantien gewährt würden. Die Gesundheit des einzelnen sei auch dann ausreichend gesichert, wenn er sich einer medizinischen Behandlung in einem anderen Mitgliedsstaat unterziehe. Auch die Wirtschaftlichkeit des luxemburgischen Gesundheitsversorgungssystems sei bereits deshalb nicht gefährdet, weil die Kostenerstattung für eine Brille bzw. Zahnbehandlung nach den in Luxemburg geltenden gültigen Sätzen erfolge. Diese Kosten wären nämlich auch beim Erwerb einer Brille im Inland bzw. bei Inlandsbehandlungen entstanden.

Auch wenn die nationalen Sozialsysteme vom Gemeinschaftsrecht unberührt bleiben, müßten die Mitgliedsstaaten bei der Ausübung ihrer Befugnisse das Gemeinschaftsrecht beachten. Die Mitgliedsstaaten müßten daher die Vorschriften über den freien Warenverkehr, Art. 30 EG-Vertrag, berücksichtigen.

\section{Konsequenzen der EuGH-Entscheidung}

Aus den beiden Urteilen geht hervor, daß der EG-Vertrag auch die Freiheiten des Waren- und Dienstleistungsverkehrs gewährleistet, indem er marktwirtschaftliche Strukturen auch für Sach- und Dienstleistungen im Gesundheitswesen durch ein wettbewerblich geprägtes System vorgibt11.

Dienstleistungsfreiheit bedeutet, daß jeder Dienstleister das Recht hat, seine von inm angebotenen Dienstleistungen nicht nur in seinem eigenen Land, sondern gleichermaßen in allen Mitgliedsstaaten der EU anzubieten und zu erbringen. Jedem Gemeinschaftsbürger steht umgekehrt das Recht zu, sich die Dienstleistungen, die er benötigt, im gesamten Gebiet der Gemeinschaft frei zu suchen 12 .

10 EuGH, Urteile vom 28.04.1998, C-120/95, C-158/96, MedR 1998, 317 (319 bzw. 321)

11 Hanika, MedR 1998, 317 (322)

12 Everling, Veränderung der Krankenkassen-Landschaft, in: Das Deutsche Gesundheitswesen im vereinten Europa, Teltower Kreis, Radebeul 1997, 6 (17) 
Fraglich ist nun, welche Auswirkungen die Urteile auf den deutschen Gesundheitsmarkt haben können und haben werden.

Zwar beschäftigen sich die Urteile ausschließlich mit Luxemburger Fällen, was einige dazu veranlaßte, die Geltung für Deutschland abzulehnen oder zumindest in Frage zu stellen. De facto kann man jedoch vor den Folgen. der Entscheidungen auch für Deutschland nicht die Augen verschließen. Auch für Deutschland gelten die im EG-Vertrag festgeschriebenen Grundfreiheiten, zu denen die Dienstleistungs- und Warenfreiheit innerhalb der EU gehört13. Dies ist insbesondere im Hinblick auf $\S 16$ Abs. 1 Nr. 1 sowie $\S 18$ Abs. 1 SGB V zu sehen, da in Deutschland grundsätzlich auch keine Kostenübernahme für Leistungen im Ausland erfolgt, es sei denn, eine Behandlung ist nur im Ausland möglich.

Festzustellen ist, daß die Urteile des EuGH nur ausdrücklich Aussagen über solche Leistungen machen, für die Kostenerstattung in Betracht kommt. Für Leistungen, die ausschließlich als Sachleistung erbracht werden, können die Ausführungen des Gerichtshofes nicht uneingeschränkt als Präjudiz gewertet werden. Diesbezüglich wäre eine eigenständige Prüfung durch den EuGH erforderlich, die die Motive für das Sachleistungsprinzip berücksichtigt14.

Im folgenden sollen die möglichen Auswirkungen auf das deutsche Gesundheitssystem dargestellt werden.

- Zunächst ist davon auszugehen, daß nach wie vor die Leistung in dem Mitgliedsstaat erbracht wird, dessen Staatsbürger betroffen ist. Nur eine geringe Anzahl von Gemeinschaftsbürgern werden voraussichtlich die sich abzeichnenden Möglichkeiten des freien Gesundheitsmarktes nutzen. Ein Massenpatiententourismus ist wegen der Sprachbarriere und der Vorleistungspflicht sowie dem Risiko erheblicher Eigenbeteiligung nicht zu befürchten ${ }^{15}$. Dies ist zumindest für den Bereich der Behandlungen zu erwarten. Bisher betrugen die Gesundheitsleistungen im Ausland weniger als 0,3\% der Gesamtausgaben der gesetzlichen Krankenversicherung16.

13 Vgl. auch Berg, Anmerkung zum Urteil des EuGH vom 28.04.1998 in der Rs. C158/96, Kohll, Pharma Recht (1998), Heft 7, 232 (233)

14 Derselbe, 235

15 Vgl. anstelle vieler: Berg, Anmerkung zum Urteil des EuGH vom 28.04.1998 in der Rs. C-158/96, Kohll, Pharma Recht (1998), Heft 7, 232 (235)

16 Die Ersatzkasse 6/98, 239 
- Was die Heil- und Hilfsmittel betrifft, könnte dies je nach Entwicklung der Preisverteilung aber eine ernstzunehmende Alternative für den Bürger der EU darstellen. Insoweit sollten die Auswirkungen der Urteile nicht unterschätzt werden.

- Die nationale Kompetenz im Gesundheitswesen wird durch die Urteile nicht angegriffen oder in Frage gestellt. Lediglich die Beschränkung durch Gemeinschaftsrecht wird erörtert.

- Die Gesundheitsmärkte sind nach wie vor zwar abgeschottet. Die hier besprochenen Urteile eröffnen nun im Hinblick auf die europarechtlich garantierten Marktfreiheiten neue Wege im Zusammenhang mit der Weiterentwicklung neuer Versorgungsmodelle - nicht nur innerhalb Deutschlands.

- Fraglich ist, ob sich die Entscheidungen auch auf andere Bereiche, wie z. B. den Arzneimittelmarkt, ausweiten werden. Davon ist mit großer Wahrscheinlichkeit auszugehen. In diesem Zusammenhang ist zu überlegen, inwiefern der Handel von Arzneimitteln davon betroffen sein kann (auch der Internethandel). Aber auch die Problematik der Re- und Parallelimporte muß in diesem Zusammenhang wieder in einem völlig neuen Licht gesehen werden. Schließlich stellt sich im Zusammenhang mit Arzneien sowie Heil- und Hilfsmitteln die Frage nach der Haltbarkeit von Budgets und Festbeträgen 17.

- Äußerst problematisch ist das durch die Urteile entstehende Spannungsverhältnis zwischen dem in Deutschland geltenden und nach dem neuen Koalitionsvertrag der SPD/Die Grünen auch weiterhin angestrebten Sachleistungsprinzip, § 13 Abs. 1 SGB V, und dem in den Urteilen festgestellten Anspruch auf Kostenerstattung.

- Das Sachleistungsprinzip ist seinem Inhalt nach durch die Unmittelbarkeit der Bedarfsbefriedigung gekennzeichnet. Die Versicherten „erhalten die Leistungen als Dienst- und Sachleistungen“, § 2 Abs. 2 Satz 1 SGB V. Das bedeutet, daß sie die Leistungen in Natur erhalten. Das Sachleistungsprinzip hat als übergreifendes, essentielles Strukturelement, tragendes Prinzip der GKV18 Steuerungsfunktion19. Durch das Sachleistungsprinzip sollen die Versicherten vor einer Kostenvorleistung geschützt werden und folglich nicht dem Risiko der

17 Hanika, Anm. zu den Urteilen des EuGH, MedR 1998, 317 (323)

18 BT-Drucks. 12/3608 zu § 13; BSG, NZS 1993, 312 (314)

19 BSG, NZS 1993, 312 (314) 
Kostenerstattung ausgesetzt werden. Darüber hinaus soll mit der Verschaffungspflicht durch die Krankenkasse eine in Qualität, Wirksamkeit und Wirtschaftlichkeit gleichermaßen effektive medizinische Versorgung erreicht werden 20 . Auch wenn durch die Einführung des $\S 13$ Abs. 2 SGB V das Sachleistungsprinzip ausdrücklich durchbrochen wird, stellt die Kostenerstattung die absolute Ausnahme dar. $\S 13$ Abs. 2 regelt: „Freiwillige Versicherte sowie ihre nach $\S 10$ versicherten Familienangehörigen können für die Dauer der freiwilligen Versicherung anstelle der Sachleistung oder Dienstleitung Kostenerstattung wählen."

- Diese gegenläufige Richtung könnte als Instrument im Wettbewerb unter Kassen und mit der privaten Krankenversicherung dienen. Mittelfristig ist ein Nebeneinander zweier Leistungsarten auch unter administrativen Gesichtspunkten uneffektiv, zu teuer und folglich nicht haltbar21.

- Mit den Urteilen muß sich der Gesetzgeber aufgefordert fühlen, das deutsche Krankenversicherungsrecht im Lichte des europäischen Dienstleistungs- und Warenverkehrsrechts zeitnah und tiefreichend wettbewerblich orientiert und marktwirtschaftlich ausgerichtet auf den Gebieten der ambulanten ärztlichen und zahnärztlichen Versorgung, der Arzneimittel-, Heilmittel- und Hilfsmittelversorgung, im Krankenhaussektor und schließlich in der Versicherungsverfassung zu überarbeiten und mit Anreizen zu versehen 22.

- In Zukunft wird mehr Flexibilität durch die Öffnung der nationalen Sozialsysteme über die Grenzen des eigenen Landes hinaus verlangt werden müssen. Neue Formen der Zusammenarbeit müssen gefunden werden. Von einer Abgabe der Kompetenzen der einzelnen Mitgliedsstaaten ist nicht auszugehen. Daher wird sich die Entwicklung voraussichtlich verstärkt auf die Suche nach neuen Modellen der Zusammenarbeit mit den Leistungserbringern richten.

- Nach den Entscheidungen des EuGH ist zu erwarten, daß die Bereitschaft, auch im Sozialrecht die Augen nicht vor den Entwicklungen in Brüssel zu verschließen, wächst 23 .

20 Noftz in: Hauck, SGB V, Kommentar, K § 13 Rz 9

21 Hanika, MedR 1998, 317 (323)

22 Hanika, MedR 1998, 317 (323)

23 So auch Benkel, NZS Heft 8/1998, 359 (364) 


\section{Fall Kohll .I. Union des caisses des maladie (Zahnbehandlung)}

EuGH Urteil vom 28.04.1998 - C-158/96:

Verstoß gegen den Grundsatz des freien Dienstleistungsverkehrs

Eine nationale Regelung, die die Erstattung der Kosten für Zahnbehandlung durch einen Zahnarzt in einem anderen Mitgliedsstaat nach den Tarifen des Versicherungsstaates von der Genehmigung des Trägers der sozialen Sicherheit des Versicherten abhängig macht, verstößt gegen Art. 59 und 60 EG-Vertrag.

\section{Fall Decker .I. Caisse de maladie des employés privés}

EuGH Urteil vom 28.04.1998 - C-120/95:

Verstoß gegen den Grundsatz des freien Warenverkehrs

Eine nationale Regelung, nach der ein Träger der sozialen Sicherheit eines Mitgliedsstaates einem Versicherten die pauschale Kostenerstattung für eine Brille mit Korrekturgläsern, die dieser bei einem Optiker in einem anderen Mitgliedsstaat gekauft hat, mit der Begründung versagt, daß der Erwerb medizinischer Erzeugnisse im Ausland der vorherigen Genehmigung bedarf, verstößt gegen die Art. 30 und 36 EG-Vertrag.

\section{§ 16 Abs. 1 Nr. 1 SGB V}

Ruhen des Anspruchs

(1) Der Anspruch auf Leistung ruht, solange Versicherte 1. sich im Ausland aufhalten, und zwar auch dann, wenn sie dort während eines vorübergehenden Aufenthalts erkranken, soweit in diesem Gesetz nichts Abweichendes bestimmt ist, ...

$\S 18$ Abs. 1 und Abs. 3 SGB V

Kostenübernahme bei Behandlung im Ausland

(1) Ist eine dem allgemein anerkannten Stand der medizinischen Erkenntnisse entsprechende Behandlung einer Krankheit nur im Ausland möglich, kann die Krankenkasse die Kosten der erforderlichen Behandlung ganz oder teilweise übernehmen. Der Anspruch auf Krankengeld ruht in diesem Fall nicht. 
(3) Ist während eines vorübergehenden Auslandsaufenthalts eine Behandlung unverzüglich erforderlich, die auch im Inland möglich wäre, hat die Krankenkasse die Kosten der erforderlichen Behandlung insoweit zu übernehmen, als Versicherte sich hierfür wegen einer Vorerkrankung oder ihres Lebensalters nachweislich nicht versichern können und die Krankenkasse dies vor Beginn des Aufenthalts festgestellt hat. ...

Die Kostenübernahme ist nicht zulässig, wenn Versicherte sich zur Behandlung ins Ausland begeben.

Sachleistungsprinzip, § 2 Abs. 2 SGB V

(2) Die Versicherten erhalten die Leistungen als Sach- und Dienstleistungen, soweit dieses Buch nichts Abweichendes vorsieht. Über die Erbringung der Sach- und Dienstleistungen schließen die Krankenkassen nach den Vorschriften des vierten Kapitels Verträge mit den Leistungserbringern.

Durchbrechung des Sachleistungsprinzips durch Kostenerstattung als Ausnahme, § 13 Abs. 2 SGB V

(2) Freiwillige Versicherte sowie ihre nach $\S 10$ versicherten Familienangehörigen können für die Dauer der freiwilligen Versicherung anstelle der Sachleistung oder Dienstleitung Kostenerstattung wählen.

\section{Begründung der EuGH- Urteile}

Der luxemburgische Genehmigungsvorbehalt verstößt gegen ịm EGVertrag gewährleistete Grundfreiheiten des freien Dienstleistungs- bzw. Warenverkehrs.

Zwar kann eine erhebliche Gefährdung des finanziellen Gleichgewichts des Systems der sozialen Sicherheit einen zwingenden Grund des Allgemeininteresses darstellen, der eine Beschränkung der elementaren Grundfreiheiten des Waren- und Dienstleistungsverkehrs rechtfertigt. Eine solche Gefährdung liegt hier aber nicht vor, weil:

- eine unterschiedliche Behandlung je nachdem, ob die Leistung im Inland oder im Ausland erbracht wird, erfolgt; 
- die Behandlung durch einen Arzt eines anderen EU-Landes wegen der bisherigen Harmonisierung auf dem Gebiet der medizinischen Berufe und Erzeugnisse keine Gefährdung der öffentlichen Gesundheit darstellt;

- die Wirtschaftlichkeit des Gesundheitsversorgungssystems nicht gefährdet ist, da die Kostenerstattung nach den im Inland gültigen Sätzen erfolgt. Diese Kosten wären auch bei Erwerb einer Brille im Inland bzw. bei Inlandsbehandlungen entstanden.

\section{Konsequenzen der EuGH-Entscheidung für den deutschen Gesundheitsmarkt}

- Massenpatiententourismus nicht zu erwarten.

- Nationale Kompetenz im Gesundheitswesen wird durch die Urteile nicht angegriffen.

- Urteile bereiten Weg für faktische Harmonisierung.

- Berücksichtigung europäischer marktwirtschaftlicher Strukturen bei der Weiterentwicklung des Krankenversicherungssystems unvermeidbar.

- Auswirkung der Urteile auf andere Bereiche, wie z. B. Arzneimittel, zu erwarten.

- Kollision des deutschen Sachleistungsprinzips mit dem Kostenerstattungsprinzip muß in Zukunft durch neue Modelle gelöst werden. Nebeneinander erscheint nicht effektiv.

- Neue Möglichkeiten für Wettbewerb der Kassen untereinander und mit der privaten Krankenversicherung. 


\section{Themenkreis II}

\section{Veränderte Versorgungsstrukturen}

Helmut Laschet

Dieses Kapitel ist eine Offenbarung. Warum? Es behandelt den Stand der Diskussion über die Organisation komplizierter Dienstleistungsprozesse in der Medizin.

Die Medizin ist der größte Wirtschaftssektor in Deutschland. Nicht für Nahrung, nicht für Autos geben die Deutschen das meiste Geld aus, sondern für die Erhaltung und vor allem für die Wiederherstellung inrer Gesundheit. Das wäre für sich genommen noch kein Problem. Sieht man einmal davon $a b$, daß der weitaus größte Teil medizinischer Leistungen durch solidarische und nichtsolidarische Versicherungssysteme und damit außerhalb individueller Eigenverantwortlichkeit finanziert wird (das ist Gegenstand anderer Kapitel dieses Buches), dann bleiben beachtliche Besonderheiten, die für die Erstellung medizinischer Dienstleistungen charakteristisch sind:

Kein anderer Wirtschaftssektor "bearbeitet" eine solche Fülle von Problemen, die ihrerseits wieder von höchst unterschiedlicher Komplexität sind.

Kein anderer Wirtschaftssektor bietet eine solche Fülle von Problemlösungen an wie die Medizin; aber: die Wirksamkeit und der Nutzen (letztlich für den Patienten) vieler medizinischer Handlungsmöglichkeiten ist eher umstritten, oft unzureichend und in jedem Fall auf höchst unterschiedliche Weise und Qualität belegt.

Je komplexer ein gesundheitliches Problem ist, desto mehr Handlungsoptionen müssen miteinander kombiniert werden-und zwar auf verschiedenen Versorgungsebenen. Diese Versorgungsebenen arbeiten bislang wenn nicht abgeschottet voneinander, so doch mehr nebeneinander als miteinander, seltener jedenfalls systematisch koordiniert.

Die an sich notwendige Koordinierung wird dadurch erschwert, daß jede Versorgungsebene und jeder Sektor des Gesundheitswesens seine eigenen Organisationsstrukturen entwickelt oder kraft Gesetzes verliehen bekommen hat: die niedergelassenen Ärzte unter dem Dach des KVMonopols, das aus dem Verständnis seiner Mitglieder mehr eine fürsor- 
gerische Funktion für Ärzte als die Pflicht zur Sicherstellung der gesundheitlichen Versorgung hat, die aber untereinander in einem teilweise ruinösen Wettbewerb stehen; die Krankenhäuser, die zu einem erheblichen Teil noch wie volkseigene Regiebetriebe geführt werden, unter denen sich aber auch gewinnträchtige Privatunternehmen finden; Hersteller von Arzneimitteln, unter denen die Großen nur noch im weltweiten Maßstab denken, unter denen es in Deutschland aber noch eine beachtliche Zahl mittelständischer Firmen gibt, und schließlich eine Fülle von Freiberuflern - vom ambulanten Pflegedienst über den Masseur bis zum Logopäden -, die auf Gedeih und Verderb von ärztlichen Indikationsstellungen unter wechselnden Budgetbedingungen abhängig sind.

Staatliche und parastaatliche Macht: Sie manifestiert sich vor allem in Krankenhäusern, beginnend bei der Krankenhausplanung über die Gestaltung der Bundespflegesatzverordnung (mit der man sich selbst, quasi im Wege des sonst verbotenen Selbstkontrahierens, die Mittel zuweist) bis hin zu den Eigentumsverhältnissen. So ist die meist unsichtbare Verschwendung knapper Ressourcen durch Universitätskliniken, Landratsdenkmäler oder Krankenhäuser der Wohlfahrtsverbände - sie machen ja alle keinen Gewinn oder streben zumindest nicht danach weitaus weniger anrüchig als das offen ausgewiesene Einkommensoder Gewinnstreben der nach erwerbswirtschaftlichen Prinzipien arbeitenden Zweige des Medizinbetriebs wie niedergelassene Ärzte oder pharmazeutische Unternehmen, die freiwillig oder durch gesetzlichen Zwang ihre Jahresabschlüsse offenlegen.

Die asymmetrische Machtverteilung hat natürlich Auswirkungen auf die Budgetzuteilung, die sich dieser Staat im Streben nach Vollkommenheit zur Aufgabe gemacht hat: stets ist es dabei die staatsnah organisierte Medizin, die sich immer größer werdende Kuchenstücke vom Bundesund Landesgesetzgeber reservieren läßt, der seinerseits stets aufs neue Belastungsfähigkeit und Flexibilität der nach erwerbswirtschaftlichen Prinzipien arbeitenden Leistungsanbieter erprobt.

Unter diesen Bedingungen findet auf den folgenden Seiten eine Diskussion darüber statt, wie die „Versäulung“ im deutschen Gesundheitswesen aufgebrochen werden kann und wie komplexe medizinische Dienstleistungen verknüpft oder vernetzt werden können - oder wie sich die Anbieter untereinander vernetzen können. So nimmt es denn nicht Wunder, daß die Debatte so gut wie nie von der medizinischen Problemstellung und den zur Verfügung stehenden Problemlösungsmöglichkeiten ausgeht - da wir in Deutschland systematisch auf eine Epidemiologie verzichtet haben, wäre eine solche Debatte ja auch nur auf der Basis düsterer Ahnungen möglich -, sondern man konzentriert sich auf 
das, was man zuverlässig weiß: der Ressourcenverbrauch (gleich Umsatz oder Einkommen) der eigenen Anbietergruppe, den es zu sichern und auszubauen gilt, indem man sich im Wege der Vernetzung Steuerungs- und Indikationsmacht sichert. Symptomatisch hierfür ist der Vergleich Peter Sauermanns vom Hausarzt als der Spinne im Netz (Wen mag sie wohl am liebsten fressen?). Diese Rolle möchten aber auch die Krankenhäuser beanspruchen, quasi als medizinisches Dienstleistungszentrum für Prävention, fachärztliche Diagnostik und Therapie, Rehabilitation und Pflege. Die zwischen diesen beiden Blöcken sitzenden Fachärzte fürchten, zwischen für die ambulante Versorgung geöffneten Krankenhäusern und den Hausärzten (beide könnten durch die nächste Strukturreform im Gesundheitswesen gestärkt werden) zerrieben zu werden, und reklamieren deshalb der Mode folgend, auch sie seien ja so etwas wie Hausärzte: etwa die Nephrologen, die Gynäkologen, ja sogar die Orthopäden.

Und die Krankenkassen? Das gedanklich wohl älteste Modell, das AOKHausarztmodell, hat bislang nicht einmal die Embryonalphase überstanden. Gescheitert ist es nicht am Widerstand der Ârzte, sondern an der mangelnden Bereitschaft von Versicherten, sich explizit für eine bestimmte Zeit für einen bestimmten Hausarzt zu entscheiden. Eine auBerordentlich schwierige Geburt erlebte das Berliner BKK/TK-Praxisnetz, das mit kombinierten Budgets arbeitet und daher schon sehr kompliziert und anspruchsvoll angelegt ist. Auch hier zeigt sich eine sehr zurückhaltende Akzeptanz bei den Versicherten: Auf 450 teilnehmende Ärzte kommen 14.000 eingeschriebene Versicherte, eine Relation von 1:30.

Trotz aller politischen, gesetzlichen und wirtschaftlichen Hemmnisse gibt es in ganz Deutschland inzwischen wohl über 300 verschiedenartige Netzwerke durchweg in der ambulanten Medizin. Es mag sein, daß die Kraft zum organisatorischen Fortschritt an der Basis und in der Peripherie in kleinen Innovationsschritten letztlich mehr bewirkt, als Spitzenfunktionäre über Top-down-Modelle zu erreichen im Stande sind. 


\section{Öffnung von Krankenhäusern für die fachärztliche Versor- gung}

Susanne Renzewitz

\section{Finanzierungssystem der GKV}

Worin liegt die Notwendigkeit, sich mit veränderten Versorgungsstrukturen im Gesundheitswesen auseinanderzusetzen? Ad hoc fallen mir zwei Gründe ein.

Der eine ist ein wirtschaftlicher Grund. Knappe Ressourcen zwingen zu neuen Überlegungen. Die finanzielle Lage der GKV war in den vergangenen Jahren trotz tendenziell steigender Beitragssätze von zum Teil erheblichen Defiziten gekennzeichnet.

Auf der Ausgabenseite haben vor allem Auswirkungen des medizinischen und medizinisch-technischen Fortschritts, der demographischen Entwicklung sowie die hohe Dienstleistungsintensität des Gesundheitssektors zu überproportionalen Ausgabensteigerungen geführt. Zukünftig werden vor allem die durch den medizinischen Fortschritt und die demographische Entwicklung induzierten Leistungen die GKV belasten.

Die Entwicklung auf der Einnahmenseite des lohnbezogenen Finanzierungssystems der GKV ist zunehmend von der rückläufigen gesamtwirtschaftlichen Lohnquote geprägt. Die Ursachen hierfür liegen in der ungünstigen gesamtwirtschaftlichen Entwicklung mit hoher Arbeitslosigkeit und niedrigen Lohnabschlüssen und in dem Trend zur Einkommensentstehung in Bereichen, die nicht der Beitragsbemessung für die GKV unterliegen. Für die nähere Zukunft ist keine nachhaltige Verbesserung der Situation auf der Einnahmenseite der GKV zu erwarten.

Bemühungen zur Stabilisierung der finanziellen Lage der GKV haben in der Vergangenheit vor allem an der Ausgabenseite angesetzt. Die Bedeutung der Regelungen zur Umsetzung des „Grundsatzes der Beitragssatzstabilität" hat bei der Budgetbemessung in allen Leistungsbereichen stetig zugenommen.

Zur Begrenzung des Ausgabenzuwachses und zur Sicherung der Beitragssatzstabilität beabsichtigt die neue Regierungskoalition, mit dem Solidaritätsstärkungsgesetz den einzelnen Leistungssektoren eine strikte Budgetierung zu verordnen. Globale Wirtschaftlichkeitsreserven werden in nennenswertem Umfang im System vermutet. Damit scheint 
die Rationalisierungsdebatte wieder zu einem zentralen Thema der Politik zu werden.

Ich möchte jetzt nicht auf die viel diskutierte Frage eingehen, ob, wo und in welchem Umfang globale Wirtschaftlichkeitsreserven - und dabei liegt die Betonung auf global - in unserem Gesundheitssystem vorhanden sind. Fest steht jedoch, daß in einer besseren Kooperation und Koordination der einzelnen Versorgungsbereiche seit Jahren Wirtschaftlichkeitsreserven vermutet werden. Dennoch kapitulierten die politisch Verantwortlichen bislang davor, insbesondere eine bessere Zusammenarbeit zwischen dem stationären und ambulanten Versorgungsbereich sinnvoll zu fördern. Es wäre verfehlt zu glauben, die Verzahnung könne allein von der Selbstverwaltung gelöst werden. Die Erfahrungen aus der Vergangenheit zeigen, daß die Verzahnungsdiskussion zwischen allen Beteiligten am Gesundheitswesen eher bissig geführt worden ist, was nicht nur an der dentalen Begrifflichkeit liegen dürfte. Vielmehr geht es hier um die Wahrung alter Pfründe und die daraus resultierenden unterschiedlichen Vorstellungen zur sektorübergreifenden Versorgung.

\section{Bedarfs- und patientenorientierte Versorgung}

Der zweite Grund, über eine Veränderung von Versorgungsstrukturen nachzudenken, ist das Ziel einer bedarfs- und patientenorientierten Versorgung. Dieses Postulat ist in der jüngsten Vergangenheit wieder vermehrt ins Blickfeld gesundheitspolitischer Überlegungen gerückt worden. Ich erinnere hier an den Paradigmenwechsel der Gesundheitspolitik zum Ausgang der letzten Legislaturperiode. Die Diskussion um eine Weiterentwicklung des Gesundheitssystems soll sich zukünftig nicht ausschließlich an ökonomisch-finanziellen Kriterien orientieren, sondern auch an medizinischen Versorgungsaspekten und am Patienten. Hier spielen sicherlich der medizinische Fortschritt und die demographische Entwicklung eine entscheidende Rolle. Eine medizinische Orientierung wird aber auch Auswirkungen auf die bestehenden Versorgungsstrukturen haben. Dies gilt insbesondere für ein verbessertes „Schnittstellenmanagement" zwischen ambulanter und stationärer Versorgung, aber auch zwischen Akutversorgung, Rehabilitation, Pflege und Hauskrankenpflege. Sie sehen, auch der Ansatz einer bedarfs- und patientenorientierten Versorgung führt zum Thema Verzahnung.

\section{Ansätze integrierter Versorgungsformen}

Zahlreiche Gedankenmodelle zur Förderung einer besseren Zusammenarbeit zwischen ambulanter und stationärer Versorgung sind entwikkelt worden. Einige möchte ich nennen. 
Als einen Ansatz, Versorgungsstrukturen durch eine bessere Integration zu verändern, wird die Einführung von Managed-Care-Strukturen in das deutsche Gesundheitswesen diskutiert. Hier muß man allerdings sagen, daß Managed Care mittlerweile zu einem Sammelbegriff geworden ist, der von den jeweiligen Interessengruppen unterschiedlich interpretiert wird. Grundsätzlich setzen Managed-Care-Überlegungen bei der Leistungssteuerung an. Die Inanspruchnahme von Leistungen soll durch das Ordnungsprinzip „Vernetzungen“ gesteuert werden. Mit dem 2. GKV-Neuordnungsgesetz sind durch Modellvorhaben und Strukturverträge Freiräume für die Erprobung von Managed-Care-Strukturen geschaffen worden. Echte Integrationslösungen sind durch die Umsetzung dieser Regelung jedoch noch nicht gelungen. Vielmehr zeichnet sich die Gefahr $a b$, daß die Gräben zwischen den Versorgungsbereichen nicht zugeschüttet, sondern vertieft werden.

So sind Modellvorhaben und Strukturverträge zur Spielwiese jedweder Vernetzungsideen im ambulanten Bereich geworden. Leider sind die Krankenhäuser dabei zum Großteil außen vor gelassen worden. Das liegt insbesondere auch daran, daß der Gesetzgeber die Regelungen so konzipiert hat, daß der ambulante Bereich den stationären Bereich im jeweiligen Sinn steuern kann, jedoch nicht umgekehrt. Krankenhäuser sind keine gesetzlich vorgesehenen Vertragspartner von Strukturverträgen nach § 73 a SGB V; Modellvorhaben, die die vertragsärztliche Versorgung betreffen, sind nur mit Zustimmung der KBV/KVen möglich. Einsparerfolge durch Strukturverträge und Modellvorhaben konnten bislang noch nicht verbucht werden. Allerdings setzt sich wohl die Erkenntnis durch, daß Modelle, die mehr als die Befriedung des kassenärztlichen Bereichs erreichen sollen, ohne Einbeziehung der Krankenhäuser wenig sinnvoll sind.

Die Krankenkassen sehen eine Möglichkeit zur Veränderung der Versorgungsstrukturen darin, die Trennung zwischen der ökonomischen und der medizinischen Verantwortung aufzuheben, indem sie Leistungen einkaufen und dann ihren Versicherten anbieten wollen - Stichwort: Einkaufsmodelle. Einkaufsmodelle würden, zusammen mit dem zwangsläufig erfolgenden Wegfall der Krankenhausplanung und des staatlichen Sicherstellungsauftrages, zu einer bedenklichen Konzentration der Nachfragemacht auf seiten der Krankenkassen führen. Bei konkurrierenden Kassenarten besteht die Gefahr, daß angestrebte Wettbewerbsvorteile zu Lasten einer bedarfsgerechten und gleichen Versorgung der Versicherten gehen.

Von seiten der Ärzteschaft soll veränderten Versorgungserfordernissen durch eine Ausweitung der Ermächtigung von Krankenhausärzten 
Rechnung getragen werden. Angesichts der Entwicklung im Ermächtigungsbereich kann dieses Konzept wenig überzeugen. Die Zahlen sprechen hier für sich. Der Anteil der ermächtigten Krankenhausärzte an der Gesamtzahl der an der vertragsärztlichen Versorgung teilnehmenden Ärzte ist allein im Zeitraum von 1991 bis 1997 um 13,3\% zurückgegangen.

Die Diskussion um veränderte Versorgungsstrukturen darf sich auch nicht ausschließlich darauf fokussieren, Krankenhausleistungen zu vermeiden und Kapazitäten im Krankenhausbereich abzubauen. Der Grundsatz „soviel ambulant wie möglich, soviel stationär wie nötig“ ist wenig aussagekräftig, wenn es darum geht, die bestehenden Versorgungsstrukturen einem veränderten Bedarf anzupassen. Im übrigen gibt es aus ökonomischer Sicht derzeit keinerlei Beweise, daß die anstelle der Krankenhausbehandlung kompensatorisch notwendigen ambulanten Leistungen insgesamt zu weniger Ausgaben für die Krankenkassen führen.

Eine Veränderung der Versorgungsstruktur kann nur dann von Erfolg gekrönt sein, wenn die Abstimmung einer bedarfsgerechten Patientenversorgung in gleichberechtigter Partnerschaft zwischen den Akteuren im Gesundheitswesen erfolgt.

\section{Weiterentwicklung der Krankenhäuser zu integrierten Dienstleistungszentren}

Wie sehen nun die Überlegungen der Deutschen Krankenhausgesellschaft dazu aus? Die DKG hat im März 1998 ein Konzept verabschiedet, bei dem die Krankenhäuser zu sogenannten integrierten Dienstleistungszentren weiterentwickelt werden sollen (Abb. 1).

Der Ansatz der DKG geht von der Notwendigkeit einer effizienteren Arbeitsteilung der Leistungsanbieter aus. Ziel ist es, nicht nur gemeinsam Aufgaben zu bewältigen, sondern auch über die Aufteilung und Umverteilung von Leistungen nachzudenken. Die Idee vom integrierten Dienstleistungszentrum ist dabei von folgenden Elementen geprägt:

- Verbesserung der Kooperationen unter den Leistungsanbietern,

- Flexibilisierung des stationären Versorgungsangebotes und

- Öffnung der Krankenhäuser für weitere ambulante Funktionen. 


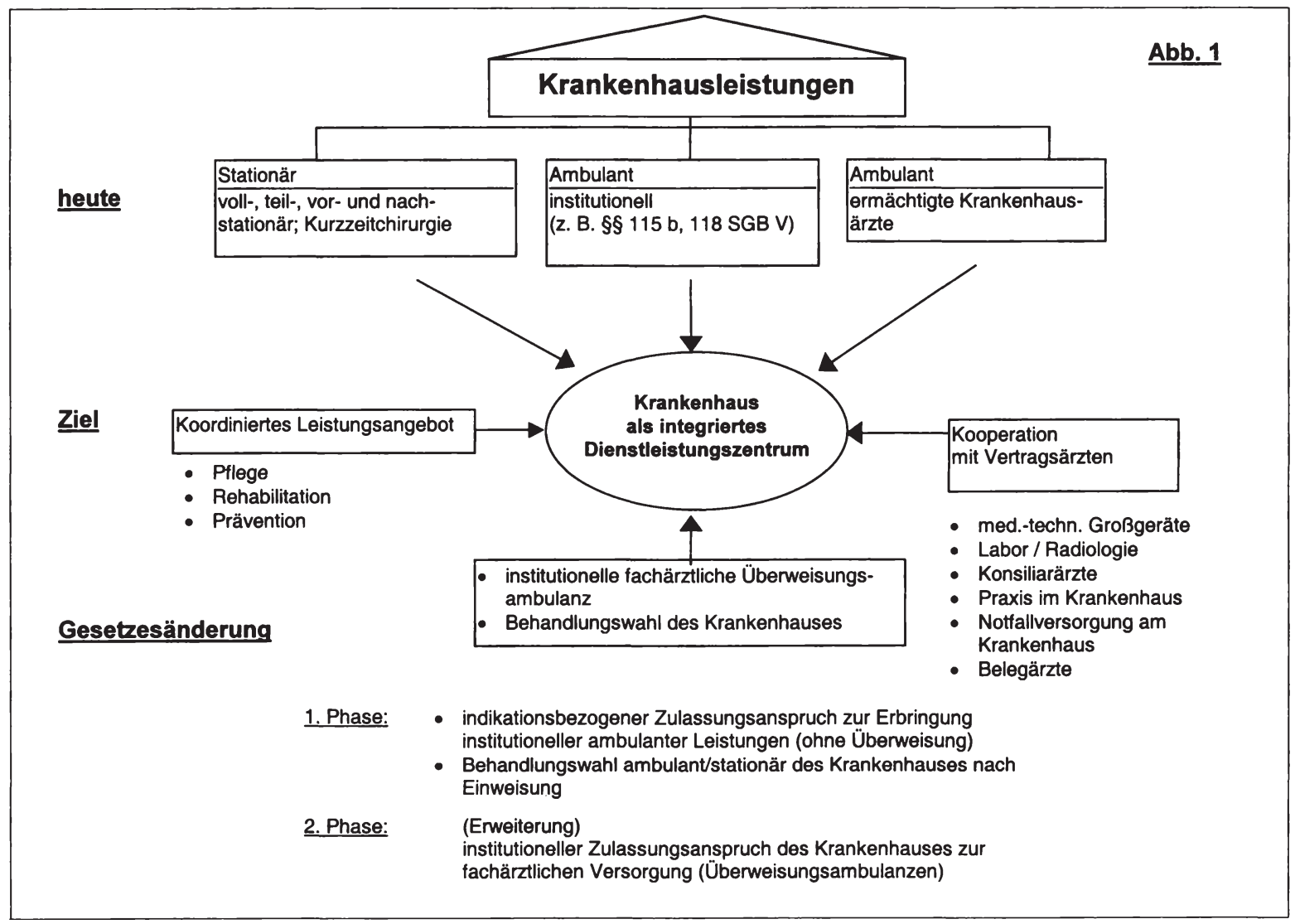




\section{Verbesserung der Kooperation unter den Leistungsanbietern}

Die Förderung von Kooperationen zwischen Krankenhäusern und niedergelassenen Ärzten trägt zu einer Verbesserung des Schnittstellenmanagementes und der Patientenversorgung sowie zur Mobilisierung von Wirtschaftlichkeitspotentialen bei. Eine wachsende Kooperationsbereitschaft ist bereits festzustellen, wie eine im Dezember 1997 in Niedersachsen durchgeführte Kooperationsuntersuchung zeigt (Abb. 2).

Abb. 2: Kooperation zwischen Krankenhäusern und Praxen in Niedersachsen

\begin{tabular}{|c|c|c|c|c|c|c|}
\hline \multicolumn{7}{|c|}{ Krankenhausgrößenklassen in Betten } \\
\hline & $1-100$ & $101-200$ & $201-400$ & über $\mathbf{4 0 0}$ & $\begin{array}{l}\text { insg. } \\
\text { geplant }\end{array}$ & \\
\hline \multicolumn{7}{|c|}{ Mitnutzung von Krankenhauseinrichtungen durch eine Praxis } \\
\hline OP-Einrichtung & 22,2 & 35,9 & 28,6 & 23,7 & 28,6 & $\bullet \bullet$ \\
\hline Radiologie & 20,0 & 28,1 & 32,7 & 15,8 & 25,0 & $\bullet$ \\
\hline Med.-techn. Großgeräte & 2,2 & 6,3 & 34,7 & 42,1 & 19,4 & $\bullet$ \\
\hline Anästhesiologie & 13,3 & 31,3 & 2,0 & 13,2 & 16,3 & $\bullet$ \\
\hline Labor & 15,6 & 21,9 & 8,2 & 13,2 & 15,3 & $\bullet$ \\
\hline $\begin{array}{l}\text { Kooperierende Krankenhäu- } \\
\text { ser }(\mathrm{KH})\end{array}$ & 37,8 & 54,7 & 65,3 & 57,9 & 54,1 & $\bullet \bullet$ \\
\hline \multicolumn{7}{|c|}{ Praxis auf dem Krankenhausgelănde mit belegărztlicher Tătigkeit } \\
\hline Kooperierende KH & 37,8 & 54,7 & 36,7 & 39,5 & 43,4 & \\
\hline \multicolumn{7}{|c|}{ Praxis auf dem Krankenhausgelände ohne belegärztliche Tätigkeit } \\
\hline Kooperierende KH & 11,1 & 20,3 & 22,4 & 23,7 & 19,4 & |• \\
\hline \multicolumn{7}{|c|}{ Medizinische Dienstleistung für das Krankenhaus durch eine Praxis } \\
\hline Laborleistung & 51,1 & 51,6 & 46,9 & 42,1 & 48,5 & $\bullet$ \\
\hline Med.-techn. Großgeräte & 31,1 & 51,6 & 46,9 & 39,5 & 43,4 & $\bullet$ \\
\hline Bakteriologie & 31,1 & 46,9 & 38,8 & 36,8 & 39,3 & \\
\hline Pathologie & 26,7 & 35,9 & 40,8 & 34,2 & 34,7 & $\bullet$ \\
\hline Röntgendiagnostik & 28,9 & 35,9 & 30,6 & 10,5 & 28,1 & $\bullet$ \\
\hline Nuklearmedizin & 24,4 & 26,6 & 24,5 & 13,2 & 23,0 & \\
\hline Dialyse & 13,3 & 20,3 & 18,4 & 13,2 & 16,8 & $\bullet$ \\
\hline Kooperierende KH & 62,2 & 76,6 & 79,6 & 71,1 & 73,0 & $\bullet \bullet$ \\
\hline
\end{tabular}

- schwächer • stärker

Quelle: E. Bruckenberger, Hannover, Dezember 1997

Das Ergebnis dieser Untersuchung belegt nicht nur Kooperationen zwischen Krankenhäusern, sondern auch zwischen Krankenhäusern und niedergelassenen Ärzten, insbesondere in den Bereichen der belegärzt- 
lichen Tätigkeit, in der gemeinsamen Nutzung medizinisch-technischer Großgeräte, aber auch in der Inanspruchnahme der Krankenhausinfrastruktur durch Vertragsärzte bei der Durchführung von ambulanten Operationsleistungen.

\section{Flexibilisierung des stationären Versorgungsangebotes}

Die bisherigen stationären Behandlungsmöglichkeiten müssen nach unserem Dafürhalten breiter gefächert werden, um dem Bedarf des Patienten an einer differenzierten Behandlung gerecht zu werden. Im Rahmen der stationären Krankenhausversorgung wird nach derzeitiger Gesetzeslage lediglich nach vor- und nachstationären, teil- und vollstationären Patienten unterschieden. Der medizinische Fortschritt eröffnet hier jedoch mehr Möglichkeiten. Dem soll z. B. das von der DKG entwickelte Konzept für kurzzeitchirurgische Behandlungen Rechnung tragen. Dieses Konzept ist auf die Patienten zugeschnitten, bei denen eine ambulante Behandlung nicht möglich ist und eine teil- bzw. vollstationäre Behandlung nicht nötig ist. Das sind in der Regel Patienten, die besondere Merkmale aufweisen, die dazu zwingen, sie unter Beobachtung zu stellen und auch für eine kurze Zeit im Krankenhaus zu behalten (Liegezeiten 1 - 3 Tage). Der von der DKG entwickelte Katalog umfaßt derzeit 17 häufig erbrachte stationäre Leistungen. Entgegen anderer Kommentierungen soll hier nicht der Versuch unternommen werden, ambulante $\mathrm{Pa}$ tienten zusätzlich in die Krankenhausversorgung zu überführen. Ganz entscheidend ist daher die klare formale Abgrenzung der kurzzeitchirurgischen Behandlung vom ambulanten Operieren. Es handelt sich bei der Kurzzeitchirurgie um eine stationäre Leistung, die eine Einweisung erfordert.

\section{Öffnung der Krankenhäuser für die ambulante fachärztliche Versorgung}

Unter einem integrierten Dienstleistungszentrum versteht die DKG schließlich auch eine schrittweise weitergehende Öffnung des Krankenhauses für ambulante Funktionen. Hierdurch würde die Wahlfreiheit der Patienten erhöht, innen die Inanspruchnahme der diagnostischen, therapeutischen und rehabilitativen Dienste und Einrichtungen des Krankenhauses ermöglicht und eine größere Wirtschaftlichkeit erreicht. Für den Patienten würde die ambulante Behandlung im Krankenhaus eine Erleichterung bedeuten, wenn Verzögerungen und umständliche Überweisungswege vermieden werden. Zielsetzung ist auch eine erhöhte Behandlungskontinuität sowie der Abbau von Informationsverlusten und Doppeluntersuchungen. Ausgehend von Erfahrungen und medizinischem Sachverstand haben wir unser Konzept zwischenzeitlich konkre- 
tisiert und Indikationen in einem Katalog zusammengestellt, bei denen eine ambulante fachärztliche Versorgung durch das Krankenhaus sinnvoll ist. In diesem ersten Schritt geht es darum, schwerkranke Patienten sowohl ambulant als auch stationär durch „eine Hand" behandeln zu lassen. Diese Patienten bewegen sich derzeit ständig zwischen Einweisung und Überweisung, wodurch die Versorgungskontinuität und Qualität sowie letztlich auch die Wirtschaftlichkeit der Leistungserbringung leidet.

Der Standort Krankenhaus bildet aufgrund der vorhandenen Einrichtung und des medizinisch-technischen Know-hows das Herzstück des integrierten Dienstleistungszentrums. Im Krankenhaus vorhandene Strukturen können so sinnvoll genutzt werden und müssen nicht in anderen Versorgungsbereichen kostenintensiv aufgebaut werden. Zudem stehen alle Krankenhausärzte verschiedener Fachrichtungen zur Verfügung.

Mit der Weiterentwicklung der Krankenhäuser zu integrierten Dienstleistungszentren könnte auch der Wettbewerbsgedanke sinnvoll ausgebaut werden. Diesen Wettbewerb wollen wir als Leistungs- und Qualitätswettbewerb verstanden wissen und nicht als Vertrags- und Preiswettbewerb. $\mathrm{Da}$ es bei Marktchancen nicht nur Gewinner geben kann, liegt auf der Hand. So wird von seiten der Vertragsärzte vehement versucht, den Vorstoß „integriertes Dienstleistungszentrum“ abzuwehren. Dies gilt natürlich in erster Linie der Forderung, mehr ambulante Leistungen im Krankenhaus zu integrieren. Sind diese Abwehrkämpfe auch verständlich, muß doch gefragt werden, warum der vertragsärztliche Bereich zu keiner Zeit die ambulante Notfallversorgung durch die Krankenhäuser in Frage gestellt hat und stellt.

Das Konzept vom integrierten Dienstleistungszentrum ist aus unserer Sicht eine Möglichkeit, notwendige Anpassungsprozesse zu beschleunigen und zu bewältigen. Die Option, daß die Selbstverwaltung von Krankenkassen, Kassenärztlichen Vereinigungen und Krankenhausträgern allein entsprechende Modellvorhaben oder Strukturverträge auf den Weg bringt, ist allerdings Illusion. Es bedarf hierzu vielmehr gesetzlicher Neuregelungen. Die neue Regierungskoalition hat sich veränderte Versorgungsstrukturen als ein Element der angekündigten „Strukturreform 2000" zum Ziel gesetzt. Der angekündigte politische Dialog mit allen Beteiligten am Gesundheitswesen wird zeigen, ob und wie ernst die Politik dieses Ziel nimmt. 


\section{Öffnung von Krankenhäusern für die fachärztliche Ver- sorgung}

Lothar Krimmel

Im Zusammenhang mit der anstehenden Diskussion um eine „Strukturreform 2000" in der gesetzlichen Krankenversicherung wird von verschiedenen Seiten wieder verstärkt auf das Problem der sogenannten „Verzahnung“ von ambulanter und stationärer Versorgung hingewiesen. Dabei werden zum Teil weitreichende Forderungen auf zwei grundlegende Annahmen gestützt:

1. Es gibt in Deutschland eine zu starke Trennung zwischen ambulanter und stationärer Versorgung.

2. Eine stärkere „Verzahnung“ von ambulanter und stationärer Versorgung führt zur Erschließung von Wirtschaftlichkeitsreserven in der GKV.

Beide Annahmen stellen sich bei näherer Betrachtung als unzutreffend heraus. So gibt es in Deutschland bereits seit langem ein geregeltes Zusammenspiel zwischen ambulanten und stationären Versorgungskapazitäten. Bekannte Beispiele hierfür sind die

- Übernahme stationärer Versorgungsaufgaben durch mehr als 5.000 belegärztlich tätige Kassenärzte,

- Ermächtigung von mehr als 10.000 Krankenhausärzten zur Teilnahme an der ambulanten Versorgung,

- Möglichkeit von Krankenhäusern zur Übernahme vor- und nachstationärer Behandlung sowie zur Durchführung ambulanter Operationen.

Auch die zweite Annahme, daß eine noch stärkere „Verzahnung“ zur Erschließung weiteren Wirtschaftlichkeitspotentials führen würde, wird durch keine empirische Studie gestützt. Im Gegenteil: In Untersuchungen durch den MDK Baden-Württemberg hat sich gezeigt, daß die stationäre Versorgung gerade dort die meisten Fehlbelegungen aufweist und damit am teuersten ist, wo die „Verzahnung“ in Gestalt einer verstärkten Teilnahme von Krankenhäusern an der ambulanten Versorgung am weitesten fortgeschritten ist. Dies bestätigen Erfahrungen aus den USA, daß erfolgreiches Kostenmanagement mit Wahl der jeweils richtigen Versorgungsstufe für den Patienten nicht etwa durch „Verzahnung“, 
sondern im Gegenteil nur durch strikte „Abschottung“ im Sinne einer Begrenzung des teuren stationären Leistungssektors auf die unbedingt notwendige Krankenhausbehandlung erreicht werden kann. Vor diesem Hintergrund scheint die Verzahnungsdiskussion zunehmend geeignet, von den eigentlichen Problemen abzulenken, die in den dramatisch zunehmenden Kosten der Krankenhausbehandlung bei gleichzeitig deutlich abnehmendem Bedarf zu sehen sind.

Die Zahlen jedenfalls sprechen für sich: So ist der Anteil der Ausgaben für die stationäre Versorgung an den gesamten Leistungsausgaben der gesetzlichen Krankenkassen von 32,1\% im Jahr 1992 auf 34,8\% im Jahr 1998 gestiegen, während im gleichen Zeitraum der Ausgabenanteil für die ambulante Versorgung, einschließlich Arzneimittelbehandlung, von $33,4 \%$ auf $31,6 \%$ zurückgegangen ist (Abb. 1). Dies geschah zudem vor dem Hintergrund einer zunehmenden Verlagerung von Aufgaben aus dem stationären Bereich in die ambulante Versorgung. Für diese Verlagerungseffekte steht nicht nur der massive Ausbau des ambulanten Operierens in Arztpraxen, sondern auch die zunehmende Möglichkeit, schwerwiegende internistische Erkrankungen durch eine intensive ambulante Therapie außerhalb des Krankenhauses zu behandeln.

Abb. 1: Ausgabenvergleich stat. und amb. Kosten für die Jahre 1992 - 1998

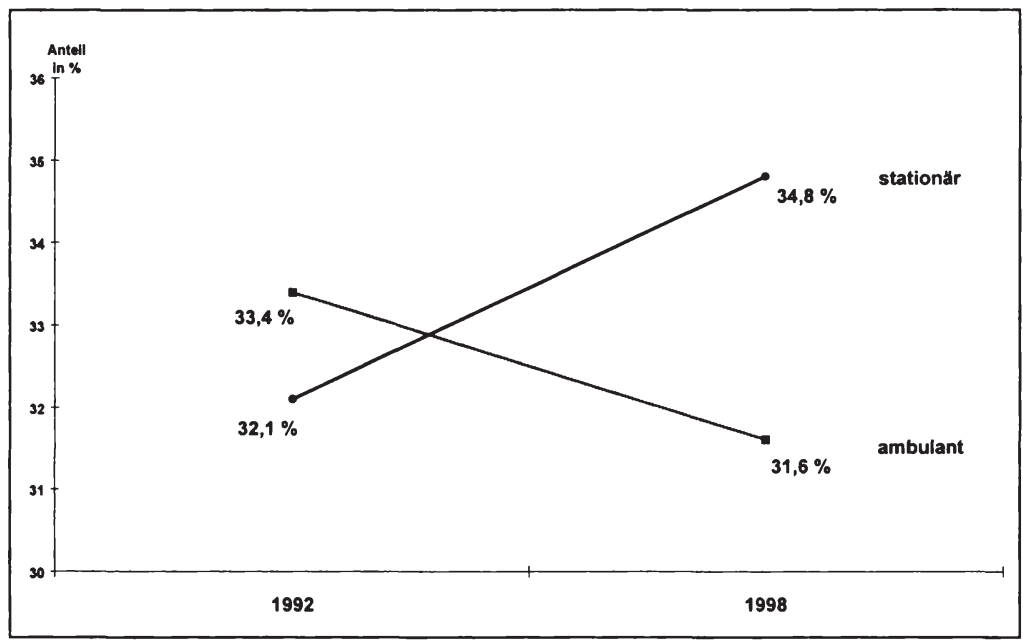

Dieser klare medizinische Trend kommt auch in dem stetigen Rückgang der Krankenhausbehandlungstage pro Jahr zum Ausdruck. Und es steht nicht zu erwarten, daß dieser Trend in Kürze gebrochen wird. Im Gegenteil: Die Zahl der Krankenhausbehandlungstage je Einwohner und 
Jahr ist in Deutschland mit rund 2,8 Tagen immer noch fast dreimal so hoch wie in den USA mit 1,1 Tagen (Abb. 2). Daher steht zu erwarten, $\mathrm{da} ß$ in den nächsten Jahren noch ganz erhebliche Abschmelzungsprozesse der enormen Überkapazitäten im Krankenhausbereich stattfinden müssen.

Abb. 2: Zahl der Krankenhausbehandlungstage je Einwohner und Jahr

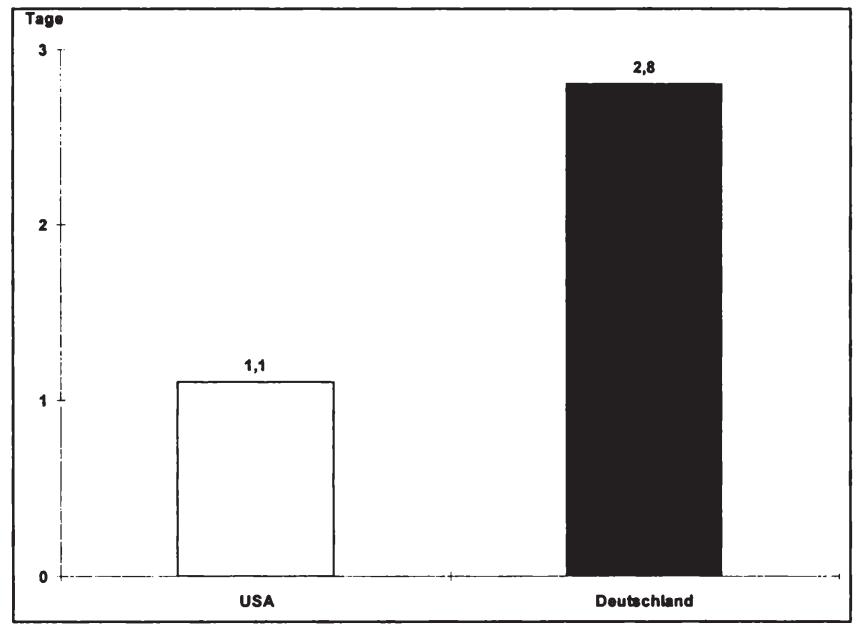

\section{„Gesundheitszentrum“ als Rettungsanker}

Diese Entwicklung wird selbstverständlich auch von der breiten Interessenvertretung der Krankenhäuser, bestehend aus Gewerkschaften, Kirchen, Ländern und Kommunen, mit Sorge wahrgenommen. Die Deutsche Krankenhausgesellschaft wird insbesondere im Vorfeld erwarteter politischer Entscheidungen nicht müde, auf eine Zunahme der Fallzahlen bei gleichzeitiger Reduzierung der durchschnittlichen Verweildauer im Krankenhaus hinzuweisen. Dabei wird geflissentlich verschwiegen, daß sich hinter den zunehmenden Fallzahlen abnehmende Patientenzahlen verbergen, da durch mehr oder weniger geschicktes zwischenzeitliches Entlassen aus der stationären Versorgung Fallzahlsteigerungen gewissermaßen „produziert" werden. Es bleibt auch unerwähnt, daß die Zahl der Krankenhausbehandlungstage als Summe aller Behandlungstage im Krankenhaus kontinuierlich zurückgehen.

Da jedem klar sein muß, daß Zahlenspiele nur noch über eine gewisse Zeit über die schwindende Bedeutung des Krankenhauses außerhalb 
der Betreuung von Hochrisikopatienten hinwegtäuschen können, haben die hinter dem Krankenhaus stehenden Interessengruppen zu einer weitreichenden Gegenbewegung ausgeholt. Ziel ist, unter Erhalt der Standorte und der Arbeitsplätze die nicht mehr benötigten Krankenhauskapazitäten durch Umwandlung weiterer Krankenhausbereiche in „Gesundheitszentren" für die ambulante Versorgung zu erhalten. Dieses Ziel scheint nunmehr greifbar nah, da die neue rot-grüne Regierungskoalition in kaum zu überbietender Eindeutigkeit mit einer Öffnung der Krankenhäuser für die ambulante fachärztliche Versorgung sympathisiert.

Die Inhalte des Vorschaltgesetzes des Jahres 1999 sprechen bereits eine eindeutige Sprache. Obwohl dieses Gesetz nur eine "Atempause“ für die Diskussion der Strukturreform 2000 schaffen sollte, wurden bereits weitreichende Weichenstellungen vorgenommen. Der ambulante Versorgungsbereich wurde unbefristet budgetiert und im Ausgangsniveau des Jahres 1999 noch unter den Ausgaben des Jahres 1998 eingefroren, während der stationäre Sektor nicht nur die erheblichen Gewinne aus dem Jahr 1998 als Sockel mitnehmen durfte, sondern darüber hinaus auch noch für 1999 massive Ausnahmeregelungen erhielt. Da diese Ausnahmetatbestände in gleicher Weise für den ambulanten Sektor zutreffen, dort aber nicht berücksichtigt wurden, gibt das sogenannte „Vorschaltgesetz 1999“ allen Anlaß, die Verfassungsmäßigkeit dieser Gesetzgebung im Hinblick auf den Gleichheitsgrundsatz gem. Art. 3 des Grundgesetzes überprüfen zu lassen.

\section{Rechtliche, finanzielle und medizinische Einwände}

Gegen die von den vereinigten Krankenhaus-Lobbyisten und der rotgrünen Regierungskoalition beabsichtigte Öffnung der Krankenhäuser für die ambulante fachärztliche Versorgung sind eine ganze Reihe von Einwänden vorzubringen, die an dieser Stelle auf rechtliche, finanzielle und medizinische Aspekte beschränkt werden sollen. Aus rechtlicher Sicht scheint eine generelle Öffnung der Krankenhäuser bereits wegen der offensichtlichen Verletzung von Art. 14 des Grundgesetzes nicht durchführbar. Die Öffnung der Krankenhäuser würde für einen Großteil der ambulant tätigen Fachärzte mit öffentlich-rechtlicher Zulassung einen enteignungsgleichen Eingriff darstellen. Auch wettbewerbsrechtliche Erwägungen sprechen gegen ein solches Vorhaben. Aufgrund der derzeit praktizierten dualen Finanzierung, also der Mitfinanzierung der Krankenhäuser durch die öffentliche Hand, würde mit dem Öffnen der Krankenhäuser gleichzeitig ein Wettbewerb zwischen privat organisierten, niedergelassenen und öffentlich subventionierten Anbietern eröffnet. Dies ist nach geltendem Recht unzulässig. Voraussetzung für die Eröffnung eines derartigen Wettbewerbs wäre daher die monistische Finan- 
zierung der Krankenhäuser alleine über die Krankenversicherung. Dies aber ist allenfalls nach Abschluß einer langwierigen Übergangsphase realistisch.

Gegen eine weitgehende Beteiligung der Krankenhäuser an der ambulanten Versorgung sprechen jedenfalls in Deutschland auch finanzielle Aspekte. Es liegt geradezu auf der Hand, daß die Öffnung der Krankenhäuser für die ambulante fachärztliche Versorgung einen Sprengsatz erster Ordnung für die Finanzen der gesetzlichen Krankenversicherung bilden würden. Bereits mit der bisherigen begrenzten Einbeziehung der Krankenhäuser im Rahmen von persönlichen oder institutionellen Ermächtigungen sowie der Möglichkeit zum ambulanten Operieren und zur vor- und nachstationären Versorgung zeigt sich, daß der öffentliche Dienst im Krankenhaus auch nicht annähernd in der Lage ist, sich aus den Vergütungssätzen zu finanzieren, welche für die ambulante Versorgung durch freiberufliche Kassenärzte derzeit gelten. Nicht anders ist es zu erklären, daß nicht nur die Möglichkeit des ambulanten Operierens, sondern insbesondere die Möglichkeit zur vor- und nachstationären Behandlung anstelle einer Krankenhausbehandlung bislang von den Krankenhäusern nur in minimalem Umfang genutzt wurde. Die jedenfalls für Deutschland unter Wirtschaftlichkeitsaspekten zu konstatierende Wettbewerbsunfähigkeit eines im öffentlichen Dienst organisierten Versorgungssystems kann schon anhand eines Vergleichs der aktuellen Arbeitsbedingungen von freiberuflich tätigen Kassenärzten und öffentlich Bediensteten gezeigt werden (Abb. 3). 
Abb. 3: Vergleich der Arbeitsbedingungen von angestellten und freiberuflich tätigen Ärzten

\begin{tabular}{|l|c|c|}
\hline & freiberuflich & angestellt \\
\hline Krankheitstage & ca. 2/Jahr & ca. 20/Jahr \\
Urlaubstage & ca. 20/Jahr & ca. 30/Jahr \\
bezahlter Fortbildungsurlaub & --- & 5 Tage/Jahr \\
Arbeitszeit & ca. 55 Std./Woche & 38,5 Std./Woche \\
Nacht- und Wochenendbereit- & bis zu & ca. 10 Std./Woche \\
schaft & 100 Std./Woche & $50 \%$ \\
Rentenversicherungsbeiträge & $100 \%$ & $50 \%$ \\
Krankenversicherungsbeiträge & $100 \%$ & nein \\
freiberufliches Risiko & ja & nein \\
Regreßrisiko für Arzneimittel & ja & nein \\
Preisverfall der ärztlichen Lei- & ja & \\
stung & \multicolumn{2}{|c|}{}
\end{tabular}

Angesichts der sich zunehmend verschlechternden Arbeitsbedingungen für niedergelassene Kassenärzte ist dieser Vergleich durchaus angetan, für den einzelnen Kassenarzt den Schrecken vor einer zwangsweisen Überführung der freiberuflichen kassenärztlichen Tätigkeit in den öffentlichen Dienst zu nehmen. Dies gilt gerade angesichts zunehmender Existenzbedrohung durch im eigentlichen Sinne berufsfremde Risiken wie Regresse aus Richtgrößenprüfungen und Überschreitungen der Arzneimittel- und Heilmittelbudgets. Es wäre interessant zu beobachten, wie bei Öffnung der Krankenhäuser für die ambulante fachärztliche Versorgung die Krankenhäuser mit den zu erwartenden Milliardenregressen umzugehen beabsichtigen, die angesichts der unter Wirtschaftlichkeitsaspekten nur als unbedarft zu bezeichnenden Verordnungspraxis der Krankenhausärzte kaum abzuwenden sein dürften.

Gegen eine weitgehende Beteiligung der Krankenhäuser an der ambulanten fachärztlichen Versorgung sprechen schließlich nicht zuletzt auch medizinische Gründe. Dabei ist zunächst davon auszugehen, daß selbst in den weitestgehenden politischen Vorstellungen eine Beteiligung der Krankenhäuser nur in solchen medizinischen Bereichen erwogen wird, die zum einen keine Überschneidung mit der hausärztlichen Versorgung aufweisen und zum anderen an einem Großteil der Krankenhäuser verfügbar sind. Dabei handelt es sich um spezifische Leistungen aus den 
internistischen Teilgebieten (z. B. Kardiologie, Gastroenterologie, Nephrologie) sowie um Leistungen aus dem chirurgischen Fachgebiet. Andere Fachgebiete eignen sich zum einen wegen der notwendigen engen Bindung zwischen Arzt und Patient (z. B. Gynäkologie) und zum anderen wegen der nur sehr geringen Verbreitung entsprechender Krankenhausabteilungen (z. B. Dermatologie) nicht für eine ambulante Versorgung am Krankenhaus.

Gegen eine Verlagerung des ambulanten Versorgungsgeschehens ans Krankenhaus spricht insbesondere die unübersehbare und inzwischen empirisch untermauerte Gefährdung des Patienten durch eine unnötige Übernahme in die stationäre Behandlung. Das Krankenhaus ist ohne jede Frage ein sehr gefährlicher Ort für alle Patienten, die dort nicht hingehören. In der folgenden Übersicht ist eine Auswahl von Gefahren wiedergegeben, die insbesondere untragbar in solchen Fällen sind, in denen die Behandlung nicht stationär hätte durchgeführt werden müssen. Angesichts einer vom Medizinischen Dienst der Krankenkassen ermittelten Fehlbelegungsquote von durchschnittlich $20 \%$ ist das entsprechende Gefahrenpotential für die Patienten nicht zu unterschätzen. Dies wird zusätzlich untermauert durch die geringen Anstrengungen der Krankenhäuser, die gesetzlich eröffneten Möglichkeiten einer ambulanten vor- und nachstationären Behandlung anstelle der Krankenhausbehandlung zu nutzen.

Das Krankenhaus ist ein sehr gefährlicher Ort für Patienten, die dort nicht hingehören

(1) Nosokomiale Infektionen

(1) Thrombo-embolische Zwischenfälle

(9) Stürze und andere Unfälle

(3) Medikamentenverwechslungen

(2) Entwicklung von Antibiotika-Resistenzen

(2) Zunahme von Pflegebedürftigkeit

Quelle: Creditor, Annals Int. Med. 118: 219 - 23 
Hinzu kommt, daß die Weiterbildung am Krankenhaus seit Jahrzehnten sehr spezialisiert ausgerichtet ist auf die stationäre Versorgung. Dies hat zur Folge, daß die spezifischen Probleme der ambulanten Versorgung auch vom weitergebildeten Arzt nach der Niederlassung als Kassenarzt erst ganz allmählich in die ambulanten Behandlungsstrategien eingebaut werden müssen. Es steht daher zu erwarten, daß mit einer weitgehenden Beteiligung von Krankenhausärzten an der ambulanten fachärztlichen Versorgung ein ganz erheblicher Qualitätsverlust eintritt, der - wenn überhaupt - nur über lange Zeiträume aufgearbeitet werden kann.

An der Grenze zwischen medizinischen Aspekten und Aspekten des Behandlungskomforts liegen weitere Bedenken gegen eine Beteiligung der Krankenhäuser an der ambulanten fachärztlichen Versorgung. Hierzu gehören

- die häufige Behandlung durch nicht weitergebildete Ärzte,

- der häufige Wechsel des behandelnden Arztes und

- die im Durchschnitt deutlich längere Anfahrt zum Krankenhaus.

Es ist äußerst fraglich, ob die an einen hohen fachärztlichen Behandlungskomfort gewohnten deutschen Versicherten vor diesem Hintergrund das Angebot einer ambulanten fachärztlichen Versorgung am Krankenhaus annehmen würden. Eine systemverändernde Öffnung der Krankenhäuser als konkurrierender fachärztlicher Versorgungsstrang ist aber gerade unter Aspekten der Wirtschaftlichkeit der Versorgung nur bei entsprechender Akzeptanz in der Bevölkerung zu rechtfertigen.

\section{Kompromiß: Optimierung durch sinnvolle Integration}

Es steht außer Frage, daß die ambulante fachärztliche Versorgung durch niedergelassene Kassenärzte derzeit angesichts entsprechender Anstrengungen der vereinigten Krankenhaus-Lobby sowie gleichgerichteter Absichten der neuen rot-grünen Bundesregierung unter massivem Druck steht. Hinzu kommt eine weitere Bedrohung der Fachärzte durch Teile der auch gegenüber der Politik ganz offen nach Primärarztstatus strebenden Hausärzte. Die ambulante fachärztliche Versorgung steht daher unmittelbar vor einem „Zwei-Fronten-Konflikt“, der trotz der beschriebenen rechtlichen, finanziellen und medizinischen Einwände ein erhebliches Bedrohungspotential entfaltet. Damit es in dieser Situation nicht zu einem „Show down" mit unabsehbaren Folgen für die medizinische Versorgung der Bevölkerung kommt, ist auf beiden Seiten die Bereitschaft zum Kompromiß gefordert. 
Ein solcher Kompromiß kann darin bestehen, anstelle einer konfrontativen Auseinandersetzung eine einvernehmliche Optimierung der Versorgung durch sinnvolle Integration bestimmter Krankenhauskapazitäten in die ambulante fachärztliche Versorgung anzustreben. Die Abstimmung auf dieser Grundlage ist innerärztlich bereits weit gediehen. So sind sich die Bundesärztekammer, die Kassenärztliche Bundesvereinigung und der Marburger Bund weitgehend darin einig, daß zur Optimierung der ambulanten und stationären Versorgung insbesondere folgende Instrumente in Frage kommen:

- ein Ausbau des kooperativen Belegarztsystems,

- die weitere Verbreitung von Praxiskliniken,

- ein Ausbau der Ermächtigung von Krankenhausärzten zur Teilnahme an der ambulanten Versorgung,

- die gemeinsame Anschaffung und/oder Nutzung von Großgeräten,

- die gemeinsame Nutzung und Betreibung von Einrichtungen,

- die Einrichtung von Praxen niedergelassener Ärzte am Krankenhaus sowie

- die Errichtung einer Notfallpraxis am Krankenhaus oder die Nutzung von gemeinsamen Notfalleinrichtungen.

So soll insbesondere die gemeinsame Nutzung von Großgeräten und kostenaufwendigen Spezialeinrichtungen in der ambulanten und stationären Versorgung so weit wie möglich gefördert werden. Dazu dienen nach Auffassung der am Konsenspapier beteiligten Organisationen vor allem

- Kooperationsverträge zwischen Krankenhausträgern, Krankenhausärzten und Vertragsärzten,

- die Zulassung von Vertragsärzten zum Betreiben einer Kassenpraxis auch als Zweigpraxis in den Räumen eines Krankenhauses sowie

- die Ermächtigung von Krankenhausfachärzten zur Teilnahme an der vertragsärztlichen Versorgung.

Insbesondere dieser letzte Punkt der Ermächtigung von Krankenhausärzten könnte durchaus im Konsens aller Beteiligten geregelt werden. Als Grundlage der Ermächtigung kann ein von der Ärzteschaft definierter Katalog spezialisierter Leistungen dienen, für deren fachgerechte $\mathrm{Er}$ bringung zur Vermeidung unnötiger gesundheitlicher Risiken für den $\mathrm{Pa}$ tienten die Infrastruktur eines Krankenhauses oder eine entsprechende intensivmedizinische Struktur vorgehalten werden muß. Für die in einen 
solchen Katalog aufgenommenen Leistungen wäre in der Regel der Bedarf für die Ermächtigung entsprechend qualifizierter Krankenhausärzte zu vermuten (sogenannte „Regelermächtigung“). Zu einem derartigen Katalog gehören nach Auffassung der am Konsenspapier beteiligten Organisationen Leistungen der

- interventionellen Kardiologie,

- interventionellen Gastroenterologie,

- interventionellen Radiologie,

- Versorgung spezieller onkologischer Patienten,

- Versorgung spezieller Formen der AIDS-Erkrankung.

Es bleibt zu hoffen, daß der von Bundesärztekammer, Kassenärztlicher Bundesvereinigung und Marburger Bund beschrittene Weg einer einvernehmlichen Lösung der „Verzahnungsproblematik“ von der Regierungskoalition im Rahmen der Gesetzesberatungen für eine Strukturreform 2000 aufgegriffen wird. Vor dem Hintergrund eines nicht nur für die niedergelassenen Fachärzte, sondern auch für die Krankenhäuser und die Gesundheitspolitik unkalkulierbaren Ausgangs einer konfrontativen Öffnung der Krankenhäuser für die ambulante Versorgung bleibt allerdings kaum eine andere Wahl als die Übernahme einer medizinisch sinnvollen und innerärztlich konsensfähigen Kompromißlösung. 


\section{Öffnung von Krankenhäusern für die fachärztliche Versor- gung}

\section{Realistische Anforderungen an eine Weiterentwicklung der Versor- gungsstrukturen}

Peter Schwoerer, Johann Freund, Elisabeth Simoes, Dr. Friedrich Gerhard

\section{Einleitung}

Die vorbehaltlose Öffnung der Krankenhäuser für die ambulante Betreuung von Patienten wird als die Rationalisierungsreserve im Gesundheitswesen diskutiert.

Der Gedanke, die ambulante fachärztliche Versorgung mit Hilfe der in den Krankenhäusern vorgehaltenen fachärztlichen Kompetenz und technischen Ausstattung quasi im Nebenschluß preiswert zu gestalten, scheint vielen Politikern als ein Ausweg aus der Kostenkrise des Gesundheitswesens.

Für die Deutsche Krankenhausgesellschaft stellte Frau Renzewitz die bekannten, in der jahrelangen Diskussion stereotyp wiederholten Argumente zusammen. Sie plädierte unter den Stichworten "Gemeinsame Nutzung der Technik, gemeinsame Inanspruchnahme der Krankenhausinfrastruktur, breite Therapieangebote, hoher Qualitätsstandard“ und unter Hinweis auf die „beispielhafte Notfallversorgung“ für eine Öffnung der Krankenhäuser zur ambulanten Versorgung.

Herr Krimmel, stellvertretender Geschäftsführer der KBV, hielt mit sattsam bekannten Argumenten dagegen. Er weist auf die negativen Aspekte eines unnötigen Krankenhausaufenthaltes für die Patienten genauso hin wie auf die überhöhten Kosten für die Lösungen von Gesundheitsproblemen durch Krankenhäuser.

Einig waren sich die Kontrahenten in der Feststellung, daß zur Zeit noch relativ viele Patienten in den Betten der Krankenhäuser liegen, die eigentlich ambulant behandelt werden könnten, wenn denn endlich geklärt wäre, welche Ärzte sie zu welchen Bedingungen behandeln dürfen. 


\section{Fakten}

Der MDK Baden-Württemberg führt seit einigen Jahren in Umsetzung des $\S 17$ a KHG umfassende, mittlerweile hochstandardisierte Fehlbelegungsprüfungen durch. Diese Prüfungen zeigen das Mißbrauchspotential einzelner Krankenhausabteilungen auf. Sie bieten jedoch nicht die Grundlage für systematische Versorgungsstrukturanalysen, die ihrerseits wieder Basis einer Weiterentwicklung in Richtung Integration der ambulant-stationären Versorgung sein können. Folgerichtig entwickelten die Referenten des MDK Baden-Württemberg auf der Basis des § 301 SGB $V$ und unter Einschluß der L1 - L5 Statistiken der Bundespflegesatzverordnung eine standardisierte DV-gestützte Auswertung zur Leistungsanalyse und zum Leistungsvergleich einzelner Krankenhausabteilungen (BIOS: MDK Baden-Württemberg ICPM orientierte Strukturanalyse/siehe MDK-Forum 02.06.98 Dr. Freund/ Dr. Simoes). Diese BIOSStrukturanalyse untersucht Krankenhausabteilungen unter mehreren Fragestellungen. Beispielsweise werden die Fragen beantwortet:

- Welcher Prozentsatz der stationär aufgenommenen Patienten könnte ambulant behandelt werden?

- Welcher Prozentsatz der stationär aufgenommenen Patienten könnte tagesklinisch 24 behandelt werden?

- Welcher Prozentsatz der vollstationär behandelten Patienten könnte praxisklinisch 25 behandelt werden?

- Welcher Prozentsatz der in einer Krankenhausabteilung erbrachten Operationen würde unter medizinischen, ökonomischen und qualitativen Aspekten sinnvoller in einer Schwerpunktabteilung versorgt werden?

- Welche Operationsarten werden in den Krankenhausabteilungen vorgehalten und welche werden dann auch tatsächlich in einer notwendigen Mindestfrequenz erbracht?

- Welche Altersstruktur weisen die Patienten auf?

- Liegen ältere Patienten unter konservativer Behandlung auf stationär operativen Abteilungen, ohne operiert zu werden?

Die BIOS-Analysen, verbunden mit den Fehlbelegungsprüfungen, führen zu einigermaßen erstaunlichen Ergebnissen, zum Beispiel:

24 1-Tagesaufenthalt

25 max. 3-Tagesaufenthalt 
- Vermeintlich wirtschaftlich arbeitende Krankenhäuser werden durch die Analyse als unwirtschaftlich entlarut, da sie zum einen potentiell ambulant $\mathrm{zu}$ versorgende Patienten im Krankenhaus festhalten und zum anderen Leistungsspektren vorhalten, die mit dem Auftrag ihrer Versorgungsstufe z. B. als Krankenhaus der Grund- oder Regelversorgung nichts zu tun haben.

- Andere operativ tätige Krankenhausabteilungen behandeln ihre über 65jährigen Patienten in hoher Prozentzahl ausschließlich konservativ, ohne daß eine plausible medizinische Begründung erkennbar wäre.

- Wiederum andere Krankenhäuser zeichnen sich durch hohe Fehlbelegungsraten aus - es ergeben sich Indizien dafür, daß sich die Fehlbelegung interessanterweise immer dann häuft, wenn die ambulant-stationäre Verzahnung nach derzeitigen Vertrags- und Gesetzesgrundlagen besonders gut funktioniert. Der Verdacht der nichtindizierten stationären Aufnahme aus einer "gut laufenden Ambulanz" muß mindestens diskutiert werden.

\section{Was muß verändert werden?}

Die von interessierter Seite propagierte schlichte Öffnung der Krankenhäuser für die ambulante Versorgung wird voraussichtlich einen Verdrängungswettbewerb mit staatlicher Investitionszulage ergeben, der in jedem Fall zu Lasten der Vertragsärzte ginge. Das würde nicht nur den finanziellen Ruin von vielen hochqualifizierten Spezialisten bedeuten, die unter Vorspiegelung eines langfristigen politischen Willens in den Jahren 1993 - 1995 aus ihren Stellungen als Oberärzte der Kliniken heraus in die Niederlassung gelockt wurden, sondern - weit bedeutender für die Patienten - würde diese Öffnung zu einem Ende des Innovationsdrucks in der Medizin führen. Zeigt sich doch, daß technische Verfahren in den letzten Jahrzehnten zwar oft in Universitätskliniken entwickelt wurden, aber ihre Einführung in die Regelversorgung der Patienten über die Praxen und durch Investitionen der niedergelassenen Ärzte stattfand.

Das reine Monopol der Kassenärztlichen Vereinigungen für die ambulante fachärztliche Versorgung würde schließlich in dem Moment, in dem alle tatsächlich ambulant zu lösenden Patientenprobleme aus den Krankenhäusern herausgeholt würden, zu einem Ausbluten der Krankenhäuser und damit zum Ende vieler strukturell notwendiger Krankenhäuser der Grund- und Regelversorgung führen (Risikoentmischung, ärztliche und pflegerische Weiter- und Ausbildung). Die für die Patienten flächen- 
deckende stationäre Versorgung wäre dann nur noch durch noch höhere Subventionen aufrecht zu erhalten - man unterläge dann tatsächlich weiter dem Zwang zu Doppelinvestitionen in ein parallel hochqualifiziertes Versorgungssystem.

\section{Sektorenübergreifende Konzeption}

Eine neue, durchgängige und logische, medizinische Versorgungsstruktur muß daher von einer Basisbetreuung durch ein vernetztes Hausarztsystem ausgehen. Hausärzte werden die fachärztliche Betreuung der Patienten genauso koordinieren, wie sie die Inanspruchnahme von Pflegeleistungen und sozialen Einrichtungen in Zusammenarbeit mit den Patienten regeln müssen.

In der nächsten Ebene müssen Hausärzte mit fachärztlichen Schwerpunktpraxen zusammenarbeiten. Diese Praxen können selbstverständlich auch an Krankenhäusern sein. Die Möglichkeit der Kooperation zwischen Krankenhausärzten und Vertragsärzten mit dem Ziel, technische Einrichtungen gemeinsam zu nutzen, muß weiter eröffnet werden. Ebenso ist für die ökonomische Kooperation zwischen Hausärzten, Fachärzten und Krankenhäusern eine belastbare sozialrechtliche Grundlage erforderlich. Ein Teil der fachärztlichen Betreuung im Krankenhaus und ambulanter Medizin wird durch ein kooperatives Belegarztsystem ermöglicht.

Die Krankenhausbehandlung kann sich dann tatsächlich auf die indizierte stationäre Behandlung schwerkranker Patienten konzentrieren, wobei das Krankenhaus bei entsprechenden Vertragsbedingungen selbstverständlich auch in den Wettbewerb um ambulante fachärztliche Betreuung eintreten kann.

Dieses System kann nur arbeiten, wenn der Gesetzgeber die nötigen Voraussetzungen schafft:

- Monistische Krankenhausfinanzierung (damit keine Preisvorteile für die Krankenhäuser im Bereich des ambulant-stationären Übergangs).

- Übergang von einer krankenhausbezogenen landesweiten Planung zu einer reinen Rahmenplanung der Länder für die Krankenhausversorgung (Kapazitäten, Versorgungsaufträge, Erreichbarkeit von Leistungen).

- Definierte Regularien für die Festlegung der Preise einzelner ambulanter und stationärer Leistungen in einem morbiditätsbezogenen Fallpauschalen-System. 
- Vertragliche Etablierung von einheitlichen Instrumenten des Qualitätsmanagements zur Verhinderung eines dem wettbewerblichen System potentiell immanenten Qualitätsdefizits („Leistungsausdünnung“ bei pauschalierten Vergütungen, numerische "Leistungsausweitung" infolge Indikationsausdehnung durch Leistungsanbieter mit Steuerungsfunktion).

- Konzeption einer verfaßten Institution mit den Aufgaben „Wettbewerbskontrolle und Qualitätsförderung" sowie "Leistungsmonitoring" in der offenbar mittelfristig erhaltenen Wettbewerbssituation zwischen Kostenträgern (GKV-Kassenarten/PKV) und Leistungsanbietern.

Im folgenden sollen einige Bedingungen für dieses Konzept aufgestellt werden.

- Ein verbindlicher Katalog derjenigen ambulanten Leistungen, die unter den Aspekten des Patientenschutzes krankenhausnah erbracht werden müssen (z. B. interventionelle Endoskopie, interventionelle Kardiologie). Dieser Katalog darf nicht nur die zu erbringenden Prozeduren enthalten, er muß auch die Indikationsstellung und die Qualitätssicherung der Indikationsstellung sowie die Qualitätssicherung der Prozesse und Behandlungsergebnisse klar erfassen.

- Ein weiterer Katalog aller übrigen ambulant zu erbringenden Leistungen, die sowohl von der fachärztlichen Praxis als auch im Krankenhaus erbracht werden können. Der Katalog muß einheitlich und preisgleich sein.

Anmerkung: Zwischen den für die Leistungsmeldungen gemäß $\S 301$ SGB $V$ durch die Leistungsanbieter erhobenen und gemeldeten ICDs und ICPMs, also zwischen Diagnosen- und Prozedurenschlüssel, muß ein klarer Zusammenhang hergestellt werden können. Andernfalls ist ein sinnvolles und sektorenübergreifendes Monitoring für eine sachgerechte Gesundheitsberichterstattung nicht möglich. ICPMs müssen differenziert, soweit möglich auch für die konservativen Fächer, weiterentwickelt werden.

Die ökonomische Steuerung innerhalb der Versorgungsstruktur kann unterschiedlich erfolgen; entweder durch ein Einkaufsmodell der Krankenkassen oder aber durch hausärztliche Versorgungsnetze mit Koordinations- und Steuerungsfunktion. 
Fazit: (Fast) alle Beteiligten haben die Wahl

Für alle am Gesundheitssystem Beteiligten sind folgenschwere Überlegungen anzustellen:

Die Krankenhäuser bisheriger Struktur werden sich überlegen, ob sie sich für kooperative Belegarztsysteme öffnen und damit als gleichberechtigte Vertragspartner dieser Ärzte und der damit entstehenden Netze agieren und nicht mehr, wie bisher, in vermeintlich festen Versorgungsaufträgen und in überholten hierarchischen Strukturen verharren.

Die niedergelassenen hochspezialisierten Fachärte haben die Wahl, in ihren technisch überrüsteten Einzelpraxen finanziell auszubluten oder sich in fachübergreifenden Gemeinschaften an Krankenhäusern oder an Krankenhäuser lokal angebundenen Ärztehäusern zu assoziieren.

Die Hausärzte haben die Wahl zwischen der Existenz als isolierte Barfußärzte in Einzelpraxen und der Kooperation in Netzstrukturen, in denen ihre allgemeinärztliche oder internistische Kompetenz genauso gefordert sein wird wie inr sozialmedizinisches Engagement.

Die Patienten haben die Wahl zwischen einem die Behandlung koordinierenden durchstrukturierten Hausarzt/Facharzt-System mit klarer Krankenhaus- und Pflegestruktur, oder sie haben die Möglichkeit, nach Gutdünken und eigenem Besserwissen sich ihre persönliche medizinische Versorgung zu wählen.

Die Politik und die Krankenkassen haben keine Wahl. Soll dem drohenden Druck hin zu einer Rationierung medizinischer Leistungen und/oder einer „Zweiklassenmedizin“ Einhalt geboten werden, so obliegt innen, die Systeme unter den o. g. Kautelen zu öffnen. Dies bedeutet, daß sie sowohl der eingangs zitierten Deutschen Krankenhausgesellschaft eine Öffnung der Krankenhäuser für kooperative Belegarztsysteme diktieren müssen als auch den Kassenärztlichen Vereinigungen einen Teil ihres Monopols zur Vertragsgestaltung abringen müssen. Die Hoffnung, daß neue Strukturen durch die Ärzteschaft selbst definiert und etabliert werden, sind angesichts der heillosen Zerstrittenheit der Ärzteschaft - aber auch wegen der zunehmenden Mitbestimmungsansprüche anderer Professionen und Anbieter im Gesundheitswesen - offenkundig irreal. 


\section{Pro und Contra von Hausarztmodellen}

\section{Peter Sauermann}

Es wird Sie sicherlich nicht überraschen, daß ich pro Hausarztmodelle bin.

In der öffentlichen Diskussion werden der zunehmende Kostendruck und die bevorstehenden massiven Einschnitte in der ärztlichen Versorgung durch das neue Gesetz zur Stärkung der Solidarität in der gesetzlichen Krankenversicherung (SolG) mehr und mehr in den Vordergrund gerückt.

So mancher Kollege fragt sich in diesen Zeiten, wie es noch weitergehen soll. Die Erfordernisse nach weiteren Rationalisierungen und Rationierungen werden auch in der Presse eingehend verfolgt. Die Aussichten eines sich neu niederlassenden Arztes werden von Jahr zu Jahr schlechter.

Seit Jahren werden Schlagworte wie „Managed Care" und „Disease Management" heiß diskutiert, freilich ohne ein erfolgversprechendes und vor allen Dingen erfolgreich umsetzbares Konzept zu beinhalten.

Als ich mich vor 25 Jahren niederließ, stand mir noch ein gut funktionierendes Netz zur Verfügung. Ich war damals sozusagen „Primärarzt“ in meinem 1.000 Einwohner zählenden Dorf. Meine Facharztkollegen, Gynäkologen, Orthopäden, Neurochirurgen, was immer Sie wollen, arbeiteten damals schon Hand in Hand mit mir zusammen. Selbst mit dem Krankenhaus kam eine gute sektorübergreifende Zusammenarbeit zustande. Das alles hat es damals schon gegeben, und es klappte hervorragend. Die Aufgeregtheiten über die Begriffe „Primärarzt“ oder „primärer Anlaufarzt" sind eigentlich hausgemacht. Und warum? Weil die Strukturen heute nicht mehr stimmen, weil im Verhältnis am Bedarf vorbei Ärzte ausgebildet werden und sich niederlassen. Heute streiten wir mit den Krankenkassen um minimale Gesamtvergütungssteigerungen, wie auch immer, das gesamte System ist an die Wand gefahren und wir sehen auch aus der Ärzteschaft eigentlich keine Struktur, keine Strategie, die konsequent in eine vernünftige Lösung münden könnte.

Auch die Situation der Hausärzte ist von dieser Entwicklung nicht verschont geblieben. 
Viele neue Wege werden derzeit beschritten, um in den verschiedenen Nischen der ärztlichen Versorgung ein finanzielles Auskommen zu erzielen.

Bereits seit 10 Jahren verpflichtet $§ 73$ SGB V die Hausärzte zur Koordination therapeutischer und pflegerischer Maßnahmen zur Zusammenführung der Behandlungsdaten aus der ambulanten und stationären Versorgung und zur Erläuterung oder Durchführung präventiver und rehabilitativer Maßnahmen sowie zur Integration nichtärztlicher Hilfen und pflegerischer Dienste.

Gerade diese Koordinierungsaufgaben prädestinierten den Hausarzt, die strukturierte ambulante ärztliche Versorgung primär zu übernehmen. Die bisherigen gescheiterten Versuche, ambulante Medizin in Netzen zu verwirklichen, ohne auf $\S 73$ und 73 a SGB $V$ einzugehen, konnten daher nur in die Sackgasse führen. Hunderte von sogenannten Praxisnetzen sind in den vergangenen Jahren wie Pilze aus dem Boden geschossen, ohne eine auch nur annähernd erfolgreiche Bilanz vorstellen zu können. Diese regionalen, durch Marketingspezialisten und Pharmafirmen entstandenen Vernetzungen zeigen zudem eine unzureichende Patientenorientierung auf, ohne eine optimale Struktur- und Qualitätsverbesserung garantieren zu können. Die Profitziele stehen in diesen industriell geförderten Vernetzungen zu sehr im Vordergrund. Auch zuletzt deshalb sind diese Modelle zum Scheitern verurteilt gewesen, weil sich diese Art von Netzen zumeist auf eine Indikation alleine gestützt hat. So z. B. auf den Bluthochdruck, auf die KHK oder die Hyperlipidämie und das auch noch mit einer einzelnen Krankenkasse für unterschiedliche Arztgruppen innerhalb einer KV!

Das durch Strukturverträge nach $\S 73$ a SGB V auf KV-Ebene erzeugte Hausarztmodell soll dieses Chaos in der ambulanten Medizin wieder in geordnete Bahnen lenken. Der Patient wird in ein gut organisiertes Geflecht miteinander kommunizierender Ärzte integriert und erhält so eine optimale Rundumversorgung. Netze können in dieser Weise auch als Qualitätszirkel, Labor- und Gerätegemeinschaften gesehen werden. Darüber hinaus bilden diese Netze in ihrer Endstufe auch Einkaufs- und Apparategemeinschaften, die im Fall der Fälle auch Notdienstringe darstellen können. Eine verbesserte Allokation der knapper werdenden Ressourcen kann somit gewährleistet werden.

Untersuchungen, die durch andere Fachgruppen erbracht werden müssen, kauft der Hausarzt sozusagen bei seinem Facharztkollegen einfach ein. Die Leistung bleibt auf das unbedingt Notwendige beschränkt. So werden in diesem Hausarztnetz Doppel- und Mehrfachuntersuchungen 
und nicht notwendige Krankenhauseinweisungen und Selbsteinweisungen vermieden. Ein permanenter Rückkopplungseffekt zwischen veranlassendem Arzt und Leistungserbringer wird implementiert. Die Effektivität wird so gewährleistet.

Die beteiligten Netzärzte könnten auf diese Weise mit einem gerechteren Honorar rechnen, denn schließlich kontrollieren sich die Ärzte gegenseitig. Eine übermäßige Fall- und Leistungsausweitung des einzelnen Arztes im System kann unmittelbar durch den veranlassenden Arzt sanktioniert werden. Zudem wird der gefürchtete Punktwertverfall verhindert. Als positive Nebenwirkung wird zusätzlich der Hamsterrad-Effekt abgebremst. Dem Patienten werden auf diese Weise keine unnötigen Untersuchungen zugemutet, so daß auch die Lebensqualität der Patienten weiter erhöht wird.

Sollten jedoch mehrere Systeme der ärztlichen Versorgung (Hausarztnetze mit Strukturverträgen nach $\S 73$ a SGB V, System der freien Arztwahl, Netze von Haus- und Fachärzten ohne Strukturverträge) miteinander konkurrieren, müssen diese im Vorfeld sauber voneinander getrennt werden. Der Patient muß dann jedoch auch bereit sein, verbindlich zu erklären, daß er zwischenzeitlich nicht aus dem Hausarztnetz in das jeweils andere System wechselt. Ansonsten wird der Sinn eines Hausarztnetzes konterkariert und weiteren Leistungssteigerungen, Fallzahlsteigerungen und damit floatenden Punktwerten Tür und Tor geöffnet. Auch der Patient muß zukünftig mehr Verantwortung tragen. Ohne seine Kooperation wird es nicht möglich sein, ein solches Modell umzusetzen.

Wichtig ist, und was auch dringend erforderlich ist, daß diese Netze, wenn sie funktionieren sollen, und die Verträge, die dann abgeschlossen werden müssen, finanziell begrenzt sind und in ihrem Risiko auch kalkulierbar bleiben müssen. Das ist etwas, was bis jetzt noch nie in den Verträgen, unter denen wir arbeiten, erreicht worden ist. Deshalb sind wir auch permanent in der Vertragsfalle. 


\section{Pro und Contra von Hausarztmodellen}

Wolfgang Brech

Nachdem die Referenten der zurückliegenden Beiträge dieses Symposiums von ihrem hochkarätigen juristischen und ökonomischen Niveau immer wieder in die Tiefen der Zoologie herabgestoßen sind-es war die Rede von Ziegen, von Schafen und gar Melkkühen -, scheint es der Veranstalter darauf abgesehen zu haben, jetzt auch noch Streithähne auf die Bühne dieses Forums zu bringen. „Pro und Contra von Hausarztmodellen": In der Tat könnte dieses Thema das zoologische Instrumentarium beträchtlich erweitern. Ich muß Sie aber enttäuschen, die Diskussion über dieses Thema scheint den Streit weniger bei den Hähnen als bei noch nicht ausgereiften Küken belassen zu haben. Sie werden diese „Entwarnung“ begreifen, wenn ich, ähnlich wie es Herr Sauermann schon getan hat, mein Referat biographisch einleite: Seit 25 Jahren niedergelassen als spezialisierter Internist betreibe ich eigentlich nichts anderes als ein Primärarztsystem in meiner Praxis, indem ich ausschließlich überwiesene Patienten akzeptiere. Das soll aber nicht heißen, daß ich nicht zum Pro und Contra eines solchen Systems einiges zu sagen hätte.

„Beziehungsfähigkeit und menschliche Begegnung kennzeichnen eine Leistung der ambulant tätigen Ärzte. Kreatives, fachlich kompetentes und qualitativ sicheres Fallmanagement für den Patienten beschreibt die zweite Leistungsebene. Individuelle Begegnung und individuelles Fallmanagement gehören zusammen. Ein verläßlicher Maßstab für die Beziehung ist die freie Arztwahl durch den Patienten."

Es wird manchen erstaunen, daß ich ein Zitat von Ellis Huber, dem Präsidenten der Ärztekammer Berlin, an den Anfang meiner Gedanken über das Primärarztsystem stelle. Es gibt aber genau das Generalthema an, unter das ich diese Diskussion stellen möchte. Ausgesprochen von einem eher freidenkerischen, zumindest unkonventionellen Geist: die freie Arztwahl. Diese stellt einen der wesentlichsten Grundzüge unseres Gesundheitssystems dar und wohl auch einen wichtigen Faktor für seinen Erfolg und für seine hohe Akzeptanz bei unserer Bevölkerung. Die freie Arztwahl ist auch ein Garant für die hohe Qualität unserer medizinischen Versorgung.

Die Freiheit des Bürgers, sich den Arzt seines Vertrauens für seine individuellen Gesundheitsbedürfnisse selbst zu wählen, sei es als Allgemeinarzt oder als Facharzt, setzt Mündigkeit voraus, welche dem Bürger 
heute auch in anderen öffentlichen Bereichen zugemutet wird, und entspricht der Forderung nach Eigenverantwortlichkeit und Selbstbeteiligung an den Kosten des Gesundheitswesens ebenso wie dem demokratischen Selbstverständnis unserer Gesellschaft. Leider müssen wir aber beobachten, daß gegenwärtig dieses eigenverantwortliche Selbstverständnis des Bürgers einer politischen Nivellierung anheimfällt.

Die freie Arztwahl ist zugegebenermaßen ein Faktor in unserem Gesundheitssystem, der sich durchaus als kostensteigernd erweisen kann - ohne daß dies aber so sein muß -, es muß aber entgegengehalten werden, ob dies nicht durch die so erreichte höhere Qualität einer gesundheitlichen Versorgung und durch ihren freiheitlichen Charakter aufgehoben wird. Die strukturell zwanghafte Vorschaltung eines Primärarztes, bei dem ein Versicherter eingeschrieben ist und der immer mehr auf eine Verteilungskompetenz herabgestuft wird, wobei Verteilung unter Umständen auch noch mit einer Finanzierungsverantwortlichkeit von veranlaßten Leistungen verknüpft wird, eine solche Primärarztstruktur muß sich durchaus auch der Frage der Qualität einer solchen Versorgung stellen. Ärztlicherseits wird ein so verstandenes System von keiner Seite, auch nicht von den hausärztlichen Berufsverbänden gewünscht. Darüber hinaus ist es bis heute keineswegs erwiesen, daß ein solches primärärztliches System, das die Wahlfreiheit der Versicherten einschränkt, tatsächlich Kosten im Gesundheitswesen einsparen kann.

Ein Primärarztsystem widerspricht den Optionen einer freiheitlich und individuell handelnden Gesellschaft. So stieß ein entsprechender Modellversuch in Frankreich auf wenig Resonanz. Nur 6.000 Patienten haben sich freiwillig einen Primärarzt gesucht, nur $11 \%$ der niedergelassenen Ärzte (in Paris waren es nur $5 \%$ ) haben sich an einem solchen Versuch beteiligt. Bei einer kürzlich durchgeführten Delphi-Umfrage sprachen sich $90 \%$ der Befragten eindeutig für das Recht aus, nach eigener Präferenz ihren Arzt wählen und in Konfliktfällen auch wechseln zu können, $43 \%$ der Befragten sagten, sie hätten auch schon einmal den Arzt gewechselt und nur zwei Drittel der Befragten meinten, daß sie grundsätzlich zuerst den Hausarzt aufsuchen würden. Prinzipiell möchte sich der Versicherte in Deutschland also die Tür zur freien Arztwahl offen halten. Wie sensibel er allerdings mit diesem Problem umgeht - wesentlich sensibler als mancher Berufspolitiker -, zeigt eine andere Umfrage, die darstellte, daß bis zu $90 \%$ der befragten Patienten tatsächlich einen Hausarzt besitzen. Hier wird also auf freiwilliger Basis, gewissermaßen als vernünftige Grundstruktur, bereits $\S 76$ Abs. 3 Nr. 2 SGB V verwirklicht, der besagt: „... der Versicherte wählt einen Hausarzt“. Diese Aussage des Gesetzgebers kann nicht als gesetzliche Grundlage oder gesetzliche Forderung für die Einführung eines Primärarztsystems ange- 
sehen werden. Desgleichen ist auch der viel zitierte $\S 73$ Abs. 1 nicht in diesem Sinne zu interpretieren, der besagt, daß sich die vertragsärztliche Versorgung in eine hausärztliche und eine fachärztliche Versorgung gliedere, und der die Aufgaben des Hausarztes definiert. Hausarzt im Sinne des $\S 73$ Abs. 1 Nr. 1 - 4 SGB V kann durchaus auch der Gynäkologe, der Urologe, der Orthopäde oder der internistische Organspezialist sein.

Ein Primärarztsystem, wie es z. B. in England oder in Holland praktiziert wird, führt zwangsläufig zu einem Qualitätsverlust in der allgemeinärztlichen bzw. hausärztlichen Praxis, welche dann notwendigerweise von ihrer Technikorientierung - dies ist ebenfalls Ausdruck eines freiheitlichen Gesundheitssystems - zur reinen Betreuungsorientierung skelettiert wird. Der „Gatekeeper" erhält Verteilungskompetenz und verliert Handlungskompetenz, weswegen inm konsequenterweise im AOKHausarztmodell ein Gesundheitsberater zur Seite gestellt wird. Ich komme darauf noch zu sprechen.

Ein in der GKV fest verankertes Primärarztsystem generiert außerdem eine Zweiklassenmedizin, da dann die freie Arztwahl nur noch dem privat versicherten Patienten oder dem, der eine höhere Prämie zahlt, gewährleistet ist. Aus Gesprächen mit verschiedenen Mitgliedern unserer Gesellschaft geht hervor, daß diese ein primärarztzentriertes Gesundheitssystem ablehnen, auch unter der Prämisse einer reduzierten Prämienzahlung für die Krankenversicherung.

Der immer wieder durchgeführte Vergleich unseres nicht in einem Primärarztsystem verankerten Gesundheitswesens mit demjenigen anderer Länder, wie Holland, Dänemark oder England, ist unzulässig. Es handelt sich dabei einerseits um größtenteils steuerfinanzierte und nicht prämien und solidarisch finanzierte Systeme, andererseits ist nur in Deutschland die neben der hausärztlichen Versorgung fundamentale zweite Säule unseres ambulanten Gesundheitswesens, nämlich die fachärztliche Versorgung, zu hohem Standard entwickelt. Die Zweigleisigkeit unseres fachärztlichen Systems, nämlich im Krankenhaus und in der freien Praxis niedergelassener Ärzte, wird vielfach als kostensteigernder Faktor angeprangert. Dies mag für hochinvestive Bereiche zutreffen, solange diese Strukturen nicht ausgelastet sind, wie in der neuen Radiologie, der interventionellen Kardiologie oder der Dialyse. Hier haben sich jedoch bundesweit bereits bereichsübergreifende Kooperationen mit gemeinsamer Nutzung vorhandener Investitionen angebahnt. Abgesehen davon ist es also gerade unsere hoch entwickelte fachärztliche Struktur, welche das Prinzip „ambulant vor stationär“ überhaupt erst ermöglicht. Nur durch in die Fläche gestreute fachärztliche Infrastruktur ist es möglich 
gewesen, in den letzten Jahren zahlreiche Patientenprobleme eben nicht mehr auf der teuren stationären Ebene, sondern ambulant zu lösen.

Den Zugang zu diesem System für den Versicherten von einer primärärztlichen Entscheidung abhängig zu machen oder durch ein Bonus- respektive Malus-System zu sanktionieren, würde einen Rückschritt für Niveau und Versorgungstiefe unserer ambulanten Versorgung bedeuten. Wir wollen den kompetenten Hausarzt, der nicht auf eine Minimaltätigkeit oder Verteilerfunktion begrenzt ist und entsprechend seiner Qualifikation auch zu gewissen technischen, diagnostischen oder therapeutischen Bereichen Zugang hat und nicht in den Verdacht oder Vorwurf gerät, als alleiniger Gatekeeper den Übergang zur höheren Versorgungsebene zu blockieren oder zu versäumen. Und wir wollen die freie Entscheidung des Patienten gewahrt wissen, auch zu dieser höheren Ebene freien Zugang zu besitzen. Eine Entscheidung, die heute um so mehr getroffen wird, je höher der Informationsgrad des Patienten zu werten ist.

Dies verpflichtet uns allerdings auch, das Chipkartenproblem zu lösen, das Patientenwanderungen von Arzt zu Arzt in unkoordinierter Weise ermöglicht. Dabei halte ich allerdings die von der KBV vorgeschlagenen Lösungen nicht für sinnvoll, daß nämlich die Chipkarte so programmiert wird, daß im Quartal jeweils nur ein Hausarzt und ein Facharzt konsultiert werden kann.

Je höher andererseits der Spezialisierungsgrad einer Praxis ist, desto mehr sollte der Zugang zu dieser Praxis durch ein Überweisungsverfahren geregelt werden und um so notwendiger ist die Kommunikation mit einem Arzt der Grundversorgung. Das Instrument des Überweisungsscheins ist ein unverzichtbares Element ärztlicher Kooperation. Seine Nutzung ist durch Honorierungsstrukturen zu subventionieren, ohne die freie Arztwahl des Patienten zu tangieren. Dies bedeutet, daß das Problem Primärarzt, Hausarzt oder Gatekeeper strukturell in unserer ärztlichen Selbstverwaltung und nicht staatlich oder gesetzlich gelöst werden muß. Das Problem darf also nicht von oben herab oktroyiert werden, sondern bedarf der innerärztlichen kooperativen Lösung durch Handlungsleitlinien, honorarpolitische Strukturen und freiwillige, jedoch nicht exklusive Vernetzungsmodelle.

Bei solchen strukturpolitischen Überlegungen stimmen allerdings neuere Untersuchungsergebnisse des Zentralinstituts bedenklich, welche gezeigt haben, daß im fachärztlichen Bereich die Überweisungsfälle im vergangenen Jahr wesentlich zurückgegangen sind und demgegenüber die Originalfälle zunehmen. Hier besteht dringend struktureller Hand- 
lungsbedarf. Die Kommunikation zwischen den Ärzten ist besser zu honorieren. Im einheitlichen Bewertungsmaßstab müssen Elemente fixiert werden, welche die Überweisungsfrequenz bei Fachärzten berücksichtigen. Dies kann auch im HVM geschehen, so daß überwiesene Krankheitsfälle einer besseren Honorierung zugeführt werden als Patienten, die "original", also direkt in die Praxis eines Facharztes gekommen sind. Ärzte, Hausärzte und Fachärzte müssen ein Interesse daran haben, das honorarpolitisch zu untermauern ist, miteinander kooperativ Krankheitsprobleme zu lösen und nicht additiv. Eine ganze Reihe solcher Elemente haben wir im vergangenen Jahr bereits in unserer Versorgungsstruktur instrumentiert. Dabei muß aber auch der direkte Zugang zum Facharzt erhalten bleiben, insbesondere um der Gefahr - wenn diese auch nur potentiell vorhanden ist - der Verzögerung oder der Verhinderung von notwendiger Diagnostik oder Therapie entgegenzuwirken. Zugleich muß die Degradierung des Hausarztes zum Verteiler verhindert werden, ebenso wie eine Abhängigkeit des Facharztes von der hausärztlichen Zuweisung. Das Primärarztsystem schafft eine neue Schnittstelle, nämlich die zwischen hausärztlicher und fachärztlicher Versorgung. Hier entstehen neue Reibungsverluste, die nicht geeignet sind, die Qualität der medizinischen Versorgung $\mathrm{zu}$ verbessern. Das Problem Hausarzt/Facharzt darf nicht im Rahmen eines Verteilungskonflikts gelöst werden. Vielmehr muß die Pluralität unseres Versorgungsangebotes aufrechterhalten werden, jedoch die Rationalität ebenso wie auch die Rentabilität einer Praxis in Richtung Vernetzung und Kooperation gelenkt werden.

Bereits 1992 beschäftigte sich der Sachverständigenrat für die Konzertierte Aktion im Gesundheitswesen in seinem Jahresgutachten mit dem Primärarztsystem. Hieraus möchte ich folgende Aussagen zitieren:

- „Das Leistungsgeschehen ist im jetzigen System nicht auf das medizinisch Notwendige beschränkt, ein medizinisch nicht indizierter Mengenanstieg durch falsche ökonomische Anreize führt zur Überversorgung."

Überversorgung ist dabei im Sinne von fachärztlicher Überversorgung zu verstehen. Der Sachverständigenrat konnte damals, 1992, nicht wissen, daß inzwischen die ärztliche Selbstverwaltung aus eigenem Antrieb in der Lage war, Instrumente zur Eindämmung der Leistungsdynamik im ambulanten Versorgungssystem zu finden, nämlich die Praxisbudgets, welche jetzt durch entsprechende gesetzlich ermöglichte mengenbegrenzende Systeme, nämlich die Regelleistungsvolumina, abgelöst werden sollen. Dabei ging es den ärztlichen Gremien nicht darum, gemäß dem Vorwurf des Sachverständigenrates, das nicht medizinisch Not- 
wendige zu begrenzen, sondern die Leistungen der Ärzte innerhalb der GKV auf das Maß der verfügbaren Ressourcen zurückzuführen.

- „Technische Leistungen sind überbewertet, eine undifferenzierte Inanspruchnahme des gebietsärztlichen Angebots durch die Versicherten beinhaltet die Gefahr, daß zu viele technische Leistungen erbracht werden."

Dem ist prinzipiell zuzustimmen, jedoch beinhaltet die „Überbewertung technischer Leistungen" eine bisher unbewiesene Behauptung, die immer wieder wiederholt wird, ohne dadurch an Wahrheitsgehalt zu gewinnen und deren Erörterung ein abendfüllendes Thema darstellen würde.

- „Die umfassende Betreuung durch den Primärarzt führt dazu, daß alle relevanten Informationen über einen Patienten (z. B. familienanamnestische Kenntnisse) Berücksichtigung finden. Die von den Spezialisten erhobenen Befunde werden vom Primärarzt zu einem Gesamtbild integriert. Er übernimmt dabei die Koordination diagnostischer und therapeutischer Maßnahmen, die Bewertung und Aufbewahrung der wesentlichen Behandlungsdaten, Befunde und Berichte aus dem ambulanten und stationären Bereich, die Indikation nichtärztlicher Hilfen und ambulanter Dienste (insbesondere der häuslichen Pflege) in die Behandlungsmaßnahmen sowie die kritische Bewertung der Lebensführung des Patienten, auch unter Berücksichtigung der Selbstmedikation."

Hier wird im wesentlichen der Inhalt des $\S 73$ Abs. 1 SGB V beschrieben, gewissermaßen eine Selbstverständlichkeit ärztlicher Kooperation.

Somit ist die Frage des Primärarztsystems meiner Meinung nach auch prinzipiell innerärztlich und strukturell zu lösen durch folgende Elemente:

- Erhalt der freien Arztwahl,

- Steuerung durch finanzielle Anreize und

- Abbau innerärztlicher Konkurrenz zwischen Haus- und Facharzt durch Strukturverträge nach $\S 73$ a SGB V, z. B. durch vernetzte Praxen mit arbeitsteiligem Zusammenwirken von hausärztlicher und fachärztlicher Versorgung.

Ein Einschreibesystem bei einem Hausarzt, wie etwa in Holland, England oder Dänemark, wird von der Ärzteschaft in Deutschland abgelehnt. Darüber hinaus muß betont werden, daß der Disease Manager eines 
chronisch Kranken, also der Arzt, der den Patienten von der Diagnose bis zur Prognose in den psychischen, somatischen und sozialen Elementen seiner Krankheit versorgt, keineswegs nur der Hausarzt, also etwa ein Primärarzt sein muß oder sein sollte, sondern durchaus ein Organspezialist, der ja für diesen Fall wesentlich höher qualifiziert ist. Ich denke dabei an den Diabetiker, an den Patienten mit chronischer Niereninsuffizienz, an den Asthmapatienten und viele andere mehr. Daher ist die bisher aufgestellte Gleichung Hausarzt = Case Manager oder Disease Manager sicherlich nicht richtig.

Der ausschließliche Zugang eines Patienten zu bestimmten Arztgruppen über eine Überweisung wird andererseits in unserem Gesundheitswesen in besonderen Fällen allgemein akzeptiert, so zum Radiologen, zur Labormedizin oder zum ermächtigten Krankenhausarzt. Eine Ausweitung dieses Prinzips auf hochtechnisierte, nicht betreuungsorientierte Praxen ist konsensfähig.

Der direkte Zugang zu solchen Praxen könnte auch dem Kostenerstattungsprinzip anstelle der Sachleistung unterworfen und so unter Beibehaltung des Prinzips der freien Arztwahl geregelt werden. Eine systemimmanente Abhängigkeit der Versicherten für fachärztliche Leistungen von einer hausärztlichen Entscheidung jedoch

- birgt die Gefahr von Verzögerung oder Verhinderung notwendiger Diagnostik und Therapie,

- degradiert den Hausarzt zum Patientenverteiler,

- führt von Technikorientierung zu Betreuungsorientierung,

- bedingt eine unmittelbare fachärztliche Abhängigkeit von Hausärzten und birgt damit die Gefahr von kartellähnlichen Fehlentwicklungen, wie wir sie im Laborsektor erlebt haben, und gefährdet die wirtschaftliche Basis der Fachärzte,

- erhöht die Haftungslast des Hausarztes,

- führt nicht zwangsläufig zur Verbilligung des Systems, vielmehr würde, wie es der Kammerpräsident von Westfalen-Lippe, Herr Flenker, ausdrückte, „die abgerundete, sich ergänzende und qualitativ auf einem hohen Niveau stehende ambulante Medizin in Deutschland unnötig monostrukturiert“.

Der Konflikt zwischen Primärarztsystem und freiem Zugang des Versicherten zu allen Ärzten hat sich durch die imperativen Forderungen des BDA zu einem Verteilungskonflikt der vorhandenen Ressourcen entwikkelt. Überlegungen zu strukturellen Lösungen in unserem Gesundheits- 
system müssen jedoch frei von Verteilungsaspekten unter verschiedenen Arztgruppen geführt werden. Desgleichen ist es notwendig, daß solche Diskussionen frei von ideologischer Färbung bleiben. So enthalten Programme verschiedener politischer Parteien und auch der Krankenkassen die Forderung, nicht nur die freie Arztwahl der Patienten, sondern auch die Autonomie der Ärzte selbst einzuschränken. Die im ambulanten Bereich angedachten Versorgungsstrukturen über Arztnetze, Managed-Care-Systeme oder andere Strukturverträge beinhalten keine freie Arztwahl. Der Zugang zum Versorgungssystem erfolgt vielmehr über einen Gatekeeper, in der Regel über den Hausarzt. Hierdurch werden angeblich die Rationalität der Inanspruchnahme und damit auch die Effizienz der Versorgung erhöht.

Neben den zum Primärarzt degradierten Hausarzt stellen verschiedene Krankenkassenverbände das von innen propagierte Modell eines $\mathrm{Ge}$ sundheitsberaters, d. $h$. eines entsprechend weitergebildeten Krankenpflegers oder einer Krankenschwester. Das ist so im AOK-Hausarztmodell vorgesehen, ich zitiere: „Gesundheitsberater sind ausgebildete Krankenschwestern oder -pfleger, die im Kern Fallmanagement durchführen“. Dabei ist zu fragen, wer soll nun der Ansprechpartner des Patienten sein, wer soll der Fallmanager oder auch Gatekeeper sein, der Hausarzt oder der Gesundheitsberater? Auf einem Presseseminar in Maria Laar im März 1998 wurde von seiten der AOK weiterhin ausgeführt: Die Gesundheitsberater unterstützten den Behandlungsplan des Hausarztes und förderten die Therapie des Patienten, indem sie sich regelmäßig mit inm in Verbindung setzen. Hier gibt es also neben dem Hausarzt noch eine regelmäßig beratende Funktion durch die Krankenkasse. „Sie erfragen krankheitsrelevante Parameter und können so krankheitsspezifische Veränderungen erkennen." Aufgabe des Gesundheitsberaters sei es auch, „bei chronisch Kranken Ineffizienzen zu entdecken und Lücken in der oft fehlenden Verzahnung von verschiedenen Gesundheitsinstitutionen zu schließen“. Der Gesundheitsberater wird so - und das wird an Fallbeispielen demonstriert - dem Hausarzt raten, wann er einen Chirurgen oder einen Diabetologen zuziehen soll, wann der Patient einen orthopädischen Schuhmacher braucht etc. So schlägt der Gesundheitsberater beispielsweise auch die Teilnahme an einer Koronarsportgruppe vor, in einem anderen Fall kümmert er sich um orthopädische Belange des Patienten. Er sorgt gewissermaßen auch dafür, daß die Wunde an einem Fuß eines Diabetikers richtig behandelt wird, daß seine Ernährung die richtige sei etc. In der Beschreibung des Fallbeispiels heißt es dann wörtlich: „Die vermittelnde und unterstützende Tätigkeit des Gesundheitsberaters trug zu dem Heilungsprozeß bei.“ 
Hier werden ganz eindeutig hausärztliche Funktionen von einem Gesundheitsberater übernommen und/oder dem Hausarzt/Gatekeeper aufgezwungen, der eben nur noch Primärarzt ist und sonst nichts. Ich frage mich, ob unsere Ärzteschaft bereit ist, diesen Schritt zu gehen.

Es ist wahr, daß der OECD-Bericht von 1997 für Deutschland zu dem Ergebnis kam, daß unser Gesundheitswesen relativ teuer sei, daß seine Kosten in den letzten Jahren sogar zugenommen haben, so daß wir neben den USA an zweiter Stelle in der Welt stehen. Die Behauptung, seine Effizienz sei nur mittelmäßig, ist aber nicht nachvollziehbar, solange Effizienz nur an reinen Mortalitätszahlen gemessen wird und nicht an der Lebensqualität der Bevölkerung und an dem gesamten Gesundheitsangebot und dem Zugang zu diesem für die gesamte Bevölkerung. Ebenso fragwürdig ist, ob die von der OECD vorgeschlagene Lösung für Deutschland, nämlich daß das Primärarztsystem die richtige Lösung ist, indem nämlich eine Hauptqualität unseres Systems, der freie Zugang zum Arzt, beschnitten würde. Um die Effizienz unseres Gesundheitssystems oder Z. B. der ambulanten Versorgung zu beurteilen, bedarf es nicht nur eines Aspektes. Es muß auch gesehen werden, wie sich hierzu Zahl der Krankenhausbetten, Zahl der niedergelassenen Fachärzte im Vergleich zu den Krankenhausfachärzten, Zahl der Krankenhaustage pro Krankheitsfall, Krankenhauskosten, ambulante Kosten und Medikamentenkosten verhalten.

Es ist zu fragen, ob wir eine solche Strukturänderung haben wollen. Dabei ist das Primärarztsystem nur ein Faktor solcher neuen Strukturen. Angedacht werden weit darüber hinausgehende Elemente; hierzu gehören Arztnetze ebenso wie Modellversuche, wobei gefordert wird, die Krankenkassen müßten endlich in der Lage sein mitzubestimmen, wie das ärztliche Honorar verteilt werde. Eine "dispositive Sozialmedizin“ wird gefordert, die bestimmt, welche Leistungen welcher Patient erhält, wieviel Medikamente, wieviel Hüftgelenke, wieviel Dialysen etc. Solche Managed-Care-Strukturen widersprechen der freien Arztwahl und sind mit unserer heutigen Auffassung einer medizinischen Versorgung nicht kompatibel. 
Christian Dierks

Bevor ich versuche, das „Pro“ und das "Contra“ meiner Vorredner zu verbinden oder gar zu versöhnen, lassen Sie mich bitte noch einmal zum Ausgangspunkt der Diskussion zurückkehren. Nach der Einführung der "Chipkarte" wurde vor vier Jahren eine sehr intensive Diskussion um Hausarztmodelle geführt. Nach einer etwa vierjährigen Pause wird dieses Thema von der Koalition der neuen Mitte nun wiederbelebt. Die Probleme, die zu dieser Erörterung Anlaß geben, sind nach wie vor dieselben: Steigende Kosten, die sich unter Budgetierung überwiegend als verminderte Vergütung der Ärzte darstellen, „Chipkarten-Tourismus“, unkontrollierte Leistungserbringung im Bereich der Großgeräte und des Labors und schließlich ein als „ungünstig" zu bezeichnendes Verhältnis zwischen Hausärzten und Fachärzten von rund $40 \%$ zu $60 \%$.

Daneben bestehen in den Metropolen ernstzunehmende Strukturprobleme: Die Allgemeinmedizin verkommt zum Auslaufmodell. In Berlin wurden im Jahr 1997 lediglich 10 Weiterbildungsassistenten im Bereich der Allgemeinmedizin beschäftigt. In Hamburg sind weniger als ein Viertel der Ärzte allgemeinmedizinisch tätig, mehr als jeder fünfte ist über 65 Jahre alt.

Freilich wird die hausärztliche Versorgung auch zu einem großen Teil von hausärztlich tätigen Internisten wahrgenommen. Die sechsjährige Weiterbildungszeit der Inneren Medizin zielt auf die internistische Behandlung unter klinischen Bedingungen, wobei der Schweregrad der Erkrankung immerhin so ernstgenommen wurde, daß eine stationäre Aufnahme den Anlaß der Behandlung bietet. Ob die Inhalte dieser Weiterbildung für die Betreuung des Patienten als „allgemeine und fortgesetzte ärztliche Betreuung bei Kenntnis des häuslichen und familiären Umfeldes" (so § 73 Abs. 1 Nr. 1 SGB V) geeignet sind, muß bezweifelt werden.

Es ist mir hier jedoch nicht daran gelegen, einer konkurrierenden Polarität zwischen Hausärzten und Fachärzten das Wort zu reden. Entscheidend ist nicht, welcher Gebietsarzt das Rennen um den Patienten gewinnt, sondern daß der sozialrechtliche Anspruch des Patienten auf hausärztliche Versorgung sichergestellt wird. Gesucht wird der Arzt der Care und Cure verbindet und dabei ohne Nachteile für den Patienten oder sich selbst weitergehende oder maximale Diagnostik und Therapie veranlassen kann. 
Es ist naheliegend, unter diesen Umständen die Etablierung eines Hausarztmodells, Primärarztsystems oder einer "Gatekeeper-Funktion“ des Allgemeinmediziners zu diskutieren. Mitnichten handelt es sich dabei um eine neue Idee. Vergleichbare Strukturen sind seit Jahren in anderen Ländern der Europäischen Gemeinschaft etabliert und auch aus deutscher Sicht schon mehrfach untersucht worden (vgl. z. B. Armann, ErsK 94, 389 ff.). Bei der Adaptation derartiger Systeme für den Bereich der gesetzlichen Krankenversicherung in Deutschland sind nicht nur die unterschiedlichen Voraussetzungen $\mathrm{zu}$ beachten. Auch die unerwünschten Entwicklungen ausländischer Hausarztmodelle müssen identifiziert und vermieden werden.

In Dänemark sind Besserverdienende in der Klasse II versicherbar, in der freie Arztwahl um den Preis einer 40\%igen Zuzahlung geboten wird. Nachdem 1976 auch diesen Versicherten die Möglichkeit eröffnet wurde, in die zuzahlungsfreie, aber primärarztgebundene Klasse I zu wechseln, sind der Klasse II nur noch wenige Prozent der versicherbaren Bürger geblieben.

In Holland schränkt das System die freie Arztwahl selbst für Privatversicherte ein. Ein landesweites Primärarztsystem vergütet die ärztlichen Leistungen im wesentlichen mit Kopfpauschalen, auch wenn diese im Detail weiterer Differenzierung unterliegen. In der Folge ist die apparative und oft personelle Ausstattung der holländischen Allgemeinarztpraxis im Vergleich zu den deutschen Kollegen eher bescheiden. Das Verhältnis zum nachgeordneten Personal beträgt in der holländischen Allgemeinpraxis $1: 0,8$, während der deutsche Kollege auf durchschnittlich $1: 2,3$ Mitarbeiter zurückgreifen kann (Angaben laut Armann a.a.O.). Dementsprechend ist der Umfang der Weiterüberweisung der Patienten zu Spezialisten mit $60 \%$ entsprechend groß.

In England führt die oftmals noch spartanischere Ausstattung der Arztpraxen ebenfalls zu einer hohen Überweisungsrate. Die fachärztliche Versorgung ist dort allerdings unter der strengen Rationierung geknebelt. Oftmals vergehen Jahre, bis fachärztliche Konsultationen oder elektive Eingriffe durchgeführt werden können. Erst durch den Community Care Act und der den Ärzten damit eingeräumten Möglichkeit des fund holding sind entscheidende neue Impulse in das System gekommen. Mittlerweile betätigen sich mehr als die Hälfte der niedergelassenen Ärzte als fund holder und bieten nach umfangreichen Investitionen in ihrer Praxis anspruchsvolle Medizin auf höherem Niveau. Der in den letzten Jahren zu verzeichnende Anstieg privater Krankenversicherungsverträge zeugt allerdings nach wie vor von der Unzufriedenheit der Briten mit ihrem steuerfinanzierten Primärarztsystem. 
In Italien ist die Allgemeinmedizin zur Domäne derjenigen Ärzte verkommen, denen eine fachärztliche Qualifikation oder die Tätigkeit in einem renommierten Krankenhaus nicht beschieden ist. Unter der strikten Festlegung von Fallzahlobergrenzen findet der Allgemeinarzt gerade in der Metropole kein Auskommen mehr - die hausärztliche Versorgung degeneriert zum Teilzeitjob. Es nimmt nicht Wunder, daß in Italien jede fünfte Lira im Bereich der Krankenversorgung privat finanziert wird.

In Deutschland ist das Hausarztmodell längst etabliert. Es existiert freilich nur auf dem Papier:

- Gemäß § 73 Abs. 1 Satz 1 SGB V ist die kassenärztliche Versorgung in die haus- und fachärztliche Versorgung gegliedert.

- Die hausärztliche Versorgung ist gesetzlich definiert. Sie beinhaltet die allgemeine und fortgesetzte ärztliche Betreuung des Patienten, die Koordination diagnostischer, therapeutischer und pflegerischer Maßnahmen, die umfassende Dokumentation der wesentlichen Behandlungsdaten und die Verantwortung für präventive und rehabilitative Maßnahmen (vgl. § 73 Abs. 1 Satz 2 SGB V).

- Das Bundessozialgericht hat diese Gliederung als rechtmäßig bestätigt (BSG vom 18.06.1997 - 6 RKa 58/96).

- Die nähere Spezifikation der hausärztlichen Versorgung und Fragen der Vergütung sind im Hausarztvertrag vom 06.09.1993 geregelt.

- Die Kommunikation zwischen Haus- und Facharzt wird durch $\S 73$ Abs. 1 b SGB V nicht nur gefordert, sondern auch datenschutzrechtlich spezifiziert und ermöglicht.

- Der Patient ist verpflichtet, einen Hausarzt zu wählen ( $§ 76$ Abs. 3 Satz 2 SGB V).

- Ein Wechsel des Hausarztes innerhalb des Quartals ist nur bei Vorliegen eines wichtigen Grundes zulässig (§ 76 Abs. 3 Satz 1 SGB V).

Trotz dieser nahezu lückenlosen Regelung eines Hausarztmodells im Sozialgesetzbuch ist eine entsprechende Umsetzung bislang ausgeblieben. Viele der genannten Vorschriften sind den beteiligten Ärzten und Patienten nicht einmal bekannt. Wie ist dieses Auseinanderfallen zwischen Gesetz und Realität zu erklären? Liegen hier Defizite des Gesetzgebers vor? Oder um mit Morgenstern zu fragen: „Ist hier die Staatskunst anzuklagen?".

Bei näherer Betrachtung erklärt sich, warum die gesetzliche Ausgestaltung des Hausarztmodells Programmsatz oder Appell geblieben ist. Hohe Rechtsgüter stehen auf dem Spiel: Das Recht des Patienten, unter 
den niedergelassenen Ärzten frei auszuwählen, ist tradiert und im Anspruchsdenken der Bevölkerung fest verankert. Es dürfte nicht an Möglichkeiten fehlen, die freie Arztwahl aus dem allgemeinen Persönlichkeitsrecht und dem Sozialstaatsprinzip herzuleiten. Aber auch die Eigentumsgarantien der über 60.000 Fachärzte dürfen nicht durch einen gesetzgeberischen Federstrich gefährdet werden. Es handelt sich dabei überwiegend um hochqualifizierte Einzelunternehmen, die den Versicherten gegenwärtig eine fachärztliche Versorgung auf hohem therapeutischen Niveau bieten. Jeder Versuch, den Zugang zu diesen Spezialisten gesetzlich von heute auf morgen abzuschneiden und von der Überweisung eines Hausarztes abhängig zu machen, würde bei den zuständigen Gerichten ein deutliches Stirnrunzeln im Sinne des $\S 100$ Bundesverfassungsgerichtsgesetz hervorrufen.

Seit dem 01.07.1997 ist es freilich auch nicht mehr erforderlich, die Verdichtung der gesetzlichen Vorgaben zu einem faktischen Hausarztmodell auf gesetzlicher Ebene herbeizuführen. Der Gesetzgeber hat mit dem zweiten Neuordnungsgesetz die Experimentierküche der Modellvorhaben und Strukturverträge eröffnet ( $\S 63$ Abs. 1 und 2, 64 Abs. 2 und 73 a SGB V). Insbesondere die Modellvorhaben nach § 64 Abs. 4 sollen dabei gezielt entwickelt werden, um eine „Mehrfachinanspruchnahme" vertragsärztlicher Versorgung durch den Mißbrauch der Versichertenausweiskarte zu verhindern. Aus den bis zum 2. NOG geltenden Erprobungsregelungen sind damit handfeste Werkzeuge geworden, die die Erprobung von Hausarztmodellen auf regionaler Ebene möglich machen. Die beteiligten Krankenkassen und Kassenärztlichen Vereinigungen haben es dabei in der Hand, die Fehler und Nachteile ausländischer Systeme zu vermeiden.

Dementsprechend sollte

- das Vergütungssystem des Hausarztes nicht leistungsfeindlich strukturiert werden, aber auch keine übermäßigen Anreize zur Leistungsausweitung bieten,

- eine intensive Kommunikation zwischen Hausarzt und Facharzt gefördert werden,

- die Breite der bestehenden fachärztlichen Versorgung gezielt genutzt werden, um eine zeitgerechte Diagnostik und Therapie zu erreichen,

- die Weiterbildung und Fortbildung in der Allgemeinmedizin auch durch strukturelle Anreize gefördert werden.

Soweit die Teilnahme der Versicherten an Modellvorhaben freiwillig ist, bietet sich die Gestaltung neuer Tarife an, die nicht auf dem vollständi- 
gen Verzicht eines initialen Facharztbesuchs basieren, sondern eine moderate Eigenbeteiligung für diesen Fall vorsehen. Die Erfahrungen der privaten Versicherungsgesellschaft AXA-Colonia mit dem "EL-Tarif“ haben zu interessanten Ergebnissen geführt. Eine Adaptation auf dem Bereich der GKV sollte nicht unversucht bleiben.

Entscheidend scheint mir dabei das Nebeneinander verschiedener Modellvorhaben, die nicht nur im Lichte der gegenwärtigen Pandemie von Praxisnetzen gesehen werden dürfen. Die Erprobung und Bewährung von Hausarztmodellen durch Modellvorhaben muß von der ärztlichen Selbstverwaltung und den beteiligten Körperschaften als Chance begriffen und genutzt werden. Wenn verhindert werden soll, daß die Einführung eines Hausarztmodells zum gesetzgeberischen Planspiel wird, müssen die Freiräume der Modellvorhaben effektiv umgesetzt und ausgewertet werden. Letztendlich muß die Förderung der allgemeinärztlichen Versorgung dabei mit einer effizienten und redundanzfreien Auslastung der fachärztlichen Versorgungskapazitäten verbunden werden. 
Axel Munte

Integration, Kooperation und Verzahnung sind seit Jahren die beliebtesten "Zauberformeln“ im deutschen Gesundheitswesen. Die Häufigkeit ihrer verbalen Nutzung steht leider jedoch im umgekehrten Verhältnis zu ihrer realen Umsetzung. Kein Zweifel, daß hier Handlungsbedarf besteht - nicht nur seitens der Politik, die bessere gesetzliche Rahmenbedingungen schaffen muß. Doch schon heute könnten die Akteure im Gesundheitswesen integrative Versorgungsstrukturen ohne weiteres realisieren.

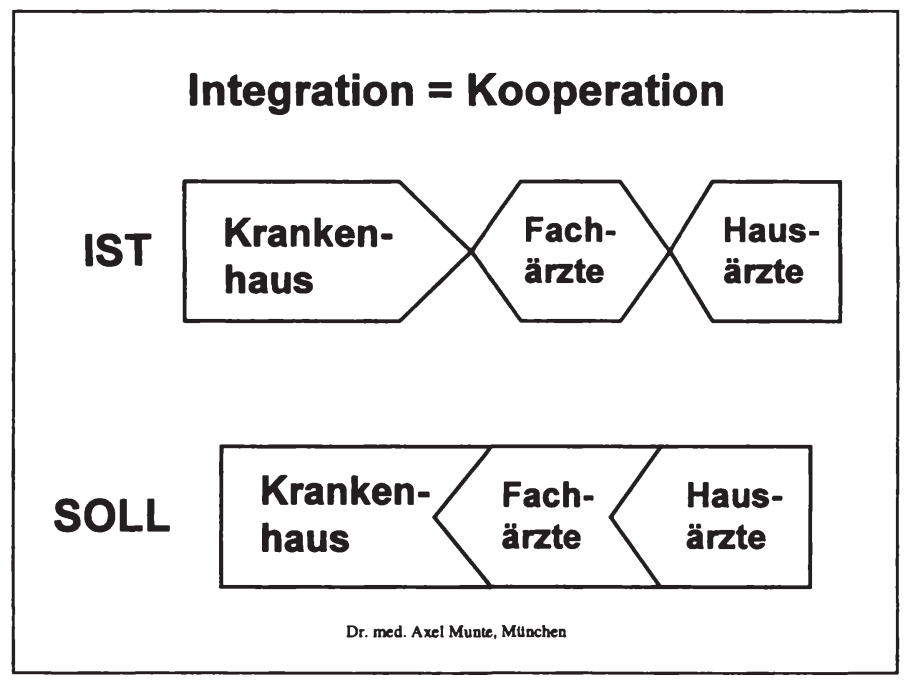

Denn Integration bedeutet nicht mehr als den systematischen und gezielten Ausbau der ambulant-stationären Schnittstelle durch gemeinsam getragene Initiativen niedergelassener Ärzte und Krankenhäuser - letztlich zum beiderseitigen Nutzen. Wer könnte auch unter den heutigen gesetzlichen Voraussetzungen Krankenhäuser und Niedergelassene daran hindern, gemeinsam den lokalen Versorgungsauftrag auszugestalten? Alles hängt immer von den Akteuren vor Ort ab: Deren Kooperationswilligkeit und Einsicht, daß nur Integration die Prozesse im Ge- 
sundheitswesen zu optimieren und die Ressourcen besser zu nutzen vermag.

Politische Ideologien wie „Öffnung der Krankenhäuser" oder „Einführung eines Hausarztmodells" sind meines Erachtens nicht die richtigen Wege zur Neustrukturierung des Gesundheitswesens. Nur die aktiv betriebene ambulant-stationäre Integration entsprechend den bestehenden lokalen Versorgungsbedürfnissen und -strukturen bietet die Chance einer schnellen Umsetzung.

Eine heutige Momentaufnahme vom deutschen Gesundheitswesen zeigt in vielen Regionen leider, zumindest in der gesundheitspolitischen Diskussion, die umgekehrte Entwicklung. Statt Integration und Kooperation kommt es häufig zu verbalen Konfrontationen und Auseinandersetzungen zwischen wesentlichen Versorgungsstrukturen, nämlich der Hausärzte, Fachärzte und Krankenhäuser. Glücklicherweise bestimmt jedoch nicht der politische Dissens den ärztlichen Alltag, sondern die gewachsenen, zum Teil sehr effektiven Kooperationsstrukturen zwischen den drei o. g. tragenden Säulen.

\begin{tabular}{|l|}
\hline Selektive Integrationsstrategien \\
- Notfallversorgung mit niedergelassenen Ärzten \\
- Gerätegemeinschaften, Einkaufsgemeinschaften \\
- Tagesklinikangebote von Vertragsärzten \\
(modifiziertes Belegarztsystem, Praxisklinik) \\
- Outsourcing von stationären Funktionsbereichen an \\
Belegärzte und Praxen in der Klinik \\
- ambulant-stationäre Stufenmodelle \\
- ambulant-stationäre Kommunikationsnetze \\
$\Rightarrow$ Rationalisierungsgewinne und Synergieeffekte \\
Dr. med. Axel Munte, Munchen
\end{tabular}

In ganz Deutschland haben sich selektive Integrationsformen entwickelt und werden selbstverständlich hingenommen.

Die Notfallversorgung der niedergelassenen Ärzte ist bundesweit im Rahmen des Sicherstellungsauftrages von den KVen integrativ und ko- 
operativ geregelt. Zunehmend wird die Kooperation niedergelassener Ärzte mit Krankenhauskollegen in gemeinschaftlich betriebenen Notfalloder Bereitschaftspraxen akzeptiert und umgesetzt.

Einkaufsgemeinschaften sind im ambulanten und auch im Krankenhaussektor keine Seltenheit mehr. Gerätegemeinschaften werden schon aus wirtschaftlichen Gründen, wie z. B. bei den Radiologen, immer häufiger zur Normalität.

Tagesklinikangebote von Vertragsärzten, die meines Erachtens noch eine große Zukunft vor sich haben, sind erst noch im Kommen.

Das Outsourcing stellt eine Sonderform, eine selektive Form der Integration dar. So werden z. B. aus stationären Funktionsbereichen Tätigkeiten an Belegärzte übergeben oder gar Praxen in Kliniken angesiedelt.

\section{Bayern-3-Modell}

Ambulant-stationäre Kooperation im

Rahmen der Großgeräteverordnung

- 88 Modell-Standorte in Bayern

- $39 \times$ Computertomographie

- $41 \times$ Kernspinresonanz-Tomographie

- $6 \times$ LMKH

- $2 \times$ Hartstrahltherapie

Dr. med. Axel Munte, Munchen

In der Zeit der Großgeräte-Zulassungsbeschränkung in Bayern war mit großem Erfolg das sog. "Bayern-3-Modell“ eingeführt worden mit dem Ergebnis, daß an fast 90 Kliniken niedergelassene Ärzte im stationären Bereich Computertomographen, MRs oder nuklearmedizinische Geräte in Betrieb genommen haben. Auf diese Weise konnten hochinvestive Anschaffungen zur Betreuung von ambulanten und stationären Patienten getätigt werden. 
Auf so normale Integrationsformen wie ambulant-stationäre Stufendiagnostik und -therapie oder auf die meist erst im Entstehen begriffenen ambulant-stationären Kommunikationsnetze sei hier nicht weiter eingegangen.

Zusammenfassend ist zu sagen, daß selektive Integrationsformen doch zu erheblichen Rationalisierungsgewinnen und zum Teil ungeahnten Synergieeffekten führen und daß diese Integrationsformen auch ohne öffentliche Diskussion und berufspolitische Auseinandersetzungen regional in aller Regel funktionieren, wobei, wie am Bayern-3-Modell nachgewiesen, eine Reintegration durch lenkende Maßnahmen möglich ist.

\section{Integrationsförderung}

- Frühzeitige Abstimmung mit der Krankenhausstrukturplanung der Länder

- Anpassung der rechtlichen Rahmenbedingungen

- Ausbau und Modifikation des Belegarztsystems

- Einbindung der Krankenhäuser in vernetzte Strukturen und Praxisnetze

- Bildung strategischer Allianzen

- Definition gemeinsamer Versorgungskonzepte

- Unternehmerische Initiative der Vertragsärzte

Dr. med. Axel Munte, Munchen

Wie betont, sind Veränderungen im Gesundheitswesen auch unter den bestehenden gesetzlichen Regelungen möglich. Aber es besteht kein Zweifel, daß günstige gesetzliche Rahmenbedingungen stark integrationsfördernd wirken können. Hier ist der Gesetzgeber gefordert und wird hoffentlich die inm jetzt gebotene Chance, Veränderungen herbeizuführen, wahrnehmen.

So ist dringend erforderlich, daß eine Abstimmung bei der Krankenhausstrukturplanung der Länder frühzeitig stattfindet und endlich die monistische Finanzierung durch die Krankenkassen in Angriff genommen wird.

Das Belegarztsystem hat eine große integrative Kraft zwischen ambulanter und stationärer Versorgung. Hier bedarf es eines weiteren Ausbaus und größerer Akzeptanz in einigen Bundesländern. 
Die Krankenhäuser haben zwar spät, aber dennoch erkannt, daß sie sich aus den vernetzten Strukturen, insbesondere aus der Entwicklung im ambulanten Bereich bis hin zu den Praxisnetzen nicht heraushalten dürfen. Selbst in Großstädten mit höchster Qualifikation im hausärztlichen und fachärztlichen ambulanten Bereich und der Möglichkeit, sehr viele diagnostische und therapeutische Leistungen patientenfreundlich und kostensparend in ambulanten Strukturen erbringen zu können, werden heute und künftig die diagnostischen und therapeutischen Versorgungs-Highlights dem stationären Bereich vorbehalten bleiben. Zur Schnittstellendefinition ist eine enge Kooperation mit dem stationären Sektor unabdingbar. Auch wenn Krankenhäuser, und künftig wohl auch Praxisnetze, untereinander im Wettbewerb stehen, gibt es keinen Zweifel, daß zur Arbeitsteilung und zur politischen Außenvertretung strategische Allianzen innerhalb der Sektoren wie auch zwischen den Sektoren geschlossen werden sollten.

Gemeinschaftliche ambulant-stationäre, d. h. sektorübergreifende, auf die regionalen Bedürfnisse zugeschnittene Versorgungskonzepte zu entwickeln, wäre heute die vornehmste Aufgabe gesundheitspolitisch aktiver Ärzte und Sozialpolitiker.

An dem Beispiel der unternehmerischen Initiative von Vertragsärzten im Raum München sei dargestellt, wie allein die Kraft des Handelns beeindruckende Veränderungen initiiert und die "Lagerstrategen“ aller Versorgungsbereiche aufgescheucht und zu Integrationsgesprächen geführt hat.

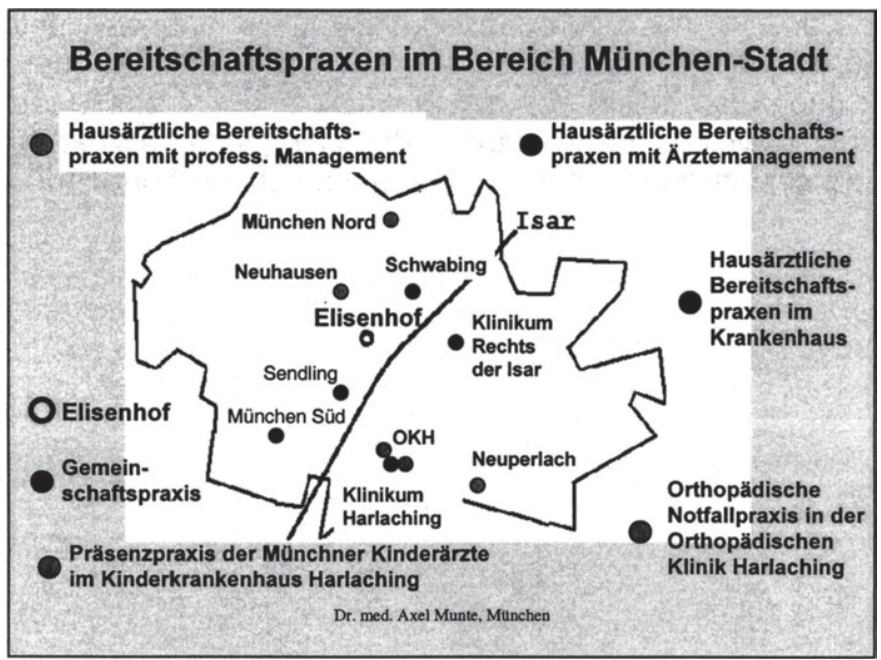


Die KVB-Bezirksstelle München hatte bereits vor 5 Jahren intensive Gespräche mit einem städtischen Krankenhaus aufgenommen, um dort eine von Kassenärzten betriebene Notfall- bzw. Bereitschaftspraxis zu etablieren. Die Verhandlungen scheiterten an den Vorurteilen der Verwaltung und der dortigen Klinikärzte. Die KV München initiierte daraufhin die mittlerweile bundesweit bekannte Bereitschaftspraxis am Hauptbahnhof im Elisenhof, die in 2 Jahren bereits über 130.000 Patienten betreut hat, und zwar hausärztlich und fachärztlich. Neben Allgemeinärzten und Internisten sind dort auch Orthopäden, Chirurgen, Kinderärzte, Gynäkologen, Augenärzte und Dermatologen tätig.

Der enorme Erfolg dieser Bereitschaftspraxis der KVB mit oft über 600 Patientenbehandlungen an einem Tag am Wochenende hatte zur Folge, $\mathrm{da} ß$ Verwaltungen und Klinikchefs von sich aus bereitwillig mit der KVBBezirksstelle Verhandlungen begonnen haben, um weitere Bereitschaftspraxen in den Kliniken selbst zu etablieren. Es zeigt sich wie so oft: Positive Fakten schaffen ist die beste Motivation zur Veränderung. Bis zur Drucklegung dieses Artikels werden in München dann bereits 2 Bereitschaftspraxen in Kliniken etabliert sein, in einer Universitätsklinik und in einer städtischen Klinik.

Aber auch die niedergelassenen Ärzte Münchens wurden durch das Elisenhof-Beispiel motiviert, in den verschiedenen Stadtteilen Bereitschaftspraxen zu gründen. Bei weitgehend hausärztlicher Besetzung dieser Praxen haben sich diese interessanterweise fast immer in den Räumen einer Facharztpraxis etabliert. Die Initiativen der Ärzte wurden weder von der KVB noch von den Krankenkassen finanziell gefördert. Sie entstanden vielmehr als Gemeinschaftsunternehmung, in der Regel als GmbH gegründet, allein aus der Finanzkraft der Ärzte. Das Management liegt bei einigen in den Händen der initiierenden Ärzte oder aber auch bei professionellen Managern, die entsprechend honoriert werden müssen. 


\section{Bereitschaftssystem München}

- 5 Ärztliche Bereitschaftspraxen mit ambulantstationärer Kooperationsvereinbarung = Bagatellfälle gehen nicht ins Krankenhaus

- 2 Ärztliche Bereitschaftspraxen im Krankenhaus = Bagatellfälle werden sofort umgeleitet

- 1 Orthopädische Notfallpraxis in der Fachklinik

- 1 Kinderärztliche Präsenzpraxis im Kinderkrankenhaus

$\Rightarrow$ Rationalisierung der Notfallversorgung und Verbesserung der vertragsärztlichen Präsenz

Dr. med. Axel Munte, München

Das Bereitschaftssystem München ist leider noch viel zu wenig publiziert worden. Es zeigt beispielhaft, wie ärztliche Initiative auch unter erschwerten wirtschaftlichen Rahmenbedingungen und keineswegs günstigen gesetzlichen Voraussetzungen sowie festgezurrten und starren Strukturen im Gesundheitswesen gewaltige Veränderungen schaffen kann. Während die Kassen diese Entwicklung quasi ignorieren, obwohl hier künftig mit enormen Einsparungen im stationären Bereich zu rechnen ist, zermartert sich die KV-Führung seit Jahren den Kopf, wie sie mit dieser unbotmäßigen Initiative der ärztlichen Basis am zweckmäßigsten umgeht. Im Definitionswirrwarr zwischen Notfallpraxis, Bereitschaftspraxis, Präsenzpraxis und Anlaufpraxis bewirkt sie bei den initiierenden Ärzten Rechtsunsicherheit und gibt innen wenig Schützenhilfe für ihre Initiativen. Dennoch ist die Entwicklung in München, die durch die Initiative der Bezirksstelle der KV München im Elisenhof angestoßen wurde, als eine hochintegrierte und integrationsfähige Versorgungsform beachtenswert.

Nach diesem Beispiel aus dem Münchner Versorgungsalltag mit erfreulichen Zukunftsperspektiven möchte ich abschließend noch einen Überblick geben über den Stand der Diskussion integrierter Versorgungsformen bei einigen prominenten Gesundheitspolitikern und den wesentlichen Verbänden und Körperschaften des Gesundheitswesens. 


\section{Positionen}

Dr. W. Schorre (KBV):

Die ambulante Behandlung im Krankenhaus ist wegen deren kostspieliger Infrastruktur deutlich teurer als bei niedergelassenen Fachärzten. Zudem gibt es nicht genügend Krankenhäuser, um eine wohnortnahe Versorgung sicherzustellen. Neben einer hohen Fluktuation beim Klinikpersonal besteht der weitere Nachteil, daß kein Arzt des Vertrauens als ständiger Ansprechpartner zur Verfügung steht. Zudem sind die Wartezeiten in den Klinikambulanzen länger als in der Arztpraxis. Hospitalismuskeime gefährden Patienten. Eine institutionelle Öffnung der Krankenhäuser kommt überhaupt nicht in Frage. Als Fazit muß es daher eine klare Aufgabentrennung geben, die durch sinnvolle Kooperation ergänzt wird, wie z. B. die gemeinsame Gerätenutzung.

\section{Dr. R. Hess (Hauptgeschäftsführer KBV):}

Integration heißt nicht Versorgung aus einer Hand (Dienstleistungszentrum Krankenhaus), sondern Steuerung in einer Hand (koordinierte Versorgungsprozesse in integrierten, kooperativen Strukturen mit eindeutiger medizinischer Aufgabenteilung). Das Steuerungsinstrument kann ein Netzwerk mit Praxen und Krankenhaus sein, das den lokalen Versorgungsbedarf gemeinschaftlich abdeckt.

\section{Position der Deutschen Krankenhausgesellschaft:}

Die DKG spricht sich für eine Öffnung der Krankenhäuser aus mit dem Stichwort: "Das Krankenhaus als integriertes Dienstleistungszentrum" (Beschäftigungssicherung durch Integration). Zudem sollte ein genereller gesetzlicher Zulassungsanspruch (wie bei psychiatrischen Institutsambulanzen) zur Erbringung der ambulanten fachärztlichen Behandlung spezieller Indikationsgruppen (Krebs- und AIDS-Patienten werden z. B. komplett im Krankenhaus versorgt statt in der Arztpraxis) gegeben sein. Hausärzte sollten eine umfassende, zentrale Vermittlungs- und Koordinierungsfunktion in der Versorgungskette übernehmen, eine Kooperation mit anderen Leistungserbringern am Krankenhaus sollte selbstverständlich werden.

\section{Position des Marburger Bundes:}

Die Zusammenarbeit vor Ort sollte sich über die fachliche Ebene, nicht über die finanzielle Ebene gestalten. Krankenhäuser sollten als gleichberechtigte Partner in die §§ 64 und 73 a SGB V aufgenommen werden. 


\section{Position der BÄK und der KBV:}

Diese haben ein Konsenspapier zur Verzahnung von ambulanter und stationärer Versorgung publiziert, in dem sie sich nicht für eine generelle Öffnung der Krankenhäuser, sondern für verstärkte Integration in neuen kooperativen Strukturen aussprechen. Krankenhäuser sollten in bestehende und zukünftig noch zu bildende Praxisnetze eingebunden werden, d. h. Krankenhausärzte sollten an Modellvorhaben und Strukturverträgen beteiligt werden. Ein koordinierter Notfall- und Rettungsdienst sollte die Vertragsärzte einbeziehen (Sicherstellungsauftrag, Vermeidung unnötiger Krankenhausaufnahmen).

Weitere Vorschläge kommen in folgenden Stichpunkten zum Ausdruck:

- Zweitmeinungsverfahren: Ermächtigung von Klinikern für Konsiliarleistungen,

- Ausbau eines kooperativen Belegarztsystems als Bindeglied zwischen ambulanter und stationärer Versorgung,

- gemeinsame Nutzung von Großgeräten und Spezialeinrichtungen,

- Definition eines Katalogs spezialisierter Leistungen, für deren fachgerechte Erbringung zur Vermeidung unnötiger Risiken für den Patienten die stationäre Infrastruktur vorgehalten werden muß.

Als sinnvolle Vereinbarungen in diesem Sinne werden Kooperationsverträge zwischen Krankenhausträgern, Klinikern und Vertragsärzten angesehen, weiterhin die Zulassung von Vertragsärzten zum Betreiben einer Kassenpraxis als Zweigpraxis oder Praxissitz in den Räumen eines Krankenhauses sowie die Ermächtigung von Krankenhausfachärzten zur Teilnahme an der vertragsärztlichen Versorgung.

Zuletzt verweisen wir auf die Basisinitiativen der Ärzteschaft wie Niederlassung im Krankenhaus (Praxissitz, Zweigpraxis, Schwerpunktpraxis), Kooperations- und Konsiliarverträge, belegärztliche Tätigkeit, interdisziplinäre Belegbetten, Bereitschaftsdienste und -praxen, Teilprivatisierung von stationären Kapazitäten oder Abtretung von Nutzungsrechten an stationären Ressourcen an niedergelassene Fachärzte (Integration einer privaten Tagesklinik in ein bestehendes Krankenhaus). 


\section{Integrierte Versorgungsformen}

\section{Qualitätsgemeinschaften und Praxisnetze}

Gerd W. Zimmermann

\section{Einleitung/Problembeschreibung}

\section{Strukturelle Defizite bei der Organisation und Steuerung der Versorgung}

Die Leistungsfähigkeit des deutschen Gesundheitssystems ist, nicht zuletzt wegen seines hohen Versorgungsniveaus bei einer im Vergleich zu anderen Industrienationen durchaus moderaten und über das letzte Jahrzehnt hinweg relativ konstanten Gesundheitsquote von etwa $10 \%$, durchaus als gut einzuschätzen. Jedoch wurde in den vergangenen Jahren zunehmend sichtbar, daß dieses System mit substantiellen Problemen behaftet ist.

Der Ausgabenentwicklung in der GKV versuchte man zwar mit diversen Gesetzen 26 entgegenzuwirken, die Organisationsstrukturen des Gesundheitssystems wurden bisher jedoch keiner umfassenderen Reform unterzogen. Ansätze hierzu existieren - z. B. in Form von Modellprojekten (wie z. B. Praxisnetzen) - erst seit relativ kurzer Zeit. Vor allem durch die mit dem 2. GKV-Neuordnungsgesetz eingeführten Änderungen des SGB V ist die Realisierung solcher Ansätze zur Verbesserung der Koordination und Steuerung in der medizinischen Versorgung deutlich vereinfacht worden.

\section{Fragmentierte Versorgungsstrukturen}

Die Organisationsformen im deutschen Gesundheitswesen standen bisher dem stetigen Wandel und der Ausdifferenzierung von Aufgaben, Inhalten, Funktionen, Qualifikationen etc. prinzipiell relativ unverändert gegenüber, was die Fähigkeiten zur Innovation, zum Wandel und zur Anpassung stark einschränkte. Gerade in der Struktur der Gesundheitsversorgung läßt sich aber ein zentrales Problemfeld der GKV ausmachen, denn kennzeichnend für den strukturellen Aufbau des deutschen Ge-

26 Krankenversicherungs-Kostendämpfungsgesetz 1977, Kostendämpfungs-Ergänzungsgesetz 1981, Krankenhaus-Kostendämpfungsgesetz 1981, Haushaltsbegleitgesetz 1982, Krankenhaus-Neuordnungsgesetz 1984, Gesundheitsreformgesetz 1988, Gesundheitsstrukturgesetz 1992 
sundheitswesens ist die einerseits zwar stark arbeitsteilig angelegte und sektorierte medizinische Versorgung, andererseits jedoch auch die eher schwach ausgeprägte Kommunikation, Kooperation und Koordination innerhalb dieses Versorgungssystems.

Diese Problematik tritt sowohl an den Schnittstellen zwischen ambulantem und stationärem Sektor, als auch innerhalb des ambulanten Versorgungsbereichs auf, da in diesem Bereich die medizinische Versorgung stark arbeitsteilig organisiert ist, z. B. in Form der Trennung zwischen fach- und allgemein- bzw. hausärztlicher Versorgung, der Trennung zwischen medizinischer, pflegerischer und psychologisch-sozialer Betreuung oder der Vielzahl von Einzelpraxen als (immer noch) vorherrschende Form der Praxisausübung.

Ferner ergab sich durch nicht obligatorische bzw. unstrukturierte Kommunikationsbeziehungen für den Arzt auch kaum die Möglichkeit, seine Vorgehensweisen in Diagnostik und Therapie zu hinterfragen und z. B. mit anderen Kollegen zu diskutieren, d. h. - um ein Schlagwort unserer Zeit zu verwenden - Qualitätssicherung zu betreiben.

\section{Resultierende Unwirtschaftlichkeiten und Probleme}

Man kann also feststellen, daß in der Bundesrepublik zwar ein weitgehend fragmentiertes Versorgungssystem existiert, jedoch kein Instrument zur systematischen Koordination der Einzelleistungen innerhalb eines Versorgungsprozesses (wie Z. B. Case oder Disease Management), das ineffektive Vorgehensweisen verhindern könnte.

Unwirtschaftlich ist dieses System z. B. durch die Existenz von:

- Doppeluntersuchungen,

- Doppelinvestitionen,

- überflüssigen Arztwechseln,

- Fehlbelegungen in Krankenhäusern wegen fehlender ambulanter pflegerischer Einrichtungen,

- vermeidbaren Einweisungen als Folge einer nicht aufeinander abgestimmten Arzneimitteltherapie.

Zudem - und dies ist zumindest für den Patienten die gravierendere Auswirkung - kann eine unkoordinierte Leistungserbringung, z. B. im Falle von nicht abgestimmten Arzneimitteltherapien, auch kontraproduktiv sein, indem gesundheitliche Schäden verursacht werden. 


\section{Überwindung der Koordinationsdefizite durch vernetzte Versorgungsstrukturen}

Wie eingangs dargestellt, ist der Bereich der ambulanten ärztlichen Versorgung durch deutliche Defizite bei der Kommunikation, Kooperation und Koordination gekennzeichnet. Folge dieses Umstandes ist eine ineffiziente und ineffektive Versorgung, z. B. durch die Existenz von Mehrfachuntersuchungen. Dieses grundlegende Problem wurde zudem durch die Einführung der Krankenversichertenkarte und die für den Patienten dadurch gegebene Möglichkeit des "Doctor-Shoppings“ oder "DoctorHoppings“ verschärft. Zusätzlich führt das „Einzelkämpfertum“ des Arztes auch zu Diagnose- und Therapieansätzen, die vielfach vor allem auf der individuellen Erfahrung beruhen und nicht - im Sinne der Qualitätssicherung - ausreichend kritisch hinterfragt werden.

Ein naheliegender Ansatz, die genannten Probleme in Angriff zu nehmen und diese ineffektiven und zudem antiquierten Strukturen und Verhaltensweisen zu überwinden, besteht darin, daß man, nicht zuletzt auch unter Einbindung moderner Kommunikationstechnologien, die Kommunikation und Kooperation der Ärzteschaft in einem überschaubaren Rahmen, also auf lokaler oder regionaler Ebene, fördert, indem sich die (Vertrags-) Ärzte „vor Ort“ zu einem Netz zusammenschließen.

Je nach dem Umfang der (angestrebten) Versorgung lassen sich solche Netze in Mikro- und Makronetze unterscheiden. Ziel eines Mikronetzes ist beispielsweise die Erweiterung der Verfügbarkeit der bestehenden ambulanten kardiologischen Versorgung. Zur Realisierung einer integrierten kardiologischen Versorgung wird das Netz dann Kooperationsverträge mit Spezialeinrichtungen (z. B. im Bereich Herzchirurgie) schließen. Ein Makronetz wird hingegen die ständige Verfügbarkeit einer ambulanten Gesamtversorgung als Voraussetzung der Substitution von stationären Behandlungen anstreben.

Im folgenden werden ausschließlich Makronetze besprochen.

\section{Zielsetzungen}

Vor dem Hintergrund der aktuellen Probleme des Gesundheitswesens lassen sich Ziele von integrierten (hier: im Sinne von „vernetzen“) Versorgungsstrukturen dementsprechend definieren: Durch die Verbesserung von Kommunikation, Kooperation und Koordination in der ambulanten Versorgung auf der Basis von klar definierten und verbindlichen Kommunikations- und Organisationsstrukturen soll die Versorgung der Patienten im Netz effektiver gestaltet werden, d. h. Outcome-Ver- 
besserungen/Qualitätssteigerungen bzw. das Eröffnen und Ausschöpfen von Rationalisierungspotentialen sollen erreicht werden. Ein wichtiges Element dieses Systems ist auch die Optimierung (Rationalisierung) von Diagnose und Therapie auf der Basis von Qualitätssicherungsmaßnahmen.

Durch das Ausschöpfen der gesamten Möglichkeiten des ambulanten Sektors zur Optimierung der Versorgung läßt sich die Hospitalisation in vielen Fällen vermeiden. Durch eine enge Kooperation sowohl mit ärztlichen (z. B. Krankenhäusern) als auch nichtärztlichen (z. B. Pflegedienste) Leistungserbringern kann die medizinische Versorgung im Sinne eines optimierten und integrierten Versorgungsprozesses gestaltet werden.

\section{Rechtsgrundlagen für Modellvorhaben und Strukturverträge}

Auch wenn es vor dem Inkrafttreten des durch das 2. GKV-Neuordnungsgesetz geänderten SGB V am 1. Juli 1997 bereits einige innovative Ansätze zur Weiterentwicklung der Versorgungsstrukturen im ambulanten Bereich in Form von Praxisnetzen gab27, so wurde jedoch erst mit dem 2. NOG einer ganzen Palette von Möglichkeiten zur Einführung neuer Versorgungsansätze der Weg geebnet.

Die durch das 2. NOG geänderten $\S \S 63$ - 65 SGB V bieten den KVen und den Krankenkassen vielfältige und weitreichende Gestaltungsmöglichkeiten zur Realisierung von zeitlich befristeten Modellvorhaben zur Weiterentwicklung von Verfahrens-, Organisations-, Finanzierungs- und Vergütungsformen. Mit der nahezu radikalen Möglichkeit der Vertragspartner, das gesamte Leistungserbringerrecht (4. Kapitel: $\S \S 69$ bis 140 SGB V) vertraglich zu suspendieren, werden neue Entwicklungen ermöglicht. Eine wissenschaftliche Begleitung und Evaluation ist vorgeschrieben 28 .

Vor allem sieht das SGB V im § 73 a die Möglichkeit zum Abschluß von Strukturverträgen vor. Hierbei können die Verbände der Krankenkassen und die KVen auf Landesverbandsebene neue (dauerhafte) Versorgungs- und Vergütungsstrukturen vereinbaren. Damit wird ein weitgehender Ansatzpunkt geboten, Anreizsysteme (z. B. Pauschalen-Ver-

27 z. B.: „Medizinische Qualitätsgemeinschaft Rendsburg", 1. Juli 1996; „Praxisnetz Berliner Ärzte und Betriebskrankenkassen", 1. Juli 1996; „Ärztliche Qualitätsgemeinschaft Ried", 1. April 1997

$28 \mathrm{Vgl}$ § 65 SGB V 
gütung, Bonussysteme), Steuerungsinstrumente (z. B. Primärarztprinzip, Case und Disease Management) sowie Organisations- und Kooperationsformen (z. B. Praxisnetze) in die Regelversorgung zu übernehmen.

\section{Strukturelemente vernetzter Versorgungsstrukturen}

Medizinische Versorgungskonzepte, die auf dem Grundprinzip der Vernetzung aufbauen, sind durch bestimmte Strukturen gekennzeichnet. Diese müssen allerdings in ihrer Gesamtheit nicht bei jedem Ansatz unbedingt in gleicher Ausprägung vorhanden sein.

\section{Verbesserung der Präsenz: Rufweiterleitung, Leitstelle etc.}

Ein zentrales Anliegen von Praxisnetzen ist die Erhöhung des Präsenzgrades der im Netz zusammengeschlossenen Ärzte, vor allem außerhalb der üblichen Praxisöffnungszeiten, um somit eine (zeitlich) umfassende Versorgung anbieten zu können.

Die Koordination des Präsenzdienstes kann z. B. einfach dadurch erfolgen, daß der Patient, der außerhalb der üblichen Praxiszeiten seinen Arzt anruft, mittels einer Ansage auf dem Anrufbeantworter über den gerade diensthabenden Arzt informiert wird. Ein weiterer (Fort-) Schritt ist die Nutzung moderner Kommunikationstechnologien (z. B. ISDN), da hierdurch die Rufweiterleitung eines eingehenden Anrufes an den jeweils präsenten Arzt möglich wird29.

Eine noch weitergehende und auch aufwendigere Lösung ist die Einrichtung einer Leitstelle. Diese Einrichtung ist in Notfällen der telefonische Ansprechpartner für die Patienten - die Leitstelle koordiniert dann die angeforderte ärztliche Versorgung. In Erweiterung dieser Aufgabe wird es möglich, sowohl haus- und fachärztliche als auch pflegerische Versorgung zu organisieren (vor allem außerhalb der üblichen Praxiszeiten), d. h., durch die Einrichtung einer Leitstelle kann sichergestellt werden, daß Patienten rund um die Uhr ausreichend und auf einer sinnvollen Stufe ambulant versorgt werden.

Eine weitere Aufgabe der Leitstelle (als Servicecenter des Netzes) kann zudem die Erbringung von Serviceleistungen für die Netzärzte sein, indem über geeignete Krankenhäuser und qualitätsgesicherte Leistungserbringer im nichtärztlichen Gesundheitsbereich, wie ambulante Pflegedienste, Reha-Einrichtungen oder Selbsthilfegruppen, Informationen be-

29 Wie das Modell „Ärztliche Qualitätsgemeinschaft Ried“ gezeigt hat, ist dieses Konzept aber in der Umsetzung problematischer als zunächst gedacht. 
reitgestellt werden. Leitstellen können auf Wunsch auch Reservierungen von freien Krankenhausbetten vornehmen oder die nichtärztliche Hilfe in der ambulanten Versorgung selbständig organisieren.

\section{Qualitätssicherung: Qualitätszirkel, Fallkonferenzen und Zweitmeinung}

Die Sicherung der Qualität soll erreicht werden, indem die Netzärzte regelmäßig an Qualitätszirkeln teilnehmen. Im Gegensatz zu den herkömmlichen fachgruppenspezifischen Qualitätszirkeln können diejenigen im Netz auch fachübergreifend und problemorientiert arbeiten. Neben der Optimierung von Diagnose und Therapie beschäftigen die Zirkel sich auch damit, qualitätsgesicherte Einsparmöglichkeiten aufzuzeigen, z. B. durch Entwicklung/Adaption von Leitlinien. Möglich ist auch die Erarbeitung netzinterner Positivlisten für Arzneimittel.

Eine nicht unwesentliche Rolle bei der Qualitätssicherung kann auch das Instrument der Zweitmeinung spielen, bei der vor der Einweisung in ein Krankenhaus die Zweitmeinung eines spezialisierten fachärztlichen Kollegen eingeholt werden soll. Eine besondere Variante ist die "second opinion ex-post" wie sie in Südbaden30 zum Einsatz gelangt: Im Rahmen von Fallkonferenzen werden Krankenhauseinweisungen fallbezogen diskutiert und mögliche Behandlungsalternativen (nachträglich) analysiert.

\section{Förderung der Kooperation durch verbesserte Kommunikation: Dokumentation und Austausch medizinischer Patientendaten}

Neben der Qualitätssicherung und der Verbesserung der Präsenz ist es ein zentrales Ziel von Praxisnetzen, die innerärztliche Kooperation zu fördern. Da Kooperation nur dort möglich und effektiv ist, wo auch die Kommunikation zwischen den Beteiligten funktioniert, muß die Kommunikation unter den Netzärzten sowie mit anderen Leistungserbringern (Krankenhäuser, Pflegedienste etc.) eine entsprechende Qualität besitzen.

Dieser Anspruch an die Kommunikation wird unterstützt, indem eine umfangreiche patientenbezogene Dokumentation erfolgt: Bei jeder Überweisung wird dem mitbehandelnden Arzt ein Begleitbrief (oder ein ähnliches Dokument) vom überweisenden Arzt zugeleitet. Möglichkeiten bestehen hier sowohl in der "konventionellen“ Form, d. h. indem dem

30 Modell "Qualität und Humanität" in Südbaden 
Patienten ein Brief mitgegeben wird, oder besser (da schneller und nicht vom Patienten abhängig) durch die Nutzung moderner Kommunikationstechnologien wie Fax oder e-mail. Die Nutzung des Angebots des „Deutschen Gesundheitsnetzes“ ist ebenfalls denkbar.

Inhalte dieses Dokuments sind Vermerke und Hinweise auf Befunde zur Medikation und zu bereits durchgeführten Leistungen. Der mitbehandelnde Arzt ergänzt dann die Eintragungen und sendet den Patientenbrief zurück an den Hausarzt (bzw. an einen u. U. hinzuzuziehenden weiteren Facharzt). Auf diese Weise erhalten alle Beteiligten alle wichtigen Informationen, die Mehrfachuntersuchungen und unerwünschte pharmakologische Interaktionen vermeiden helfen.

$\mathrm{Zu}$ diesen Kommunikationsinstrumenten gehört auch das „Patientenbuch", das in einigen Netzen eingesetzt wird31. Mit diesem Instrument soll erreicht werden, daß zumindest in Notfällen dem Arzt bestimmte Grundinformationen, z. B. über vorliegende Erkrankungen, Medikationen und andere Besonderheiten des Patienten, sofort zur Verfügung stehen und der Notfallversorgungsprozeß so optimiert werden kann. In besonderem Maße notwendig - und deshalb auch gerade hier eingesetzt - ist ein solches Patientenbuch bei chronisch Kranken.

\section{Kooperation mit verschiedenen (z. T. auch nichtärztlichen) Leistungserbringern}

Eines der fundamentalen Ziele vernetzter Versorgungsstrukturen ist die Kooperation mit anderen, z. T. auch nichtärztlichen Leistungserbringern, da sich erst auf diese Weise ein bereichsübergreifender "integrierter" Versorgungsprozeß erreichen läßt. Speziell die Kooperation mit dem stationären Bereich birgt einige konzeptimmanente Probleme in sich, denn die Optimierung der ambulanten Versorgung bringt es zwangsläufig mit sich, daß das Praxisnetz in partielle Konkurrenz zu (zumindest den nächstgelegenen) Krankenhäusern tritt. Hier bedarf es also eines Konsenses zwischen der Klinik und dem Netz, um nicht knappe Ressourcen durch einen unnötigen Konkurrenzkampf zu verschwenden.

Weitere "Leidtragende" eines Praxisnetzes sind u. U. die ortsansässigen Apotheken, denn eine Optimierung der Pharmakotherapie ist in der Regel gleichbedeutend mit einem Umsatzrückgang bei den Apotheken. Aber auch hier sind Kooperationen möglich, wie das Beispiel „Ärztliche Qualitätsgemeinschaft Ried“ zeigt.

31 z. B.: „Ärztliche Qualitätsgemeinschaft Ried“; „Medizinisches Qualitätsnetz Hofheim“; geplant auch für das Hausarztmodell 
Weniger problematisch ist die Kooperation mit anderen, vor allem den nichtärztlichen Leistungserbringern, da diese die ärztlichen Leistungen ergänzende Dienste erbringen 32 . Hierbei ist an die Optimierung der Versorgung im Sinne von Versorgungsprozessen zu denken, indem z. B. die Pflege zu Hause durch ambulante Dienste anstatt einer nicht notwendigen stationären Pflege realisiert werden kann. Ferner können z. B. Kooperationen bei Rehabilitationsmaßnahmen mit entsprechenden Einrichtungen, mit Sanitätshäusern bei der Heil- und Hilfsmittelbeschaffung oder Z. B. auch mit Pflegediensten erfolgen.

\section{Nutzung von Mengeneffekten und Rationalisierungspotentialen aufgrund der Größe 33}

Netze sind gegenüber einer Einzelpraxis oder Gruppenpraxis in der Lage, ihre Marktmacht als Nachfrager auszunutzen. So sind Netze potentiell in der Lage, Rabatte durch den Einkauf größerer Mengen, beispielsweise beim Praxisbedarf, zu reatisieren. Ebenso sind z. B. günstige Konditionen bei der Beschaffung von Heil- und Hilfsmitteln (z. B. Vertrag mit einem Sanitätshaus) möglich.

Ferner besteht die Möglichkeit eines gemeinsamen Praxis-Managements. Praxisnetze sind in der Lage, Wissen (Know-how) zu transferieren, z. B. bezüglich einer optimierten Organisation der Praxis oder bezüglich der Praxis-EDV. Dabei ist sowohl an Hilfestellungen der Netzärzte untereinander als beispielsweise auch an den - etwa verglichen mit der Einzelpraxis - wirtschaftlicheren „Einkauf" von Expertenwissen zu denken. Standardisierte Lösungen verringern zudem die „Reibungsverluste" im Netz.

\section{(„Neue“) Aufgaben von Ärzten in Praxisnetzen}

Entsprechend der hier dargestellten Strukturelemente und dem Ausmaß, in dem sie in einem Netz Anwendung finden, läßt sich unmittelbar auch ein entsprechendes Aufgabenprofil für den Netzarzt ableiten, d. h., es ergibt sich zwangsläufig aus dem eben Festgestellten. Kurz zusammengefaßt läßt sich dieses durch folgende Schlagworte kennzeichnen:

32 d. h., sie bieten Komplementärgüter an, während z. B. Krankenhäuser Substitutgüter anbieten ( $\Rightarrow$ Konkurrenz)

33 „economies of scale“ 
- Präsenz

- Qualitätssicherung

- Kommunikation und Kooperation

- Gemeinsames Praxismanagement

\section{Honorierung und Finanzierung}

Nicht zuletzt die Änderungen des 2. NOG erlauben eine Vielfalt an Varianten zur Finanzierung von Netzstrukturen und zur Vergütung der im Netz erbrachten Leistungen.

Um ein Netz erst einmal „zum Laufen zu bringen“, ist eine Anschubfinanzierung seitens der Kassen, z. B. für die Teilnahme an den Qualitätszirkeln, sinnvoll, um den Anfangsaufwand des Netzes zu reduzieren. Da ein Ziel von Praxisnetzen die Realisierung von Einsparpotentialen ist, ist es naheliegend, daß die Vertragspartner sich über die Verteilung der erzielten Einsparungen, ggf. unter Berücksichtigung von Garantiesummen, einigen.

Die Vergütung der erbrachten Leistungen ist prinzipiell frei gestaltbar, so daß z. B. netzeigene Budgets oder auch (was für den Arzt besonders interessant ist) Einzelleistungsvergütung bei festen Punktwerten möglich sind, ebenso wie Mischformen.

\section{Der organisatorische Rahmen eines Netzes}

Ein Praxisnetz ist durch seine Zielsetzungen bzw. Aufgaben und die hierzu notwendigen Abläufe ein recht komplexes Gebilde und kann deshalb im unorganisierten anarchischen Zustand nicht ernsthaft funktionieren. Es braucht deshalb gewissermaßen als Rückgrat eine Organisationsstruktur und zudem eine geeignete Rechtsform.

\section{Organe und Verwaltung des Netzes}

Das Netz verwaltet sich innerhalb dieser Struktur selbst. Um Entscheidungen herbeizuführen, muß deshalb ein Organ geschaffen werden, das hierzu berechtigt und in der Lage ist, z. B. ein "Netzvorstand" und eine „Netz-(Voll-)Versammlung“. Ferner wird - je nach Größe des Netzes die Einrichtung einer Geschäftsstelle sinnvoll sein, um die notwendige Verwaltungsarbeit zu übernehmen. 


\section{Rechtsform}

Die Rechtsform des Netzes ist vor allem hinsichtlich der Haftungsfrage von Bedeutung. Sofern hierüber keinerlei gesonderte Regelung existiert, ist ein Netz eine Gesellschaft bürgerlichen Rechts (GbR). Folge dieser Gesellschaftsform ist, daß jedes Mitglied voll mit seinem Privatvermögen haftet. Um dies auszuschließen, bietet sich die Rechtsform einer $\mathrm{GmbH}$ an. Ebenfalls denkbar ist ein Praxisnetz als eingetragener Verein.

Modellprojekte: „Qualitätsgemeinschaften“, „Praxisnetze“ etc.

\section{Einige Beispiele}

Praxisnetze, Qualitätsgemeinschaften etc. sprießen derzeit „wie die Pilze aus dem Boden“. Im Moment gibt es in der Bundesrepublik etwa 140 Praxisnetze, die installiert sind bzw. sich in einer fortgeschrittenen Projektphase befinden.

Frühe Ansätze zu diesen Versorgungsformen sind beispielsweise die folgenden Projekte:

- Modell „Qualität und Humanität“ der KV Südbaden und der AOK Baden-Württemberg, Start: 1. Januar 1996

- Medizinische Qualitätsgemeinschaft Rendsburg der KV und der Ersatzkassenverbände Schleswig-Holstein, Start: 1. Juli 1996

- Praxisnetz Berliner Ärzte der KV und des BKK-Landesverbands Berlin, Start: 1. Juli 1996

- Ärztliche Qualitätsgemeinschaft Ried der KV und des VdAK Hessen, Start: 1. April 1997 (erstes derartiges Projekt in Hessen)

\section{Netzstrukturen in Hessen}

\section{Ärztliche Qualitätsgemeinschaft Ried}

Seit dem 1. April 1997 (bzw. dem 1. Januar 1997, wenn man die Vorlaufphase mitzählt) existiert im südhessischen Ried das erste Praxisnetz im Bereich der KV Hessen. Vertragspartner sind die KV Hessen und die Ersatzkassenverbände.

Gewissermaßen die Keimzelle diese Netzes ist der „Hausärztliche Qualitätszirkel Ried“, der seit Mai 1994 besteht. 
Zielsetzungen dieses Netzes sind - entsprechend der Grundideen von Praxisnetzen -, Qualitätsverbesserungen in der ambulanten Versorgung zu realisieren und Wirtschaftlichkeitspotentiale zu erschließen, insbesondere auch an den Nahtstellen zwischen den Versorgungsbereichen. Durch verstärkte Kooperation, Kommunikation und Koordination der niedergelassenen Ärzte in Ried, aber auch der Versorgungsbereiche untereinander sollen

- insbesondere eine Rund-um-die-Uhr-Versorgung der Patienten erreicht,

- nicht notwendige Doppel- und Mehrfachuntersuchungen vermieden,

- Leitlinien und Standards erarbeitet, Arznei-, Heil- und Hilfsmittel rationaler eingesetzt,

- nicht notwendige vollstationäre Behandlungen vermieden sowie

- die Zusammenarbeit zwischen den teilnehmenden Ärzten und an den Schnittstellen zu anderen Versorgungsbereichen verbessert

werden. Zur Umsetzung dieser Ziele haben die beteiligten Ärzte verschiedene „Bausteine" entwickelt:

- Qualitätszirkel und Arbeitsgruppen bilden die Basis für die Kommunikation der teilnehmenden Leistungserbringer. Die Arbeitsgruppen befassen sich z. B. mit

- Kommunikationstechnik, gemeinsamer Notdienstrufnummer und der Entwicklung des Patientenbuchs (AG Technik),

- fallbezogenen Diskussionen in Zusammenarbeit mit speziellen Fachgruppen, z. B. Onkologen, Nuklearmedizinern (AG Fallkonferenz),

- Pharmakotherapie (AG Pharmakotherapie),

- Kooperation mit Krankenhausärzten, z. B. zur Abstimmung der prästationären Diagnostik oder zu gemeinsamen Leitlinien (AG Krankenhaus).

- Ein quartalsweiser "Verordnungsspiegel" verschafft Transparenz über die eigene Verordnungsweise eines Arztes,

- ein eigenes Honorarbudget vermindert die Anreize zur medizinisch nicht notwendigen Leistungsausweitung,

- ein Patientenbuch für chronisch Kranke ist zu einem wichtigen Instrument des Disease Managements gereift, 
- das Zweitmeinungsverfahren (ex-post) ermöglicht die Reflexion des Einweisungsverhaltens und

- die Einbeziehung der über Patientenbefragungen festgestellten Bedürfnisse der Patienten.

An der ärztlichen Qualitätsgemeinschaft nehmen z. Z. 34 Ärzte unterschiedlicher Fachrichtungen aus dem Gebiet "Riedstadt" teil, wobei Haus- und Fachärzte etwa je die Hälfte stellen.

Das ÄQ Ried wird wissenschaftlich begleitet und evaluiert (AQUAInstitut); im Mai 1998 wurde nach einem Jahr Laufzeit ein erster Bericht präsentiert.

\section{Ärztenetz Rhein-Main in Rüsselsheim und Umgebung}

Seit dem 1. Oktober 1998 gibt es ein Praxisnetz in Rüsselsheim und Umgebung:

Vertragspartner der KV Hessen bei diesem Netz ist die BKK Opel.

Ziel des Modells ist die Erprobung des Gesundheitsmanagements unter Führung des Arztes. Wie für das Praxisnetz-Konzept typisch geht es auch hier um die Verbesserung der Versorgungsqualität und die Erschließung von Wirtschaftlichkeitspotentialen durch

- rationaleren und rationelleren Einsatz von Arznei-, Heil- und Hilfsmitteln,

- die Verbesserung der Kommunikation zwischen Netzärzten, Notdienst, Krankenhausärzten und nichtberuflichen Heilberufen,

- die Verkürzung von Verweildauern im Krankenhaus sowie

- die Vermeidung von Doppeluntersuchungen, stationärer Pflege und Krankenhausbehandlungen allgemein.

Unter dieser Zielsetzung haben sich ca. 100 Ärzte unterschiedlicher Fachgruppen aus Rüsselsheim, Raunheim, Trebur, GinsheimGustavsburg, Bischofsheim und Flörsheim zusammengeschlossen.

Zu den Aufgaben dieser Ärzte gehören die Teilnahme an Qualitätszirkeln, die gemeinsame Erarbeitung von Standards der Diagnostik und Therapie, die Durchführung von Fallkonferenzen, die Unterstützung der Begleitforschung, die Entwicklung eines Patientenbuchs und die Ab- 
sprache mit anderen Institutionen, wie Pflegediensten oder stationären Einrichtungen.

Das Modell wird wissenschaftlich evaluiert, wobei gegenüber den beteiligten Ärzten ein zeitnahes Feedback gewährleistet werden soll. Die Ärzte hingegen sind verpflichtet, die Begleitforschung zu unterstützen.

Abhängig von den Ergebnissen der wissenschaftlichen Begleituntersuchung ist eine Ausweitung des Modells auf weitere Bereiche in Hessen nicht ausgeschlossen, weshalb auch ein Rahmenvertrag mit dem BKKLandesverband abgeschlossen wurde.

\section{„Medizinisches Qualitätsnetz Hofheim“ (MQH)}

Mit dem MQH steht ein (weiteres) Modell in den Startlöchern, das auf gewachsenen guten Kommunikationsbeziehungen von Ärzten in Hofheim im Taunus aufbaut. Trotz der Nähe zu und der relativ guten Verkehrsanbindung an Frankfurt ist Hofheim bezüglich der Patientenströme ein regional eher abgrenzbares Gebiet. Dies darf wohl vor allem auch auf das breite Angebot an ambulanter medizinischer Versorgung (Praxen aus 16 Fachgruppen) zurückgeführt werden.

Vertragsverhandlungen laufen in weit vorangeschrittenem Zustand derzeit mit allen Primärkassen. Hierin unterscheidet sich das MQH auch von den vorgenannten Modellen in Hessen und auch von den meisten derartigen Projekten in anderen Teilen der Bundesrepublik Deutschland, denn diese werden in der Regel in Zusammenarbeit mit nur einem Kassenverband gestartet (z. B. Ried: VdAK, Rüsselsheim: BKK Opel). Informationsgespräche mit den Ersatzkassen haben außerdem ebenfalls bereits stattgefunden.

Beim MQH handelt es sich um ein Projekt auf Basis eines Strukturvertrages nach $\S 73$ a SGB V. Die Zielsetzungen des Netzes sind „netztypisch":

- Durch den Strukturvertrag soll die Versorgungsqualität für den Patienten erhöht werden. Die Versorgung soll auf der jeweils adäquaten medizinischen Stufe erfolgen. Dabei ist in der Regel der Versorgung in der häuslichen Umgebung Vorrang zu geben.

- Die verstärkte kollegiale Zusammenarbeit der niedergelassenen Ärzte soll Behandlungsstrategien zusammenführen und rationale Verordnungen unterstützen. 
- Durch die Kooperation mit allen Leistungserbringern im Gesundheitswesen sollen Rationalisierungsreserven erschlossen werden.

Zur Realisierung dieser Ziele haben sich inzwischen 42 Ärzte (davon 12 Allgemeinmediziner) aus 34 Praxen und 16 Fachgruppen zu einem Netz zusammengeschlossen.

Zu den Aufgaben dieser Ärzte gehört die Einrichtung von Arbeitsgruppen und Qualitätszirkeln. Die Arbeitsgruppen werden sich u. a. mit folgenden Themen befassen:

\section{- Kommunikation Arzt - Patient - Arzt}

- Die Ärzte des MQH geben an die Patienten (vor allem an chronisch Kranke) einen Patientenpaß aus, der die wichtigsten Behandlungsdaten enthält.

- Die Zusammenarbeit wird über ein nach der Dringlichkeit abgestimmtes Überweisungssystem optimiert.

- Kommunikation Arzt - Patient - Krankenhaus

Die Kommunikation mit den Krankenhäusern der Region soll durch schnellen Informationsaustausch zwischen ambulant und stationär tätigen Ärzten verbessert werden.

- Kommunikation Arzt - Arzt

Die Kommunikation zwischen den Ärzten soll durch Nutzung von ISDN und Fax, mittelfristig auch durch den Einsatz eines adäquaten EDV-Systems standardisiert werden.

- Notdienst

Zur Optimierung des ärztlichen Notfalldienstes sind einheitliche Rufnummern in der Region und patientenorientierte Standortwahl der Notdienstzentrale vorgesehen.

- Zusammenarbeit mit anderen Leistungserbringern

Der rationale und rationelle Einsatz therapiebegleitender Maßnahmen soll durch Kooperation mit anderen Leistungsanbietern (z. B. Apotheken, Physiotherapeuten, Pflegediensten, Sanitätshäusern) erreicht werden.

Die Qualitätszirkel werden sich u. a. mit den Themen Pharmakotherapie, Notfallmedizin, Gerätesicherheit in der Praxis etc. befassen. 
Das Modell „Medizinisches Qualitätsnetz Hofheim“ wird umfassend evaluiert werden.

\section{„Frankfurter Arztnetz": Hausarztmodell}

Der AOK-Bundesverband hatte sein Konzept einer hausärztlichen Versorgung Mitte 1994 der Öffentlichkeit präsentiert und - auch durch eigene Veranstaltungen 34 - öffentlich zur Diskussion gestellt. Das AOKHausarztmodell verfolgte zwei zentrale Ziele:

Erstens sollte der Hausarzt als Gatekeeper die Schlüsselposition für die optimale Ausnutzung des Versorgungssystems besitzen. Da ein großer Anteil der Behandlungsanlässe lediglich solche Leistungen erfordert, die auch ein Hausarzt qualifiziert erbringen kann, verhindert eine obligatorische Erstkonsultation dieses Arztes die Inanspruchnahme von nicht notwendigen Leistungen einer höheren Versorgungsstufe.

Zweitens sollte der Hausarzt die Kosten von Behandlungsprozessen senken und deren Rationalität erhöhen, indem er in der Funktion als "Lotse" durch das Versorgungssystem die starke Disintegration der ambulanten Versorgung, die auf die Koordinationsdefizite bei der Arbeitsteilung von Primär- und Fachärzten zurückzuführen ist, reduziert.

Nach einem inhaltlich sehr stark reduzierten Pilotprojekt in Frankfurt wird das Hausarztmodell inzwischen als „Frankfurter Arztnetz“ zu einem Primärarzt- und Praxisnetzmodell weiterentwickelt, welches im Bereich der Bezirksstelle Frankfurt stattfinden soll (hätte stattfinden sollen!). In dem Merkmal des Primärarztes als "Gatekeeper" (wenn auch hier nicht in einer strengen Form) unterscheidet sich dieses Modell auch von anderen Ansätzen.

Aufgrund der Entstehungsgeschichte des Modells steht fest, daß zunächst nur die AOK als Vertragspartner in Frage kommt.

Ziel des Modellprojektes ist eine verbesserte Versorgung von AOKVersicherten durch eine intensive hausärztliche Betreuung und eine verbesserte Zusammenarbeit zwischen Haus- und Fachärzten. Die Verbesserung der Kommunikation und die Einrichtung von Qualitätszirkeln sind ebenso vorgesehen wie die Vermeidung von Krankenhausaufenthalten und die Kooperation mit weiteren Leistungserbringern.

34 z. B. durch die Veranstaltungen der „AOK im Dialog"-Reihe im November 1994, April 1995 etc. 
Zu den Aufgaben des Hausarztes gehören

- die Koordination der medizinischen Versorgung des Patienten. Der Funktion des Primärarztes wird Rechnung getragen, indem der freie Facharztzugang für den Patienten nur auf Ausnahmen (Augenärzte, Frauenärzte, Psychotherapeuten) beschränkt ist.

- die Teilnahme an Qualitätszirkeln und anderen qualitätsverbessernden Maßnahmen,

- die Teilnahme an der technischen Vernetzung der Praxen,

- das Angebot zusätzlicher Leistungen für die teilnehmenden AOKVersicherten:

- individuelles Gesundheitsgespräch (einmal jährlich)

- Terminvergabeservice bei Überweisungen

- Teilnahme an Arzt-Patienten-Abenden (1 - 2 pro Jahr).

Neben diesen Zusatzleistungen soll auch ein "Gesundheitsbuch" (Patientenbuch) eingesetzt werden, aus dem ein Arzt die wesentlichsten Behandlungsdaten entnehmen kann.

Ferner muß ein teilnehmender Arzt gewisse Mindeststandards in Sachen technische Praxisausstattung erfüllen (Fax, Praxissoftware mit ADT-Schnittstelle, EKG, Akutlabor).

Die AOK will ihrerseits weitere begleitende Leistungen anbieten, so z. B. Kurse zur Sekundär- und Tertiärprävention und den „AOK-Gesundheitsberater" als Beratungsleistung bei bestimmten Krankheitsbildern.

Geplant ist die Teilnahme von ca. 400 Ärzten, maximal jedoch 600 Ärzten und etwa 10.000 Patienten.

Das Modellprojekt soll wissenschaftlich evaluiert werden.

\section{Ein weiteres Projekt in Hessen: Gießen}

In Gießen laufen derzeit Sondierungsgespräche mit beiden Kassenarten zu einem Praxisnetz-Projekt „im großen Stil“. Es existiert bereits eine Gesellschaftstruktur in Form einer GmbH. Der Zusammenschluß von ca. 125 Ärzten soll auch ein professionelles Netzmanagement erhalten. Die Kooperation (oder Konfrontation?) mit dem stationären Sektor dürfte sich aufgrund des Vorhandenseins von einem Uni-Klinikum und drei Krankenhäusern auch eher aufwendig gestalten. 


\section{Fazit}

"Qualitätsgemeinschaften“, „Praxisnetze" etc. sind eigentlich die naheliegendste Lösung, die Koordinations- und Kooperationsproblematik in der GKV zu lösen. Dabei zielen derartige Versorgungsformen zunächst darauf ab, die bestehenden Defizite innerhalb der ambulanten vertragsärztlichen Versorgung zu reduzieren, indem die Zusammenarbeit der Ärzte in einem regionalen Rahmen verbessert und durch z. B. Maßnahmen zur Qualitätssicherung der Behandlungsprozeß optimiert wird.

In einer weiteren Ebene kann eine Verbesserung der Kooperation mit anderen Leistungserbringern sowohl aus dem ambulanten, als auch aus dem stationären Bereich den Integrationsgrad der Versorgung erhöhen: Durchorganisierte und "nahtlos“ aufeinander abgestimmte Versorgungsleistungen können zu einem Versorgungsprozeß integriert werden. Durch solche Optimierungsprozesse kann die Versorgung nicht nur qualitativ hochwertiger, sondern auch effektiver gestaltet werden.

Eine wesentliche Voraussetzung für das „Funktionieren“ solcher Netze scheint die Überschaubarkeit zu sein, denn für gute Kommunikationsbeziehungen ist wohl auch die Größe bzw. Anzahl der Mitglieder eines solchen Netzes ausschlaggebend - der einzelne Arzt soll nicht anonym in der Masse verschwinden. Daher ist es auch wichtig, daß solche Ansätze „Von unten“ also „bottom-up" entstehen, weil Ärztegruppen, die sich selbst organisieren - und dies funktioniert vorzugsweise in kleinen überschaubaren Gruppen -, viel eher (gemeinsam formulierte) Ziele akzeptieren und mit dem nötigen Nachdruck verfolgen. Hieran krankt z. B. auch das Hausarztmodell, das als Modell "von oben“ also „top-down“ viel stärker mit Akzeptanzproblemen zu kämpfen hat.

Probleme wird ein solches Netz vor allem mit (partiell) konkurrierenden Leistungsanbietern, also dem stationären Sektor bekommen, zumal hier an dieser Schnittstelle (aus verständlichen Gründen) die individuellen Interessen überwiegen und auch noch weiterhin überwiegen werden, solange die GKV auf sektorbezogenen Anreizsystemen (d. h. Vergütungssystemen) aufbaut.

\section{Zukunftsperspektiven}

- Regionale Praxisnetze zukünftig der „Normalzustand“ in der ambulanten Versorgung?

- Praxisnetze als Einstieg in Managed-Care-Systeme in der GKV?

- Funktionen der KVen/Einkaufsmodelle? 


\section{Integrierte Versorgungsleistungen}

\section{Integration 'Vernetzter Praxen' in die GKV}

Karl-Heinz Schönbach

Auch in der 14. Legislaturperiode gehört eine grundlegende Strukturreform des Gesundheitswesens zu den vorrangigen Zielen einer neuen Bundesregierung. Doch schon das Vorschaltgesetz, mit dem bis dahin die Neuordnungsgesetze der alten Koalition korrigiert und die Ausgabenentwicklung stabilisiert werden soll, wirft die Gräber rückwärtsgewandter Verteilungsdebatten auf. Die Ärzteverbände mobilisieren inre Mitglieder mit der Parole, die Versorgung der Patienten werde gefährdet. Das Klima für eine Diskussion, medizinisch fragwürdige Leistungen und unwirtschaftliche Angebotsstrukturen abzustellen, wird a priori belastet. Wie angesichts der inszenierten Polarisierung eine offene, die Innovationsbereitschaft der Beteiligten aufnehmende Debatte entstehen kann, steht dahin. Zumindest wird aber offensichtlich, daß es eine Flexibilisierung und Vertragsrechte unterhalb der Ebene formierter Verbände geben muß, soll das Gesundheitswesen Reformfähigkeit zurückgewinnen.

Den einzelnen Anbietern im Medizinbetrieb stehen meist nur gleichförmige, ihrem Leistungsvermögen und ihrer Innovationsbereitschaft in keinerlei Differenzierung folgende Vertragsbedingungen mit den Krankenkassen zur Verfügung. Auf dem relevanten regionalen Markt sind selbst untereinander im Wettbewerb stehende Ärzte, Apotheker und Krankenhäuser usw. darauf angewiesen, daß - so es die gesetzlichen Vorgaben überhaupt zulassen - entfernte Mehrheiten einer landesweiten oder bundesweiten Vertreterversammlung neue Vertragsoptionen mit den Krankenkassen - für alle Beteiligten gemeinsam - auch nur in Betracht ziehen. Daß unter diesen Bedingungen der kleinste gemeinsame Nenner das Maß aller Dinge wird, überrascht nicht. Die Innovation des potentiellen Konkurrenten ist eine Herausforderung, die Mehrheiten regelmäßig vermeiden. Im Ergebnis bleibt das Gesundheitswesen hinter dem Modernisierungstempo der Gesellschaft zurück.

Dies gilt um so mehr, als beständig steigende Anbieterzahlen, insbesondere bei den niedergelassenen Ärzten, die Verteilungsspielräume verringern und den Konkurrenzdruck erhöhen: Das Honorarvolumen je Arzt stagniert, obwohl das Honorarvolumen der Krankenkassen Jahr für Jahr steigt. Da sich die Ärzte im Wettbewerb in erster Linie durch den Ausstattungsstand ihrer Praxen differenzieren können, nimmt der Fixkostenanteil zu. Werden dann sinkende Verteilungspunktwerte mit zunehmen- 
den Einzelleistungen beantwortet (Hamsterrad-Effekt), steigen auch die variablen Kosten an. Aufgrund dieses Dilemmas erzeugen die KVen eine Honorarreform nach der anderen und erhöhen die Unsicherheit der Vergütung einmal mehr, statt den Weg für strukturelle Reformen frei zu machen und neue Kooperationsformen entschieden zu fördern. Neben zahlreichen medizinischen Vorteilen, die auch in der zunehmenden Differenzierung ärztlicher Spezialisten liegen, würde eine Kooperation ökonomische Vorteile eröffnen (Gerätepool, Helferinnen-Pool). Sie würde darüber hinaus bei überschaubaren Praxisnetzen durch die Kollegialität in der Gruppe nichtrationale Hamsterrad-Effekte vermeiden. Gleichwohl leisten die meisten KVen über einige Ankündigungen hinaus bisher kaum praktisch relevante Unterstützung für Praxisnetze. Sie halten vielmehr unbeirrbar an der Einzelarztpraxis und dem auf diesem Typus aufbauenden, engmaschigen Geflecht kassenarztrechtlicher Restriktionen fest.

Das Ergebnis dieser gesundheitspolitischen Konzeption ist ein hektischer, von ritualisierten Auseinandersetzungen geprägter Prozeß, der vielfach Innovation und Leistung "bestraft" und beträchtliches politisches Legitimationspotential verbraucht. Stillstand und Fehlentwicklungen im Gesundheitswesen werden der Gesundheitspolitik zugeschrieben und mit zunehmender Apathie und Verweigerung der Akteure beantwortet. Abrechnungsskandale und Proteste der Ärzte und anderer Gesundheitsberufe mehren sich. Der Gesetzgeber steht mithin vor der Alternative, das staatliche Regulierungs- und Interventionssystem noch weiter zu verästeln oder dem Wettbewerb größere Spielräume zu geben.

\section{Gesundheitsziele leisten Orientierung im Wettbewerb}

Allerdings ist das in Gesetzen und Verordnungen über Jahrzehnte hinweg formulierte Regulierungssystem des Gesundheitswesens inzwischen für viele selbst zu einer "black box" geworden. Ungeachtet der "dynamischen Komplexität" des Gesundheitswesens, das vielstufigen und vielfältigen Regelungen und Einflüssen unterliegt, wurden in immer kürzeren Abständen Gesetzgebungsverfahren und Einzelmaßnahmen auf den Weg gebracht, die zum Teil paradoxe Züge zeigen und deren offensichtliche Umsetzungsdefizite selbst wiederum Gesetzgebungsbedarf hervorrufen. Die Verknüpfung staatlicher, verbandlicher und wettbewerblicher Steuerungselemente und entsprechender Anpassungsund Ausweichreaktionen innerhalb und über die Stufen des föderalen Systems hinweg lassen die Wirkung staatlicher Einzelmaßnahmen inzwischen nur noch bedingt kalkulierbar erscheinen. 
Die raunende Skepsis, die Anbietervereinigungen den Chancen des Wettbewerbs als vermeintliche „Risiken“ entgegenbringen, müßte dieser Sicht nach der "black box" einer interventionistischen Gesundheitspolitik gelten, die sich auf ein nahezu undurchdringliches Geflecht verschiedener Steuerungsebenen richtet. Diese Politik dekretiert „top down“ für alle Versicherten bestimmte DM-Beträge pro Packung als Zuzahlung bei Arzneimitteln, ringt aber vergebens um die Verbesserung der Diabetikerversorgung. Sie suspendiert für alle Versicherten „top down“ metallkeramische Verblendungen im Seitenzahnbereich, verfehlt aber bis heute ein qualitätsgesichertes Mammographie-Screening. Sie verkürzt für alle Versicherten „top down" die Dauer von Reha-Maßnahmen und scheitert doch, den Stellenwert der Rehabilitation gegenüber der Kuration angesichts des geänderten Krankheitspanoramas zu erhöhen.

Über die notwendige Globalsteuerung hinaus sollte die Gesundheitspolitik zielbildend statt interventionistisch handeln können. Daher plädiert der BKK Bundesverband für eine weitere Akzentverschiebung zugunsten wettbewerblicher Spielräume, wobei der Erfolg einer entsprechenden Gesundheitspolitik an ihren Ergebnissen gemessen werden sollte. Ergebnisse sind - über die ökonomischen Stabilitätsziele hinaus - an der Erreichung von Gesundheitszielen zu bewerten. Nur sofern der Wettbewerb - als Instrument der Gesundheitspolitik-diesen Zielen besser dienen kann als andere Instrumente, kann er tatsächlich größere Priorität beanspruchen. Diese Auffassung schließt ein, daß die Spielräume dazu bisher in hohem Maße ungenutzt geblieben sind.

Als vordringliche Gesundheitsziele nennt der Sachverständigenrat für die Konzertierte Aktion z. B. die Verbesserung der genetischen Beratung, der Immunlage, Vermeidung von Alkohol-Embryopathie, Verbesserung der Versorgung chronisch Kranker (Asthma, Diabetes als Beispiel), Bekämpfung des Bluthochdrucks, des Medikamentenmißbrauchs, chronischer Niereninsuffizienz, Lebererkrankungen usw.

Eine so akzentuierte Gesundheitspolitik bildet auf europäischer, nationaler, landesweiter und regionaler Ebene epidemiologisch fundierte $\mathrm{Ge}$ sundheitsziele heraus und leistet damit eine maßgebliche Orientierungsfunktion für die Akteure der gesundheitlichen Versorgung. Diese Orientierungs- und Motivationsfunktion ist nicht mit einer bürgerfernen, von Technokraten wissenschaftlich legitimierten Kommandostruktur der Gesundheitspolitik zu verwechseln. Eine moderne, dezentrale und wettbewerbliche Gesundheitspolitik initiiert Netzwerke integrierter Versorgung, in denen Patienten, Ärzte, medizinische Hilfsberufe und Krankenhäuser mit den Krankenkassen und den sozialen Diensten kooperieren. Sie unterstützt und zertifiziert medizinische Leitlinien und schlägt Quali- 
tätsindikatoren vor, anhand derer z. B. Praxisnetze Gesundheitsziele operationalisieren. Dieser Prozeß ist notwendigerweise partizipativ und pluralistisch. Er kommt der Suche nach bedarfsgerechten wirtschaftlichen Lösungen und ihrer „ständigen Verbesserung“ gleich, die nur in einer neuen Wettbewerbskultur gelingen kann. Er erneuert damit nach der Einführung der freien Kassenwahl im übrigen auch die legitimatorischen Grundlagen einer gegliederten Krankenversicherung.

\section{Betriebskrankenkassen entwickeln integrierte Versorgung}

Die Betriebskrankenkassen wollen in Kooperation mit den Gesundheitsberufen eine langfristig glaubwürdige, an Gesundheitszielen orientierte Produktpolitik betreiben. Dieses Konzept erfordert allerdings management- und kooperationsfähige Ansprechpartner vor Ort. Deshalb initiieren und unterstützen auch die Betriebskrankenkassen neue Formen integrierter Versorgung. Eine integrierte bzw. integrativ ausgerichtete Produktpolitik bezieht die maßgeblichen Akteure innerhalb der Versorgungskette auf der Grundlage umfassender Information in eine zielgerichtete Kommunikation und Kooperation ein. Es wird ein „Unternehmenskonzept" entwickelt, in dem die Beteiligten gemeinsam Gesundheitsziele und ökonomische Ziele vereinbaren. Nach dem Stand der Diskussion müssen grundsätzlich 6 Voraussetzungen geschaffen werden:

- Verbesserung der Kommunikation und Kooperation zwischen den Akteuren in der Versorgungskette, insbesondere aber auch zwischen Kostenträgern und Leistungsanbietern.

- Vereinbarung von Gesundheitszielen und Behandlungsleitlinien auf der Grundlage von Konsensuskonferenzen.

- Einführung von kombinierten Budgets, um die zielorientierte und wirtschaftliche Zusammenarbeit der Akteure zu fördern.

- Vermeidung von Doppelaktivitäten und Nutzung komparativer Vorteile der Beteiligten unter Einbezug ausgewählter externer Partner.

- An Qualitätskriterien angepaßte Dokumentation und Bewertung der Behandlungsprozesse und Gesundheitsergebnisse.

- Dokumentation und Bewertung der erreichten Gesundheitsziele, um die gemeinsamen Verfahrensgrundsätze weiterzuentwickeln.

Zur Realisierung dieser Strategie hat der BKK Bundesverband mit Kassenärztlichen Vereinigungen das Konzept „vernetzter Praxen mit kombinierten Budgets" entwickelt (BKK-Praxisnetz) und gemeinsam mit der Techniker Krankenkasse eingeführt. Es ist das bisher einzige Konzept, 
mit dem eine ökonomische Gesamtverantwortung vernetzter Praxen erreicht wird und gleichzeitig

- eine risikoäquivalente Bereinigung der kassenärztlichen Gesamtvergütung erfolgt,

- tatsächlich realisierte Ersparnisse im Krankenhausbereich und bei veranlaßten Leistungen (z. B. Arzneimittel) dem Modellansatz nach und nicht erst durch ggf. strittige aufwendige Berechnungen im nachhinein gemessen werden,

- und eine entsprechende Beteiligung an den Rationalisierungsgewinnen nicht nur für die Ärzte, sondern auch für die Versicherten erfolgt.

Zum Beispiel hatten sich rund 14.000 Versicherte aller Altersgruppen der BKK/TK bis Ende 1998 in das Berliner Praxisnetz mit rund 450 Ärzten eingeschrieben. Weitere 45.000 Versicherte werden von den Leistungsangeboten des Praxisnetzes indirekt erfaßt. Der Netzpunktwert lag bereits im ersten Quartal, in dem das kombinierte Budget eingeführt wurde, bei 11,3 Pfennig.

Die Relevanz dieser Strategie wird sich künftig in zweierlei Hinsicht erhöhen. Einerseits wird die Reichweite integrierter Netzwerke innerhalb der Versorgungskette durch den Einbezug medizinischer Hilfsberufe und stationärer Versorgungsangebote zunehmen, andererseits wird die Managementdichte innerhalb der Netzwerke durch die Organisation medizinischer und ökonomischer Gesamtverantwortung gesteigert:

- Gesundheitsziele und darauf aufbauende Behandlungsleitlinien und Qualitätsindikatoren beschränken sich gerade bei den im Mittelpunkt stehenden multimorbiden und chronisch kranken Patienten nicht auf Hausarzt oder Facharzt. So wird die Integrationswirkung eines Hausarztmodells hinter der eines Praxisnetzes zurückbleiben. Aber auch das Praxisnetz wird die Kooperation zu Pflegediensten, Therapeuten, Krankenhäusern, Fachkliniken und Reha-Einrichtungen aufbauen müssen (vertikale Kooperation). Weil dabei nicht nur eine medizinische, therapeutische und pflegerische Abstimmung erfolgen muß, sondern auch deren ökonomische und vertragliche Verknüpfung, sind die Krankenkassen als verbindendes Element und im Hinblick auf die Unterstützung eines umfassenden Fallmanagements der Ansprechpartner per se.

- Nimmt die Managementdichte innerhalb der Netzwerke zu, entstehen Vorläufer moderner Unternehmensformen im ambulanten (und stationären) Bereich. Sie bilden alternative Versorgungsangebote mit 
spezifischen Vorteilen und Qualitätsmerkmalen. Es muß aber sichergestellt sein, daß für die Patienten im regional relevanten Markt identifizierbare Alternativen bestehen. Wer die freie Arztwahl hochhält, wird die freie Wahl der Netze nicht geringschätzen können. Schließlich hat der Gesetzgeber die Forderung nach flexiblen Vertragsstrukturen und innovativen Versorgungsformen auch insofern als „Wahlmodell“ aufgegriffen, als er nicht nur für die Ärzte, sondern auch für die Versicherten von einer freiwilligen Wahl ausgeht. Daher kommt man erstens bei Wahlmodellen bzw. für die nächste Legislaturperiode angestrebten Wahltarifen nicht an der Beitrittserklärung der Versicherten vorbei. In den meisten Modellen gibt es bisher nur den Beitritt der Ärzte - die Versicherten werden nicht einmal gefragt. Und daher darf zweitens die Kooperation der Ärzte oder auch der Krankenhäuser (horizontale Kooperation) zumindest die legale Mißbrauchsvermutung einer marktbeherrschenden Stellung des Gesetzes gegen Wettbewerbsbeschränkungen nicht überschreiten. Auch dies gibt systematisch größere Spielräume für eine Kooperation der Versorgungsnetzwerke mit den Krankenkassen und Kassenarten als mit monopolistischen Anbietervereinigungen.

Gegen eine auf die Krankenkassen zentrierte Kooperation im Rahmen integrierter Versorgung ist gelegentlich eingewandt worden, die Krankenkassen könnten im Wettbewerb nur an der Konkurrenz um „gute Risiken" interessiert sein. Sie betrieben einen Wettbewerb um Gesunde. Es sei dagegen nicht zu erwarten, daß Krankenkassen Z. B. optimale Versorgungskonzepte für chronisch Kranke entwickeln und einführen würden. Diese These ist nicht nur durch die Praxis vielfach eindrucksvoll widerlegt. Sie verkennt auch mutwillig die Anreizwirkungen des Risikostrukturausgleichs. Im übrigen können die Krankenkassen im Wettbewerb langfristig nur durch eine glaubwürdige Produktphilosophie bestehen. Krankenkassen, die nicht als Problemlöser für Kranke auftreten und den Lauf der Versicherten durch das Gesundheitssystem unter Qualitäts- und Kostengesichtspunkten beeinflussen, verspielen das Image und die Grundlagen ihres Unternehmens. Ein solches Verhalten bedeutet bei Unternehmen, die im Wettbewerb untereinander in der Öffentlichkeit stehen, den Einstieg in den Marktaustritt. Dies ist eine gesundheitspolitisch gewollte Sanktion von Marktversagen, der monopolistische Anbietervereinigungen nicht ausgesetzt sind.

So hat denn auch die Diskussion um Risikoselektion beim Versicherungsschutz in keiner Weise die Relevanz der Risikoselektion in der Gesundheitsversorgung. In einem in zahlreiche Versorgungsstufen gegliederten Angebotssystem, dessen Elemente einerseits rechtlich und ökonomisch gegen Kooperation abgeschottet, das andererseits aber für die 
Verweisung und Überweisung von Patienten völlig offen gestaltet ist, findet Risikoselektion unter dem Schutz medizinisch individualisierter, nicht an Behandlungsleitlinien und Qualitätskriterien orientierter Entscheidungen nicht nur im Ausnahmefall statt:

- Ein meist zu wenig beachtetes Problem liegt in der „Internalisierung von Erträgen“ bzw. der „Externalisierung von Kosten“. Zum Gebietsarzt und von dort weiter in den stationären Sektor wird längst nicht nur aufgrund der medizinisch gebotenen Kooperation überwiesen, sondern auch aufgrund einer "Risikoselektion“ nach dem Deckungsbeitrag für die Praxen. Die für eine „punkteintensive“ Dauerbehandlung zumindest dem Honorarverteilungsmaßstab (HVM) und der Wirtschaftlichkeitsprüfung nach unkritischen Patienten-zum Beispiel solche mit definierten Wirbelsäulenbeschwerden-bleiben im eigenen Praxiskreislauf, während Patienten mit unklaren, z. B. psychosomatisch bedingten Beschwerden, die kein "punkteintensives“ und doch gleichzeitig zuwendungsarmes Handeln erlauben, mitunter in den externen Kreislauf gelenkt werden.

- Bei Behandlungsleitlinien unter Einschluß medizinischer Überweisungskriterien hat Deutschland im internationalen Vergleich einen erheblichen Rückstand. Allen Gesetzen zum Trotz sind bisher nicht einmal die Grenzen zwischen der hausärztlichen und der gebietsärztlichen Versorgung inhaltlich definiert. Darüber hinaus hat z. B. die massive Ausweitung ambulanter Operationen bisher kaum eine Entlastung des stationären Sektors gezeigt. Offenbar werden in nicht zu vernachlässigendem Umfang "selektiv“ minderschwere, krankenhausuntypische Fälle ambulant operiert.

- Unter Budgetbedingungen haben Krankenhäuser Patienten gegen Jahresende abgewiesen, ohne auch nur den Ausgleich von Abteilungsbudgets innerhalb des Hauses zu nutzen. Inzwischen wird teilweise nach Fallpauschalen abgerechnet. Dadurch ist die Verweildauer drastisch gesunken. Patienten werden allerdings hier und da trotz bestehendem Bedarf der Akutbehandlung vorzeitig in RehaKliniken verlegt.

- Und schließlich hat die Diskussion um einen neuen EBM auf der Grundlage betriebswirtschaftlicher Kalkulationen gezeigt, wie weit die einzelwirtschaftliche Ökonomisierung der Gesundheitsversorgung fortgeschritten ist. Ist da Risikoselektion ein Fremdwort? Zahlreiche Arztgruppen machen freimütig deutlich, daß sie den Behandlungs- 
umfang einschränken könnten, wenn ihre Vergütungsanliegen nicht erfüllt werden.

Kurzum: Die versuchte Abwertung des Kassenwettbewerbs ist interessengeleitet. Fehlentwicklungen in der Startphase der freien Kassenwahl sind inzwischen bedeutungslos. Der Wettbewerb entwickelt sich gerade auch im Hinblick auf Innovationen in der Gesundheitsversorgung in die gesundheitspolitisch gewünschte Richtung.

Die Entwicklung neuer Versorgungsformen bedarf eigenständiger, in der Versorgungskette über die Grenzen der ambulanten Versorgung hinausgehender Vertragsoptionen der Krankenkassen im Sinne einer integrierten Versorgung.

Zur Einführung neuer Versorgungsformen hat der Gesetzgeber die Regelungen über Modellvorhaben nach $\S \S 63 \mathrm{ff}$. SGB V geschaffen. Er hat diese Möglichkeiten jedoch - soweit die ambulante Versorgung tangiert ist - auf Verträge mit den Kassenärztlichen Vereinigungen verengt und den Kassenärztlichen Vereinigungen mit Strukturverträgen nach $\S 73$ a SGB $V$ parallel eine Vertragsgrundlage auf den ambulanten ärztlichen Bereich beschränkt eingeräumt.

Seit Inkrafttreten des GKV-Neuordnungsgesetzes konnten die Spitzenverbände der Krankenkassen mit der Kassenärztlichen Bundesvereinigung die geforderten bundesmantelvertraglichen Regelungen weder für die Grundlagen zur Durchführung von Modellvorhaben nach § 63 SGB V noch zur Durchführung von Strukturverträgen nach $\S 73$ a SGB V schließen. Insbesondere Regelungen über das Zustandekommen von Modellvorhaben sollen über unklare, weit über die gesetzlichen Bestimmungen hinausgehende Restriktionen behindert werden. Aber auch die seit Jahresfrist verhandelten Rahmenvereinbarungen für Strukturverträge kamen nicht zustande. Vielmehr wenden sich die Kassenärztlichen Vereinigungen inzwischen vehement gegen „selektive Verträge“ und reklamieren ihr Monopol. Geworben wird mit der These, bessere Qualität innerhalb von Erprobungen müsse allen Versicherten zugute kommen. Dies verkennt völlig, daß es im Gesundheitswesen kein Patent auf Innovationen gibt, erfolgreiche Verträge nachgeahmt werden und die Zeit in der gesetzlichen Erprobungsfrist von 8 Jahren nicht stehen bleibt. Wettbewerb ist kein Intermezzo. Mit dieser Auffassung fällt die institutionalisierte Ärzteschaft hinter den Stand des NOG zurück.

Im Ergebnis bleiben Vertragsrechte für den einzelnen Arzt oder Arztgruppen innerhalb und außerhalb von Modellvorhaben ausgeschlossen. Durch die überragende Marktstellung der Anbietervereinigungen wird 
damit auch die Nachfragerfunktion der Krankenkassen im Gesundheitswesen behindert. Der Nutzen des Wettbewerbs, der den Versicherten auf dem Versicherungsmarkt zugute kommt, bleibt den Patienten in der Gesundheitsversorgung weithin verwehrt.

\section{Folgerungen für eine funktionsfähige Wettbewerbsordnung}

Eine moderne Gesundheitspolitik, die sich an Gesundheitszielen orientiert und auf neue integrierte Versorgungsformen stützt, wird sich auf der Grundlage strikter Rahmenvorgaben konzeptionell der Funktionen des Wettbewerbs bedienen. Dabei ist der Wettbewerb nicht das alleinige, aber doch ein zentrales Steuerungsinstrument. Die Tradition der deutschen Gesundheitspolitik, die der Selbstverwaltung immer schon große Freiräume eingeräumt hat, bietet exzellente Voraussetzungen für diese Modernisierungsstrategie. Jedenfalls waren die konzeptionellen Sprünge für die Gesundheitspolitik in den USA oder der Schweiz z. B. weitaus größer. Dennoch sind gerade diese Länder in der Entwicklung voraus. Die Herausforderung für die deutsche Gesundheitspolitik besteht nun im wesentlichen darin, die Innovationsblockaden der Selbstverwaltung durch funktionalen Wettbewerb aufzulösen.

Aus Sicht des BKK Bundesverbands sind für eine solche Modernisierungsstrategie vor allem drei Schritte erforderlich:

a) Die Weiterentwicklung der Grundlagen medizinischer Transparenz über die ökonomische Transparenz hinaus durch die gemeinsame Selbstverwaltung.

b) Die Gewährung von Vertragsrechten über die landesweiten Anbietervereinigungen hinaus auf der regionalen Ebene in Verbindung mit Wettbewerbsregeln zum Ausschluß marktbeherrschender Stellungen.

c) Die Ergänzung der Rechtsaufsicht im Gesundheitswesen um eine Wettbewerbsaufsicht, die sich auch auf neue Versorgungsformen erstreckt.

\section{a) Gemeinsame Grundlagen}

Die entscheidende Voraussetzung für einen funktionsfähigen, an Gesundheitszielen orientierten Wettbewerb ist hinreichende „Markttransparenz". Der Transparenz dienen Standards und anerkannte Methoden, mit denen die Vergleichbarkeit medizinischer Konzepte hinsichtlich der erreichten Gesundheitsziele und hinsichtlich der Qualität der Versorgung erhöht wird. Dies ist eine "einheitliche und gemeinsame“ Aufgabe der 
Selbstverwaltung im Gesundheitswesen, in deren Zentrum heute der Bundesausschuß der Ärzte und Krankenkassen steht.

Im Bundesausschuß der Ärzte und Krankenkassen fließen vielfältige praktische Erfahrungen und wissenschaftliche Erkenntnisse zusammen. Seine Aufgabe besteht nicht zuletzt darin, therapeutische Verfahren nach Stufen der Evidenz und diagnostische Methoden nach ihrer Aussagekraft zu bewerten. In seinem Umfeld arbeiten die Bundesärztekammer, die Kassenärztliche Bundesvereinigung und die Spitzenverbände der Krankenkassen an der Beurteilung und Zertifizierung medizinischer Leitlinien sowie an der Bewertung von Qualitäts- und Ergebniskriterien. Diese Entwicklungen, mit denen der Rückstand im Hinblick auf die medizinische Transparenz und Qualitätssicherung im internationalen Vergleich aufgeholt wird, erleichtern die rationale Beurteilung medizinischer Prozesse im Gesundheitswesen. Sie gefährden gleichwohl nicht die Pluralität in Therapie und Versorgung, sondern sichern sie gegenüber Polypragmasie und strukturieren die medizinische Arbeitsteilung.

Diese Arbeit des Bundesausschusses und korrespondierender Institutionen sollte unter Einbezug der medizinischen Fachgesellschaften auf den stationären Sektor erstreckt werden. Auch für den stationären Bereich ist die Bewertung von Verfahren und Methoden nach ihrer Evidenz sowie die Zertifizierung von Methoden zur Qualitätssicherung und Outcome-Indikatoren im Sinne der Transparenz in hohem Maße erforderlich. Die Forschungsfunktionen der Krankenhäuser bleiben unberührt.

Angesichts der Vielfalt und Bedeutung der Aufgaben sollte der so ergänzte Bundesausschuß aus Sicht der Betriebskrankenkassen institutionell von der Geschäftsführung einer Partnerorganisation verselbständigt und in höherem Maße professionalisiert werden. Damit ließe sich das Tempo der bereits erzielten Fortschritte weiter steigern.

Die gewonnene Transparenz erleichtert die Formulierung alternativer Versorgungsformen, die Krankenkassen ihren Versicherten auf vertraglicher Grundlage mit den Anbietern zur Auswahl stellen können. Gegen unterschiedliche Verträge der Krankenkassen gerichtete Formeln wie „Die Medizin ist unteilbar" sind interessengeleitet: Wer die freie Arztwahl und die Therapiefreiheit hochhält, wer zu Recht selbst die Pluralität von Leitlinien reklamiert und die Ausbreitung neuer Versorgungsstrukturen als Suchprozeß versteht, der wird mit der Formel von der „Unteilbarkeit der Medizin" nicht erfolgreich Monopole rechtfertigen können.

Ebenso wie in medizinischer Hinsicht durch Qualitätsstandards die Transparenz gesichert wird, ist sie in wirtschaftlicher Hinsicht zu sichern. 
Dadurch werden zudem gemeinsame Wettbewerbsbedingungen definiert. Dies gilt insbesondere für die Festlegung von Leistungskomplexen als Grundlage für die Vergütung, für die Festlegung von Fallpauschalen, die Maßstäbe der allgemeinen Wirtschaftlichkeitsprüfung einschließlich der Richtgrößen usw. Auch dies sind aus Sicht der Betriebskrankenkassen weiterhin „einheitliche und gemeinsame“ Aufgaben der gemeinsamen Selbstverwaltung im Gesundheitswesen.

Schließlich bleibt der Katalog von Kernleistungen der GKV „einheitlich und gemeinsam" festzulegen, während die Satzungsleistungen von den Krankenkassen individuell gestaltet werden. Eine Differenzierung des Katalogs der Kernleistungen würde einen sich aufschaukelnden Leistungswettbewerb zur Folge haben, der alle fiskalischen Restriktionen ad absurdum führte. Hierin unterscheidet sich das Gesundheitswesen von anderen Bereichen. Verwiesen sei an dieser Stelle nur auf die Fortschrittsfalle der Medizin.

\section{b) Wettbewerbsregeln}

Forderungen etwa der Wirtschaftsweisen oder der Monopolkommission, insbesondere einzelnen Ärzten und Arztgruppen sollten gegenüber den Krankenkassen generell Vertragsrechte eingeräumt werden, scheinen auch im Rahmen der hier zur Diskussion gestellten Positionen der Betriebskrankenkassen absolut folgerichtig. Die Monopolkommission war bei ihrem jüngsten Gutachten davon ausgegangen, dies forciere von ihr angemahnte Ansätze von „Managed Care“. Die gesundheitspolitisch gewünschte "vertikale Kooperation“ entlang der Versorgungskette bleibt allerdings soweit und solange illusionär, wie die jeweiligen Versorgungsstufen regional keine management- und kooperationsfähigen Ansprechpartner aufweisen. Statt Wettbewerbsprozesse auf der Grundlage bestehender atomistischer Angebotsstrukturen in Gang zu setzen, böte das Anknüpfen an Formen der kooperativen Selbstorganisation der Anbieter nicht zuletzt Akzeptanzvorteile. Solche Formen entstehen zur Zeit mit Praxisnetzen.

Praxisnetze leisten eine fachübergreifende Vernetzung in der ambulanten ärztlichen Versorgung (horizontale Kooperation). Da sie eine medizinische und ökonomische Gesamtverantwortung zum Ziel haben, bieten sie eigenständige, der bestehenden Einzelarztpraxis fremde Leistungsund Vertragsinhalte. Vertragsrechte diesem Bereich vorzuenthalten, führt zur erforderlichen Dynamik bei vertretbaren Transaktionskosten. Es entstehen neue Unternehmensformen, für die im Interesse der Wahlfreiheit der Versicherten/Patienten und des Wettbewerbs zwischen Praxisnetzen Regeln entwickelt werden müssen. 
Aus Sicht des BKK Bundesverbandes sollten regional kooperierende Anbieter (Ärzte, Arztgruppen) und Nachfrager (Krankenkassen, Verbände der Krankenkassen) eigenständige Vertragsrechte ausüben können, soweit sie keine marktbeherrschende Stellung haben, für die im Gesetz gegen Wettbewerbsbeschränkungen die Legalvermutung mißbräuchlicher Marktmacht gilt. Diese Legalvermutung gilt ab einem Marktanteil $\geq$ $33 \frac{1}{3} \%$. Für die übrigen Anbieter bleibt es - soweit und solange keine neuen Versorgungsformen gebildet werden-bei dem bestehenden Vertragssystem.

Als Voraussetzung dafür hätten die Partner der Selbstverwaltung die Mindestkriterien (Fachgruppenmix, Rechtsformen, Managementstandards usw.) für regionale Kooperationsformen (z. B. Praxisnetze) festzulegen und die Bereinigung der Gesamtvergütungen um die entsprechenden regionalen Verträge zu regeln. Der Gesetzgeber hätte dieser Aufgabe der Selbstverwaltung mit der Festlegung von Ersatzvornahmefristen Nachdruck zu verleihen und im übrigen die gesetzliche Befristung neuer Versorgungsformen aufzuheben. Hiervon ginge ein nachhaltiger Modernisierungsimpuls für das deutsche Gesundheitswesen aus.

\section{c) Wettbewerbsaufsicht}

Die bestehenden gemeinsamen Wettbewerbsgrundsätze der Aufsichtsbehörden für die gesetzliche Krankenversicherung vom 3. November 1994 erstrecken sich nur auf die Krankenkassen. Sie sprechen zwar das Gesetz gegen unlauteren Wettbewerb (UWG) an, nicht jedoch das Gesetz gegen Wettbewerbsbeschränkungen (GWB). Soweit die Krankenkassen im Rahmen sozialrechtlicher Normen "hoheitliche" Aufgaben erfüllen - z. B. mit den KVen Gesamtverträge schließen -, ist dieser Ausschluß des GWB notwendig und gerechtfertigt. Dies gilt aus Sicht der Betriebskrankenkassen bei neuen "wettbewerblichen“ Aufgaben - z. B. bei Wahltarifen mit integrierter Versorgung - nicht in gleicher Weise. Die Angebotsdefinition neuer Versorgungsformen ist aus Sicht der Versicherten/Patienten ungleich stringenter als die globaler Gesamtverträge mit den Kassenärztlichen Vereinigungen. Daher sind für die Versicherten/Patienten mehrere Angebote unverzichtbar. Dies erfordert einerseits die systematische Verhinderung marktbeherrschender Stellungen und andererseits unbeschränkte Informationsrechte der Versicherten:

- Die Versicherten müssen sich über im Wettbewerb stehende Angebote möglichst vollständig informieren können. Entsprechend sind für regionale Kooperationsformen und ihre Verträge Werbeverbote auf- 
zuheben, die an das ärztliche Berufsrecht anknüpfen. Ausreichend ist eine strikte Anwendung des UWG.

- Zudem sollten die Versicherten nicht nur vom medizinischen, sondern auch vom wirtschaftlichen Erfolg regionaler Kooperationen profitieren. Entsprechend sollen für Wahltarife nach dem Versicherungsprinzip differenzierte Beitragssätze zugelassen werden.

Eine Anwendung des GWB durch die Aufsichtsbehörden im Hinblick auf die Abgrenzung relevanter Märkte und die Erfassung von Behinderungsund Konzentrationsstrategien könnte durch die Zusammenarbeit mit dem Bundeskartellamt und den Landeskartellämtern unterstützt werden. In jedem Fall aber ist durch eine nahtlose Abstimmung der Bundes- und Landesaufsichtsbehörden auszuschließen, daß für unterschiedliche, in ihrem jeweiligen Zuständigkeitsbereich tätige Krankenkassen bestehende Regelungen unterschiedlich praktiziert werden. Nach Auskunft der Krankenkassen ist dies bereits heute ein nicht zu vernachlässigendes Problem. 


\section{Die Pharmaindustrie als Vertragspartner}

Ulrich Vorderwülbecke

Schon seit einigen Jahren bieten die neuen Versorgungsformen ein beliebtes Diskussionsfeld, wobei man sich allerdings heute fragen mag, ob es sich dabei um ein modernes oder um ein modisches Thema handelt. Nicht nur dem externen Betrachter fällt auf, daß viele Begriffe durch den Raum schwirren, deren Definition und Inhalt oft unklar sind. Da neue Versorgungsformen in erster Linie in den USA praktiziert werden und von dort aus inzwischen auch auf europäische Länder ausstrahlen, haben sich auf diesem Gebiet naturgemäß zahlreiche Anglizismen eingebürgert. Zusätzlich erschwert wird die Kommunikation noch dadurch, daß oft Abkürzungen verwendet werden, die nur Insidern verständlich sind. So bedeutet DM nicht Deutsche Mark, sondern Disease Management, MC nicht Musikcassette, sondern Managed Care, EPO nicht Erythropoeitin, sondern Exklusive Provider Organisation. Allerdings sind auch in Deutschland deklaratorische Unschärfen zu verzeichnen. Das Sozialgesetzbuch umreißt Modellvorhaben und Strukturverträge. In der Realität werden freilich Vereinbarungen und Kooperationsformen mit diesen Begriffen belegt, ohne den gesetzlichen Regelungen Rechnung zu tragen. Ein bemerkenswerter Wildwuchs macht sich auch unter dem Titel „Praxisnetz" breit, wobei lästerliche Zungen behaupten, neuerdings reklamiere schon jeder ärztliche Stammtisch für sich, ein Praxisnetz zu sein. Wenn man vor diesem Hintergrund nun behauptet, gerade auf dem Sektor der neuen Versorgungsformen gebe es eine babylonische Sprachverwirrung, so ist das natürlich übertrieben; ein wahrer Kern steckt jedoch darin.

Abseits aller Definitionen und terminologischen Feinheiten läßt sich jedoch konstatieren, daß bei vielen Akteuren im Gesundheitswesen ein großes Interesse an neuen Versorgungsstrukturen besteht.

Auch der VFA ist für neue Versorgungsformen aufgeschlossen. Die forschenden Arzneimittelhersteller stehen für Innovation. Der Innovationsgedanke ist aber nicht auf das Produkt Arzneimittel beschränkt, sondern bezieht sich in weiterem Sinne auch auf neue und moderne Versorgungsformen. Dementsprechend ist bei VFA-Mitgliedsunternehmen das Interesse für neue Versorgungsformen groß, zumal manche Unternehmen in anderen Ländern bereits aktiv in neuartigen Versorgungsstrukturen mitwirken. Insofern sind neue Versorgungsformen für VFA-Firmen nicht nur von akademischem Interesse, sondern vielmehr von ganz praktischer Bedeutung. 
Das übergeordnete Ziel neuer Kooperationsformen besteht darin, effizientere Versorgungsstrukturen zu erreichen. Die Relation von Qualität und Kosten der Versorgung soll verbessert werden. So wird etwa in den USA versucht, die Versorgungsqualität und die therapeutischen Ergebnisse für die Versicherten zu verbessern, indem speziell auf Krankheiten und Patienten zugeschnittene Managementtechniken verwendet werden, die möglichst alle Aspekte einer Krankheit berücksichtigen. Dabei bilden klinische und ökonomische Kriterien die wesentlichen Entscheidungsgrundlagen.

Generell gehen neue Versorgungsformen von einem breiten Ansatz aus. In das Aktionsbündel fließen ein:

- Ursachen, Merkmale und Kostenfaktoren einer Krankheit besser zu verstehen,

- Diagnose und Therapie noch stärker am Krankheitszustand und -verlauf zu orientieren,

- den Einsatz von Ressourcen ganz gezielt auf die qualitativ besten und kosteneffektivsten Interventionsmodalitäten auszurichten,

- ein effektives Daten- und Ergebnismanagement zu betreiben.

Aus einer anderen Perspektive betrachtet wird darauf abgestellt, die verschiedenen Stufen in einer Behandlungskette zu optimieren. Dies beginnt bei der Krankheitsaufklärung und Prävention, setzt sich bei der Diagnosestellung fort, wirkt bei der Therapieeinstellung mit, gilt für die Therapietreue und mündet schließlich in die Therapiekontrolle ein. Somit bezieht sich der Optimierungsansatz sowohl auf die einzelnen Schritte als auch auf inre Verknüpfung und letztlich auf den gesamten Prozeß.

Eine Bestandsaufnahme der neuen Versorgungsformen zu wagen, wäre ein geradezu verwegenes Unterfangen. Zwar besteht landläufig der Eindruck, die USA seien das Paradies für neue Versorgungsformen, doch gießen manche Beobachter durchaus Wasser in diesen Wein. Obgleich in den USA neue Versorgungsformen seit vielen Jahren praktiziert werden und mehr als 50 Unternehmen Programme laufen haben, gilt dort die „Beziehungskiste“ der Beteiligten als noch nicht geklärt. Schon wegen der komplexen Interaktionen erscheine das Zusammenspiel von Arzneimittelherstellern mit den anderen Akteuren immer noch wie ein vorsichtiger „Balztanz“. Vor überzogenen Illusionen sei also gewarnt.

Arzneimittelhersteller sind im Ausland indessen nicht nur in neue Versorgungssysteme eingeschaltet, sie sind auch Partner von Verträgen, die hierzulande - aus meiner Sicht glücklicherweise - unbekannt sind. 
Teils handelt es sich dabei um Vereinbarungen, bei denen Risiken abgewälzt oder verteilt werden (Risk Sharing). In anderen Fällen geht es um eine Art von Erfolgsgarantie bei der Produktanwendung oder die Position als Hoflieferant. Ob die beteiligten Pharmaunternehmen sich tatsächlich einen Gefallen getan haben, solche Verträge abzuschließen, braucht hier nicht weiter erörtert zu werden. Gelinde Zweifel erscheinen jedoch angebracht.

Was im Ausland gedacht und getan wird, läßt sich für die Situation in Deutschland natürlich nicht 1:1 umsetzen, sondern könnte allenfalls systementsprechend adaptiert werden.

In Deutschland sind Arzneimittelhersteller derzeit erst in relativ wenigen Fällen an neuen Versorgungsformen beteiligt.

In diesem Zusammenhang ist beispielsweise das Projekt mit dem Namen „Fokus Diabeticus“ zu nennen, an dem eine Krankenkasse, Ärzte und ein Pharmaunternehmen beteiligt sind. Zielsetzung dieses Projektes ist es, eine flächendeckende Qualitätsentwicklung und -sicherung bei effizienterer Nutzung der Ressourcen in der ambulanten Diabetikerbetreuung zu erreichen. Geschehen soll dies durch Qualitätszirkel und Fortbildung der Ärzte, Handlungsempfehlungen und Überweisungskriterien für die teilnehmenden Ärzte, eine Notdienstregelung, die vollständige Untersuchung und Dokumentation aller Patienten und eine Patientenschulung und -anleitung zur Blutzuckerselbstkontrolle.

In einem anderen Modellprojekt mit dem Titel „Diabetiker-Zentrum Rostock" sind alle an der Gesundheitsversorgung Beteiligten einschließlich eines Arzneimittelherstellers engagiert. Durch ein integriertes Versorgungskonzept wird eine Verbesserung der Qualität der ambulanten Diabetikerversorgung und die Vermeidung von Krankenhauseinweisungen angestrebt. Zudem sollen die Spätfolgen des Diabetes reduziert werden.

Es zeigt sich also, daß auch in Deutschland schon der Anfang gemacht ist, Pharmaunternehmen in neue Versorgungsformen einzubeziehen. Weitere Projekte dürften folgen. Daß man sich vorsichtig in dieses Feld hineintastet, ist nicht einmal schlecht. Versuch und Irrtum sind wesentliche Bestandteile eines dynamischen Wettbewerbsprozesses auf der Suche nach besseren Versorgungslösungen - auch im Gesundheitswesen. Man sollte nicht darauf setzen, gleich im ersten Versuch den groBen Wurf zu landen. Eher sind "Trial and Error" die Realität. Wieder einmal bestätigt sich das Wort vom Wettbewerb als Entdeckungsverfahren. 
Für die Frage, wie sich die neuen Versorgungssysteme in Deutschland auch und gerade unter Einbeziehung von Pharmaunternehmen - entwickeln werden, spielen die gesetzlichen Rahmenbedingungen eine entscheidende Rolle.

Von besonderer Bedeutung sind dabei die sozialrechtlichen Vorschriften. Mit dem Gesundheitsstrukturgesetz von 1993 wurden neue Elemente - und zwar wettbewerblicher Natur - in die gesetzliche Krankenversicherung eingeführt. Die GKV-Neuordnungsgesetze haben diese wettbewerbliche Orientierung aufgenommen und die Handlungsmöglichkeiten für die Akteure im Gesundheitswesen erweitert. Zum einen wurden die Modellvorhaben zur Weiterentwicklung der Versorgung geregelt ( $\$ \S 63$ ff. SGB V), zum anderen können sogenannte Strukturverträge geschlossen werden (§ 73 a SGB V).

In den einschlägigen gesetzlichen Vorschriften ist die Einbeziehung von Arzneimittelherstellern allenfalls randständig geregelt. Insofern besteht in dieser Beziehung noch einiger Nachholbedarf. Grundsätzlich sollte der Zugang zu Modellvorhaben und Strukturverträgen nicht zu restriktiv gefaßt sein. Die Dominanz der ärztlichen Organisationen und der Krankenkassen sollte nicht übertrieben werden. Prinzipiell sollte den Systemen ein möglichst hoher Grad an Offenheit zugestanden werden. Statt eines einheitlichen Leistungskatalogs, eines gemeinsamen und einheitlichen Verhaltens und festgefügter Strukturen sind Differenzierungsmöglichkeiten angesagt. Zentralistische Positionen sind zugunsten dezentraler Einheiten aufzugeben.

Neben den sozialrechtlichen sind auch datenschutzrechtliche Vorschriften nicht außer acht zu lassen. Dabei ist von Bedeutung, welche Daten erfaßt, weitergeleitet und ausgetauscht werden. Die Erforderlichkeit und Zumutbarkeit von Datenflüssen steht ebenso zur Diskussion wie die Frage, wer Eigentümer von Daten ist und wem Daten zugänglich gemacht werden dürfen.

Schließlich kommt das Vertragsrecht ins Spiel, wenn Arzneimittelhersteller neuen Versorgungsstrukturen in rechtlich gefestigter Form beitreten. Der Grundsatz der Vertragsfreiheit läßt viele Variationsmöglichkeiten zu. Je nach Ausgestaltung eines Projekts im einzelnen ist sowohl ein zweiseitiger Vertrag möglich als auch ein mehrseitiger. Die Bandbreite reicht von einer schlichten bilateralen Vereinbarung bis hin zu einem mehrstufigen Vertragssystem mit vielen Beteiligten in unterschiedlichsten Funktionen. Daß ein Vertrag meistens eine Leistung und eine Gegenleistung enthält, sei an dieser Stelle der guten Ordnung halber erwähnt. 
Im Rahmen neuer Versorgungsformen kommen für die Industrie- und Herstellerseite diverse potentielle Partner in Betracht. Kooperationen können - ohne Anspruch auf Vollständigkeit - einbeziehen: einen Arzt, ein Praxisnetz, eine Kassenärztliche Vereinigung, ein Krankenhaus, eine Krankenkasse, einen Apotheker sowie nahezu jede denkbare Kombination dieser Partner.

Inwieweit die Herstellerseite als Partner für neue Versorgungsformen in Betracht kommt, bedarf einer differenzierten Betrachtungsweise. Die Arzneimittelhersteller, die in ihrer Gesamtheit als Pharmaindustrie bezeichnet werden, haben sich in Verbänden organisiert. Über die Verbände nehmen die Hersteller als Industrie auf die Rahmenbedingungen Einfluß, innerhalb derer die Akteure sich dann bewegen (können). Als Pharmaindustrie, also als Gesamtheit von Pharmaunternehmen, aufzutreten, ist problematisch. Ein gemeinsamer und einheitlicher Auftritt im Markt paßt schwerlich in eine Zeit, in der einzelne Krankenkassen ein eigenes Profil gewinnen wollen und behauptete Monopolstrukturen auf seiten der Ärzteschaft in Frage gestellt werden.

Auch käme ein Pharmaverband in eine ausgesprochen heikle Lage, wenn er als Organisation der Pharmaindustrie für die Pharmaindustrie Kooperationen eingehen und Verträge schließen sollte. Zum einen sind die Mitgliedsfirmen Individuen und haben individuelle Interessen; so sind Arzneimittelhersteller in ganz unterschiedlichem Grade daran interessiert, sich an neuen Versorgungsformen zu beteiligen. Davon abgesehen sind die Unternehmen Wettbewerber, und der Verband hat strikt darauf zu achten, sich wettbewerbsneutral zu verhalten. Gerade vor dem Hintergrund des Wettbewerbs hätte ein Verband - wäre er eine Kooperationsvereinbarung für die Pharmaindustrie eingegangen - interne Umsetzungsprobleme - im Innenverhältnis gegenüber den einzelnen Mitgliedsunternehmen. Schließlich kommt bei allen markt- und wettbewerbsrelevanten Punkten das Kartellrecht ins Spiel. Unter diesen Aspekten wird man kaum davon sprechen können, daß die Pharmaindustrie als solche Vertragspartner bei neuen Versorgungsstrukturen sein mag.

Partner bei neuen Versorgungsformen werden wohl nur (individuelle) Pharmaunternehmen sein können.

Pharmaunternehmen sind - so sie es wollen - in der Lage, mehr als nur die Ware Arzneimittel anzubieten. Die Firmen produzieren neben der Hardware auch Software, nämlich neues Wissen um die Möglichkeiten, die das Arzneimittel bietet. Diese Software könnte womöglich-noch besser als bisher - in das Gesundheitssystem eingebracht werden. 
So können international tätige Firmen beispielsweise ihre Erfahrungen aus den USA oder anderen Ländern offerieren. Sie verfügen oftmals über Know-how, das vor allem für diagnosebezogene Auswertungen und - darauf aufbauend - für die Entwicklung optimierter Behandlungsabläufe benötigt wird. Konkret auf die aktuellen Verhältnisse und Bedingungen in Deutschland bezogen, könnten sich Pharmaunternehmen z. B. bei Patientenschulungen, Therapieleitsystemen und Datendokumentationen und -auswertungen engagieren. Andere Möglichkeiten für Pharmaunternehmen, Input zu leisten, könnten darin bestehen, spezifisches Know-how zur Arzneimittelversorgung zur Verfügung zu stellen, das sich beispielsweise in Therapiehinweisen niederschlagen könnte. Multimedial ließen sich Querverbindungen zwischen Ärzten herstellen; auch ist an einen Internetservice für Ärzte sowie bedingt für Patienten zu denken.

Von diesen mehr inhaltlichen Aspekten abgesehen, scheinen die Möglichkeiten, sich von Herstellerseite in neue Versorgungsstrukturen einzubringen, noch längst nicht durchdekliniert zu sein. Nicht überraschend erschiene es mir, wenn Hersteller sich im Laufe der Zeit zu Systemanbietern hin entwickeln würden; womöglich tritt dies am ehesten bei der Krankenhausversorgung sowie womöglich auch gegenüber Praxisnetzen ein. Auf der anderen Seite erscheint es als nicht unwahrscheinlich, daß sich Konsortien von Herstellern bilden, die vielleicht mit ganz unterschiedlichen Projektbeiträgen aufwarten.

Die Haltung der anderen etablierten Akteure gegenüber den Angeboten von Herstellerseite ist etwas ambivalent. Eine Kooperation dergestalt, das Know-how von Pharmaunternehmen entgegenzunehmen und zu akzeptieren, wird punktuell vorsichtig eröffnet. Mit Gegenleistungen für die Leistungen von Herstellerseite tut man sich dagegen schwer. Namentlich der Gedanke an eine Bezahlung wird verdrängt.

Auf die Frage, warum Pharmaunternehmen sich denn überhaupt in neuen Versorgungsformen engagieren sollten, hört man die Antwort, daß der Nutzen für Hersteller beispielsweise im Erkenntnisgewinn über Krankheitsverläufe und in der Nähe zu Patienten liege. Ob auf längere Sicht eine monetäre Vergütung stattfinden wird, ist schwer abzuschätzen. Rein altruistisch werden sich Pharmaunternehmen auf Dauer nicht verhalten können. Entscheidend mag letztlich sein, welchen Vorteil Arzneimittelhersteller darin sehen, Partner - womöglich Vertragspartner - in einer neuartigen Versorgungsstruktur zu sein, und wie sie diesen Vorteil bewerten. 
Peter Dewein

Die politischen Rahmenbedingungen sind für die Versorgungsqualität im Gesundheitswesen wie auch für die Planungssicherheit der pharmazeutischen Unternehmer von herausragendem Interesse. Doch wie auch immer sich die Politik, die Wirtschafts- und Gesundheitspolitik, der neuen Regierung gestalten wird, eines ist sicher: Die Finanzierungsprobleme der gesetzlichen Krankenversicherung werden ganz unabhängig von den politischen Rahmenbedingungen zunehmen.

Das demographische Szenario für das erste Quartal des kommenden Jahrhunderts ist in zahlreichen Vorträgen und Publikationen skizziert worden. Der Bevölkerungsanteil der über 60jährigen wird in den kommenden zwei Jahrzehnten auf etwa ein Drittel der Gesamtbevölkerung anwachsen. Ebenfalls ist allseits bekannt, daß es sich bei diesem Alterssegment um die Versichertengruppe handelt, die aufgrund der altersbedingten überdurchschnittlichen Inanspruchnahme von Leistungen die höchsten Kosten verursacht. Das Segment der aktiven Beitragszahler nimmt relativ und absolut gesehen ab. Hinzu kommt der kostensteigernde medizinisch-technische Fortschritt, der - und das muß stets betont werden - mit Blick auf die Gesundheitsziele erwünscht ist.

Wie jedoch sollen sich in der Zukunft die Strukturen gestalten, innerhalb derer die effiziente und gerechte Allokation knapper Ressourcen vorgenommen wird?

Wir müssen zu völlig neuen Denkstrukturen im Gesundheitswesen finden. Die rigiden Organisationsformen, von denen die Gesundheitslandschaft gegenwärtig geprägt ist, haben keine Zukunft. Vernetzte Strukturen, wie sie sich in unabhängigen Organisationen erfolgreich entwickeln, müssen auch Eingang in sämtliche Leistungsbereiche des sozialen Gesundheitswesens finden. Barrieren und Konflikte zwischen Leistungserbringern und Kostenträgern, die auf systemimmanente Kommunikationslücken zurückzuführen sind, bedeuten Reibungs- und Effizienzverluste. Insbesondere der Arzneimittelbereich hat sich - da er sich im Gegensatz zu anderen Leistungsbereichen durch ein Höchstmaß an Transparenz auszeichnet - für regulatorische Eingriffe mißbrauchen lassen müssen. Die Leistungserbringer, nämlich die Arzneimittelhersteller, sind nicht oder nur vordergründig in die Ausgestaltung der Rahmenbedingungen eingebunden worden. 
Die Finanzierungsprobleme der gesetzlichen Krankenversicherung können jedoch nicht im Konflikt mit der pharmazeutischen Industrie gelöst werden.

Arzneimittel stellen, wie Sie alle wissen, nicht den Motor für Ausgabensteigerungen im Gesundheitswesen dar. Vielmehr können die Arzneimittelhersteller in Deutschland innerhalb des Systems beanspruchen, einen besonders wirtschaftlichen Beitrag zur gesundheitlichen Versorgung der Patienten zu leisten. Das Medikament ist nicht nur patientenfreundlich - dies spiegelt sich beispielsweise in einer hohen Compliance im Rahmen der Arzneimitteltherapie wider -, vielmehr ist es auch kosteneffizient. In internationalen Vergleichen hat das deutsche Gesundheitssystem unter anderem deshalb über einen langen Zeitraum eine Spitzenposition bezogen, weil Arzneimittel innerhalb der Gesundheitsversorgung einen hohen Stellenwert eingenommen haben. Ausweichstrategien ohne die aktive Mitwirkung der pharmazeutischen Industrie wirken verteuernd und qualitätsmindernd.

Dem Bereich der Arzneimittelversorgung drohen weiterhin - die ersten gesundheitspolitischen Schritte der neuen Regierung lassen dies erkennen - empfindliche Einschnitte. Hierdurch sind substantielle Schäden am System insgesamt zu erwarten. Es ist daher notwendig, die Arzneimittelindustrie als einen der wichtigsten Leistungserbringer im System der gesetzlichen Krankenversicherung in das Entscheidungsgeschehen partnerschaftlich einzubinden.

Ich wende mich an die Politik mit der Forderung, sich auf Instrumente und Strukturen zu besinnen, die den Partnerschaftsgedanken aufgreifen, transparent sind und ökonomisch rationale Gestaltungsalternativen im System mit dem Ziel medizinisch-ethisch vertretbarer und effizienter Ergebnisse hervorbringen. Die pharmazeutische Industrie hat ihre Bereitschaft gezeigt, in konstruktiver Weise Lösungswege mitzugestalten.

Vor diesem Hintergrund geht es uns um ein vertragspartnerschaftliches Konzept. Vertragspartnerschaft ist die ökonomische Übersetzung von pluraler Demokratie.

Hier werden Interessenkonflikte im Konsens gelöst. Die beiden Grundvoraussetzungen eines derartigen Ansatzes sind "Waffengleichheit" und Transparenz des Handelns. Weder die eine noch die andere Voraussetzung sehen wir gegenwärtig gegeben. Deshalb versehe ich die Vertragspartnerschaft mit einem großen Fragezeichen. 
Natürlich werden wir die neue Regierung und ihre gesundheitspolitischen Akzente daraufhin überprüfen, ob es positive Ansätze für Vertrags- oder wenigstens partnerschaftliche Verhältnisse gibt. Positive Stichworte in der Koalitionsvereinbarung sind Patientenorientierung, Gesundheitsberichterstattung, Stärkung des Hausarztes und Vernetzung (stärkere Zusammenarbeit).

Wir, die pharmazeutische Industrie, setzen uns schon lange für die aktive Integration aller Arbeitsfelder im Gesundheitswesen ein. Vorrangig gehört dazu auch die Einbeziehung der Patienten. Denn der Zielerreichungsgrad einer effizienten Gesundheitsversorgung (Qualität) kann allein an der gesundheitsbezogenen Lebensqualität der Versicherten gemessen werden. Der Weg hierhin kann nur über vernetzte kleinräumige und dezentrale Versorgungsstrukturen führen, in der die pharmazeutische Industrie eingebunden ist. Aber, wie wir wissen, eben nicht als Partner, sondern lediglich als Zahlmeister! Wie die neue Mehrheit mit der Pharmaindustrie umgehen will, wird sich nicht nur bei der Positivliste usw. zeigen, sondern im heutigen Zusammenhang vor allem bei den Gesundheitskonferenzen, die in beiden Regierungsparteien diskutiert und teilweise realisiert werden. Natürlich müssen solche Veranstaltungen und Konstrukte unter Einbeziehung aller Beteiligten stattfinden. Parallel dazu ist eine Gesundheitsberichterstattung und die Formulierung von Gesundheitszielen vonnöten. Grundsätzlich sehe ich die politischen Voraussetzungen hierfür als gut an. Ich kann nur dafür plädieren, daß die pharmazeutische Industrie die Idee der Partnerschaft als positives Signal transportiert und sich für ihre Einbindung mit Blick auf den Arzneimittelsektor einsetzt. Es besteht schneller Handlungsbedarf, denn infolge einer politisch gewollten Reduzierung der Zuzahlungen und wegen anderer finanzwirksamer Maßnahmen wird sich der finanzielle Druck im Bereich der GKV-Arzneimittelausgaben schnell erhöhen.

Nur nebenbei:

Die Forderung nach partnerschaftlichen Strukturen kann sich jedoch nicht allein an die Politik richten. Auch auf Unternehmensebene muß das Denken in dezentralen Strukturen Einzug halten. Es kann sich in Zukunft als tödlich erweisen, auf dem Status quo zu verharren. Rigide Organisationsstrukturen, die über lange Zeit in weiten Marktbereichen straffrei geblieben sind, müssen durch den Vernetzungsgedanken abgelöst werden. Nur dann wird der Begriff der "Apotheke der Welt" auch in Zukunft positiv besetzt sein.

Die Zukunft wird keinen Platz für idealistische Einzelkämpfer bieten. Dem regulatorischen Druck wie auch dem stärker wehenden Wind des 
internationalen Wettbewerbs werden Unternehmen nur standhalten können, wenn sie in allen denkbaren Bereichen kooperieren. Diese Notwendigkeit besteht in den einzelnen Geschäftsbereichen wie Einkauf, Vertrieb oder Forschung und Entwicklung, besonders aber auch im Auftreten gegenüber den Kostenträgern und der Politik. Es muß über völlig neue Kooperationsformen nachgedacht werden. In manchen Marktbereichen beobachten wir bereits ein erstaunliches Maß an Kreativität in dieser Hinsicht. Wir sehen auch, daß zahlreiche Ansätze erfolgreich sind. Manche Segmente hingegen sind eher von Skepsis geprägt. Es ist für individuell strukturierte mittelständische Unternehmen schwer, sich von einer auf den Einzelerfolg ausgerichteten Organisation zu lösen.

Vertragspartnerschaften, nennen Sie die meinetwegen Einkaufsmodelle, sind also nur akzeptabel, wenn die Partner auf GKV-Seite den Vorrang des Arzt-Patienten-Verhältnisses, die Therapiefreiheit und in ihrer Folge die abgestufte Therapie als Spiegelbild der humanen Versorgungsrealitäten akzeptieren. So könnte der Mangel an Transparenz und Waffengleichheit im Vertragsverhältnis einigermaßen kompensiert werden.

Der Partnerschaftsgedanke ist grundlegend demokratisch. Das Miteinander auf der Basis umfassender Information ist der einzig legitime Weg, Effizienz in der sozialen Krankenversicherung zu erreichen. Einzelne Ansätze, die mit dem Ziel einer rationalen Ausgestaltung von Anreizstrukturen diskutiert werden, stehen hierzu nicht im Gegensatz.

So kann der Aufbau eines Primärarztsystems durchaus die Erkenntnis beschleunigen, daß Therapiefreiheit und Ökonomie keine Widersprüche darstellen.

Der effiziente Markt spiegelt ein System von Rechten wider. Nicht zuletzt gilt: Die Freiheit der Leistungserbringer ist das Recht des Patienten.

Ich möchte dafür plädieren, daß wir uns im weiteren gemeinsam gezielt folgenden Fragen widmen:

- Wie erreichen wir schnellstmöglich den Aufbau einer soliden Informationsbasis als Grundlage rationaler Zielsetzungen und an welchen Indikatoren können sich diese orientieren? (Dabei ist mir grundsätzlich bewußt, daß von der Zielsetzung allein kein Hinweis auf die Steuerung von Mitteln ausgeht.)

- Wie läßt sich ein Zielsetzungsprozeß als Konsensprozeß gestalten und wo liegt die Rechenschaftspflicht? 
- Wie definieren wir Qualität und wie kann dabei der künstliche Widerspruch zwischen Ökonomie und Medizin überwunden werden?

- Wie kann die Vertragspartnerschaft mit dem Leitgedanken der Ergebnisorientierung ausgestaltet werden?

- Welche Organisationsformen setzen sich durch, um sämtliche Beteiligten auf Basis des Partnerschaftsgedankens in ein Geflecht effizienter dezentraler Strukturen einzubinden?

Mit diesen Aspekten müssen wir uns intensiv und schnell auseinandersetzen, um die Systemsklerose zu stoppen und den Erfolg des Gesundheitssektors, der einen der gesellschaftlich und ökonomisch wertvollsten Bereiche unserer Volkswirtschaft darstellt, gewährleisten zu können. Es ist keine Frage, daß die pharmazeutische Industrie der Ergebnisorientierung unseres Gesundheitswesens und dem Wachstumsmarkt Gesundheit partnerschaftlich und zielgerichtet zum Erfolg verhelfen will. 


\section{Die Pharmaindustrie als Vertragspartner}

\section{Christoph Straub}

Am Ende eines langen und sehr interessanten Tages hier in Bad Orb referiere ich zum Thema "Die Pharmaindustrie als Vertragspartner". Ich habe diesen Vortrag kurzfristig von meinem Kollegen Herrn Dr. Glaeske übernommen, und anders als bei diesem bildet der Bereich „Pharma und Vertragsbeziehungen" bei mir nicht das Hauptarbeitsgebiet. Ich kann deshalb eine etwas aus dem Zentrum gerückte Perspektive wählen und vielleicht - so wie wir es gestern gehört haben - als Makler von Ideen auftreten. Im Moment beschäftigt die Diskussion um das Solidaritätsstärkungsgesetz alle Beteiligten im Gesundheitswesen und wohl auch alle heute hier Anwesenden. Jeder möchte gerne wissen, welche Weichenstellungen im Solidaritätsstärkungsgesetz vorgenommen werden und was dies für die im nächsten Jahr zu erwartende, „durchgreifende Strukturreform" bedeuten kann. Ich möchte den amerikanischen Gesundheitsökonomen Uwe Reinhardt von der Princeton University zitieren: "The Future is difficult to predict, especially in advance" und auf diesen klugen Rat hin, unabhängig von den zirkulierenden Gerüchten und Mutmaßungen, einige grundsätzliche Probleme direkter Vertragsbeziehungen zwischen der gesetzlichen Krankenversicherung und der Pharmaindustrie ansprechen.

Es ist eine Grundforderung der Ersatzkassen und ihrer Verbände, mehr wettbewerbliche Möglichkeiten im Gesundheitswesen zu schaffen. Wettbewerb vor dem Hintergrund einer solidarischen Grundordnung ist der Begriff für das vorgeschlagene Konzept. Es bedeutet die Auflösung monopolistischer Strukturen zugunsten konkurrierender Einheiten, um einen „kollektiven Suchprozeß“ zu ermöglichen, der Planungen ersetzt und schneller und besser als diese Entwicklungen der Versorgungsstrukturen und Prozesse ermöglicht. Grundsätzlich gilt dies auch für den hier in Rede stehenden Bereich. In den meisten Industrienationen ist aber gerade der Arzneimittelmarkt in besonderer Weise reguliert. Dies liegt wohl u. a. daran, daß es sich bei Arzneimitteln um Produkte und nicht um Dienstleistungen handelt und noch dazu um hübsche kleine bunte Packungen, die sich leicht zählen lassen und deren Preis sich in überschaubaren Größenordnungen bewegt. All dies fordert geradezu heraus zu regulierendem Eingriff in ökonomisch angespannten Situationen. Es hängt dabei stark davon ab, welche Instrumente in welchem Rahmen zur Regulierung eingesetzt werden, ob man mit ihnen auch die ursprünglich gesteckten Ziele erreicht bzw. mehr Nutzen als Schaden stiftet. Bezogen auf die hier diskutierte Option heißt dies, nicht die Ein- 
führung direkter Vertragsbeziehungen an sich wird ein Gesundheitssystem verbessern, sondern nur die Integration dieses Instruments in ein ordnungspolitisch schlüssiges Gesamtkonzept. Gerade in diesem Punkt gibt es im Blick auf die in der Vergangenheit entwickelten Konzepte gewisse Zweifel. Die SPD-Bundestagsfraktion hat zum Thema „Direkte Vertragsbeziehungen zwischen Pharmaindustrie und gesetzlicher Krankenversicherung" in ihrem Vorschlag für ein GSG II folgendes Konzept entworfen: Die Spitzenverbände der Krankenkassen könnten demnach in direkte Preisverhandlungen mit den Arzneimittelherstellern treten, aber nur, nachdem die Bundesregierung in Abstimmung mit dem Bundesrat bereits einen Konsens über Festbeträge für Medikamente erzielt und diese als Verordnung erlassen hätte. Es ist eher unwahrscheinlich, daß die Pharmahersteller, nachdem sie bereits dem schmerzvollen Prozeß eines staatlichen Preisdiktats unterzogen wurden, große Bereitschaft zeigen würden, sich nochmals mit den in diesem Punkt gemeinsam und einheitlich handelnden Spitzenverbänden an einen Tisch setzen würden, um eine weitere Absenkung der diktierten Preise zu verhandeln.

Es gibt zwei weitere Punkte auf der sozialpolitischen Agenda der neuen Regierungsparteien, die prinzipiell freien Verhandlungen von Marktteilnehmern, das heißt dem Prinzip einer Deregulierung, entgegenstehen: die Positivliste für Arzneimittel und die Globalbudgetierung. Ausgehend von diesen beiden Eckpunkten ist es schwierig, ein Szenario zu entwerfen, in dem freiere Vertragsbeziehungen zu einer Effizienzsteigerung des Systems führen könnten. Diese Aussage ist stark verkürzt, man müßte zur Erläuterung viele Aspekte und reale Erfahrungen mit Budgets diskutieren, um die Aussage näher zu erläutern. Dazu ist hier jedoch kein Raum. Aus der entgegengesetzten Perspektive, also unter der Bedingung freierer Vertragsbeziehungen zwischen GKV und Arzneimittelherstellern, ist ein anderer wesentlicher Punkt zu diskutieren. Es gibt innerhalb des GKV-Systems mehrere Grundwerte, die nicht in Frage gestellt werden. Dazu gehört der einheitliche Leistungskatalog sämtlicher gesetzlicher Krankenkassen. Nun werden im Moment im Zusammenhang mit freieren Vertragsbeziehungen immer nur Preisverhandlungen angesprochen, es ist aber die Frage, welche Folgewirkungen sich eher unbeabsichtigt einstellen könnten. Dies wäre z. B. angesichts einer im Wettbewerb agierenden gesetzlichen Krankenversicherung mit unterschiedlich wirtschaftsstarken Kassen durchaus auch die Differenzierung des Leistungsangebots, das heißt der zur Verfügung gestellten bzw. bezahlten Medikamente. Eine solche Entwicklung widerspricht aber nach gegenwärtigem Stand den Intentionen von GKV und Politik. Ein weiterer Punkt in diesem Zusammenhang ist die sozialrechtliche Option der Versicherten zur Teilhabe am Fortschritt. Es könnte durchaus sein, daß 
durch direkte Vertragsbeziehungen die Teilhabe am Fortschritt schneller realisiert würde, besser als es das derzeitige System leistet. Es wäre aber auch das Gegenteil möglich, dann nämlich, wenn der oder die Kostenträger als Vertragspartner auf der Krankenkassenseite sich unter dem Einfluß von Budgetierungen diesem Fortschritt verschließen würden. Grundsätzlich ist die Festlegung des Leistungsumfangs der GKV eine politische Aufgabe, basierend idealerweise auf einem gesamtgesellschaftlichen Konsens, und nicht in das Ermessen einzelner Krankenkassen oder aber auch der gesamten GKV gestellt. Jedes Konzept freier Vertragsbeziehungen müßte diese Einschränkung berücksichtigen.

Ein weiterer Punkt, den ich kurz diskutieren möchte, ist der logistische Aspekt freier Verhandlungen in einem deregulierten System: Derzeit gibt es in Deutschland rund 430 Krankenkassen, denen 600 Hersteller von Arzneimitteln mit einem Angebot von ca. 45.000 Fertigarzneimitteln gegenüberstehen. Es ist unmittelbar einzusehen, daß nicht alle Anbieter und Nachfrager in direkte, z. B. jährliche Verhandlungen treten könnten. Unter den 450 Krankenkassen und wohl auch unter den Herstellern von Arzneimitteln sind einige, deren Personalstärke und Expertise wohl kaum ausreichen dürfte, um die notwendigen Verhandlungen zu führen. Vor diesem Hintergrund und unter Beachtung der oben bereits genannten politischen Selbstbindung der GKV auf ein einheitliches Leistungsangebot auch bei Arzneimitteln erscheint nur die Option eines gemeinsamen und einheitlichen Handelns der gesetzlichen Krankenversicherung evtl. eines Verbändewettbewerbs wahrscheinlich.

Eine Alternative unter Aufgabe der Einheitlichkeit des Leistungsangebots wäre eine Entwicklung, wie sie im amerikanischen Gesundheitswesen zu beobachten war. Die Entwicklung unterschiedlichster "Nachfragegemeinschaften" auf seiten der Krankenkassen, die ihrerseits die Dienste neuartiger intermediärer Unternehmen in Anspruch nehmen, die zwischen Herstellern und Kostenträgern eine spezifische zusammengestellte Arzneimittelliste und deren Preise vermitteln. Zweifellos hat diese Organisationsform die Kosten für Arzneimittel im amerikanischen Gesundheitsmarkt in Teilen „kontrolliert"; über die Auswirkungen auf die Qualität der Versorgung lassen sich keine Aussagen machen. Vom Standpunkt der GKV und der geltenden Rechtslage aus betrachtet, wäre dieses Modell aber zumindest primär mit zusätzlichen Kosten verbunden. Die außergewöhnlich niedrigen Verwaltungskosten der GKV beruhen nicht zuletzt auf dem „Systemverzicht“ auf direkte Verhandlungen über Leistungen und Preise.

Ein weiterer Diskussionspunkt im Zusammenhang mit einer Deregulierung des Arzneimittelmarktes sind die juristischen Folgewirkungen. Jede 
Einführung von Marktelementen in das System der GKV macht es schwieriger, deren korporatistisches System auf der EU-Ebene wettbewerbsrechtlich bzw. kartellrechtlich zu rechtfertigen. Ohne weiter ins Detail gehen zu wollen und zu können, ist dies ein gewichtiges Argument gegen die Einführung freier Verhandlungen zwischen Krankenkassen und Arzneimittelherstellern.

Ein weiterer Aspekt der Deregulierung soll an einem Beispiel erläutert werden: Im vergangenen Jahr mußte ein neues Präparat gegen Hypertonie wegen tödlicher Komplikationen kurz nach der Einführung vom Markt genommen werden. In einem System, in dem einzelne Krankenkassen oder Teile der GKV eigenständig über die Aufnahme neuer Präparate in ihren Leistungskatalog entscheiden könnten, ergäbe sich wohl eine unternehmerische Mitverantwortung für derartige Vorkommnisse. Wahrscheinlich ist, daß diese Konstellation die Einführung innovativer Arzneimittel gegenüber der gegenwärtigen Situation eher verzögern würde. Welche Krankenkasse möchte schon in die Presse kommen als Finanzier einer tödlichen Innovation?

Den Versicherten der GKV steht derzeit ein umfassendes Arzneimittelangebot zur Verfügung. Das relative, durchschnittliche Preisniveau im Vergleich zu anderen Industrienationen ist in den vergangenen Jahren unter dem Einfluß der Festbetragsregelung von einer Spitzenposition in Richtung des Durchschnitts gesunken. Eine abstrakte Diskussion der Einführung freier Verhandlungen ergibt für die Option „Verhandlungen auf Spitzenverbandsebene" keine erkennbaren kurz- oder mittelfristigen Vorteile. Freie Verhandlungen auf Ebene der einzelnen Krankenkassen würden zwangsläufig zu einer gegenwärtig weder von der GKV noch von der Politik gewünschten Differenzierung des Leistungsangebots und zu beträchtlichen juristischen Risiken auf der Ebene des Gesamtsystems (Wettbewerbsrecht und korporatistische Verfassung der GKV) und der Einzelkasse (eventuell Mithaftung für Produktrisiken) führen. Was ist dagegen die Forderung der Spitzenverbände der gesetzlichen Krankenkassen an die Politik? Derzeit sind gemessen am Umsatzvolumen ca. $50 \%$ des Arzneimittelmarktes von Festbetragsregelungen erfaßt. Eine sinnvolle Ausweitung dieser Regelung unter Einbeziehung weiterer Arzneimittelgruppen und eine klare Rechtswegezuweisung zu den Sozialgerichten werden favorisiert, um die Einheit des Leistungsangebots und ein aus Sicht der Solidargemeinschaft angemessenes Preisniveau zu gewährleisten. Darüber hinaus könnte das Festbetragssystem durch die Verhandlung von Erstattungshöchstgrenzen für andere verschreibungspflichtige Arzneimittel ergänzt werden. In jedem Fall wird es schon im Jahr 1999 auf diesem Feld zu bedeutenden Änderungen kommen (müssen): Das Urteil des OLG Düsseldorf zur (Un-)Gültigkeit einer Festbe- 
tragsfestsetzung durch den Bundesausschuß von Ärzten und Krankenkassen und der politische Wille der Regierungskoalition, im Rahmen einer durchgreifenden Strukturreform der GKV eine Positivliste einzuführen, werden den Arzneimittelmarkt in Deutschland und seine Mechanismen stark verändern. 


\section{Themenkreis III}

\section{Ausgewählte Finanzierungsalternativen}

Dieter Cassel

Die bisher behandelten Themenkreise haben sich mit dem Niveau und der Struktur der Gesundheitsversorgung in Deutschland generell wie auch unter den speziellen Aspekten des EU-Binnenmarktes befaßt. Dabei wurde deutlich, daß weder die beginnende Liberalisierung der europäischen Märkte für die deutsche Gesundheitsversorgung noch die Suche nach veränderten Versorgungsstrukturen, die seit 1997 durch die gesetzgeberische Freigabe von Strukturverträgen und Modellvorhaben verstärkt eingesetzt hat, kaum etwas zur Lösung des Finanzierungsproblems des deutschen Gesundheitswesens beitragen können. Europaweit und unabhängig von den Versorgungsstrukturen gilt vielmehr, daß die bisher wirksamen Treibsätze für die progressive Entwicklung der Gesundheitsausgaben auch künftig nicht an Schubkraft einbüßen dürften: Der per Saldo leistungsausweitende medizin-technische Fortschritt, die Vergreisung der Bevölkerung und der damit verbundene Anstieg der ausgabentreibenden Multimorbidität, eine zunehmende Präferenz der Bevölkerung für Gesundheitsgüter sowie nicht zuletzt das Gesundheitswesen als ein arbeitsintensiver, durch geringe Produktivitätssteigerungen geprägter Dienstleistungssektor, sind quasi "systemindifferente“ Faktoren, die in den entwickelten Industriegesellschaften beobachtbar sind und auch künftig jenseits aller gesundheitspolitischer Weichenstellungen die Ausgabendynamik im Gesundheitswesen prägen dürften.

Für die Bundesrepublik Deutschland ergibt sich daraus ein besonderes Finanzierungsproblem, weil über $90 \%$ der Bevölkerung in der gesetzlichen Krankenversicherung (GKV) versichert sind, in der die entstehenden Gesundheitsausgaben durch einkommensproportional erhobene Beiträge gedeckt werden. Dabei wird der im Umlageverfahren kalkulierte Beitragssatz hälftig vom Mitglied und von seinem Arbeitgeber getragen, wodurch jede Erhöhung der GKV-Beitragssätze entsprechend auf die Lohnzusatzkosten durchschlägt. Immerhin ist der GKV-durchschnittliche Beitragssatz trotz aller reformpolitischen Bemühungen in den letzten 25 Jahren von gut $8 \%$ auf inzwischen 13,5\% angestiegen. Entscheidend dafür war, daß die Entwicklung der Arbeitseinkommen als Beitragsbemessungsgrundlage wegen der vielfältigen Probleme des Beschäftigungssystems nicht mit der Ausgabendynamik im Gesundheitswesen mithalten konnte. Die daraus resultierende "Beitragssatzexplosion“ 
macht die Finanzierungsproblematik speziell in Deutschland zu einem gesundheitspolitischen Dauerbrenner, dem sich auch die neue Bundesregierung nicht entziehen kann.

Wie teilweise schon unter der letzten Bundesregierung läuft die Gesundheitspolitik zunehmend Gefahr, sich in eine schier ausweglose Situation hineinzumanövrieren: Unter dem Druck der mit dem Lohnzusatzkostenargument geführten Standortdiskussion verkürzt sich die gesundheitspolitische Zielsetzung auf die Erhaltung der "Beitragssatzstabilität“, was letztlich darauf hinausläuft, die Gesundheitsausgaben an die zu erwartenden Einnahmen anzupassen. Ausgabenrestriktionen auf der Makroebene sind jedoch nicht ohne administrative Maßnahmen durchsetzbar. Da es sich hierbei stets nur um ein „Krisenmanagement“ handeln kann, das die genannten Ursachen für die Ausgabendynamik nicht beseitigt, ist der Mißerfolg der Ausgabendämpfung vorprogrammiert. Mehr noch: Die zunehmenden administrativen Eingriffe in den ökonomisch gesehen ohnehin nicht rationalen Steuerungsmechanismus des GKVSystems beeinträchtigen zusätzlich die Effizienz und Effektivität der Gesundheitsversorgung und verstärken die ohnehin bestehenden strukturellen Verwerfungen. Letztlich wird also der Versuch, die Finanzierungsproblematik durch eine administrative Ausgabensteuerung anzugehen, in einem Lenkungschaos enden, was in einem Sektor, in dem 1996 immerhin rd. 250 Mrd. DM ausgegeben wurden, nicht nur die Versicherten, sondern auch Millionen von Leistungserbringern und ihre Beschäftigten gegen die Politik aufbringen muß.

Nicht zuletzt vor diesem Hintergrund ist die nun folgende Diskussion der vom Veranstalter im Themenkreis 3 ausgewählten Finanzierungsalternativen zu sehen: Sicherlich gibt es in der anhaltenden Reformdiskussion eine ganze Reihe von Stimmen, die fordern, "mehr Geld in das System" zu bringen. Prominentester Reformvorschlag in diesem Zusammenhang ist die Erhöhung der Beitragsbemessungs- und Versicherungspflichtgrenze. Wie sich jedoch zeigen wird, gerät dieser Vorschlag leicht in Widerspruch zu den gewachsenen Solidarprinzipien der GKV und berührt überdies das sensible Verhältnis zwischen GKV und PKV nicht unerheblich. Da letztlich auch quantitativ kaum ein nennenswerter Finanzierungsbeitrag hiervon zu erwarten ist, wird sich die Diskussion rasch der Ausgabenseite zuwenden und damit die „Budgetierung“ in ihren verschiedensten Varianten zum zentralen Thema machen.

Dabei sollte klar sein, daß Budgetierung im Sinne der Aufstellung eines Budgets bzw. Haushaltsplans für individuelle Haushalte, Unternehmen und jedwede Organisationen Ausdruck rationaler Verwendung von knappen Ressourcen ist und insofern nicht nur wirtschaftlich notwendig, 
sondern auch völlig unproblematisch erscheint. Zum Problem wird die „Budgetierung" jedoch immer dann, wenn Ausgaben für ganze Wirtschaftszweige wie das Gesundheitswesen politisch festgelegt werden und administrativ bis auf die individuelle Handlungsebene heruntergebrochen werden müssen. „Budgetierung“ gehört mit diesem Anspruch, unabhängig von der konkreten Realisierungsform, immer in den Instrumentenkasten zentraladministrativer Planung und muß sich deshalb in einer Marktwirtschaft auch stets das Argument der Systeminkonformität entgegenhalten lassen. Konkret: Es macht einen gewaltigen Unterschied, ob sich z. B. im Berliner BKKTTK-Praxisnetz die beteiligten Leistungserbringer auf die Einhaltung der von innen selbst aufgestellten kombinierten Budgets verpflichten, oder ob für Arzneimittel, Arzthonorare, Krankenhausleistungen oder ähnliches feste Ausgabenblöcke administrativ vorgegeben werden und diese, je nach Art der Budgetierung, letztlich beim Leistungserbringer als einzuhaltende "Planauflage“ ankommen. Wir dürfen gespannt sein zu hören, wie sich diese Problematik aus der Sicht der Kassen und der Gesundheitspolitik darstellt. 
Franz Knieps

Ein Gespenst geht um im deutschen Gesundheitswesen. Und Ärzte, Apotheker, Krankenhäuser, pharmazeutische Unternehmen, ja selbst Krankenkassen zittern offenbar vor Angst und heulen laut. Der Name des Gespenstes ist Budgetierung. Mal trägt es das alte Linnen der strukturierten Budgets, mal das zerfledderte Hemd der sektoralen Budgets, mal das Patchwork der kombinierten Budgets, künftig wohl das wallende Kleid der globalen Budgetierung. Erstaunlich ist, daß diese Gespenster, deren Schöpfer an dieser Veranstaltung teilnehmen, nach mehr als 20 Jahren Kostendämpfungspolitik die Interessengruppen zu erschrecken vermögen. Oder sollte hinter dem lauten Wehklagen doch der alte Bonner Lobbygrundsatz stehen „Lerne klagen, ohne zu leiden!“? Denn unterschiedliche Formen der gesetzlich angeordneten Budgetierung sind-spätestens seit dem Krankenversicherungs-Kostendämpfungsgesetz aus dem Jahr 1977-im deutschen Gesundheitswesen gängige Praxis. Erst recht sind Budgets keine Neuigkeit für diejenigen, die komplexe Systeme und Einrichtungen politisch und ökonomisch steuern müssen. Sie müßten sich an vorgegebene oder ausgehandelte, virtuelle oder reale Budgets längst gewöhnt haben.

Budgets sind selbstverständliche, ökonomische Steuerungsinstrumente im Management-Regelkreislauf. Sie sind logische Folge knapper Ressourcen. Vergleichsweise neu ist offenbar für viele Beteiligte die Erkenntnis, daß auch im Gesundheitswesen Knappheitsgesetze gelten. Noch Ende der achtziger Jahre konnte es vorkommen, daß Gesundheitsökonomen auf dem Deutschen Ärztetag ausgepfiffen wurden, wenn sie das Gesundheitswesen als Wirtschaftszweig betrachteten und deshalb-je nach ordnungspolitischer Präferenz-ökonomisch basierte Steuerungsvorschläge machten.

Dabei arbeiten die niedergelassenen Ärzte doch seit langem mit Budgets. Seit mehr als 15 Jahren ist die ärztliche Gesamtvergütung mehr oder minder strikt gedeckelt und wirken sich Mengensteigerungen punktwertmindernd aus. Auch die Krankenhäuser arbeiten seit der Reform der Krankenhausfinanzierung im Jahr 1972 mit Budgets, die mit den Krankenkassen jährlich ausgehandelt werden. Und die Krankenkassen haben spätestens seit Einführung des Risikostrukturausgleichs (RSA) zumindest virtuelle Budgets. Diese Budgets orientieren sich an den Normkostenprofilen des RSA. Wer diese Normkostenprofile überschreitet, ist zu höheren Beitragssätzen als bei Konkurrenzkassen ge- 
zwungen und wird damit weniger wettbewerbsfähig. Bei den Kassen hat es allerdings einige Zeit gedauert, bis die meisten Führungskräfte diese eigentlich simple Mechanik begriffen haben. Speziell die Kassen, die in der Vergangenheit Knappheit nicht gewohnt waren oder die ihre finanzielle Situation schicksalhaft beklagt haben, tun sich nach innen und auBen schwer, mit den virtuellen Budgets des RSA zu arbeiten. Nicht zuletzt deshalb hat die alte Bundesregierung - in sehr umstrittener Art und Weise - der Flucht in Beitragssatzerhöhungen schmerzhafte Hindernisse entgegengesetzt.

Die Sorge vor steigenden Beitragssätzen treibt wohl auch die neue Regierungskoalition um. Zwar will die neue Bundesgesundheitsministerin Andrea Fischer Beitragssatzstabilität nicht als Fetisch verstanden wissen. Wer jedoch die Koalitionsvereinbarungen zwischen der SPD und Bündnis 90/DIE GRÜNEN sorgfältig liest, kann unschwer erkennen, daß dem Gebot der Beitragssatzstabilität herausragende Bedeutung beigemessen wird. Es ist das erklärte politische Ziel der Koalition, die Sozialabgabenquote unter $40 \%$ zu drücken. Hierzu wird die gesetzliche Krankenversicherung ihren Beitrag leisten müssen. Zumindest will die Politik keine höhere Belastung der Arbeitnehmer und Betriebe durch steigende Beitragssätze der Krankenkassen hinnehmen. Deshalb greift sie erneut auf das Instrument der Budgetierung zurück, das nach Auffassung der politischen Mehrheit im Gesundheitsstrukturgesetz wertvolle Dienste geleistet hat. Wer seinen Blick nicht durch Standes- oder Interessenpolitik trüben läßt, wird nicht umhinkönnen, dem Einsatz von Budgets in der GKV Innovationskraft und Steuerungswirkung beizumessen.

Die sektoralen Budgets haben nach Inkrafttreten des GSG spürbare Bremswirkungen bei den Ausgaben hinterlassen, ohne daß es zu gesundheitspolitisch relevanten Versorgungsengpässen gekommen wäre. Die immer wieder behaupteten Leistungsverweigerungen infolge erschöpfter Budgets erwiesen sich bei konkreten Nachforschungen sehr schnell als Propaganda zur Einschüchterung von Politik, Kassen und Patienten. Das Verordnungs- und Überweisungsverhalten der Ärzte hat sich dort erheblich verändert, wo Kassenärztliche Vereinigungen zur Steuerung von zwar nicht geliebten, aber politisch akzeptierten Budgets die Beratung inrer Mitglieder als legitime und wichtige Aufgabe angesehen haben und ansehen. Die Bindungswirkung der Budgets hat dort jedoch schnell nachgelassen, wo Kassenärztliche Vereinigungen nach dem Motto „Je höher die Überschreitungen, desto geringer die Wahrscheinlichkeit der Rückzahlung" eine bewußte Anti-Budget-Politik fuhren und liebedienerische Sekundanten aus der Politik dieses Verhalten goutierten. Die Budgetdisziplin wurde aber vor allem schwächer, weil der Krankenhaussektor von vornherein in den Budgetdeckel so viele Löcher 
gebohrt hatte, daß in diesem Kessel keinerlei Druck entstand. Wer will es dann anderen Leistungserbringern verdenken, daß sie Geldverlagerungen in den stationären Sektor nicht tatenlos zusehen wollten.

Wohl kaum jemand wird bezweifeln, daß die Phase der sektoralen Budgetierung die gesundheitspolitisch innovativste der letzten Jahre war. Die meisten Modellüberlegungen zur Reform von Versorgungsstrukturen und Versorgungsprozessen wurden in der Zeit von 1993 bis 1996 bis zur Projektreife konkretisiert. Das gilt sowohl für Strukturveränderungen von Hausarztmodellen über Qualitätsgemeinschaften bis zu vernetzten Praxen als auch für die systematische Prozeßsteuerung, speziell bei chronischen Erkrankungen und besonders teuren Fällen (Case Management und Disease Management). Ohne den ökonomischen Druck der Budgetierung wären weder Krankenkassen noch Leistungserbringer bereit, die Diskussion um neue Versorgungsstrukturen, die mindestens zehn Jahre lang ohne praktische Resonanz geblieben ist, in konkretes Handeln zu überführen. Ich wage sogar die These, daß der nachlassende Budgetdruck mit dazu geführt hat, Modellpläne nicht weiter zu konkretisieren oder sie gar völlig in der Schublade verschwinden zu lassen.

Man muß nach diesen - hier nur sehr gerafft dargestellten - Erfahrungen leider festhalten, daß eine sektorale Budgetierung, die mit dem Vorschaltgesetz noch einmal verlängert werden soll, dem realen Leistungsund Kostengeschehen im deutschen Gesundheitswesen nicht gerecht wird. Wenn es beispielsweise richtig ist, daß rund ein Drittel der in Deutschland stationär erbrachten Leistungen auch teilstationär oder ambulant erbracht werden kann, muß dies Konsequenzen für den Mittelfluß in die Versorgungsbereiche haben. Eine sektorale Budgetierung und eine strukturkonservative Krankenhausfinanzierung verhindern heute, daß der Mittelfluß entsprechend umgelenkt wird. Deshalb ist es schon für das Vorschaltgesetz bedeutsam, daß Budgets dort flexibel ausgestaltet werden, wo neue Versorgungsformen erprobt werden. Ansonsten besteht die Gefahr, daß das zarte Pflänzchen der mittelfristigen Investitionsplanung für neue Versorgungsformen, das die Akteure des Gesundheitswesens gerade mühsam eingeführt haben, von den groben Instrumenten der Globalsteuerung plattgewalzt wird.

Die neue Regierungsmehrheit will zumindest für 1999 an die alten sektoralen Budgets anknüpfen. Dies ist keine optimale Lösung, wenn man Strukturveränderungen im Gesundheitswesen will. Dies ist aber zu verstehen, wenn man in wenigen Wochen ein Vorschaltgesetz durch die parlamentarischen Gremien schleusen muß, das den Boden für umfassendere Reformen bereitet. Sektorale Budgets für das Jahr 1999 können deshalb nur Übergangslösungen sein. Es wird von der Reformbereit- 
schaft und der Innovationskraft der Akteure in der Diskussion um die Ausgestaltung der nächsten strukturellen Reform abhängen, wann und wie diese Budgets durch andere Steuerungsinstrumente abgelöst werden können. Darüber hinaus halte ich es für sinnvoll und im Vorschaltgesetz ohne Probleme für praktikabel, sektorale Budgets durch flexiblere Instrumente zwischen den Sektoren zu ergänzen.

Im Rahmen der für 1999 geplanten Strukturreform halte ich eine globale Ausgabengrenze in der gesetzlichen Krankenversicherung ohne Abstriche für erforderlich und umsetzbar. Sie ist für mich in erster Linie eine politische Größe. Diese Größe markiert den finanziellen Rahmen, den die Gesellschaft für eine solidarisch finanzierte Gesundheitsversorgung auszugeben bereit ist. Solche Obergrenzen existieren mehr oder weniger strikt in allen Staaten, die sich der Gewährleistung umfassender sozialer Sicherheit verpflichtet fühlen.

Das Plädoyer für eine Ausgabenobergrenze, die politisch verantwortet werden muß, sagt noch nichts darüber aus, wie hoch diese Obergrenze ist und wie sie sich im Laufe der Zeit zu verändern hat. Die gesellschaftspolitische Bedeutung der Obergrenze macht es aber zwingend, daß inre Veränderung nicht dem Interessenspiel der Akteure im Gesundheitswesen ausgeliefert ist. Das Machtspiel von Kassenverbänden oder Kassenärztlichen Vereinigungen, die ökonomischen Interessen von Krankenhäusern oder pharmazeutischen Unternehmen sollten als Bestimmungsfaktoren von Budgetveränderungen auf der globalen Ebene ausscheiden. Vielmehr erscheint mir nur eine epidemiologisch fundierte und politisch legitimierte Konsensfindung geeignet, globale Obergrenzen an tatsächliche Veränderungen im Gesundheitswesen anzupassen. Solange die Finanzierung der sozialen Krankenversicherung am Faktor anknüpft, wird als Anknüpfungspunkt der Budgetfortschreibung die Veränderung der beitragspflichtigen Einnahmen zu wählen sein. Ändert man die Finanzierungsgrundlagen, so wäre eine Orientierung an der Entwicklung des Bruttoinlandsprodukts denkbar. Damit würde der Anteil solidarisch finanzierter Gesundheitsausgaben stabilisiert.

Die mittel- und langfristig wichtigste Frage bei der Ausgestaltung der Budgetierung ist die Bestimmung des Budgethalters. Da die Konsequenzen unterschiedlicher Optionen (Krankenkasse, Kassenart, GKV) noch längst nicht ausgeleuchtet sind, stehe ich einer vorschnellen Lösung skeptisch gegenüber, die - wie im SPD-Entwurf des GSG II- die Krankenkassen zum Budgethalter für globale Ausgabenobergrenzen im Gesundheitswesen machen will. Dies mag eine praktikable Lösung für eine Übergangsphase sein. Langfristig erscheint mir weder Interessenlage noch Struktur der wettbewerblich orientierten Kassenorganisation 
geeignet, solche Steuerungsfunktionen zu übernehmen. Auch erscheinen mir die Instrumentarien der GKV nicht ausreichend, globale Ausgabengrenzen im Kräftespiel des Gesundheitswesens auch tatsächlich so durchzusetzen, daß eine bedarfsgerechte Versorgung der Bürgerinnen und Bürger gewährleistet ist. Vielmehr ist die Gefahr groß, daß die ökonomische und organisatorische Macht der Leistungserbringerorganisationen dann die Verteilung der Finanzmittel bestimmt. Ohne eine (kassenbezogene) Einkaufsmacht und eine Verbesserung der Steuerungskompetenz ist die Einzelkasse mit Budgetsteuerung und -haftung überfordert. Die korporatistische Ausgestaltung des deutschen Gesundheitswesens und die strukturelle Anbieterdominanz spricht vielmehr dafür, das Globalbudget (regional) einer Arbeitsgemeinschaft der Krankenkassen zuzuordnen. Selbstverständlich müssen dann Regelungen gefunden werden, wie die Budgets heruntergebrochen werden.

Eine globale Ausgabengrenze - etwa auf der Landesebene - sagt noch nichts darüber aus, wie denn das Geld tatsächlich verteilt wird. Zwischen den in der Region vertretenen Kassen kann der Risikostrukturausgleich möglicherweise diese Verteilungsfunktion übernehmen. Es wäre reizvoll zu überlegen, ob die Normkostenprofile auch zur Budgetsteuerung bei den Leistungserbringern genutzt werden können. Ohne Gesundheitsziele und eine epidemiologisch fundierte Evaluation von Versorgungsstrukturen und -prozessen wird Budgetierung ein Steuerungsprozeß werden ohne Bezug zur Versorgungsrealität mit erheblichen Gefahren der gesundheitspolitischen Fehlsteuerung. Die Ressourcensteuerung über Budgets muß sich also in einen neuen Regelkreislauf der gesundheitspolitischen Systemsteuerung einfügen.

Die Einführung eines Globalbudgets ohne hinreichend flexible Steuerungsinstrumente auf den Ebenen, wo das reale Versorgungsgeschehen stattfindet, wird außer Kostendämpfungswirkungen nur begrenzte Veränderungen im Gesundheitswesen erzeugen. Im SGB V muß also ein hinreichend flexibler Rahmen geschaffen werden, mit neuen Instrumenten den Finanzfluß in und zwischen den Versorgungsbereichen zu steuern. Reale und virtuelle Teilbudgets werden künftig wesentlicher Verhandlungsgegenstand zwischen Krankenkassen und Leistungserbringern sein. Im stärkeren Umfang als bisher werden Kombinationen zwischen den Budgetformen die Integration von Strukturen und Prozessen fördern und positive wie negative Anreize zur Erreichung von Versorgungszielen setzen müssen. Sektorale Budgets können unterhalb der globalen Ausgabenbegrenzung schrittweise von den Vertragspartnern kombiniert und flexibilisiert werden. Dies setzt voraus, daß in einer Strukturreform starre Normvorgaben für die Steuerung von Kapazitäten, 
Mengen, Preisen und Inhalten der Leistungserbringung zugunsten fiskalischer und gesundheitlicher Ziele aufgelockert werden.

Eine ausdifferenzierte Versorgung wird nur dann Qualitätsgewinne erzeugen und Wirtschaftlichkeitsreserven erschließen, wenn Leistungserbringer auch in die ökonomische Verantwortung für eigene und veranlaßte Leistungen, speziell für Überweisungen und Weiterbehandlungen einbezogen werden. Ein Blick in andere Länder, speziell in die Niederlande, in die Schweiz, Kanada oder die USA, könnte den Horizont auch in Fragen der Budgetierung weiten und Anregungen für einen innovativen Umgang mit dem neuen Steuerungsinstrument geben. Der Diskussionsprozeß um neue Formen der Ressourcensteuerung ist also mit der politischen Grundsatzentscheidung für die Einführung von Globalbudgets keineswegs beendet, sondern hat gerade erst begonnen. Die Akteure des Gesundheitswesens haben die Chance, bei der Schaffung neuer Rahmenbedingungen mitzuwirken und neue Freiräume zu schaffen. Wer allerdings glaubt, mit aggressiver Lobbypolitik nach dem Motto „In die Ecke Besen, Besen, sei's gewesen, sei's gewesen!" sei das Gespenst der Budgetierung zu bändigen, dürfte sich aus der Diskussion bald verabschieden. 
Hartmut Reiners

\section{Weshalb brauchen wir ein Globalbudget in der gesetzlichen Krankenversicherung?}

Die praktische Politik wird von Schlagworten geprägt, die in der Öffentlichkeit für eine ganz bestimmte inhaltliche Orientierung in der Sache stehen sollen. Der CDU-Politiker Heiner Geißler stellte einmal fest, die hohe Schule der Politik bestehe darin, Begriffe zu besetzen. Er selbst ist ein anerkannter Meister dieser Kunst. Ein Begriff, der die Gesundheitspolitik in Deutschland seit 25 Jahren prägt, stammt von ihm: die „Kostenexplosion“. In der von inm herausgegebenen Studie „Das Krankenversicherungsbudget" (Geißler, 1974) wurde erstmals das Szenario einer ausufernden Entwicklung der Krankenkassenausgaben entwickelt.

Der unstreitig besonderen Ausgabendynamik des Gesundheitswesens wurde seither mit verschiedenen Ansätzen einer Budgetierung zu begegnen versucht. $\mathrm{Da}$ ist seit 20 Jahren von kombinierten, sektoralen oder strukturierten Budgets sowie - immer wieder - vom Globalbudget die Rede, ohne daß eigentlich so richtig klar ist, was damit genau gemeint ist. Franz Knieps hat mit seiner Charakterisierung der Budgetierung als einem "Gespenst" gezeigt, wie sich Fachbegriffe in Reizvokabeln verwandeln können. Überhaupt wird die gesundheitspolitische Diskussion von Schlagworten überlagert, die in den Medien ein faktenresistentes Eigenleben entwickelt haben.

\section{Mythos Kostenexplosion 35}

Ausgangspunkt aller Konzepte zur Budgetierung von Krankenkassenausgaben ist die Überzeugung, daß ohne eine administrativ festgelegte oder vertraglich vereinbarte Begrenzung die Ausgaben aus dem Ruder zu laufen drohen. Dem scheint die Tatsache entgegenzustehen, daß sich die Gesundheitsausgaben in Deutschland seit 20 Jahren in etwa im Gleichschritt mit dem allgemeinen Wirtschaftswachstum bewegt haben. Auf jeden Fall kann von einer Kostenexplosion keine Rede sein. Wenn die Gesundheitsausgaben in den letzten Jahren wieder stärker gestiegen sind als das Bruttoinlandsprodukt (BIP), dann hängt dies vor allem

35 Vgl. hierzu Braun/Kühn/Reiners 1998. Soweit nicht anders erwähnt, stammen alle nachfolgend angeführten Daten aus dieser Publikation. 
mit den Folgen der deutschen Einigung und der schlechten wirtschaftlichen Lage der neuen Länder zusammen:

- Die Infrastruktur des DDR-Gesundheitswesens war wegen chronischer Unterfinanzierung in einem miserablen Zustand. Daraus resultierte ein investiver Nachholbedarf insbesondere bei den Krankenhäusern, wie wir inn in Westdeutschland in durchaus vergleichbarer Form in den späten 60er und frühen 70er Jahren hatten; seinerzeit sprang der Anteil der Gesundheitsausgaben am Bruttosozialprodukt von $6,4 \% 1970$ auf 9,1\% im Jahr 1975.

- Die Gesundheitsausgaben können sich nicht automatisch der wirtschaftlichen Entwicklung anpassen, zumal dann, wenn sie - wie in den neuen Ländern - rückläufig ist. So sind die Wachstumsraten des BIP von durchschnittlich $8,1 \%$ in den Jahren 1990 - 92 auf durchschnittlich 2,7\% in den Jahren 1993 - 94 gesunken. Dieser Trend hat sich weiter fortgesetzt, was den Anteil der Gesundheitsausgaben am BIP in Deutschland nach Berechnungen der OECD derzeit über die 10\%-Marke getrieben hat. Dabei muß deutlich zwischen Ost- und Westdeutschland differenziert werden. 1994 lagen nach Berechnungen des BASYS-Instituts die Gesundheitsausgaben in Westdeutschland bei $9,23 \%$ des BIP, in Ostdeutschland hingegen bei $13,31 \%$ (Schneider u. a., 1998).

Man sieht, der Anteil der Gesundheitsausgaben am BIP hängt mindestens ebenso von der allgemeinen wirtschaftlichen Entwicklung ab wie von der Kostenentwicklung im Gesundheitswesen. In Irland z. B. gab es in den letzten Jahren wegen eines überdurchschnittlich hohen BIPWachstums einen sinkenden Anteil der Gesundheitsausgaben, obwohl auch diese absolut gestiegen waren.

\section{Einnahmeschwäche: Ursache für die Finanzkrise der GKV}

Noch brüchiger wird die gängige These von der Kostenexplosion, wenn wir uns nur die GKV-Ausgaben anschauen. Deren BIP-Anteil ist in Westdeutschland seit 1980 so gut wie konstant. Er stieg von durchschnittlich 5,87\% im Jahrfünft $1980-84$ auf $5,95 \%$ in den Jahren 1991 - 95. Das ist eine Steigerung um ganze 0,08 Prozentpunkte. Wie kommt es aber dann zu anhaltend steigenden Beitragssätzen? Sie sind in den alten Bundesländern zwischen 1980 und 1997 von durchschnittlich $11,38 \%$ auf $13,42 \%$ gestiegen, in den neuen Ländern von 1991 bis 1997 von $12,80 \%$ auf 13,81 \%. Das sind stolze Zuwachsraten, die sich mit der moderaten Entwicklung bei den Leistungsausgaben nicht erklä- 
ren lassen. Es bleibt nur eine Ursache für die steigenden Beitragssätze der Krankenkassen übrig: sinkende Einnahmen.

Da sich die Krankenkassen aus Beiträgen, d. h. aus Lohn- bzw. Gehaltsanteilen ihrer Mitglieder finanzieren, hängt die Einnahmeentwicklung grundsätzlich von zwei Faktoren ab: von der Zahl der Beitragszahler, und damit der versicherten Arbeitnehmer, sowie dem Niveau der Arbeitnehmereinkommen. Hier liegen die eigentlichen Gründe für die wirtschaftlichen Probleme der GKV in den letzten Jahren. Der Bruttolohnanteil am Volkseinkommen sank von $75,0 \%$ im Jahresdurchschnitt von 1975 - 80 auf $70,46 \%$ im Jahrfünft von 1989 - 93. Berücksichtigt man den gleichzeitig gestiegenen Anteil der Arbeitnehmer an der Gesamtzahl der Erwerbstätigen, dann ging die so bereinigte Lohnquote von $71,75 \%$ auf $65,7 \%$ des Volkseinkommens zurück. Da die Lohnquote die Finanzierungsbasis der GKV ist, hat ihr Sinken selbst bei konstanten Ausgaben steigende Beitragssätze zur Folge. Wären Beschäftigung und Lohnquote heute noch auf dem Niveau der frühen 80er Jahre, läge der aktuelle durchschnittliche Beitragssatz in der GKV bei unter $12 \%$, d. h. um fast 2 Prozentpunkte niedriger als heute. Die steigenden Krankenkassenbeiträge der letzten Jahre sind also vor allem auf makroökonomische Verteilungseffekte zu Lasten der Arbeitnehmer zurückzuführen.

\section{Mythos Lohnnebenkosten}

Insofern ist es schon ein wenig verrückt, wenn z. B. der Sachverständigenrat zur Begutachtung der gesamtwirtschaftlichen Entwicklung regelmäßig seine Litanei vom wettbewerblichen Zwang zu sinkenden Löhnen verkündet und im selben Atemzug die steigenden Sozial- und Krankenversicherungsbeiträge beklagt, wo es sich doch um zwei Seiten ein und derselben Medaille handelt. Außerdem sind die Lohnkosten kein sachlich begründeter Maßstab für die Entwicklung der Krankenkassenausgaben. Mal abgesehen davon, daß sie eine betriebswirtschaftliche und keine gesundheitsökonomische Kategorie darstellen, ist die Behauptung, die Höhe der Lohnnebenkosten sei ein entscheidender Wettbewerbsnachteil für den Standort Deutschland, ohne empirische Grundlage. Zum einen liegt die deutsche Wirtschaft mit ihrer Abgabenbelastung im internationalen Vergleich recht günstig; nach Angaben der OECD haben lediglich Japan und die USA niedrigere Werte. ${ }^{36}$ Wie sonst wären auch

36 Insbesondere für die USA müssen die OECD-Daten relativiert werden. Als Sozialversicherungsabgaben gelten in den OECD-Statistiken ex definitione nur Steuern und Sozialversicherungsbeiträge. In den USA sind die Arbeitnehmer nicht durch eine Sozialversicherung oder den Staat gegen gesundheitliche Risiken abgesichert, sondern über betriebliche "health plans“, die von privaten oder - wie 
die nach wie vor hohen Exportüberschüsse der deutschen Wirtschaft zu erklären? Zum zweiten wird die Bedeutung der Krankenkassenbeiträge für die Kostenbelastung der Unternehmen völlig überschätzt. Der Sachverständigenrat für die Konzertierte Aktion im Gesundheitswesen ist 1995 in einer Modellrechnung zu dem Ergebnis gekommen, daß eine Beitragssatzsteigerung um einen Prozentpunkt - das wäre eine Ausgabensteigerung um etwa $20 \mathrm{Mrd}$. DM! - die Arbeitskosten im produzierenden Gewerbe um knapp 0,4\% anheben würde. Die Arbeitskosten machen aber insgesamt nur gut ein Viertel der Gesamtkosten aus, so daß der tatsächliche Kosteneffekt bei $1 \%$ liegt. Es ist wenig überzeugend, einer solchen Größenordnung eine wettbewerbsentscheidende Funktion beizumessen. Und ob die neue Bundesregierung sich mit der insbesondere von den Grünen postulierten Formel "Ökosteuer = Absenken der Lohnnebenkosten $=$ mehr Arbeitsplätze" einen Gefallen getan hat, möchte ich bezweifeln.

\section{Die besondere Ausgabendynamik des Gesundheitswesens}

Wenn aber die Finanzkrise der Krankenkassen vornehmlich einnahmebedingt ist und auch die Lohnnebenkosten für die GKV-Ausgaben keine sachlich zu begründende Orientierung bieten, wozu brauchen wir dann eine Ausgabenbegrenzung? Wir dürfen in diesem Zusammenhang eines nicht übersehen: Die relativ konstante Entwicklung der Leistungsausgaben der GKV wäre ohne die zahlreichen politischen Eingriffe - von den Kostendämpfungsgesetzen bis zum Gesundheitsstrukturgesetz - nicht denkbar. Es kann kein Zweifel daran bestehen, daß die Krankenkassenausgaben ohne die gesetzlich vorgegebenen bzw. vertraglich vereinbarten Begrenzungen ungleich stärker gestiegen wären. Seit über 20 Jahren hat es praktisch keine Zeit ohne Budgetierungen gegeben. Wenn sie nicht gesetzlich geregelt waren, wurden und werden sie in Form von Pauschal- oder Gesamtvergütungen praktiziert. Und was, bitte schön, sind z. B. Regelleistungsvolumina anderes als spezifische Budgetdefinitionen?

Die Gesundheitsausgaben haben unstreitig eine immanente Tendenz zu überproportionalen Steigerungsraten. Das ist unter Fachleuten weitgehend unbestritten. Für dieses Phänomen werden diverse Erklärungsversuche geliefert, die jedoch von unterschiedlicher Stichhaltigkeit sind. In

z. B. Blue Shield/Blue Cross - Non-profit-Versicherungen angeboten werden. Dabei handelt es sich um betriebliche Sozialleistungen, die zwar in die Lohnkosten einfließen, aber in den OECD-Statistiken nicht als Sozialversicherungsabgaben geführt werden. 
den Medien, aber auch bei den meisten Standesfunktionären der Ärzteschaft dominieren drei Argumente:

- Der medizinische Fortschritt sei ausgesprochen kostenintensiv, seine Umsetzung für die Patienten nicht ohne zusätzliches Geld zu haben.

- Die steigende Lebenserwartung der Menschen und die zunehmende Zahl älterer Menschen erhöhten den gesellschaftlichen Bedarf an medizinischer Betreuung.

- Das umfassende Leistungsangebot der GKV führe zu einer „Vollkasko-Mentalität" und provoziere mißbräuchliche Inanspruchnahme.

Diese für den unbefangenen Betrachter plausibel erscheinenden Begründungen sind jedoch eher Vorurteile als wissenschaftlich belegbare Aussagen. Gleicht man vorhandene wissenschaftliche Erkenntnisse mit diesen Thesen ab, ergibt sich folgendes Bild (vgl. Braun/Kühn/Reiners, 1998):

- Der medizinisch-technische Fortschritt kann die Gesundheitsausgaben in die Höhe treiben, muß dies aber nicht. Die ökonomischen Auswirkungen bzw. Kosten derartiger Innovationen hängen von dem Zusammenhang $a b$, in dem sie eingesetzt werden. Es handelt sich um ein Mengen- und Qualitätsproblem vor dem Hintergrund handfester wirtschaftlicher Interessen, nicht um eine Art Naturereignis. So entwickelt sich z. B. die medizin-technische Diagnostik um ein Vielfaches schneller als die entsprechenden therapeutischen Möglichkeiten, die solche Verbesserungen für die Patienten überhaupt erst sinnvoll machen. Außerdem können heute dank der Entwicklung in der Mikrochirurgie und der Anästhesie Eingriffe kostengünstig ambulant bzw. teilstationär durchgeführt werden, die vor Jahren noch mit einem wochenlangen Krankenhausaufenthalt verbunden waren. Selbst das Argument, viele Eingriffe und Behandlungsmethoden wären früher technisch nicht möglich gewesen, ist nicht unbedingt stichhaltig. Die entsprechenden Patienten wurden womöglich nicht operiert oder weniger effektiv therapiert, aber deswegen waren sie ja nicht ohne medizinische Betreuung ihres Leidens; und die neuen Behandlungsmethoden sind nicht a priori teurer als die alten. Hier wären kritische Wirkungsforschung, transparente Steuerung und Planung anstelle unreflektierter Bewunderung neuer Techniken erforderlich. Wenn wir z. B. in Deutschland eine dreimal so hohe Versorgungsdichte mit Linksherzkathetermeßplätzen haben wie in den Niederlanden, so ist dies weniger ein Beleg für die Modernität unseres Gesundheitswesens als vielmehr ein harter Indikator für unwirtschaftlichen Ressourceneinsatz. 
- Zweifelsohne hat die steigende Zahl älterer Menschen Auswirkungen auf den Bedarf an medizinischen Leistungen. Jedoch sind die daraus womöglich resultierenden Kosteneffekte weit weniger dramatisch, als allgemein suggeriert wird. Der Sachverständigenrat für die Konzertierte Aktion im Gesundheitswesen kam in seinem 1995er Gutachten auf demographiebedingte Steigerungen der Gesundheitsausgaben von zwischen $0,74 \%$ und $0,86 \%$ pro Kopf, also keine besorgniserregenden Werte. Auch läßt sich zeigen, daß die Ausgaben für medizinische Leistungen nicht mit zunehmendem Alter steigen, sondern mit der Nähe zum Tod. Da jeder Mensch nur einmal stirbt und ein hoher Behandlungsaufwand dem letzten Lebensjahr zugerechnet werden muß, wächst dieser nicht automatisch dadurch, daß das Sterbealter sich erhöht. Es gibt sogar in verschiedenen internationalen Studien deutliche Hinweise, die auf eher geringere Krankheitskosten im hohen Alter schließen lassen.

- Die unter Ökonomen und Sozialwissenschaftlern unter dem Begriff „Moral hazard" diskutierte These, das über Umverteilung finanzierte Sozialversicherungssystem führte notwendigerweise zu mißbräuchlicher Inanspruchnahme und damit unangemessenen Kosten, ist für die Krankenversicherung noch nicht einmal als Plausibilitätsüberlegung geeignet. Diese These geht nämlich implizit davon aus, daß die Inanspruchnahme medizinischer Leistungen so erstrebenswert ist, daß die Versicherten gar nicht genug davon bekommen können. Diese Annahme läßt sich eigentlich schon mit dem Hinweis auf zeitraubende Wartezeiten beim Arzt, die zweifelhaften Freuden eines Zahnarztbesuches oder den reichlich herben Genuß einer BypassOperation als sachfremd verwerfen. Natürlich kennen wir alle jemanden, der oder die zum Arzt geht, ohne nennenswerte Beschwerden zu haben, oder schlicht und einfach „krank feiert“. Mal abgesehen davon, daß der Krankenstand in Deutschland seit Jahren rückläufig ist und zum Teil unter dem Niveau vergleichbarer Länder liegt, ist die hier zur Diskussion stehende Personengruppe kein kostenmäßig wirklich relevantes Phänomen. $75 \%$ der GKV-Ausgaben entfallen auf nur $10 \%$ der Versicherten, allesamt Schwer- bzw. Chronischkranke, d. h. Menschen, von denen man wohl nicht behaupten kann, sie mißbrauchten die Leistungen der Krankenkassen.

Die immanente Tendenz zu steigenden Gesundheitsausgaben läßt sich also nicht mit den Verweisen auf den medizinisch-technischen Fortschritt, die demographische Entwicklung oder das Anspruchsverhalten der Versicherten begründen. Wenn diese gängigen Behauptungen nicht stimmen, dann entfällt zugleich die Grundlage für das von interessierter 
Seite immer wieder vorgetragene Menetekel einer Rationierung der Medizin, falls nicht deutlich mehr Geld ins Medizinsystem fließt. Diese Parole ist entweder irreführend, solange wir insbesondere in der stationären Versorgung, der Apparatemedizin und der Arzneimittelversorgung noch große Rationalisierungspotentiale haben. Oder sie ist banal, da angesichts prinzipiell nicht unbegrenzt zur Verfügung stehender Ressourcen immer entschieden werden muß, welche Leistungen Vorrang haben - was ja auch eine Art Rationierung ist.

\section{Angebotsinduzierte Nachfrage}

Der Schlüssel zum Verständnis der besonderen Ausgabendynamik im Gesundheitswesen liegt in der ökonomischen Funktion der Ärzte als Anbieter bzw. Erbringer medizinischer Leistungen. Der Medizinsoziologie ist das seit längerem bekannt, auch in der Gesundheitsökonomie macht sich diese Erkenntnis allmählich breit. Kein anderer Wirtschaftszweig wird so von einer Berufsgruppe dominiert wie das Gesundheitswesen von den Ärzten. Sie haben - egal, ob als frei niedergelassene oder im Krankenhaus angestellte Ärzte-grundsätzlich das gesellschaftliche Mandat, den Gesundheitszustand ihrer Patienten und die daraus abzuleitenden therapeutischen Maßnahmen zu definieren. Das ist kein kritikwürdiges Phänomen, sondern ein alternativloser Sachverhalt. Irgend jemand muß diese Aufgaben wahrnehmen, und wem sollte das sonst zufallen als dem Berufsstand, der dafür ausgebildet wurde, den Ärzten?

Daraus erwächst jedoch ein erhebliches ökonomisches Problem, da im Gesundheitswesen praktisch die Anbieter von Leistungen zwar nicht unbedingt die Preise, so aber doch Art und Menge der von ihnen zu erbringenden Leistungen in erheblichem Umfang selbst bestimmen können. Der laut Lehrbuchökonomie einen Gleichgewichtszustand von Angebot und Nachfrage zwar nie vollständig erreichende, aber doch in seiner Tendenz anstrebende Marktmechanismus funktioniert im Gesundheitswesen grundsätzlich nicht. Es herrscht strukturelle und nicht nur temporäre Anbieterdominanz und damit das, was Ökonomen als "Marktversagen" zu bezeichnen pflegen. Der Umgang mit diesem Phänomen ist das zentrale Steuerungsproblem im Wirtschaftszweig Gesundheitswesen. Es war der große strategische Fehler von Herrn Seehofer, mit seinen GKVNeuordnungsgesetzen einen - wie er es nannte - „Paradigmenwechsel“ zu verkünden und den ökonomischen Hebel bei den Patienten und Versicherten anzusetzen anstatt bei den Leistungserbringern. Das war nicht nur eine politische Steilvorlage für den bekanntlich sehr erfolgreichen Wahlkampf der früheren Opposition. Es war auch aus gesundheitsökonomischer Sicht eine krasse Fehlentscheidung. 
Soll die Entwicklung der Gesundheitsausgaben nicht ausufern und mit erheblicher Ressourcenverschwendung verbunden sein, bedarf es einer entsprechenden Stärkung der Nachfragemacht als einer „countervailing power". Insofern kann es in der Gesundheitspolitik nicht um reine Angebotssteuerung nach dem Muster einer Kommandowirtschaft gehen. Es kommt vielmehr darauf an, das Verhältnis von Anbietern und Nachfragern durch politisch gesetzte Rahmenbedingungen u. a. im Vertragsrecht zu steuern. Das ist zwar a priori keine Garantie für eine wie auch immer definierte optimale Ressourcenverteilung. Die Steuerung über Recht und Politik sowie kollektive Vertragssysteme hat im Gesundheitswesen aber unstreitige Vorteile gegenüber privat- bzw. einzelwirtschaftlichen Steuerungsformen. Am Beispiel der USA läßt sich zeigen, daß ironischerweise gerade durch die Privatisierung der Nachfrage und Risikoverteilung im Gesundheitswesen sich eine unglaublich aufgeblähte Bürokratie entwickelt hat. Da stehen - bildlich ausgedrückt - neben jedem Arzt zwei Rechtsanwälte und drei Versicherungsangestellte, die damit beschäftigt sind, Wirtschaftlichkeit und Qualität der ärztlichen Leistungen zu kontrollieren. Anders ist in einem privatwirtschaftlichen System die dominante Stellung der Ärzte auch gar nicht in den Griff zu bekommen. Die kostenmäßigen Auswirkungen sind erheblich. In den USA wird ein Drittel der Krankenhauskosten von den Verwaltungen der Kliniken und Versicherungen verschlungen; in Deutschland sind dies nur $10 \%$. Bei uns sind auch die Verwaltungskosten der gesetzlichen Krankenversicherungen deutlich niedriger als bei der privaten Konkurrenz. Zwar behaupten letztere, sie seien mit einem Verwaltungskostenanteil von ca. $4 \%$ wirtschaftlicher als die GKV, die etwa $5 \%$ ihres Budgets dafür ausgibt. Jedoch verschweigt die PKV dabei inre enormen Akquisitionskosten in Form von Provisionen und Vertreterhonoraren, die - je nach Versicherung - bis zwischen $7 \%$ und $15 \%$ ihrer Ausgaben verschlingen. Soviel zum Thema „Abbau der Bürokratie durch Privatisierung“.

Dieser Sachverhalt begründet die ökonomischen Vorteile einer politischen Regulierung des Gesundheitswesens durch eine gesetzliche Krankenversicherung, jedoch noch nicht die Notwendigkeit eines Globalbudgets. Letzteres ist dann gerechtfertigt, wenn die Krankenkassen nicht von sich aus in der Lage sind, ihre Ausgaben in einem akzeptablen Rahmen zu halten. Die entscheidende Frage ist dabei: Welche Ausgabenhöhe ist akzeptabel? In anderen Wirtschaftszweigen wird diese Frage vom Markt bzw. der zahlungsfähigen Nachfrage beantwortet. Dieser Parameter ist im Gesundheitswesen aus guten gesellschaftspolitischen Gründen weitgehend ausgeschaltet. Es gehört zu den Merkmalen einer zivilisierten Gesellschaft, daß der Zugang zur medizinischen Versorgung nicht oder nur in begründeten Ausnahmefällen von der individuellen Finanzkraft der Bürger abhängt und statt dessen über eine staatliche Ver- 
sorgung oder - wie in Deutschland - über eine gesetzliche Krankenversicherung reguliert wird.

Dementsprechend übernimmt faktisch die GKV die Funktion des Nachfragers und trägt im Rahmen ihrer gesetzlich vorgegebenen Kompetenzen die finanzielle Verantwortung. Dabei ist es in einem selbstverwalteten, gegliederten Krankenversicherungssystem nicht von vornherein zwingend, daß der Staat bzw. die Politik über die leistungsrechtlichen Ansprüche der Versicherten und die Vertragsbeziehungen zwischen Kassen und Leistungserbringern hinaus auch die Budgethöhe der Krankenkassen festlegt. Theoretisch wäre auch der Wettbewerb unter den Krankenkassen in der Lage, die Höhe der GKV-Ausgaben in den gesellschaftlich erwünschten Dimensionen zu belassen. Dieses Modell ist bekannt und in der gesundheitsökonomischen Literatur hinreichend gewürdigt worden. Bei freier Kassenwahl haben die Krankenkassen ein elementares Interesse an möglichst niedrigen Beitragssätzen. Dementsprechend hoch wird ihre Motivation eingeschätzt, bei den Vertragsverhandlungen mit den Ärzten, Krankenhäusern usw. zu kostengünstigen Abschlüssen zu kommen.

Das Funktionieren dieses Modells setzt zweierlei voraus. Zum einen benötigen die Krankenkassen die erforderlichen Freiheiten in der Vertragsgestaltung. Davon kann in dem gegebenen GKV-System nicht die Rede sein. $\mathrm{Zu}$ viele hinreichend bekannte Hindernisse stehen den Krankenkassen im Weg. Als Stichworte seien nur die duale Finanzierung der Krankenhäuser, die strikte Trennung zwischen ambulanter und stationärer Versorgung und das Behandlungsmonopol der Kassenärzte in der ambulanten Versorgung genannt. Bislang war die Politik nicht in der Lage, diese seit jeher beklagten Systemmängel unseres Gesundheitswesens zu beheben. Der zwischen SPD und Bündnis 90/Die Grünen geschlossene Koalitionsvertrag verspricht zwar, sich diesen Problemen vorrangig zu widmen. Aber ein in Jahrzehnten gewachsenes System läßt sich nicht von heute auf morgen in seinen arbeitsteiligen Strukturen verändern, von dem auch aus den eigenen Reihen der Regierungsparteien zu erwartenden Widerstand einmal abgesehen. Aber selbst wenn die Krankenkassen den gewünschten Spielraum bei Vertragsverhandlungen bekommen, werden sich wettbewerbliche Strukturen nur in Teilbereichen erfolgreich implementieren lassen. So ist es z. B. in Ballungszentren kein größeres Problem, Versorgungsverträge mit Krankenhäusern zu kündigen. Die Versorgung ist nicht gefährdet, nur weil ein Stadtteil kein eigenes Hospital hat. Das sieht auf dem flachen Land schon ganz anders aus, wo die Patienten oft auf nur ein Krankenhaus angewiesen sind. Wettbewerb bedeutet Auswahlmöglichkeiten, und davon kann da nicht die Rede ein. Überhaupt habe ich den Eindruck, daß die in der 
Diskussion befindlichen Wettbewerbsmodelle implizit unterstellen, Deutschland sei eine Agglomeration von Ballungszentren, wo den Patienten eine bunte Auswahl verschiedener medizinischer Versorgungseinrichtungen zur Verfügung steht. In Ländern wie Brandenburg kann die Schließung eines Krankenhauses bedeuten, daß die Patienten ab sofort ein über $50 \mathrm{~km}$ entferntes Haus aufzusuchen haben. Wahlmöglichkeiten gibt es für sie nicht.

Die zweite Voraussetzung für einen funktionierenden Wettbewerb unter den Kassen ist auch nur bedingt gegeben: die Orientierung auf die Vertragsgestaltung mit den Leistungserbringern. Da sich die Höhe des Beitragssatzes einer Kasse aus den Ausgaben und den Beitragseinnahmen ergibt, ist die wirtschaftliche Leistungsfähigkeit einer Kasse von der Einkommensstruktur ihrer Mitglieder einerseits, der Morbidität der Versicherten andererseits abhängig. Es lohnt sich für eine Kasse, sich bei der Akquisition ihrer Versicherten an deren Risiken zu orientieren. Das ist auch erheblich einfacher, als über das schwierige Geschäft der Vertragsverhandlungen mit Ärzten und Krankenhäusern zu günstigen Beitragssätzen zu gelangen, zumal diese Bemühungen vergeblich sein können, wenn sich in einer Kasse die „schlechten“ Risiken anhäufen. Der Gesetzgeber hat mit dem Gesundheitsstrukturgesetz von 1993 den Risikostrukturausgleich (RSA) in der GKV nicht nur deshalb eingeführt, weil die Risikoselektion mit den Grundsätzen der sozialen Krankenversicherung nicht vereinbar ist. Der RSA sollte auch die Funktion haben, das Augenmerk der Kassen ganz auf die Wirtschaftlichkeit und Qualität bei der Vertragsgestaltung zu lenken. Das ist nur bedingt gelungen. Zum einen haben die einzelnen Kassenarten nach wie vor unterschiedliche Möglichkeiten der Risikoselektion. Die Betriebskrankenkassen haben ganz andere Einflußpotentiale bezüglich der Struktur ihrer Klientel als die AOK oder die Ersatzkassen; und sie machen davon auch fleißig Gebrauch. Zum zweiten finden bestimmte Risikotatbestände keinen Eingang in den RSA, z. B. die Härtefallregelungen, was die Kassen mit einer sozial schwachen Versichertenstruktur deutlich benachteiligt. Zum dritten werden Morbiditätsrisiken nur zum Teil berücksichtigt. Als Morbiditätskriterium kennt der RSA nur Alter und Geschlecht der Versicherten, wobei man sich an den damit verbundenen durchschnittlichen Krankheitsrisiken orientiert. Nun ist es aber so, daß ein 60jähriger Bankangestellter für die Kassen ein weit "besseres“ Risiko darstellt als ein gleichaltriger Stahlarbeiter mit 45 Jahren Konti-Schicht auf dem Buckel. Sozial bedingte Morbiditätsunterschiede finden in dem RSA keine Berücksichtigung. Risikoselektion ist also nach wie vor möglich, wovon die Kassen auch nachweislich Gebrauch machen. 
Man sieht, die Voraussetzungen für einen patientenorientierten, an der Qualität und Wirtschaftlichkeit der medizinischen Versorgung ausgerichteten Wettbewerb sind nur sehr bedingt gegeben. Das soll nicht heißen, daß man besser auf dieses Instrument verzichten sollte. Das ist in einer gegliederten Krankenversicherung ex definitione unmöglich, und eine Einheitsversicherung ist aus verschiedenen, hier nicht zu thematisierenden Gründen eine weit schlechtere Lösung als das gegebene GKV-System. Es wäre jedoch eine verhängnisvolle Illusion zu glauben, man könne über den Wettbewerb und eine entsprechende Deregulierung der GKV die Politik bzw. den Staat entlasten. Nicht nur marxistische oder keynesianische Ökonomen, sondern auch die ordoliberalen Väter der sozialen Marktwirtschaft wie Eucken oder Röpke haben hinreichend deutlich machen können, daß der Markt keine sich selbst störungsfrei regulierende Veranstaltung ist, sondern schon aus Selbstschutz klarer staatlicher Rahmenbedingungen bedarf. Wenn dies schon für „normale“ Märkte gilt, dann erst recht für das von Marktversagen gekennzeichnete Gesundheitswesen. Hier muß die Politik Vorgaben an die Akteure machen, und dazu gehört auch die Festlegung der Höhe des Krankenversicherungsbudgets. In diesem Sinn ist das Globalbudget eine von der Politik vorgegebene, verbindliche Orientierungsgröße, aber kein starres Korsett wie die zwischen 1993 und 1995 geltende sektorale Budgetierung.

\section{Literatur}

Braun, Bernard/Kühn, Hagen/Reiners, Hartmut (1998), Das Märchen von der Kostenexplosion. Populäre Irrtümer zur Gesundheitspolitik, Frankfurt am Main

Schneider, Markus u. a. (1998), Gesundheitsausgaben im internationalen Vergleich - Übersichten 1997, Augsburg 
Gerhard Schulte

Für die anwesenden Experten der Gesundheitsversorgung enthält die Koalitionsvereinbarung zum Thema Gesundheitspolitik wenig Geheimnisse. Wir finden einige allgemeine Absichtserklärungen, die wir im wesentlichen in allen Regierungserklärungen seit 1949 wiederentdecken können. Wir finden einige sehr konkrete Hinweise, etwa die Abschaffung sog. Instrumente der privaten Krankenversicherung, aber doch auch ein Geheimnis. Dieses Geheimnis ist das „Globalbudget". Deswegen ist der Zeitpunkt günstig, die Frage zu vertiefen: Was ist ein Globalbudget und wie funktioniert es? Ich habe gerade im Vortrag von Herrn Reiners etwas über die Vorgeschichte des Globalbudgets erfahren, aber doch noch relativ wenig über die Funktionsfähigkeit und die Instrumente dieses Budgets.

Nun haben wir in Deutschland seit einigen Jahren Erfahrungen mit Budgets, die wir entweder gleich so nennen, die aber auch „Gesamtvergütung“ heißen können, „Richtgrößen“, „Regelleistungsvolumen“ oder „kombinierte Budgets“. Es macht deshalb Sinn, sich in begrenztem Umfang auch mit der Geschichte der Instrumente zu beschäftigen, die wir zur Verfügung haben. Zunächst einmal sind Budgets Instrumente der Planung. Das Problem der letzten Jahre aber war, das muß der Ehrlichkeit halber gesagt werden, daß die vorhandenen Budgets Instrumente der Kostenbegrenzung waren. Und deswegen wäre es auch zu einfach, wenn die weitere Entwicklung eines notwendigen Planungsinstrumentes weiterhin auf den Aspekt der Kostenbegrenzung eingeschränkt würde. Planungsinstrumente brauchen allerdings Verantwortlichkeit der Handelnden, und Handelnde wiederum müssen haften für ihr Handeln. Und daran hat es, wie Sie alle wissen, in der Vergangenheit gemangelt. Man kann vielleicht in den ersten Jahren der Budgetierung den Handelnden zugute halten, daß die Steuerungsinstrumente unterentwickelt waren und jedenfalls in den Jahren 1993 oder 1994 einige Probleme in der Erfassung relevanter Daten bestanden, was sich aber im Laufe der Zeit gewaltig verbessert hat. Andererseits sind gerade diejenigen Budgets, die nicht einfach auf einer Steigerungsdynamik beruhen, die sich am Wachsen der beitragspflichtigen Einnahmen der gesetzlichen Krankenversicherung orientiert, sondern durchaus sinnvolle Gegenstände der Planung zum Inhalt hatten, in der Umsetzungsphase am schwersten zu handhaben - nicht zuletzt, da diese Instrumente auch eine Intelligenz der Anwender voraussetzen. An dieser Intelligenz auf seiten der Anwender hat es weitgehend gefehlt. 
Ich möchte das am Beispiel des Arzneimittelbudgets des Gesundheitsstrukturgesetzes erläutern. Das Arzneimittelbudget des Gesundheitsstrukturgesetzes kennt das Wort „Steigerung der beitragspflichtigen Einnahmen" nicht, sondern beruht auf folgenden Vorgaben: Veränderung der Zahl und der Altersstruktur der Versicherten, Veränderung der Preise der Arzneimittel, Veränderung der gesetzlichen Leistungspflicht der Krankenkassen, bestehende Wirtschaftlichkeitsreserven und Innovationen. Da sind im Kern eigentlich all diejenigen Dinge genannt, die intelligente Anwender zugrunde legen würden, wenn sie Steigerungsoder auch Minusraten in einem Versorgungssegment verantwortlich für einen Zeitraum vorgeben wollen. Was vielleicht in dieser Aufzählung fehlt, ist die Veränderung der kurativen Fallzahlen. Dieses Element würde bei einer Wechselbewegung von der stationären Versorgung in die ambulante Versorgung auch diesem Sachverhalt Rechnung tragen. Sie wissen um das Schicksal der Arzneimittelbudgets. Es ist leider eine unintelligente Strategie verfolgt worden, auch wenn sie einzelne für intelligent gehalten haben, mit der Folge, daß die Politik auf der Suche nach einem anderen Instrument war, das aber wiederum auch nicht die gewünschten Erfolge gebracht hat. Auch wenn die Formulierung des Arzneimittelbudgets im SGB V über ausreichende Hinweise des Gesetzgebers zu einem vernünftigen Umgang mit diesem Budget verfügte, muß man doch festhalten, daß es jedenfalls an einer Korrespondenz der sektoralen Budgets untereinander gefehlt hat. Und deshalb ist die interessante Frage, ob das versprochene Globalbudget dieses Problem lösen kann.

Nun ist ja die Frage bei einem Globalbudget, wer ist der Globus. Da könnte man zunächst einmal den einzelnen Arzt als Globus bezeichnen. $\mathrm{Er}$ ist ausgestattet mit einem kombinierten Budget, das die Elemente des Globalbudgets aus der untersten Ebene enthält. In seiner Verantwortung und auch in seiner Steuerungsfähigkeit veranlaßt er diejenigen medizinischen Maßnahmen, die er im Einzelfall für die richtigen hält, und weiß zugleich, daß er die Verantwortung für eine bestimmte finanzielle Obergrenze zu tragen hat, die abhängig ist von den Fallzahlen, die er zu betreuen hat.

Die zweite Möglichkeit wäre, den Globus in der einzelnen Krankenkasse zu sehen. Die Krankenkasse hat heute ein Budget nach Risikostrukturausgleich. Dieses ist weitgehend unabhängig von der Einnahmesituation der einzelnen Krankenkasse nach Ausgleich der Risikofaktoren. Die Krankenkasse würde allerdings, wenn sie das "Globalbudget" wäre, Steuerungsinstrumente brauchen, um zur Einhaltung des Budgets kommen zu können. Im Augenblick hat sie annähernd keine solchen Steuerungsinstrumente, es sei denn, man sieht die Möglichkeit, in weiten Be- 
reichen Preise zu verhandeln, als Steuerungsinstrument. Aber das ungelöste Problem der gesetzlichen Krankenversicherungen ist die Steuerung der Fallzahlen in den verschiedenen Bereichen. Dazu stehen der Krankenkasse wiederum keine Instrumente zur Verfügung. Man könnte ihr, um ein solches Instrument für die Krankenkasse einzuführen, ein konsequentes Einkaufsrecht oder auch ein konsequentes Vertragsrecht an die Hand geben. Dann könnte die Krankenkasse für den Fall, daß $20 \%$ der stationären Angebote nicht erforderlich wären, weil die Leistungen in der Ambulanz stattfinden könnten, den Sektor Krankenhaus eingrenzen und den Vertragsbereich ambulante Versorgung einschließlich Arznei-, Heil- und Hilfsmittel erweitern, also ein Steuerungsmoment, das als „Einkaufsmodell“ bekannt ist.

Die dritte Möglichkeit, sich einen Globus vorzustellen, ist die gesetzliche Krankenversicherung in Deutschland. Hier wäre es besonders einfach, eine Obergrenze für die Leistungsausgaben der gesetzlichen Krankenversicherung von Jahr zu Jahr festzusetzen, auf der Ebene des Bruttoinlandsprodukts oder der Entscheidung des Gesetz- oder Verordnungsgebers. Hier können durchaus auch politische Prioritäten einfließen. Allerdings wäre damit das zentrale Problem keineswegs gelöst. Wie kommt es im Rahmen der gesetzlichen Krankenversicherung in Deutschland zu einer Steuerung vorhandener sektoraler Budgets? Und dies ist das Kernproblem überhaupt, weil wir in einem System zweiseitiger Verträge keine Institutionen haben, die Entscheidungen treffen könnten über Verlagerungen von einem sektoralen Budget in das andere. Wenn man den Versuch macht, sich vorzustellen, wie dies organisiert werden könnte, dann könnte man zunächst einmal auf die bescheidenen Ansätze der Systematik dreiseitiger Verträge verweisen. Dieses System könnte man ausweiten auf alle relevanten Bereiche. Man müßte dann ggf. sechsseitige Verträge schließen, wobei die Mehrheitsfindung in diesem Gremium allerdings ein Problem wäre. Ordnet man jeder Gruppe eine Stimme zu, würde sich die gesetzliche Krankenversicherung in der Situation sehen, daß die Leistungserbringer untereinander Mehrheiten bilden und die Entscheidungen darüber treffen, von welchem Bereich in den anderen umverlagert werden soll. Und wenn man konsequenterweise der Krankenversicherung die Hälfte der Stimmen einräumen würde, dann bräuchte sich die gesetzliche Krankenversicherung nur einen Partner auszusuchen, um mehrheitlich Entscheidungen gegen die anderen Vertragspartner zu treffen. Es ist ein meines Erachtens kaum aufzulösendes Problem. Insoweit würde eine Überlegung aktuell, die wir in der Gesundheitspolitik der letzten Jahre häufiger schon unter verschiedenen Stichworten gehört haben. Herr Reiners hat gerade mit seinem Stichwort „dezentrale Steuerung“ angedeutet, wie so etwas aussehen könnte. Es würde zunächst einmal naheliegen, die Ebene vom Bund auf die Länder 
zu verschieben, und in großen Flächenländern wiederum könnte man die Ebene noch weiter regionalisieren. Im Vertragssystem der Krankenversicherung stellt sich dann die gleiche Frage für die Entscheidungsträger wie zuvor dargestellt. Es würde sich an der Problematik nichts ändern, man könnte allerdings, um zu mehr Gerechtigkeit zu kommen, diese Entscheidungsebenen um andere Verantwortliche ergänzen, die sich um den Gesundheitsbereich sorgen, zu Recht oder zu Unrecht. Dann landen wir möglicherweise bei einer Ländergesundheitskonferenz, in der die Kassenärztlichen Vereinigungen, die Landeskrankenhausgesellschaften und die gesetzliche Krankenversicherung zwar auch noch vertreten sind, die aber wahrscheinlich politisch angereichert würde durch Selbsthilfegruppen, Patientenanwälte, Behindertenverbände und weitere Organisationen, die mit einem gewissen Recht sagen können, sie seien Interessenvertreter einer spezifischen Gruppe, die in einem hohen Maße Gesundheitsleistungen in Anspruch nehmen muß. Dies wäre sicher auch eine Möglichkeit, um zu einer Entscheidungsfindung zu kommen.

Bei einer dezentralen Steuerung, die ja bei der Größe der Bundesrepublik Deutschland zweifelsohne Sinn macht, muß aber bedacht werden, daß wir im System der gesetzlichen Krankenversicherung keine dezentrale Finanzierung haben. Aus der Sicht des Landes Bayern etwa ist ein hohes Mißtrauen zu Entscheidungen bei Krankenhausfinanzierungsfragen im Land Berlin angemessen. Denn wenn eine dezentrale Berliner Konferenz Fragen der Notwendigkeit und Wirtschaftlichkeit der Gesundheitsversorgung zu entscheiden hätte, dann weiß sie ja, daß das Geld zu einem beträchtlichen Teil aus den restlichen Teilen der Bundesrepublik kommt. Das ist die Ursache dafür, daß die Situation in Berlin so ist, wie sie ist, weil die Berliner noch nie ihre eigene Gesundheitsversorgung allein finanziert haben. Insofern habe ich eine sehr große Skepsis gegenüber dezentralen Steuerungselementen, die nicht konsequent auch die dezentrale Finanzierung vorsehen. Dies trifft nicht auf die Faktoren zu, die im Risikostrukturausgleich ausgeglichen werden. Die Frage der beitragsfrei mitversicherten Familienangehörigen oder die Höhe der Gehälter ist weder eine Leistung noch eine Fehlleistung der Region. Aber andere Sachverhalte wie Inanspruchnahmeverhalten der Versicherten, wie Überversorgung, die im wesentlichen auch politisch indiziert ist, das sind schon Verantwortlichkeiten der Regionen. Dafür mag es gute oder weniger gute Gründe geben. Das Problem ist jedenfalls, daß diejenigen, die aus dem vollen schöpfen, die Fülle nicht zu bezahlen haben.

Es bleibt die Frage: Wer entscheidet über die Höhe des Budgets für ein laufendes Jahr? Zweifelsohne gibt es eine Präferenz außerhalb der Verantwortlichkeiten der gesetzlichen Krankenversicherung. Dies könnte der Deutsche Bundestag oder das Bundesgesundheitsministerium sein. 
Oder man kann schlicht und einfach als Kriterium die Steigerung des Bruttoinlandsproduktes des Vorjahres bestimmen. Jedenfalls wäre es zu einfach, an die beitragspflichtigen Einnahmen der Krankenversicherung anzuknüpfen. Denn niemand wird behaupten, daß die beitragspflichtigen Einnahmen der Krankenversicherung etwas mit der Entwicklung der Gesundheitsversorgung oder dem medizinischen Fortschritt und der Altersstruktur der Versicherten zu tun haben. Dies wäre eine rein ökonomische Betrachtungsweise.

Vielleicht ist deutlich geworden, wie schwierig die Umsetzung eines seriösen globalen Budgets ist, bei allem Reiz, der dahinter steht. Ich wünsche denjenigen, die dies im nächsten Jahr zu entscheiden haben, viel Erfolg im Interesse aller, die gespannt darauf warten müssen. 


\section{Beitragsbemessungs- und Versicherungspflichtgrenzen}

Martin Pfaff, Susanne Busch

\section{Szenarien zur Anhebung der Versicherungspflicht und Beitragsbemessungsgrenze der gesetzlichen Krankenversicherung}

"Szenarien im Gesundheitswesen in den kommenden Jahren" können vielfältige Themenkreise betreffen: Zusätzlich zu dem aktuellen Komplex der Auswirkungen der Liberalisierung der europäischen Märkte sind im Rahmen der wissenschaftlichen und politischen Diskussion im Gesundheitswesen - sieht man einmal von Selbstbeteiligungsregelungen ab zwei Hauptansatzpunkte zu unterscheiden, nämlich die Seite des Leistungsangebots und die der Finanzierung. Unser Beitrag wird sich konkret mit letzterer befassen, indem zunächst kurz die Ausgangslage geschildert und auf die Mittelaufbringung und Finanzverfassung des Gesundheitswesens generell eingegangen wird. Diese breite Perspektive wird dann sukzessive verengt, von einem Überblick über unterschiedliche Ansatzpunkte, die Veränderungen der Finanzierungsbasis der gesetzlichen Krankenversicherung (GKV) bedingen, bis hin zu dem ganz speziellen Szenario "Anhebung der Versicherungspflicht- und Beitragsbemessungsgrenze der gesetzlichen Krankenversicherung". Besondere Relevanz erhält diese Alternative im Hinblick einerseits auf die Diskussion der Lohnnebenkosten und Beitragssatzstabilität und andererseits vor dem Hintergrund der sogenannten Friedensgrenze zwischen der gesetzlichen und privaten Krankenversicherung. Demgemäß greifen auch die vorliegenden Modellrechnungen diese Variante auf und kommen - wie weiter unten gezeigt wird - zu durchaus vergleichbaren Ergebnissen, zumindest wenn vergleichbare Modellparameter und -szenarien herangezogenen werden. In der politischen Diskussion erfolgt die Auswahl der Modellvarianten allerdings eher sehr interessengeleitet, getreu dem Motto: "If you let me choose the assumptions I'll prove you anything".

\section{Ausgangslage}

Eine Analyse der Finanzierungsalternativen im Gesundheitswesen umfaßt bei einem breiten Zugang ein weites Spektrum an Maßnahmen, die an der Mittelaufbringung und Finanzverfassung des Gesundheitswesens ansetzen. Neben der Neueinstellung von Parametern, die an der Beitragsfinanzierung ansetzen, können eine Vielzahl weiterer Ansatzpunkte angeführt werden, die sich teils mittelbar, teils unmittelbar z. B. über verminderte Leistungsausgaben auswirken. Der Sachverständigenrat für 
die Konzertierte Aktion im Gesundheitswesen führt hierzu in seinem Jahresgutachten 1997 etwa folgende Maßnahmen an (SVR 1997: 263):

- Reale Eingrenzung des Pflichtleistungskataloges (z. B. Ausgliederung versicherungsfremder Leistungen aus dem Leistungskatalog der GKV, Einführung von Wahltarifen).

- Finanzielle Eingrenzung des Leistungsanspruchs (z. B. Selbstbeteiligung für Patienten, risikoabhängige Beitragskalkulation).

- Verlagerung der Finanzierung auf öffentliche Haushalte und andere Sozialversicherungsträger (z. B. mittels Einnahmen aus Steuern oder Zuweisung an andere Sozialversicherungsträger).

- Neuordnung des Solidarausgleichs in der Krankenversicherung (z. B. mehr Kapitaldeckung zur Absicherung des Krankheitsrisikos, demographiebezogene Sozialfonds zur Sicherung des Umlageverfahrens).

Ergänzend sind bei einer umfassenden Bestandsaufnahme der Finanzierungsalternativen Veränderungen der Finanzierungsmodalitäten zwischen den Akteuren im Gesundheitswesen anzuführen, etwa die Einführung von Vertragsmodellen - wie sie im Kontext der veränderten Versorgungsstrukturen (vgl. Themenkreis 2) von Relevanz sind-oder die Einführung kombinierter Budgets, wie sie im Vorschaltgesetz im Hinblick auf die zum Jahr 2000 vorgesehene Strukturreform im Gesundheitswesen angesprochen sind (Bundestagsdrucksache 14/24, S. $63 \mathrm{ff}$.). Die bisherige Reformgesetzgebung hat derart gestaltete Maßnahmen bislang mit Ausnahme von Selbstbeteiligungsregelungen und Verlagerungen zwischen Sozialversicherungsträgern - die aber von der Verteilungswirkung eher zu Lasten der GKV gingen - weniger aufgegriffen. Lediglich seit den zwei GKV-Neuordnungsgesetzen (NOGs) aus dem Jahr 1997 besteht die Möglichkeit, mittels Modellversuchen nach $\S \S 63 \mathrm{ff}$. SGB V und Strukturverträgen nach § 73 a SGB V die Finanzierungsmodalitäten zwischen Leistungserbringern und Finanzierungsträgern grundlegender zu verändern 37 .

Der engere Zugang zur Analyse der Finanzierungsalternativen in der GKV umfaßt Parameter der Beitragsfinanzierung der GKV. Im SGB V, 8. Abschnitt "Finanzierung", sind alle relevanten Tatbestände zur Finanzierung und Beitragserhebung und in SGB V, 2. Abschnitt, der versicherte Personenkreis abschließend geregelt. Hiermit sind die Faktoren, die Beitragseinnahmen der gesetzlichen - und somit zumindest teilweise auch der privaten - Krankenversicherung seitens der Einnahmen - un-

37 Siehe hierzu u. a. die Diskussion im Rahmen der Bad Orber Gespräche des Themenkreises 2 im vorliegenden Band und in Wille/Albring 1998, S. 94 ff. 
geachtet des kassenindividuellen ausgabendeckenden Beitragssatzes determiniert ${ }^{38}$. Im Hinblick auf die Themenstellung interessiert insbesondere der Kreis der versicherungspflichtigen bzw. -berechtigten Personen einerseits, die Höhe (und Bemessungsgrundlage) der beitragsrelevanten Einkommen andererseits. Beide Parameter sind im wesentlichen durch die Beitragsbemessungs- und Versicherungspflichtgrenze zur GKV (BBG $\left.\mathrm{GKV}_{\mathrm{G}}\right)$ und (VPG $\mathrm{GKV}_{\mathrm{G}}$ ) bestimmt, die $75 \%{ }^{39}$ der Beitragsbemessungsgrenze der gesetzlichen Rentenversicherung $\left(\mathrm{BBG}_{\mathrm{GRV}}\right)^{\mathbf{4 0}}$ beträgt.

\section{Ansatzpunkte zur Veränderung der Finanzierungsbasis}

Maßnahmen, die nun konkret die Parameter, die maßgeblich die Einnahmenseite der GKV - und so auch die konkrete Beitragshöhe und daraus auch die Lohnnebenkostenbelastung - verändern, setzen $\mathrm{z}$. B. an der Höhe der Beitragsbemessungs- und/oder Versicherungspflichtgrenze, an der Definition der beitragspflichtigen Einkommensarten, an der Aufteilung der hälftigen Finanzierung des Beitrages oder an der Abgrenzung des versicherungsfähigen, -berechtigten und -pflichtigen Personenkreises an. Zur Quantifizierung bzw. Monetarisierung von entsprechenden Effekten auf die Versichertenbestände, auf die Einnahmen und Ausgabenstrukturen und demgemä $ß$ auf die Beitragshöhe sind in den vergangenen Jahren umfassende Modellrechnungen durchgeführt worden, die sich dieser Thematik mit unterschiedlicher Schwerpunktsetzung angenommen haben. Der Sachverständigenrat für die Konzertierte Aktion im Gesundheitswesen hat sich in seinem Sondergutachten 1997 intensiv mit der zukünftigen Finanzierung des Krankenversicherungsschutzes befaßt (SVR 1997, TZ 408 ff.). Die konkrete Grenzziehung zwischen GKV und PKV im Hinblick auf die Anhebung der $B_{B} G_{G K V}$ und/oder $V_{P G} G_{G K V}$ - Stichwort Friedensgrenze - wurde formal (juristisch) diskutiert und empirisch auch unter Berücksichtigung von statischen Anpassungsreaktionen seitens der PKV (Boetius/Wisemann, 1998) quantifiziert (im weiteren zitiert als PKV-Studie).

38 Ergänzend hinzu kommen die positiven oder negativen Transfers aus dem Risikostrukturausgleich. Diese gleichen allerdings nur kassenindividuelle Unterschiede in der Mitgliederstruktur und der Grundlohnsumme aus und sind von daher bei einer Gesamtbetrachtung der GKV-Einnahmen zu vernachlässigen.

39 Im Jahr 1999 betrug die BBG GRV DM 8.500 monatlich in den alten Bundesländern, dementsprechend die BBG $\mathrm{GKV}$ DM 6.375.

$40 \mathrm{Da}$ alle sozialversicherungspflichtigen Arbeiter und Angestellten der Rentenversicherungspflicht unterliegen, gibt es demgemäß keine Versicherungspflichtgrenze der Rentenversicherung. 
INIFES (Internationales Institut für Empirische Sozialökonomie gGmbH) hat eine Reihe von Szenarien im Hinblick auf die Identifikation maximaler Beitragssatzeffekte im Rahmen einer komparativen statischen Analyse simuliert. In Übersicht 1 sind für ausgewählte Modellvarianten die Anzahl der potentiell betroffenen Versicherten, der hypothetische Beitragssatzeffekt sowie daraus abgeleitet der durchschnittliche jährliche Entlastungsbetrag (Arbeitgeber- und Arbeitnehmeranteil) in DM ausgewiesen. Diese Angaben beruhen auf Modellrechnungen für das Jahr 1994; sie beschränken sich auf die alten Bundesländer und basieren auf einem durchschnittlichen rechnerisch kostendeckenden Beitragssatz von $12,89 \%$ beim Status quo. Die BBG GKV $_{\text {hat }}$ sich im Referenzjahr auf $5.700 \mathrm{DM}$ monatlich belaufen (vgl. zur methodischen Vorgehensweise und zur Diskussion dieser Ergebnisse im Detail: Pfaff, 1998 a, Pfaff, 1998 b, Busch/Pfaff/Rindsfüßer, 1996). 
Übersicht 1: Ergebnisse ausgewählter simulierter Modelle zu Finanzierungsalternativen für die gesetzliche Krankenversicherung für das Jahr 1994, nur alte Bundesländer

\begin{tabular}{|c|c|c|c|c|}
\hline $\begin{array}{l}\text { Vari- } \\
\text { ante }\end{array}$ & $\begin{array}{l}\text { Kurzbeschreibung der Modellie- } \\
\text { rung }\end{array}$ & $\begin{array}{l}\text { Verände- } \\
\text { rung der } \\
\text { Anzahl der } \\
\text { Versicher- } \\
\text { ten }\end{array}$ & $\begin{array}{l}\text { Beitrags- } \\
\text { satzeffekt } \\
\text { in Bei- } \\
\text { tragssatz- } \\
\text { prozenten }\end{array}$ & $\begin{array}{l}\varnothing \text { Vermin- } \\
\text { derung } \\
\text { des Jah- } \\
\text { resbeitra- } \\
\text { ges }\end{array}$ \\
\hline $1 \mathrm{a}$ & $\begin{array}{l}\text { Anhebung der } V_{P G} G_{G K V} \text { und } \\
B_{B B G} \text { auf } B B G_{G R V}\end{array}$ & +0,88 Mio. & $-0,67 \%$ & $237 \mathrm{DM}$ \\
\hline $1 \mathrm{~b}$ & \begin{tabular}{|l|} 
Anhebung der $\mathrm{BBG}_{\mathrm{GKV}}$ auf \\
$\mathrm{BBG}_{\mathrm{GRV}}$ und Wegfall der $\mathrm{VPG}_{\mathrm{GKV}}$ \\
\end{tabular} & +1,23 Mio. & $-0,79 \%$ & 279 DM \\
\hline 2 & $\begin{array}{l}\text { Anhebung der } B_{B B G_{G K V}} \text { auf } \\
B_{B G} G_{G R V} \text { und generelle Versiche- } \\
\text { rungspflicht für die gesamte Be- } \\
\text { völkerung (Beibehaltung der Son- } \\
\text { dersysteme) }\end{array}$ & +7,05 Mio. & $-0,54 \%$ & $191 \mathrm{DM}$ \\
\hline 3 & $\begin{array}{l}\text { Wegfall der } \mathrm{BBG}_{\mathrm{GKV}} \text { und Bei- } \\
\text { tragsbelastung des gesamten } \\
\text { Volkseinkommens, der Renten } \\
\text { und der BA-Leistungen }\end{array}$ & +10,48 Mio. & $-4,68 \%$ & $1.654 \mathrm{DM}$ \\
\hline $4 a$ & $\begin{array}{l}\text { Gesonderte Beitragsleistung für } \\
\text { mitversicherte Ehepartner ohne } \\
\text { Kinder unter } 7 \text { Jahren (50\% des } \\
\text { jeweiligen Beitrages) }\end{array}$ & $\begin{array}{l}\text { keine Ver- } \\
\text { änderung }\end{array}$ & $-0,88 \%$ & $311 \mathrm{DM}$ \\
\hline $4 \mathrm{~b}$ & $\begin{array}{l}\text { Gesonderte Beitragsleistung für } \\
\text { mitversicherte Ehepartner (50\% } \\
\text { des jeweiligen Beitrages) }\end{array}$ & $\begin{array}{l}\text { keine Ver- } \\
\text { änderung }\end{array}$ & $-1,20 \%$ & $424 \mathrm{DM}$ \\
\hline $4 \mathrm{c}$ & $\begin{array}{l}\text { Ausgliederung (und Steuerfinan- } \\
\text { zierung) der Leistungsausgaben } \\
\text { von mitversicherten Kindern unter } \\
18 \text { Jahren }\end{array}$ & -10,79 Mio. & $-0,88 \%$ & $311 \mathrm{DM}$ \\
\hline
\end{tabular}

Quelle: Busch/Pfaff/Rindstüßer 1996, S. 49 ff.

Ein Vergleich der berücksichtigten Varianten der Finanzierungsalternativen zeigt, daß sich die durchschnittliche finanzielle Beitragsentlastung für GKV-Mitglieder ohne Berücksichtigung von Anpassungsreaktionen (Befreiungsanträge, Kündigungsverhalten im Übergangszeitraum) rein hypothetisch für Maßnahmen, die an der BBG bzw. VPG ansetzen (Varianten $1 \mathrm{a}$ und $1 \mathrm{~b}$ ) auf maximal $279 \mathrm{DM}$ beläuft. Die Einführung einer generellen Versicherungspflicht unter Beibehaltung der Sondersysteme (Variante 2) würde diese Effekte reduzieren, da für einen Großteil der dann hinzukommenden ehemals PKV-Versicherten - insbesondere 
Beamte und Selbständige - die plausible Annahme getroffen wurde, daß die Struktur ihrer Einkommen der der GKV-Mitglieder entsprechen würde. Variante 3 beinhaltet eine weitreichende Ausdehnung der Bemessungsgrundlage auf das gesamte Volkseinkommen, der Renten und BALeistungen bei gleichzeitiger Aufhebung der $\mathrm{BBG}_{\mathrm{GKV}}$ und bedingt so den maximalen jährlichen Entlastungsbetrag in Höhe von 485 DM.

Modellvarianten, die an der Modifikation der unentgeltlichen Mitversicherung von Familienangehörigen (Varianten $4 \mathrm{a}, 4 \mathrm{~b}, 4 \mathrm{c}$ ) ansetzen, haben einen hypothetischen Entlastungsbetrag von maximal 424 DM jährlich ermittelt, wenn z. B. bislang unentgeltlich mitversicherte Ehegatten generell einen Beitrag in Höhe von 50 \% des Mitgliederbeitrages entrichten müßten. Mit einem Entlastungsbetrag in Höhe von 311 DM würde den Modellrechnungen zufolge eine Steuerfinanzierung der Leistungsausgaben für Kinder unter 18 Jahren zu geringeren Effekten führen.

Die angeführten Varianten zum Status quo der Finanzierungsgrundlagen haben aufgezeigt, daß unter Beachtung der Restriktionen der Modellrechnungen der rechnerische kostendeckende Beitragssatz zwischen 0,67 Prozentpunkten und maximal 4,68 Prozentpunkten ceteris paribus gesenkt werden könnte. Hierbei handelt es sich allerdings um hypothetische Werte, die keine Handlungsempfehlungen implizieren. Keinesfalls sollen die Grundprinzipien der GKV in Frage gestellt werden - so z. B. hinsichtlich der unentgeltlichen Mitversicherung von Kindern in der GKV. Sie sollen auch nicht darüber hinwegtäuschen, daß eine Vielzahl von Rationalisierungs- und Wirtschaftlichkeitsreserven im Gesundheitswesen vorhanden ist, die es primär auszuschöpfen gilt.

\section{Anhebung der Versicherungspflicht- und Beitragsbemessungs- grenze der gesetzlichen Krankenversicherung}

Im weiteren wird detailliert auf Basis der Modellrechnungen von INIFES und der PKV auf die Variante Anhebung der $\mathrm{BBG}_{\mathrm{GKV}}$ und der $\mathrm{VPG}_{\mathrm{GKV}}$ um $33 \%$ auf das Niveau der $B_{B B} G_{\text {GRV }}$ eingegangen. Konkret würde eine solche Änderung zu folgenden Reaktionen führen: Ein wesentlicher Teil der Personen, die bislang freiwillig in der GKV versichert waren, würde, ebenso wie bislang privat versicherte Arbeitnehmer in der PKV, versicherungspflichtig, solange ihr Einkommen die $\mathrm{BBG}_{\mathrm{GRV}}$ nicht überschreitet. Dies würde zu einem Zuwachs der Zahl der GKV-Versicherten und entsprechend zu einem Rückgang der PKV-Versicherten führen. Zur Ermittlung der beitragssatzrelevanten Auswirkungen auf die GKV muß zunächst die Anzahl der potentiell betroffenen Versicherten ermittelt werden. Im Endeffekt würden Veränderungen der Einnahmen und Ausgaben der GKV resultieren, deren konkreter Saldo dann den rechneri- 
schen Beitragssatzeffekt (Differenz des rechnerisch kostendeckenden Beitragssatzes im Status quo und nach Anhebung der $B_{B G} G_{G K V}$ und VPG $\mathrm{GKV}_{\text {) }}$ determiniert.

Konkret werden die Einnahmen ansteigen, da

- die Anhebung der $\mathrm{BBG}_{\mathrm{GKV}}$ zu höheren Beitragszahlungen der bislang freiwillig versicherten GKV-Mitglieder führt,

- die Anhebung der VPG $\mathrm{GKV}_{\mathrm{Gu}}$ zusätzlichen Beitragseinnahmen von bislang privat Versicherten führt.

Auch die Ausgaben werden ansteigen, da

- für neu hinzugekommene Versicherte Leistungsausgaben finanziert werden müssen und

- durch die Anhebung der $\mathrm{BBG}_{\mathrm{GKV}}$ höhere (einkommensabhängige) Krankengeldzahlungen für GKV-Mitglieder mit Einkommen oberhalb der alten $\mathrm{BBG}_{\mathrm{GKV}}$ resultieren werden.

Um diese Zahlungsströme monetarisieren zu können, muß einerseits eine Abschätzung der sozialversicherungspflichtigen Einkommen der PKV-Versicherten und GKV-Mitglieder mit Einkommen über der $B_{B G} G_{G R V}$ bzw. weiterer Einkommensarten erfolgen sowie Berechnungen der voraussichtlichen zusätzlichen Leistungsausgaben der vormals PKV-Versicherten durchgeführt werden. Sollen in den Modellrechnungen zusätzlich Anpassungsreaktionen von Versicherten im Übergangszeitraum berücksichtigt werden, welche wohl de facto nicht zu vermeiden sein würden, muß zusätzlich abgeschätzt werden, welche Personen die Möglichkeit nutzen, sich von der Versicherungspflicht befreien zu lassen (Befreiungsquote) bzw. die Mitgliedschaft in der GKV zu kündigen (Kündigungsquote).

Die Befreiungsquote drückt hierbei quasi vor dem Hintergrund eines "Bestandsschutzes" aus, welche PKV-Versicherten, die als Konsequenz der angehobenen $V_{P G}$ GKV versicherungspflichtig in der GKV werden würden, sich hiervon dauerhaft befreien lassen würden. Es steht zu vermuten, daß Personen mit geringem persönlichen Gesundheitsrisiko bzw. ohne Familienangehörige, für die in der PKV ja eigene Beiträge zu entrichten wären, davon Gebrauch machen würden (Befreiungsquote). Dementsprechend ist es nicht unplausibel, daß vor allem PKV-Versicherte mit schlechten Risiken, also insbesondere diejenigen, die in der GKV die unentgeltliche Familienversicherung in Anspruch nehmen könnten, die Gelegenheit nützen würden, in die Solidargemeinschaft der 
GKV zurückzukehren oder einzutreten (Rückkehrquote $=1$-Befreiungsquote).

Der zweite wichtige Parameter im Übergangszeitraum betrifft die Mitglieder der GKV, die bislang, bedingt durch die niedrigere $V P G_{G K V}$, freiwillig versichert waren. Sofern ihre Einkommen unterhalb der neuen $V_{P G} G_{G K}$ liegen, werden sie, wenn sie nicht von einem Kündigungsrecht im Übergangszeitraum Gebrauch machen, versicherungspflichtig in der GKV. Da der zu leistende Beitrag um bis zu $33 \%$ ansteigen wird41, wird ein Wechsel in die PKV insbesondere für gesunde, jüngere Mitglieder ohne unentgeltlich mitversicherte Familienangehörige interessant, da der höheren Beitragsbelastung mit Ausnahme eines höheren Krankengeldanspruchs keine Leistungssteigerungen gegenüberstehen (Kündigungsquote 1). Dieses Argument gilt in noch stärkerem Maße für die Mitglieder, deren Einkommen auch die neue $V_{P G} G_{G k V}$ übersteigt, die also nach wie vor freiwillig versichert wären, die aber in jedem Fall um $33 \%$ höhere Beiträge zu zahlen hätten (Kündigungsquote 2).

\section{Modellierungsparameter und Vorgehensweise}

Grundsätzlich steht die Simulation der Auswirkungen der Anhebung der $\mathrm{BBG}_{\mathrm{GKV}}$ und $\mathrm{VPG}_{\mathrm{GKV}}$ vor dem Problem, daß die intendierten Wechselbewegungen sowohl Mitglieder als auch Versicherte betreffen. In der GKV stehen durch die unentgeltliche Mitversicherung von Familienangehörigen einem Beitragszahler - dem Mitglied - unterschiedlich viele Versicherte mit Leistungsanspruch gegenüber. In der PKV muß jeder Versicherte individuelle risikoabhängige Beiträge bezahlen. Um diesem Aspekt Rechnung zu tragen, wurden in der INIFES-Studie die zusätzlichen Leistungsausgaben versichertenbezogen nach Alter und Geschlecht ermittelt; so konnte eine Konstruktion von Mitgliederhaushalten bei Wechslern von der PKV in die GKV vermieden werden. Da zur Ermittlung des Beitragssatzeffektes nur der Gesamtsaldo relevant ist, interessieren die individuellen familienbezogenen Transfersalden nicht. Der Ansatz der PKV-Studie geht demgegenüber bei der Ermittlung der zusätzlichen Leistungsausgaben von der Mitgliederebene aus, d. h., es müssen Annahmen über die Mitversichertenstrukturen der von der PKV in die GKV wechselnden Mitglieder getroffen werden. Zudem unterscheiden sich die beiden Studien insbesondere in folgender Hinsicht:

41 Bei einem geschätzten Höchstbeitrag von ca. 800 DM monatlich kann sich dies auf bis zu DM 3.168 (Arbeitgeber- plus Arbeitnehmeranteil) belaufen. 
- Bezugsjahr: INIFES: 1994, PKV: 1995

- Bezugsregion: INIFES: West, PKV: Ost und West,

- Ausgabenprofile: INIFES: INIFES RSA42 94, PKV: IGES RSA 95,

- berücksichtigter Personenkreis: INIFES: Arbeiter und Angestellte, PKV: Angestellte und Selbständige.

Bei der weiteren Vorgehensweise stützen sich die Untersuchungen weitgehend auf vergleichbare bzw. identische Datenquellen. Angaben zur Zahl der Personen mit Einkommen über der $\mathrm{BBG}_{\mathrm{GKV}}$ und $\mathrm{VPG}_{\mathrm{GKV}}$ werden aus dem Mikrozensus (Statistisches Bundesamt (Hrsg.), „Versicherte in der Kranken- und Rentenversicherung", Fachserie 13, Reihe 1) entnommen; Angaben zur Einkommensverteilung aus der Statistik „Aktiv Versicherte" des Verbandes Deutscher Rentenversicherungsträger. Die Berechnung der zusätzlichen durch neu hinzukommende Versicherte verursachten Ausgaben (INIFES) bzw. der Mitglieder (PKV-Studie) in der GKV wurde jeweils auf Basis von RisikostrukturausgleichsKostenprofilen durchgeführt. Übersicht 2 faßt die wesentlichen Modellparameter und deren geschätzte Auswirkungen im Überblick für die INIFES- und PKV-Studie zusammen.

42 Dies sind nach Alter und Geschlecht differenzierte Pro-Kopf-Kostenprofile der Versicherten der gesetzlichen Krankenversicherung. Die Profile gleichen sich in ihrer Struktur und Höhe annähernd (vgl. Pfaff/Pfaff/Rindsfüßer, 1993). 
Übersicht 2: Modellparameter und Ergebnisse zu den Auswirkungen einer Anhebung der Beitragsbemessungsgrenze und der Versicherungspflichtgrenze der gesetzlichen Krankenversicherung auf das Niveau der Beitragsbemessungsgrenze der gesetzlichen Rentenversicherung

\begin{tabular}{|c|c|c|}
\hline Modellparameter & INIFES-Studie & PKV-Studie \\
\hline Bezugsjahr & 1994 & 1995 \\
\hline Bezugsraum & GKV West & GKV Ost + West \\
\hline $\begin{array}{l}\text { Betroffener Personen- } \\
\text { kreis }\end{array}$ & $\begin{array}{l}\text { Alle abhängig beschäftigten Arbeiter und Angestellten, } \\
\text { deren Einkommen } \geq B B G_{G K V} \\
\text { Keine Selbständigen, keine Beamten, keine Personen } \\
\text { mit sonstigen Einkommen }\end{array}$ & $\begin{array}{l}\text { Alle abhängig beschäftigten Angestellten und } \\
\text { Selbständigen, deren Einkommen } \geq B B_{G K V} \text {. } \\
\text { Keine Arbeiter, keine Personen mit sonstigen } \\
\text { Einkommen. }\end{array}$ \\
\hline $\begin{array}{l}\text { Datenbasis für potentiell } \\
\text { betroffene Personen }\end{array}$ & $\begin{array}{l}\text { Mikrozensusangaben für das Jahr } 1994 \text { nach Alter } \\
\text { und Geschlecht } \\
\text { VDR-Statistik Aktiv Versicherte für Einkommen }\end{array}$ & $\begin{array}{l}\text { Mikrozensus für das Jahr 1995, nach Alter und } \\
\text { Geschlecht } \\
\text { VDR-Statistik Aktiv Versicherte für Einkommen }\end{array}$ \\
\hline $\begin{array}{l}\text { Berechnungsbasis zur } \\
\text { Ermittlung der Strukturen } \\
\text { der Wechsler }\end{array}$ & $\begin{array}{l}\text { Wechsler werden auf Basis einzelner Versicherter } \\
\text { berechnet. } \\
\text { Alle Arbeiter und Angestellten, die It. Mikrozensus pri- } \\
\text { vat versichert waren (differenziert nach Alter und Ge- } \\
\text { schlecht). } \\
\text { Alle "mitversicherten Angehörigen" und nicht Er- } \\
\text { werbstätigen unter } 60 \mathrm{~J} ., \text { die It. Mikrozensus privat } \\
\text { versichert waren, wurden als potentielle zukünftige } \\
\text { Mitversicherte definiert (differenziert nach Alter und } \\
\text { Geschlecht) }\end{array}$ & $\begin{array}{l}\text { Wechsler werden auf Basis von Mitgliedern mit } \\
\text { evtl. mitversicherten Angehörigen berechnet. } \\
\text { Alle Angestellten, deren Bruttoeinkommen } \geq \\
\text { BBMGKv und alle Selbständigen, die in der GKV } \\
\text { versichert sind. } \\
\text { Mitversicherte werden über Annahmen bezüglich } \\
\text { der typischen Versichertenhaushalte simuliert. } \\
\text { Annahmen = zusätzliche GKV-Mitglieder (Rück- } \\
\text { kehrer) } \\
25 \% \text { Mitglieder mit Ehepartner (m } 56 \text { Jahre, w } \\
54 \text { Jahre); } \\
75 \% \text { Mitglieder mit Familienangehörigen (Ehe- } \\
\text { paar m. } 35 \text { Jahre, w. } 33 \text { Jahre, } 2 \text { Kinder, } 2 \text { und } 4 \\
\text { Jahre). }\end{array}$ \\
\hline
\end{tabular}




\begin{tabular}{|c|c|c|c|}
\hline \multirow{8}{*}{ 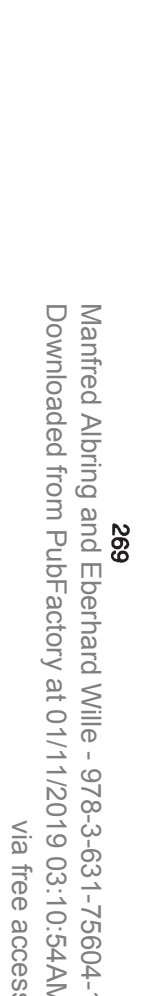 } & Modellparameter & INIFES-Studie & PKV-Studie \\
\hline & $\begin{array}{l}\text { Berechnungsbasis zur } \\
\text { Ermittlung der zukünfti- } \\
\text { gen Einnahmen }\end{array}$ & $\begin{array}{l}\text { Da nur Arbeiter und Angestellte unter den PKV- } \\
\text { Versicherten berücksichtigt wurden, Schätzung der } \\
\text { sozialversicherungspflichtigen Einkommen auf Basis } \\
\text { der VDR-Statistik (Aktiv Versicherte). Jahreseinkom- } \\
\text { men liegen in 5.000-DM-Abstufung vor. }\end{array}$ & $\begin{array}{l}\text { VDR-Statistik (Klassifizierung nach 100-DM- } \\
\text { Klassen bei Annahme einer Gleichverteilung bei } \\
\text { der vorliegenden Abstufung in 5.000 DM). } \\
\text { Für Selbständige werden Nettoeinkommensan- } \\
\text { gaben It. Mikrozensus auf Bruttoeinkommen } \\
\text { hochgerechnet. Diese Ergebnisse werden auch } \\
\text { für die Berechnungen von Wechslern PKV-GKV } \\
\text { herangezogen. }\end{array}$ \\
\hline & $\begin{array}{l}\text { 1994: } \mathrm{BBG}_{\mathrm{GKV}} \\
\text { 1994: } \mathrm{BBG}_{\mathrm{GRV}} \\
\end{array}$ & $\begin{array}{l}\text { West: } 5.700 \mathrm{DM}(1994) \\
\text { West: } 7.600 \mathrm{DM}(1994)\end{array}$ & $\begin{array}{l}\text { West: } 5.850 \text {; Ost: } 4.800 \text { (1995) } \\
\text { West: } 7.800 \text {; Ost: } 6.400 \text { (1995) } \\
\end{array}$ \\
\hline & \begin{tabular}{|l} 
Leistungsausgaben der \\
neu hinzukommenden \\
Versicherten
\end{tabular} & $\begin{array}{l}\text { Basis: Versichertenbezogene Kostenprofile ohne } \\
\text { Krankengeld auf Basis RSA-Profile } 1994 \text { (INIFES), } \\
\text { differenziert nach Alter und Geschlecht }\end{array}$ & $\begin{array}{l}\text { Basis: Versichertenbezogene Kostenprofile ohne } \\
\text { Krankengeld auf Basis -RSA-Profile } 1995 \\
\text { (IGES), differenziert nach Alter und Geschlecht }\end{array}$ \\
\hline & Krankengeldausgaben & $\begin{array}{l}\text { Berechnung eines durchschnittlichen Krankengeld- } \\
\text { quotienten (je verdienter Markt durchschnittlich 1,15 } \\
\text { Pfg. Krankengeld) jeweils für die zusätzlichen KG- } \\
\text { Ausgaben für alten Mitgliederbestand und für neue } \\
\text { Mitglieder. }\end{array}$ & $\begin{array}{l}\text { Wird in einem Szenario berücksichtigt, unter Be- } \\
\text { rücksichtigung der Kündigungs- und Befreiungs- } \\
\text { quote (siehe unten), Ansatz beruht auf INIFES- } \\
\text { Werten. }\end{array}$ \\
\hline & Weitere Annahmen & $\begin{array}{l}\text { Formalinzidenz, Langfristwirkung, d. h. Annahme, daß } \\
\text { diese Wirkung der Modellrechnung sofort, ohne Kün- } \\
\text { digungsmöglichkeiten für bisherige PKV-Versicherte } \\
\text { und ohne Befreiungsquote für neu hinzukommende } \\
\text { Versicherungspflichtige, zutrifft. }\end{array}$ & $\begin{array}{l}\text { Übergangszeitvarianten: Berücksichtigung von } \\
\text { Modellvarianten bezüglich bisher PKV-Versicher- } \\
\text { ter mit Befreiungsrecht sowie bislang noch frei- } \\
\text { willig versicherter GKV-Mitglieder mit Kündi- } \\
\text { gungsrecht (siehe unten). }\end{array}$ \\
\hline & Methodischer Ansatz & \multicolumn{2}{|c|}{ Statische Analyse } \\
\hline & \begin{tabular}{|l|} 
Ergebnisse: \\
Vorbemerkung
\end{tabular} & \multicolumn{2}{|c|}{$\begin{array}{l}\text { Die Ergebnisse sind rein zeitpunktbezogen. Zukunftsprojektionen werden nicht erstellt. Bei Übergangs- } \\
\text { regelungen werden - ceteris paribus - erst mittelfristig die Verschiebungen im Versichertenbestand und } \\
\text { dementsprechend auch der Beitragssatzeffekt, der die maximale theoretische Höhe ausweist, resultie- } \\
\text { ren. }\end{array}$} \\
\hline
\end{tabular}




\begin{tabular}{|c|c|c|c|}
\hline & Modellparameter & INIFES-Studie & PKV-Studie \\
\hline & $\begin{array}{l}\text { Maximaler rechnerischer } \\
\text { Beitragssatzeffekt }\end{array}$ & 0,67 Prozentpunkte & 0,58 Prozentpunkte \\
\hline & $\begin{array}{l}\varnothing \text { Entlastung für bis- } \\
\text { herige GKV-Mitglieder }\end{array}$ & DM 237 & DM 205 \\
\hline 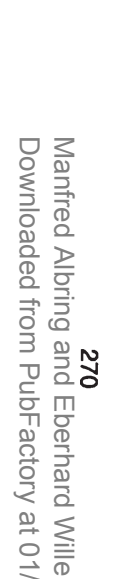 & Szenarien & $\begin{array}{l}\text { Veränderungsparameter } \\
\text { Weitere Ausweitung des Versichertenkreises } \\
\text { Verbreiterung der Bemessungsgrundlage } \\
\text { Neugestaltung der Familienhilfe } \\
\text { (Ergebnisse dieser Varianten vgl. im Überblick } \\
\text { Busch/Pfaff/Rindsfüßer 1996: } 79 \text { und Übersicht } 1 \\
\text { oben) }\end{array}$ & $\begin{array}{l}\text { Veränderungsparameter } \\
\text { Befreiungsquote (Anteil der zukünftigen GKV- } \\
\text { Versicherungspflichtigen bisherigen PKV- } \\
\text { Mitglieder, die zum Zeitpunkt des Rechtswech- } \\
\text { sels einen Befreiungsantrag stellen); } \\
\text { Kündigungsquote (Anteil bisheriger GKV- } \\
\text { Mitglieder, die kündigen, weil der Beitragssatz } \\
\text { ansteigt) } \\
\text { K1 (Personen mit Einkommen zwischen BBGGKV } \\
\text { und BBGGRV) } \\
\text { K2 (Personen mit Einkommen über der BBG } \\
\text { Alternative Ausgabenansätze für Mitgliederhaus- } \\
\text { halte (Konstruktion von Mitgliedereinheiten vs. } \varnothing \\
\text { Ausgaben eines Mitgliedes). } \\
\text { (Ergebnisse dieser Szenarien vgl. im Überblick } \\
\text { Boetius/Wiesemann 1998: 75, 77, 121ff. und } \\
\text { Übersicht } 3 \text { unten) }\end{array}$ \\
\hline
\end{tabular}

Quellen: Busch/Pfaft/Rindsfüßer 1996, Boetius/Wiesemann 1998. 


\section{Vergleich der Studienergebnisse}

Ungeachtet des individuell unterschiedlichen Zuganges bzw. Modellansatzes kommen beide Berechnungen zu ähnlichen Schätzungen des rechnerischen Beitragssatzeffektes (INIFES 0,67\% und PKV 0,58\%), wenn unterstellt wird, daß bei Anhebung der $B_{B B} G_{G K V}$ und $V_{P G} G_{G K V}$

- keine Befreiungsmöglichkeiten bestehen für alle rentenversicherungspflichtigen Arbeiter und Angestellten sowie in der GRV freiwillig versicherte Selbständige, die durch die Anhebung der $B_{B} G_{G K V}$ und $V_{P G_{G K V}}$ versicherungspflichtig würden, und

- keine Kündigungsmöglichkeiten für bislang in der GKV freiwillig Versicherte eröffnet würden.

Bestätigt werden diese Angaben auch von den Modellrechnungen des Sachverständigenrates für die Konzertierte Aktion im Gesundheitswesen, der zu einem Wert von 0,74 Prozentpunkten (SVR 1997, TZ 441) kommt. Dementsprechend ist ein maximaler hypothetischer Beitragssatzeffekt bei Anhebung der $\mathrm{BBG}_{\mathrm{GKV}}$ und $\mathrm{VPG}_{\mathrm{GKV}}$ um $33 \%$ auf das $\mathrm{Ni}$ veau der $B B G_{G R V}$ bei einer statischen komperativen Betrachtung von ca. 0,6 Prozentpunkten realistisch. Der geringere Beitragssatzeffekt des Ansatzes der PKV-Studie wird insbesondere aus den höheren zusätzlichen Leistungsausgaben resultieren, die durch die Konstruktion von Mitgliederhaushalten bedingt sind43. Durch den Ansatz von Leistungsausgaben auf Versichertenebene kann die INIFES-Studie die zusätzliche finanzielle Belastung durch hinzukommende Leistungsausgaben realitätsgerechter abbilden.

Bei beiden Modellrechnungen handelt es sich - ungeachtet der teilweise simulierten Kündigungs- und Befreiungsquoten - um Modellrechnungen, die eine theoretische - beziehungsweise erst nach einer Übergangszeit realisierbare - Obergrenze des Beitragssatzeffektes ausweisen. Relevant für die zukünftige Entwicklung der Versichertenanteile im Vergleich GKV und PKV werden $u$. a. auch die allgemeine Einkommensentwicklung, die Erwerbsbeteiligung der Frauen, der EU-Binnenmarkt und vieles mehr sein. Gleichermaßen wird sich auch die Entwicklung des Leistungskatalogs der GKV und des Anbieterverhaltens der Akteure des Gesundheitswesens mittel- und unmittelbar auf die Wahlmöglichkeiten

43 Auch die berücksichtigte Variante, die durchschnittliche Ausgaben der zusätzlichen Mitglieder unterstellt, überschätzt die Ausgaben, da davon auszugehen ist, $\mathrm{da} ß$ auch die aktuell betroffenen PKV-Versicherten vergleichsweise unterdurchschnittliche Ausgabenrisiken darstellen. 
bzw. -entscheidungen und Ausgabenentwicklungen der beiden Zweige der Krankenversicherung auswirken.

\section{Befreiungs- und Kündigungsquoten im Übergang}

Wie bereits angedeutet, erscheint es - ungeachtet der vielfältigen generellen Einwände und Bedenken (vgl. hierzu etwa ausführlich Boetius/Wiesemann, 1998, insbesondere S. $33 \mathrm{ff}$.) - nicht realistisch, daß Maßnahmen zur Veränderung der "Friedensgrenze" zwischen GKV und PKV ohne vorherige Ankündigungseffekte und Übergangsregelungen umgesetzt werden könnten. Kurz- und mittelfristig wird die Berücksichtigung von Befreiungs- und Kündigungsquoten die ausgewiesenen Effekte mindern. Die Ausgestaltung dieser Quoten wird naturgemäß interessengeleitet beurteilt und begründet, so etwa bei Boetius/Wiesemann mittels der Frage der Verfassungskonformität derartiger Vorhaben. Zur Abschätzung der Effekte im Übergangszeitraum simulieren sie des weiteren in ihren Modellrechnungen 12 unterschiedliche Szenarien zur Höhe der Befreiungs- und Kündigungsquoten. Extreme Szenarien bezüglich der "Ein- und Auswanderer" in die bzw. aus der PKV und der damit einhergehenden finanziellen Be- bzw. Entlastungen, können so-wie aus Übersicht 3 hervorgeht - zu extremen Ergebnissen führen. 
Übersicht 3: Berechnete Szenarien der PKV-Studie unter Berücksichtigung unterschiedlicher durchschnittlicher Leistungsausgaben, Befreiungsquoten und Kündigungsquoten

\begin{tabular}{|c|c|c|c|c|c|c|c|}
\hline \multirow[t]{2}{*}{$\begin{array}{l}\text { Szenario- } \\
\text { typ }\end{array}$} & \multirow{2}{*}{$\begin{array}{c}\text { Szena- } \\
\text { riona- } \\
\text { me }\end{array}$} & \multirow[t]{2}{*}{\begin{tabular}{|l|} 
Beson- \\
derheit
\end{tabular}} & \multirow{2}{*}{$\begin{array}{c}\text { GKV- } \\
\text { Ausga- } \\
\text { benansatz } \\
\text { je } \\
\text { Mitglied* }^{*}\end{array}$} & \multirow{2}{*}{$\begin{array}{l}\text { Befrei- } \\
\text { ungs- } \\
\text { quote }\end{array}$} & \multicolumn{2}{|c|}{$\begin{array}{l}\text { Kündigungs- } \\
\text { quote }^{\star \star}\end{array}$} & \multirow{2}{*}{$\begin{array}{c}\text { GKV- } \\
\text { Beitrags- } \\
\text { satzsen- } \\
\text { kung }\end{array}$} \\
\hline & & & & & K1 & $\mathrm{K} 2$ & \\
\hline \multirow{3}{*}{$\begin{array}{l}\text { Grundsze- } \\
\text { narien }\end{array}$} & $\bar{A}$ & & $\vec{D}$ & $70 \%$ & $10 \%$ & $20 \%$ & $0,14 \%$ \\
\hline & B & & D & $70 \%$ & $20 \%$ & $40 \%$ & $-0,09 \%$ \\
\hline & C & & D & $50 \%$ & $5 \%$ & $10 \%$ & $0,29 \%$ \\
\hline \multirow{2}{*}{$\begin{array}{l}\text { Varianten } \\
\text { von Sze- } \\
\text { nario A }\end{array}$} & A KG & Mit Kran & D & $70 \%$ & $10 \%$ & $20 \%$ & $0,09 \%$ \\
\hline & $\mathrm{A} 1$ & & U & $70 \%$ & $10 \%$ & $20 \%$ & $0,18 \%$ \\
\hline \multirow{3}{*}{\begin{tabular}{|l} 
VPG- \\
Szenari- \\
en $n^{\star \star \star}$
\end{tabular}} & VPG A & & D & $70 \%$ & $0 \%$ & $0 \%$ & $0,05 \%$ \\
\hline & VPG B & Ankün- & D & $70 \%$ & $10 \%$ & $20 \%$ & $-0,06 \%$ \\
\hline & VPG C & & D & $0 \%$ & $0 \%$ & $0 \%$ & $0,15 \%$ \\
\hline \multirow{4}{*}{$\begin{array}{l}\text { Vegleichs- } \\
\text { szenarien }\end{array}$} & V1 & & $\mathrm{D}$ & $50 \%$ & $0 \%$ & $0 \%$ & $0,40 \%$ \\
\hline & V2 & & U & $50 \%$ & $0 \%$ & $0 \%$ & $0,46 \%$ \\
\hline & V3 & & D & $0 \%$ & $0 \%$ & $0 \%$ & $0,48 \%$ \\
\hline & V4 & & U & $0 \%$ & $0 \%$ & $0 \%$ & $0,58 \%$ \\
\hline
\end{tabular}

* D: Differenzierte GKV-Ausgaben je Mitglied (entsprechend der Konstruktion der Mitversichertenstrukturen), U: Undifferenzierte GKV-Ausgaben je Mitglied

** K1: Personen mit Einkommen zwischen $B_{B B} G_{G K V}$ und $B B G_{G R V}$ K2: Personen mit Einkommen über der $B_{B B} G_{G V}$

*** Aufhebung der $V P G_{G K V}$ und Anhebung $B B G_{G K V}$ auf Niveau $B B G_{G R V}$

Quelle: Boetius/Wiesemann 1998, S. 77

Dies gilt etwa für die Unterstellung, daß $70 \%$ der bislang PKVVersicherten von ihrem Befreiungsrecht Gebrauch machen und von den bislang freiwillig in der GKV Versicherten $20 \%$ bzw. $40 \%$ (Einkommen größer als die $B_{B B} G_{G K V}$ aber kleiner als die $B_{B B} G_{G R V} b z w$. Einkommen größer als die $B_{B B} G_{G R V}$ ) von ihrem Kündigungsrecht Gebrauch machen, da der zusätzlichen Beitragsbelastung in Höhe von bis zu 33 \% nur marginal verbesserte Leistungen bei eventuellem Krankengeldbezug gegenüberstünden. Bezogen auf die BBG $_{\mathrm{GRV}}$ im Jahr 1998 in Höhe von mo- 
natlich $8.400 \mathrm{DM}$ würden sich die zusätzlichen Beitragsbelastungen bei einem durchschnittlichen Beitragssatz zur GKV in Höhe von 13,56 \% im Monat auf maximal 285 DM (jährlich also auf 3.420 DM) belaufen.

Wenn zudem annahmegemäß der Großteil der zusätzlichen GKVVersicherten schlechte Risiken und der der abwandernden GKVVersicherten gute Risiken repräsentieren, sind zusätzliche Beitragsbelastungen in der GKV in Höhe von 0,09 Beitragssatzpunkten rechnerisch durchaus möglich. Zwar sind die Annahmen, daß eher schlechte Risiken in die GKV wechseln und (vorerst) gute Risiken die GKV verlassen, nicht unplausibel, allerdings dürften die Konstellationen bezüglich der unterstellten Haushaltsstrukturen (siehe Übersicht 2) in der PKV selten anzutreffen sein, zumindest wenn das Beihilfesystem nicht mit einbezogen ist. Außerdem gilt auch für diese Modellrechnung, daß zwar zeitlich befristete Wechselmöglichkeiten zu berücksichtigen sind, die im Extremfall zu Lasten der GKV gehen könnten, die Effekte durch die erhöhte BBG und VPG aber gleichermaßen mittel- bis langfristig eintreten werden.

Da die Problematik der Befreiungs- und Kündigungsquoten in der Übergangsphase nicht ganz von der Hand zu weisen ist, wird z. B. seitens des VdAK gefordert, eine Aufhebung der $\mathrm{VPG}_{\mathrm{GKV}}$ mit der Beibehaltung der $B_{B B} G_{G K v}$ zu verbinden (vgl. Minn 1997, Rebscher/Walzik, 1998). Eine generelle Versicherungspflicht für alle abhängig Beschäftigten, analog zur Regelung in der GRV, würde die Solidargemeinschaft und damit auch die Finanzierungsbasis der GKV erweitern. Dadurch würde zwar die Verbreiterung der Beitragsbemessungsgrundlage deutlich geringer ausfallen als bei einer gleichzeitigen Anhebung der $\mathrm{BBG}_{\mathrm{GKV}}$, allerdings würde im Gegenzug voraussichtlich von den bisher freiwillig versicherten GKV-Mitgliedern nur ein wesentlich kleinerer Anteil kündigen. Somit würden auch deutlich weniger gute Risiken von der GKV in die PKV wechseln, da die individuelle Beitragsbelastung nicht anstiege. Diese Reformvariante wird vom VdAK auch mit dem Äquivalenzargument begründet. Mittels dieser Variante kann jedoch die zusätzliche finanzielle Belastung der GKV durch neu hinzukommende schlechte Risiken aus der PKV nicht ausgeschlossen werden. Eine solche Entwicklung ist nach Meinung des VdAK nicht von der Hand zu weisen, da insbesondere im Gefolge der mit dem Gesundheitsreform- und Gesundheitsstrukturgesetz eingeführten Restriktionen beim Wechsel von der PKV (zurück) in die GKV, im PKV-Versichertenbestand eine Anzahl von Personen vorhanden ist, die diese Möglichkeit nutzen würde, um den (alters- oder familienbedingten) hohen Prämien der PKV zu entgehen und sich kostengünstiger in der GKV abzusichern. 


\section{Perspektiven der Reform der Beitragsbemessungsgrenze}

Die bisher diskutierten Varianten einer Anhebung der Beitragsbemessungs- und Versicherungspflichtgrenzen stellen nur einige. Beispiele dar, wie die Solidarität der wirtschaftlich Stärkeren für die Sicherung der Finanzierung der GKV in Zukunft in größerem Umfang als bisher eingefordert werden kann.

Zweifelsohne gibt es andere Varianten, bei denen dies ebenfalls angestrebt wird, beispielsweise die Ausweitung des Risikostrukturausgleichs auf die PKV-Versicherten. So haben Modellrechnungen von IGES (Institut für Gesundheits- und Sozialforschung $\mathrm{GmnH}$ ) beispielsweise ergeben, daß allein ein Grundlohnausgleich zu einem BeitragssatzEntlastungseffekt bei der GKV von 0,8 \% führen könnte (Bohm/Jacobs/ Reschke, 1996). Allerdings wirt auch diese Variante eine Vielzahl von Fragen auf, die vor einer politischen Entscheidung sorgfältig abzuwägen sind.

Wie bei allen politischen Entscheidungen, die die zukünftige Finanzierung der GKV betreffen, werden die wirtschaftlichen Interessen von Gruppen der Bevölkerung nachhaltig betroffen: Eine Finanzierungsreform, die alle besser- und niemanden schlechterstellt, ist nicht zu realisieren: Bei allen Varianten sind deshalb Anpassungsreaktionen im Übergang zu erwarten. Solche Anpassungsreaktionen werden aber in der praktischen Politik nicht als Argumente ausreichen, um als sinnvoll erachtete Reformen grundsätzlich zu verhindern.

\section{Literaturverzeichnis}

Boetius J, Wiesemann HO (1998), Die Finanzierungsgrundlage in der Krankenversicherung - Zur Grenzziehung zwischen GKV und PKV. PKV-Dokumentation 22, Köln 1998

Bohm S, Jacobs P, Reschke P (1996), Notwendigkeit und Möglichkeiten eines Umbaus der Finanzierung in der gesetzlichen Krankenversicherung. Graue Reihe der Hans-Böckler-Stiftung, Bd. 110, Düsseldorf 1996

Busch S, Pfaff AB, Rindsfüßer C (1996), Die Finanzierung der gesetzlichen Krankenversicherung. Möglichkeiten zur Umgestaltung und Ergebnisse ausgewählter Modellrechnungen. Graue Reihe der Hans-Böckler-Stiftung, Bd. 110, Düsseldorf 1996 
Entwurf eines Gesetzes zur Stärkung der Solidarität in der gesetzlichen Krankenversicherung. Bundestagsdrucksache 14/24 vom 09.11.98

Minn N (1997), Quo vadis GKV? Eine Lösung der drängenden Finanzprobleme ist (noch) nicht in Sicht. In: Die Ersatzkasse, 77. Jg., 1997, Heft 12; S. 437 - 443

Pfaff AB (1998 a), Einige Ansatzpunkte für die Finanzierung des Gesundheitswesens nach der Dritten Stufe der Gesundheitsreform. In: Schönig W, Schmale I, Gestaltungsoptionen in modernen Gesellschaften. Transfer Verlag Regensburg 1998 a: S. $199-213$

Pfaff AB (1998 b), Finanzierungsoptionen der gesetzlichen Krankenversicherung. In: Wille E, Albring M (Hrsg.) Reformoptionen im Gesundheitswesen. Peter Lang Verlag Frankfurt a. M. u. a. 1998 b: S. 59 - 74

Pfaff AB, Pfaff M, Rindsfüßer C (1993), Der Einfluß von Risikostrukturprofilunterschieden auf den Risikostrukturausgleich, dargestellt am Beispiel der Landesverbände der Betriebskrankenkassen. In: Paquet R, König W, Der Risikostrukturausgleich und die Konsequenzen für den Wettbewerb. Essen 1993; S. 72 - 89

Rebscher H, Walzik E (1998), Finanzierungsoptionen aus der Sicht der Ersatzkassen. In: Wille E, Albring M (Hrsg.) Reformoptionen im Gesundheitswesen. Peter Lang Verlag Frankfurt a. M. u. a. 1998; S. 40 - 58

Sachverständigenrat für die Konzertierte Aktion im Gesundheitswesen (SVR 1997), Sondergutachten 1997 Gesundheitswesen in Deutschland. Kostenfaktor oder Zukunftsbranche. Band II Fortschritt und Wachstumsmärkte, Finanzierung und Vergütung. Nomos Verlag Baden-Baden 1997 


\section{Beitragsbemessungs- und Versicherungspflichtgrenzen}

Norbert Klusen

Die Finanzsituation der gesetzlichen Krankenversicherung (GKV) ist abhängig von den Arbeitseinkommen ihrer Mitglieder und der Entwicklung der Ausgaben. Wir alle wissen um die Einnahmeausfälle in der GKV durch hohe Arbeitslosigkeit, stagnierende Arbeitseinkommen und um die stetig steigenden Leistungsausgaben. Eine Trendwende, die auf absehbare Zeit dauerhaft Abhilfe schafft, ist nicht in Sicht, und man kann das Gesundheitssystem auch nicht zero-base neu aufbauen.

Findige Wissenschaftler und Politiker haben sich deshalb auf die Suche nach neuen Einnahmequellen für die GKV gemacht.

Im vergangenen Jahr haben wir in Bad Orb über mögliche neue Finanzquellen diskutiert. Heftig umstritten war dabei die Frage der Veränderung von Beitragsbemessungs- und Versicherungspflichtgrenze, um der GKV neue attraktive Mitgliederpotentiale zu erschließen und zu erhalten. Im letzten Jahr war auch ein Vertreter des PKV-Verbands anwesend, und es mutete schon merkwürdig an, daß ich mit inm in diesen wesentlichen Punkten übereinstimmte. Und das, obgleich die Techniker Krankenkasse als einzige große Krankenkasse mit ihren nahezu $40 \%$ freiwillig Versicherten in einem direkten und intensiven Wettbewerbsverhältnis zur PKV steht (und viel weniger zu anderen gesetzlichen Krankenkassen).

Als Ergebnis dieser Versichertenstrukturen zahlt die Techniker Krankenkasse jährlich 7 Milliarden Mark in den Risikostrukturausgleich ein. Ihre freiwillig versicherten Mitglieder sollten also der GKV erhalten bleiben, denn sie erbringen diesen Beitrag. Übrigens zahlen sie auch kräftig in den gemeinsamen Topf der Pflegeversicherung ein (über 2 Milliarden Mark bei Leistungsausgaben von $300-400$ Millionen Mark).

Die folgenden Äußerungen mache ich als Praktiker und aus der Interessenlage der TK heraus, die aber seit der Einführung des Risikostrukturausgleichs wesentlich mehr als früher auch der Interessenlage der gesamten GKV entspricht.

Vordergründig betrachtet hat der Gedanke Charme, einen größeren Personenkreis als Pflichtmitglieder in das solidarisch finanzierte System der GKV einzubeziehen. Sicher sind für diese Überlegung auch Gerechtigkeitsaspekte von Bedeutung. Soll es denn einem Kreis von Personen 
ermöglicht werden, sich der Solidarität zu entziehen, nur weil er mehr verdient als andere?

Noch mehr Charme hätte eigentlich die von einigen Politikern und Verbänden erwogene Lösung, den unangenehmen Wettbewerber, der der GKV attraktive Mitglieder entzieht, per Gesetz durch Aufhebung der Versicherungspflichtgrenze beim Vollversicherungsschutz völlig aus dem Verkehr zu ziehen.

Da dieser Meuchelakt jedoch nicht in Sekundenschnelle und ohne, daß der Betroffene etwas merkt, zu vollziehen ist, wende ich mich realistischeren Alternativen zu - der Anhebung der Beitragsbemessungs- und der Versicherungspflichtgrenze (VPG).

Jede Anhebung der Beitragsbemessungsgrenze (BBG) führt zu Mehrbelastungen für die GKV-Mitglieder, deren Einkommen oberhalb der heutigen BBG liegt, ohne daß dem wesentliche Mehrleistungen gegenüberstehen (Ausnahme: Krankengeld).

Dies schwächt die Akzeptanz des Gesamtsystems gerade bei denen, die ganz erheblich zu dessen Finanzierbarkeit beitragen. Welche Auswirkungen dieser Akzeptanzverlust haben kann, zeigen die Abwanderungen in Richtung PKV nach der Zuzahlungserhöhung im vergangenen Jahr.

Eine Anhebung der Versicherungspflichtgrenze führt zu einer Ausweitung des versicherungspflichtigen Personenkreises, und zwar gerade um diejenigen, die heute aus freien Stücken im solidarischen System GKV sind. Außerdem werden mit heute PKV-Versicherten solche Menschen einbezogen, die sich in früheren Jahren aufgrund persönlicher Vorteilserwägungen gegen eine solidarische Krankenversicherung entschieden haben.

Mehr noch als bei einer Anhebung der BBG handelt es sich bei Veränderungen der VPG um einen Systemwechsel, und jeder Systemwechsel ruft Anpassungsreaktionen der Beteiligten zur Verbesserung der eigenen Chancen hervor.

Dies gilt um so mehr, weil eine Anhebung der VPG nicht ohne Übergangsfristen bzw. ohne Bestandsschutz umzusetzen wäre. Ungeachtet dessen haben die Reformgesetze der vergangenen Jahre die GKVMitglieder so sensibilisiert (und kritisch gemacht), daß sich schon der reine Ankündigungseffekt spürbar auswirken würde. 
Die erwähnten Anpassungsreaktionen bekäme die GKV sowohl quantitativ wie auch qualitativ nicht zu unterschätzende Wanderungsbewegungen zu spüren.

Ein großer Teil derjenigen, für die es finanziell lohnenswert ist, wird die GKV verlassen und sich privat versichern (junger, gutverdienender, gesunder Single). Das gilt konsequenterweise für die Personengruppen, die heute im GKV-System einen positiven Deckungsbeitrag aufweisen und damit zur Finanzierbarkeit der GKV beitragen. Ein umlagefinanziertes Krankenversicherungssystem kann durch solche Verschiebungen aus dem Gleichgewicht geraten.

Eine Anhebung der Versicherungspflichtgrenze in Verbindung mit Bestandsschutz und Übergangsfristen führt zu umgekehrten Wanderungsbewegungen; es werden diejenigen wieder unter das Dach der GKV schlüpfen, die durch eine private Absicherung des Krankheitsrisikos in den Vorjahren finanzielle Vorteile hatten, die sich aber nach Veränderungen des Lebensalters und Familienstandes in das Gegenteil verkehrt haben.

Dadurch, daß Menschen sich über Jahre der Solidarität entzogen haben und sie erst wieder suchen, wenn sie persönlich profitieren können (nicht umsonst ist heute eine Rückkehr aus der PKV in die GKV von Ausnahmen abgesehen nicht möglich), werden die in der GKV gewollten Umverteilungsprozesse konterkariert.

Zusammenfassend läßt sich sagen, daß diese Wanderungsbewegungen (die bei entsprechenden Berechnungen häufig vernachlässigt werden) zu adversen Effekten führen. Je nach persönlicher Vorteilslage ist die Flucht aus bzw. in die Solidarität möglich, und die gewachsene und z. Z. noch stabile Akzeptanz des Systems der gesetzlichen Krankenversicherung wird geschwächt.

Wer einen solch massiven Systemwechsel herbeiführt und das Gleichgewicht gesellschaftlichen Konsenses verändert, braucht dafür sehr gute Gründe. Wenige Zehntel Beitragssatz, wobei die Wanderungsbewegungen noch nicht berücksichtigt sind, reichen nicht aus - zumal es ein Zurück nicht mehr geben wird.

Gestatten Sie mir an dieser Stelle einen Exkurs zum Wettbewerbsverhältnis zur und innerhalb der privaten Krankenversicherung.

Bei den Wanderungsbewegungen zu und von der PKV dürfen nicht nur die finanziellen Auswirkungen ab dem Zeitpunkt des Wechsels betrach- 
tet werden, sondern auch die Zeit vor dem Wechsel von einem System zum anderen gilt es zu berücksichtigen.

Für jeden PKV-Versicherten sind Altersrückstellungen gebildet worden, die heute beim Wechsel in die GKV bei dem entsprechenden privaten Krankenversicherungsunternehmen verbleiben, ohne daß für diesen Versicherten künftig noch Leistungsausgaben anfallen. Diese werden dann von der GKV getragen.

Daher wäre es folgerichtig, wenn diese Altersrückstellungen beim Wechsel in die gesetzliche Krankenversicherung mitgegeben würden. Eine solche Regelung wäre nicht nur kompatibel im Hinblick auf die Unterschiedlichkeit der beiden Systeme, sondern sachlogisch sogar zwingend.

Im Kapitaldeckungsverfahren der PKV werden diese Rückstellungen nicht mehr für den Versicherten benötigt, und im Hinblick auf das Umlageverfahren der GKV würde dem Umstand Rechnung getragen, daß sich der bislang PKV-Versicherte am Ausgleich (jung für alt, gesund für krank und Singles für Familien) nicht beteiligt hat.

Im Binnenverhältnis der privaten Krankenversicherungsunternehmen findet aufgrund desselben Umstands nur ein sehr begrenzter Wettbewerb statt, der sich ausschließlich auf die Akquisition von Neukunden beschränkt. Haltearbeit - wie sie wegen der Wahlfreiheit in der GKV erforderlich und üblich ist - findet kaum statt, da es für einen Privatversicherten aus finanziellen Gründen fast ausgeschlossen ist, zu einem anderen privaten Anbieter zu wechseln. Die Altersrückstellungen „wandern" nicht mit ihm, entsprechend hoch wären die neuen Prämien.

Der Wettbewerb in der GKV hat sehr viele positive Entwicklungen angestoßen und gefördert. Auch für die private Krankenversicherung sollte dringend und ernsthaft erwogen werden, wettbewerbliche Verhältnisse zu ermöglichen. Dies geht nur über eine Mitgabe gebildeter Altersrückstellungen. Im übrigen lassen sich die Gründe, aus denen die Wahlfreiheit in der GKV eingeführt wurde, gut auf die PKV übertragen.

Auch beim Wechsel von der GKV in die PKV gilt es, die Zeiten vor dem Eintritt in ein privates Krankenversicherungsunternehmen zu berücksichtigen.

Im allgemeinen liegen vor diesem Wechsel Jahre, in denen der Versicherte keine oder nur geringe Beiträge gezahlt hat (beitragsfrei mitversichert, Studienzeit etc.). Dies alles ist durch das Umlageverfahren ge- 
deckt und gewollt und Bestandteil des solidarischen Ausgleichs in der GKV. Schließlich geht man davon aus, daß er später selbst zahlt und damit die anderen unterstützt.

Üblicherweise findet der Wechsel in die PKV in relativ jungen Jahren statt, und die Zeitspanne, in der der Betreffende ein eigenes Einkommen erzielt, ist bis dahin relativ kurz. Insofern wäre es - analog der Mitgabe der Altersrückstellungen - konsequent, daß die PKV für die Zeiten vor dem Wechsel eine „Ablösesumme“ an die abgebende gesetzliche Krankenversicherung zahlt. Auch dies ist kompatibel mit den unterschiedlichen Systematiken und auch in sich logisch.

Soweit dieser Exkurs.

Auch in bezug auf die Leistungserbringer und damit die Leistungsausgaben der GKV ist eine Anhebung der BBG und/oder der VPG mit Konsequenzen verbunden:

Die Anhebung von Beitragsbemessungs- und/oder Versicherungspflichtgrenze hat automatisch auch eine Erhöhung der GKV-relevanten Grundlohnsumme zur Folge.

In der heutigen Systematik, in der die Grundlohnsumme ein entscheidender Parameter für die Entwicklung der Vergütung der Leistungserbringer ist, führt dies dazu, daß ein Teil der Mehreinnahmen „durchgewunken" wird, ohne daß es zu Qualitätsverbesserungen käme, sondern zu fehlsteuernden und kostentreibenden Effekten.

Wer argumentiert, die heutigen Beitragsbemessungs- und Versicherungspflichtgrenzen seien willkürlich auf $75 \%$ der Grenzen in der gesetzlichen Rentenversicherung festgelegt, hat natürlich recht.

Unbestritten gilt aber auch, daß eine Neufestlegung, auf welchen Prozentsatz auch immer, ebenso willkürlich wäre und zudem völlig außer acht läßt, daß es sich bei der gesetzlichen Renten- und der gesetzlichen Krankenversicherung um zwei Systeme handelt, die sich in einem wesentlichen Punkt unterscheiden:

Die gesetzliche Rentenversicherung folgt dem Äquivalenzprinzip mit der Folge, daß derjenige, der mehr einzahlt, auch mehr bekommt, während dies bei der GKV für $95 \%$ der Leistungen nicht zutrifft. Hier ist das Krankengeld die einzige Ausnahme. 
Es gibt also gute systematische Gründe für unterschiedliche Bemessungs- bzw. Pflichtgrenzen. Zwar kann qua definitionem eine Festsetzung nicht willkürlicher sein als die andere, doch gilt es zu berücksichtigen, daß dem Status quo eine größere innere Logik zugrunde liegt, als es bei einer Anhebung der Grenzen auf das Niveau der gesetzlichen Rentenversicherung der Fall wäre.

Das Ziel, mehr "Gerechtigkeit“ zu erreichen, wird verfehlt, da verteilungspolitisch wenig sinnvolle Prozesse in Gang gesetzt werden und die Akzeptanz des Systems geschwächt wird. Eine noch schwerer zu begründende Willkür tritt an die Stelle von „Gerechtigkeit“.

Die TK steht, aufgrund ihres hohen Anteils an freiwillig Versicherten, in einem besonderen Wettbewerbsverhältnis zur PKV und wäre von Änderungen der BBG und/oder der VPG besonders betroffen.

Ein großer Teil unserer Versicherten hat sich freiwillig für die gesetzliche Krankenversicherung entschieden und von der Option, sich privat zu versichern, (bislang) keinen Gebrauch gemacht. Veränderungen zu Lasten der Versicherten bekommen wir „seismographisch“ deutlich zu spüren. Wenn diese Versicherten innerhalb der GKV wechseln, tut uns als TK das zwar weh, aber zumindest bleibt das Beitragsaufkommen innerhalb des Systems.

Da gerade dieser Versichertenkreis hochmobil ist, wäre bei einer Veränderung der Beitragsbemessungs- und Versicherungspflichtgrenze der Weggang des größten Teils dieser Mitglieder aus dem System sehr wahrscheinlich. Der Schaden, der über den Risikostrukturausgleich für die gesamte GKV entstünde, dürfte sich im Milliardenbereich bewegen und damit die Nachteile, die die TK erleiden würde, bei weitem überwiegen.

Trotz vordergründigen Charmes birgt eine Anhebung der Beitragsbemessungs- und Versicherungspflichtgrenze daher erhebliche Gefahren für die gesetzliche Krankenversicherung, die um so schwerer wiegen, weil die Konsequenzen unumkehrbar sind.

Mein Fazit ist: Wer die gesetzliche Krankenversicherung nicht dauerhaft und nachhaltig schwächen will, darf keine Maßnahmen ergreifen, die das System für diejenigen unattraktiv macht, ohne deren finanzielle Beiträge es nicht existieren kann. 


\section{Resümee: Die Suche nach einem effizienten und adäquat finanzierten Gesundheitswesen in einem zusammenwach- senden Europa}

Eberhard Wille

\section{Europäische Gesundheitspolitik zwischen Harmonisierung und Subsidiarität}

Von den drei Themenkreisen standen die Effekte, die von der europäischen Integration auf die deutsche Gesundheitsversorgung ausgehen können, deutlich im Mittelpunkt des Interesses. Dies lag weniger daran, daß es bei den beiden anderen Themenkreisen, $d$. h. bei den „Veränderten Versorgungsstrukturen“ und den „Ausgewählten Finanzierungsalternativen", kaum bedeutsame Probleme und kontroverse Ansichten gab, aber das "Europa-Thema“ bot für die meisten Teilnehmer den höchsten Informationsgehalt im Sinne neuer Aspekte und Herausforderungen. Die Überlegungen konzentrierten sich dabei nicht so sehr auf eine Bewertung der europäischen Gesundheitspolitik als solcher, sondern auf die wahrscheinlichen und möglichen Anpassungserfordernisse im Rahmen der deutschen Gesundheitsversorgung. Aus dieser Perspektive bestehen dann auch wieder Interdependenzen zu den beiden übrigen Themenkreisen, denn ein effizientes und adäquat finanziertes Gesundheitswesen braucht den Wettbewerb auf europäischer Ebene weniger zu fürchten.

Die Maßnahmen der Europäischen Gemeinschaft (EG) entfalteten nicht erst mit den Urteilen des EuGH in den Rechtsstreitigkeiten Kohll und Decker, sondern seit Anbeginn der europäischen Integration Auswirkungen auf die nationalen Gesundheitssysteme (siehe ausführlicher Hanika H., 1998, und Schulte B., 1998). Infolge der eingeschränkten Kompetenzen der EG im Bereich des Gesundheitswesens zielten diese Vorschriften zunächst weniger auf eine einheitliche europäische Gesundheitspolitik ab, sondern dienten primär dem Abbau von Handelshindernissen und Wettbewerbsbeschränkungen, d. h. der Verwirklichung des gemeinsamen Marktes (Schulte B., 1998, S. 2).

Der Vertrag von Maastricht, der im Januar 1993 in Kraft trat, schuf für die Gesundheitspolitik der EG eine eigenständige Rechtsgrundlage und ermöglichte der Gemeinschaft, damit „eine kohärente Strategie für den Bereich der öffentlichen Gesundheit zu entwickeln" (Kommission der Europäischen Gemeinschaften 1998, S. 7). Nach Artikel 2 des EGV gehört 
es zu den Aufgaben der Gemeinschaft, „ein hohes Maß an sozialem Schutz" zu gewährleisten und gemäß Artikel 3 „einen Beitrag zur Erreichung eines hohen Gesundheitsschutzniveaus" zu leisten. Um ein "hohes Gesundheitsschutzniveau“ sicherzustellen, ergänzt die Tätigkeit der Gemeinschaft die Politik der Mitgliedsstaaten und zielt gemäß Artikel 152 EGV (ex-Artikel 129) „auf die Verbesserung der Gesundheit der Bevölkerung, die Verhütung von Humankrankheiten und die Beseitigung von Ursachen für die Gefährdung der menschlichen Gesundheit" ab. Artikel 152 räumt dem Gesundheitswesen somit nicht nur einen speziellen Titel XIII im EGV ein, sondern überantwortet der Gemeinschaft auch einen Sicherstellungsauftrag für ein hohes Gesundheitsschutzniveau und einen Präventionsauftrag (ähnlich Hanika H., 1998, S. 193). Auf dieser Rechtsgrundlage erarbeitete die Kommission der EG acht Aktionsprogramme im Bereich der öffentlichen Gesundheit, von denen die Programme zur Prävention von Aids und anderen übertragbaren Erkrankungen, Krebsbekämpfung, Suchtprävention, Gesundheitsförderung und Gesundheitsberichterstattung angenommen wurden (Kommission der Europäischen Gemeinschaften, 1998, S. 9).

Die Kommission der Europäischen Gemeinschaften (1998, S. 12 ff.) beabsichtigt auch weiterhin, die Möglichkeiten, die der EGV, und hier insbesondere der neue Artikel 152 bieten, „so umfassend wie möglich zu nutzen“. Sie setzte für die zukünftige Gesundheitspolitik der EU die folgenden drei „Aktionsschwerpunkte“:

- die Verbesserung der Information zur Entwicklung der öffentlichen Gesundheit,

- die rasche Reaktion auf Gesundheitsgefahren und

- die Berücksichtigung der für die Gesundheit bestimmenden Faktoren durch Gesundheitsförderung und Prävention.

Trotz der zunehmenden Kompetenzen und Aktivitäten der EG im Bereich der öffentlichen Gesundheit sieht der EGV kein Harmonisierungsgebot vor, sondern überläßt die inhaltliche Ausgestaltung der nationalen Gesundheitssysteme im Sinne des Subsidiaritätsprinzips den einzelnen Ländern. So unterliegen gemäß Artikel 137 Abs. 3 EGV (ex-Artikel 118) "soziale Sicherheit und sozialer Schutz der Arbeitnehmer" dem Einstimmigkeitsprinzip des Ministerrats. Bei der Tätigkeit der Gemeinschaft im Bereich Gesundheit bleibt auch nach Artikel 152 Abs. 5 EGV „die Verantwortung der Mitgliedsstaaten für die Organisation des Gesundheitswesens und die medizinische Versorgung in vollem Umfange gewahrt". Schließlich gilt gemäß Artikel 5 EGV (ex-Artikel 3 b) für alle Bereiche, die 
nicht in die ausschließliche Zuständigkeit der Gemeinschaft fallen, das Subsidiaritätsprinzip.

Herr Dr. Zipperer betont insofern zu Recht die Kompetenz der einzelnen Länder für die Ausgestaltung inrer Sozialsysteme und damit auch ihrer Gesundheitsversorgung. Von dem Ordnungsrahmen, den das Gemeinschaftsrecht setzt, und den vielfältigen Tätigkeiten der EG können jedoch faktische Einflüsse auf die nationale Sozial- und Gesundheitspolitik ausgehen. Zwischen EG-Recht und -Politik einerseits und nationalem Sozialrecht und -politik andererseits existieren zahlreiche Wechselwirkungen, die sich zunehmend intensivieren und dahin tendieren, die nationale Steuerungskapazität im Bereich der Sozial- und Gesundheitspolitik einzuschränken (Schulte B., 1998, S. 4 ff.). Die Vorstellung, in den Mitgliedsstaaten alle ökonomischen Teilsysteme im Sinne eines gemeinsamen Markts zu harmonisieren und dabei die Sozial- und Gesundheitspolitik völlig auszusparen, beruht auf einer „Trennungsfiktion“ (Wismar M., 1998, S. 29), die von den Interdependenzen abstrahiert, die sowohl zwischen nationaler und europäischer Sozialpolitik als auch zwischen den Teilsystemen einer Wirtschafts- und Gesellschaftsordnung bestehen. Die EG und die EWU erzwingen in rechtlicher Hinsicht keine Harmonisierung der nationalen Sozialsysteme, sie lösen aber mannigfaltige Konvergenzprozesse zwischen den Teilnehmerländern aus und verstärken bereits vorhandene Tendenzen dieser Art.

Es herrschte in der Diskussion weitgehend Übereinstimmung, daß unabhängig von faktischen und im Sinne der europäischen Integration auch teilweise notwendigen Konvergenzprozessen eine völlige Harmonisierung der Sozial- und Gesundheitssysteme der Teilnehmerländer weder rechtlich geboten noch ökonomisch und sozial wünschenswert erscheint. In gesamtwirtschaftlicher Hinsicht sprechen vor allem folgende Aspekte gegen eine völlige bzw. weitreichende Harmonisierung:

- Wie Tab. 1 belegt, geben die einzelnen Teilnehmerländer einen sehr unterschiedlich hohen Anteil ihres Bruttoinlandsprodukts für die Gesundheitsversorgung aus. Die deutsche Gesundheitsquote liegt mit $10,4 \%$ z. B. über 3 Prozentpunkte über den entsprechenden Quoten von Luxemburg und Finnland. Die Rolle, die das Gesundheitswesen für die wirtschaftliche Entwicklung und damit auch für die inländische Beschäftigung spielt, weicht somit von Land zu Land spürbar ab.

- Die divergierenden Gesundheitsquoten deuten zudem - sogar bei wirtschaftlich ähnlich leistungsfähigen Volkswirtschaften - auf unterschiedliche Präferenzen im Hinblick auf den Stellenwert der Gesund- 
heitsversorgung hin. Dies schlägt sich auch in dem Umfang des Leistungskatalogs der jeweiligen gesetzlichen Krankenkassen bzw. öffentlichen Gesundheitssysteme nieder und erzeugt innerhalb der Teilnehmerländer eine unterschiedliche Erwartungshaltung gegenüber der medizinischen Grund- bzw. Mindestversorgung.

- Eine Absenkung der Gesundheitsquote in Richtung des Durchschnitts der Teilnehmerländer in Höhe von ca. $8 \%$ liefe den Präferenzen der Bürger in den betreffenden Ländern zuwider, und viele Versicherte und Patienten würden diese Art der Harmonisierung als sozialen Abbau empfinden. Umgekehrt entzieht eine Anhebung der Gesundheitsquote den anderen Wirtschaftsbereichen knappe Ressourcen, so daß die betreffenden Länder dadurch möglicherweise eine Einschränkung ihrer internationalen Wettbewerbsfähigkeit erleiden. Dieser Nachteil wiegt dann in der EWU um so schwerer, als eine Abwertung als Anpassungsmechanismus entfällt. 
Tab. 1: Gesundheitsquoten im internationalen Vergleich"

\begin{tabular}{|c|c|c|c|c|c|c|c|c|}
\hline Land Jahr & 1960 & 1970 & 1980 & 1990 & 1991 & 1995 & $1996^{21}$ & $1997^{2}$ \\
\hline Australien & 4,9 & 5,7 & 7,3 & 8,3 & 8,6 & 8,4 & 8,5 & 8,3 \\
\hline Belgien & 3,4 & 4,1 & 6,5 & 7,5 & 7,9 & 7,9 & 7,8 & 7,6 \\
\hline Dänemark & 3,6 & 6,1 & 8,7 & 8,2 & 8,2 & 8,0 & 8,0 & 7,7 \\
\hline Deutschland & 4,8 & 6,3 & 8,8 & 8,7 & 9,4 & 10,4 & 10,5 & 10,4 \\
\hline Finnland & 3,9 & 5,7 & 6,5 & 8,0 & 9,1 & 7,6 & 7,4 & 7,3 \\
\hline Frankreich & 4,2 & 5,8 & 7,6 & 8,9 & 9,1 & 9,9 & 9,7 & 9,9 \\
\hline Griechenland & 2,4 & 3,3 & 3,6 & 4,2 & 4,2 & 5,8 & 6,8 & 7,1 \\
\hline Großbritannien & 3,9 & 4,5 & 5,6 & 6,0 & 6,5 & 6,9 & 6,9 & 6,7 \\
\hline Irland & 3,8 & 5,3 & 8,7 & 6,7 & 7,0 & 7,0 & 7,0 & 7,0 \\
\hline Island & 3,3 & 5,0 & 6,2 & 7,9 & 8,1 & 8,2 & 8,2 & 8,0 \\
\hline Italien & 3,6 & 5,2 & 7,0 & 8,1 & 8,4 & 7,7 & 7,8 & 7,6 \\
\hline Japan & - & 4,4 & 6,4 & 6,0 & 6,0 & 7,2 & 7,2 & 7,3 \\
\hline Kanada & 5,5 & 7,1 & 7,3 & 9,2 & 9,9 & 9,7 & 9,6 & 9,3 \\
\hline Luxemburg & - & 3,7 & 6,2 & 6,6 & 6,5 & 6,7 & 6,8 & 7,1 \\
\hline Neuseeland & 4,3 & 5,2 & 6,0 & 7,0 & 7,5 & 7,3 & 7,3 & 7,6 \\
\hline Niederlande & 3,8 & 5,9 & 7,9 & 8,3 & 8,6 & 8,8 & 8,6 & 8,5 \\
\hline Norwegen & 2,9 & 4,5 & 7,0 & 7,8 & 8,1 & 8,0 & 7,9 & 7,4 \\
\hline Österreich & 4,3 & 5,3 & 7,7 & 7,2 & 7,2 & 8,0 & 8,0 & 7,9 \\
\hline Portugal & - & 2,8 & 5,8 & 6,5 & 7,2 & 8,2 & 8,3 & 8,2 \\
\hline Schweden & 4,7 & 7,1 & 9,4 & 8,8 & 8,7 & 8,5 & 8,6 & 8,6 \\
\hline Schweiz & 3,1 & 4,9 & 6,9 & 8,3 & 8,9 & 9,6 & 10,2 & 10,2 \\
\hline Spanien & 1,5 & 3,7 & 5,6 & 6,9 & 7,0 & 7,3 & 7,4 & 7,4 \\
\hline Türkei & - & 2,4 & 3,3 & 3,6 & 3,8 & 3,3 & 3,8 & - \\
\hline USA & 5,2 & 7,3 & 9,1 & 12,6 & 13,4 & 14,1 & 14,0 & 14,0 \\
\hline Durchschnitt ${ }^{3}$ & 3,9 & 5,1 & 6,9 & 7,6 & 7,9 & 8,1 & 8,2 & 8,3 \\
\hline
\end{tabular}

Quelle: Zusammengestellt und berechnet nach OECD Health-Data 98, Paris 1998 a.

1) Die gesamten Gesundheitsausgaben des jeweiligen Landes in vH des entsprechenden Bruttoinlandsprodukts.

2) Schätzungen

3) Für 1960 ohne Japan, Luxemburg, Portugal und Türkei

- Die Gesundheitssysteme der einzelnen Teilnehmerländer unterscheiden sich sowohl hinsichtlich des Verhältnisses von Sachleistungs- und Kostenerstattungsprinzip als auch bezüglich ihrer Finanzierung mit Hilfe von Steuern, Beiträgen oder Prämien. Entsprechend besitzt auch die private Krankenversicherung in den einzelnen Ländern ein höchst unterschiedliches Gewicht.

- In nahezu allen Ländern finden sich zwar Elemente von staatlichplanerischen, korporativen und marktwirtschaftlichen Koordinationsinstrumenten, aber jeweils mit sehr unterschiedlicher Reichweite und Stringenz bzw. Tiefe. 
Die zentrale Problematik einer weitergehenden Harmonisierung der Sozial- und Gesundheitssysteme besteht im Kontext der oben skizzierten Unterschiede darin, daß es sich hier um national gewachsene Strukturen handelt, die in den jeweiligen Ländern überwiegend auf Akzeptanz stoBen. Es gibt aus gesamtwirtschaftlicher Sicht auch keine Normvorgabe, die als Orientierung bzw. Meßlatte für eine Vereinheitlichung der Sozialbzw. Gesundheitssysteme dienen könnte. In diesem Kontext plädierte die überwiegende Mehrheit der Teilnehmer dafür, das schwergewichtig beitragsfinanzierte deutsche Gesundheitswesen, das sich durch eine flächendeckende Versorgung und vergleichsweise geringe Rationierungen auszeichnet, zwar europäischen Integrationserfordernissen anzupassen, aber gleichzeitig als System auf EG-Ebene offensiv zu vertreten.

\section{Zur Liberalisierung der europäischen Gesundheitsmärkte und ihren Wirkungen}

Der EuGH wies schon vor den jüngsten Urteilen in den Rechtsstreitigkeiten Kohll und Decker einige Male darauf hin, daß die Vorschriften über den freien Waren- und Dienstleistungsverkehr nach Artikel $27 \mathrm{ff}$. und 49 EGV (ex-Artikel $29 \mathrm{ff}$. und 59) im Bereich der sozialen Sicherheit zwar nicht uneingeschränkt, aber doch im Prinzip gelten. Die nationale Ausgestaltung des Sozialschutzes darf nicht die Grundfreiheiten außer Kraft setzen. Das Verbot der Diskriminierung des Marktzugangs von ausländischen Leistungserbringern besitzt grundsätzlich auch im Gesundheitsbereich seine Gültigkeit (Pitschas R., 1994, S. 117). Diesen Vorschriften über die Marktfreiheiten stehen gemäß Artikel 30 EGV (exArtikel 36) nur Handelsbeschränkungen nicht entgegen, die u. a. „zum Schutze der Gesundheit und des Lebens von Menschen ... gerechtfertigt sind“. Entsprechend können die Mitgliedsstaaten auch nach dem Urteil des EuGH im Rechtsstreit Kohll (Nr. 51) den freien Dienstleistungsverkehr im Bereich der ärztlichen und klinischen Versorgung einschränken, „soweit die Erhaltung eines bestimmten Umfangs der medizinischen und pflegerischen Versorgung oder eines bestimmten Niveaus der Heilkunde im Inland" dies erfordert. Zudem konzediert der EuGH (Urteil Kohll, Nr. 41; Urteil Decker, Nr. 39), daß „eine erhebliche Gefährdung des finanziellen Gleichgewichts des Systems der sozialen Sicherheit" eine Beschränkung des freien Warenverkehrs mit Gesundheitsleistungen rechtfertigen kann. „Rein wirtschaftliche Gründe“ reichen jedoch als Legitimation für „eine Beschränkung des elementaren Grundsatzes des freien Dienstleistungsverkehrs" nicht aus (Urteil Kohll, Nr. 41).

Da die Bedingungen für die Ausübung ärztlicher Tätigkeiten ebenso wie die Produktion medizinischer Güter auf EG-Ebene zahlreichen Harmoni- 
sierungsrichtlinien unterliegen, besteht kein Grund zu der Annahme, daß Gesundheitsleistungen in anderen Teilnehmerländern a priori einen niedrigeren als den inländischen Qualitätsstandard aufweisen. Eine solche Einstellung würde auch, wie mehrere Teilnehmer betonten, von einer gewissen nationalen Hybris zeugen. Der EuGH gelangt hier ebenfalls zu dem Schluß, daß ,in anderen Mitgliedsstaaten niedergelassene Ärzte und Zahnärzte für die Zwecke des freien Dienstleistungsverkehrs als ebenso qualifiziert anerkannt werden müssen wie im Inland niedergelassene" (Urteil Decker, Nr. 48). Eine finanzielle Gefährdung der betreffenden nationalen Gesundheitssysteme liegt in der Regel auch nicht vor, wenn die Krankenkassen im Ausland erworbene Gesundheitsgüter oder dort vorgenommene Behandlungen höchstens nach den im Inland geltenden Tarifen erstatten. Insofern bleibt schwer einsichtig, warum sich ein Versicherter nicht gezielt ins Ausland begeben kann, um dort Gesundheitsgüter preiswerter zu erwerben oder um eine in seinen Augen bessere Behandlung zu erhalten. Die europarechtlich garantierte Freizügigkeit, länderübergreifend Gesundheitsleistungen auswählen zu können, kommt schließlich den sozialversicherten Patienten in mehrfacher Hinsicht zugute. Ein Patient in Nordrhein-Westfalen dürfte z. B. eine Behandlung in Nijmwegen statt in Münster vorziehen, wenn das ebenso leistungsfähige holländische Krankenhaus gut $100 \mathrm{~km}$ näher zum Wohnort liegt (Schmöhe F., 1997). Darüber hinaus können solche Optionen der Versicherten einen EG-weiten Preis- und Qualitätswettbewerb der Leistungserbringer zum Wohle der Patienten und zum Vorteil ihrer Krankenkasse bzw. -versicherung auslösen. Letzteres setzt allerdings voraus, daß die Krankenkassen bzw. -versicherungen höchstens die inländischen Preise und Tarife und, sofern diese niedriger liegen, die faktischen ausländischen Entgelte zahlen.

Unabhängig von den zuweilen übertriebenen Bedenken schafft eine weitgehende Liberalisierung der Gesundheitsmärkte für das stark korporatistisch geprägte deutsche Gesundheitswesen $u$. a. folgende Anpassungsprobleme (vgl. Bundesministerium für Gesundheit 1998 sowie den Beitrag von Dr. Zipperer in diesem Band):

- Das Sachleistungsprinzip muß zumindest als Option durch eine teilweise verwaltungsaufwendigere Kostenerstattung ergänzt werden.

- Bedarfsplanung und Zulassungsbeschränkungen für Vertragsärzte in Deutschland könnten ohne die anvisierten Wirkungen bleiben.

- Budgetierungen und vor allem Mengenbegrenzungen lassen sich kaum noch bzw. nur mit einem höheren Planungsaufwand durchführen. Die Kassenärztlichen Vereinigungen können das Volumen der veranlaßten Leistungen nicht mehr in toto steuern. 
- Ein Vergütungssystem mit floatenden Punktwerten eignet sich kaum für die Honorierung ausländischer Anbieter.

- Die duale Krankenhausfinanzierung führt zu einer Subventionierung ausländischer Patienten und zu einer Wettbewerbsverzerrung gegenüber monistisch finanzierten ausländischen Krankenhäusern.

- Eine Qualitätskontrolle durch deutsche Instanzen entfällt im Ausland, d. h. sie müßte europaweit erfolgen.

- Im europäischen Wettbewerb benötigen die deutschen Leistungserbringer ähnliche Instrumente wie ihre Konkurrenten, u. a. auf dem Gebiet der Werbung.

Eine weitgehende Liberalisierung des Warenverkehrs auf den Gesundheitsmärkten zieht nicht zwingend die Abschaffung des territorialen Sachleistungsprinzips nach sich. Es reicht in diesem Zusammenhang aus, wenn Versicherte, die Gesundheitsleistungen im Ausland in Anspruch nehmen möchten, für das Kostenerstattungsprinzip votieren können. Überdies würde eine gesetzliche Öffnung in Richtung einer grenzüberschreitenden, EG-weiten Vertragserlaubnis für die inländischen Krankenkassen die Voraussetzungen schaffen, um ausländische Leistungsanbieter - vor allem in grenznahen Gebieten - in Kollektivverträge einzubinden (Neumann-Duesberg R., 1998, S. 25). Insofern bestünde durchaus die Möglichkeit, deutschen Patienten Gesundheitsleistungen auch im Ausland als Sachleistungen zu gewähren. Herr Danner wies darauf hin, daß in Holland schon entsprechende Konzepte für grenzüberschreitende Verträge mit ausländischen Leistungsanbietern existieren. Schließlich stellt sich in diesem Kontext die Frage, ob die verbleibende Inanspruchnahme ausländischer Leistungsanbieter, die dann nach dem Kostenerstattungsprinzip erfolgt, im Bereich der ambulanten und stationären Behandlung quantitativ überhaupt ins Gewicht fällt.

Es liegen bisher meines Wissens noch kaum empirisch fundierte Analysen darüber vor, ob und inwieweit die Versicherten bzw. Patienten von der Möglichkeit, Gesundheitsgüter im Ausland zu erwerben und ausländische Leistungserbringer aufzusuchen, Gebrauch machen werden. Aus theoretischer Perspektive bieten sich jedoch einige Kriterien bzw. Determinanten an, die zumindest Tendenzaussagen über mögliche Nachfrageverschiebungen innerhalb der einzelnen Behandlungsarten erlauben. Zentrale Einflußgrößen möglicher Nachfrageverschiebungen bilden zunächst die Preisdifferenzen der Gesundheitsgüter in Verbindung mit den entsprechenden Qualitätsunterschieden. Dabei spielen die Preisdifferenzen nur dann eine entscheidende Rolle, wenn sie zumindest teilweise auch dem Versicherten zugute kommen. Letzterer dürfte kaum gezielt im Ausland Gesundheitsgüter gleicher Qualität nachfragen, wenn 
dadurch lediglich seine Krankenkasse Ausgaben spart. In dieser Hinsicht enweisen sich prozentuale Selbstbeteiligungen gegenüber fixen Selbstbehalten als anreizkompatibler.

Eine Determinante der Wettbewerbsintensität stellt auch die Homogenität der Leistungen dar. Bei weitgehend homogenen Gesundheitsleistungen, wie z. B. Arzneimitteln und Medizinprodukten, herrscht in der Regel ein wesentlich intensiverer Preiswettbewerb als bei stärker inhomogenen Leistungen, wie z. B. der ambulanten und stationären Behandlung. Da die Bürger in entwickelten Volkswirtschaften dem „Gut Gesundheit“ eine hohe Wertschätzung entgegenbringen, dürften Preisdifferenzen bei vermuteten Qualitätsunterschieden zuungunsten der preiswerteren Leistungen noch keine nennenswerten Nachfrageverschiebungen auslösen. Bei der ärztlichen Behandlung handelt es sich z. B. um ein vergleichsweise inhomogenes Gut, denn bei der Arzt-Patient-Beziehung kommt es nicht zuletzt auf die persönliche Bindung und das Vertrauensverhältnis an. Was sollte einen Patienten, der sich seit vielen Jahren von seinem Arzt gut behandelt und betreut fühlt, dazu bewegen, zu einem Anbieter jenseits der Grenze zu wechseln?

Die Nachfrageverschiebungen, die bei freiem Waren- und Dienstleistungsverkehr auf den europäischen Gesundheitsmärkten eintreten, finden auch in der eingeschränkten Mobilität der Versicherten eine Grenze. Neben Sprachbarrieren dürften zumeist schon Zeit- und Wegekosten sowie Abschläge bei der verwaltungsaufwendigeren Kostenerstattung ausreichen, um selbst preisgünstigere Angebote im Ausland zu kompensieren. Bei der stationären Behandlung und der Rehabilitation tritt dann noch die Erwartung bzw. Befürchtung hinzu, in deutlich geringerem Umfang Besuche von Verwandten und Freunden zu erhalten. Letztlich wird sich ein Patient wohl nur dann gezielt zu einer medizinischen Behandlung ins Ausland begeben, wenn er sich davon eine erhebliche Ersparnis verspricht oder die dortige Leistungsqualität für eindeutig besser hält. Die Nachfrageverschiebungen hängen somit auch von der Reputation der nationalen Gesundheitssysteme bzw. der jeweiligen Leistungsanbieter ab. Da das deutsche Gesundheitssystem international einen recht guten Ruf genießt, steht aus dieser Perspektive nicht zu erwarten, daß seine Versicherten scharenweise ins Ausland wandern, um sich dort medizinisch behandeln zu lassen.

Im Detail bestimmen auch die spezifischen Eigenheiten der jeweiligen Gesundheitsleistung ihre Wettbewerbsintensität auf europäischer Ebene. Die Wettbewerbsintensität stößt an Grenzen, wenn die Leistungen ständig wiederkehren, einer intensiven Nachsorge vor Ort bedürfen oder eine weitreichende Qualitätsgarantie enthalten. In diesen Fällen kom- 
men bestenfalls grenznahe ausländische Leistungserbringer als ernsthafte Konkurrenten in Frage. Neben der bereits erwähnten Bedeutung von Umfang und Art der Selbstbeteiligung gewinnt im Bereich der Pflege auch das Verhältnis von Sach- und Geldleistungen an Relevanz.

Die Liberalisierung der europäischen Gesundheitsmärkte birgt aber nicht nur Planungsprobleme für die Krankenkassen und Gefahren für die heimischen Leistungsanbieter, sondern eröffnet auch vielfältige Chancen. So können z. B. die deutschen Krankenhäuser in nennenswertem Umfang ausländische Patienten attrahieren. Dies gilt insbesondere für hochspezialisierte Einrichtungen. Schon heute suchen z. B. zahlreiche italienische Patienten französische Krankenhäuser auf, weil sie diese für leistungsfähiger als die einheimischen halten. Die deutschen Leistungseinheiten im ambulanten, stationären sowie Kur- und Rehabilitationsbereich könnten sich dem europäischen Wettbewerb mit Erfolg stellen und per saldo Nachfragezuwächse verbuchen. Die Attrahierung ausländischer Patienten dürfte überwiegend im Rahmen eines verschärften Wettbewerbs mit den Leistungserbringern dieser Länder erfolgen. Die Patientenwanderungen können aber auch mit den Interessen der Herkunftsländer harmonieren, wenn diese in den betreffenden Leistungsbereichen temporäre Engpässe aufweisen. Die liberalisierte Gesundheitsversorgung trägt dann auf EG-Ebene dazu bei, allfällige Überund Unterversorgungen teilweise auszugleichen.

Die Gesundheitsausgaben nehmen schon heute, auch ohne Transferzahlungen bzw. Krankheitsfolgeleistungen, in entwickelten Volkswirtschaften einen Anteil von über $8 \%$ am Bruttoinlandsprodukt ein. Die meisten Prognosen gehen davon aus, daß dieser Anteil in den nächsten 30 bis 40 Jahren vor allem infolge des demographischen Wandels und des medizinisch-technischen Fortschritts eher noch zunimmt. Vor diesem Hintergrund kann unbeschadet der jeweiligen rechtlichen Auslegung des EGV der freie europaweite Waren- und Dienstleistungsverkehr aus ökonomischer Sicht nicht auf Dauer vor der Wachstumsbranche Gesundheitswesen haltmachen (so auch Danner J., 1998, S. 38). Die deutschen politischen Entscheidungsträger gehörten auf europäischer Ebene fast immer zu den engagiertesten Verfechtern eines freien Waren- und Dienstleistungsverkehrs, so daß es andernorts überraschen muß, wenn sie sich ähnlichen Bestrebungen auf den Gesundheitsmärkten widersetzen würden. Wenngleich einige Anpassungsprobleme existieren, braucht ein effizientes und effektives Gesundheitssystem den internationalen Wettbewerb auf den jeweiligen Märkten im Prinzip nicht zu fürchten. 


\section{Veränderte Versorgungsstrukturen als innovative Erprobungsmodelle}

Die bereits bestehenden Modellvorhaben und Strukturverträge und auch noch nicht realisierte Vorschläge zu veränderten Versorgungsstrukturen zielen im wesentlichen darauf ab, Managed-Care-Elemente in die GKV zu integrieren, um die Effizienz und Effektivität der deutschen Gesundheitsversorgung zu erhöhen. Dabei vermuten nicht nur externe Beobachter, sondern auch unmittelbar beteiligte Leistungsanbieter vor allem in einer besseren Kooperation und Koordination zwischen den einzelnen Versorgungsbereichen noch beachtliche Wirtschaftlichkeitsreserven. Ein sektorübergreifendes Schnittstellenmanagement, das den ambulanten, stationären, Pflege- und Rehabilitationsbereich outcome-orientiert verknüpft, könnte u. a. dazu beitragen, medizinisch-technische Großgeräte besser zu nutzen, Doppeluntersuchungen, Informationsverluste, unnötige Krankenhauseinweisungen und Patientenselektionen abzubauen und das Postulat „ambulant vor stationär“ effektiver umzusetzen.

Obgleich über den Befund, daß die medizinische und ökonomische Abschottung des ambulanten und des stationären Sektors vielfältige Ineffizienzen erzeugt, weithin Einigkeit herrscht, divergieren die Lösungsvorschläge der Beteiligten beträchtlich. Dabei fällt auf, daß im Prinzip beide Seiten, d. $h$. die ambulanten Ärzte und die Krankenhäuser, nach einer Ausdehnung ihres Behandlungs- und Budgetvolumens streben, d. h. die Leistungen an den Schnittstellen für sich reklamieren und/oder in bisher für sie unzugängliche Versorgungsbereiche vordringen möchten. Die zentrale Ursache für diesen Expansionsdrang bilden auf beiden Seiten Überkapazitäten, die man durch Substitutionen, die den eigenen Leistungsumfang erweitern, wenigstens teilweise auslasten kann. Die ambulanten Ärzte bzw. ihre Kassenärztlichen Vereinigungen betonen in diesem Kontext wohl zu Recht, daß zahlreiche Behandlungen, die heute in Akutkrankenhäusern erfolgen, ambulant kostengünstiger und patientengerechter vorgenommen werden können. Im Falle einer solchen Substitution enwarten sie verständlicherweise eine entsprechende Umschichtung der finanziellen Mittel zu ihren Gunsten.

Diesen Argumenten halten die Krankenhäuser bzw. hält die Krankenhausgesellschaft entgegen, daß auch sie Behandlungsleistungen ambulant bzw. im Zuge einer Kurzzeithospitalisation erbringen können und z. B. im Hinblick auf ambulante Operationen über die größere Stand-bySicherheit verfügen. Die Krankenhäuser streben, wie Frau Renzewitz in inrem Beitrag ausführt, eine Erweiterung in Richtung eines integrierten medizinischen Dienstleistungszentrums an, das schon in der ersten Phase einen indikationsbezogenen Zulassungsanspruch zur Erbringung 
institutioneller ambulanter Leistungen auch ohne Überweisung besitzt. Die zweite Phase sieht dann einen institutionellen Zulassungsanspruch zur fachärztlichen Versorgung vor. Die Krankenhäuser treten nach diesen Vorstellungen auf dem Gebiet der fachärztlichen Versorgung mit den ambulanten (Fach-)Ärzten in einen Preis- und Qualitätswettbewerb. Um Wettbewerbsverzerrungen zu vermeiden, setzt dieses Konzept allerdings, worauf auch Herr Dr. Schwoerer verweist, eine monistische Finanzierung der Krankenhäuser und eine aufwandsgerechte Kalkulation inrer ambulanten Leistungen voraus. Die duale Krankenhausfinanzierung würde, ebenso wie eine Mischkalkulation von ambulanten und stationären Leistungen, die ambulanten Fachärzte im Wettbewerb diskriminieren.

In einem ähnlichen Argumentationsschema bewegen sich in medizinischer und gesundheitsökonomischer Hinsicht die Meinungsverschiedenheiten zwischen Haus- und Fachärzten über die Vor- und Nachteile von Hausarztsystemen bzw. -modellen. Ihre Befürworter heben die in vielen Fällen bei gleichem Patienten-outcome kostengünstigere Behandlung hervor. Letztere resultiert zum einen aus Informationsvorsprüngen der Gatekeeper gegenüber den seltener kontaktierten Fachärzten und zum anderen aus der bescheideneren technischen Ausstattung, die weniger Anreize in Richtung auslastungsinduzierte Untersuchungen setzt. Andererseits besteht die Gefahr, daß der Hausarzt seine diagnostischen und therapeutischen Möglichkeiten überschätzt und den Patienten zu spät an eine spezialisierte Behandlungseinheit abgibt. In solchen Fällen stehen den anfänglichen Einsparungen erhebliche Folgeaufwendungen durch eine verzögerte oder falsche Behandlung gegenüber. Induktiv betrachtet gibt es für die Vor- und Nachteile von Hausarztsystemen eine Fülle von Einzelbeispielen, die alle zutreffen mögen, aber weder epidemiologisch noch gesundheitsökonomisch weiterhelfen. Ob per Saldo in medizinischer und ökonomischer Hinsicht die Vor- oder Nachteile überwiegen, kann nur eine repräsentative Evaluation auf empirischer Grundlage beantworten. Die Modellvorhaben und Strukturverträge bieten hierzu im Prinzip geeignete Ansatzpunkte.

Obgleich die Modellvorhaben und Strukturverträge aussichtsreiche Erprobungen alternativer Versorgungs- und Vergütungsstrukturen erlauben, bleiben aufgrund mangelnder Flexibilitäten noch immer mögliche Kooperationen und damit Innovationschancen ungenutzt. So behindern die gesetzlichen Bestimmungen eine intensivere Kooperation zwischen den Krankenkassen sowie zwischen diesen und den privaten Krankenversicherungen. Es gibt kaum überzeugende Gründe dafür, daß Krankenkassen unterschiedlicher Kassenarten nicht im Rahmen der Strukturverträge gemeinsam kontrahieren oder Krankenkassen Leistungen, 
die sich nicht im Katalog der GKV befinden, nicht zumindest in Kooperation mit einer privaten Krankenversicherung anbieten können.

An den neuralgischen Schnittstellen zwischen ambulanter und stationärer Behandlung liegt die Initiative im Rahmen der Erprobungsmodelle weitgehend bei den Kassenärztlichen Vereinigungen. Die Strukturverträge klammern die Mitwirkung der Krankenhäuser ebenso wie die der Arzneimittelhersteller sogar völlig aus. Die Durchführung von Modellvorhaben steht zwar auch den Krankenhäusern offen, bezieht sich dabei aber primär auf den stationären Sektor. Insoweit die Krankenhäuser im Rahmen dreiseitiger Verträge Modellvorhaben durchführen möchten, die auch den vertragsärztlichen Bereich tangieren, benötigen sie die Zustimmung der Kassenärztlichen Vereinigungen. Insofern überrascht es nicht, daß sich die Modellvorhaben im vertragsärztlichen Bereich einer Hochkonjunktur erfreuen, während diesbezüglich im Krankenhaussektor eine Flaute herrscht. Eine intensivere Beteiligung der Krankenhäuser an den Erprobungsmodellen zielt auf eine noch bessere Verzahnung von ambulanter und stationärer Behandlung ab. Im Idealfall stehen dann Verbünde aus Haus- und Fachärzten sowie Krankenhäusern miteinander in Leistungs- und Qualitätskonkurrenz.

Ein aussichtsreiches Feld für innovative Erprobungsmodelle bietet vor allem das Disease Management, das sich um neue outcome-erhöhende Versorgungsformen für chronisch oder Schwerstkranke bemüht. Eine Krankenkasse muß hier im Falle eines offenkundigen medizinischen Erfolgs allerdings befürchten, besonders viele Kranke zu attrahieren, so daß ihr Beitragssatz steigt, denn der Risikostrukturausgleich berücksichtigt nicht deren spezifische Morbidität. Unbeschadet ihrer Existenz schwächt sich diese Problematik gegenüber den amerikanischen Verhältnissen insofern etwas $a b$, als es sich bei den bundesdeutschen Krankenkassen nicht um gewinnorientierte Unternehmen, sondern um Körperschaften des öffentlichen Rechts handelt. Zudem könnte eine Krankenkasse mit solchen prima vista „unrentablen“ Projekten eine medizinische Kompetenz und ein gesundheitsökonomisches Prestige erwerben, so daß sie möglicherweise trotz eines etwas höheren Beitragssatzes weiterhin kompetitiv bleibt. Gleichwohl bedarf es bei Modellvorhaben und Strukturverträgen, die chronisch und Schwerstkranke betreffen, nach Möglichkeit eines kooperativen Vorgehens mehrerer betroffener Krankenkassen. Dies schließt allerdings unterschiedliche dezentrale innovative Suchprozesse nicht aus, denn Modellvorhaben und Strukturverträge dürften sich in der Regel auf räumlich abgegrenzte Regionen beziehen. Es stehen dann keine Krankenkassen miteinander in Konkurrenz, sondern dezentrale regionale Versorgungskonzepte. Dieser Aspekt spricht ebenfalls für ein breites Spektrum von Vertragsmöglichkeiten 
zwischen Krankenkassen sowie zwischen diesen und den Leistungserbringern.

\section{Finanzierungs- und Allokationsprobleme in der GKV}

Die Diskussionen um das Globalbudget, das im Mittelpunkt des Themenkreises 3 stand, verdeutlichten die engen Beziehungen, die zwischen Finanzierungsregelungen und der Mittelallokation in der GKV existieren. Das Globalbudget gewann insofern stark an Aktualität, als die neue Bundesregierung nach den sektoralen Budgets des Jahres 1999 den Übergang auf ein solches Instrument ankündigte. Zwischenzeitlich löste das Globalbudget - obwohl inhaltlich noch kaum präzisiert, geschweige denn final ausgelotet - bereits begrifflich bei manchen Vertretern von Krankenkassen Euphorien und bei vielen Leistungsanbietern Phobien aus. Insofern erscheint gerade auf diesem Gebiet eine vorurteilsfreie, sachbezogene Analyse besonders wichtig.

Zunächst bildeten in den neunziger Jahren, was auch Herr Schulte in seinem Beitrag betont, im Bereich der GKV Budgetierungen, wenn auch in den unterschiedlichsten Formen und terminologischen Gewändern, eher die Regel als die Ausnahme. Ein Globalbudget legt, für sich betrachtet, lediglich das Ausgabenvolumen fest, das der GKV in einem bestimmten Zeitraum zur Finanzierung der Gesundheitsversorgung zur Verfügung steht. Da es sich bei diesen Ausgaben einnahmenseitig um solidarisch aufgebrachte Zwangsbeiträge handelt, erscheint eine solche Festlegung nicht nur politisch legitim, sondern auch, ähnlich wie beim Staatshaushalt, ökonomisch naheliegend. Diese Feststellung läßt allerdings offen, ob es nicht im Rahmen von erweiterten Modellen des Krankenkassenwettbewerbs allokativ überlegene Alternativen gibt.

Die Leistungsanbieter fürchten im Rahmen einer Globalbudgetierung auch weniger den Mechanismus als solchen, denn ein stark steigendes Globalbudget würde hier kaum auf Widerstand stoßen, sondern eine zu starke Einengung des Ausgabenspielraums und damit, neben Einkommenseinbußen, zunehmende Rationierungen in der Gesundheitsversorgung. Es geht insofern in diesem Kontext primär um die konkrete Wachstumsrate des Globalbudgets, d. h. um die Wahl einer geeigneten Bezugsgröße. Eine Orientierung des Globalbudgets an der Steigerungsrate der Grundlohnsumme sichert zwar die Stabilität der Beitragssätze, dürfte aber vom Ausgabenspielraum her selbst bei weitgehender Ausschöpfung des Rationalisierungspotentials kaum ausreichen, um das Niveau der Gesundheitsversorgung von seinem heutigen Stand aus entsprechend dem medizinisch-technischen Fortschritt und dem demographischen Wandel weiterzuentwickeln. Nimmt das Globalbudget mit der 
Rate des Bruttoinlandsprodukts zu, so führt dies sehr wahrscheinlich zu steigenden Beitragssätzen, denn es steht noch auf absehbare Zeit zu befürchten, daß die Lohnsumme als dominante Bemessungsgrundlage der GKV-Einnahmen schwächer als das Bruttoinlandsprodukt wächst. Für eine konzeptionell adäquate Festlegung des Globalbudgets bietet sich, worauf auch Herr Knieps verweist, ein aufeinander abgestimmtes System von epidemiologischen und gesundheitsökonomischen Indikatoren an. Für diesen „Königsweg der Globalbudgetierung“ fehlt es allerdings derzeit an theoretisch überzeugenden Konzepten, an gesichertem instrumentalem Wissen und der notwendigen Datenbasis.

Das Globalbudget entfaltet, wie auch seine Befürworter konzedieren, keine sektorale Steuerungswirkung, d. h. unterhalb des Plafonds bleibt die Aufteilung der Mittel auf die einzelnen Versorgungsbereiche noch völlig offen. Einigkeit herrschte im Teilnehmerkreis darüber, daß die einzelnen Versorgungsbereiche bzw. Behandlungsarten in keinem Falle pari passu mit dem Globalbudget ansteigen sollten. Eine solche Parallelentwicklung liefe auf eine Zementierung der derzeitigen Strukturen und damit auf eine Fortschreibung der bestehenden Fehlallokationen hinaus. Unklarheiten bestanden aber dahingehend, ob ein Globalbudget nicht doch sektorale Budgets, die allerdings unterschiedliche Wachstumsraten aufweisen, voraussetzt und welcher Allokationsmechanismus die Mittelverteilung an den Schnittstellen steuert. Sofern die Gesundheitsversorgung in diesem Kontext künttig im Bereich der GKV mit stärkeren Einschränkungen einhergeht, erwarten vor allem die Ärzte von der Politik die Übernahme der Rationierungsverantwortung. Dabei geht es nicht um ethisch fragwürdige Rationierungen, sondern um die Ablehnung von nicht essentiellen, aber durchaus verständlichen Patientenwünschen. Die Ärzte befürchten, daß innen vor allem bei einer restriktiven Budgetierung letztlich diese unpopulären Rationierungsentscheidungen zufallen.

Die Diskussionen über die Beitragsbemessungs- und Versicherungspflichtgrenze mündeten in die Frage nach den Grenzen der solidarischen Finanzierung in der GKV ein. Die derzeitigen historisch gewachsenen Grenzen vermögen konzeptionell nicht zu überzeugen und erscheinen z. B. unter Solidaritätsaspekten als zu niedrig, im Sinne des Subsidiaritätsprinzips aber als zu hoch. Eine Anhebung von Versicherungspflicht- und Beitragsbemessungsgrenze auf das Niveau der gesetzlichen Rentenversicherung führt in der GKV u. a. zu einer Ausweitung des (Pflicht-) Versichertenkreises, der die solidarische Finanzierung auf eine breitere Grundlage stellt. Das derzeitige System nimmt nur Bürger mit einem Arbeitsentgelt bis zu dieser Grenze in die Solidaritätspflicht, was distributiven Normen eher zuwiderläuft. Gleichwohl blieben die Meinungen über die Vor- und Nachteile dieser Finanzierungsoption 
auch unter den Vertretern der Krankenkassen geteilt. Vertreter von Krankenkassen mit einem hohen Anteil an freiwillig Versicherten befürchten infolge der gestiegenen Beiträge ein Abwandern jener Mitglieder in die private Krankenversicherung, die auch weiterhin mit einem Arbeitsentgelt oberhalb der angehobenen Pflichtversicherungsgrenze diese Option besitzen. Der Verband der Ersatzkassen plädiert wohl auch unter diesem Aspekt nur für eine entsprechende Anhebung der Pflichtversicherungs- bei gegebener Beitragsbemessungsgrenze. Einige Teilnehmer sahen vor allem bei Versicherten, die über Jahre keine Gesundheitsleistungen in Anspruch nehmen, mit den derzeitigen Höchstbeiträgen die Grenzen einer zumutbaren Solidarität erreicht.

Eine andere Finanzierungsalternative besteht gerade im Hinblick auf eine europäische Harmonisierung in einem stärkeren Rückgriff auf Steuermittel. Wie bereits angedeutet, finanzieren die europäischen Staaten ihre Sozialsysteme in höchst unterschiedlichem Maße mit Hilfe von Steuern und Sozialabgaben. So beträgt der steuerfinanzierte Anteil der öffentlichen Gesundheitsversorgung in Großbritannien und Schweden über $75 \%$, während z. B. in Deutschland, Frankreich und den Niederlanden die Beitragsfinanzierung dominiert. Entsprechend weisen, wie Tab. 2 im Überblick zeigt, Deutschland (15,5\%), Frankreich $(19,7 \%)$ und die Niederlande $(17,1 \%)$ im Vergleich zum europäischen Durchschnitt (12,2\%) deutlich höhere Anteile der Sozialabgaben im Verhältnis zum Bruttoinlandsprodukt auf. Die entsprechenden Quoten liegen im OECD-Durchschnitt sogar nur bei $9,8 \%$ und in Großbritannien bei $6,2 \%$.

Beitragsfinanzierte Systeme besitzen gegenüber steuerfinanzierten bei isolierter Betrachtung Nachteile im Hinblick auf die Lohnnebenkosten und damit auf die unmittelbare Abgabenbelastung des Faktors Arbeit. Die beitragsfinanzierten Systeme geraten daher im internationalen Standortwettbewerb unter Druck, und es könnte aus dieser Perspektive naheliegen, die Beitrags- teilweise durch eine Steuerfinanzierung zu ersetzen. Sofern die Steuerfinanzierung allerdings über einen Ersatz versicherungsfremder Leistungen hinausgeht, verlieren die betreffenden Leistungen ihren Versicherungscharakter und entfernen sich immer mehr von der individuellen bzw. gruppenmäßigen Äquivalenz, die auch in sozialen Sicherungssystemen zwischen den Beiträgen der Versicherten und den innen zufließenden Leistungen besteht.

Mit dem Übergang von beitrags- zu steuerfinanzierten Sozialversicherungssystemen verlieren die Bürger ihre Versicherungsrechte bzw. -ansprüche, denn das Wesen einer allgemeinen Steuer liegt darin, daß kein Steuerpflichtiger mit seiner Abgabe einen Anspruch auf irgendeine 
staatliche Gegenleistung erwirbt. Bei der beitragsfinanzierten Sozialleistung handelt es sich nicht, wie z. B. bei der Sozialhilfe, um eine staatliche Transferleistung, der kein Äquivalent des Empfängers gegenübersteht, sondern um eine mit spezifischen Abgaben bezahlte Versicherungsleistung. Das Bundesverfassungsgericht sprach z. B. den Rentenversicherungsansprüchen einen eigentumsähnlichen Charakter zu. Der Übergang von der beitrags- zur steuerfinanzierten Gesundheitsversorgung mindert insofern die Gestaltungs- und Souveränitätsrechte der Bürger, die dann nicht mehr als Versicherte, sondern nur noch als staatliche Leistungsempfänger fungieren. Die im Rahmen der Standortdebatte zuweilen recht einseitig geführte Diskussion um eine Reform der Sozialsysteme scheint diese Aspekte zu vernachlässigen. Auch aus diesem Grund erscheint es notwendig, die deutsche Gesundheitsversorgung unter Wettbewerbsaspekten „europafest“ zu machen, um sie dann auf EG-Ebene als Konzept offensiv zu vertreten. 
Tab. 2: Steuern und Sozialabgaben im Verhältnis zum Bruttoinlandsprodukt im internationalen Vergleich

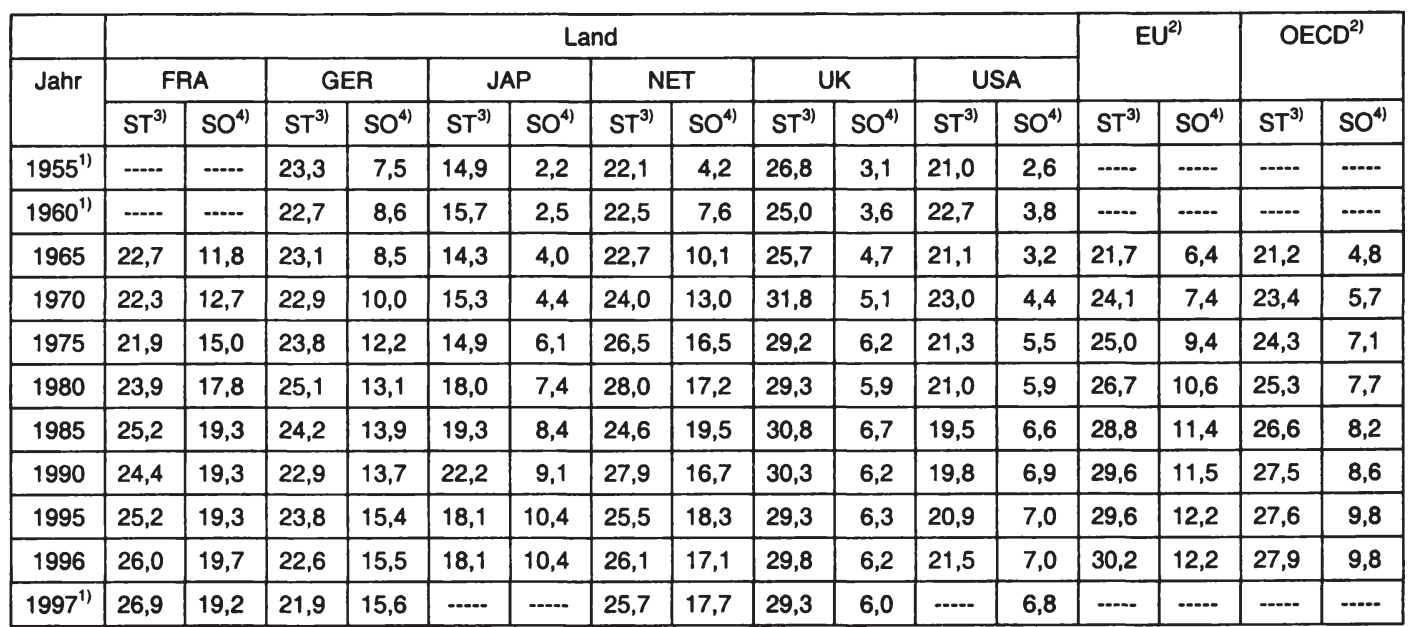

Quelle: Zusammengestellt und errechnet aus OECD (1998).

1) Schätzungen

2) Ungewichtetes Mittel

3) Steuereinnahmen in Relation zum Bruttoinlandsprodukt (in Prozent)

4) Sozialabgaben in Relation zum Bruttoinlandsprodukt (in Prozent) 


\section{Literatur:}

Arnold, Michael, Lauterbach, Karl W. und Preuß, Klaus-Jürgen, Hrsg. (1997), Managed Care. Ursachen, Prinzipien, Formen und Effekte, Stuttgart und New York

Baumann, Manfred und Stock, Johannes (1996), Managed-Care-Impulse für die GKV? Erfahrungswerte mit alternativen Formen der Steuerung in der Gesundheitsversorgung, Düsseldorf

Bundesministerium für Gesundheit (1998), Zur Anwendung des Rechts der Europäischen Gemeinschaft im Hinblick auf die Urteile des Europäischen Gerichtshofes vom 28.04.1998, in: Pressemitteilung Nr. 41 vom 05.06.1998

Busch, Susanne, Pfaff, Anita B. und Rindsfüßer, Christian (1996), Die Finanzierung der gesetzlichen Krankenversicherung. Möglichkeiten zur Umgestaltung und Ergebnisse ausgewählter Modellrechnungen, Düsseldorf

Danner, Günter (1998), Der EuGH: Ein Schreckgespenst? In: Gesundheit und Gesellschaft, 10/1998, S. 36 - 39

Hanika, Heinrich (1998), Europäische Gesundheitspolitik. Stärkung der Kompetenzen der Europäischen Union im Gesundheitswesen durch die Neufassung des Artikels 152 EG-Vertrag. In: Medizinrecht 1998, Heft 5, S. 193 - 196

Knappe, Eckhard, Hrsg. (1997), Reformstrategie „Managed Care“, Baden-Baden

Kommission der Europäischen Gemeinschaften, Entwurf, Mitteilung der Kommission an den Rat, das europäische Parlament, den Wirtschafts- und Sozialausschuß und den Ausschuß der Regionen über die Entwicklung der Gemeinschaftspolitik im Bereich der öffentlichen Gesundheit, Brüssel, den 14.04.1998

Merke, Klaus, Hrsg. (1998), Umbau oder Abbau im Gesundheitswesen? Finanzierung, Versorgungsstrukturen, Selbstverwaltung, Berlin

Münnich, Frank E. (1998), Wettbewerbsdruck durch Konvergenz der Gesundheitssysteme in der EU? In: Recht und Politik im Gesundheitswesen, Bd. 4, Heft 3, S. 81 85

Neumann-Duesberg, Rüdiger (1998), Die EuGH-Position ist angreifbar. In: Gesundheit und Gesellschaft, 10/1998, S. 22 - 27

OECD (1998a), OECD Health-Data 98, Paris

OECD (1998b), Revenue Statistics 1965 - 1997, Paris 
Pitschas, Rainer (1994), Die Weiterentwicklung der sozialen Krankenversicherung in Deutschland im Gegenlicht europäischer Gesundheitspolitik. In: Vierteljahresschrift für Sozialrecht, 2/1994, S. 85 - 118

Schmöhe, Franziska (1997), Nijmwegen statt Münster? In: Europa-Kontakt e.V. (Hrsg.), Informationsbrief Gesundheit 3/97, S. 55 - 58

Schulte, Bernd (1998), Europäische Gesundheitspolitik nach dem Vertrag von Amsterdam. In: Staatswissenschaften und Staatspraxis

Wille, Eberhard, und Albring, Manfred, Hrsg. (1998), Reformoptionen im Gesundheitswesen, Frankfurt et al.

Wismar, Matthias (1998), Europa regiert schon lange heimlich mit. In: Gesundheit und Gesellschaft, 10/1998, S. 28 - 35 
Albring, Dr. med. Manfred

Bluschke, Friedrich Wilhelm

Brech, Prof. Dr. med. Wolfgang

Cassel, Prof. Dr. rer. pol. Dieter

Danner, M. A. PhD Günter

Dewein, Peter

Diener, Dr. rer. pol. Frank

Dierks, Dr. med. Dr. iur. Christian

Ehlers, Dr. med. Dr. iur. Alexander Rechtsanwalt und Arzt, RechtsanwaltsP. F.

Esche, Dr. rer. nat. Joachim

Gitter, Prof. em. Dr. iur. Dr. h. c. Wolfgang

Granitza, Dr. iur. Axel

Großpietzsch, Dr. med. Rüdiger
Leiter der Abteilung Gesundheitswesen, Schering Deutschland GmbH, Berlin

Vorstandsvorsitzender der AOK Mecklenburg-Vorpommern, Schwerin

Vorsitzender der Kassenärztlichen Vereinigung Südwürttemberg, Reutlingen

Lehrstuhl für Wirtschaftspolitik an der Universität Duisburg

stellvertretender Direktor der Europavertretung der Deutschen Sozialversicherung, Brüssel

Geschäftsführer des Bereichs Wirtschafts-, Gesundheits- und Sozialpolitik beim Bundesverband der Pharmazeutischen Industrie, Frankfurt

Leiter der Abteilung Gesundheitsökonomie und Politik der ABDA, Eschborn

Rechtsanwalt und Arzt, Dierks \& Bohle, Rechtsanwälte, Berlin sozietät Ehlers, Ehlers \& Partner, München

Geschäftsführer der Schering Deutschland $\mathrm{GmbH}$, Berlin

Universität Bayreuth

Bereichsleiter Konzernstäbe, Schering Aktiengesellschaft, Berlin

Geschäftsführer des Medizinischen Dienstes der Krankenversicherung Mecklenburg-Vorpommern, Schwerin 
Klemm, Dr. med. Helmut

Klusen, Dr. oec. Norbert

Knieps, Franz

Koring, Hans-Dieter

Krimmel, Dr. med. Lothar

Laschet, Helmut

Lehr, Andreas

Munte, Dr. med. Axel

Naase, Birgit

Neubauer, Prof. Dr. Günter

Pfaff, Prof. Dr. phil. Martin

Pfeiffer, Dr. rer. pol. Doris stellvertretender Vorsitzender der Kassenärztlichen Vereinigung Bayern, Bezirksstelle Oberbayern, München; Vorsitzender der Vertreterversammlung der Kassenărztlichen Bundesvereinigung, Köln

Vorstandsvorsitzender der Techniker Krankenkasse, Hamburg

Leiter der Abteilung Verbandspolitische Planung, AOK Bundesverband, Bonn

stellvertretender Vorsitzender des Vorstandes der Techniker Krankenkasse, Hamburg

stellvertretender Hauptgeschäftsführer der Kassenärztlichen Bundesvereinigung, Köln

stellvertretender Chefredakteur der Ärzte Zeitung, Neu-Isenburg

Herausgeber Gesundheitspolitischer Informationsdienst - Broll \& Lehr, Bonn

Vorsitzender der Bezirksstelle München Stadt und Land der Kassenärztlichen Vereinigung Bayern, München

Gesundheitspolitische Referentin der FDP-Bundestagsfraktion, Bonn

Direktor des Instituts für Gesundheitsökonomik, München

Mitglied des Deutschen Bundestages, stellvertretender gesundheitspolitischer Sprecher der SPD-Bundestagsfraktion, Bonn

Abteilungsleiterin für Verbandspolitik, Verband der Angestellten-

Krankenkassen e. V., Siegburg 
Reiners, Hartmut

Reischl, Wilfried

Renzewitz, Susanne

Sauermann, Peter

Schlenker, Dr. iur. Rolf-Ulrich

Schmeinck, Wolfgang

Schönbach, Karl-Heinz

Schulte, Gerhard
Referatsleiter der Abteilung Gesundheit im Ministerium für Arbeit und Soziales, Gesundheit und Frauen, Land Brandenburg, Potsdam

Referent der Arbeitsgruppe Gesundheit der CDU/CSU, Bonn

Leiterin des Vorstandsbüros der Deutschen Krankenhausgesellschaft, Düsseldorf

Sanitätsrat, Vorsitzender der Kassenärztlichen Vereinigung Trier

Vorsitzender des Vorstandes des Landesverbandes der Betriebskrankenkassen Baden-Württemberg, Kornwestheim

Vorsitzender des Vorstandes des Bundesverbandes der Betriebskrankenkassen, Essen

Leiter der Hauptabteilung Verträge des Bundesverbandes der Betriebskrankenkassen, Essen

Vorstandsvorsitzender des Landesverbandes der Betriebskrankenkassen in Bayern, München

Schulte-Sasse, Dr. med. Hermann Leiter des Stabsbereichs Medizin des AOK Bundesverbandes, Bonn

Schwoerer, Dr. med. Peter Leitender Arzt des Medizinischen Dienstes der Krankenversicherung BadenWürttemberg, Lahr

Alfter

Stillfried, Dr. rer. pol. Dominik Graf von
Leiter der Abteilung Grundsatzfragen, Kassenärztliche Bundesvereinigung, Köln 
Straub, Dr. med. Christoph

Verhees, Hans Günter

Vorderwülbecke, Dr. iur. Ulrich

Weber, Mechthild

Wille, Prof. Dr. rer. pol. Eberhard

Winkler, Dr. phil. Ute

Zimmermann, Dr. med. Gerd
Leiter der Abteilung Grundsatzfragen der medizinischen Versorgung und Gesundheitswissenschaften, Verband der Angestellten-Krankenkassen e. V., Siegburg

stellvertretender Vorsitzender des Vorstandes der AOK Sachsen, Dresden

Geschäftsführer des Bereichs Marktordnung - Gesundheitssysteme, Verband der Forschenden Arzneimittelhersteller e. V. (VFA), Bonn

Leiterin Konzerngesundheitspolitik, Schering Aktiengesellschaft, Berlin Lehrstuhl für Volkswirtschaftslehre, Planung und Verwaltung, öffentliche Wirtschaft an der Universität Mannheim

Referentin des Arbeitskreises IV Gesundheit und Soziales der Bundestagsfraktion Bündnis 90/Die Grünen, Bonn

Vorsitzender der Bezirksstelle Frankfurt der Kassenärztlichen Vereinigung Hessen, Frankfurt 


\section{STAATLICHE ALLOKATIONSPOLITIK IM MARKTWIRTSCHAFTLICHEN SYSTEM}

Band 1 Horst Siebert (Hrsg.): Umweltallokation im Raum. 1982.

Band 2 Horst Siebert (Hrsg.): Global Environmental Resources. The Ozone Problem. 1982.

Band 3 Hans-Joachim Schulz: Steuerwirkungen in einem dynamischen Unternehmensmodell. Ein Beitrag zur Dynamisierung der Steuerüberwälzungsanalyse. 1981.

Band 4 Eberhard Wille (Hrsg.): Beiträge zur gesamtwirtschaftlichen Allokation. Allokationsprobleme im intermediären Bereich zwischen öffentlichem und privatem Wirtschaftssektor. 1983.

Band 5 Heinz König (Hrsg.): Ausbildung und Arbeitsmarkt. 1983.

Band 6 Horst Siebert (Hrsg.): Reaktionen auf Energiepreissteigerungen. 1982.

Band 7 Eberhard Wille (Hrsg.): Konzeptionelle Probleme öffentlicher Planung. 1983.

Band 8 Ingeborg Kiesewetter-Wrana: Exporterlösinstabilität. Kritische Analyse eines entwicklungspolitischen Problems. 1982.

Band 9 Ferdinand Dudenhöfer: Mehrheitswahl-Entscheidungen über Umweltnutzungen. Eine Untersuchung von Gleichgewichtszuständen in einem mikroökonomischen Markt- und Abstimmungsmodell. 1983.

Band 10 Horst Siebert (Hrsg.): Intertemporale Allokation. 1984.

Band 11 Helmut Meder: Die intertemporale Allokation erschöpfbarer Naturressourcen bei fehlenden Zukunftsmärkten und institutionalisierten Marktsubstituten. 1984.

Band 12 Ulrich Ring: Öffentliche Planungsziele und staatliche Budgets. Zur Erfüllung öffentlicher Aufgaben durch nicht-staatliche Entscheidungseinheiten. 1985.

Band 13 Ehrentraud Graw: Informationseffizienz von Terminkontraktmärkten für Währungen. Eine empirische Untersuchung. 1984.

Band 14 Rüdiger Pethig (Ed.): Public Goods and Public Allocation Policy. 1985.

Band 15 Eberhard Wille (Hrsg.): Öffentliche Planung auf Landesebene. Eine Analyse von Planungskonzepten in Deutschland, Österreich und der Schweiz. 1986.

Band 16 Helga Gebauer: Regionale Umweltnutzungen in der Zeit. Eine intertemporale Zwei-Regionen-Analyse. 1985.

Band 17 Christine Pfitzer: Integrierte Entwicklungsplanung als Allokationsinstrument auf Landesebene. Eine Analyse der öffentlichen Planung der Länder Hessen, Bayern und Niedersachsen. 1985.

Band 18 Heinz König (Hrsg.): Kontrolltheoretische Ansätze in makroökonometrischen Modellen. 1985.

Band 19 Theo Kempf: Theorie und Empirie betrieblicher Ausbildungsplatzangebote. 1985.

Band 20 Eberhard Wille (Hrsg.): Konkrete Probleme öffentlicher Planung. Grundlegende Aspekte der Zielbildung, Effizienz und Kontrolle. 1986.

Band 21 Eberhard Wille (Hrsg.): Informations- und Planungsprobleme in öffentlichen Aufgabenbereichen. Aspekte der Zielbildung und Outputmessung unter besonderer Berücksichtigung des Gesundheitswesens. 1986.

Band 22 Bernd Gutting: Der Einfluß der Besteuerung auf die Entwicklung der Wohnungs- und Baulandmärkte. Eine intertemporale Analyse der bundesdeutschen Steuergesetze. 1986.

Band 23 Heiner Kuhl: Umweltressourcen als Gegenstand internationaler Verhandlungen. Eine theoretische Transaktionskostenanalyse. 1987. 
Band 24 Hubert Hornbach: Besteuerung, Inflation und Kapitalallokation. Intersektorale und internationale Aspekte. 1987.

Band 25 Peter Müller: Intertemporale Wirkungen der Staatsverschuldung. 1987.

Band 26 Stefan Kronenberger: Die Investitionen im Rahmen der Staatsausgaben. 1988.

Band 27 Armin-Detlef Rieß: Optimale Auslandsverschuldung bei potentiellen Schuldendienstproblemen. 1988.

Band 28 Volker Ulrich: Preis- und Mengeneffekte im Gesundheitswesen. Eine Ausgabenanalyse von GKV-Behandlungsarten. 1988.

Band 29 Hans-Michael Geiger: Informational Efficiency in Speculative Markets. A Theoretical Investigation. Edited by Ehrentraud Graw. 1989.

Band 30 Karl Sputek: Zielgerichtete Ressourcenallokation. Ein Modellentwurf zur Effektivitätsanalyse praktischer Budgetplanung am Beispiel von Berlin (West). 1989.

\section{ALLOKATION IM MARKTWIRTSCHAFTLICHEN SYSTEM}

Band 31 Wolfgang Krader: Neuere Entwicklungen linearer latenter Kovarianzstrukturmodelle mit quantitativen und qualitativen Indikatorvariablen. Theorie und Anwendung auf ein mikroempirisches Modell des Preis-, Produktions- und Lageranpassungsverhaltens von deutschen und französischen Unternehmen des verarbeitenden Gewerbes. 1991.

Band 32 Manfred Erbsland: Die öffentlichen Personalausgaben. Eine empirische Analyse für die Bundesrepublik Deutschland. 1991.

Band 33 Walter Ried: Information und Nutzen der medizinischen Diagnostik. 1992.

Band 34 Anselm U. Römer: Was ist den Bürgern die Verminderung eines Risikos wert? Eine Anwendung des kontingenten Bewertungsansatzes auf das Giftmüllrisiko. 1993.

Band 35 Eberhard Wille, Angelika Mehnert, Jan Philipp Rohweder: Zum gesellschaftlichen Nutzen pharmazeutischer Innovationen. 1994.

Band 36 Peter Schmidt: Die Wahl des Rentenalters. Theoretische und empirische Analyse des Rentenzugangsverhaltens in West- und Ostdeutschland. 1995.

Band 37 Michael Ohmer: Die Grundlagen der Einkommensteuer. Gerechtigkeit und Effizienz. 1997.

Band 38 Evamaria Wagner: Risikomanagement rohstoffexportierender Entwicklungsländer. 1997.

Band 39 Matthias Meier: Das Sparverhalten der privaten Haushalte und der demographische Wandel: Makroökonomische Auswirkungen. Eine Simulation verschiedener Reformen der Rentenversicherung. 1997.

Band 40 Manfred Albring / Eberhard Wille (Hrsg.): Innovationen in der Arzneimitteltherapie. Definition, medizinische Umsetzung und Finanzierung. Bad Orber Gespräche über kontroverse Themen im Gesundheitswesen 25.-27.10.1996. 1997.

Band 41 Eberhard Wille / Manfred Albring (Hrsg.): Reformoptionen im Gesundheitswesen. Bad Orber Gespräche über kontroverse Themen im Gesundheitswesen 7.-8.11.1997. 1998.

Band 42 Manfred Albring / Eberhard Wille (Hrsg.): Szenarien im Gesundheitswesen. Bad Orber Gespräche über kontroverse Themen im Gesundheitswesen 5.-7.11.1998. 1999. 


\section{Medizin - Recht - Ethik}

Frankfurt/M., Berlin, Bern, New York, Paris, Wien, 1998. 159 S.

Rechtsphilosophische Hefte. Beiträge zur Rechtswissenschaft, Philosophie und Politik. Herausgegeben von Giuseppe Orsi, Kurt Seelmann, Stefan Smid und Ulrich Steinvorth. Bd. VIII

ISBN 3-631-33124-X · br. DM 53.-*

Die Medizin zieht heute aus zweierlei Gründen eine gesteigerte

Aufmerksamkeit des Rechts und der Ethik auf sich: Sie erschließt neue Bereiche des technisch Machbaren, von der Fortpflanzung bis zur Organtransplantation. Daraus resultieren bisher unbekannte Problemstellungen, die unsere moralischen Intuitionen und unser Judiz verunsichern. Zugleich droht die Medizin aber zu einem knappen Gut zu werden, dessen Verwaltung neuartige Fragen der Verteilungsgerechtigkeit aufwirft.

Aus dem Inhalt: Recht auf Gesundheit · Ethikkommissionen · Fortpflanzungsmedizin · Rationierung · Sterbehilfe · Organtransplantation

Frankfurt/M - Berlin - Bern - New York · Paris - Wien

Auslieferung: Verlag Peter Lang AG

Jupiterstr. 15, CH-3000 Bern 15

Telefax (004131) 9402131

*inklusive Mehrwertsteuer

Preisänderungen vorbehalten 
Manfred Albring and Eberhard Wille - 978-3-631-75604-1

Downloaded from PubFactory at 01/11/2019 03:10:54AM

via free access 